\title{
A new generation of high-power, waveform controlled, few-cycle light sources
}

Marcus Seidel

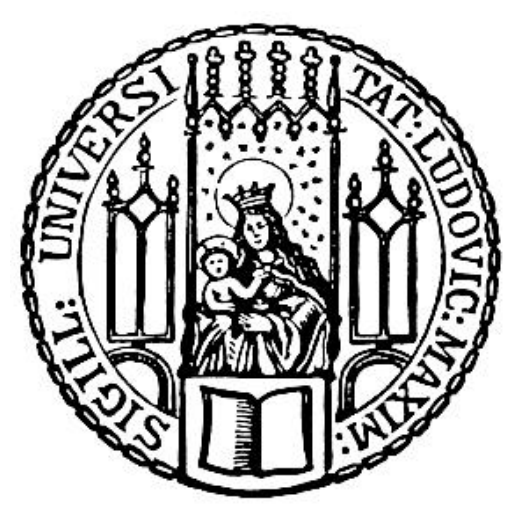

München 2018 



\title{
A new generation of high-power, waveform controlled, few-cycle light sources
}

Marcus Seidel

\author{
Dissertation \\ an der Fakultät für Physik \\ der Ludwig-Maximilians-Universität \\ München
}

vorgelegt von

Marcus Seidel

aus Marienberg

München, den 9. Februar 2018 
Erstgutachter: Prof. Dr. Ferenc Krausz

Zweitgutachter: Prof. Dr. Thomas Udem

Tag der mündlichen Prüfung: 9. April 2018 


\section{Zusammenfassung}

Mit der zunehmenden Verbreitung von in Spitzen- und Durchschnittsleistung skalierbaren Femtosekundenlasern, insbesondere von modengekoppelten Dünnscheibenoszillatoren, entstand auch die Nachfrage nach ebenso leistungsskalierbaren Methoden der Pulskompression, Träger-Einhüllenden-Phasenstabilisierung und Frequenzumwandlung. Diese Techniken werden routinemäßig bei ultraschnellen Lasern mit kleineren Durchschnittsleistungen angewandt, zum Beispiel den Titan:Saphir-basierten. Jedoch mussten sie für die stärksten Femtosekundenlichtquellen mit etwa $100 \mathrm{~W}$ Durchschnittsleitung und $10 \mathrm{MW}$ Spitzenleistung neu erfunden werden.

Diese Dissertation zeigt, wie die von einem modengekoppelten Dünnscheibenoszillator mit $45 \mathrm{~W}$ Durchschnittsleistung emittierten Pulse zum ersten Mal auf eine Dauer von nur wenigen optischen Zyklen komprimiert worden sind. Die lediglich 7.7 fs kurzen Pulse wurden durch zwei sequentielle spektralen Verbreiterungs- und Kompressionsstufen mit gechirpten Spiegeln erzielt.

Selbige Lichtquelle war auch der erste Dünnscheibenoszillator, sowie gleichzeitig der erste Laseroszillator mit mehr als $10 \mathrm{~W}$ Durchschnittsleistung, von dessen emittierten Pulszügen die Träger-Einhüllenden-Phase stabilisiert wurde. Zwei Stabilisierungsschemata werden vorgestellt: Das erste beruht auf der Modulation der Leistungsverluste des Lichts in der Laserkavität durch einen akustooptischen Modulator. Der Ansatz resultierte in einem verbleibenden Phasenrauschen von $125 \mathrm{mrad}$, gemessen im Regelkreis, sowie $270 \mathrm{mrad}$, außerhalb des Regelkreises gemessen. Die zweite Methode verwendete ein zusätzliches Netzgerät zur Modulation des Pumplaserdiodenstroms. Hierbei wurde ein verbleibendes, im Regelkreis gemessenes Phasenrauschen von 390 mrad erreicht.

Während die Methoden zur Stabilisierung der Träger-Einhüllenden-Phase leistungsskalierbar sind, wird die Skalierbarkeit des ursprünglichen Ansatzes zur Kompression der Oszillatorpulse auf nur wenige optische Zyklen durch die Zerstörschwelle von photonischen Kristallfasern mit festem Kern eingeschränkt. Die Dissertation beschreibt detailliert die Limitierungen der Fasern bezüglich maximal durchlässiger Spitzenleistungen sowie erreichbarer spektraler Verbreitungsfaktoren. Weiterhin wird ein alternativer Ansatz demonstriert. Dieser beruht auf spektraler Verbreiterung in Hohlkernfasern mit einer Kagomé-artigen photonischen Kristallstruktur. Ein zweistufiges Verbreiterungs- und Kompressionsexperiment erzielte Pulsdauern von nur 9.1 fs. Allerdings zeigte sich auch eine signifikante Überhöhung des Intensitätsrauschens der ultrakurzen Pulse im Vergleich zu denen, die direkt vom Laseroszillator emittiert wurden.

Daher wurde spektrales Verbreitern in Kristallfenstern untersucht. Durch das Ausnutzen des optischen KERR-Effekts wurden Spektren erzeugt, deren Bandbreite Pulsdauern von 15 fs erlaubt. Dieses Ergebnis motiviert die ausschließliche Anwendung von Kristallfenstern in der spektralen Verbreiterung von kurzen Pulsen. Simulationsergebnisse für die Lichtpropagation durch eine Reihe von KERR-Medien versprechen eine gute Effizienz dieser Methode. Darüber hinaus wurde die Kompression von $190 \mathrm{fs}$ Pulsen mit einer Durchschnittsleistung von $90 \mathrm{~W}$ zu 30 fs und einer Durchschnittsleistung von $70 \mathrm{~W}$ experimentell demonstriert. Hierzu wurden selbst-defokussierende BBO-Kristalle verwendet. Die umfassende Studie über spektrale Verbreiterungs- und Pulskompressionstechniken ist wegbereitend für die Erzeugung von kurzen Pulsen mit Dauern von wenigen optischen 
Zyklen bei Spitzenleistungen von einigen hundert Megawatt und Durchschnittsleitungen von mehr als einhundert Watt.

Schließlich befasst sich die Dissertation mit der Aufgabe die breitbandigen, leistungsstarken Spektren in einen Wellenlängenbereich zu übertragen, der eine riesige Anzahl an charakteristischen molekularen Absorptionen beherbergt - dem mittleren Infrarot. Die Umwandlung des Nahinfrarots zu kleineren Frequenzen durch optische parametrische Verstärkung resultierte in Strahlung mit bis zu $5 \mathrm{~W}$ bei 4,1 Mikrometer und $1.3 \mathrm{~W}$ bei 8,5 Mikrometer, was einer Durchschnittsleistungssteigerung von einer Größenordnung gegenüber anderen kompakten Femtosekundenlichtquellen mit über 5 Mikrometer Wellenlänge entspricht.

Zusätzlich zu den Leistungsmessungen werden Durchstimmbarkeit und Superkontinuumserzeugung durch aufeinanderfolgende quadratische Nichtlinearitäten demonstriert. Dies führte insgesamt zu einer spektralen Abdeckung der Wellenlängen von 1,6 bis 11 Mikrometer mit Leistungsdichten, welche $1 \mu \mathrm{W} / \mathrm{cm}^{-1}$ im gesamten Bereich übersteigen.

Die demonstrierten Methoden zur Pulskompression sowie Träger-Einhüllenden-Phasenstabilisierung werden als grundlegende Techniken für die weitere Entwicklung einer neuen Generation von Hochleistungslichtquellen dienen. Durch Wellenform-kontrollierte Pulse, die nur wenige optische Zyklen dauern, können extrem nichtlineare Effekte bei bisher noch nicht erreichten Durchschnittsleistungen und Wiederholraten erzeugt werden. Die mehrere Oktaven überspannende Femtosekundenlichtquelle im mittleren Infrarot verspricht einzigarte Anwendungen beim Detektieren von molekularen Fingerabdrücken, insbesondere durch die Möglichkeiten zur Zurückwandlung der Frequenzen in den Nahinfrarotbereich oder die Nutzung der Frequenzkammspektroskopie. 


\section{Abstract}

With the advent of peak and average power scalable femtosecond lasers, in particular mode-locked thin-disk oscillators, the need for equally scalable pulse compression, carrierenvelope-phase stabilization and frequency conversion schemes arose. These techniques have been routinely applied to lower average power ultrafast lasers, for instance the widely used Ti:sapphire based ones. But they had to be reinvented for cutting-edge femtosecond sources with $100 \mathrm{~W}$ level average and $10 \mathrm{MW}$ level peak powers.

This dissertation presents how pulses emitted from a $45 \mathrm{~W}$ average power mode-locked thin-disk oscillator were compressed for the first time to a duration of only a few optical cycles. Pulses as short as $7.7 \mathrm{fs}$ were attained from two sequential spectral broadening and chirped mirror pulse compression stages.

The same light source was also the first thin-disk oscillator, and simultaneously the first oscillator with an average power of more than $10 \mathrm{~W}$, which was carrier-envelope-phase stabilized. Two stabilization methods are presented: The first one utilized intracavity loss modulation by means of an acousto-optic modulator. This resulted in $125 \mathrm{mrad}$ inloop and $270 \mathrm{mrad}$ out-of-loop residual phase noise. The second one employed pump diode current modulation by means of an auxiliary power supply. This approach yielded a $390 \mathrm{mrad}$ residual in-loop phase noise.

Whereas the presented carrier-envelope-phase stabilization schemes are power-scalable, the scalability of the initial few-cycle pulse generation approach is restricted by the damage threshold of solid-core photonic crystal fiber. The thesis reports in detail on the limitations of these fibers with respect to maximally transmittable peak powers and attainable spectral broadening factors. Moreover, an alternative approach utilizing hollowcore Kagomé-type photonic crystal fibers is demonstrated. A double-stage broadening and compression setup yielded pulse durations of only $9.1 \mathrm{fs}$, but also showed a significant intensity noise increase in comparison to the thin-disk oscillator output.

Therefore, spectral broadening in bulk crystals was studied. By exploiting the optical KERR effect, spectra with FOURIER transform-limits of 15 fs were achieved, opening the perspective for all solid-state spectral broadening in bulk material. Simulation results for a sequence of thin KERR media predict a good power efficiency of the method. Furthermore, an experimental realization of pulse compression from $190 \mathrm{fs}$ pulses with $90 \mathrm{~W}$ average power to $30 \mathrm{fs}$ pulses with $70 \mathrm{~W}$ average power in self-defocusing BBO crystals is reported. The presented comprehensive study on spectral broadening and pulse compression techniques paves the way to few-cycle pulse generation at hundreds of MW peak power and hundreds of Watts average power.

Eventually, the dissertation addresses the issue of transferring broadband, powerful spectra to a wavelength region with a huge variety of characteristic molecular absorptions - the mid-infrared. Frequency down-conversion via optical parametric amplification resulted in radiation with up to $5 \mathrm{~W}$ at 4.1 microns and $1.3 \mathrm{~W}$ at 8.5 microns, corresponding to an order-of-magnitude average power increase for compact femtosecond light sources operating at wavelengths longer than 5 microns.

In addition to the power measurements, both wavelength tunability and supercontinuum generation by means of cascaded quadratic nonlinearities are reported, resulting in 
overall spectral coverage from 1.6 to 11 microns with power spectral densities exceeding $1 \mu \mathrm{W} / \mathrm{cm}^{-1}$ over the entire range.

The pulse compression and carrier-envelope-phase stabilization schemes demonstrated in this dissertation will serve as fundamental techniques for the further development of a new generation of waveform-controlled few-cycle pulse lasers which are capable of triggering extreme nonlinear effects at unprecedented average powers and repetition rates. The multi-octave spanning, mid-infrared femtosecond source offers exciting opportunities for molecular fingerprinting, in particular by means of frequency up-conversion and fieldsensitive techniques as well as frequency comb spectroscopy. 


\section{Contents}

Zusammenfassung $\quad$ v

Abstract vii

List of Figures $\quad$ xi

List of Tables $\quad$ xiv

Acronyms $\quad$ Xv

Thesis outline $\quad$ xvi

1 INTRODUCTION 1

1.1 What Does "Generation" Refer to? . . . . . . . . . . . . . . . . . 1

1.2 A Short History of Short Pulses . . . . . . . . . . . . . . . . . . 5

1.3 The Thin-Disk Concept - Power-scalable Ultrashort Pulse Oscillators . . . 9

1.4 Fundamentals . . . . . . . . . . . . . . . . . . . . . . . 12

1.4.1 Mode-beating . . . . . . . . . . . . . . . . 12

1.4 .2 Mode-locking . . . . . . . . . . . . . . . . . 13

1.4.3 Self-phase-modulation . . . . . . . . . . . . . . . . 15

1.4 .4 Dispersion compensation . . . . . . . . . . . . . . . . . 18

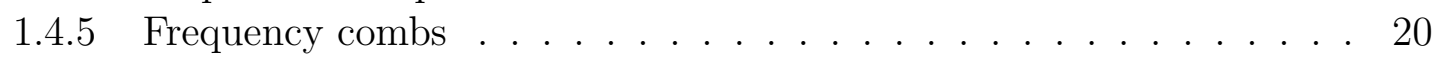

1.4.6 Nonlinear frequency down-conversion . . . . . . . . . . . . . . 22

2 PROOF OF CONCEPT: FEW-CYCLE PULSE GENERATION AND CARRIERENVELOPE-PHASE STABILIZATION

2.1 An Ultrafast Workhorse: The Kerr-lens Mode-locked Thin-disk Oscillator . 26

2.2 Entering the Few-cycle Pulse Regime with Mode-locked Thin-disk Oscillators 30 2.2.1 Solid-core Fiber-based Pulse Compression . . . . . . . . . . . . . . 30

2.2.2 Few-cycle Pulse Generation in Bulk Material . . . . . . . . . . . . . 35

2.3 Stabilizing Kerr-lens Mode-locked Thin-disk Oscillators . . . . . . . . . . . 41

2.3.1 Passive Stabilization . . . . . . . . . . . . . . . . . . 41

2.3.2 Active Carrier-Envelope-Phase Stabilization . . . . . . . . . . . 45

2.4 Chapter Summary . . . . . . . . . . . . . . . . . . . . . 55

3 POWER SCALABLE CONCEPTS $\quad \mathbf{5 7}$

3.1 Fiber-based Pulse Compression . . . . . . . . . . . . . . . . 58

3.1 .1 Limitations of Solid-core Fiber . . . . . . . . . . . . . . . . . 58

3.1.2 Kagomé-type Hollow-core Photonic Crystal Fibers . . . . . . . . . . 71

3.2 All Solid-state Spectral Broadening in Bulk Material . . . . . . . . . . . . 82

3.2.1 Compression by means of the Optical Kerr Effect of Dielectrics . . . 82

3.2.2 Efficient Pulse Compression in Self-Defocusing Bulk Media . . . . . 95 
3.3 Power-scaling Carrier-Envelope-Phase Stabilization . . . . . . . . . . . . 108

3.4 Chapter Summary . . . . . . . . . . . . . . . . . . . 115

4 FROM THE NEAR- TO THE MID-INFRARED 119

4.1 Optical Parametric Amplifiers for Frequency Down-Conversion . . . . . . . 120

4.1.1 Frequency Down-Conversion with Periodically Poled Lithium Niobate123

4.1.2 Frequency Down-Conversion with LGS . . . . . . . . . . . . . . . . 133

4.1.3 Comparison to Down-Conversion via Difference Frequency Generation141

4.2 Supercontinuum Generation in the Mid-Infrared . . . . . . . . . . . . . . 145

4.3 Chapter Summary . . . . . . . . . . . . . . . . . . . . 153

5 OUTLOOK AND CONCLUSIONS 155

5.1 Near-Future Applications . . . . . . . . . . . . . . . . 155

5.1 .1 The attosecond oscillator . . . . . . . . . . . . 155

5.1 .2 High-speed, high-rate optical switching . . . . . . . . . . . . 156

5.1.3 Field-resolved optical spectroscopy in the mid-infrared . . . . . . . 158

5.2 Have ultrafast thin-disk oscillators matured? . . . . . . . . . . . . . . . 162

$\begin{array}{lc}\text { APPENDIX } & 163\end{array}$

A.1 FROG measurements . . . . . . . . . . . . . . . . . . . . . . . . 164

A.2 RMS spectral width of a prechirped Gaussian pulse . . . . . . . . . . . 166

A.3 Transforming the differential equation (3.21) to an integral. . . . . . . . 168

A.4 Publications, etc. . . . . . . . . . . . . . . . 169

A.5 Data archiving . . . . . . . . . . . . . . . . . 173

$\begin{array}{ll}\text { List of References } & \mathbf{1 7 5}\end{array}$

$\begin{array}{ll}\text { Acknowledgements } & 194\end{array}$ 


\section{List of Figures}

1.1 Key elements of the four generations of mode-locked lasers. . . . . . . . . . 4

1.2 Thin-disk scheme . . . . . . . . . . . . . . . . . . . . 10

1.3 Beating of two modes . . . . . . . . . . . . . . . . . . . . . . . . . . . . . .

1.4 Multi-mode interferences . . . . . . . . . . . . . . . . . . . . . . . . 13

1.5 Ray tracing KERR-lens mode-locking . . . . . . . . . . . . . . . . . 15

1.6 Self-phase modulation . . . . . . . . . . . . . . . . . . . . 16

1.7 Four-wave mixing schemes . . . . . . . . . . . . . . . . . . . . . 17

1.8 Introduction chirped mirrors. . . . . . . . . . . . . . . . . . . 19

1.9 Scheme frequency comb . . . . . . . . . . . . . . . . 20

1.10 Difference Frequency generation and optical parametric amplification . . . 21

1.11 Frequency down-conversion via three-wave mixing. . . . . . . . . . . . . 22

2.1 Setup of the utilized Kerr-lens mode-locked thin-disk oscillator . . . . . . . 26

2.2 Power characteristics of the utilized Kerr-lens mode-locked thin-disk oscillator 27

2.3 Spectrum and autocorrelation of the utilized Kerr-lens mode-locked thindisk oscillator . . . . . . . . . . . . . . . . . . . . 28

2.4 Intensity noise characteristics of the KERR-lens mode-locked thin-disk oscillator . . . . . . . . . . . . . . . . . . 28

2.5 Simulation of pulse propagation in LMA-35 . . . . . . . . . . . . . 31

2.6 Spectral broadening in LMA-35 fibers of different lengths . . . . . . . . . . 31

2.7 Solid-core fiber compression setup . . . . . . . . . . . . . . . . 32

2.8 Simulated, directly measured and retrieved spectra after LMA-35 compression setup . . . . . . . . . . . . . . . . . . . . 32

2.9 Measured pulses after LMA-35 compression setup . . . . . . . . . . . . 33

2.10 Setup bulk broadening. . . . . . . . . . . . . . . . . . . . . . . . . . . . . . . . .

2.11 Broadband spectra after bulk broadening. . . . . . . . . . . . . . 36

2.12 FROG characterization 9.9 fs pulses after bulk broadening. . . . . . . . . . 37

2.13 FROG characterization $7.7 \mathrm{fs}$ pulses after bulk broadening. . . . . . . . . 38

2.14 Beam characterization after bulk broadening. . . . . . . . . . . . . . . 39

2.15 Generic intensity noise measurement setup. . . . . . . . . . . . . . . . 42

2.16 Noise properties before and after passive stabilization. . . . . . . . . . . . 42

2.17 Comparison commercial power supplies. . . . . . . . . . . . . . . . . . 43

2.18 Comparison of noise to commercial lasers. . . . . . . . . . . . . . . . . . . . . . . . . 44

2.19 Setup of the f-to-2f interferometers. . . . . . . . . . . . . . 46

2.20 AOM locking scheme. . . . . . . . . . . . . . . . . . . 47

2.21 In-loop residual phase noise intracavity AOM stabilization. . . . . . . . . . 48

2.22 Acoustic noise in out-of-loop measurements. . . . . . . . . . . . . . 50

2.23 In- and out-of-loop residual phase noise intracavity AOM stabilization. . . 51

3.1 Setup solid-core fiber broadening experiments and dispersion curves . . . . 59

3.2 Measured spectra after broadening in different PCFs. . . . . . . . . . . 60 
3.3 Broadening factor, peak irradiance and peak power in dependence of fiber mode-field diameter . . . . . . . . . . . . . . . . . . . . 61

3.4 Simulation peak irradiance enhancement in sub-critical self-focusing regime 62

3.5 Microscope images of the front fiber facets after damage . . . . . . . . . 63

3.6 Mode-field diameters and broadening factors for various pulse peak powers 64

3.7 Simulated broadening factors for positively chirped input pulses without pulse duration dependent damage . . . . . . . . . . . . . . . 65

3.8 Restrictions of chirped pulse spectral broadening . . . . . . . . . . . . 66

3.9 Mode-field diameters and broadening factors for various pulse peak powers for chirped pulse spectral broadening . . . . . . . . . . . . . . 67

3.10 Chirped pulse spectral broadening example . . . . . . . . . . . . . 68

3.11 Hollow-core photonic crystal fiber cross-sections. . . . . . . . . . . . . . . . 72

3.12 Kagomé tuning paramters. . . . . . . . . . . . . . . . . . . . 73

3.13 Setup double-stage hollowcore fiber compression. . . . . . . . . . . . . . 75

3.14 FROG characterization after first Kagomé-type HC-PCF compression stage. 76

3.15 FROG characterization after second Kagomé-type HC-PCF compression stage. . . . . . . . . . . . . . . . . . . 77

3.16 Kagomé-type HC-PCF noise measurements. . . . . . . . . . . . . . . . 78

3.17 Signs of ionization in xenon filled Kagomé-type HC-PCF. . . . . . . . . . . 79

3.18 Means of avoiding critical self-focusing within a nonlinear crystal. . . . . . 83

3.19 Setup of the KERR effect-based bulk broadening experiments. . . . . . . . 85

3.20 Spectra after single-stage bulk broadening. . . . . . . . . . . . . . . 86

3.21 Pulse compression after single-stage bulk broadening. . . . . . . . . . . . . 87

3.22 Double stage spectral broadening in quartz crystals. . . . . . . . . . . . . . 87

3.23 Spatiotemporal effects of bulk broadening in quartz. . . . . . . . . . . . 88

3.24 Intensity noise measurements after the first bulk broadening stage for different broadening factors. . . . . . . . . . . . . . . . . . 89

3.25 Simulation of the spectral broadening in a $15 \mathrm{~mm}$ crystalline quartz crystal. 90

3.26 Spatially integrated and radially resolved spectra after propagation through a $15 \mathrm{~mm}$ quartz plate. . . . . . . . . . . . . . . . . . . 91

3.27 Simulation of propagation of a $10 \mu \mathrm{J}$ pulse through ten $0.5 \mathrm{~mm}$ quartz plates. 92

3.28 Model for 100 rays describing a Gaussian beam . . . . . . . . . . . . 96

3.29 Magnitude and dispersion of the effective nonlinear refractive index. . . . . 98

3.30 Simulations with an incoming $180 \mathrm{fs} \mathrm{sech}^{2}$-pulse, focused to a beam diam-

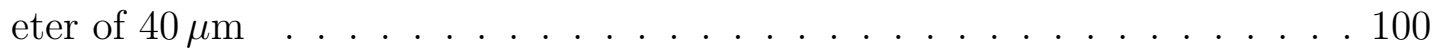

3.31 Parameter tuning in BBO spectral broadening. . . . . . . . . . . . . . . . . 101

3.32 Setup pulse compression by means of cascaded quadratic nonlinearities . . 102

3.33 Spectra and pulses after spectral broadening in BBO . . . . . . . . . . 103

3.34 FROG traces, spectra and optimized pulses after the third BBO compression stage . . . . . . . . . . . . . . . . . . . . . . 104

3.35 Setup of the dual wavelength pumping stabilization scheme. . . . . . . . 108

3.36 Integrated phase noise of the free-running beat note. . . . . . . . . . . . 110

3.37 Transfer functions of pump diodes, oscillator and PLF. . . . . . . . . . . 111

3.38 Residual in-loop phase noise achieved by the dual wavelength approach. . . 111

4.1 Generic OPA setup. . . . . . . . . . . . . . . . . . . 120

4.2 Transmission mIR beam splitters. . . . . . . . . . . . . . . . . . . 121

4.3 Seed generation in ANDi fiber. . . . . . . . . . . . . . . 123 
4.4 Microscope images of ANDi-pm fiber. . . . . . . . . . . . . . . . . . . 124

4.5 Seed and signal stability after the PPLN OPA . . . . . . . . . . 125

4.6 PPLN OPA simulations. . . . . . . . . . . . . . . . . . . . . 127

4.7 PPLN OPA power and tuning curve. . . . . . . . . . . . . . . . . . . 128

4.8 Mid-infrared stability and beam profiles after the PPLN OPA . . . . . 130

4.9 OPA setup with FROG . . . . . . . . . . . . . . . . . 130

4.10 FROG measurements after the PPLN OPA . . . . . . . . . . . . . 131

4.11 Transmission and dispersion of the LGS crystal . . . . . . . . . . . . . 134

4.12 LGS OPA seed spectrum . . . . . . . . . . . . . . . . . . . . . . . . . . . . . . . . . . . . . . .

4.13 LGS OPA simulations . . . . . . . . . . . . . . . 136

4.14 LGS OPA tuning . . . . . . . . . . . . . . . . . . . 137

4.15 LGS OPA power measurements without dichroic beam splitters . . . . . 138

4.16 Occurrence of LGS crystal damage at various input powers . . . . . . . . . 139

4.17 Intra-pulse DFG principle. . . . . . . . . . . . . . . . . . 141

4.18 Intra-pulse DFG simulations. . . . . . . . . . . . . . . . . . 142

4.19 LGS DFG results. . . . . . . . . . . . . . . . . . . . 143

4.20 Magnitude and dispersion of the effective nonlinear refractive index of ZGP. 145

4.21 Continuum generation from the OPA idler by means of cascaded quadratic nonlinearities in the mid-IR . . . . . . . . . . . . . . . 146

4.22 Bulk spectral broadening with the compressed PPLN OPA idler . . . . . . 148

4.23 FROG measurements of compressed mid-infrared pulses . . . . . . . . . . . 148

4.24 Supercontinuum generation setup . . . . . . . . . . . . . . . . 149

4.25 Coverage of the mid-infrared spectral region from $1.6 \mu \mathrm{m}-10.2 \mu \mathrm{m}$. . . . . 150

4.26 Simulation of continuum generation in ZGP with $67 \mathrm{fs}$ input pulses . . . . 151

5.1 Selection of materials for current injection experiments. . . . . . . . . . . 157

5.2 Generic setup of field-resolved spectroscopy. . . . . . . . . . . . . . . 158

5.3 Multi-channel OPA setup. . . . . . . . . . . . . . . . . . 159

A.1 Developed FROG setup . . . . . . . . . . . . . . . . . . . 164

A.2 Example of an ultrabroaband X-FROG measurement. . . . . . . . . . 165 


\section{List of Tables}

1.1 Generations of mode-locked oscillators - an overview. . . . . . . . . . . . 2

1.2 Advantages of the thin-disk architecture. . . . . . . . . . . . . . . 9

3.1 Summary of FTLs and spatial losses in experiment and simulations with $1.2 \mu \mathrm{J}$ and $10 \mu \mathrm{J}$ pulses . . . . . . . . . . . . . . . . . 93

3.2 Summary of the presented pulse compression experiments. . . . . . . . . . 104

3.3 Comparison compression techniques . . . . . . . . . . . . . 116

4.1 Examples of GVDs at pump and idler wavelengths ${ }^{a}$. . . . . . . . . . . . 122

4.2 Generated mid-IR powers and efficiencies for different pump spot sizes. . . 129

4.3 mid-infrared (mid-IR) source comparison . . . . . . . . . . . . . 153 


\section{Acronyms}

AC alternating current.

ANDi all-normal dispersion.

AOI angle of incidence.

AOM acousto-optic-modulator.

APD avalanche photodiode.

AR anti-reflection.

BBO beta barium borate, $\beta-\mathrm{BaB}_{2} \mathrm{O}_{4}$.

CCD charge coupled device.

CEP carrier-envelope phase.

COLTRIMS cold target recoil ion momentum spectroscopy.

CPA chirped pulse amplification.

CPSB chirped pulse spectral broadening.

CSP cadmium silicon phosphide, $\mathrm{CdSiP}_{2}$.

CW continuous wave.

DC direct current.

DFG difference frequency generation.

DPD digital phase detector.

EOM electro-optic modulator.

EOS electro-optic sampling.

FOM figure of merit.

FROG frequency resolved optical gating.

FS fused silica.

FTIR FOURIER transform infrared spectrometer.

FTL Fourier transform-limit.

FWHM full width at half maximum.

GDD group dispersion delay.

GVD group velocity dispersion.

HC-PCF hollow-core photonic crystal fiber.

HHG high harmonic generation.

HR high reflection (dielectric coating).

IN intensity noise.

IPN integrated phase noise.

KLM Kerr-lens mode-locked.

LGS lithium gallium sulfide, $\mathrm{LiGaS}_{2}$.

LMA large mode area.

MFD mode-field diameter.

OC output coupler.

OPA optical parametric amplifier.

OPCPA optical parametric chirped pulse amplification.

OPO optical parametric oscillator.

OSA optical spectrum analyzer.

OWB optical wave-breaking.

PBG photonic-bandgap.
PCF photonic crystal fiber.

PEEM photoemission electron microscopy.

PLF phase lead filter.

PLL phase-locked loop.

PM polarization maintaining.

PPLN periodically-poled $\mathrm{MgO}$-doped lithium niobate.

PSD power spectral density.

$\mathbf{R F}$ radio-frequency.

RIN relative intensity noise.

RMS root mean square.

SCG supercontinuum generation.

SESAM semiconductor saturable absorber mirror.

SHG second harmonic generation.

SH second harmonic.

SNR signal-to-noise ratio.

SPM self-phase modulation.

TD thin-disk.

TOD third order dispersion.

Ti:sapph titanium doped sapphire.

UV ultraviolet.

VUV vacuum ultraviolet.

WLC white-light continuum.

X-FROG cross-correlation frequency resolved optical gating.

XUV extreme ultraviolet.

YAG yttrium-aluminumgranate, $\mathrm{Y}_{3} \mathrm{Al}_{5} \mathrm{O}_{12}$.

Yb:YAG ytterbium doped yttrium-aluminumgranate.

ZGP zinc germanium phosphide, $\mathrm{ZnGeP}_{2}$.

mid-IR mid-infrared.

near-IR near infrared. 


\section{Thesis outline}

The subject of this dissertation is the development of high-average power ultrafast lasers. The main methods are spectral broadening and pulse compression, carrier-envelope-phase stabilization as well as difference frequency generation and optical parametric amplification.

The dissertation is structured as follows:

Chapter 1 firstly specifies the research area and defines the research objectives of this thesis. Secondly, it introduces the main methods from a historical point of view, outlines the shortcomings of currently prevailing laser technologies and explains possible applications for a new generation of mode-locked femtosecond oscillators. Thirdly, some important properties of the thin-disk technology are introduced. In section 4 of chapter 1, fundamental physical concepts are explained in an illustrative manner.

Chapter 2 demonstrates a proof-of-principle experiment towards thin-disk-based highpower waveform-controlled few-cycle pulse light sources. Firstly, the mainly used Kerrlens mode-locked thin-disk oscillator is characterized. Secondly, pulse compression with a solid-core fiber and a bulk material broadening stage is reported. Thirdly, carrierenvelope-phase stabilization by means of an intracavity acousto-optic modulator is demonstrated. All results are summarized at the end.

The subject of chapter 3 is the power-scalability of the results presented in chapter 2 . In section 1 solid-core and hollow-core photonic crystal fiber spectral broadening is investigated. Section 2 focuses on pulse compression techniques that rely on bulk crystals, either exhibiting only the optical KERR effect or additionally quadratic optical nonlinearities. Section 3 presents an alternative, power-scalable approach on carrier-envelope-phase stabilization. Eventually, section 4 gives a comprehensive overview about all studied techniques.

Chapter 4 focuses on frequency down-conversion of the near-infrared radiation emitted by the ultrafast oscillator to the mid-infrared. In section 1, two powerful optical parametric amplifiers are demonstrated and a comparison to an alternative down-conversion approach via intrapulse difference frequency generation is presented. The subject of section 2 is supercontinuum generation in the mid-infrared by means of large cascaded quadratic nonlinearities. In the final section the chapter is summarized.

Chapter 5 firstly proposes what can be done next in order to apply the achievements of this thesis. Three applications are discussed. Secondly, the main results of the dissertation are highlighted once more in concluding statements. 


\section{INTRODUCTION}

\subsection{What Does "Generation" Refer to?}

The relatively young history of the laser began in 1960, when T. H. MAIMAN demonstrated Light Amplification by Stimulated Emission of Radiation for the first time ${ }^{1}$. His achievement was based on the theoretical work of V. A. FABRIKANT and the NobeL prize laureates A. Einstein, A. M. Prohorov, N. G. Basov, A. L. Schawlow and $\mathrm{CH}$. H. Townes ${ }^{2-5}$. During the past 58 years, the research and development field has proliferated in many respects. Today, laser technology has become a multi-billion dollar business with a continuous growth rate on the order of $5 \%$ during the past years ${ }^{6}$. Moreover, the invention led to numerous NOBEL prize awarded inventions in physics as well as in chemistry ${ }^{7}$.

The work presented in this dissertation is focused on the development of ultrashort pulse solid-state thin-disk (TD) laser oscillators. Although solid-state lasers have only a small market share in comparison to diode lasers, the technology is fairly interesting for the biggest laser market segment, material processing ${ }^{6}$. Its key features, which are average power, intensity and laser wavelength ${ }^{8}$, are in-fact addressed in this work. However, the main goal of the thesis is to present a novel light source which enables cutting-edge applications in field-sensitive nonlinear optics as well as frequency comb spectroscopy, research fields with tremendous potential to be employed in life sciences or signal processing, for instance. This target requires the consideration of additional key elements, which are the generation of pulses with a duration on the order of an optical cycle and the full phasecontrol of the light field, i.e. waveform-control enabled by carrier-envelope phase (CEP) stabilization. These features are closely related to the emergence of femtosecond titanium doped sapphire (Ti:sapph) oscillators which have been prevailing in ultrafast optics laboratories since their advent in $1990 / 91^{9,10}$, i.e. for more than 25 years. This is a huge era in the young history of the laser. Nevertheless, the unprecedented power-scalability of TD oscillators and its most recent developments, which are at least partly presented in this dissertation, promise to gradually replace the established bulk solid-state technology within the next decades.

The "new generation of high-power, waveform controlled, few-cycle light sources" which is discussed in this thesis must be distinguished from the recently proclaimed "thirdgeneration femtosecond technology" ${ }^{11,12}$. Although both developments aim for enor-

\section{Remark: Optical cycle}

The duration of an optical cycle is calculated by the carrier (or central) wavelength of the optical pulse $\left(\lambda_{c}\right)$ divided by the speed of light $\left(c_{0} \approx 299.8 \mathrm{~nm} / \mathrm{fs}\right)$. The central wavelength of the utilized laser oscillator is about $1030 \mathrm{~nm}$ which corresponds to a $3.4 \mathrm{fs}$ $=0.0000000000000034 \mathrm{~s}$ cycle duration. 
Table 1.1: Generations of mode-locked oscillators - an overview.

\begin{tabular}{lclc}
\hline time & architecture & selected milestones & references \\
\hline 1964-1970 & multiple & first active mode-locking & 14 \\
& & first passive mode-locking & 15,16 \\
1970-1990 & organic dyes & first CW mode-locking & 17 \\
& & sub-10 fs pulses with external compression & 18 \\
$1990-?^{a}$ & bulk solid-state & demonstration of KLM Ti:sapph oscillator & 9,10 \\
& & demonstration of CEP stabilization & 19 \\
& & first few-cycle pulse oscillator with octave- & 20 \\
& spanning spectrum & \\
& & first mode-locked TD oscillator & 21 \\
$?^{a}$ & thin-disk & first > 100 W fs oscillator & 22 \\
& & demonstration of KLM TD oscillator & 23 \\
& & CEP stabilization and few-cycle pulse generation & 24 \\
& & by external compression & \\
\hline
\end{tabular}

CW - continuous wave, KLM - KERR lens mode-locked, CEP - carrier-envelope phase, TD - thin-disk

${ }^{a}$ A question mark is set here because the breakthrough of TD oscillators in a scale of previous generations has not yet come. In the final chapter of this dissertation the recent progress will be reviewed in order to conclude on how the technology will advance.

mously increasing both peak and average power, the light sources presented here are rather considered as the front end of the amplifier systems described in refs. 11 and 12 . The distinct advantages of using the oscillators as stand alone systems are higher $(\mathrm{MHz})$ pulse repetition rates and hence data acquisition rates as well as the vastly reduced complexity of the systems ${ }^{13}$.

The term "generation" has been adapted from the historical reviews provided in refs. 36 and 37. They refer to generations of mode-locked lasers. Mode-locking is the fundamental principle of every table-top ultrafast laser. It will be briefly explained in section 1.4. The classification of generations, which both papers use, is shown in table 1.1. Some important milestones of the evolving TD technology have been added. The first generation of mode-locked lasers was called the generation of picosecond (ps) lasers and may be also referred to as the pioneering era of ultrashort pulse generation since ps lasers are still quite common. Many gain materials were explored. Fundamental research on modelocking was conducted and its principles were described for the first time. The second generation was characterized by fs dye lasers. This period led to the first demonstration of few-cycle pulses, i.e. the ultimate duration limits of visible pulses were approached. Ahmed ZEWAIL's "studies of the transition states of chemical reactions using femtosecond spectroscopy", which were rewarded with the 1999 NOBEL prize in chemistry ${ }^{38,39}$, were initially based on dye lasers, but also strongly benefited from the following generation of solid-state femtosecond lasers which enabled further breakthroughs in creating nature's shortest events outside the atomic nucleus. Attosecond (as) pulses ${ }^{40-42}$ and light field transients $^{43,44}$ were demonstrated for the first time. However, these concepts do not necessarily require solid laser gain media since they originate from optical nonlinearities. They rather require light sources which are highly reliable and fairly easy to handle. That 


\section{Remark: Mode-locked fiber lasers}

In the early 1990s a tremendous development of ultrafast fiber lasers started in parallel to the proliferation of solid-state bulk oscillators as, for instance, reviewed in ref. 25. This development is still rapidly progressing, and thus should be mentioned within the context of this section. By means of increasing mode-field areas, chirped pulse amplification (CPA) and eventually coherent combination of multiple beams, fs lasers with $\mathrm{kW}$ of average power and GW of peak power have been demonstrated ${ }^{26-28}$. Moreover, single-cycle pulses have been synthesized ${ }^{29}$ and tight locking of carrier-envelope-offset frequency was achieved ${ }^{30}$. Although thousands of $\mathrm{kW}$ average and TW peak power have been envisioned ${ }^{31}$, the scalability of the technology relies so-far on coherent combining where firstly, power scales only linear with the number of beams, and secondly, the combination of thousands of lasers would be required while eight channels have been demonstrated ${ }^{28}$. The approach could be also transferred to any other laser architecture, and hence, the scalability is in that sense inferior to disk or innoslab technology where $\mathrm{kW}$ ultrafast sources were demonstrated without combining laser beams ${ }^{32-34}$. Ultrafast fiber oscillators have reached average powers of $66 \mathrm{~W}^{35}$ which is akin to the TD oscillator that was mostly used in the experiments presented here. However, resorting to the positive dispersion regime was necessary to not sustain peak power induced damages which indicates that relying on the TD concept is beneficial for power scaling. Nevertheless, all-fiber lasers exhibit certainly many advantages in terms of applicability which are high wall-plug efficiency, robustness due to the absence of free-standing optics, compactness, low vulnerability to thermal load (up to moderate fiber diameters) and cost-efficiency ${ }^{31}$. Consequently, their development has been also driven by a variety of companies and not only by research institutions ${ }^{26}$.

is what really made the difference between dye and solid-state femtosecond lasers. Not by coincidence the Kerr-lens mode-locked (KLM) Ti:sapph lasers have been commercialized only one year after they were reported for the first time in $1990^{9,45}$. Contrary to KRAUSz et $a l .{ }^{36}, \mathrm{FRENCH}^{37}$ even introduces a fourth generation which he calls "useful ultrafast lasers". He derives this name from the following consideration:

"The extremely rapid development of tunable femtosecond solid-state lasers has brought the field to the point where it is not always necessary to design an experiment around the available laser source: rather, it is now reasonable to expect that a suitable ultrafast laser will exist for a particular application."

The categorization that KRAUSz et al. proposed shall be utilized here since the fourth generation of FRENCH does not introduce a new laser architecture. Nevertheless, the quotation points out an additional important property of the third mode-locked laser generation: This is tunability or, in more general wording, the freedom to adapt the central wavelength and the bandwidth of the light pulses to the targeted application. Although dye lasers in principle already covered the spectral range from $320 \mathrm{~nm}$ to $1800 \mathrm{~nm}$, they were lacking stability in particular at near infrared (near-IR) wavelengths ${ }^{37}$. Solid-state gain materials allow lasing from $700 \mathrm{~nm}$ to about $3 \mu \mathrm{m}^{36}$. Furthermore, nonlinear frequency conversion and supercontinuum generation give access to frequencies from several $\mathrm{THz}$ up to the deep ultraviolet (UV) region and grant access to partially even octavespanning bandwidths ${ }^{43,46-49}$.

Another key feature has massively increased the importance of fs lasers. It emerged shortly after the turn of the last millennium. The phase, which describes the shift between the 


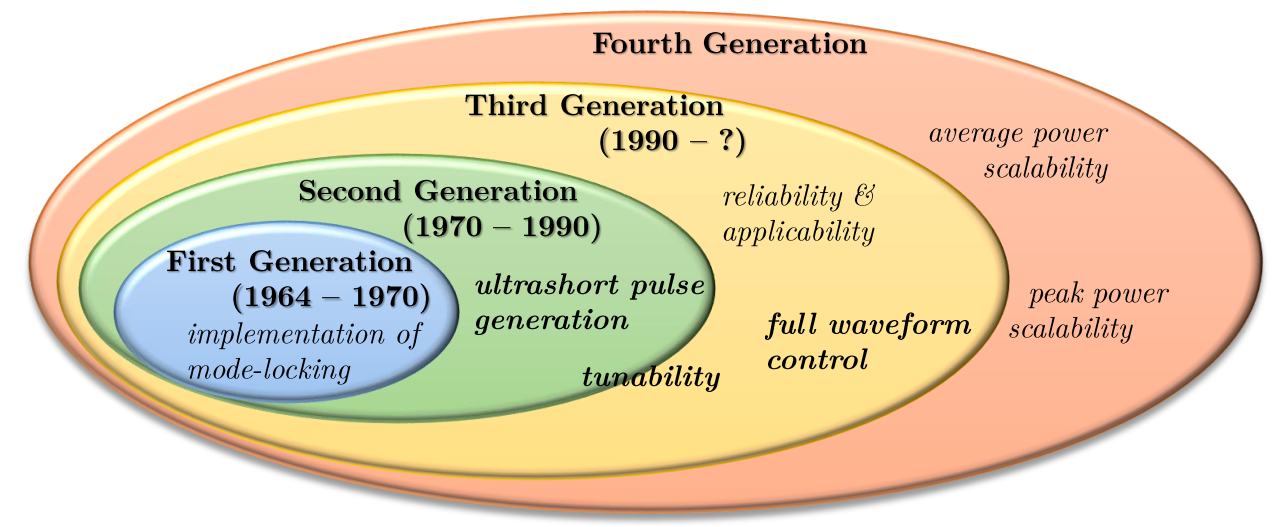

Fig. 1.1. Key elements of the four generations of mode-locked lasers described in the main text. The graphic indicates that all features of the preceding generations must be embedded into the new ones. The thesis covers the key elements printed in bold type.

peaks of the carrier wave and pulse envelope was controlled for the first time ${ }^{19,50}$. This has linked the domain of optical frequencies $(\mathrm{THz}-\mathrm{PHz})$ to the domain of radio-frequencies $(\mathrm{MHz}-\mathrm{GHz})$ and revolutionized metrology. Therefore, the third generation of mode-locked lasers is also closely linked to the 2005 NoBEL prizes in physics for JoHN L. HALL's and TheODOR W. HÄNSCH's "contributions to the development of laser-based precision spectroscopy, including the optical frequency comb technique" 51 . Moreover, the ability to precisely control the carrier-envelope phase (CEP) opened the door to explore nonlinear optical effects which do not only depend on the cycle-averaged intensity of the light-field but also on its temporal phase ${ }^{52}$. These are so-called extreme or field-sensitive optical nonlinearities. Most prominent among them is probably the generation of phase-coherent high harmonics ${ }^{53,54}$.

Since these new avenues of exploiting extreme nonlinear optics set a huge demand on both peak power to drive the effects and average power to maintain high data rates despite low efficiencies in the nonlinear conversion processes, the bulk solid-state laser architecture came up against its limitations ${ }^{11}$. Despite the efforts to extend the laser cavity lengths in order to increase the pulse energy and the peak power, resp. while keeping the average power at a Watt-level ${ }^{55}$ and to operate the laser in the positive dispersion regime, i.e. with temporally stretched pulses, to avoid detrimental nonlinearities, Ti:sapph oscillators remained at sub- $\mu \mathrm{J}$ pulse energy and sub- $10 \mathrm{~W}$ average output power levels ${ }^{56,57}$. By contrast, the first mode-locked TD oscillator, demonstrated in $2000^{21}$, already delivered more than $16 \mathrm{~W}$ of average power at about $0.5 \mu \mathrm{J}$ pulse energy. Today, roughly 18 years later, average powers of more than $250 \mathrm{~W}^{58,59}$, multiple tens of $\mu \mathrm{J}^{59-61}$ and peak powers of more than $60 \mathrm{MW}^{59,61}$ are directly extracted from the oscillators. Moreover, up to $230 \mathrm{MW}$ after external pulse compression ${ }^{62}$ have been obtained. In spite of these highly impressive numbers, mode-locked TD lasers have only been employed in a few spectroscopic applications ${ }^{13,63,64}$ which did not even clearly reveal the advantages of the oscillators. A reason is that until recently the key features of the second and third generations of mode-locked lasers have not been realized with the new, power-scalable technology. However, to constitute a novel generation of femtosecond oscillators, it is essential that all main advantages of the older technologies are transferred to the new one. Otherwise, the development may be interesting for specific applications but will not be capable of replacing a technology 
which has been established for decades. The key elements of the four mode-locked laser generations are displayed in Fig. 1.1.

A very important step towards applicability of mode-locked TD oscillators was made through the first demonstration of KLM in $2011^{23}$. Maybe similar to the replacement of slow saturable absorbers in dye lasers with fast saturable absorbers in bulk lasers, the advance from semiconductor saturable absorber mirror (SESAM) to KERR media as primary mode-locker yielded a huge gain in reliability ${ }^{65}$. In-fact, the first KLM oscillator has been utilized for the research presented in this dissertation. It has been running for about five years on a daily basis without the need for any major replacements.

Three key elements of Fig. 1.1 remained to be realized in order to proclaim a next generation of mode-locked oscillators. They are highlighted in bold type, being firstly, the demonstration of few-cycle pulse operation, secondly waveform stabilization, and thirdly, the ability to transfer all the advantageous properties to other wavelength regions. This thesis presents the realization of the first two features and shows examples of transferring all properties from the near-IR to the mid-IR which is extremely attractive for frequency and time-domain applications ${ }^{41,66,67}$.

The next subsection will take a look at the addressed key elements from a historical perspective. This will motivate the experimental methods presented in this dissertation.

\subsection{A Short History of Short Pulses}

In 1961, already one year after the first demonstration of the laser, the controlled pulsed operation of a laser was used to generate light of unprecedented intensity ${ }^{68}$. This socalled "Giant Optical Pulsation" was estimated to be $0.12 \mu$ s long, coming with a "total peak output intensity" of about $600 \mathrm{~kW}$. The still very common technique of quality-, or briefly, Q-switching by means of a POCKELs cell was employed to achieve the pulsed operation. Temporally increasing the losses (lowering the quality) of the laser resonator allows an enhanced inversion build-up in the active medium which is depleted quickly if the resonator quality is high. The Pockels effect describes a direct current (DC)-field dependent polarization rotation of the laser field ${ }^{69}$, i.e. actively switching a high static voltage led to the pulse formation. Further information about Q-switching can be found in laser textbooks, for instance in refs. 70 and 71.

By tailoring the loss modulation frequency to the longitudinal mode spacing of the resonator, the formation of much shorter pulses is achieved. The periodic modulation leads to side-band formation of the lasing longitudinal modes and thus to a broad emission spectrum. Moreover, all modes oscillate in phase, they are locked, and hence interfere constructively at a certain time $t_{0}$ which consequently leads to the formation of ultrashort pulses (cf. section 1.4). The more modes oscillate and the better their relative timing, the shorter the generated pulses. In 1964, this principle of mode-locking was described and realized, resp. for the first time by W. E. LAmB ${ }^{72}$ and L. E. Hargrove et al. ${ }^{14}$.

\section{Remark: Pulse duration}

The definition of "pulse duration" is not unique. Unless explicitly specified differently, it refers to the full width at half maximum (FWHM) of the pulse intensity envelope. 
The latter paper describes a He-Ne-laser with an acousto-optic-modulator (AOM) inside the resonator. The optical wave is diffracted from the acoustic wave which is driven at the doubled laser repetition rate. At the zero-crossing time of the acoustic wave, the resonator losses are minimal, and hence photons preferably pass the modulator at this instant of time. Consequently, the modes are forced to oscillate in phase which enabled the generation of $5 \mathrm{~ns}$ pulses in the first demonstration of the method ${ }^{14}$.

Up to six orders of magnitude shorter pulses can be generated if the mode-locking is not achieved by an active device such as an AOM but through a passive mechanism which is in general triggered by some (slow or fast) saturable absorber ${ }^{73}$. The saturation behavior results in lower resonator losses for intense pulses than for waves continuously spread in time. Since the most intense pulses are generated by a constructive interference of a broad band of resonant cavity modes, the laser seeks the mode-locking regime if some chaotic light is launched in the cavity (cf. section 1.4). While the active approach requires the synchronization of the repetition rate and the driving frequency of the mode-locker, the passive approach is intrinsically synchronized. Mode-locking is, for instance, explicitly described in the textbooks 74 and 75 . First passively mode-locked lasers were demonstrated in 1965. A Q-switched ruby laser was used and an additional dye was inserted which acted as the saturable absorber and initiated the mode-locking ${ }^{15}$. A dye also served as passive mode-locker in the first femtosecond laser which utilized flowing Rhodamine 6G as active medium. It was reported in $1974^{76}$. The same gain medium was employed in the first sub-100 fs laser ${ }^{77}$. Contrary to their previous work, the group around Charles V. SHANK separated the gain medium from the saturable absorber and arranged them in the colliding pulse geometry. The technique is, for instance, explained in ref. 78. Moreover, all bandwidth limiting elements inside the cavity were removed. Additional balancing of intracavity group dispersion delay (GDD) and self-phase modulation (SPM) as well as saturable absorption and gain led to the shortest pulses which have been directly generated from a dye laser. A pulse intensity autocorrelation measurement ${ }^{79,80}$ revealed a duration of $27 \mathrm{fs}{ }^{81}$.

In general, the minimal pulse duration achievable from an oscillator is limited by the gain bandwidth of its active medium (although a few exceptions have been demonstrated, e.g. refs. 82, 83). Therefore, external spectral broadening is utilized to extend the spectral width of the pulses, and consequently to compress them to shorter durations. In the majority of experiments, this is achieved by applying SPM in combination with negative GDD to the pulses. First broadening experiments were conducted in fused silica fiber ${ }^{84,85}$ and negative dispersion was applied through grating or fiber pairs ${ }^{86,87}$. The principle was also used to generate the first sub-10 fs pulses ${ }^{18}$. In 1987, the shortest pulses of the second generation of mode-locked oscillators were achieved. They exhibited a duration of only $6 \mathrm{fs}$, corresponding to three optical cycles at the central wavelength of $620 \mathrm{~nm}^{88}$.

\section{Remark: Dispersion}

The term "negative dispersion" is ambiguous since group velocity dispersion (GVD) and the "dispersion parameter", which is more common in fiber optics, have opposite signs. In this dissertation, positive means normal dispersion, while negative means anomalous dispersion. 
By this time, the unique properties for ultrashort pulse generation of Ti:sapph crystals have already been discovered ${ }^{89}$. Of course, many of the optical techniques and models which have been studied with dye lasers, such as balancing of GDD and SPM in the laser cavity (e.g. described by the so-called master equation for fast saturable absorbers ${ }^{90}$ ) or external pulse compression, could be transferred to the solid-state architecture. For example, the shortest pulse record of the second mode-locked laser generation was beaten 10 years later for the first time with a similar fiber-prism-grating pulse compression scheme ${ }^{91}$. Therefore, it is rather surprising that probably the most common solid-state laser modelocking technique, KLM, was not discovered before the advent of Ti:sapph oscillators. Rather by coincidence, it was firstly experimentally observed ${ }^{9,10}$ and based on earlier proposals $^{92}$ explained shortly after its realization ${ }^{93-95}$. Nowadays, the extremely broad gain bandwidth of Ti:sapph has supported octave-spanning spectra generated directly from the oscillator ${ }^{20}$. Broadband pulses, exhibiting a FOURIER transform-limit (FTL) of only $3.7 \mathrm{fs}$, could be compressed to $4.4 \mathrm{fs}$, less than two optical cycles ${ }^{96}$. To generate such ultrashort pulses, the control of higher-order phase terms becomes inevitable ${ }^{54}$, and thus the achievement is inherently linked to the advances of dielectric multi-layer coatings ${ }^{97}$. The invention of chirped mirrors ${ }^{54,98}$ has provided a huge degree of freedom in tailoring the phase of the light fields, being not restricted anymore to the control of GDD and third order dispersion (TOD) which was possible with grating-prism-type compressors. Further reductions of pulse durations even to sub-cycle regime ${ }^{43,44}$ have been achieved through the synthesize of multiple ultrashort pulses by spectral broadening of nearly $\mathrm{mJ}$ pulses with $\mathrm{kHz}$ repetition rate in a hollow-core capillary followed by three or four parallel chirped mirror compression stages.

The shortest isolated light pulses which have been generated up to now, rely on the technique called high harmonic generation $(\mathrm{HHG})^{53,99-102}$. In 2001, the method opened the gate to the sub-fs regime ${ }^{40-42}$. Nowadays, even pulses of less than 100 as have been demonstrated $^{103-105}$. Attosecond pulse generation originally required the light field causing HHG to fulfill three conditions ${ }^{42}$ :

(i) Its peak intensities must be on the order of $10^{13} \mathrm{~W} / \mathrm{cm}^{2}-10^{15} \mathrm{~W} / \mathrm{cm}^{2}$ to initiate the extreme nonlinear effect.

(ii) Its duration must be in the few-cycle regime to generate only a single as pulse per shot.

(iii) Its waveform must be controlled to control temporal and spectral shape of the high harmonics.

It is to note that meanwhile modified attosecond generation techniques have been developed that somewhat soften the second condition, but those are less efficient than the original technique ${ }^{106}$.

Condition (iii) was recognized early, ${ }^{52}$ but the means to achieve precise control came with the revolution of frequency metrology, ${ }^{107}$ namely the full stabilization of the frequency comb emitted by a mode-locked laser. The review of ref. 50 describes that the realization of a stable frequency comb, as a clockwork for linking a precisely known frequency reference to an atomic or molecular transition of interest, had been envisioned for decades. However, only after the emergence of the Ti:sapph technology in the 1990s and the invention of microstructured photonic crystal fibers (PCFs), the technical preconditions, namely the routine generation of ultrabroadband (octave-spanning) spectra, were fulfilled to actually realize an universal, precise link. 
While for frequency domain applications, the nJ pulses emitted from bulk solid-state or fiber oscillators are sufficient for conducting experiments, reaching $>10^{13} \mathrm{~W} / \mathrm{cm}^{2}$ (condition (i)) for field-sensitive nonlinear optics was hardly possible with ultrafast oscillators of the first three generations. A few field-sensitive experiments in gases with oscillators were demonstrated. They exhibit, however, very low conversion efficiencies and do not fulfill conditions (ii) and (iii), ${ }^{57,108,109}$ i.e. can, for instance, not be employed in as pulse generation with $\mathrm{MHz}$ repetition rates. For frequency comb spectroscopy applications in the vacuum ultraviolet (VUV) and extreme ultraviolet (XUV), enhancement cavities were utilized to boost the pulse energies and average powers inside a resonator in order to make up for the low conversion efficiencies in $\mathrm{HHG}^{110}$. This approach has allowed to generate frequency combs with up to about $100 \mathrm{eV}$ photon energies ${ }^{111,112}$ and power levels of tens to hundreds of $\mu \mathrm{W}$ per harmonic ${ }^{113-115}$. But the concept exhibits also several drawbacks. Firstly, the setup is rather complex and expensive. Secondly, output coupling of the UV while maintaining high enhancement factors of the near-IR is a highly complex issue where no "ideal" solution for a wide parameter range has been found, yet ${ }^{116-119}$. Thirdly, enhancing large bandwidths comes with increasing difficulty and reduced enhancement factors. The shortest enhanced pulses exhibited a duration of about $20 \mathrm{fs}^{120}$, being still too long to satisfy condition (ii).

For extracavity experiments exploiting field-sensitive nonlinear optical effects, multi-pass or regenerative amplifiers are utilized to boost the pulse energies of the oscillator pulses by several orders of magnitude ${ }^{121-123}$. To avoid nonlinear effects in the additional gain media chirped pulse amplification $(\mathrm{CPA})^{124}$ has been established. It stretches pulses with fs FTLs to ns in order to strongly reduce their peak power. Recompression after amplification has allowed to reach PW peak power levels ${ }^{125,126}$. However, due to storage of pump power in the gain materials of the amplifiers and the consequent thermal load, there is a trade-off between repetition rate and peak power, in particular in Ti:sapph based CPA systems ${ }^{11}$. This trade-off is eliminated by switching from real to parametric gain materials, i.e. crystals with quadratic $\left(\chi^{(2)}\right)$ nonlinearities, exploiting so-called optical parametric chirped pulse amplification (OPCPA $)^{127}$. In this case, the quantum defect between pump and laser photon energy is not thermally dissipated but stored in a third, idle beam, i.e. in optical power. The bandwidth of OPCPAs is determined by the spectrum of the low power beam seeding the amplifier and the phase-matching bandwidth of the nonlinear crystals, which can be very broad, particularly in a noncollinear geometry ${ }^{46}$. Therefore, the powerful pulses pumping the OPCPA process can exhibit ps durations ${ }^{11}$ which are typically reached by power-scalable laser architectures. With fiber, ${ }^{28}$ innoslab, ${ }^{128}$ and thin-disk amplifiers, ${ }^{34} \mathrm{~kW}$ average power levels with pulse durations between 0.26 to $1.1 \mathrm{ps}$ have already been demonstrated.

Nevertheless, CPA and OPCPA systems are highly complex, expensive and often also noisy. They will be needed for applications in particle acceleration, relativistic optics and plasma physics ${ }^{125}$ but not necessarily to fulfill the three conditions which have been stated on the previous page. Instead, power-scalable ultrashort pulse oscillators could be utilized to generate CEP-stabilized few-cycle pulses which can be focused to peak irradiances of $10^{15} \mathrm{~W} / \mathrm{cm}^{2}$. Apart from employing such sources in HHG, time-domain applications involving free charge carriers would highly benefit. For instance, time resolved photoemission electron microscopy (PEEM) aims for high temporal and spatial resolution, but if multiple electrons are released through a single laser shot, the spatial resolution will be strongly reduced due to space charge effects ${ }^{129}$. Hence, high repetition rates and 
Table 1.2: Advantages of the thin-disk architecture ${ }^{135}$.

\begin{tabular}{ll}
\hline property & consequence \\
\hline $\begin{array}{l}\text { minimal transversal temper- } \\
\text { ature gradient }\end{array}$ & $\begin{array}{l}\text { good beam quality even at high average powers and negligible } \\
\text { stress-induced birefringence }\end{array}$ \\
$\begin{array}{ll}\text { large surface area } \\
\text { can brightness pump sources can be efficiently used and power }\end{array}$ \\
$\begin{array}{ll}\text { large surface-to-volume ratio } \\
\begin{array}{l}\text { multi-pass pumping scheme } \\
\text { with reimaging }\end{array}\end{array}$ & $\begin{array}{l}\text { more than } 90 \% \text { of pump power can be absorbed by the disk } \\
\text { despite its thickness }\end{array}$ \\
\hline
\end{tabular}

moderate intensities, just enough to free a single electron per shot, would be ideal for time-resolved PEEM. Similar arguments hold for ultrafast electron diffraction where the shortest electron pulses contain only a single particle ${ }^{130,131}$. Moreover, high repetition rate lasers employed in cold target recoil ion momentum spectroscopy (COLTRIMS) near the ionization threshold have proven to detect improbable events which can hardly be investigated with $\mathrm{kHz}$ systems ${ }^{132}$. However, the targeted studies of complex dynamics like non-sequential double ionization would strongly benefit from few- (or better single-) cycle pulses and CEP control ${ }^{133}$. The TD technology is most promising for realizing the stated applications ${ }^{13}$. It will be introduced in the next section.

\subsection{The Thin-Disk Concept - Power-scalable Ultrashort Pulse Oscillators}

In order to scale the average power achievable from solid-state lasers, Adolf GiEsen suggested to utilize only very thin gain media which act as active cavity mirrors ${ }^{134}$. For this idea and its realization, AdOLF GIESEN has received the 2017 CHARLes HARD Townes Award of the Optical Society of America. The setup of a thin-disk module is sketched in Fig. 1.2. The disks are usually very thin $(0.1 \mathrm{~mm}-0.4 \mathrm{~mm})$ and can be cooled very efficiently and homogeneously across the beam plane (Fig. 1.2(a)). Therefore, thermal effects in the gain medium can be strongly suppressed. Consequently, the laser architecture is average power scalable. Moreover, the disk diameters of $>20 \mathrm{~mm}^{2}$ allow large laser spot sizes. Hence, the fluence in the gain medium can be kept sufficiently low to avoid detrimental nonlinearities, and thus the concept is also peak power scalable. The advantages of the technology, stated in ref. 135, are summarized in Table 1.2. The success of TD manifests itself by the development of continuous wave (CW), ns, ps and fs lasers, the commercialization of the architecture by companies like Dausinger + Giesen GmbH, Jenoptik AG or TRUMPF Laser GmbH + Co. KG and the power records which have been achieved with the technology. The average powers of the first TD oscillator was $4.4 \mathrm{~W}^{134}$. Today, $\mathrm{CW}$ disk lasers routinely reach tens of $\mathrm{kW}$ of average power ${ }^{136,137}$ in multi-transversal mode operation and up to $4 \mathrm{~kW}$ in near fundamental transversal mode operation ${ }^{138}$. Ultrashort pulse amplifiers emitting ps pulses with more than $1 \mathrm{~kW}$ and good beam quality have been demonstrated as well ${ }^{33,34}$. They reach peak powers of more than $150 \mathrm{GW}$, exceeding the intracavity peak power of the oscillator presented in section 2.1 by more than 4000 times. 
(a)

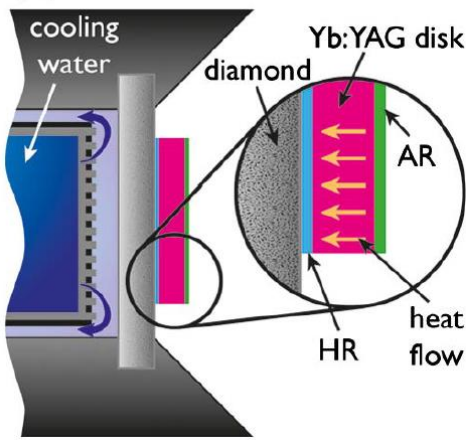

(b)

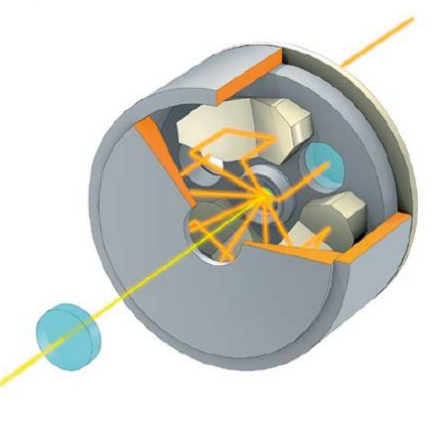

Fig. 1.2. (a) Schematic side view on a TD. The disk is anti-reflection (AR) coated at the front side and high reflection (HR) coated on the rear side, and thus acts as a turning mirror (with gain) inside the laser resonator. The yellow arrows show the coaxial heat flow which ensures low thermal gradients across the laser beam. In this case, the gain medium is ytterbium doped yttrium-aluminumgranate (Yb:YAG), but other active media are utilized as well. The sketch shows that the TD is bonded to a diamond heat sink. This is usually done by the disk supplier TRUMPF Laser GmbH. In most of the experiments presented in this thesis, the used disk is bonded to a copper heat sink (disk was provided by Dausinger + Giesen $\mathrm{GmbH})$. Both materials exhibit extraordinary heat conductivity, and thus the thermal power $(\approx 150 \mathrm{~W}$ for the oscillator used in most of the experiments) can effectively be dissipated in the cooling water circuit. (b) Sketch of a simple TD laser. The pump light is entering the disk head from the rear side and passes the disk multiple times by virtue of an imaging system consisting of parabolic mirrors. Multiple passes are necessary to let the thin disk absorb a large share of the pump light. The simple resonator consists only of the gain medium and an output coupler (OC) which is located in front of the disk head. Picture taken from ref. 11 .

The first mode-locking of a TD laser was demonstrated in $2000^{21}$. An average power of $16.2 \mathrm{~W}$ was reported. The pulse energy was $0.47 \mu \mathrm{J}$ and the duration $730 \mathrm{fs}$. Modelocking was realized with SESAM, i.e. a slow saturable absorber. These types of fs- to ps-laser oscillators have been continuously developed during the past 18 years. They now reach average powers of far more than $100 \mathrm{~W}$, pulse energies of several tens of $\mu \mathrm{J}$ and peak powers of more than $60 \mathrm{MW}^{58,60,61}$. Contrary to CW lasers and ps amplifiers, fs oscillators have not been commercialized, yet. This might be due to the shortcomings of the SESAMs which have been pointed out in ref. 65. In 2011, a first KLM TD oscillator was demonstrated ${ }^{23}$. It operated at $45 \mathrm{~W}$ average power and emitted pulses of only $270 \mathrm{fs}$ duration. With a lower output coupling ratio, which reduced the intracavity losses, pulses of only $200 \mathrm{fs}$ were reported. This immediately revealed an important advantage of the fast saturable absorber over SESAM: the ability to generate clearly shorter pulses. A comparison of the different mode-lockers is, for instance, presented in ref. 65. Despite the eleven years advance of SESAM mode-locked oscillators, KLM TD oscillators quickly caught up in their development. In 2014, a $270 \mathrm{~W}$ average power laser was demonstrated ${ }^{59}$, very comparable to the $275 \mathrm{~W}$ stated for the highest power SESAM fs oscillator presented in $2012^{58}$. Moreover, peak power levels above $60 \mathrm{MW}$ were reached for SESAM modelocked oscillators in $2014^{61}$ and for KLM in $2016^{139}$. Intracavity pulse energies clearly exceeding the record of $320 \mu \mathrm{J}$ generated from a SESAM mode-locked oscillator, were also enabled by a KLM TD (ring) oscillator. This was however, only reported in a conference proceeding ${ }^{140}$. Unfortunately, the stability and the beam quality of the laser has not allowed any application of the light source until today and a journal publication of the group only claimed a clearly reduced intracavity pulse energy ${ }^{141}$. 
All the stated records have been achieved with ytterbium ion $\left(\mathrm{Yb}^{3+}\right)$ doped yttriumaluminumgranate, $\mathrm{Y}_{3} \mathrm{Al}_{5} \mathrm{O}_{12}$ (YAG) as the gain medium, presenting a nearly perfect material for high-power solid-state lasers ${ }^{142}$. It is optically isotropic, mechanically robust and possesses a high thermal conductivity. Furthermore, its absorption lines at $969 \mathrm{~nm}$ (zero-phonon line) and $940 \mathrm{~nm}$ (first-phonon line) overlap with the emission wavelengths of InGaAs laser diodes. These can be stacked to deliver up to multiples of $\mathrm{kW}$ optical power. Fiber coupled diode units are available from commercial suppliers like DILAS Diodenlaser GmbH (now part of Coherent) or Laserline GmbH. Yb:YAG lasers typically emit at $1030 \mathrm{~nm}$ central wavelength, i.e. if pumped with $969 \mathrm{~nm}$, the quantum defect (or STOKES shift) is about $76 \mathrm{meV}$, corresponding to only $6 \%$ of the (pump) photon energy. For comparison, if a Ti:sapph laser is pumped at $532 \mathrm{~nm}$ and emits at a central wavelength of $790 \mathrm{~nm}$, the quantum defect is $761 \mathrm{meV}$ and $33 \%$, resp. Although a first TD Ti:sapph oscillator has been recently demonstrated, ${ }^{143}$ the much higher relative quantum defect basically disqualifies the gain material from being nearly as power scalable as Yb:YAG. Additionally, green laser diodes, which are desirable for efficient pumping, are clearly inferior to InGaAs diodes as they come with significantly lower wall-plug efficiencies and lifetimes ${ }^{144-146}$. On the other hand, the fluorescence linewidth of Yb:YAG is, for instance, only about $\Delta \lambda_{f}=9 \mathrm{~nm}$ FWHM at room temperature ${ }^{147}$, compared to $\Delta \lambda_{f}=230 \mathrm{~nm}$ for Ti:sapph ${ }^{142}$. Although near emission bandwidth limited $140 \mathrm{fs}$ pulses have been generated at high efficiency ${ }^{139}$ and going even beyond the FWHM of the fluorescence band was demonstrated $^{82}$, direct few-cycle pulse generation from Yb:YAG oscillators seems impossible. This motivates to retrace the early approach on sub- $10 \mathrm{fs}$ pulse generation with dye lasers and employ external compression schemes. Just "copying" the existing principles is, however, not possible. Typical fs oscillators operate at sub- or low MW peak power levels where solid-core silica fibers can be employed. By contrast, amplifiers operate at multiple hundreds of MW or GW peak power. The gap between these two regimes, where the latest mode-locked TD oscillators fall into, is hardly explored and requires to go beyond the established compression schemes. Different approaches will be discussed in chapter 3 of this thesis.

It is to note that other gain materials have been envisioned or utilized in the TD geometry as well. Two (slightly outdated) overviews are, for instance, presented in refs. 148 and 149 . A few years ago, a $\mathrm{Yb}: \mathrm{CaGdAlO}_{4}$ (Yb:CALGO) oscillator was SESAM mode-locked ${ }^{150}$. It emitted $62 \mathrm{fs}$ pulses, but only delivered $5 \mathrm{~W}$ of average power and could not be powerscaled so-far. A recent publication presented 35 fs pulses emitted from a KLM Yb: $\mathrm{Lu}_{2} \mathrm{O}_{3}$ thin-disk oscillator, however, also only at $1.6 \mathrm{~W}$ average power ${ }^{83}$. Another very recent development reports on a first ultrafast TD oscillator emitting at $2 \mu \mathrm{m}$ with an average power of $20 \mathrm{~W}^{151}$. The result is highly interesting for applications in the mid-infrared. 


\subsection{Fundamentals}

So far, the methods which will be discussed in this thesis have been considered from a historical perspective. In this section their physical backgrounds shall be introduced in an illustrative way without going into great detail. The reader may be referred to many excellent graduate level textbooks where all concepts have been explained in a comprehensive and mathematically neat form. This is beyond the scope of this dissertation which will just present a few mathematical derivations where particularly helpful.

\subsubsection{Mode-beating}

As the section is called "fundamentals", it starts with a very basic experiment which is usually taught in middle or high school physics courses. Two tuning forks emitting acoustic waves at slightly different frequencies shall be considered, or, since this is a thesis about lasers, two perfect CW lasers emitting optical waves at slightly different frequencies (cf. Fig. 1.3). While a single tuning fork gives a constant clear tone which is used as a reference for singers, two slightly detuned forks give a similar tone which, however, is periodically modulated in volume (cf. Fig. 1.3(c)). Mathematically, this is shown as follows:

$$
\begin{aligned}
E(t) & =\cos ([\omega-\Delta \omega] \cdot t+\underbrace{\varphi_{1}}_{=0})+\cos ([\omega+\Delta \omega] \cdot t+\underbrace{\varphi_{2}}_{=0}) \\
& =\frac{1}{2}\left(e^{i(\omega-\Delta \omega) t}+e^{-i(\omega-\Delta \omega) t}\right)+\frac{1}{2}\left(e^{i(\omega+\Delta \omega) t}+e^{-i(\omega+\Delta \omega) t}\right) \\
& =\frac{1}{2}\left(e^{i \Delta \omega t}+e^{-i \Delta \omega t}\right)\left(e^{i \omega t}+e^{-i \omega t}\right) \\
& =\underbrace{2 \cos (\Delta \omega t)}_{A(t)} \underbrace{\cos (\omega t)}_{\operatorname{car}(t)},
\end{aligned}
$$

where $\omega$ is the angular frequency, $t$ the time, $E(t)$ the field amplitude, $A(t)$ is the (slowly varying) envelope of the wave and $\operatorname{car}(t)$ its carrier. A wave does not only evolve in time but also in space, and hence the time and space dependent field reads:

$$
E(t, \vec{r})=2 \cos (\Delta \omega t-\Delta \vec{k} \cdot \vec{r}) \cos (\omega t-\vec{k} \cdot \vec{r}),
$$

(a)

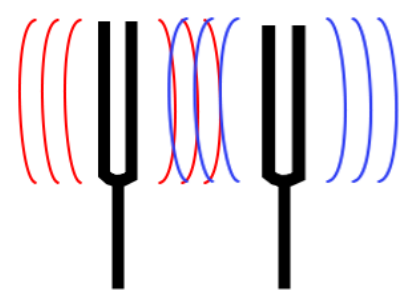

(b)

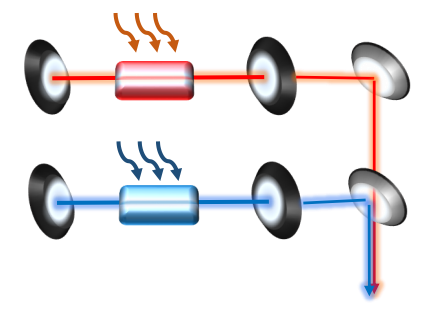

(c)

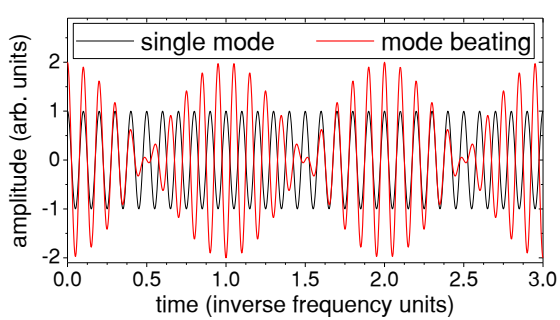

Fig. 1.3. (a) Two tuning forks emitting acoustic waves at slightly different frequencies. (b) Two perfect CW lasers emitting optical waves at slightly different frequencies. (c) The functions $\cos (10 \cdot t)$ (black line) and $\cos ([10-0.5] \cdot t)+\cos ([10+0.5] \cdot t)$ (red line). 
where $\vec{k}$ is the wave vector and $\vec{r}$ the space coordinate. The velocity of the carrier phase fronts ("phase velocity", $v_{p}$ ), which fulfill $\omega t-\vec{k} \cdot \vec{r}=$ const., can be readily derived:

$$
\vec{v}_{p}:=\frac{d \vec{r}}{d t}=\frac{d}{d t}(\omega t-\text { const. }) \frac{\vec{k}}{|k|^{2}}=\frac{\omega}{|k|} \hat{k}
$$

where $\hat{k}$ is the unit vector in propagation direction. In the same way the velocity of the envelope ("group velocity", $v_{g}$ ) is determined, yielding $\vec{v}_{g}=\Delta \omega /|\Delta k| \Delta \hat{k}$. If the wave packet consists of a quasi-continuous set of modes, this expression is generalized to

$$
v_{g}:=\frac{1}{|d \vec{k} / d \omega|_{\omega_{0}}},
$$

where $\omega_{0}$ is the carrier angular frequency.

\subsubsection{Mode-locking}

The case of a wave packet consisting of a quasi-continuous set of modes describes the pulses emitted by a mode-locked oscillator. The laser cavity which is used in most of the experiments has a roundtrip time of about $25 \mathrm{~ns}$ for a light pulse with a $3 \mathrm{~dB}$ bandwidth of about $5 \mathrm{~nm}$ at $1030 \mathrm{~nm}$ central wavelength, corresponding to a frequency width of 1400/ns. Consequently, about $N=1400 \cdot 25 \approx 35000$ modes (or "perfect CW lasers") oscillate in the utilized resonator within the FWHM of the laser pulse spectrum. In Fig. 1.3(c) and Eq. (1.4) it became apparent that already the existence of two modes allows the description of the wave in terms of carrier and envelope. Constructive interference of the modes doubled the amplitude (quadrupled the intensity) in comparison to the single mode while destructive interference led to an instantaneous amplitude of zero. Fig. 1.4(a)

(a)

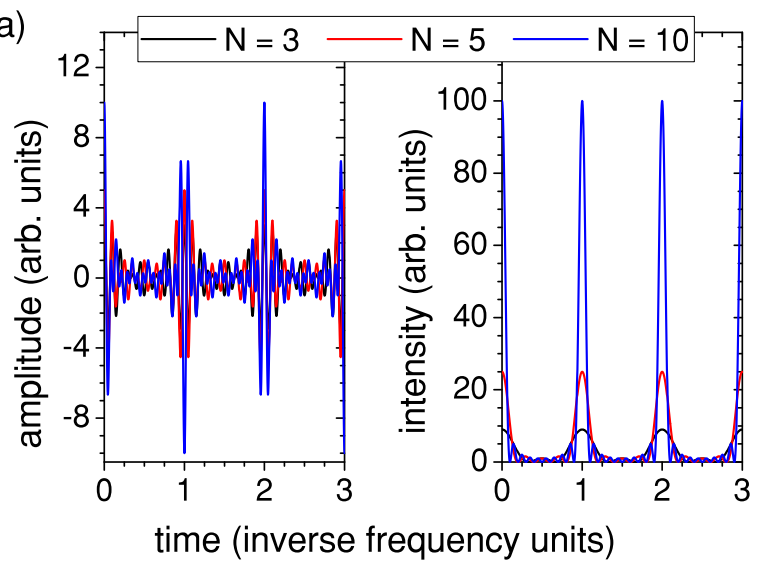

(b)

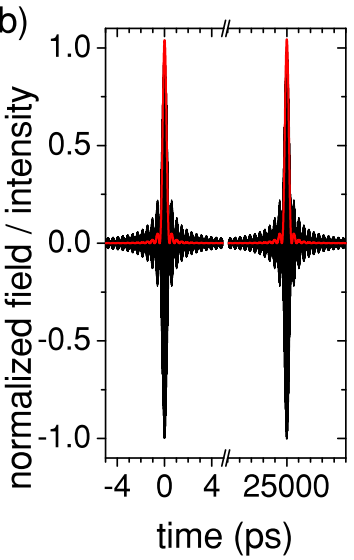

(c)

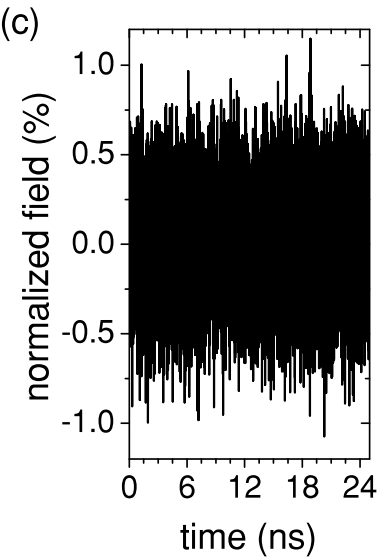

Fig. 1.4. (a) Field (left graph) and cycle-averaged intensity (right graph) of 3,5 and 10 modes oscillating in phase, $\omega=10, \Delta \omega=1$ like in Fig. 1.3(c). The more modes, the higher the amplitude, the narrower the FWHM and the longer the dead-time. (b) Normalized field (black line) and cycle-averaged intensity (red line) for $\omega=2 \pi \cdot 291 \mathrm{THz}, \Delta \omega=2 \pi \cdot 40 \mathrm{MHz}$ and $N=60000$. The modes are phase-locked. Note the long dead-time of about $25 \mathrm{~ns}$, about five orders of magnitude longer than the FWHM of the pulse. The situation is comparable to the utilized oscillator. However, a constant mode amplitude (i.e. flat gain) has been assumed which is in reality not the case. (c) Same parameters like in (b), but each mode has a random phase. The field is normalized as in (b). One spike at around $18 \mathrm{~ns}$ reaches slightly more than $1 \%$ of the maximal field strength. It will undergo the highest gain during a cavity roundtrip. 


\section{Remark: What determines the pulse duration?}

The description in this section might be somewhat misleading in the sense that the number of oscillating modes does not directly determine the pulse duration. Like the mode spacing $\Delta \omega$, it is set by laser repetition rate which is in general independent of the pulse duration. Instead, the spectral width $(N \cdot \Delta \omega)$ is decisive for the pulse duration. In the shown examples, it increases with $N$ by assuming constant $\Delta \omega$. In a real mode-locked oscillator it is, however, determined by the gain medium in the first place, and furthermore by intracavity nonlinearities and GDD ${ }^{78}$.

shows how these effects get enhanced if additional modes are added. Fig. 1.4(b) displays an example which is close to the situation in a real laser. In this case, 60000 modes were constructively added and the ratio of $\Delta \omega / \omega_{0}$ is on the order of $10^{-7}$. This results in a very narrow pulse $(\approx \mathrm{fs})$ and a long dead-time $(\approx \mathrm{ns})$ where the optical intensity is practically zero. Fig. 1.4(c) shows that the field behaves completely different if a random (time-independent) phase term is added to the thousands of cosine functions which have been introduced for two modes in Eq. (1.1). The modes are not locked in this case, but oscillate freely. Many textbooks introduce mode-locking in almost exactly the same way (e.g. refs. 71, 152-155). They may be consulted for further details about the technique. Here, it is only briefly discussed how to get from the random phase case displayed in Fig. 1.4(c) to the phase-locked situation of Fig. 1.4(b).

In section 1.2, it has been already mentioned that passive mode-locking requires some kind of saturable absorber in the laser cavity. In the experiments presented in this dissertation, a fast saturable absorber based on the optical KERR effect has always been exploited. The response of a dielectric medium to an external intense light field is usually described well by bound electrons which follow the light field quasi-instantaneously. Consequently, the dispersion of the material polarization $P$ plays a negligible role. It can be described as ${ }^{156}$ :

$$
P(t)=\epsilon_{0}\left[\chi^{(1)} E(\vec{r}, t)+\chi^{(2)} E(\vec{r}, t)^{2}+\chi^{(3)} E(\vec{r}, t)^{3}+\mathcal{O}\left\{E(\vec{r}, t)^{4}\right\}\right],
$$

where $\epsilon_{0}$ denotes the vacuum permittivity, $\chi^{(n)}$ the formally TAYLOR expanded $n^{\text {th }}$ order susceptibility and $E(t)$ the real electric field. The linear term determines the material's refractive index, the second term the so-called three-wave mixing processes (two waves in the $E^{2}$ term of the polarization and the third in the wave equation), the third term the socalled four-wave mixing processes and so on. The used KERR medium, sapphire (c-cut), is centrosymmetric, and thus three-wave mixing processes are suppressed ${ }^{156}$. The higherorder terms exhibit only a negligible influence on the material polarization. The $\chi^{(3)}$-term remains. It includes a self-action contribution $\Re\left\{\chi^{(3)}\right\}|A(t)|^{2} E(t)$ ( $\Re$ denotes the real part and $|A|^{2}$ is proportional to the cycle averaged intensity) from which a nonlinear refractive index $\left(n_{2}\right)$ :

$$
\Delta n(\vec{r}, t)=n_{2} I(\vec{r}, t)
$$

is derived ${ }^{157}$, where $\Delta n(r, t)$ is the intensity $(I)$ dependent refractive index change. Typical $n_{2}$ values of dielectrics are on the order of $10^{-16}-10^{-15} \mathrm{~cm}^{2} / \mathrm{W}^{158}$, i.e. even at high peak irradiances of $1 \mathrm{TW} / \mathrm{cm}^{2}$, the maximal refractive index change is only on the order of $10^{-4}-10^{-3}$. Nevertheless, due to the Gaussian profile of the laser pulses, the KERR effect induces a wavefront curvature which leads to a self-focusing effect ${ }^{159,160}$. Fig. 1.5 

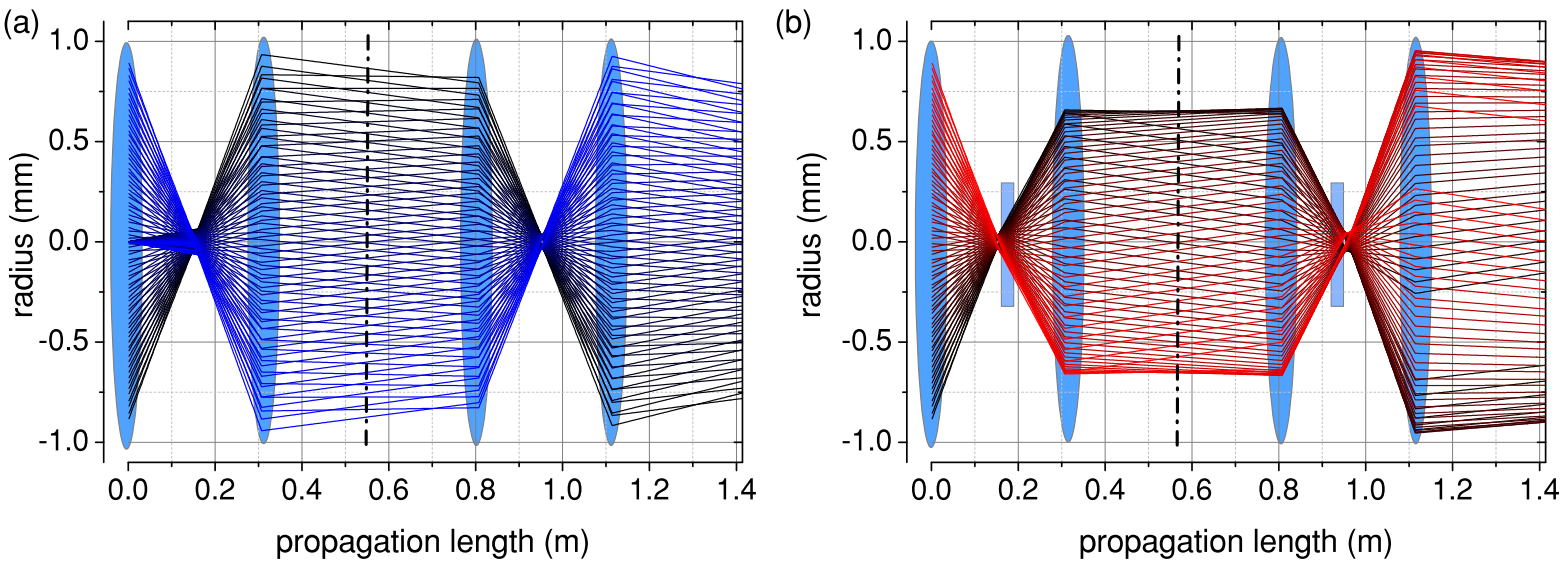

Fig. 1.5. Ray tracing of the unfolded telescope setup inside the oscillator (cf. Fig. 2.1(b)) which acts as a fast saturable absorber. (a) CW operation. The peak irradiance is low, and hence there is no KERR lensing effect. (b) Mode-locked operation. The KERR-effect introduces a third lens in the telescope which reduces the beam size near the folding mirror and improves the collimation at the exit of the telescope. Focal lengths $(150 \mathrm{~mm})$, peak power $(36 \mathrm{MW})$ and KERR medium thickness $(1 \mathrm{~mm})$ have been adapted from the experimental situation. Most of the ray tracing was done with ABCD matrices, ${ }^{163}$ only the propagation inside the KERR medium was based on FERMAT's principle. It will be explained in more detail in section 3.2.2. The intensity inside the medium was assumed to be constant. The telescope length $(307 \mathrm{~mm})$ and the distance between focal plane and KERR medium input facet $(8 \mathrm{~mm})$ were free parameters of the model. They are also close to the experimental situation. For simplicity, normal incidence on the KERR plate was assumed.

indicates how to utilize this self-lensing effect to create a fast saturable absorber which initiates and maintains mode-locking. It shows the propagation of ray packet equivalents of Gaussian beams ${ }^{161}$ through a telescope consisting of two spherical mirrors (depicted as lenses in the graphs), a folding mirror (dashed-dotted line in the graphs) and a $1 \mathrm{~mm}$ thick fused silica (FS) plate acting as a KERR-lens for intense beams. Fig. 1.5(a) considers the propagation of CW radiation. Due to the low irradiance, it does not undergo self-focusing. Therefore, the beam size close to the folding mirror is larger than for an intense mode-locked beam (Fig. 1.5(b)) and the collimation when exiting the telescope is worse. Consequently, by putting a hard aperture close to the folding mirror, the CW beam experiences higher losses during the roundtrip than the mode-locked beam. Furthermore, CW light and pulsed light are diverging differently which also introduces an additional "soft aperture" by virtue of changing the overlap of laser and pump spot in the gain medium. This also leads to an improved gain-loss-ratio for the pulsed operation ${ }^{65,155}$. The behavior forces the oscillator to amplify the most intense spikes of chaotic light which leads to a transition from the random phase output shown Fig. 1.4(c) to the mode-locked operation illustrated in Fig. 1.4(b). Such a transition has been recently resolved in time and frequency, ${ }^{162}$ presenting a more detailed picture of the outlined procedure.

\subsubsection{Self-phase-modulation}

After having discussed self-focusing, which originates from the spatial gradient of an intense laser beam, self-phase modulation, which originates from the temporal gradient of an intense laser pulse, is introduced. It will be studied in parts of the chapters $2-4$ and is of strong importance for this thesis. The simplest way to look at this phenomenon is to 

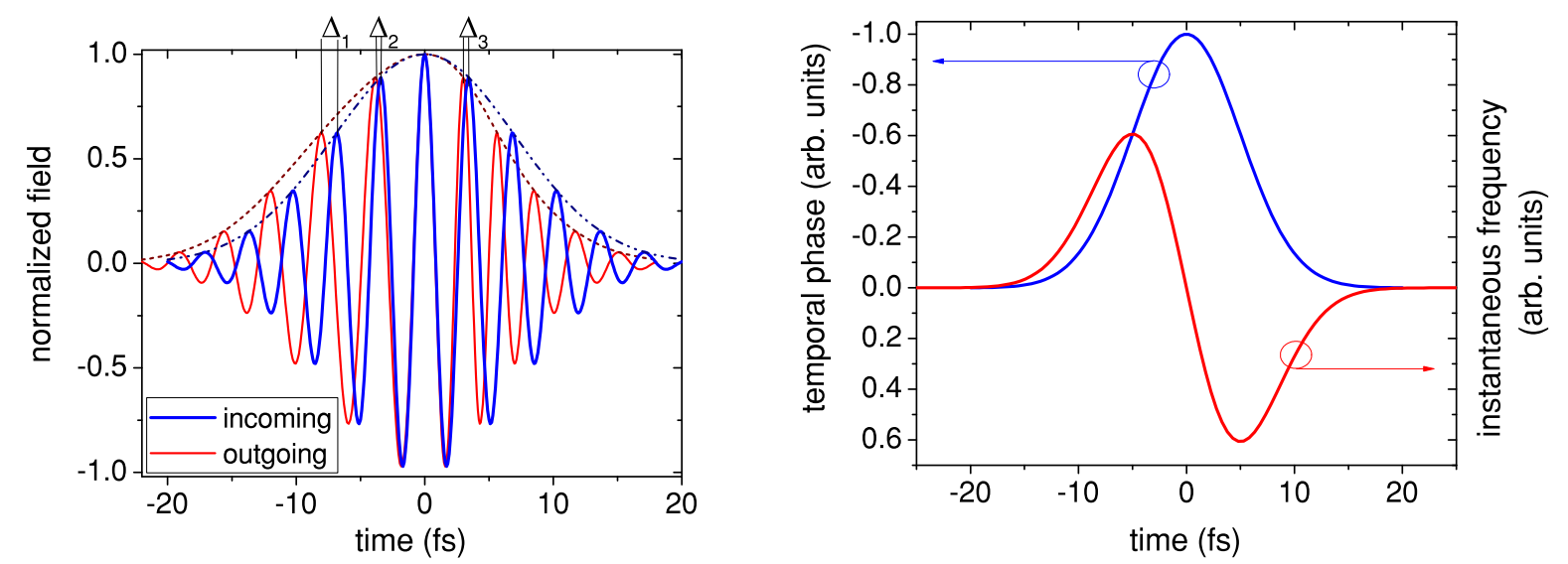

Fig. 1.6. (a) A FouRIER transform-limited $10 \mathrm{fs}$ pulse with a peak irradiance of $1 \mathrm{TW} / \mathrm{cm}^{2}$ (blue line) travels through $2 \mathrm{~mm}$ of a material with $n_{2}=3 \times 10^{-4} \mathrm{~cm}^{2} / \mathrm{TW}$. The red line shows the pulse shape after propagation. It is simply evaluated by means of Eq. (1.10). The central field peak is overlapped in time. The dashed lines show the pulse envelope which becomes slightly asymmetric. Due to symmetric velocity profile, the pulse duration stays the same, however. While for the incoming pulse the cycle periods are constant, they get increasingly shorter at later times after propagation as the $\Delta$ 's indicate. The pulse is chirped. (b) SPM induced temporal phase and its time derivative, the instantaneous frequency. The frequency sweep in the pulse center is nearly linear, corresponding to linear chirp. Each frequency appears at two instances of time, yielding the characteristic periodic modulation of a self-phase modulated spectrum. The $\mathrm{y}$-axis is inverted which accounts for the minus sign in the $\omega t-\vec{k} \cdot \vec{r}$ term. Hence, like in (a), the longer wavelengths precede the shorter ones near the pulse center.

incorporate Eq. (1.9) into the expression for the speed of light $c$ :

$$
c(t)=\frac{c_{0}}{n_{0}+n_{2} I(t)}
$$

where $c_{0}$ is the vacuum speed of light and $n_{0}$ the linear refractive index. Obviously, the center of the laser pulse is propagating slower than the wings, yielding a characteristic shift of the phase fronts that is exemplary shown in Fig. 1.6(a). While in the FOURIER transform-limited case, the peaks of the electric field are equidistant in time, which corresponds to a constant instantaneous frequency equal to the carrier frequency, the self-phase modulated pulse exhibits a frequency chirp where the long wavelengths precede the shorter ones. The width of purely self-phase modulated pulse remains constant, however. As indicated in the previous section, the shortest pulses possible with a fixed bandwidth are achieved if all modes oscillate in phase. Subsequently, the spectral width of the self-phase modulated pulse must be broader than that of the initial one. This is the main application of SPM which will be employed in sections 2.2, 3.1, 3.2.1 and 4.2. The simple picture of Fig. 1.6(a) also indicates what happens if prechirped pulses undergo SPM. If the pulses are initially down-chirped, the effect described above is reversed, i.e. the SPM induced chirp counteracts the prechirp and the outgoing pulse can thus be nearly Fourier transform-limited. But since the pulse duration stays the same, SPM induces spectral narrowing ${ }^{164,165}$. Applying positive input chirp modifies the spectral shape of a self-phase modulated pulse, but still leads to spectral broadening ${ }^{165}$. This will be investigated in more detail in section 3.1.1.

The more formal and most common approach on SPM (cf. the comprehensive description in ref. 165) is adding temporal phase $(\Delta \varphi(t))$ to the time-dependent electric field of the 
(a)

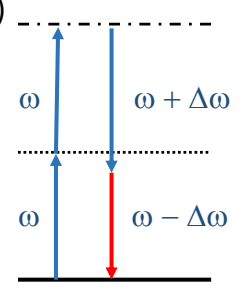

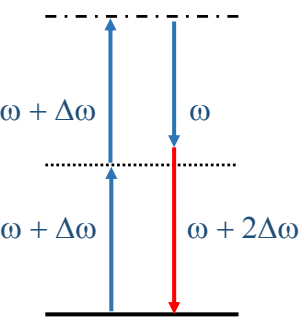

(b)

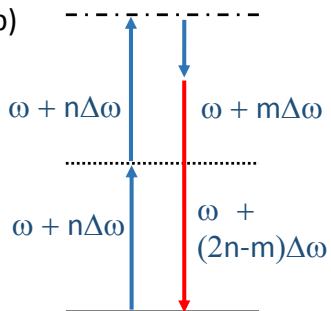

(c)

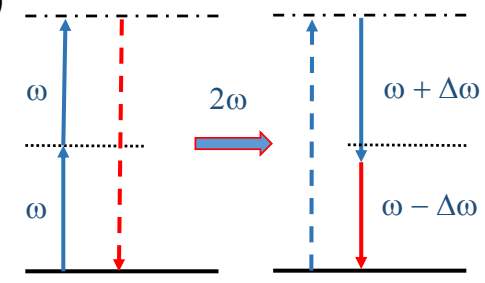

Fig. 1.7. Four-wave mixing processes. The blue arrows show the input waves (in the polarization term) and the red arrows the output wave. The arrows heading upwards indicate a plus sign, the arrows heading downwards a minus sign. (a) Four-wave mixing of two neighboring comb modes. The incoming modes at $\omega$ and $\omega+\Delta \omega$ mix and generate new modes at $\omega-\Delta \omega$ and $\omega+2 \Delta \omega$. The comb spacing is maintained.

(b) Example of four-wave mixing at the pulse edges where strongly non-degenerate frequencies overlap. (c) Cascaded $\chi^{(2)}$-nonlinearities leading to an effective $\chi^{(3)}$-process. The input- and the output are effectively the same like in the left diagram in (a). However, the process is mediated by the generation and consequent destruction of a photon at $2 \omega$.

pulse. This follows from Eqs. (1.5) and (1.9):

$$
\begin{aligned}
\omega t-\vec{k} \cdot \vec{r} & =\omega t-\left(n+n_{2} I(t)\right) k_{0} z, \\
\Rightarrow \Delta \varphi(t) & =-n_{2} I(t) k_{0} z
\end{aligned}
$$

where the z-axis was chosen as the propagation direction and $k_{0}=\omega / c_{0}$. It is to note that the minus sign in Eq. (1.12) depends on the convention in the carrier term which can be either $\omega t-\vec{k} \cdot \vec{r}$ or $\vec{k} \cdot \vec{r}-\omega t$. If ultrashort pulses are considered, the $\vec{k} \cdot \vec{r}$ term is often dropped, making the origin of $\Delta \varphi(t)$ unclear which frequently leads to a wrong sign of it. Since $\Delta \varphi(t)$ is a pure phase term, it does not change the pulse shape, but only the spectral shape. The instantaneous angular frequency is defined as the time derivative of the temporal phase. Both quantities are shown in Fig. 1.6(b) (excluding the carrier term). A Gaussian pulse shape was assumed. Like in (a), the frequency chirp is apparent, it is nearly linear in the vicinity of the pulse center where most of the optical power is concentrated. Another feature of SPM becomes readily apparent. Each instantaneous frequency occurs at two instances of time. This leads to a beating signature in the spectrum, comparable to the beat signal in time shown Fig. 1.3. Eq. (1.12) will be used in supplement 2 of section 3.1.1 where the spectral broadening factor for positively prechirped pulses is predicted.

Finally, SPM shall be interpreted as a four-wave mixing process. From the time-domain treatment it becomes clear that a perfect $\mathrm{CW}$ laser cannot undergo spectral broadening since a continuous wave does not exhibit a temporal gradient. Consequently, at least two modes must be present. Fig. 1.7(a) shows diagrams which illustrate SPM in the frequency domain. The incident two modes symmetrically spectrally broaden to four modes. This picture indicates that the equidistant mode spacing dictated by the laser cavity is preserved although the spectral shape changes. Maintaining the initial frequencies comb structure and mutual pulse coherence, respectively, is essential for stabilizing the carrier-envelope-offset frequency and employing the spectrally broadened light source in field-sensitive experiments ${ }^{166,167}$. While in the pulse center of a self-phase modulated pulse the chirp is linear which favors the interaction of neighboring modes, in the pulse wings stronger non-degenerate frequencies temporally overlap after a sufficiently long propagation distance. This results from the SPM-induced down-chirp in combination 
with the normal dispersion of the optical medium. In other words, the trailing red-shifted frequencies catch up with leading blue shifted frequencies, and may thus mix to new frequency components. This phenomenon is illustrated in Fig. 1.7(b). The effect, called optical wave-breaking (OWB), is common in fiber-based spectral broadening. It is described in more detail in ref. 165. Among the plurality of $\chi^{(3)}$ processes $^{168}$, an effect leading also to spectral broadening shall be pointed out which is of relevance in this thesis (cf. section 3.2.2). It is actually not a four-wave mixing process in the stricter sense, but a sequence of two three-wave mixing processes (cascaded $\chi^{(2)}$ ). A second harmonic (SH) photon is generated first and after a short propagation length destroyed again. Since the photon is neither present at in- nor output, the process is effectively also on the order of $\chi^{(3)}$. The creation and the destruction process of the SH does not need to involve exactly the same frequencies. If, for instance, two $\omega$ photons create a $2 \omega$ photon $(\omega+\omega=2 \omega)$ which interacts with a $\omega+\Delta \omega$ photon, a new $\omega-\Delta \omega$ photon is generated $(2 \omega-\omega-\Delta \omega=\omega-\Delta \omega)$ as it is illustrated in Fig. 1.7(c). Refs. 169 and 170 explain cascaded quadratic nonlinearities in more detail.

So-far, the effects derived from Eq. (1.9) resulting from the temporal and spatial gradient of the irradiance have been considered separately. In section 3.2, the interplay between self-focusing and SPM will be investigated and discussed explicitly.

\subsubsection{Dispersion compensation}

In the previous paragraph, it was shown that a self-phase modulated pulse is chirped. Therefore, dispersive elements must be utilized to compress the spectrally broadened pulse to its FTL. In the beginning of this fundamentals section, the group velocity of two propagating modes was derived. The term $\Delta \omega / \Delta k$ can be rewritten to $c_{0}\left(\omega_{i+1}-\right.$ $\left.\omega_{i}\right) /\left(n_{i+1} \omega_{i+1}-n_{i} \omega_{i}\right)$ where $i$ is the mode number and $n_{i}$ the linear refractive index at the frequency of the $i^{\text {th }}$ mode. Consequently, the group velocity would only be a constant over the whole laser spectrum if $n$ would exhibit no or a linear wavelength dependence. This is only the case in vacuum, and hence the group velocity is generally dispersive. An intuitive definition for the GVD would thus be ${ }^{171}: \mathrm{GVD}^{*}:=d v_{g} / d \lambda$, where $\lambda$ is the wavelength. However, for pulse propagation, it is more convenient to define the GVD as follows ${ }^{171}$ :

$$
\operatorname{GVD}:=\left.\frac{\partial^{2} k}{d \omega^{2}}\right|_{\omega_{0}}=-\left.\frac{1}{v_{g}^{2}} \frac{d v_{g}}{d \omega}\right|_{\omega_{0}} .
$$

This is the definition which will be used throughout this dissertation. It is to note that the GVD is, like the group velocity, evaluated at the carrier frequency $\omega_{0}$. Of course, also the GVD is dispersive and its derivative is then called TOD and so on. Expanding the angular wavenumber $k$ in this manner into a TAYLOR series is often useful because terms higher than second order usually influence the pulse duration only for few-cycle pulses ${ }^{54}$. The expansion is discussed explicitly in refs. 171 and 172 .

The GDD describes the linear chirp an optical element applies to an ultrashort pulse. It is just the GVD multiplied by the propagation length for pulse propagation through a homogenous medium. Up to about $1.2 \mu \mathrm{m}$ wavelength, it is not possible to utilize bulk media in order to compress self-phase modulated pulses since all common materials exhibit normal dispersion, and thus can only compensate for down-chirp. In the mid-IR, 
(a)

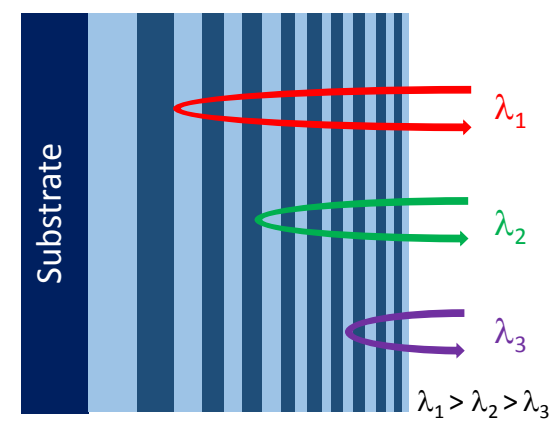

(b)

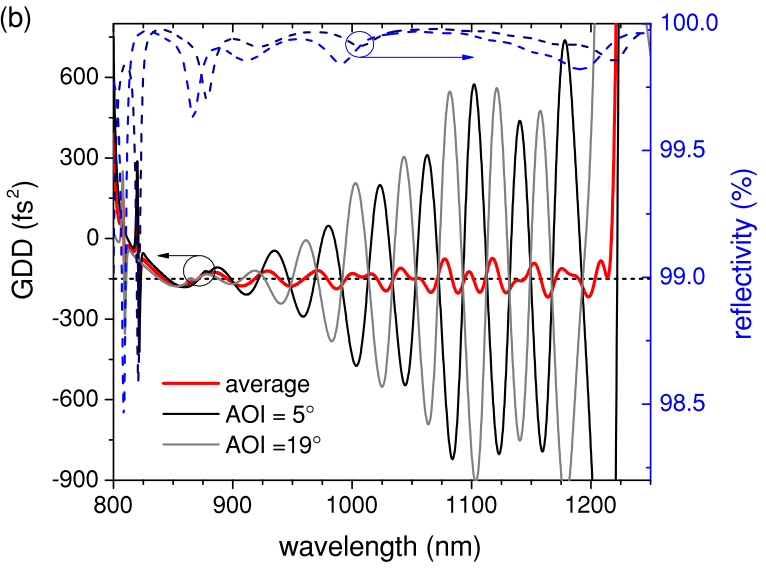

Fig. 1.8. (a) The chirped mirror principle. The longer wavelength penetrate deeper into the multilayer structure and thus get delayed with respect to the shorter wavelengths which leads to effective negative GDD. (b) Chirped mirror example - "PC1305". The GDD of the single mirrors oscillates strongly, in particular for longer wavelengths due to the cavity effects in the multilayer structures. The oscillations can, however, be largely compensated by using one mirror with $5^{\circ}$ angle of incidence (AOI) and one with $19^{\circ} \mathrm{AOI}$, as the red solid line shows. The GDD is nearly constant over the range from $825 \mathrm{~nm}$ to $1200 \mathrm{~nm}$. For reference, the black dashed line shows GDD $=-150 \mathrm{fs}^{2}$. The reflectivity (blue dashed lines) is about $99.8 \%$ over the whole range of flat GDD. (Courtesy: Vladimir Pervak)

both normally and anomalously dispersive materials exist, offering at least full control over the quadratic spectral phase of an ultrashort pulse which is related to the GVD of a material. Optical elements like prism or grating pairs can also introduce anomalous dispersion in the near-IR by firstly, spatially separating the frequency components of the pulse, secondly, delaying the long with respect to the short wavelengths by letting them travel a longer optical path, and thirdly, spatially recombining the frequencies. The techniques are described in refs. 172 and 173. They offer somewhat limited control over the spectral phase of the ultrashort pulses - only over GDD or GDD and TOD if prisms and gratings are combined ${ }^{88,174}$.

In this dissertation, chirped mirrors are used for dispersion compensation. Through the ability to precisely tune the penetration depth of the wavelength components into a multilayer structure, they combine the highest degree of phase control with low losses and large bandwidths ${ }^{54,97,172}$. The concept of a chirped mirror is sketched in Fig. 1.8(a). The picture, however, oversimplifies the working principle since the individual wavelength components are not totally reflected at a specific penetration depth, but undergo partial FRESNEL reflections on all interfaces of the multilayer structure. This leads to interference effects comparable to those in a FABRY-PEROT cavity ${ }^{172}$. Therefore, numerical tools are utilized ${ }^{175,176}$ to optimize the multilayer design for an anticipated phase and reflectivity. Moreover, among other approaches, complementary mirror pairs ${ }^{177}$ and double-angle configurations ${ }^{178}$ smooth the inevitable dispersion oscillations that arise from the cavity effects in the layer structure. Fig. 1.8(b) shows an example for chirped mirrors which are utilized in multiple experiments presented in this thesis. They exhibit almost $100 \%$ reflectivity over the whole operation range from $825 \mathrm{~nm}$ to $1200 \mathrm{~nm}$. By combining two AOIs the GDD oscillations are strongly suppressed and the mirror pair exhibits a nearly perfectly flat GDD with an average of $-150 \mathrm{fs}^{2}$ per bounce. 


\subsubsection{Frequency combs}

In the previous paragraph, it was noted that the group velocity is generally dispersive. In particular, all of the oscillator modes travel with a different (phase) velocity through the laser cavity. Nevertheless, it suffices to describe the emitted femtosecond pulses composed of thousands of modes by the carrier and the envelope term (at least at pulse durations which are obtained in this thesis), similar to Eq. (1.4) where only two modes were considered. Ref. 179 has demonstrated with a precision of better than $10^{-15}$ that the spacing between the individual modes is equidistant and equal to the repetition rate of the laser. Therefore, the output of a mode-locked oscillator has been considered as a perfect frequency ruler which is, however, not intrinsically calibrated. Obviously, the line spacing varies with the roundtrip time of the laser pulse which is directly related to the resonance frequencies of the laser cavity. Consequently, full stabilization of a frequency comb needs firstly, stabilization of the repetition rate which is usually done by means of a piezoelectric-actuated mirror ${ }^{180}$. Secondly, the zero-offset of the frequency ruler must be stabilized. It originates from the difference of the (phase) velocity of the carrier and the (group) velocity of the envelope ${ }^{181}$ and is therefore called carrier-envelope-offset frequency $\left(f_{\text {ceo }}\right)$.

On the one hand, for the envelope, a roundtrip takes the time $T=2 L / v_{g}=1 / f_{\text {rep }}$ where $L$ is the physical length of the (linear) laser resonator and $v_{g}$ is averaged over $L$. On the other hand, for the carrier, a roundtrip takes $T_{c}=2 L / v_{p}$ where $v_{p}$ is again averaged over $L$. Consequently, a phase slip after every period $\mathrm{T}$ arises:

$$
\Delta \varphi=2 \pi T \Delta f=2 \pi T \frac{\left(v_{p}-v_{g}\right)}{2 L}=2 \pi\left(\frac{T}{T_{c}}-1\right)=2 \pi f_{\text {ceo }} / f_{\text {rep }}+2 \pi m,
$$

where $0 \leq f_{\text {ceo }} \leq f_{\text {rep }}$ and $m$ is an integer. It is shown in ref. 182 that the FouRIER transformation of a complex pulse train:

$$
\tilde{E}(\omega)=\int_{-\infty}^{\infty} \sum_{n} A(t-n T) e^{i\left[\omega_{0}(t-n T)+n \Delta \varphi+\varphi_{0}\right]} e^{-i \omega t} d t
$$

(a)

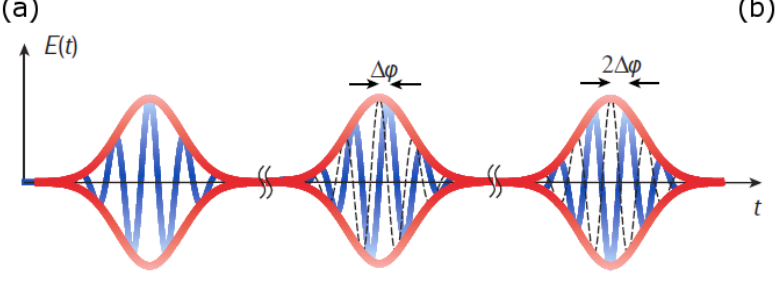

(b)

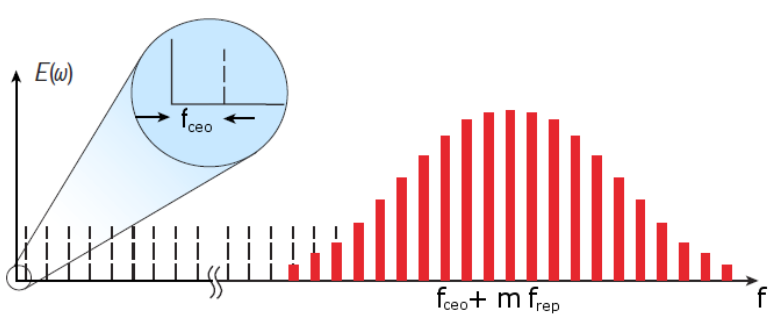

Fig. 1.9. (a) Pulse train like shown in Fig. 1.4(c). The periodic phase slip $\Delta \varphi$ is emphasized. It is constant if the carrier-envelope-offset frequency is stabilized. (b) FouRIER transformation of the pulse train from (a). The carrier-envelope-offset frequency is shown which does not carry any power. The comb modes within the laser gain medium (red columns) lie at much higher frequencies. They are equally spaced by $f_{\text {rep }}$ and can be expressed by $f_{\text {ceo }}+m f_{\text {rep }}$. The picture is simplified for clarity. A real mode-locked laser spectrum typically spans over thousands of comb modes. Adapted from ref. 50. 
where $n$ is the pulse number, $\omega_{0}$ the angular carrier frequency and $\varphi_{0}$ a constant phase shift, allows only the frequencies

$$
f=f_{\text {ceo }}+m f_{\text {rep }}
$$

to interfere constructively, and thus to compose the laser spectrum. This discrete mode structure is called the "frequency comb". It is illustrated in Fig. 1.9 in time and frequency domain. While $f_{\text {rep }}$ can be directly detected through the beating of neighboring modes when laser light is sent onto a photodiode, the detection of the radio frequency $f_{\text {ceo }}$ is not straight forward. It will be discussed in section 2.3.2. The detection and stabilization of the offset frequency is particularly interesting for time domain applications. Eq. (1.15) indicates that the term $n \Delta \varphi+\varphi_{0}$ determines the relative position of the carrier with respect to the peak of the envelope of the $n^{\text {th }}$ pulse (it is therefore called "carrier-envelopephase"), and hence has decisive influence on the maximal field strength of few-cycle pulses.

The considerations of a frequency comb so-far only referred to the output of a modelocked oscillator. Mathematically, Eq. (1.15) is, however, much more general. Basically, two conditions have to be fulfilled: The carrier as well as envelope terms must be periodic in $T$ and must be independent of the pulse number $n$, i.e. the pulses must be identical up to the CEP. This means that the comb nature is maintained if $T$ is changed, for instance, by a pulse picker, if the pulses accumulate temporal or spectral phase, amplitude modulation or if the comb frequencies are mixed through nonlinear effects. The frequency domain pictures of SPM in Fig. 1.7 indicate that the generated frequencies will obey the comb spacing dictated by the initial pulse train. This robustness of the frequency comb description is of fundamental importance for almost all applications of the tool. For example, it enabled self-referencing of a frequency comb by generating the $\mathrm{SH}$ with twice the offset frequency, ${ }^{19}$ attosecond pulse generation with $\mathrm{kHz}$ repetition rates but phase control on the oscillator level, ${ }^{42}$ frequency combs in the $\mathrm{XUV}^{110}$ and mid-IR which will be discussed extensively in chapter 4 .

(a)

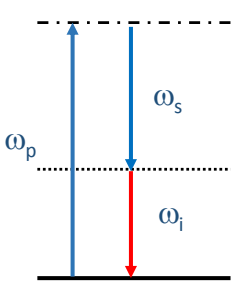

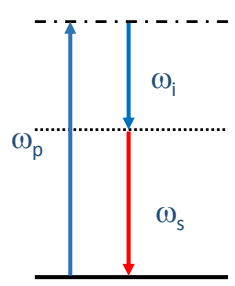

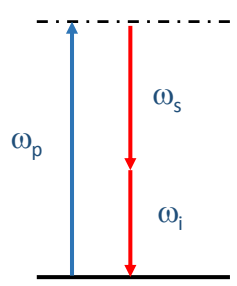

(b)

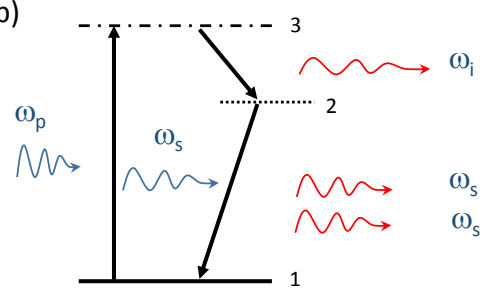

Fig. 1.10. (a) Difference frequency generation schemes. The first two graphs are taken from ref. 183. The leftmost scheme illustrates the generation of an idler photon $\left(\omega_{i}\right)$, from a pump photon $\left(\omega_{p}\right)$. The process is seeded by a signal photon $\left(\omega_{s}\right)$. Vice versa, the middle graph shows the generation of a signal photon seeded by an idler photon. The rightmost graph illustrates the spontaneous decay of a high energy pump photon into lower energy signal and idler photons. In this case, energy conservation is obvious. In the other graphs, the two outgoing seed photons are not explicitly shown. This stimulated process becomes clearer in (b) where the analogy to a laser gain medium is drawn. The pump photon excites the virtual laser level 3 which instantaneously relaxes to the virtual level 2 under the emission of an idler photon. At the same time, a seed photon causes the emission of a second identical (in phase and energy) signal photon. 


\subsubsection{Nonlinear frequency down-conversion}

Reaching the mid-IR spectral range is accomplished in this thesis by frequency downconversion via three-wave mixing. Similar to the diagrams of Fig. 1.7, the process is illustrated in Fig. 1.10(a). The first two graphs are taken from ref. 183 where the mathematical description of the nonlinear phenomenon can be found. Two incoming photons, called "pump" and "seed", generate a third photon at the difference frequency of both ("difference frequency generation" - DFG). The terminology is taken from refs. 184, 185 which also give a detailed overview about different down-conversion techniques. It will become clearer when Fig. 1.10(b) is described. The rightmost graph of (a) shows a spontaneous, and thus highly unlikely process. Only one input photon decays into two lower energy output photons. The process is triggered by vacuum fluctuations which can, within the uncertainty of energy and time, create a seed photon for DFG. The process is exploited in optical parametric generators or OPOs. One may think of a virtual excited state that is populated by the pump photon and is spontaneously deexcited like a real transition.

The analogy is extended in Fig. 1.10(b) where a stimulated emission-like process is depicted. The scheme shows a three-level system which can be found in every laser textbook (e.g. in ref. 186). Energy is stored by a pump source in an upper energy level (3) and released again by a photon which stimulates the emission of an identical second photon from a lower lying state 2. It is populated by a fast relaxation process. Crucially, the upper two levels (2 and 3 ) are virtual. This has important consequences. Firstly, contrary to a real gain medium, excitation, relaxation and stimulated emission must happen at the same instance of time (again within the time-energy uncertainty), i.e. the process depends on intensity, not fluence. Secondly, no energy is stored in the gain medium and the relaxation process is purely optical, not thermal. This has been exploited in OPCPAs as described in section 1.2 and is utilized in this thesis as well. While in OPCPAs, the optical relaxation produces an "idle" photon which is dumped again, this long-wavelength radiation makes up the mid-IR sources that are presented in chapter 4 . Thirdly, virtual

(a)

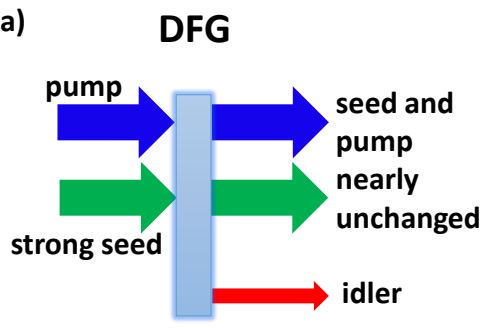

thin $\chi^{(2)}$ crystal (b)

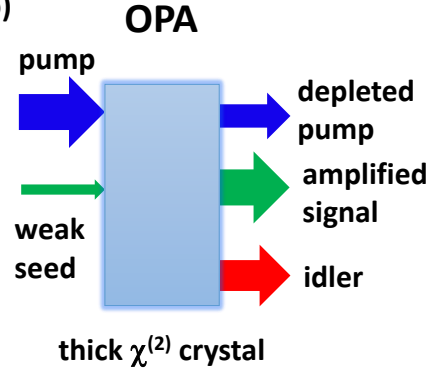

(c) OPO

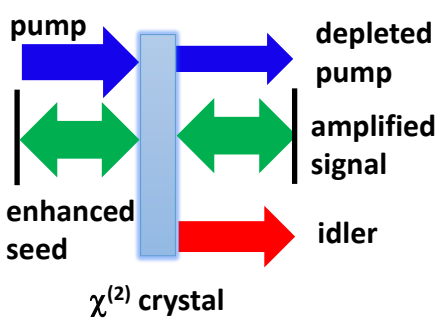

Fig. 1.11. Frequency down-conversion via three-wave mixing. (a) difference frequency generation (DFG): Pump and seed radiation are of similar power. The nonlinear crystal is typically fairly short such that the idler irradiance scales proportional to pump and signal irradiance ${ }^{184}$. This yields only moderate conversion efficiencies but usually very broadband mid-IR radiation. (b) optical parametric amplifier (OPA): The pump power is much higher than the seed power but the seed undergoes a coherent build-up in a typically long nonlinear crystal. Conversion efficiencies are higher than in a DFG setup but usually the bandwidth is narrower due to the long interaction lengths. (c) (singly resonant) optical parametric oscillator (OPO): Power of pump and seed in the nonlinear crystal are high because a weak seed is enhanced in a resonator. Pump and seed pulses must be synchronized. Crystal lengths differ. Typically, the conversion efficiencies are the highest. More details on the conversion schemes are presented, for instance, in refs. 183-185. Also many hybrid forms of the sketched schemes exist. 
states form a continuum resulting in tunability of the outgoing photons which typically clearly exceeds the gain bandwidth of a real laser medium. Tuning is accomplished by phase-matching pump-, signal- and idler-waves. The velocities of each wave are adjusted such that photons, which are created at different crystal positions, constructively interfere. This is equivalent to preserving the total photon momentum ${ }^{183}$. The seed photons replicate themselves during the three-wave mixing process. This is comparable to stimulated emission in real gain media. Therefore, one speaks of optical parametric oscillators in analogy to laser oscillators and optical parametric amplifiers in analogy to laser amplifiers. The latter ones will be investigated in more detail in section 4.1. At this point, only the basic principles of typical DFG, OPA and OPO schemes are summarized in Fig. 1.11. 



\section{PROOF OF CONCEPT: FEW-CYCLE PULSE GENERATION AND CARRIER-ENVELOPE-PHASE STABILIZATION}

Parts of this chapter have been published in:

O. Pronin, M. Seidel, F. Lücking, J. Brons, E. Fedulova, M. Trubetskov, V. Pervak, A. Apolonski, Th. Udem, and F. Krausz, "High-power multi-megahertz source of waveformstabilized few-cycle light," Nat. Commun. 6, 6988 (2015).

The first chapter has pointed out important breakthroughs of solid-state laser architectures. In particular Ti:sapph lasers have revolutionized frequency metrology and ultrafast optics. The introduction has also emphasized the potential of the thin-disk technology to become the foundation of the upcoming power-scalable laser oscillator generation. However, when I started my PhD work, neither compression of thin-disk laser pulses to the few-cycle regime nor carrier-envelope phase stabilization of any TD oscillator were demonstrated.

These two achievements present important milestones in the development of the technology towards new workhorses in ultrafast optics laboratories. This chapter reports on spectral broadening and pulse compression experiments where pulses with durations of only 2.3 carrier-wave cycles were attained. Moreover, carrier-envelope phase stabilization by means of intracavity loss modulation is presented. For the $40 \mathrm{~W}$ average power oscillator a residual carrier-envelope phase noise of less than $300 \mathrm{mrad}(1 \mathrm{~Hz}-500 \mathrm{kHz}$ bandwidth) was measured. 


\subsection{An Ultrafast Workhorse: The Kerr-lens Mode-locked Thin-disk Oscillator}

Most of the experiments presented in this dissertation have been performed with the KLM TD oscillator which was developed by OLEG PRONIN when he was a PhD student in the laboratories of FERENC KRAUSz. His dissertation explains the development and the working principle of the oscillator in detail ${ }^{65}$. In the context of this thesis, just a short characterization of the light source is presented. A picture and the setup of it are shown in Fig. 2.1. Although it is the first KLM TD oscillator which has been demonstrated ${ }^{23}$, it has served as a true "ultrafast workhorse" because it has been operated for about 5 years on a daily basis and without the need for major replacements or changes.

Over the time, however, some minor modifications were made to improve the handiness and stability of the laser. Firstly, most of the mirror mounts were exchanged. The new ones were water-cooled to avoid thermal drifts of the oscillator. After an initial cavity alignment with a weak CW laser, only the end mirror and the outcoupling mirror were still

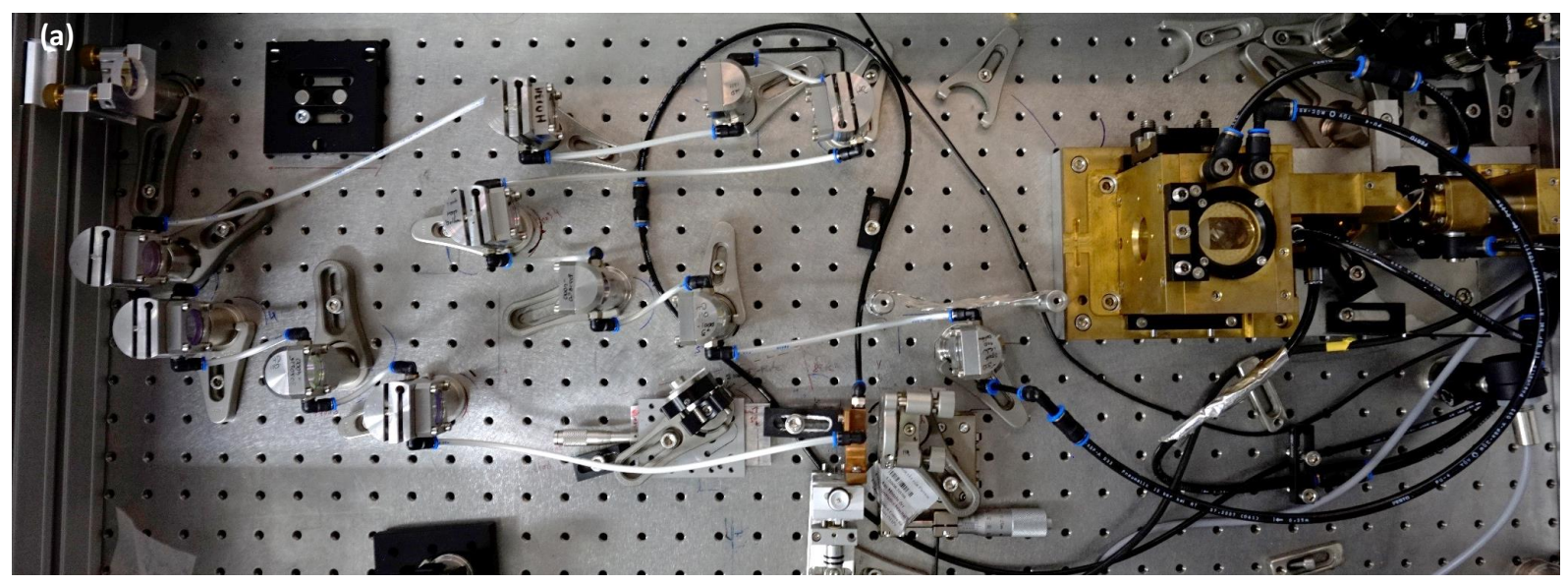

(b)

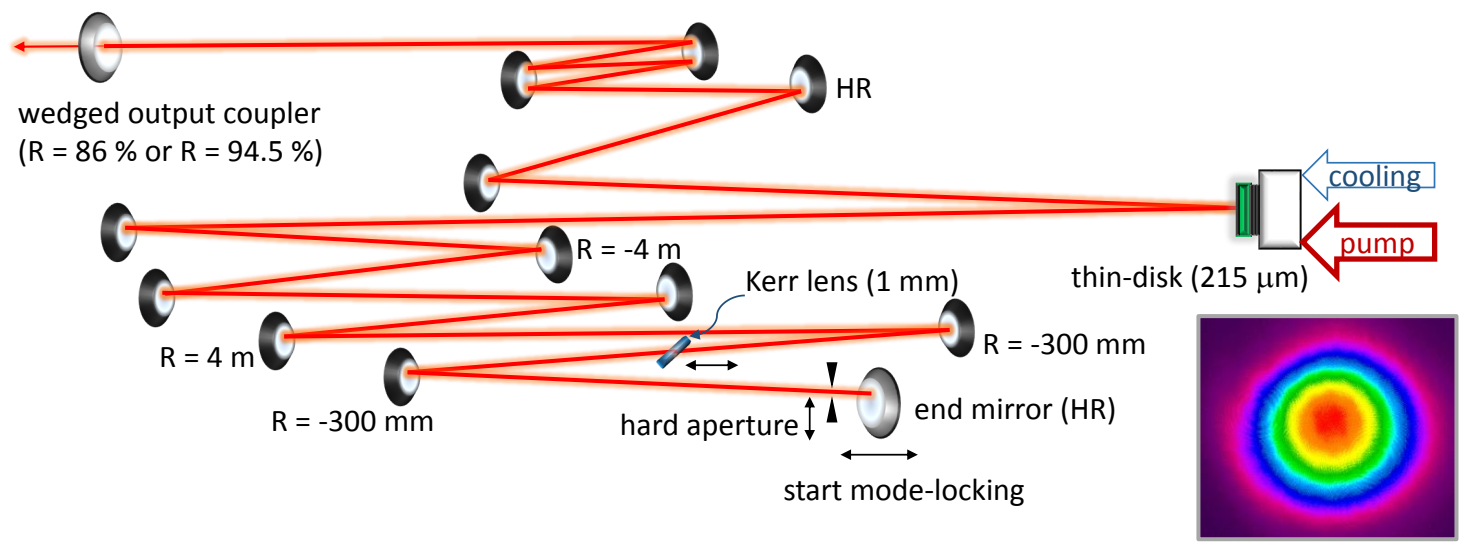

Fig. 2.1. (a) Top view photograph of the latest setup. (b) Sketch of the beam path inside the oscillator and the optical components. The high reflection (dielectric coating) (HR) mirrors are marked. If not flat, their radii of curvature (R) are stated. All unspecified mirrors are flat and highly dispersive. Their GDD at $1030 \mathrm{~nm}$ is either $-1000 \mathrm{fs}^{2}$ or $-3000 \mathrm{fs}^{2}$. The Kerr medium is a $1 \mathrm{~mm}$ thick sapphire plate. The disk (Dausinger + Giesen $\mathrm{GmbH}$ ) is only $215 \mu \mathrm{m}$ thin. It is cooled from its rear side. The collimated pump light passes the disk 24 times. Mode-locking is initiated by gently shifting the end mirror forth and back again. The hard aperture is a copper plate with a $4.5 \mathrm{~mm}$ hole drilled into it. The oscillator operated at about $17 \mathrm{~W}$ output power with the $5.5 \%$ OC, which was only used for initial CEP stabilization experiments, and usually between 45 and $50 \mathrm{~W}$ with the $14 \%$ output coupler. The inset shows a typical output beam profile of the mode-locked KLM TD oscillator measured with a CCD camera. 
adjusted, whereas the other mirrors remained fixed. Moreover, no SESAM was utilized to start the mode-locking as it has been described in refs. 23 and 65. Solely a water cooled hard aperture made from copper was placed directly in front of the end mirror. A $1 \mathrm{~mm}$ thick sapphire plate has served as the KERR medium instead of an initially used $1 \mathrm{~mm}$ thick FS plate due to the higher heat conductivity of the crystalline material. Although the plate has not been replaced for more than three years, it had to be frequently rotated by a few degrees, circa after a couple of weeks, since increased scattering indicated microscopic damages. Rotating the plate was, however, quite convenient and did not involve a major realignment of the oscillator. Gently pushing the end mirror instead of one of the $R=-300 \mathrm{~mm}$ spherical mirrors to start mode-locking greatly improved the handiness of the oscillator. This is because the end mirror was aligned exactly at $0^{\circ}$ AOI, and hence shifting it introduced just a slight but sufficient perturbation of the laser operation. The chaotic starting process was often the cause for damaged optics inside the oscillator. Reducing the external perturbation helped also to encounter damage less often. Another precaution was taken to avoid the need of frequent realignment of the laser. Mirrors with $-3000 \mathrm{fs}^{2}$ GDD were removed from the beam path between thin-disk and output coupler where the beam size was relatively small. Instead, HR mirrors as well as mirrors with $-1000 \mathrm{fs}^{2}$ were inserted owing to the higher damage threshold of these optics in comparison to highly dispersive mirrors ${ }^{187}$. It was iteratively tried to reduce the length of the telescope, i.e. the distance between the two $R=-300 \mathrm{~mm}$ spherical mirrors, in order to move closer to the stability center of the resonator. Average power and spectral width after mode-locking were measured to ensure that the pulse energy and duration were not negatively affected when changing the resonator length. In the final configuration, the telescope length was $305 \mathrm{~mm}$.

Characteristic output powers for the $14 \%$ output coupler are shown in Fig. 2.2. Depending on the alignment, the output power values varied by $\approx \pm 5 \%$ The emitted pulses exhibited durations between $220 \mathrm{fs}$ and $270 \mathrm{fs}$. Increasing the output power through the diode current usually yielded slight shortening of the emitted pulses. The oscillator was operated in the so-called soliton mode-locking regime (cf. ref. 65 for more details). It exhibited a nearly perfect $\operatorname{sech}^{2}$-spectral and temporal shape as the fits of the spectrum and autocorrelation measurements of Fig. 2.3 demonstrate. The output beam was nearly ideally Gaussian. The $\mathrm{M}^{2}$ factors in horizontal and vertical direction were 1.05. These

(a)

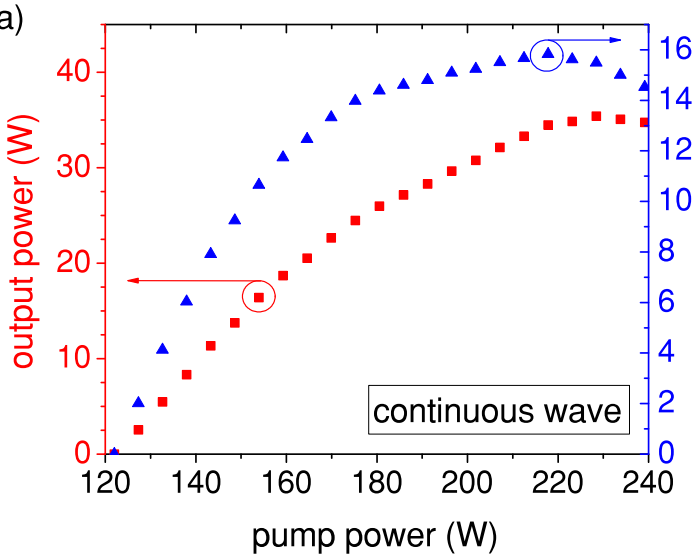

(b)

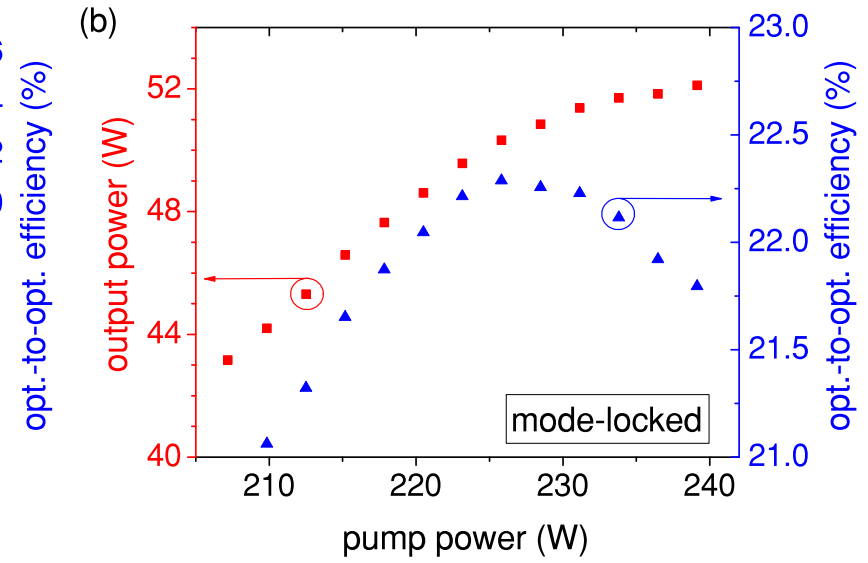

Fig. 2.2. Average power and optical-to-optical efficiency of the TD oscillator in (a) continuous wave and (b) mode-locked operation. The laser was usually operated with $200-230 \mathrm{~W}$ pump power, depending on the application. The presented data was taken without intermediate realignment. 

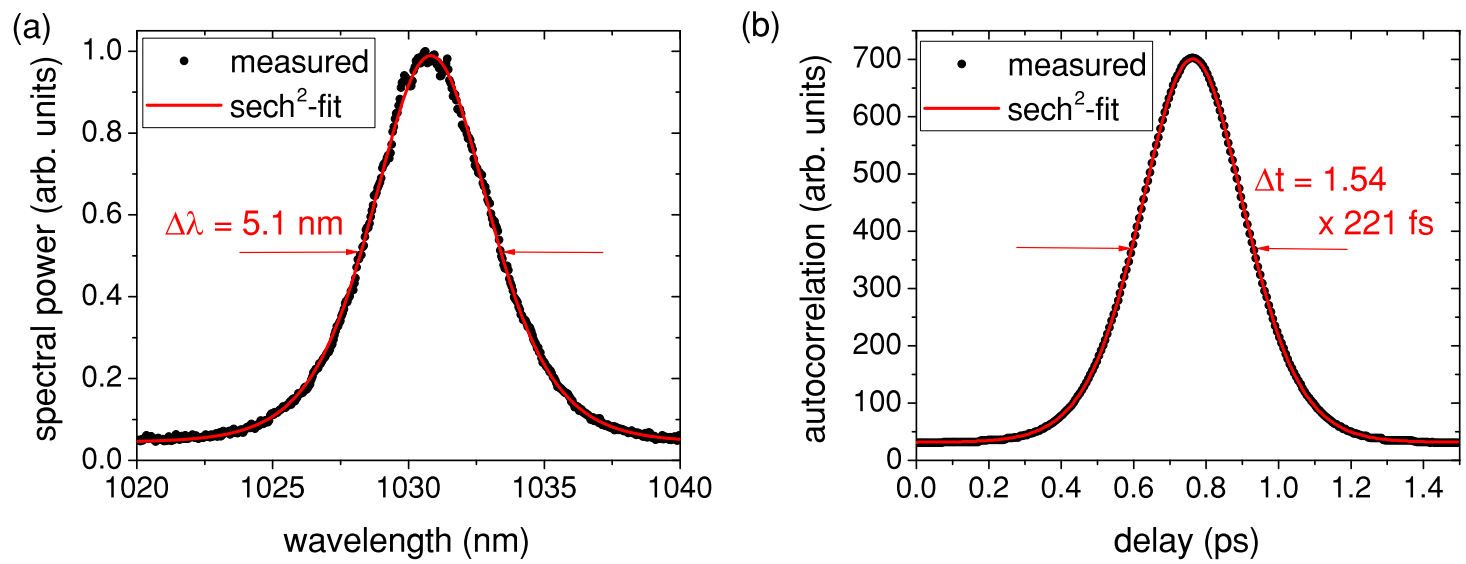

Fig. 2.3. (a) Spectrum measured with a grating spectrometer and fitted to the characteristic sech ${ }^{2}$-pulse shape ${ }^{171}, S(\Omega) \propto \operatorname{sech}^{2}\left(\pi \Omega \tau_{s} / 2\right)$, where $\Omega=2 \pi c_{0}\left(1 / \lambda-1 / \lambda_{c}\right), c_{0}$ is the speed of light, $\lambda$ the wavelength, $\lambda_{c}=1030.8 \mathrm{~nm}$ the central wavelength and $\tau_{s}=t_{p} / 1.763$. A pulse duration of $t_{p}=220$ fs is retrieved from the fit. The spectral width is $5.1 \mathrm{~nm}$. (b) Intensity autocorrelation measured with an APE PulseCheck. The sech ${ }^{2}$-fit yields a pulse duration of $221 \mathrm{fs}$. Data was taken at $52 \mathrm{~W}$ output power.

"textbook characteristics" of pulses and beam presented an ideal starting point for comparing the experiments, which will be presented later in this dissertation, to nonlinear beam propagation simulations.

Eventually, relative intensity noise (RIN) measurements of short- and longtime laser fluctuations are presented in Fig. 2.4 to complete the characterization of the KLM TD oscillator. They were measured after the oscillator had warmed up and do not include initial thermal drifts. Usually, half an hour after the oscillator was started, mode-locking was easily possible, but slight adjustments of the beam height with the end mirror were still
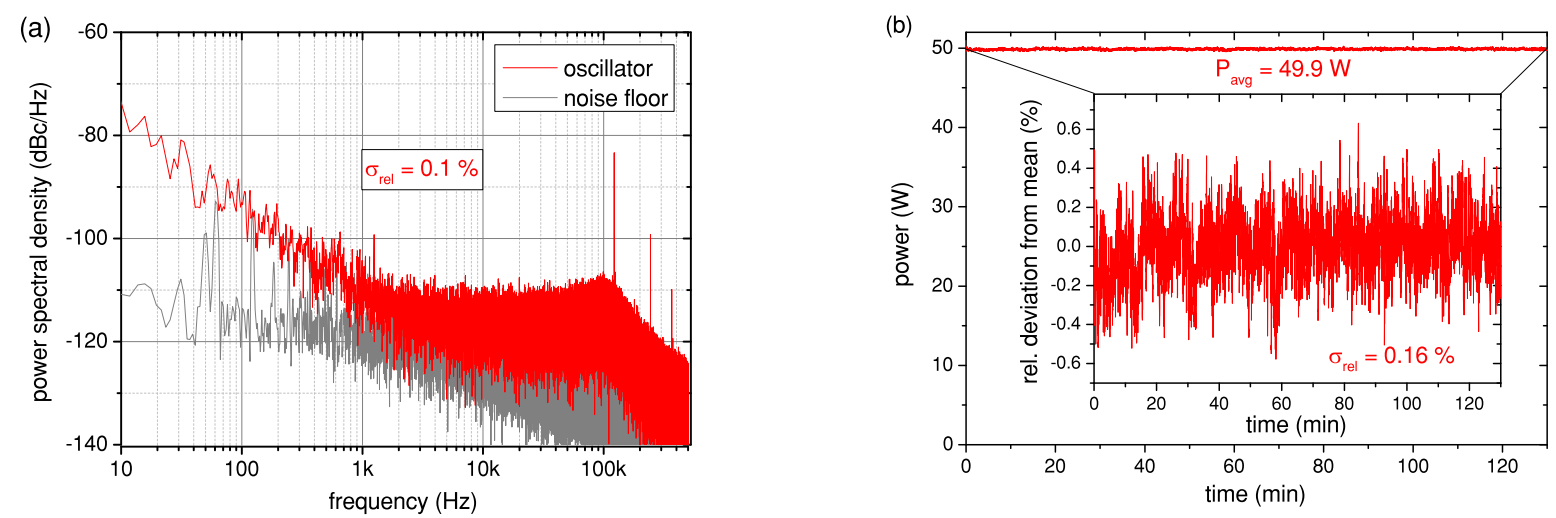

Fig. 2.4. (a) Short-term power fluctuations measured with a radio-frequency (RF) spectrum analyzer in the range from $10 \mathrm{~Hz}$ to $500 \mathrm{kHz}$. The power spectral density (PSD) is normalized to the power of the carrier which was also measured with the RF spectrum analyzer. The root mean square (RMS) is only $0.1 \%$ of the carrier power. (b) Long-term fluctuations of the oscillator power measured over more than 2 hours with 1 sample per second. The sensitivity seems limited by the noise of the thermal power meter which was used to measure the power fluctuations. The outer graph shows the fluctuations in scale while the inset shows the fluctuations relative to the mean average power of the laser. The derived RMS is only $0.16 \%$. It is to note that the fluctuations were also alignment-dependent. In section 3.3 a noise spike was measured at $7 \mathrm{kHz}$ while in section 4.1 slow fluctuation with $\mathrm{a} \approx 9$ minute period were observed. The exact origins of these perturbations have not been investigated. 
necessary within the following 2-3 hours of operation to sustain the initial average power level. Afterwards, the oscillator was running without any need for realignment. During daily operation only the end mirror was used to correct for thermal drifts. The very low RMS values $<0.2 \%$ of the RIN measurements demonstrate an important advantage of amplification-free systems. Active optical elements of laser amplifiers do not only enhance pulse energy, they introduce additional noise to the light source. Consequently, their RIN figures without active stabilization are hardly on the sub-percent level. Already small differences may, for instance, have a huge impact in experiments involving extreme nonlinear optics where field amplitude fluctuations may be enhanced exponentially.

Section 2.3 presents more details on the passive stabilization of this KLM TD oscillator. Beforehand, first pulse compression experiments are discussed which enable to reduce the pulse duration from more than $200 \mathrm{fs}$ to less than $10 \mathrm{fs}$. 


\subsection{Entering the Few-cycle Pulse Regime with Mode-locked Thin-disk Oscillators}

\subsubsection{Solid-core Fiber-based Pulse Compression}

As mentioned in the introduction section 1.2, the first pulses with durations below $10 \mathrm{fs}$ were generated by means of spectral broadening in solid-core fiber and post-compression by applying negative GDD ${ }^{18}$. This approach was also transferred to first compression experiments of SESAM mode-locked TD oscillators. In 2003 SÜDMEYER et al. accomplished to reduce the duration of $810 \mathrm{fs}$ pulses to $33 \mathrm{fs}$ by spectral broadening in a large mode area (LMA) PCF with about $16 \mu \mathrm{m}$ mode-field diameter (MFD) and post-compression by a prism pair ${ }^{188}$. Two years later the group of URSULA KELLER reported obtaining 24 fs pulses from a similar compression scheme ${ }^{189}$, but never published the experiment in a peer-reviewed journal. To the best of the author's knowledge, these results present still today the shortest pulses achieved from a SESAM mode-locked TD oscillator. For first compression experiments with KLM TD oscillators, LMA PCFs were chosen as well, because they allow single mode operation over a broad bandwidth despite MFDs of several tens of microns ${ }^{190-193}$. Therefore, they are also able to strongly reduce the influence of mostly anomalous waveguide dispersion, and thus let ultrashort pulses centered at $1030 \mathrm{~nm}$ undergo the normal dispersion of FS. The consequent pulse broadening leads to a saturation of the irradiance driven nonlinear effects, and hence to a relatively stable and coherent power output if compared to fiber with anomalous dispersion ${ }^{48,194}$. Furthermore, large core areas enable to increase pulse peak powers up to the critical power of the fiber material. The peak power limitations of LMA PCFs will be investigated and explained in more detail in section 3.1.1 of the dissertation.

\section{Spectral broadening in LMA-35 and post-compression to sub-20 fs}

In the initial fiber compression experiments, LMA-35 with a MFD of $26 \mu \mathrm{m}$ was used. It was, contrary to fibers with even larger core sizes, readily commercially available when the experiments were performed (supplied by NKT Photonics). A fiber length of about $8 \mathrm{~cm}$ was chosen. The simulations in Fig. 2.5(a) show that the SPM-based broadening is fully saturated at this point since the peak powers of the ultrashort pulses have dropped to about one forth of their initial value. Consequently, the gain in RMS spectral width per unit length is relatively low and mainly originates from $\mathrm{OWB}^{195}$, a four-wave mixing process briefly described in the fundamental section 1.4. OWB exhibits two obvious differences in comparison to SPM. Firstly, the spectrum is not modulated but forms a flat pedestal structure. Secondly, the resulting frequency components are not delayed in time with respect to the edges of the pulse as the spectrogram in Fig. 2.5(b) shows. Subsequently, imposing only linear chirp on the broadened pulses would lead to a significant pedestal structure. A choice of an $8 \mathrm{~cm}$ fiber length therefore also simplified pulse compression after the fiber stage. The predictions of the discussed simulations have been experimentally confirmed as the spectra of Fig. 2.6 indicate. The presented measurements were taken with 32 - $33 \mathrm{~W}$ of pump power. For permanent operation only $27 \mathrm{~W}$ of power were sent into the fiber. An empirical study of MARCHESE ${ }^{196}$ showed an exponential increase of fiber degradation with increasing pump power. Although a quantitative explanation was missing and the relation was extracted from only a single data set, the reduction of pump power well below $30 \mathrm{~W}$ also extended the fiber life times from sev- 
(a)

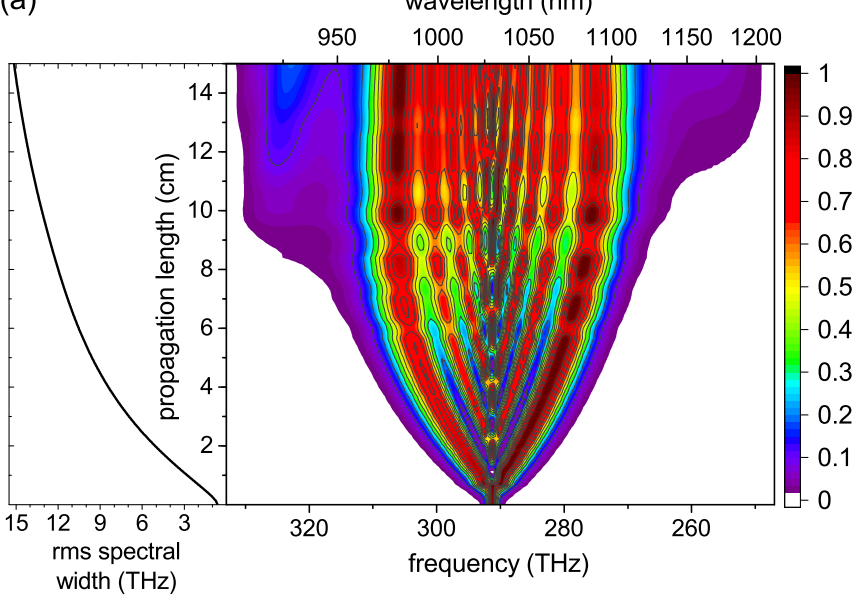

(b)

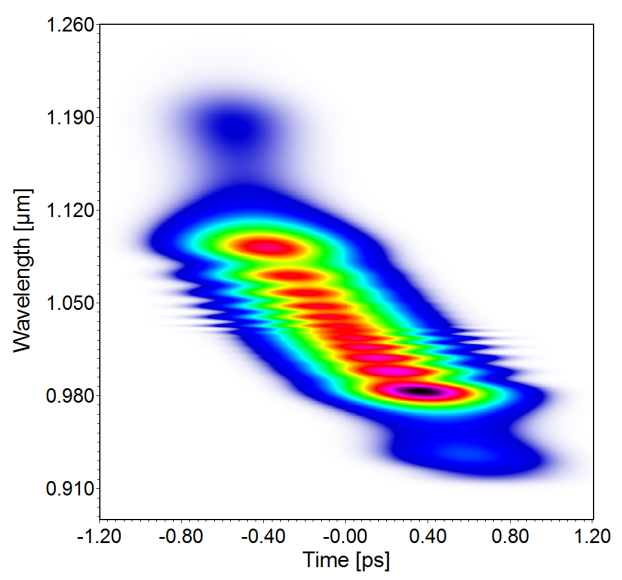

Fig. 2.5. (a) Propagation of $550 \mathrm{~nJ}, 250 \mathrm{fs}$ pulses in a $15 \mathrm{~cm}$ long LMA-35 fiber with $26 \mu \mathrm{m}$ MFD. The pulse parameters are close to the experimental ones. Only the input energy is slightly lower than in the experiment $(595 \mathrm{~nJ})$. This can be attributed to uncertainties in the exact MFD of the fiber as well as the $n_{2}$ value at $1030 \mathrm{~nm}$. The simulation considered second and third order dispersion as well as SPM and OWB. Raman scattering and self-steepening hardly affect the simulation outcome. The plotted spectra were normalized for each propagation length. The dominating SPM contributions exhibit strong spectral modulations while the OWB contributions at the spectral wings are rather flat. The RMS spectral width is increased from about $650 \mathrm{GHz}$ to $12 \mathrm{THz}$ after $8 \mathrm{~cm}$ of propagation, yielding a remarkable broadening factor of 18.4. (b) Spectrogram of the pulses after $15 \mathrm{~cm}$ of propagation. While the SPM contributions exhibit nearly linear chirp, the OWB contributions are hardly delayed with respect to the wings of the SPM broadened spectrum. The simulations were conducted with the "fiberdesk" software, version 2.0 (www.fiberdesk.com).

eral hours to several weeks. The usage of fiber end caps ${ }^{197}$ has led to the best long term stability results to the best of the author's knowledge and is now implemented in the midinfrared generation setup of ref. 198, the first application of the fiber-based compression scheme which is presented in this section.

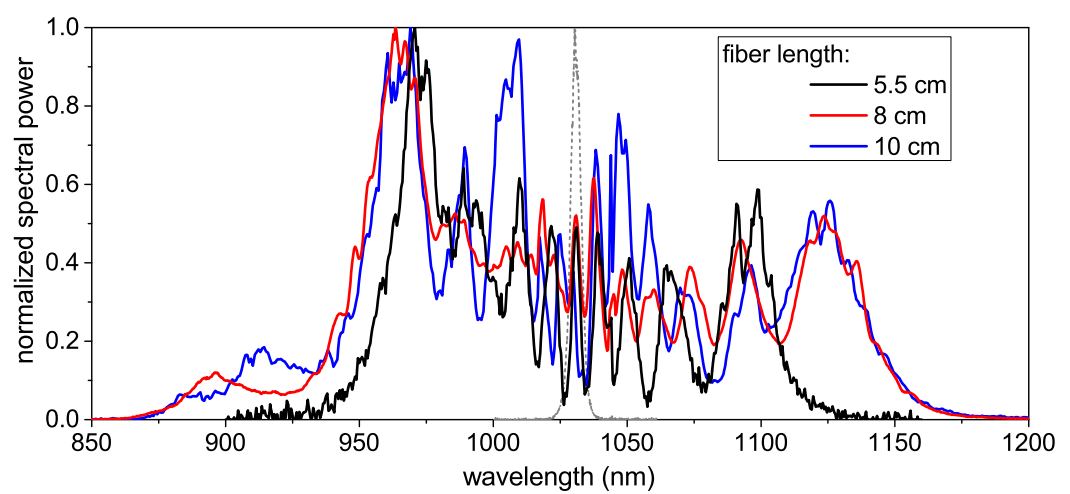

Fig. 2.6. Spectra measured with an optical spectrum analyzer (OSA) after LMA-35 fibers of different lengths. The pump powers were $32-33 \mathrm{~W}$. After $5.5 \mathrm{~cm}$ of propagation the spectrum looks purely broadened by SPM. The OWB features become visible after $8 \mathrm{~cm}$ of propagation but also the SPM contributions are clearly stronger than after $5.5 \mathrm{~cm}$. Hardly any change in spectral width is noticeable when comparing the spectra after 8 and $10 \mathrm{~cm}$ of propagation. For comparison, the oscillator spectrum is shown (dashed gray line). 


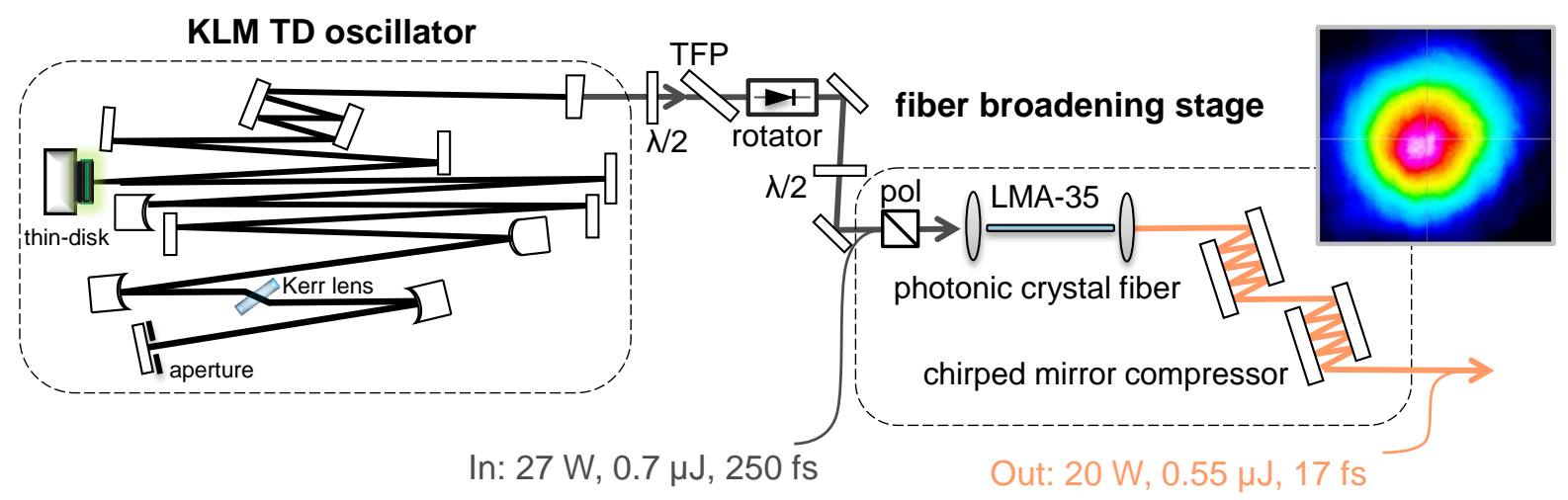

Fig. 2.7. Solid-core fiber compression setup. The oscillator output was attenuated to $27 \mathrm{~W}$ through a half-wave plate $(\lambda / 2)$ and a thin-film polarizer (TFP). Back reflections from the fiber facet into the oscillator were avoided by means of a FARADAY rotator. A second half-wave plate and a polarizing beam splitting cube (pol) yielded a clean p-polarization of the beam at the fiber entrance. Coupling into and collimation after the fiber was achieved by plano-convex FS lenses. After the chirped-mirror compressor, which was set up in double-angle configuration, $20 \mathrm{~W}$ of average power and $17 \mathrm{fs}$ pulses were measured. The inset shows the beam profile recorded after the compression stage with a charge coupled device (CCD) camera (WinCamD).

The experimental setup is sketched in Fig. 2.7. Using an $f=35 \mathrm{~mm}$ plano-convex lens, coupling efficiencies of up to $85 \%$ were achieved. They were measured directly behind the fiber and may include residual contributions of light coupled into the cladding. The beam was collimated with an $f=50 \mathrm{~mm}$ thin FS lens. The output profile exhibited the
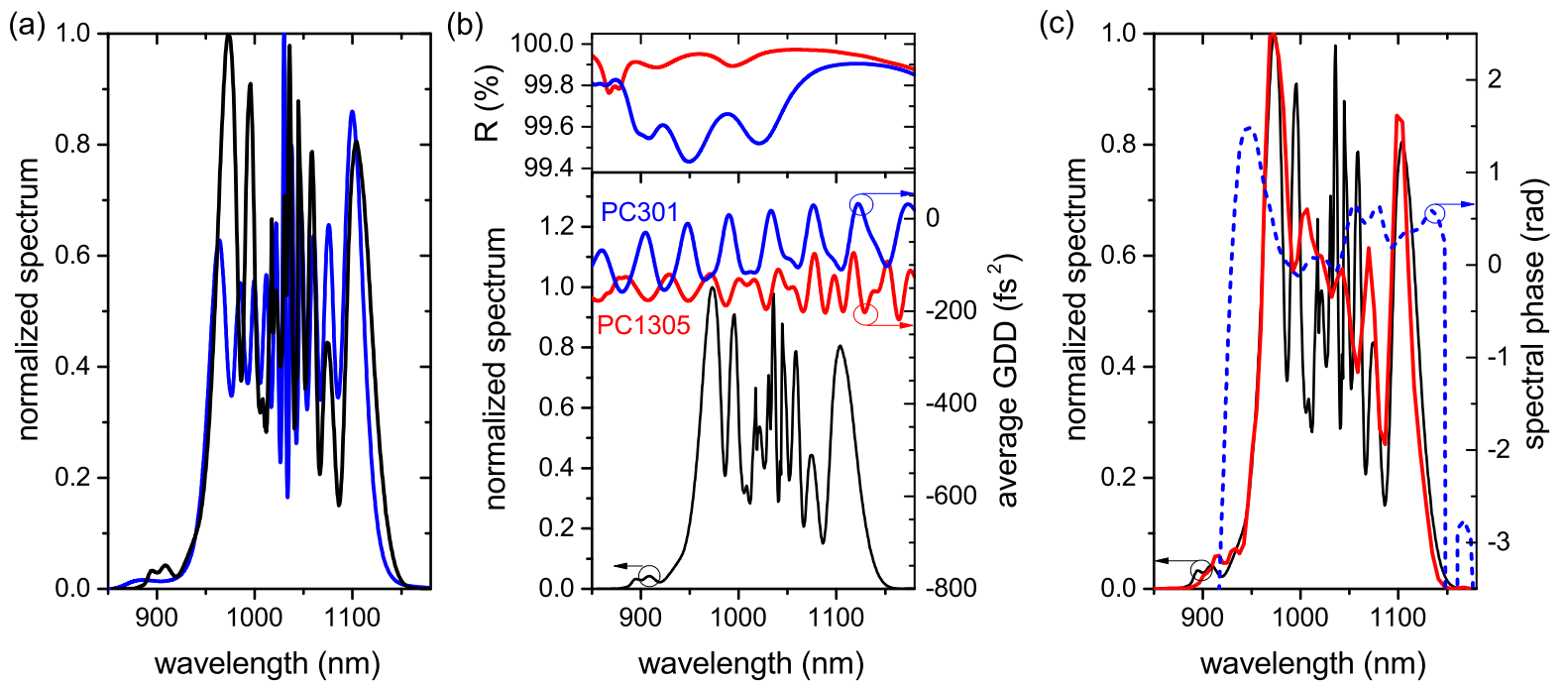

Fig. 2.8. (a) Spectrum measured with an OSA (black solid line) in comparison to the spectrum extracted from the simulation of Fig. 2.5 (blue solid line). Their agreement is excellent. (b) Theoretical reflectivity (R) and average GDD of the chirped mirrors PC1305 and PC301 for p-polarized light. The displayed GDD plots refer to the average of pairwise reflections under an AOI of $5^{\circ}$ and $19^{\circ}$ for PC1305 as well as $5^{\circ}$ and $20^{\circ}$ for PC301. Those are the design AOIs of the double-angle mirror pairs. Both mirror sets cover the full bandwidth of the broadened spectrum. (c) Comparison of the directly measured (black solid line) spectrum and the spectrum retrieved from a frequency resolved optical gating (FROG) measurement (red solid line). The excellent agreement indicates the reliability of the FROG retrieval. The dashed blue line shows the retrieved spectral phase. The phase of the SPM broadened spectrum is basically contained within an $[-\pi / 2, \pi / 2]$ interval, the OWB contribution is, however, clearly out of phase. 
typical hexagonal pattern of the LMA core (cf. Fig. 2.7). The mode did not exhibit spectral inhomogeneities. Moreover, an $M^{2}$ factor of 1.3 was measured in horizontal (x-) and vertical $(\mathrm{y}-)$ direction of the beam. For comparison, the $M^{2}$ value of the oscillator was 1.05 in $\mathrm{x}$ - and $\mathrm{y}$-direction, i.e. the beam quality was slightly reduced but still good.

The broadened spectrum, measured with an OSA, is shown in Fig. 2.8(a). It is compared to the simulation results from Fig. 2.5. As expected, the spectrum is mainly formed by SPM but it also exhibits a weak OWB contribution on the short wavelength side. The FTL of the spectrum is $13.6 \mathrm{fs}$, and thus in excellent agreement with the computed broadening factor of 18. The spectrogram of Fig. 2.5(b) indicates that linear up-chirp dominates the spectrally broadened pulses. To compensate for it, two sets of chirped mirrors were utilized. They were labeled PC1305 and PC301. Both were arranged in a double-angle configuration to minimize the GDD oscillations which are inherent to broadband chirped mirrors ${ }^{178}$. Moreover, they were designed for p-polarized light. Most of the positive dispersion was compensated by 12 bounces off PC1305, 3 from each of the 4 utilized mirrors. The average GDD of those mirrors was about $-150 \mathrm{fs}^{2}$ per bounce. PC1305 did not exhibit TOD. This was in contrast to PC301. Those mirrors exhibited a GDD of about $-60 \mathrm{fs}^{2}$ and a TOD of about $-120 \mathrm{fs}^{3}$. The pulses were reflected twice from each of the 4 mirrors used. Fig. 2.8(b) presents an overview of bandwidth, reflectivity and dispersion of both mirror sets. In sum with the collimation lens, a GDD of $-2100 \mathrm{fs}^{2}$ and a TOD of $-1100 \mathrm{fs}^{3}$ was applied to the pulses, reducing their duration to only $17 \mathrm{fs}$ (cf. Fig. 2.9(b)). The difference between the FTL and the measured pulse duration originates from the OWB contribution to the spectrum. Fig. 2.8(c) shows that the spectral phase on the short wavelength edge of the spectrum is not compensated. Pulse duration and phase were measured with a home-built second harmonic FROG setup (cf. Appendix A.1). A reflection from a FS wedge provided sufficient power for the pulse duration measurements.
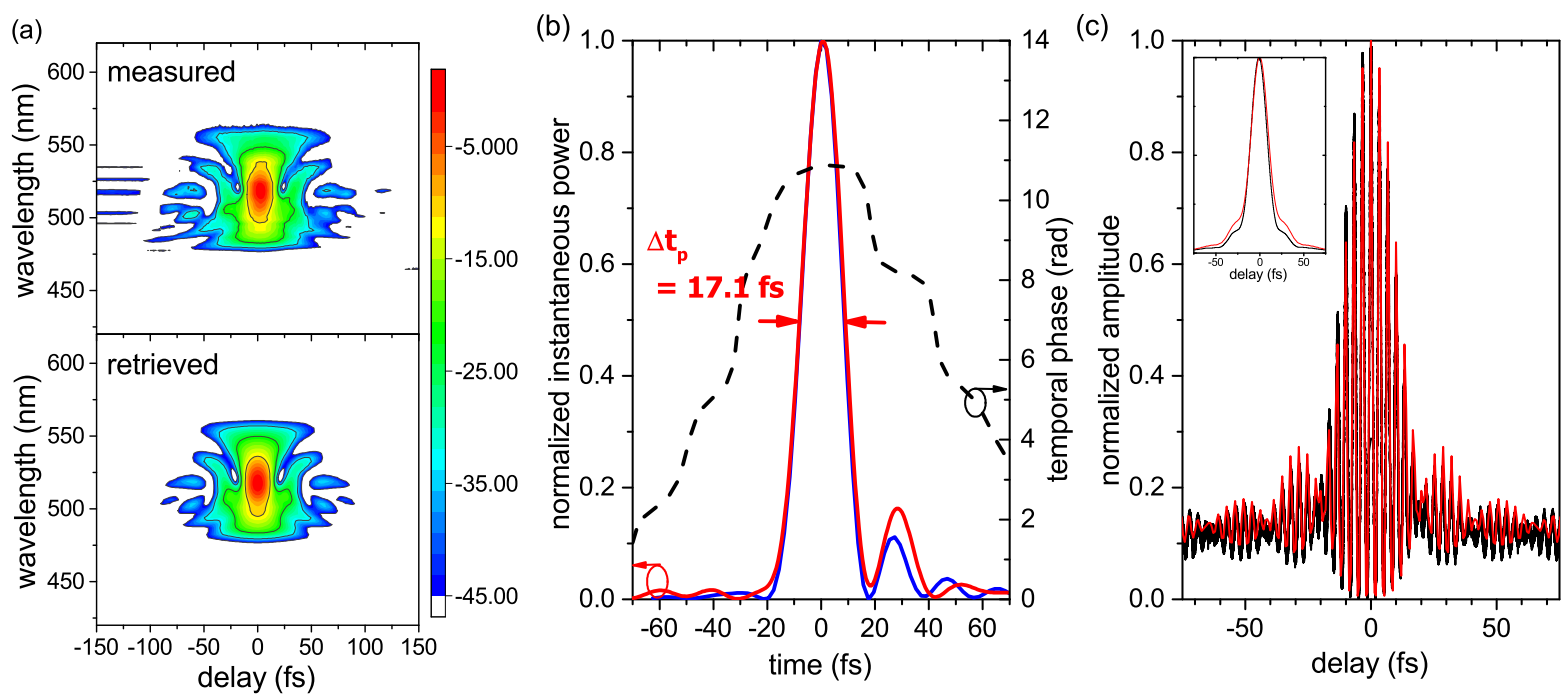

Fig. 2.9. (a) Measured (top) and retrieved (bottom) FROG traces of the pulse after the chirped mirror compressor. The color scale is in units of $\mathrm{dB}$ with respect to the maximum of the trace. The RMS FROG error was $0.38 \%$ for a $512^{2}$ grid size. (b) Retrieved ultrahort pulse (red solid line) and temporal phase (black solid line). The blue solid line shows the compression of the simulated spectrum of Fig. 2.8(a) with GDD only. The simulation of the short pulse is in excellent agreement with the measured pulse. (c) Fringe resolved autocorrelations measured with a Femotchrome autocorrelator (black solid line) and derived from the retrieved pulse of (b). The inset shows the intensity autocorrelations which were computed by low-passing the fringe resolved traces. 
The spectrometer could be easily saturated from the $\mathrm{SH}$ generated in a $10 \mu \mathrm{m}$ beta barium borate, $\beta-\mathrm{BaB}_{2} \mathrm{O}_{4}(\mathrm{BBO})$ crystal.

Several cross-checks confirmed the FROG results. Firstly, the retrieved FROG spectrum reproduces the directly measured one well (Fig. 2.8(c)). Secondly, the previously discussed simulations show excellent agreement with the measured pulses (Fig. 2.9(b)). Finally, the autocorrelation trace measured with a two-photon-conduction-based device (Femtochrome FR-103PD) can be reproduced by a numerical autocorrelation of the pulses extracted from FROG (Fig. 2.9(c)). It is to note that the small asymmetry of directly measured autocorrelation stems from the asymmetric beam splitter in the autocorrelator ${ }^{199}$.

\section{Discussion}

To the best of the author's knowledge, the demonstrated compression experiments already yielded pulses shorter than from any Yb-based system at the time they were reported. In the meantime, hollow-core capillary based compression schemes after fiber amplifiers have been also able to generate sub-20 fs pulses ${ }^{200,201}$. The experiments were, however, performed with mJ-level pulses. The success of compression experiments reported here is attributed to two major advantages of the presented scheme. Firstly, direct pumping by a KLM TD oscillator provides relatively short input pulses. KERR-lens mode-locking allows to exploit a large share of the $\mathrm{Yb}$-ion gain bandwidth - about $4.5 \mathrm{~nm}$ in the presented setup, nearly the full bandwidth of $9 \mathrm{~nm}$ in ref. 139 and even up to $40 \mathrm{~nm}$ in a distributed KERR-lens setup ${ }^{82}$ which was, however, realized at sub-10 W average power. For comparison, the initially mentioned SESAM TD oscillator solid-core fiber compression experiment was performed with 810 fs input pulses, the most powerful SESAM mode-locked oscillator generated pulses of about $580 \mathrm{fs}^{58}$, the most energetic mode-locked TD oscillator emitted ps pulses ${ }^{61}$. Other TD gain materials have allowed the emission of sub- 100 fs pulses but only generated about $5 \mathrm{~W}$ of average power ${ }^{150,202}$. While SESAM mode-locked osillators suffer from the finite bandwidth of the saturable absorber, fiber amplifiers cannot directly generate ultrashort pulses due to gain narrowing. However, broadband oscillators, CPA grating stretchers and combiners as well as active phase shapers enabled to extract sub-300 fs pulses from the amplifiers, and consequently also led to sub-20 fs pulses after nonlinear pulse compression ${ }^{200}$. The second advantage of the presented scheme stems from the large broadening factors achievable with fiber and the resulting dominating linear chirp. The long nonlinear interaction lengths, $8 \mathrm{~cm}$ here, up to $\approx 100 \mathrm{~m}$ in other experiments (e.g. ref. 203), enabled a broadening factor of 18 in our case which can be even increased to about 50 for the same input pulse duration as section 3.1.1 will demonstrate. Large broadening factors in bulk or filamentation based spectral broadening experiments usually result in reduced efficiency and compressibility which will be shown in section 3.2.

Another favorable property of the compression scheme shall be highlighted: The input peak power of about $2.5 \mathrm{MW}$ was increased to $25 \mathrm{MW}$, i.e. by factor of ten. Increasing peak power by means of pulse compression is in particular important for $\mu \mathrm{J}$ pulse energy levels because extreme nonlinear optical effects in gases, like HHG, usually require peak powers on the order of at least $100 \mathrm{MW}$. Such powerful pulses have not been emitted from any oscillator, yet. On the other hand, the presented scheme is very limited in peak power scalability as section 3.1.1 will discuss in more detail. This is certainly the most severe drawback of solid-core fiber compression. Only $27 \mathrm{~W}$ average power of the $40 \mathrm{~W}$ emitted from the KLM TD oscillator could be utilized in the compression experiment. 
The latest generation of mode-locked TD oscillators delivers pulses with peak powers of about $60 \mathrm{MW}^{61,139}$. Achieving a further increase of this value by means of solid-core fiber pulse compression would be very difficult. The only successfully demonstrated applicable concept, coherent combining, ${ }^{204,205}$ would require tens of fibers operating in parallel, and thus would be highly impractical. A few other minor drawbacks are the degradation of the fiber facets which has been mentioned before and the daily need for optimizing the coupling into the fiber (if no expensive automated system is employed). These features reduce the applicability of solid-core fiber compression which is also of major importance of a new generation of ultrahort pulse sources as pointed out in the introduction section 1.1.

In conclusion, sub-20 fs pulses have been demonstrated for the first time from any highpower amplification-free system. The concept of solid-core fiber compression has revealed strongly favorable properties, i.e. high compression factors and high compression efficiencies, but also severe limitations with respect to the peak power scalability of the method. Approaches to overcome this drawback will be presented in chapter 3 of this dissertation.

\subsubsection{Few-cycle Pulse Generation in Bulk Material}

While 17 fs pulses were the shortest pulses ever generated from a TD oscillator, they do not fully suffice the initial target to reach the few-cycle regime. For a duration of five optical cycles the maximal field strength of a sine pulse would be only about $0.35 \%$ larger than that of a cosine pulse (assuming the same Gaussian envelope for both). If the pulses would be compressed by another factor of two, the contrast was already about $1.35 \%$. For a single cycle pulse, it would be $7.88 \%$, i.e. the CEP dependence of extreme nonlinear effects would become much stronger.

Two major challenges arise upon going to sub-10 fs pulse durations. Firstly, as discussed in the previous section, the peak power of the $17 \mathrm{fs}$ pulses is about $25 \mathrm{MW}$, i.e. about a factor of six larger than the critical power of FS. Consequently, the use of a second solid-core fiber for further spectral broadening is not possible. Chirped pulse broadening ${ }^{206}$ is also not applicable as section 3.1.1 will explain. The second challenge arises from the spectral bandwidth of the short pulses and the impact of material dispersion, resp. While $290 \mathrm{~cm}$ of FS were needed to double the duration of the pulses emerging from the oscillator, only

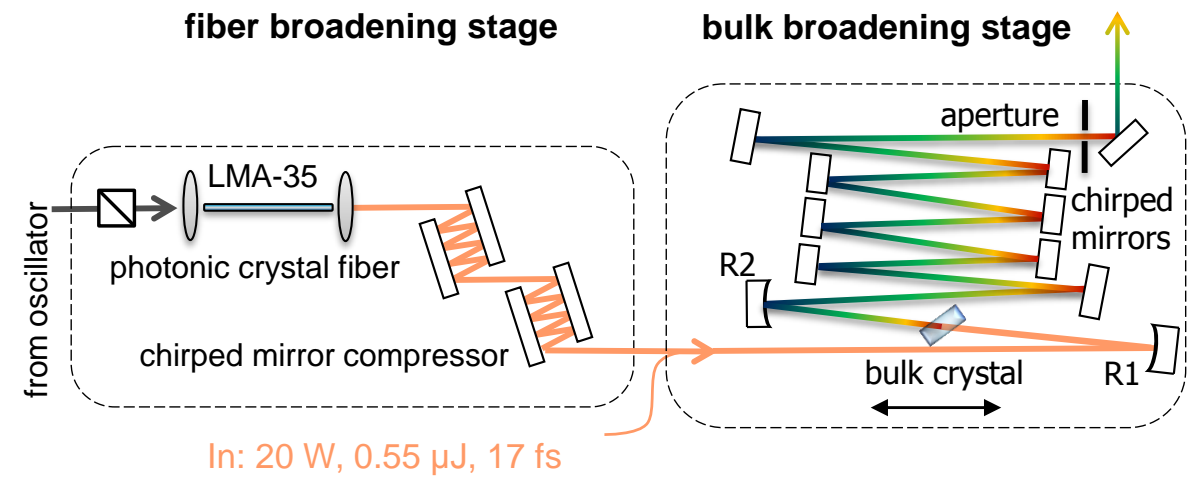

Fig. 2.10. The setup of the compression experiment is sketched. The output of the fiber compression stage is focused with a spherical mirror R1 into a dielectric medium which can be translated along the propagation direction. The spherical mirror R2 collimates the spectrally broadened beam which is steered into a chirped mirror compressor. A hard aperture cleans the beam profile. 
$1.9 \mathrm{~cm}$ are sufficient to lengthen the $17 \mathrm{fs}$ pulses by a factor of two (RMS duration, c.f. supplement 2). Subsequently, for further spectral broadening experiments, it becomes necessary to either shorten the interaction length between the intense pulses and the nonlinear medium or to reduce material nonlinearity and dispersion. The former approach is addressed in this section while the latter approach will be discussed in section 3.1.2. A short interaction length will apparently reduce the total GDD the pulses undergo in the broadening medium. Furthermore, beam collapse happens after a certain propagation length (cf. section 3.2.1), and hence, the material thickness can be made shorter than the critical self-focusing distance. These properties, together with the target of demonstrating only a moderate compression factor of $\approx 2$ for reaching the few-cycle regime, motivated the approach of spectral broadening in a dielectric bulk medium.

\section{Spectral broadening in bulk material}

The $17 \mathrm{fs}, 500 \mathrm{~nJ}$ pulses emerging from the fiber compression stage were focused by means of an enhanced silver mirror (R1) into a dielectric window to achieve additional spectral broadening (Fig. 2.10). The window was placed behind the focus of $\mathrm{R} 1$ and was afterwards slowly shifted by means of a mechanical translation stage towards the focal plane while the spectrum emerging from the nonlinear medium was monitored with an OSA. If the material was moved too close to the focus, where peak irradiances in the range of 1$10 \mathrm{TW} / \mathrm{cm}^{2}$ were reached, beam filamentation ${ }^{49,207}$ set in. This process, involving the generation of an electron-hole plasma, was avoided due to the high thermal load of the nonlinear medium. Instead, the spectral broadening was solely based on the optical KERR effect which does not involve absorption, and hence generally leads to better power stability. By applying the described routine, several parameters were varied to maximize SPM in the bulk medium: Firstly, different materials were tested. While YAG and sapphire tended to form filaments or were damaged before exhibiting broad spectra, the $n_{2} \approx 1.9 \times 10^{-16} \mathrm{~cm}^{2} / \mathrm{W}$ of $\mathrm{CaF}_{2}$ was too low to reach best broadening results which were obtained with fused silica and crystalline quartz. The latter has much better heat conductivity and generated steadily broad spectra over several hours. Neither slight variation of the crystal thickness ( $3-5 \mathrm{~mm}$ for crystalline quartz), nor of the input chirp

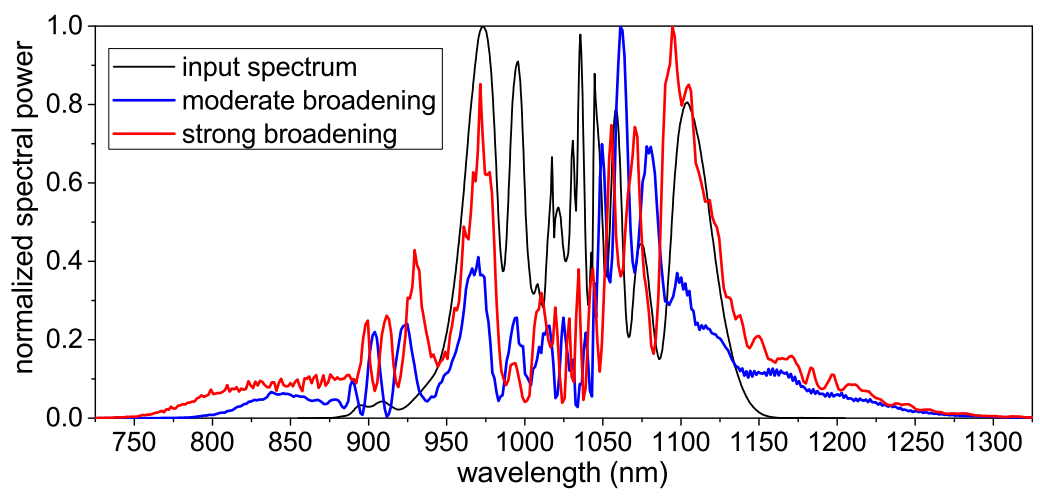

Fig. 2.11. Spectra measured with an OSA after spectral broadening in a $5 \mathrm{~mm}$ thick crystalline quartz plate for different distances between focal plane and nonlinear medium. The blue solid line shows moderate spectral broadening. The FTL is $7.5 \mathrm{fs}$. After moving the quartz plate closer to the focus, the red solid line was measured. The FTL is $6.4 \mathrm{fs}$. For comparison, the spectrum of the pulses emerging from the fiber compression stage is shown (black solid line). 
had significant impact on the achievable spectral widths. Instead, balancing the Gaussian beam divergence and the nonlinear self-focusing turned out to be decisive for reaching the broadest spectra. This will be investigated in more detail in section 3.2. Spherical mirrors (R1) with focal lengths of $50 \mathrm{~mm}$ and $150 \mathrm{~mm}$ were tested. With a $f=50 \mathrm{~mm}$ focal length, a FTL of 6.4 fs was achieved (red spectrum Fig. 2.11). In this configuration, a slight red glow became visible, being a precursor of filamentation, and thus marking the maximally achievable spectral bandwidth.

These ultrabroadband pulses required, however, tailored dispersive mirrors in order to reach durations on the order of two optical cycles. Consequently, in a first step, a slightly narrower spectrum (blue curve Fig. 2.11) was compressed by six bounces off complementary chirped mirrors, labeled PC247. These compensated for the dispersion of $2 \mathrm{~mm}$ FS per bounce (Fig. 2.12(b)). A pulse duration of 10 fs was retrieved (Fig. 2.12(d)) by means of a SH-FROG measurement. The autocorrelator used to cross-check the $17 \mathrm{fs}$ pulse measurements could not be used for these very short pulses. It was noticed that inserting a $2 \mathrm{~mm}$ thin FS window did not change the autocorrelation trace although the short pulses must be sensitive to it. Therefore, only the RMS FROG error and the agreement between measured and retrieved spectrum could be used to verify the correctness of the FROG measurement (Fig. 2.12(a)+(c)). Although these cross-checks are not quite as extensive as in the fiber-compression study, they exhibit clear evidence for the reliability of the pulse measurement.

The spectral phase of Fig. 2.12(c) reveals that the PC247 mirrors are not ideal for the compression of even shorter pulses. At the long wavelength edge, around $1300 \mathrm{~nm}$, both the GDD of PC247 and the spectral phase are uncontrolled, and hence the spectrum is close to the bandwidth limits of the mirrors. Moreover, the oscillations of the spectral
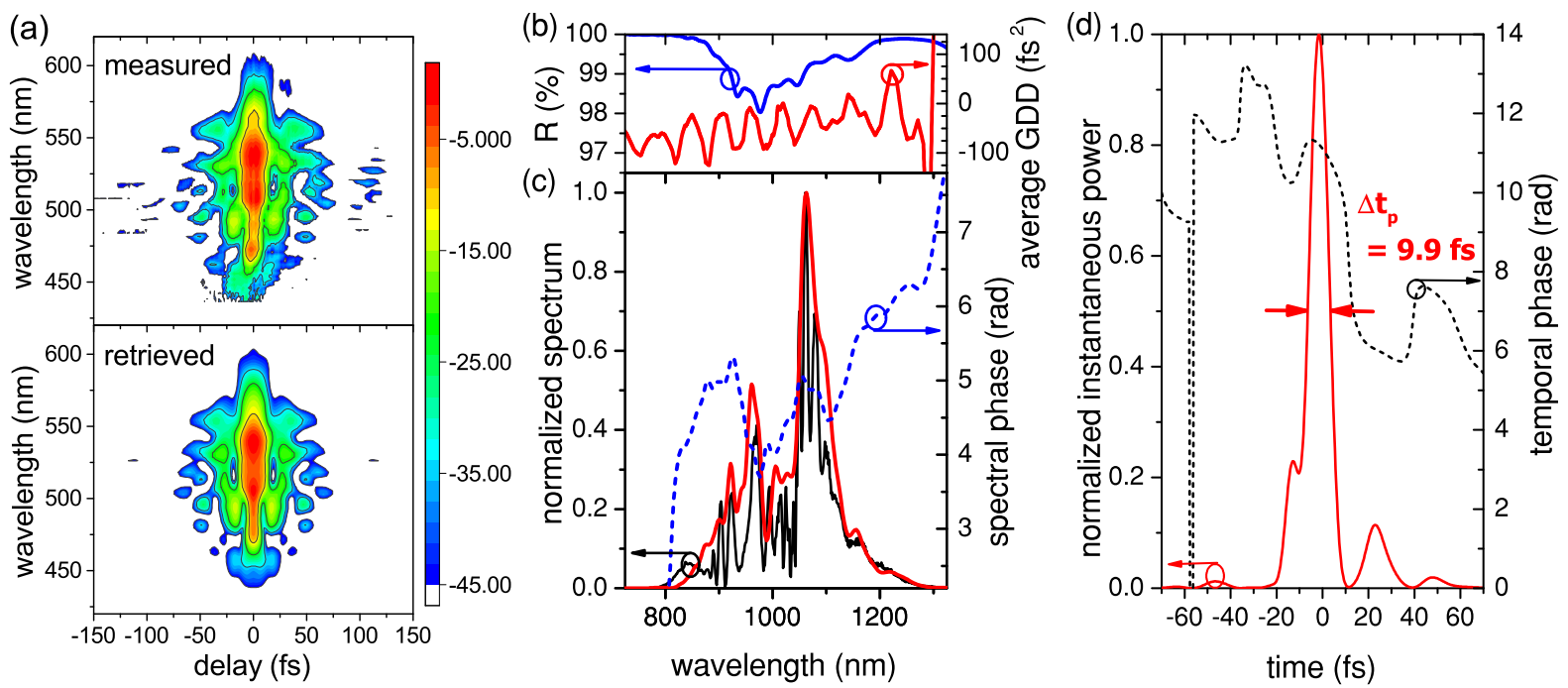

Fig. 2.12. (a) Measured (top) and retrieved (bottom) FROG traces of the pulses after the PC247 mirror compressor. The color scale is in units of $\mathrm{dB}$ with respect to the maximum of the trace. The RMS FROG error was $0.77 \%$ for a $512^{2}$ grid size. (b) Reflectivity (R, blue solid line) and average GDD (red solid line) of PC247. "Average" refers to the mean GDD of a complementary mirror pair. The dispersion of about $2 \mathrm{~mm}$ FS is compensated by one bounce off the mirrors in the spectral range from $700-1300 \mathrm{~nm}$. (c) Spectrum measured with an OSA (black solid line) and retrieved from FROG (red solid line). The blue dashed line shows the retrieved spectral phase. (d) Retrieved ultrahort pulse of only $10 \mathrm{fs}$ duration (red solid line) and corresponding temporal phase (black dashed line). 
phase in the most powerful parts of the spectrum are quite strong. This results in about $25 \%$ of the energy being located in the pulse pedestals. For comparison, only $15 \%$ of the energy was located in the pedestals of the $17 \mathrm{fs}$ pulse.

Subsequently, new mirrors were designed to compress the pulses with the maximal bandwidth as clean as possible. In order to do so, the spectral phase of the uncompressed pulses was measured by means of SH-FROG. It is represented by the dark blue dotted line in Fig. 2.13(b). Different thin $\mathrm{CaF}_{2}$, FS and sapphire plates of thicknesses from 1 to $5 \mathrm{~mm}$ were inserted into the collimated beam to verify the phase sensitivity of the FROG. Afterwards, the measured phase was fed into a multilayer design software (www . optilayer.com) which calculated an optimal layer structure for double-angle chirped mirrors. Their reflectivity and average GDD are shown in Fig. 2.13(b). It is remarkable that the reflectivity is larger than $99.8 \%$ for the whole pulse spectrum and that the GDD exhibits very strong excursions from -800 to $+800 \mathrm{fs}^{2}$, i.e. hardly implies a phase that can be readily decomposed into GDD, TOD and higher-order terms. Nevertheless, the predicted spectral phase of the ultrashort pulses, which is plotted as a dashed dark gray line in Fig. 2.13(c), is very flat. The pulses were compressed by means of two bounces off the tailored mirrors. Their measured spectral phase (blue dashed line Fig. 2.13(c)) is in very good agreement with the prediction. This demonstrates on the one hand, the capability of FROG to precisely determine the spectral phases of ultrashort pulses, and on the other hand, the powerfulness of both state-of-the-art multilayer-mirror design software and coating facilities. The measured pulses exhibit a duration of $7.7 \mathrm{fs}$, corresponding to only 2.2 optical cycles at $1030 \mathrm{~nm}$ central wavelength (Fig. 2.13(d)). If the spectral phase and the spectrum mea-

(a)

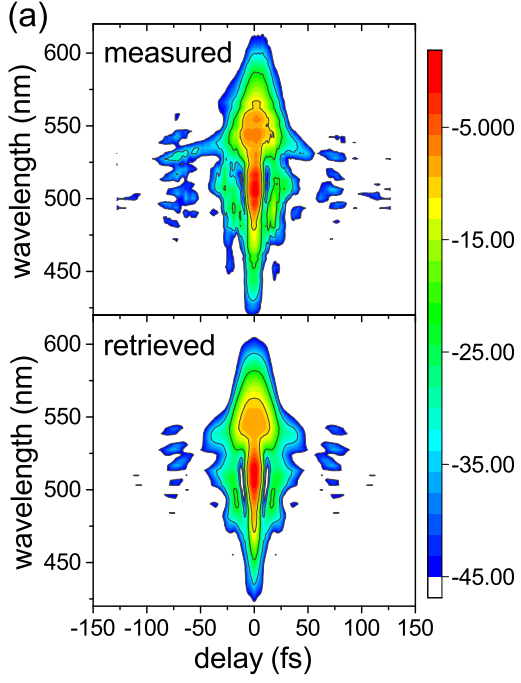

(b)

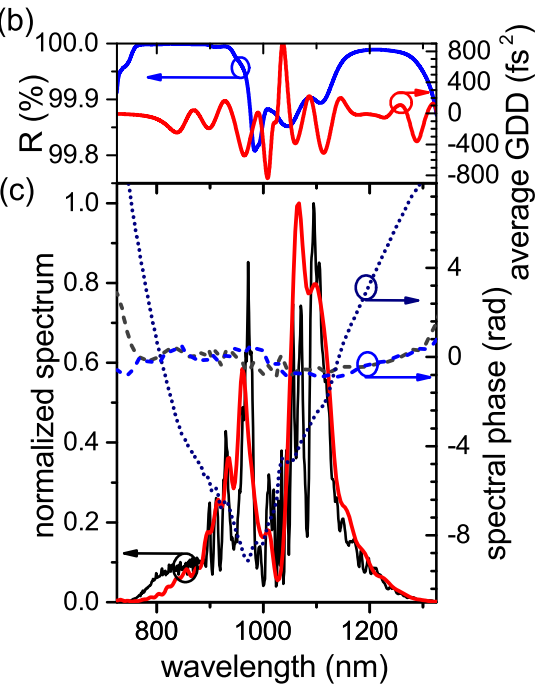

(d)

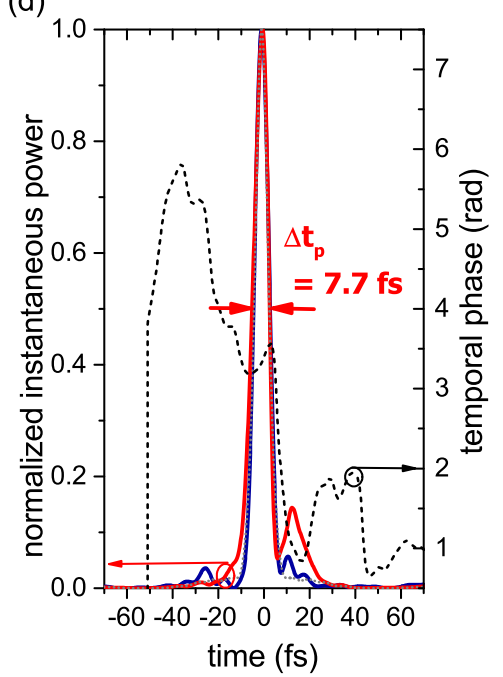

Fig. 2.13. (a) Measured (top) and retrieved (bottom) FROG traces of the pulses after the tailored mirror compressor. The color scale is in units of $\mathrm{dB}$ with respect to the maximum of the trace. The RMS FROG error was $0.48 \%$ for a $512^{2}$ grid size. (b) Reflectivity (R, blue solid line) and average GDD (red solid line) of the tailored mirrors. "Average" refers to the mean GDD of a double-angle mirror pair. (c) Spectrum measured with an OSA (black solid line) and retrieved from FROG (red solid line). The blue dashed line shows the corresponding retrieved spectral phase of the compressed pulses, the dark blue dotted line the retrieved spectral phase of the uncompressed pulses and the dark gray dashed line the spectral phase predicted from the uncompressed phase and the tailored mirror design. (d) Retrieved ultrahort pulse of only $7.7 \mathrm{fs}$ duration (red solid line) and corresponding temporal phase (black dashed line). For comparison, the FouRIER transform-limited pulse of the retrieved spectrum from (b) (dashed gray line, mostly hidden behind the measured pulse) and the predicted pulse from the tailored mirror design (solid blue line, also mostly hidden behind the measured pulse) are shown. 
(a)

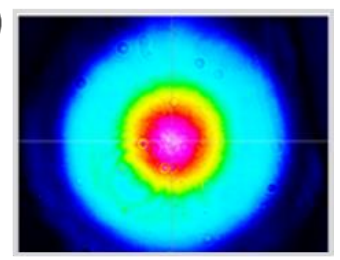

(b)

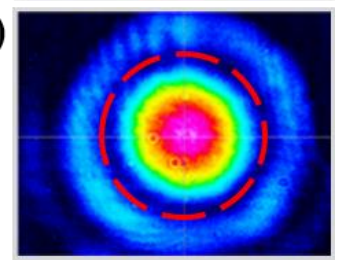

(c)

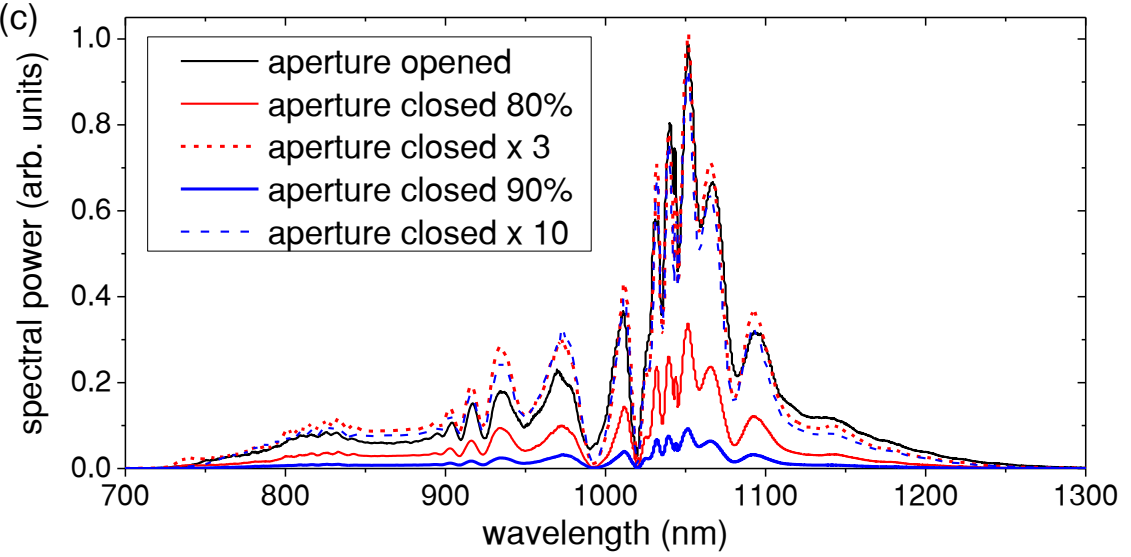

Fig. 2.14. (a) Measured beam profile with a CCD camera without additional spectral filtering. (b) Beam profile after a $1064 \mathrm{~nm}$ laser line bandpass filter. (c) Spectra measured with an OSA in dependence on the hard aperture transmission. The solid lines show the recorded spectra. The dashed lines have been scaled in order to normalize all spectra. The spectral shapes are very similar, the FTLs nearly identical.

sured with the OSA are combined, the pulse duration would be only $6.6 \mathrm{fs}$, and hence very close to the FTL of $6.4 \mathrm{fs}$, showing very good compression quality. If the retrieved pulse is compared to the predicted one, just a trailing pedestal is more pronounced than expected from the design. The main pulse, however, contains $80 \%$ of the total energy.

Contrary to fiber, bulk material does not provide a guiding structure which leads to homogeneous spectral broadening across the beam area ${ }^{208}$. Therefore, beam profile and spectral homogeneity of the beam are of particular interest in bulk broadening. The connection between spatial and temporal nonlinearities will be investigated in more detail in section 3.2. For now, the studies mainly refer to experimental observations: The beam profile after the broadening stage (Fig. 2.14(a)) exhibits higher spatial frequencies which were generated analogously to the new spectral frequency components in the nonlinear material. This becomes particularly evident by filtering a narrow band of incoming wavelengths as demonstrated in Fig. 2.14(b). To clean the beam from higher spatial frequencies, the aperture indicated in Fig. 2.10 was set such that only the part within the red dashed circle of Fig. 2.14(b) was transmitted. In the case of the blue spectrum of Fig. 2.11 about $60 \%$ of the power was contained in the encircled part, while about $40 \%$ of the power is transmitted in case of the broadest spectrum (red solid line of Fig. 2.11). An $\mathrm{M}^{2}$-measurement according to the ISO 11146 standard was performed with the spatially filtered beam after moderate broadening (i.e. blue curve Fig. 2.11). A scanning slit beam profiler with a pyroelectric detector was used to quantify the beam quality. The measured $\mathrm{M}^{2}, 1.4$ in both directions, was only slightly larger than the $\mathrm{M}^{2}$ value behind the PCF of the first broadening stage $\left(\mathrm{M}^{2}=1.3\right)$. To investigate the homogeneity of the broadened spectra, the aperture was gradually closed while the spectrum of the transmitted beam was measured. Fig. 2.14(c) shows that closing the aperture does not affect the spectral width of the pulse. The FTLs of all three curves are $6.1 \mathrm{fs} \pm 0.1 \mathrm{fs}$.

\section{Discussion}

The experiments have shown that spectral broadening in bulk material is capable of generating ultrabroadband spectra with FTLs down to 6 fs. The relatively short crystal 
length of $5 \mathrm{~mm}$ resulted in solely SPM-based broadening despite the fact that the peak power of the incident pulses was multiple times higher than the critical power of the nonlinear material. By utilizing a tight focusing geometry, the additional free parameter of divergence, which is not accessible in fiber schemes, was exploited to achieve significant spectral broadening without beam filamentation. The relatively low broadening factors of 1.7 - 2.3 sufficed to reach the few-cycle regime. Consequently, bulk broadening has proven to be a viable method for ultrashort pulse compression which works in pulse parameter regimes where solid-core fiber techniques are not applicable.

A considerable drawback of the approach was, however, the fact that the peak power of the pulses could not be further increased due to the spatial nonlinearities which led to ring formation and subsequently power losses. The peak power of the $10 \mathrm{fs}$ and the $7.7 \mathrm{fs}$ pulses were $20 \mathrm{MW}$ and $16 \mathrm{MW}$, resp., i.e. both values were lower than the peak power of the input pulses. Furthermore, only $15 \%$ of the oscillator power and $30 \%$ of the average power after the fiber stage were transferred to the most broadband pulses, highlighting that succeeding research must concentrate on improving the efficiency of the method. Section 3.2 will demonstrate techniques to make bulk broadening more efficient.

An additional disadvantage of the bulk scheme was the need for tailored chirped mirrors. As previously discussed, chirp compensation could be mainly accomplished by applying quadratic phase to the spectrally broadened pulses emerging from LMA-35. By compensating GDD and TOD only, minimal pulse durations of $10 \mathrm{fs}$ could be reached after the quartz crystal while the generation of shorter pulses required tailored mirrors. Fortunately, cutting-edge multilayer technology was available for accomplishing sub-10 fs compression. Such an advanced dispersion control is, however, only accessible for a small number of ultrafast optics laboratories worldwide. A first reason for the increased difficulty of high quality pulse compression is the nearly octave-spanning bandwidth of the pulses which was very hard to handle before the emergence of the chirped mirror technology ${ }^{54,98}$. Another reason, however, is connected to the free beam propagation in the bulk material which leads to a coupling of beam transformation and pulse shape ${ }^{209,210}$ (cf. Fig. 3.28).

Otherwise, bulk broadening has revealed many practical advantages over fiber-based broadening. The output spectrum of the compression stage could simply be adjusted by means of a translation stage that shifted the quartz window with respect to the focal plane. No daily coupling optimization was necessary, no degradation of the nonlinear medium was observed. If the bulk plate was moved too close to the focus and got damaged, it could be simply rotated in order to reuse it. By contrast, fiber damage required at least recleaving, which is not straight forward due to the large air content of LMA-35, or even fiber exchange in order to maintain a length of about $8 \mathrm{~cm}$. Consequently, the $5 \mathrm{~mm}$ quartz plate was never replaced while performing the experiments whereas meters of LMA-35 were used for the compression experiments. This constitutes an important complexity and cost advantage of the bulk scheme in comparison to the fiber setup.

In conclusion, for the first time, sub-10 fs, sub-3 cycle pulses have been generated from the output of a TD oscillator. This proof-of-principle experiment has demonstrated that power-scalable lasers that emit pulses of clearly more than $100 \mathrm{fs}$ can be transformed to few-cycle sources by virtue of external pulse compression schemes. Bulk broadening was applied to successfully overcome the peak power limitations of fiber broadening. The technique has very practical advantages, but still requires concepts to operate with higher efficiencies. These concepts will be discussed in section 3.2 of this thesis. 


\subsection{Stabilizing Kerr-lens Mode-locked Thin-disk Oscillators}

Compressing the output pulses of a KLM TD oscillator to durations of only a few optical cycles opens a new gateway to extreme nonlinear optics at $\mathrm{MHz}$ repetition rates. However, another imperative prerequisite to efficiently exploit field sensitive effects is the control over the optical waveform. Therefore, it was aimed for CEP stabilization as a next milestone in mode-locked thin-disk laser development. It was targeted in earlier experiments ${ }^{65,211}$ where beat signals in f-to- $2 \mathrm{f}$ interferometers were detected but could not be stabilized.

The CEP is highly sensitive to any kind of external laser perturbations. It could, for example, be easily shifted by gently manipulating the flow of the disk cooling water. Therefore, an initial investigation of noise sources was performed as a prerequisite of ensuing carrier-envelope-offset frequency locking experiments. Although mode-locked laser noise manifests itself in many ways, ${ }^{212,213}$ only intensity noise (IN) in the following section and subsequently CEP noise will be discussed in detail. The a priori suppression of IN is of utmost importance for achieving active CEP stabilization owing to amplitude-to-phase coupling in nonlinear elements, e.g. in the KERR lens inside the oscillator or the PCFs utilized for spectral broadening.

\subsubsection{Passive Stabilization}

A typical setup of short-term $(\mathrm{t}<100 \mathrm{~ms})$ IN measurements is sketched in Fig. 2.15. Essentially, a photodiode was placed in an attenuated laser beam. It converted optical power to electric current which was proportional to the voltage drop across a load resistor that was usually analyzed in frequency domain by an RF spectrum analyzer. The Agilent E4447A has a very low noise floor and can measure precisely down to $10 \mathrm{~Hz}$. Taking a time domain trace with an oscilloscope and performing the frequency analysis via FouRIER transformation is an alternative to direct frequency domain measurements. This was done in the experiments reported in section 3.1.2. Typical digital oscilloscopes exhibit, however, clearly higher noise floors than spectrum analyzers, and hence they are better

\section{Remark: carrier-envelope-offset frequency, carrier-envelope phase and carrier-envelope phase shift}

The following sections describe the stabilization of the carrier-envelope-offset frequency and the carrier-envelope phase without explicitly discriminating between the distinct quantities. In section 1.4.5, the linear relation between carrier-envelope-offset frequency and carrier-envelope phase shift per round trip was explained. Consequently, if the carrierenvelope-offset frequency is stabilized, the carrier-envelope phase shift is stabilized as well. This, however, does not imply that the carrier-envelope phase of every pulse is the same. This would require to stabilize carrier-envelope-offset frequency to an integer of the repetition rate. The absolute carrier-envelope phase is also unknown and cannot be measured by means of an f-to- $2 \mathrm{f}$ interferometer but only by field-sensitive nonlinear optics ${ }^{214,215}$. Nevertheless, a carrier-envelope-offset frequency stabilized source will be also called CEP stabilized because the carrier-envelope phase is, up to a residual jitter, controllable by means of the reference frequency of the servo loop and dispersive elements in the beam path (e.g. thin wedges). 


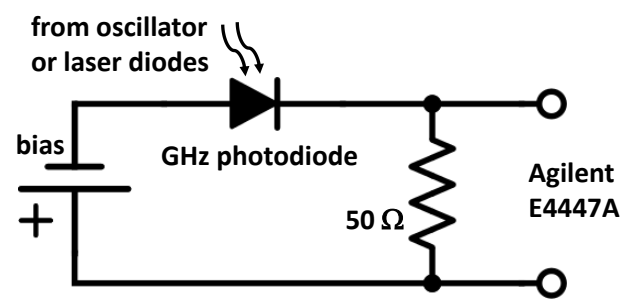

Fig. 2.15. Generic intensity noise measurement setup.

suited for analyzing fluctuation in the low $\mathrm{Hz}$ and $\mathrm{mHz}$ range which can hardly be reached by RF spectrum analyzers. In this section, the IN in the frequency range from $10 \mathrm{~Hz}$ to $500 \mathrm{kHz}$ is investigated. Typically, an optical power which corresponded to a peak-peak voltage between $500 \mathrm{mV}$ and $1 \mathrm{~V}$ was measured by the fast photodiode. The noise figures of the utilized KLM TD oscillator measured in 2017 are shown in Fig. 2.4 of section 2.1. These properties have been reached after identifying noise sources which did not allow CEP stabilization in the first experiments that are described in ref. 65. Fig. 2.16(a) compares the short term fluctuations of Fig. 2.4 with a measurement which was taken in August 2012 before the oscillator was passively stabilized. The graph shows very significant improvement in the IN performance that has mainly been reached because of (I) the usage of a highly stable power supply to drive the laser diodes and (II) the strong reduction of turbulence in the cooling circuits of thin disk and laser diodes.

The importance of (I) becomes very clear upon considering Fig. 2.16(b). The laser diodes (Laserline LDM 500-1000) had to be purchased with an integrated chiller as well as an integrated power supply. If solely the IN of the laser diodes (and not of the KLM TD oscillator) was measured, massive excess noise was detected in whole $\mathrm{kHz}$ range (black curve Fig. 2.16(b)). This was partially filtered through the active $\mathrm{Yb}^{3+}$ ions hosted by the YAG disk as the comparison to Fig. 2.16(a) indicates. The gain medium's upper

(a)

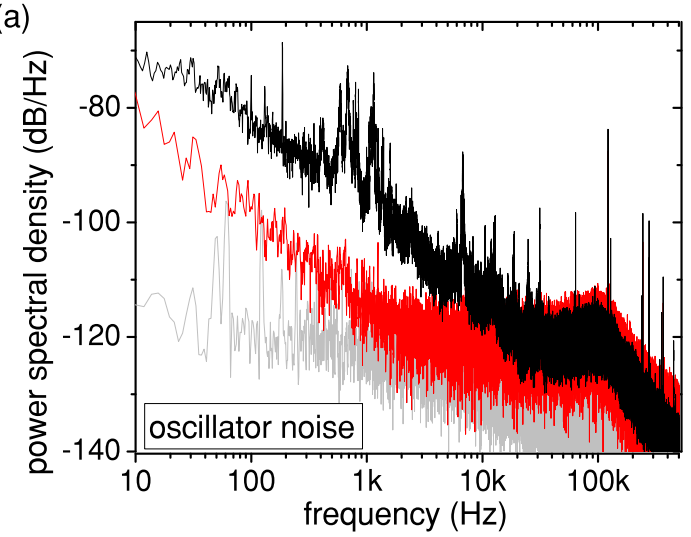

(b)

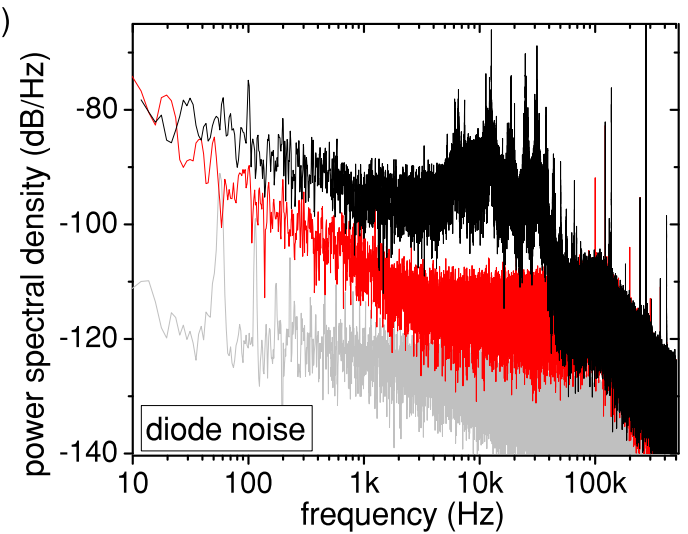

Fig. 2.16. (a) Gray and red curve correspond to the noise measurements shown in Fig. 2.4(a) (3 averages). The black curve shows the measured oscillator IN before additional means to stabilize the KLM TD oscillator were taken (10 averages). A massive excess noise is visible at frequencies lower than $10 \mathrm{kHz}$. The PSDs have been normalized to the value at $500 \mathrm{kHz}$ where the noise floor of the Agilent PSA E4447 spectrum analyzer is reached. (b) Measured IN of the fiber-coupled laser diodes of Laserline LDM 500-1000. The black curve shows a measurement that was taken when the laser diodes were driven with the integrated power supply. The red curve was taken after the laser diodes have been connected to an external power supply (Delta Electronika). The gray curve shows the noise floor. 
(a)

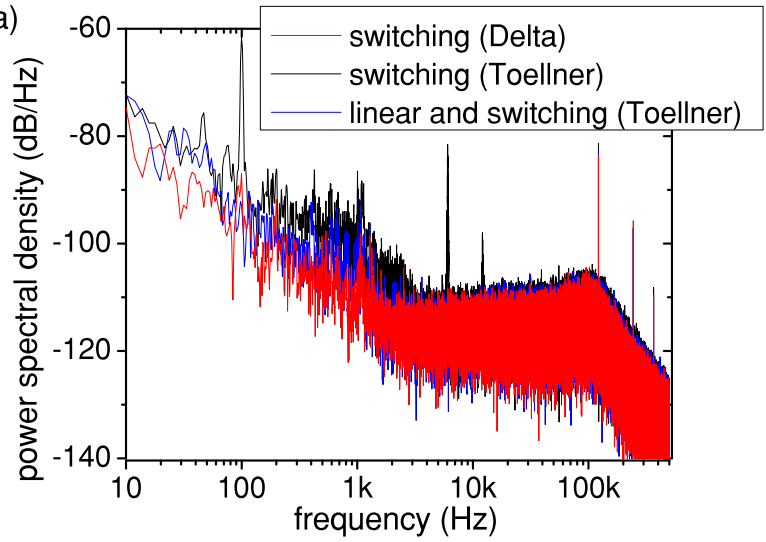

(b)

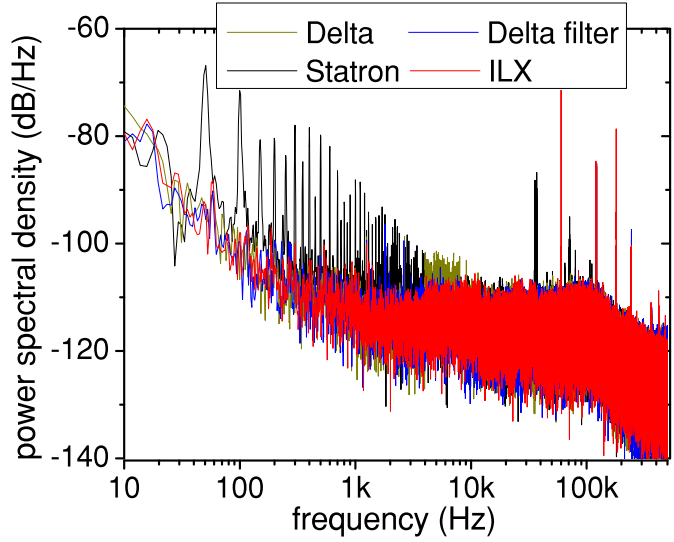

Fig. 2.17. (a) Comparison between IN measurements of laser diodes pumped with different switching (red line: Delta Elektronika, black line: Toellner Electronics) or a series connection of linear and switching (blue line, Toellner Electronics) power supplies. The Delta Elektronika supply demonstrates the best noise performance. The linear/switching combination exhibits only excess noise around $1 \mathrm{kHz}$. Using solely the Toellner Electronics switching power supply resulted in the highest integrated IN. (b) Comparison between IN measurements of laser diodes pumped with high-end switching power supplies (Delta Elektronika, ILX Lightwave) or a cost-efficient linear power supply (Stratron). The blue curve represents a measurement with the Delta Elektronika supply where power output was additionally passed through a $0.47 \mu \mathrm{F}$ feedthrough filter. This reduced the noise in the lower $\mathrm{kHz}$ range (cf. dark yellow curve). The devices of ILX lightwave and Delta Elektronika exhibited a comparable good performance while many noise spikes were observed if the cost-efficient power supply of Statron was connected to the laser diodes. In both plots laser diodes of the company Dilas, which required lower currents than the LDM 500-1000, were driven by the power supplies.

state lifetime is $\approx 1 \mathrm{~ms}^{142}$, resulting in an effective low pass filter of the pump light. Nevertheless, the noisy power supply severely affected the measured PSD of the KLM TD oscillator. Therefore, several other power supplies were tested. The measurements are partly presented in Fig. 2.17. It was expected that linear power supplies exhibit better noise performance than switching power supplies which generate a $\mathrm{kHz}$ frequency modulated signal before current rectification. The need of a typically heavier and more expensive linear power supply does, however, not seem imperative. Fig. 2.17(a) shows IN measurements of lower power laser diodes (supplied by DILAS) that were taken with a switching power supply of the company Delta Electronika as well as a switching and a series connection of linear and switching supply of the company Toellner. Toellner could, unfortunately, not offer a single linear power supply for the required electric current, and thus two sources had to be connected. The figure demonstrates firstly, that all supplies yielded better noise properties than those obtained with the integrated supply (cf. Fig. 2.16(b)). Secondly, if only the Toellner devices are compared, the use of a linear supply led to IN reduction at frequencies between $100 \mathrm{~Hz}$ and $3 \mathrm{kHz}$. Nonetheless, the Delta Elektronika device exhibited the lowest noise level although it was a switching power supply. Therefore, the choice of the power supply type (linear/switching) appears not as important as proper alternating current (AC) suppression by adequate low pass filtering.

This claim is manifested by Fig. 2.17(b) which shows that the high-end switching supplies of the specialized companies Delta Elektronika and ILX Lightwave exhibit a similar low power spectral density over the full measurement bandwidth, whereas the cost-efficient linear device of Statron is fairly noisy. Consequently, discriminating only between linear 
(a)

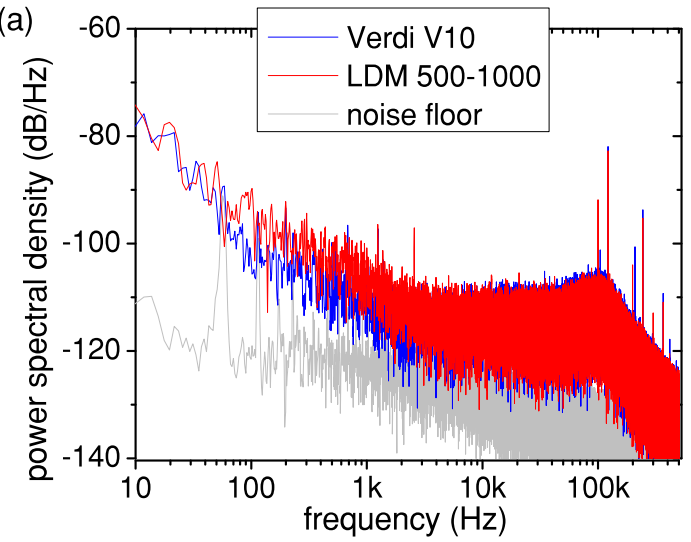

(b)

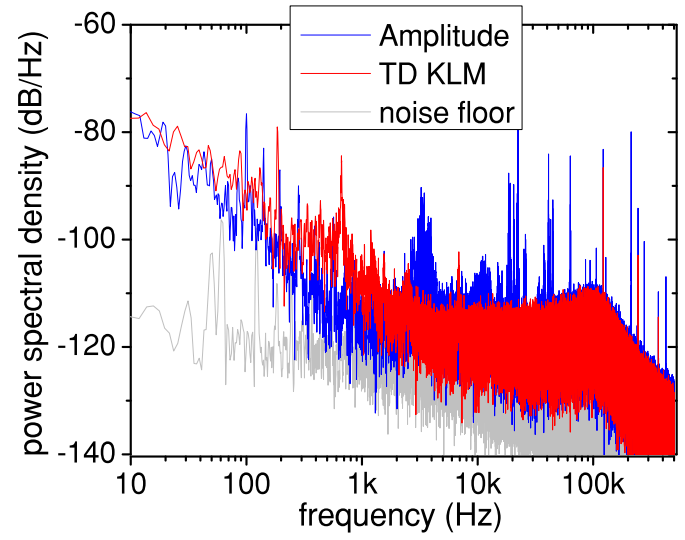

Fig. 2.18. (a) IN comparison of the a typical low-power Ti:sapph pump source (Verdi V10) and the utilized high-power laser diodes (LDM 500-1000) driven by a stable linear power supply. (b) IN comparison of the a commercial femtosecond oscillator (Amplitude t-pulse 50) and the utilized KLM TD oscillator with about 20 times higher power than the commercial oscillator. The thin-disk oscillator still exhibits two pronounced noise features in the shown measurement. One is located around $1 \mathrm{kHz}$, corresponding to the inverse of the upper state lifetime, and hence it can be attributed to relaxation oscillations. The second one is located around $7 \mathrm{kHz}$. It is not clear where this feature originates from. In some experiments it showed up (e.g. Fig. 3.38(b)), in some it did not (e.g. Fig. 3.38(a)). Without knowing further details, it is therefore believed that proper oscillator alignment may remove this contribution to the total IN.

and switching types of power supplies is not sufficient for the choice of an appropriate laser pump source. Proper electronic noise filtering is at least as important as the choice of the supply type. It is, however, difficult to extract detailed information on the noise performance of the power supplies from the companies' data sheets, and thus the test of different devices was inevitable. Eventually, it was decided to utilize a power supply of ILX Lightwave, a company which specializes on laser diode control. Nonetheless, it is to note that other supplies performed similarly well. Fig. 2.18(a) demonstrates that the search for a suited power supply was successful. Noise levels of a common low power Ti:sapph pump source, Coherent Verdi V10, were achieved with the high-power diodes pumping the KLM TD oscillator. The frequency doubled solid-state laser is well-suited for carrier-envelope-offset frequency stabilization, ${ }^{216}$ and thus served as a good reference.

After having addressed the noise in the $\mathrm{kHz}$ range ("electronic noise"), reducing the fluctuations in the $\mathrm{Hz}$ range ("acoustic noise") was targeted. At first, the setup was entirely covered to minimize the air flow in the vicinity of the laser beam. If this was not fully possible, the air conditioning was turned off for a few minutes in order to perform measurements. Moreover, changes were introduced to the cooling circuits (II). The integrated chiller of the pump diodes was removed. It contained a temperature dependent switch which alternatingly turned off and on the coolant flow. Instead, an external laser chiller was used that constantly circulated water with a temperature of $24.0^{\circ} \mathrm{C}$ and a flow of about $5 \mathrm{l} / \mathrm{min}$. Furthermore, the coolant flow in the disk head was strongly reduced from $3 \mathrm{l} / \mathrm{min}$ to only about $0.5 \mathrm{l} / \mathrm{min}$. The impingement cooling scheme (cf. Fig. 1.2(a)) otherwise induced vibrations of the disk. The improvement in the IN after having performed the described changes becomes obvious in Fig. 2.16. Further potential noise sources were not considered at that point, since the KLM TD oscillator IN power spectral density became very comparable to that of the commercial oscillator Amplitude t-pulse 50 which 
only provided $5 \%$ of the TD laser output power. Therefore, ensuing research was targeted to reach the primary goal of the presented investigations, the first CEP stabilization of a mode-locked thin-disk oscillator.

\subsubsection{Active Carrier-Envelope-Phase Stabilization}

\section{Beat signal detection}

Contrary to the IN, which can easily be measured with a photodiode and some analyzing device, the carrier-envelope-offset frequency is not accessible in a straight forward way since not even the best optical spectrum analyzers can nearly measure with $\mathrm{MHz}$ resolution. Therefore, schemes were employed that generate a beat signal of two frequencies which are shifted by carrier-envelope-offset frequency. Generally, this is achieved by spatially and temporally overlapping the $\mathrm{j}^{\text {th }}$ and $\mathrm{k}^{\text {th }}$ harmonic of the comb lines $\mathrm{m}, \mathrm{n}$ :

$$
\Delta f=j \cdot\left(m f_{\text {rep }}+f_{\text {ceo }}\right)-k \cdot\left(n f_{\text {rep }}+f_{\text {ceo }}\right),
$$

where $f_{\text {rep }}$ is the laser repetition rate and $f_{\text {ceo }}$ is the carrier-envelope-offset frequency. If $j \cdot m=k \cdot n$, a beat signal with the radio frequency

$$
f_{0}=|j-k| f_{\text {ceo }}
$$

can be detected. Typically $|j-k|=1$, e.g. in so-called 0-to-f-interferometers ${ }^{217,218}$ $(\mathrm{j}=1, \mathrm{k}=0)$ or 2 -to-3f-interferometers ${ }^{219,220}(\mathrm{j}=3, \mathrm{k}=2)$. The most common configuration, namely an f-to-2f interferometer ${ }^{19,221}(\mathrm{j}=2, \mathrm{k}=1)$ was implemented here. A quasi common-path interferometer was utilized which typically introduces less artificial noise, due acoustically or thermally induced optical path fluctuations, than in the originally used Michelson-type interferometers ${ }^{222,223}$. Its setup is sketched in Fig. 2.19(a) while Fig. 2.19(b) shows an optimized free running beat signal which was detected with an RF spectrum analyzer. Obviously, a beat signal can only be detected if the frequency doubled $\mathrm{m}^{\text {th }}$ comb line (2f component) and the $\mathrm{n}^{\text {th }}$ comb line (f compenent) carry optical power, i.e. if a coherent octave spanning spectrum is generated. Therefore, a small part of the ultrashort pulses, which were emitted from the fiber compression stage presented in section 2.2.1, were sent into a highly nonlinear PCF at the input of the interferometer. It was operated in the anomalous dispersion regime, and hence sub- $100 \mathrm{fs}$ pulses were needed to maintain coherence at the fiber output ${ }^{48}$. If longer pulses are used, quantum fluctuations may initiate amplified spontaneous RAMAN scattering while laser intensity fluctuations lead to extremely nonlinear soliton-fission and modulation instabilities resulting in the destruction of the pulse-to-pulse phase coherence which is essential for frequency combs. The temporal overlap in the avalanche photodiode was accomplished by a delay stage consisting of two prisms which resolved the optical spectrum in space and two D-shape mirrors which created an optical path difference between the blue and the red wing of the continuum. Since only the spectra around $680 \mathrm{~nm}$ and $1360 \mathrm{~nm}$ were of interest for the beat note detection, a precise phase control was not necessary. The fundamental wavelength of $1360 \mathrm{~nm}$ carried relatively high spectral power in the fiber continuum (cf. spectrum in ref 65). It was doubled in a periodically-poled MgO-doped lithium niobate (PPLN) crystal which exhibits a high effective quadratic nonlinearity $d_{\text {eff }} \approx 16 \mathrm{pm} / \mathrm{V}$ and can be type 0 phase-matched, i.e. fundamental and $\mathrm{SH}$ exhibit the same polarization, and 

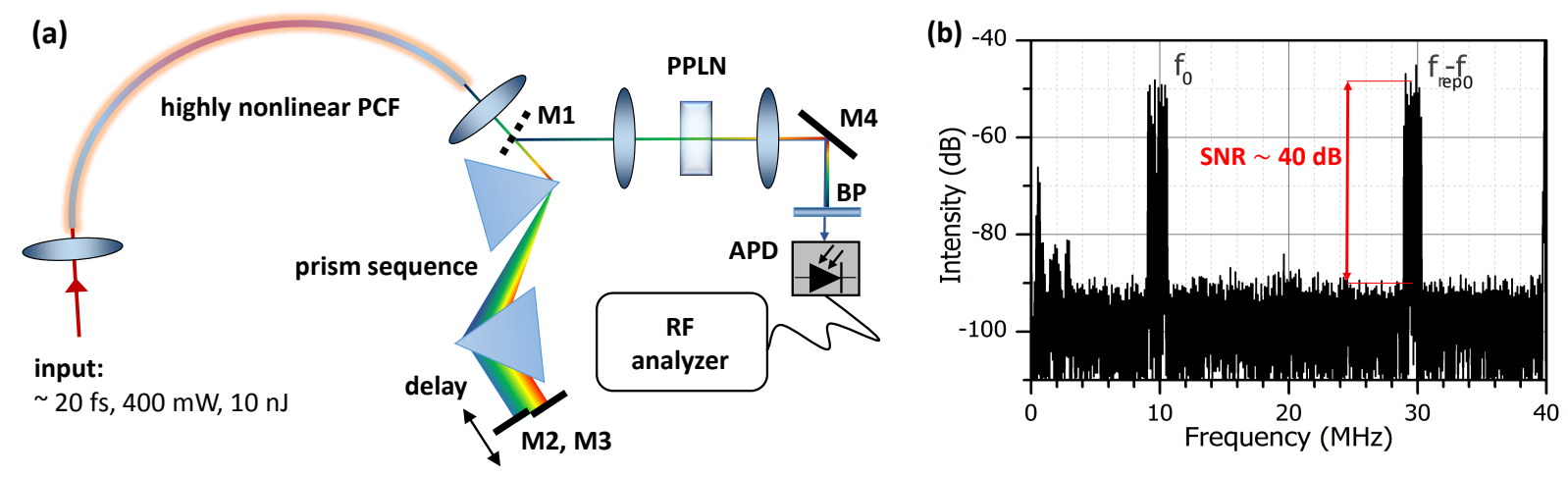

Fig. 2.19. (a) Setup of the f-to-2f interferometers. The highly nonlinear PCF (SC-3.7-975, NKT Photonics) generated a coherent continuum which is angularly dispersed by the prism sequence. The Dshape mirrors M2 and M3 introduce a delay between long and short wavelengths in order to temporally overlap the $\mathrm{f}$ and the $2 \mathrm{f}$ component of the interferometer. They are also set to reduce the beam height at the D-shape mirror M1 such the the beam returning from the delay line is reflected into a lens that focuses into a PPLN crystal which frequency doubles the continuum wavelengths around $1360 \mathrm{~nm}$. The lens behind the PPLN gently focused the beam on a APD. To get a high SNR, the beam passed a filter (BP) with a $10 \mathrm{~nm}$ transmission band centered at $680 \mathrm{~nm}$ before. In a first step the beat signal was detected with an analog RF spectrum analyzer. All mirrors (M1-4) were silver-coated. All lenses were asphers. (b) Optimizing input power, fiber coupling and overlap of $f$ and $2 \mathrm{f}$ components typically yielded $40 \mathrm{~dB}$ SNR within a $10 \mathrm{kHz}$ resolution bandwidth. The frequency at $f_{\text {rep }}+f_{0}$ originates from the beating of the $2 \mathrm{f}$ component with a neighboring comb line of the $\mathrm{f}$ component since the bandpassed spectrum still hosts many thousands of comb lines. No active stabilization of the beat signal is applied in the graph, yielding a $\approx 1 \mathrm{MHz}$ excursion of the signal.

thus can interfere on the detector. Allowing only the comb lines with frequency doubled counterparts to hit the avalanche photodiode (APD) was imperative to keep the noise floor of the detector low. Therefore, a narrow frequency band around $680 \mathrm{~nm}$ was filtered. Since a high signal-to-noise ratio (SNR) of the beat signal resulted in tighter locking of the carrier-envelope-offset frequency, ${ }^{216}$ optimization of the interferometer alignment was performed to a minimum $35 \mathrm{~dB}$ difference between noise floor and beat note. Typically, even $40 \mathrm{~dB}$ could be reached (cf. Fig. 2.19(b)). Mainly the coupling into the highly nonlinear PCF was repeated often. But also the AOI of the beam on the bandpass filter, which slightly manipulated the transmission spectrum, and the input power were varied to maximize the SNR.

\section{Stabilizing the beat signal}

After having established means to measure the carrier-envelope-offset frequency, its strong, quasi-random excursions (cf. Fig. 2.19(b)) were supposed to be reduced as much as possible. For this purpose a phase-locked loop (PLL) was implemented ${ }^{224}$. The setup is shown in Fig. 2.20. The main challenge to achieve a tight lock is indicated in Fig. 2.16. It is related to the $\approx 1 \mathrm{~ms}$ upper state lifetime of the active medium. In Fig. 2.16, this effective low-pass led to a strong suppression of the laser diode IN. By contrast to this desirable effect, the low-pass will be detrimental for the purpose of actively controlling the carrier-envelope phase because it severely reduces the bandwidth of a pump power regulated feedback loop. For comparison, the upper state relaxation time of Ti:sapph is only about $3 \mu \mathrm{s},{ }^{142}$ and hence feedback up to the $100 \mathrm{kHz}$ range is possible. This is usually more than sufficient to achieve tight locking of the carrier-envelope-offset frequency. 
(a)

Feedback to AOM

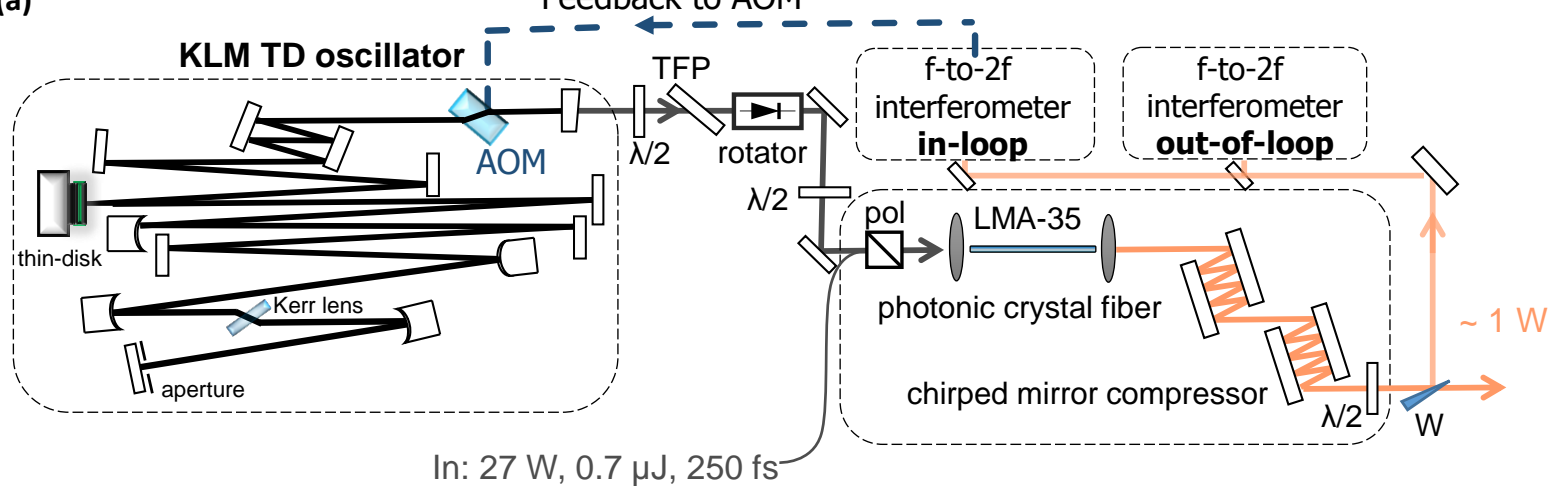

(b)

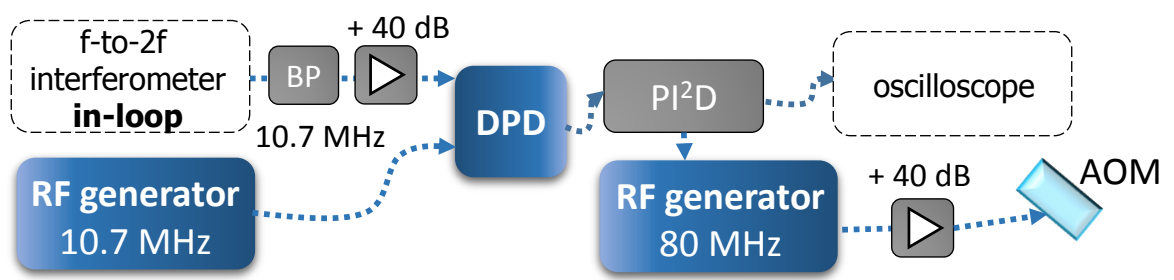

Fig. 2.20. (a) CEP stabilization setup. Oscillator and fiber compression stage are almost as before (cf. Fig. 2.7, $\lambda / 2$ - half-wave plate, TFP - thin-film polarizer, pol - polarizing beam splitting cube), just an AOM was inserted into the oscillator next to the output coupler. It was placed at BREWsTER's angle. For initial experiments the $5.5 \%$ OC was used, otherwise the $14 \%$ OC. In the former case, the pulses emerging from the compression stage were about $40 \mathrm{fs}$ long. This was still short enough to the generated a coherent supercontinuum in the highly nonlinear PCFs. The power sent into the interferometers was adjusted by a half-wave plate and a wedge $(\mathrm{W})$ with $45^{\circ}$ to $55^{\circ}$ AOI. Two f-to- $2 \mathrm{f}$ interferometers were set up. The in-loop interferometer provided feedback to the AOM while the out-of-loop interferometer analyzed the residual CEP noise. The dashed line indicating the electronic feedback is explained in more detail in (b). The beat signal was electronically band-passed (central frequency $10.7 \mathrm{MHz}$, bandwidth $2 \mathrm{MHz}$ ) to suppress the signal originating from the laser repetition rate. The beat signal was amplified afterwards and compared to a reference stemming from a RF generator by a digital phase detector (DPD) in the in-loop interferometer and a analog phase-detector in the out-of-loop interferometer. The error signal originating from the phase-difference was sent to a $\mathrm{PI}^{2} \mathrm{D}$ controller and monitored in parallel. The signal of the servo controller modulated the $80 \mathrm{MHz}$ RF signal that drove the AOM.

Consequently, pump power modulation via an extra-cavity AOM or electro-optic modulator (EOM) is routinely employed in carrier-envelope phase locking schemes ${ }^{216,225-227}$. Diode-pumped mode-locked oscillators (bulk or fiber) can even be carrier-envelope phase stabilized by direct current modulation of the laser diodes ${ }^{228,229}$. This approach is, however, not straight-forward for the KLM TD oscillator as will be discussed in more detail in section 3.3.

The challenge to overcome the reduced feedback bandwidth that pump power modulation offers can be avoided by modulating the oscillator losses instead of the gain. Both approaches basically target to access control over the carrier-envelope-offset frequency via amplitude-to-phase coupling which, for instance, manifests itself through SPM in the KERR medium ${ }^{52}$. LEE et al. demonstrated with a $20 \mathrm{~mW}$ fiber oscillator that intracavity loss modulation can in-fact significantly extend the bandwidth of the PLL ${ }^{230}$. They reached an in-loop phase jitter of less than $150 \mathrm{mrad}$, a record for fiber lasers. However, the approach of using an absorbing modulator was not applicable to the KLM TD oscillator which operated at more than 2800 times higher intracavity average power than 

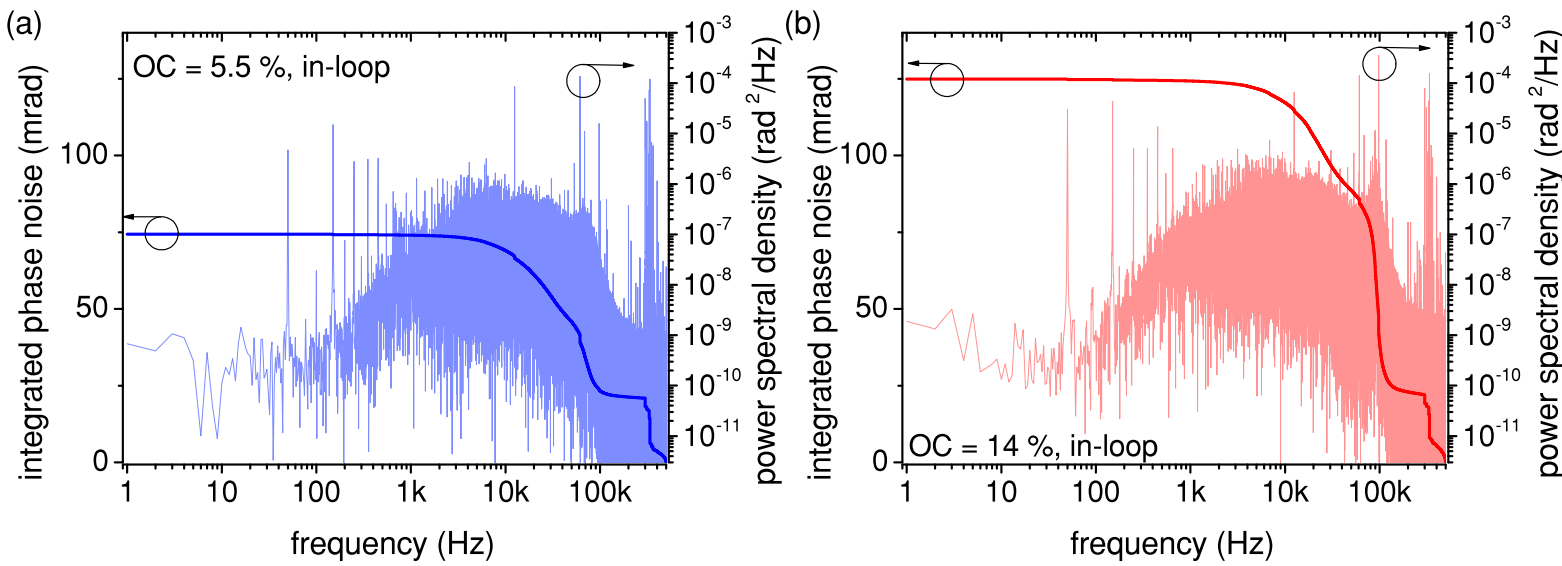

Fig. 2.21. (a) In-loop beat signal stabilization of the KLM TD oscillator with a 5.5\% OC and (b) with a $14 \%$ OC. Residual phase noise as low as $75 \mathrm{mrad}$ for the lower output coupling and $125 \mathrm{mrad}$ for the higher output coupling were reached in the bandwidth from $1 \mathrm{~Hz}$ to $500 \mathrm{kHz}$. The main difference in phase noise accumulates around $90 \mathrm{kHz}$. Due to the increasing phase lag of the feedback, the servo loop becomes resonant at this frequency. The noise spike around $330 \mathrm{kHz}$ originated from the DPD electronics. The best corner frequency settings were $f_{\mathrm{I} 1}=20 \mathrm{kHz}, f_{\mathrm{I} 2}=5 \mathrm{kHz}$ or $f_{\mathrm{I} 2}=10 \mathrm{kHz}$ and $f_{\mathrm{D}}=50 \mathrm{kHz}$.

the fiber laser in ref. 230. Therefore, loss modulation was targeted with a $3 \mathrm{~mm}$ thick intracavity AOM which does not absorb power itself, but introduces losses by diffracting light into the first acoustic grating order $(\approx 14 \mathrm{mrad}$ deflection angle $)$. The water-cooled AOM was placed at BREWSTER's angle directly in front of the output coupling mirror (Fig. 2.20(a)) where the beam waist is relatively small $(\approx 1 \mathrm{~mm})$. The $4 \mathrm{~mm}$ aperture of the modulator was large enough that no additional cavity losses were detected when no acoustic wave was launched. If a $80 \mathrm{MHz}$ signal at $6 \mathrm{~W} \mathrm{RF}$ power was applied to the transducer, the intracavity power was reduced by $2 \%$, corresponding to the modulation depth of the EOM in ref. 230. The RF power was varied by means of the modulation input of the RF generator that received the feedback signal from a servo controller (D2-125, Vescent Photonics). The locking electronics are sketched in Fig. 2.20(b). A digital phase detector ${ }^{224}$ compared the band-passed beat signal with an RF reference and generated an error signal for the $\mathrm{PI}^{2} \mathrm{D}$ controller.

First locking experiments were performed with the 5.5\% OC which required considerably less pump power $(\approx 100 \mathrm{~W})$ than the $14 \%$ OC $(\approx 200 \mathrm{~W})$. Consequently, the passive carrier-envelope-offset frequency stability was expected to be better, ${ }^{231}$ promising easier locking. Moreover, the pump power and the KERR-lens position were varied such that the free-running carrier-envelope-offset frequency excursions were relatively low (Fig. 2.19(b), $\mathrm{RMS} \approx 350 \mathrm{kHz}$ ) and the beat signal was located around the central frequency of the electronic bandpass $(10.7 \mathrm{MHz})$. For the $14 \%$ OC, the carrier-envelope-offset frequency sensitivity to the output power modulations was about $10 \mathrm{MHz} / \mathrm{W}$. Consequently, a $1 \mathrm{MHz}$ shift of the beat signal was introduced by a change of the output power by only about $0.25 \%$. In the final laser configuration, even $20 \%$ of output power variation did not interrupt mode-locking (cf. Fig. 2.2(b), note: in earlier experiments mode-locking sometimes stopped when the servo loop could not be closed). This demonstrates that the KLM TD oscillator was highly robust against the modulations introduced by the AOM. The corner frequencies of the $\mathrm{PI}^{2} \mathrm{D}$ controller were optimized on a trial and error basis although plenty of feedback loop theory exists (cf. ref. 232 and references therein). Firstly, relatively low 
cut-off frequencies of the integral parts were set $(100 \mathrm{~Hz}-1 \mathrm{kHz})$. The differential part, whose cut-on frequency was set between $10 \mathrm{kHz}$ and $50 \mathrm{kHz}$, helped to capture the beat signal but did lead to a significantly tighter lock. This was accomplished mainly by optimizing the proportional gain. After a minimal error signal was achieved, the cut-off frequencies of the integrators were varied and the optimization procedure was repeated. The phase of the beat signal and the $10.7 \mathrm{MHz}$ reference were initially compared by a 12 bit $\mathrm{DPD}^{224}$ which offered a huge capture range of $\pm 2^{12} \cdot 2 \pi \approx \pm 25000 \mathrm{rad}$. On the other hand, the lock performance was limited by the intrinsic detector noise. Connecting the reference signal to both inputs of the DPD resulted in a radian-level residual phase noise. Therefore, after having found a optimal servo controller setting, the detector range was reduced to $\pm 2^{4} \cdot 2 \pi \approx \pm 100 \mathrm{rad}$. In this case, the residual noise was only several tens of mrad. The servo controller optimization routine was repeated for the 4 bit detector. Best results for tight locking of the in-loop beat signal are shown in Fig. 2.21. The error signal was recorded with a digital oscilloscope. The voltage-to-phase conversion factor was determined by locking both RF generators to each other and slightly detuning them by $\Delta f=1 \mathrm{kHz}$. This resulted in a sawtooth wave. Fitting the slope $d V / d t$ gave the conversion factor:

$$
\frac{\Delta V}{\Delta \varphi} \approx \frac{d V / d t}{d \varphi / d t}=\frac{d V / d t}{2 \pi \Delta f}
$$

The power spectral densities shown on the right-hand axes of the plots in Fig. 2.21 was derived by FOURIER transforming the recorded time signal and the integrated phase noise (IPN) at frequency $f$ is computed by

$$
\operatorname{IPN}(f)=\left[\int_{f}^{f_{\max }} \operatorname{PSD}_{\varphi}(\nu) d \nu\right]^{1 / 2}
$$

where $\operatorname{PSD}_{\varphi}$ is the power spectral density of the measured phase $\varphi$. The upper integration boundary was usually set to $f_{\max }=500 \mathrm{kHz}$ where the phase error is already corrupted by the DPD electronics and where the noise floor of the detection scheme is hit (cf. Fig. 3.38).

\section{Out-of-loop characterization}

It remains to clarify if the measured phase noise results only from the beating comb lines as implied by Eq. (2.2). FORTIER et al. claimed that the carrier-envelope phase slippage in the in-loop f-to-2f interferometer is different from that of the pulse train emerging from the mode-locked oscillator ${ }^{233}$. Therefore, the usage of an additional out-of-loop interferometer was proposed which is able to quantify the artificially inscribed jitter of the feedback loop. The interferometer-induced carrier-envelope phase noise was attributed to amplitude-to-phase coupling in the highly nonlinear fiber which generated the continuum by means of SPM, i.e. by the same physical mechanism that also induces carrier-envelopeoffset frequency noise in the oscillator ${ }^{52}$. The servo-loop cannot distinguish the origins of jitters, and hence adds the inverse of the artificial fiber-induced phase noise to the one originating from the oscillator. Variations of the paths between $f$ and $2 \mathrm{f}$ component in the interferometer are also interpreted as carrier-envelope-offset frequency noise. The DPD counts the zero-crossings of the beat signal. If its AC term is proportional to (assuming 
$f_{0}$ being perfectly stable)

$$
\cos \left\{2 \pi\left(f_{0} t-f_{b} \cdot \tau(t)\right)\right\},
$$

where $f_{b}$ is the central wavelength of the bandpass, $t$ is the time and $\tau(t)$ is the delay between the $\mathrm{f}$ component and the $2 \mathrm{f}$ component, then the phase shift of the zero-crossing $\Delta \varphi_{0}(t)$ is given by

$$
\Delta \varphi_{0}(t)=4 \pi \frac{\Delta l(t)}{\lambda_{b}}
$$

where $\Delta l(t)$ is the time-dependent path difference and $\lambda_{b}$ is the central wavelength of the bandpass, i.e. $680 \mathrm{~nm}$ in the presented experiments. This explains why quasi-commonpath interferometers exhibit better out-of-loop phase stability ${ }^{222}$. The first out-of-loop measurements in-fact revealed a strong discrepancy to the in-loop values as Fig. 2.22(a) shows. Most prominently, in the acoustic range from $100 \mathrm{~Hz}$ to about $1.1 \mathrm{kHz}$ significant excess noise was detected by the second interferometer. The origin of it was investigated by means of IN measurements allowing conclusions on the phase noise by virtue of amplitudeto-phase coupling as described above. Fig. 2.22(b) demonstrates that both the highly nonlinear PCFs in the in- and out-of-loop interferometers deliver a very noisy output if compared to the thin-disk oscillator. The most dominant spikes in the PSD of the outof-loop fiber in Fig. 2.22(b), around 160 and $280 \mathrm{~Hz}$, appear as well in the PSD of the out-of-loop beat signal in Fig. 2.22(a). In-fact, they were already introduced by the LMA fiber and got enhanced in the interferometers due to the highly nonlinear behavior of the supercontinuum generating PCFs. The acoustic resonances turned out to be very sensitive to the coupling of the laser beam into the fiber. Hence, they were reduced as much as possible after the LMA-35 by slightly changing its alignment. Remarkably, the best noise performance was not reached at the highest coupling efficiency. The reason for this has not been investigated further. The degrees of freedom to align the highly nonlinear PCFs were rather limited since it was usually optimized for reaching a high SNR of the beat note (cf. Fig. 2.19(b)) which was also important to achieve a tight lock. Nevertheless, the reduction of the IN emerging from the LMA was already sufficient to demonstrate much better out-of-loop performance. The carrier-envelope phase noise measurements

(a)

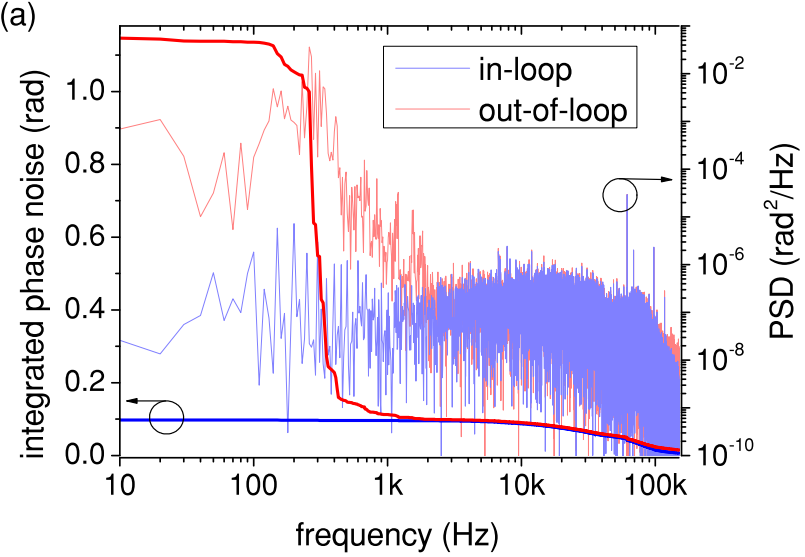

(b)

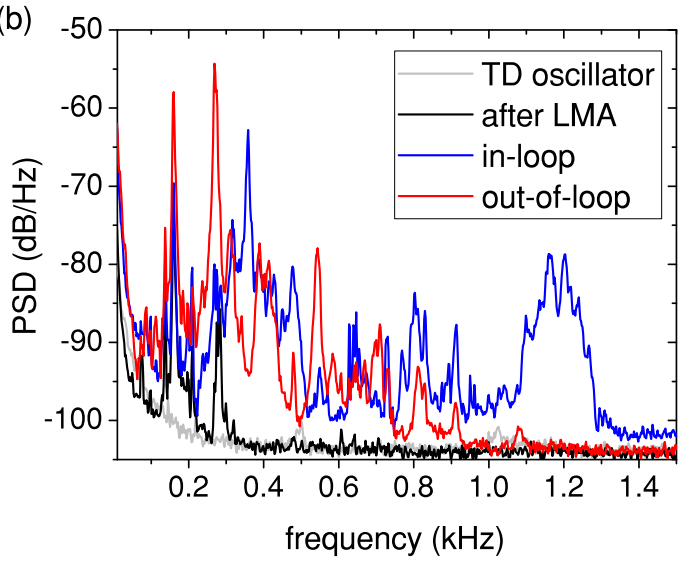

Fig. 2.22. (a) First in-loop and out-of-loop beat note measurement. It reveals strong excess phase noise of the out-of-loop signal in the acoustic range between $100 \mathrm{~Hz}$ and $1.1 \mathrm{kHz}$. The analysis was done with the numerical routine which in explained in more detail in section 3.3. (b) IN measurements behind the three PCFs of the setup shown in Figs. 2.19 and 2.20, resp. The measurements were done with a digital oscilloscope. The signal from the fast photodiode was low-passed by a filter with $1.9 \mathrm{MHz}$ cut-off frequency to reduce the noise floor of the oscilloscope. 

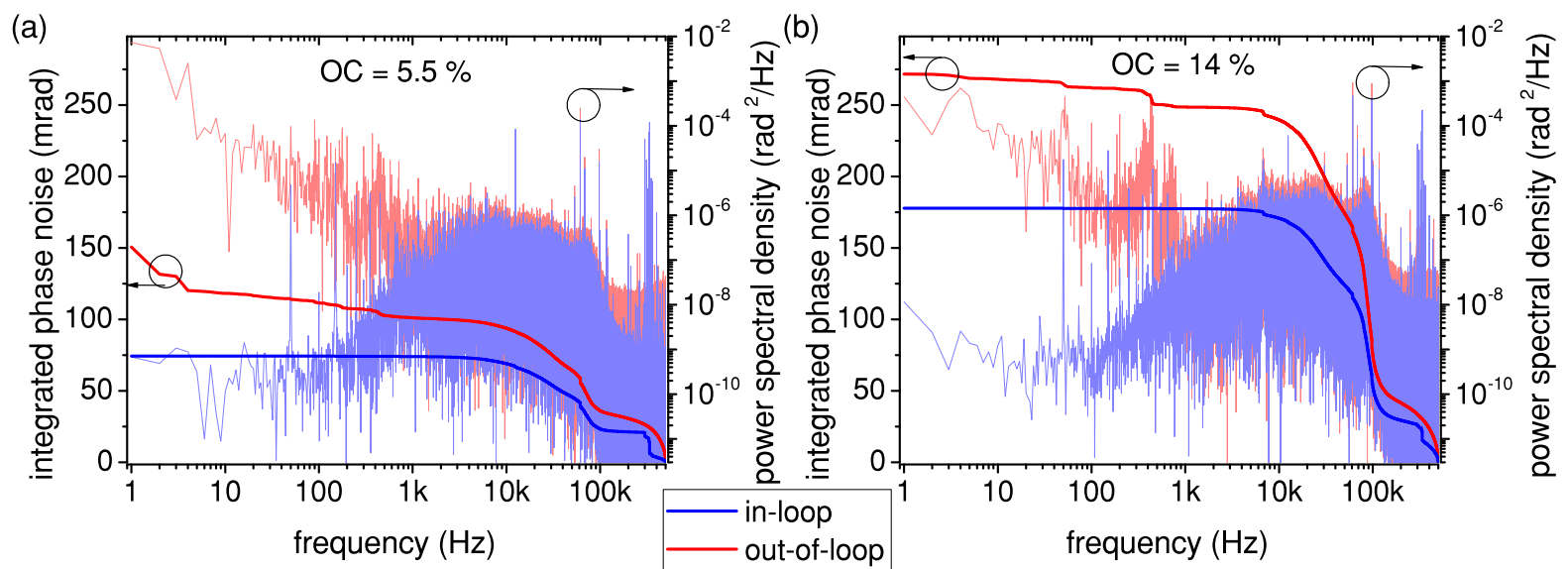

Fig. 2.23. (a) In-loop and out-of-loop beat signal stabilization of the KLM TD oscillator with a $5.5 \%$ OC and (b) with a $14 \%$ OC. Residual out-of-loop phase noise of $150 \mathrm{mrad}$ for the lower output coupling and $270 \mathrm{mrad}$ for the higher output coupling were reached in the bandwidth from $1 \mathrm{~Hz}$ to $500 \mathrm{kHz}$. The in-loop measurements were done as described before. The out-of-loop phase error was measured with an analog phase detector. It has a lower capture range than the DPD and is also sensitive to amplitude fluctuations but does not show noise spike around $330 \mathrm{kHz}$.

are shown in Fig. 2.23. The acoustic resonances which were present in Fig. 2.22(a) were eliminated for the most part. Different phase detectors were used for the in-loop and out-of-loop measurements. The analog detector in the out-of-loop interferometer did not reveal a noise spike around $330 \mathrm{kHz}$ but displayed a generally higher residual noise in the $\mathrm{kHz}$ domain (ca. 20 and $70 \mathrm{mrad}$, resp.). The other noise features $(<4 \mathrm{~Hz}$ for the $5.5 \%$ OC and $425 \mathrm{~Hz}$ for the $14 \%$ OC) were not present in all measurements and were probably again related to the coupling into the highly nonlinear PCFs.

\section{Discussion}

For the first time carrier-envelope phase stabilization of a mode-locked thin-disk oscillator was demonstrated. This is considered as an important step towards both MHz-rate phase-sensitive high-field experiments and the generation of high photon-flux XUV frequency combs which do not require complex enhancement cavities ${ }^{110,114}$. After the first presentation of the preliminary stabilization results ${ }^{234}$, a first carrier-envelope phase stabilized SESAM mode-locked TD oscillator was demonstrated ${ }^{235}$. It operated, however, only at average power levels of about $2 \mathrm{~W}$. Higher power SESAM mode-locked TD oscillators could not be stabilized so-far ${ }^{236}$. Consequently, the presented KLM TD oscillator is at the moment the only carrier-envelope phase stabilized oscillator operating at average power levels above $10 \mathrm{~W}$.

The intracavity AOM has manifested itself as a very good means to control the carrierenvelope-offset frequency. The resonance bump of the servo loop is situated around $90 \mathrm{kHz}$ which is way beyond the inverse of the upper state lifetime of the active medium $(\approx 1 \mathrm{kHz})$. By contrast to the intracavity EOM approach presented in ref. 230, the AOM does not modulate via absorbing light but through diffracting light out of the resonator. Therefore, the modulator works at about $35 \mathrm{~dB}$ higher average power. If the in-loop residual noise is compared (no out-of-loop measurement was done in ref. 230), the stability of the carrierenvelope-offset frequency of the high-power TD oscillator is even slightly better than that 
of the low-power fiber oscillator $(140 \mathrm{mrad})$. This is due to the better passive phase stability of solid-state lasers ${ }^{230}$. The best PLLs for Ti:sapph oscillators reached residual noise levels well below $100 \mathrm{mrad}$ (out-of-loop) in the investigated frequency bandwidth and also provided better long term stability ${ }^{218}$. This is certainly due to the usage of a completely fiber-free stabilization schemes. The PCFs have been identified as significant noise sources in the presented experiments. It will be shown in section 3.2 that spectral broadening in bulk material does not lead to such a noise enhancement, and may thus be a better platform for improving the noise performance of the lock. The lowest RMS values for the carrier-envelope phase jitter has been reached with the so-called "feed-forward" approach $^{237,238}(30 \mathrm{mrad}, 50 \mathrm{mHz}-500 \mathrm{kHz})$. The method is, however, not suited for highpower oscillators since firstly, the carrier-envelope phase stabilized pulses are diffracted into the first order of an acoustic grating introduced by an acousto-optic frequency shifter which results in low efficiencies of the scheme (30\% in ref. 238). This contradicts the purpose of accessing the high-field regime without the need of amplification. Secondly, high peak powers would require large spot sizes in order to avoid detrimental nonlinear effects in the modulator. Consequently, diffraction efficiency or modulation bandwidth would be clearly lower than for Ti:sapph systems ${ }^{239}$.

This concern limits also the power scalability of the demonstrated AOM-based stabilization approach. In the presented experiments the intracavity peak power was about $30 \mathrm{MW}$. It is expected that the method can readily be scaled at least by an order of magnitude. Supplement 1 explains why. Nevertheless, avoiding additional intracavity elements as sources of laser losses and potentially also damage is certainly attractive. Section 3.3 presents a scheme which targets carrier-envelope phase stabilization via direct pump current modulation.

\section{Supplement 1: Peak power scaling limitations of intracavity AOMs}

In the presented experiments an AOM made of FS with an effective aperture of $4 \mathrm{~mm}$ and a thickness of $3 \mathrm{~mm}$ was used. It was supplied by Gooch \& Housego. While the GDD of the modulator is negligible in comparison to the total GDD a pulse experiences during a round trip, the AOM presents an additional KERR medium inside the cavity which may counteract the function of the actual KERR lens in the telescope. Consequently, it might introduce additional (nonlinear) losses and complicate mode-locking. To prevent this, the spot size in the AOM should be kept sufficiently large which could, however, not be feasible due to limited apertures and the reduced modulation bandwidth which decreases with spot size due to the propagation speed of the acoustic wave.

The nonlinear losses introduced by the modulator are estimated in the following through the power which is diffracted into higherorder transversal modes. Since the $\mathrm{M}^{2}$ factor of the oscillator beam is close to 1 , higher-order modes are mostly absorbed during a round trip.

The model uses the coupled-mode theory presented in ref. 208. The publication investigates the self-diffraction losses of an ultrashort pulse that passes a KERR medium under negligible dispersion and self-steepening. It assumes that only the fundamental and the first allowed higher-order LAGUERREGaUssian modes are coupled which is also a good approximation here due to the very low losses. The following normalized complex field-amplitudes $\mathcal{A}_{0 p}(z, t)$ for $0^{\text {th }}$ angular and $\mathrm{p}^{\text {th }}$ radial LAGUERRE-GAUSSIAN modes were derived in the pulse reference frame for an initially fundamental mode: 


$$
\begin{aligned}
\mathcal{A}_{00} \approx & \exp \left\{i \kappa\left|H_{0}(t)\right|^{2}\right. \\
& \left.\times\left[\arctan \left(\frac{z}{z_{R}}\right)-\arctan \left(\frac{z_{0}}{z_{R}}\right)\right]\right\},
\end{aligned}
$$

$\mathcal{A}_{01} \approx \frac{\kappa\left|H_{0}(t)\right|^{2}}{4\left(2+\kappa\left|H_{0}(t)\right|^{2}\right)}\left[F(z, t)-F_{0}\left(z_{0}, t\right)\right]$,

$$
\Gamma_{01}=\left|\mathcal{A}_{01}(z, t) / \mathcal{A}_{00}(z, t)\right|^{2}
$$

where $t$ denotes the time relative to the pulse center, $z$ the position in the nonlinear medium relative to the focal plane, $z_{0}$ the position of the front facet of the nonlinear medium, $H_{0}(t)$ the normalized pulse shape of the fundamental mode and $z_{R}$ the RAYLEIGH length. $\Gamma_{01}(z, t)$ denotes the instantaneous power transfer from the fundamental to the first higherorder mode. The parameter $\kappa$ and the function $F$ are defined as follows:

$$
\begin{aligned}
\kappa & =\pi \frac{z_{0}}{\lambda} n_{2} I_{p} \\
F & =\exp \left\{2 i\left[1+\kappa\left|H_{0}(t)\right|^{2}\right] \arctan \left(\frac{z}{z_{R}}\right)\right\}
\end{aligned}
$$

where $n_{2}$ is the nonlinear refractive index and $I_{p}$ the peak irradiance. The losses, i.e. the power transfer to the first higherorder mode, are maximal at $H_{0}(t)=1$, the pulse peak. Since $\Gamma_{01}(z, t)$ increases with $H_{0}(t)$, the AOM introduces nonlinearly rising losses which counteract the saturable absorption of the KERR lens, at least in the presented configuration. In the following, only the loss at $t=0$ will be considered as an upper limit for the energy transfer to higher-order modes.

Firstly, $\Gamma_{01}$ for the presented oscillator configuration is computed. It is assumed that the center of the AOM is in the focal plane of the beam which is a reasonable assumption since the modulator length is much smaller than the RAYLEIGH length of the beam. The positioning at BREWSTER's angle, and hence the discrimination between sagittal and tangential plane is neglected. A peak power of $30 \mathrm{MW}$ and a waist of $1 \mathrm{~mm}$ are set, resulting in a peak irradiance of $1.9 \mathrm{GW} / \mathrm{cm}^{2}$. The nonlinear refractive index is set to $2.8 \times$ $10^{-16} \mathrm{~cm}^{2} / \mathrm{W}^{240}$ and the wavelength to $1030 \mathrm{~nm}$. This results in the following graph:

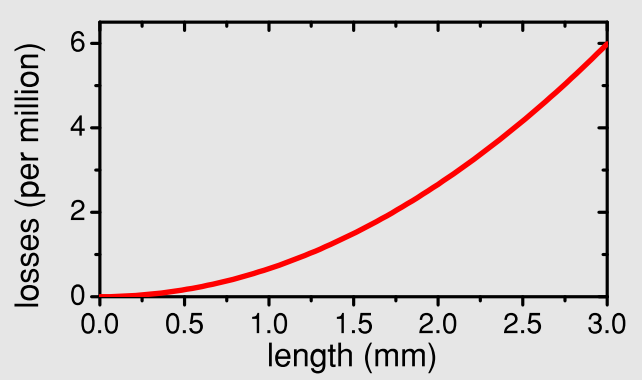

If the pulses travel through the $3 \mathrm{~mm}$ thick modulator only about $6 \times 10^{-6}$ of the power at the peak (less in the wings) is transferred to higher-order modes in a single pass, i.e. the losses are completely negligible which agrees with the experimental observation.

It has not been investigated how much nonlinear losses the oscillator will tolerate until it stops mode-locking. This, of course, strongly depends on the modulation depth of the fast saturable absorber and the configuration of the oscillator within the stability zone of the resonator. Two examples are presented where the maximal spot sizes are determined in dependence on the intracavity peak power by fixing the losses to $0.1 \%$ and $0.5 \%$ per pass:

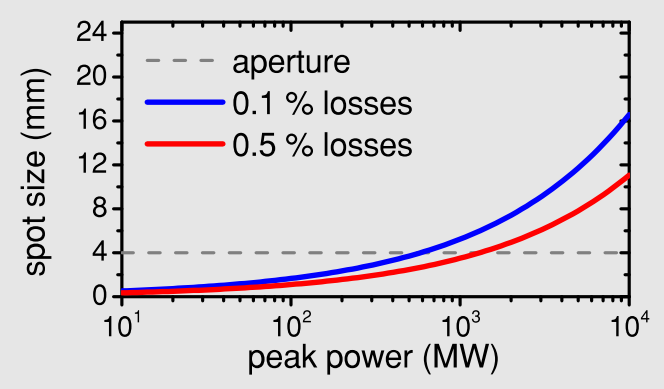


The term "spot size" was defined by the beam area which contains $99.5 \%$ of the optical power. This corresponds to a diameter of about 3.3 times the beam waist. The gray dashed line shows the aperture of the AOM which was used in the carrierenvelope phase stabilization experiments. If peak power losses of $0.1 \%$ per pass are tolerated by the oscillator, the modulator can be used up to an intracavity peak power of about $600 \mathrm{MW}$ which is 20 times higher than what has been experimentally demonstrated here. If $0.5 \%$ losses per pass are tolerated even peak power of 1.4 GW would be acceptable.

Eventually, the upscaling of spot sizes results also in a reduced modulation bandwidth of the AOM owing to the finite acoustic velocity $(5.7 \mathrm{~mm} / \mu \mathrm{s}$ for quartz $\left.{ }^{241}\right)$. The bandwidth is related to the characteristic rise time of the acoustic wave $\left(t_{r}\right)$ by ${ }^{239}$ :

$$
\Delta f_{\beta}=0.29 \sqrt{\beta} / t_{r},
$$

where $\beta$ is the frequency roll-off in $\mathrm{dB}$. The rise time is proportional to the spot size. It was specified by the supplier as $t_{r}=220 \frac{\mathrm{ns}}{\mathrm{mm}} \cdot w_{0}$, where $w_{0}$ is the Gaussian beam waist. For this value and the spot sizes shown in the previous graph, the $3 \mathrm{~dB}$ modulation bandwidth was calculated:

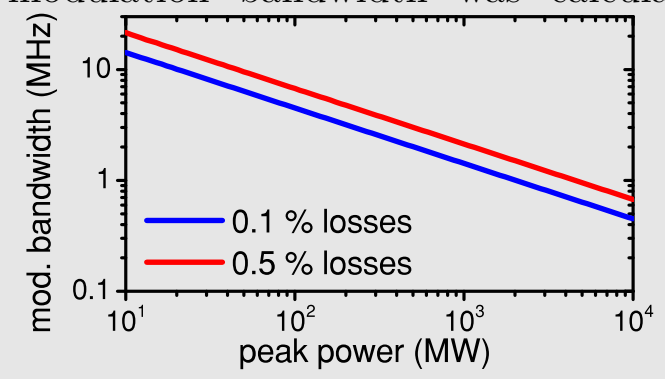

It is well above $90 \mathrm{kHz}$, which was the resonance frequency of the presented PLL, even for the largest considered peak power of $10 \mathrm{GW}$. For comparison, the highest intracavity peak powers that have been demonstrated are about $400 \mathrm{MW}^{139}$.

In conclusion, carrier-envelope phase stabilization by means of intracavity loss modulation via an $\mathrm{AOM}$ is expected to be readily scalable to the latest generation of mode-locked thin-disk oscillators which deliver an order of magnitude more peak power than the oscillator utilized here. Apertures larger than $4 \mathrm{~mm}$ promise further scalability. Moreover, the limiting self-focusing effect of the AOM could be also utilized in different resonator geometries, for instance, as an (additional) saturable absorber or in distributed KERR lens setups ${ }^{82}$. This would offer further scalability of the approach. The modulation bandwidth of the AOM does not limit the method in its scalability.

It remains to be clarified how thermal effects (thermal lensing) adversely affect the AOM approach, i.e. how average power scalable it is. In the experiments presented in ref. 139, a $5 \mathrm{~mm}$ thick sapphire plate was used as a KERR medium at $1 \mathrm{~kW}$ average power. The KLM TD oscillator still exhibited a very good longterm stability $(\mathrm{RMS}<1 \%$ measured over 1 hour). Although the thermal conductivity of (crystalline) quartz is a factor 3-4 lower than that of sapphire, the results hold promise that intracavity acoustooptic modulation can also be scaled to the kW average power level. 


\subsection{Chapter Summary}

In this chapter, two important achievements on the route to establish thin-disk technology in ultrafast optics laboratories have been demonstrated. On the one hand, the fewcycle pulse regime has been reached for the first time by means of double-stage external pulse compression. On the other hand, a first TD oscillator (and also the first modelocked oscillator with more than $10 \mathrm{~W}$ of average power) has been carrier-envelope phase stabilized.

Intracavity loss modulation by means of an AOM has proven to be an excellent approach for carrier-envelope-offset frequency stabilization of high-power oscillators. The residual CEP noise of $270 \mathrm{mrad}(\approx \pi / 12,1 \mathrm{~Hz}-500 \mathrm{kHz}$ ) is suited for many field-sensitive experiments. The lock-performance was comparable to the best results for low-power fiber oscillators at the time they were reported ${ }^{230}$. It is to note that in the meantime fiber oscillators have reached sub-100 mrad residual CEP noise ${ }^{30,242}$ which is comparable to the lowest values that have been achieved for Ti:sapph, or more general solid-state oscillators. The monolithic scheme of ref. 218 would certainly lead to improved phase-stability of the high-power KLM TD oscillator. The additional spectral broadening that is needed in bulk materials can be achieved by the methods which are explained in section 3.2. The "feedforward" approach ${ }^{237,238}$ is not particularly promising for mode-locked thin-disk oscillators due to the trade-off between diffraction efficiency and modulation bandwidth.

At the shortest pulse durations of $7.7 \mathrm{fs}$, the average power was about $6 \mathrm{~W}$ and the pulse energy about $150 \mathrm{~nJ}$. State-of-the-art commercial few-cycle Ti:sapph oscillators deliver at least one order of magnitude less average power ${ }^{243,244}$ while lower Watt-level Ti:sapph oscillators operate at longer pulse durations $(\geq 50 \mathrm{fs})^{56,57,245}$. Consequently, the presented proof-of-principle experiment has already demonstrated a significant advance in scaling amplification-free few-cycle pulse and CEP-stabilized sources to unprecedented average power levels.

Yet, to reach peak irradiances of at least $10^{14} \mathrm{~W} / \mathrm{cm}^{2}$, which are typical for $\mathrm{HHG}$ in gases, the laser beam would have to be focused down to a waist of less than $3 \mu \mathrm{m}$. This is feasible but rather inconvenient. Also, short RAYLEIGH lengths would lead to small interaction volumes, and thus low conversion efficiencies. The situation is different for solids where peak irradiances on the order of $10^{12} \mathrm{~W} / \mathrm{cm}^{2}$ are sufficient to trigger extreme nonlinear effects ${ }^{246,247}$. Nevertheless, applications like XUV frequency comb spectroscopy or attosecond pulse generation would certainly require HHG in gases. Although high harmonics can be generated in solids ${ }^{248-250}$, the (targeted) high photon flux would lead to a rapid degradation of the nonlinear medium. Therefore, further power scaling of the methods discussed in this chapter is highly desirable. In particular the fiber broadening scheme, which already did not allow to utilize the full oscillator power, needs to be substituted by an alternative power-scalable method, and, at best, maintain the high efficiencies of the technique. The next chapter demonstrates different power-scalable approaches on pulse compression and will also present a method how conventional pump power modulation is employed in CEP stabilization of KLM TD oscillators. 



\section{POWER SCALABLE CONCEPTS}

This chapter of the dissertation will discuss the average and peak power scalability of the achievements described in chapter 2, i.e. few-cycle pulse generation and CEP stabilization. In the thesis' introduction, this has been proclaimed as the general goal of current femtosecond laser development. However, there are often application dependent preferences in scaling either pulse energy or repetition rate, rather than both. A few examples shall be sketched to enable a more specific discussion of the results presented in the ensuing sections.

For time domain applications, high pulse energies are typically desired. They are directly proportional to the peak power and laser fluence, and thus grant easy access to nonlinear phenomena. Moreover, if matter is in some way excited by light, it needs a finite time to relax back into its ground state. These time scales range from a few femtoseconds, e.g. for lattice vibrations in solids, up to thousands of seconds, e.g. for lifetimes of metastable atomic states $^{251}$. The relaxation time of the sample hence sets an upper limit on the repetition rate of the laser exciting the matter under test. For instance, in ultrafast electron diffraction ${ }^{252}$ or multi-photon microscopy ${ }^{253}$ repetition rates between $100 \mathrm{kHz}$ and $1 \mathrm{MHz}$ are preferred. Another concrete example is found in the work of HACHÉ et $a l .{ }^{254,255}$. Their experiments investigated single and two-photon-absorption interferences in the conduction band of GaAs. Two light sources were utilized: one with $82 \mathrm{MHz}$ and the other one with $250 \mathrm{kHz}$ repetition rate. The $\mathrm{kHz}$ source allowed most of the free carriers in GaAs to relax before the sample was excited by the following pulse, and thus readily enabled to observe fringe patterns. By contrast, interferences could only be observed with the $\mathrm{MHz}$ source after additional defects, reducing the carrier lifetime, were added to the GaAs crystal.

On the contrary, for a variety of frequency domain applications very high repetition rates are desired whereas nonlinear effects must be avoided. In frequency comb spectroscopy, for instance, repetition rate does not only raise the average power of the mode-locked laser, but also the spacing between the comb teeth. Therefore, assuming fixed laser bandwidth and pulse energy, the power per comb line is additionally increased by the reduced number of modes which make up the optical spectrum. Finally, if a frequency comb in the XUV shall be generated both high peak power, to enable HHG, and high repetition rate, to achieve high powers per comb line, are required.

These brief examples shall motivate the following considerations on scaling average and peak power in pulse compression and CEP stabilization schemes. The next section will examine pulse compression techniques in solid- and hollow-core photonic crystal fiber. A detailed study of pulse compression in bulk materials follows. Eventually, a second CEP stabilization method is presented that avoids possibly limiting intracavity optical elements. 


\subsection{Fiber-based Pulse Compression}

\subsubsection{Limitations of Solid-core Fiber}

Parts of this section have been published in:

M. Seidel, X. Xiao, and A. Hartung, "Solid-Core Fiber Spectral Broadening at its Limits"

IEEE J. Sel. Top. Quant. Electron. 24 (2018)

In the previous chapter, pulse compression experiments with LMA-35 were discussed. Large compression factors of about 15 with $75 \%$ power efficiency were achieved. But if more than $30 \mathrm{~W}$ of average power emerging from the KLM TD oscillator were coupled into the fiber, damage occurred. This section will examine the origin of damage and infer to which extend the damage threshold can be manipulated by pre-chirping pulses or the use of PCFs with different mode-field diameters (MFDs).

Furthermore, the sub-10 fs regime could not directly be reached with the LMA-35 compression stage presented in the last chapter. Instead, bulk-based spectral broadening was utilized to generate few-cycle pulses. That, however, reduced the average and peak power of the ultrafast light source. Therefore, the scalability of solid-core fiber broadening, which promises highly efficient few-cycle pulse generation, will be investigated. A final discussion will identify possible applications of solid-core fiber pumped by high-power thin-disk oscillators.

\section{Spectral broadening factors for different photonic crystal fibers}

In the LMA-35 experiment of section 2.2.1, pulse compression was accomplished by utilizing chirped mirrors. The amount of GDD needed for chirp compensation was determined by both the spectral phase induced by spectral broadening and by fiber dispersion. The latter also saturated the SPM effect and basically determined the broadening factor $\beta$ which is, for initially unchirped Gaussian pulses and flat normal GVD, proportional to the square root of the ratio between nonlinear $\left(L_{n}=\lambda /\left(\pi n_{2} I_{p}\right)\right)^{256}$ and dispersion lengths $\left(L_{d}=t_{p, i n}^{2} / \mathrm{GVD}\right)^{257}$ :

$$
\beta \approx \kappa_{1} \sqrt{L_{d} / L_{n}}=\kappa_{1} \sqrt{\left(\frac{t_{p, i n}^{2}}{\mathrm{GVD}}\right) /\left(\frac{\lambda}{\pi n_{2} I_{p}}\right)} \propto \sqrt{\frac{n_{2} I_{p}}{\lambda \cdot \mathrm{GVD}}} t_{p, i n}
$$

where $\kappa_{1}$ is a constant, $\lambda$ denotes the vacuum wavelength, $n_{0}$ the linear and $n_{2}$ the nonlinear refractive index, $t_{p, \text { in }}$ the input pulse duration, and $I_{p}$ the peak irradiance. The prefactor $\kappa_{1}$ may vary depending on the application. For maximal broadening it is about 0.5 , for efficient pulse compression with linear chirp it is about $0.37^{257}$. The lengths $L_{d}$

\section{Remark: Nonlinear length}

The nonlinear length is often defined as $L_{n}=\left(\gamma P_{p}\right)^{-1}$ where $P_{p}$ is the peak power while $\gamma=\frac{2 \pi n_{2}}{\lambda A_{\text {eff }}}$ (cf. e.g. ref. 256). The effective area of a Gaussian beam is $A_{\text {eff }}=\pi w_{0}^{2}$ where $w_{0}$ is the beam waist. For fibers, the definition is reasonable because the waist is defined by the MFD. Since bulk broadening is also discussed in the context of this thesis, the definition by means of peak irradiance and $n_{2}$ is preferred. 
and $L_{n}$ can be interpreted in the following way: By scaling $L_{d}$ with $\sqrt{3} /(4 \ln 2)$, it would be the propagation length that doubles the duration of a Gaussian pulse in absence of nonlinearity ${ }^{171}$. Scaling $L_{n}$ with 1.974 would be the propagation length that doubles the RMS width of Gaussian pulse in absence of dispersion ${ }^{165}$. These characteristic lengths also determine the optimal physical fiber length which can be approximated by ${ }^{257}$ :

$$
L_{f} \approx \kappa_{2} \sqrt{L_{d} L_{n}}
$$

where the prefactor $\kappa_{2}$ is about 1.4 for efficient pulse compression with linear chirp ${ }^{257}$. As an example, Eqs. (3.1) and (3.2) with $\kappa_{1}=0.37$ and $\kappa_{2}=1.4$ would lead to broadening factors of 16.7 and fiber lengths of $10 \mathrm{~cm}$ for the input parameters of section 2.2.1. This is close to the experimental values of 18 and $8 \mathrm{~cm}$, and hence indicates that the simple Gaussian pulse model can be utilized for discussing scalability of solid-core fiber broadening.

At first, Eq. (3.1) exhibits a linear dependence of $\beta$ on the input pulse duration. If perfect compressibility is assumed, i.e. if the compression factor is equal to the broadening factor, then $\beta=t_{p, \text { in }} / t_{p, o u t}$, and thus the output pulse duration $t_{p, \text { out }}$ would be independent of the input pulse duration. Although neglecting many pulse width dependent effects like RAMAN scattering ${ }^{258}$, optical breakdown in $\mathrm{FS}^{259,260}$ or impact of higher-order dispersion, this indicates why broadening factors of 35 could be reached in a comparable fiber broadening experiment with a TD oscillator emitting 810 fs pulses ${ }^{188}$ and why broadening factors of only about 2 were achieved in the second stage of the compression experiments presented in section 2.2. Secondly, since $n_{2}$ as well as the GVD are determined by the fiber material (for LMAs), and $\lambda$ as well as $t_{p, i n}$ are given by the light source, the only remaining free parameter of Eq. (3.1) is the peak irradiance. This motivates investigations of the fiber damage threshold dependence on the MFD which determines $I_{p}$.

Spectral broadening in four different PCFs (all supplied by NKT photonics) was examined. LMA-35, LMA-25 and LMA-12 were tested. These LMA fibers exhibit MFDs between 10 and $26 \mu \mathrm{m}$ and basically the dispersion of FS (Fig. 3.1(b)). Moreover, an ANDi fiber
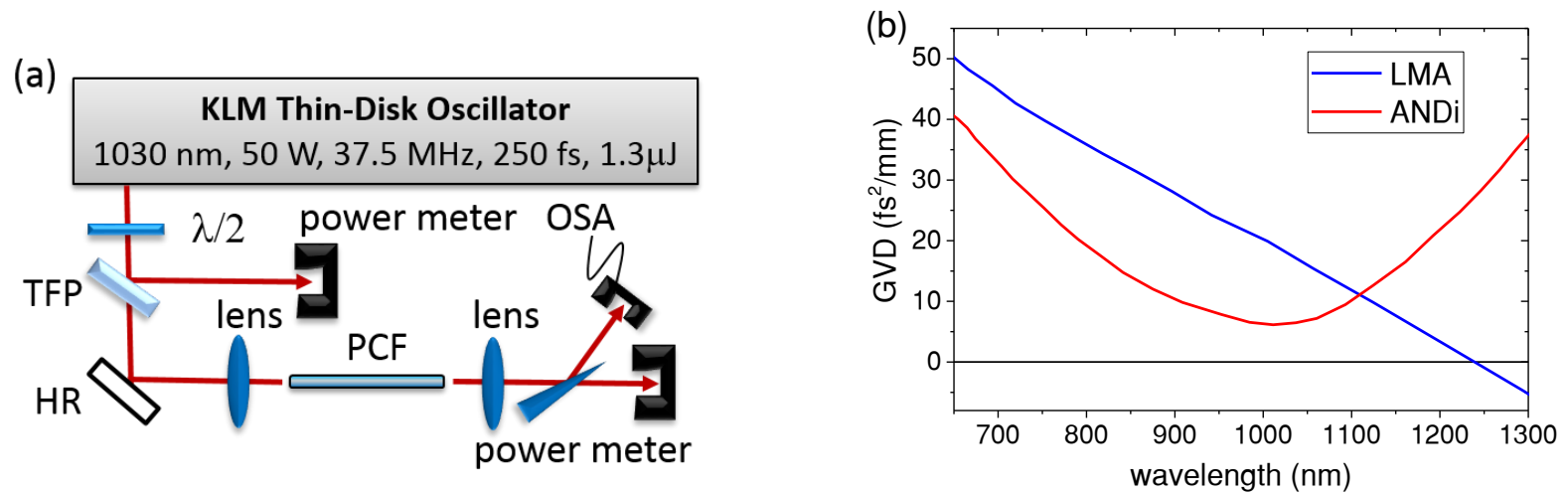

Fig. 3.1. (a) Setup solid-core fiber broadening experiments. The input power into the PCFs was adjusted with a half-wave plate $(\lambda / 2)$ and a thin-film polarizer (TFP). HR denotes a highly reflective mirror that steers the beam towards the focusing lens which couples light into the fiber. After collimation, a wedge reflection is sent to the OSA. Moreover, the power of the fiber output is monitored behind the wedge. (b) Fiber dispersion curve for the LMA fibers (blue solid line, provided by Thorlabs) which is basically the GVD of FS and the tailored dispersion of the all-normal dispersion (ANDi) fiber (red solid line, from ref. ${ }^{261}$ ) which is always positive and minimal around $1050 \mathrm{~nm}$. 


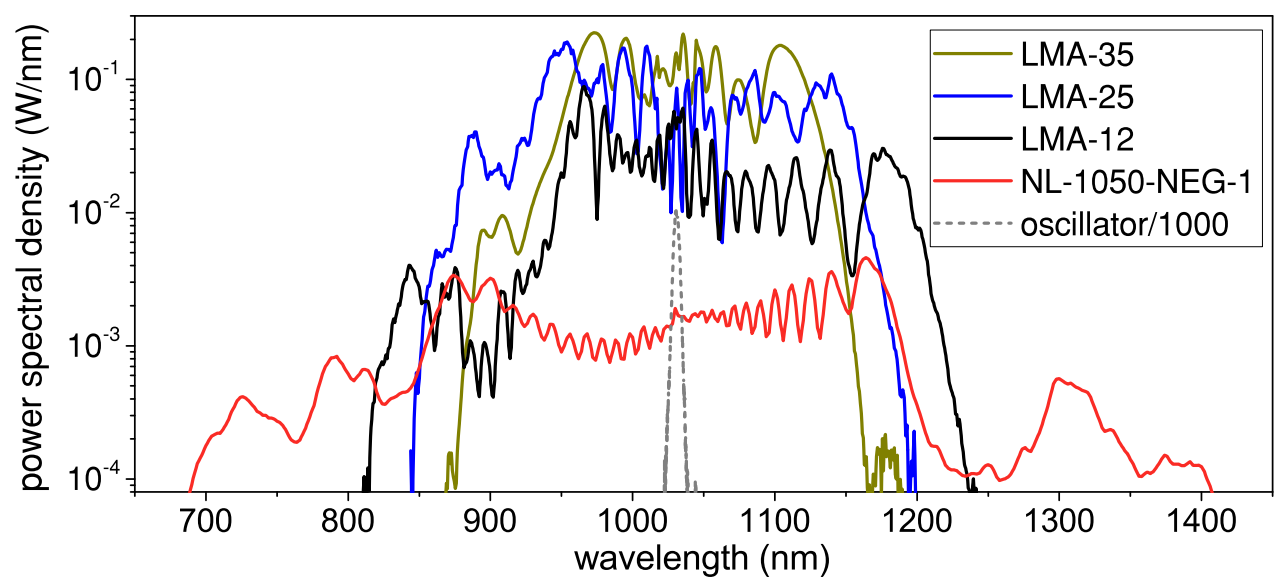

Fig. 3.2. Spectra measured with an OSA. Four fibers of different MFDs were tested: According to the data sheets provided by NKT photonics, LMA-35 has an MFD of $26.0 \mu \mathrm{m}$, LMA-25 of $20.9 \mu \mathrm{m}$ and LMA-12 of $10.3 \mu \mathrm{m}$. The ANDi fiber (NKT NL-1050-NEG-1) with a tailored waveguide dispersion has an MFD of about $3.0 \mu \mathrm{m}^{261}$. The power spectral density of the ANDi spectrum does not exceed $5 \mathrm{~mW} / \mathrm{nm}$ whereas the LMA fibers generate power spectral densities of slightly more than $200 \mathrm{~mW} / \mathrm{nm}$ at the $37.5 \mathrm{MHz}$ repetition rate of the oscillator. As a reference, the TD oscillator spectrum is shown (gray dashed line). It has been measured with a grating spectrometer and is plotted in units of $\mathrm{kW} / \mathrm{nm}$.

(NKT NL-1050-NEG-1) with an MFD of about $3 \mu \mathrm{m}$ was investigated. The small mode area allows to introduce significant waveguide dispersion which was tailored to minimize the GVD near $1050 \mathrm{~nm}$ and to keep it positive for all wavelengths ${ }^{261-263}$ (Fig. 3.1(b)). According to Eq. (3.1), this allows large broadening factors. All fibers were manually cleaved by means of a ruby fiber scribe to avoid micro-cracks on the fiber core surface. Those were usually visible for LMA-25 and LMA-35 when an automated fiber cleaver was used. They potentially reduce the surface damage threshold ${ }^{264}$. Manual cleaving resulted in fiber length variations between 8 and $10 \mathrm{~cm}$ which had, however, only a small impact on the broadening factors (cf. Fig. 2.5 and 3.3(a)). To couple the output of the oscillator to the fiber modes, lenses with focal lengths $f$ were chosen which approximately sufficed the equation:

$$
f=\frac{\pi d_{i n} \mathrm{MFD}}{4 \lambda}
$$

The equation refers to the focus size of a Gaussian beam ${ }^{163}$ where $d_{\text {in }}$ is the diameter of the collimated laser beam before the lens and $\lambda$ the wavelength. The setup is sketched in Fig. 3.1(a). Output spectra and transmitted powers were measured while the input power was slowly increased until fiber damage was observed by means of reduced spectral width and transmission. Fig. 3.2 shows spectra which were measured at input powers slightly below the damage threshold. From the graph, it becomes obvious that smaller MFDs lead to broader spectra which span over more than one optical octave in the extreme case of the ANDi fiber. Fig. 3.3(a) displays the broadening factors and the FTLs of the measured spectra. For the LMA fibers, FTLs between 9 and 13 fs were extracted. The ANDi fiber generates spectra with sub-5 fs FTLs, resulting in broadening factors of more than 50. This would directly allow few-cycle pulse generation although control of higherorder phase terms would become necessary to compress the OWB contributions to the spectrum ${ }^{265}$. 

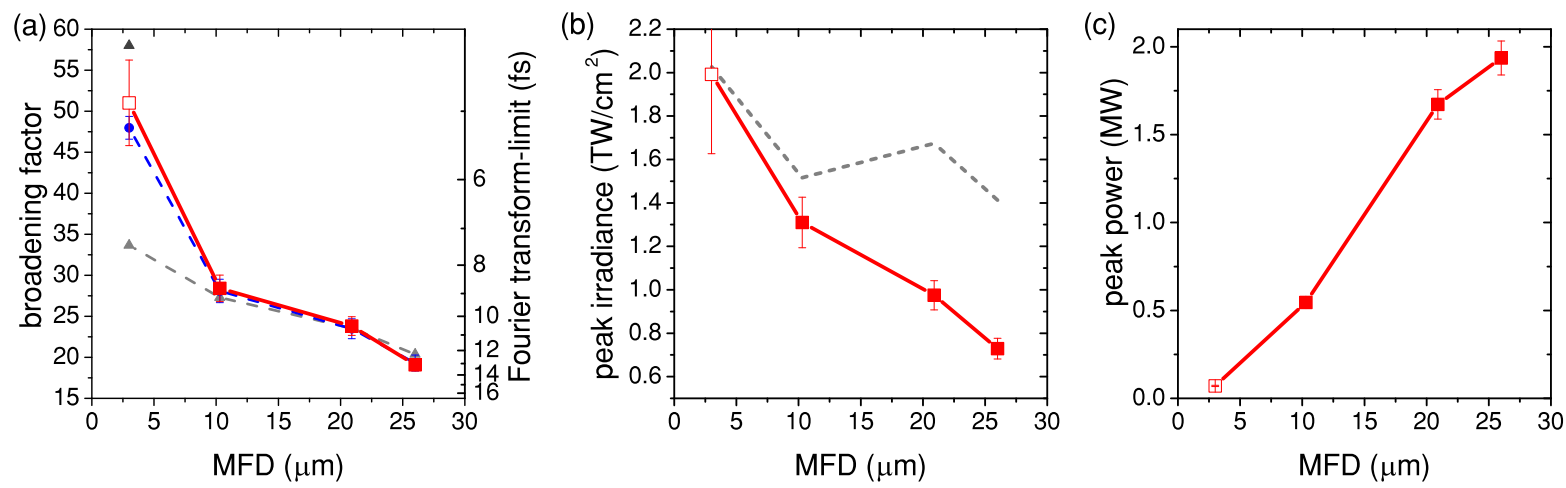

Fig. 3.3. (a) Dependence of the broadening factors and FTLs, resp. on different MFD of the utilized PCFs. Multiple fibers of each type were tested, resulting in a transform-limit uncertainty of $\pm 0.5 \mathrm{fs}$ (red error bars). The dashed gray curve shows the calculated broadening factors derived from Eq. (3.1) inserting the peak irradiances of (b) and $\kappa_{1}=0.45$. The dark gray triangle in the top-left corner represents the calculated broadening factor for GVD $=6.4 \mathrm{fs}^{2} / \mathrm{mm}$ (ANDi fiber) instead of GVD $=19 \mathrm{fs}^{2} / \mathrm{mm}$ (LMA fibers). The blue dashed line shows simulation results obtained with fiberdesk V. 2.0. The blue error bars (partly hidden behind the experimental results) show the impact of fiber length variations between $8 \mathrm{~cm}$ and $10 \mathrm{~cm}$. The fiber dispersions shown in Fig. 3.1(b) were used for the simulations. The effects of selfsteepening and RAMAN scattering were included, but hardly affected the simulation outcome. (b) Peak irradiances derived from the power measurements behind the fiber. The red solid curve is predicted from pulse energy, pulse duration and MFD, the gray dashed curve takes into account non-critical self-focusing in the fiber. (c) Peak powers tolerated by the fibers. The values were derived from the measured output power and the input pulse duration. The hollow red dots in (a)-(c) represent the ANDi fiber, the solid red dots the LMA fibers.

By virtue of the power measurements, the peak irradiances inside the fibers were calculated. The red curve in Fig. 3.3(b) shows that the lower the MFD the higher the peak irradiance the fiber seems to tolerate. The results of Fig. 3.3(a) and (b) are qualitatively in agreement with the expectations from Eq. (3.1). The gray dashed curve in Fig. 3.3(a) indicates that the FTLs determined after the LMA fibers are close to the $\sqrt{I_{p}}$ dependence. For a good agreement with the measured broadening factors, $\kappa_{1}$ in Eq. (3.1) was set to 0.45 which is close the values for optimal compression (0.37) and maximal broadening $(0.5)^{257}$. The discrepancy in the broadening factor of the ANDi fiber stems from its tailored dispersion entering also Eq. (3.1). Whereas the gray curve in Fig. 3.3(a), derived from a flat dispersion of $19 \mathrm{fs}^{2} / \mathrm{mm}$, yields a smaller broadening factor than measured, the actual 3-fold lower GVD at $1030 \mathrm{~nm}$ of the ANDi fiber would result in a somewhat overestimated broadening factor of 58 due to the non-flat GVD over the optical octave (dark gray triangle in Fig. 3.3(a)). In simulations, whose results are plotted with a blue dashed curve in Fig. 3.3(a), the broadening factor of the ANDi fiber agrees well with the measured one. Moreover, the $\sqrt{I_{p}}$ dependence predicted in Eq. (3.1) is also confirmed.

The origin of the varying peak irradiances displayed in Fig. 3.3(b) will be discussed in the next paragraph. Beforehand, it is to note that smaller MFDs, on the one hand, allow larger peak irradiances. On the other hand, they tolerate lower peak power, and thus also lower pulse energies as Fig. 3.3(c) demonstrates. Consequently, the choice of the MFD results in a trade-off between achievable spectral bandwidth and maximal pulse energy. 


\section{Origins of damage}

Self-phase modulation and to some extend four-wave mixing have been identified as the physical mechanisms which caused spectral broadening in the LMA-35 experiment (section 2.2.1). Within the framework of perturbative nonlinear optics, these are considered as third-order, i.e. $\chi^{(3)}$, effects ${ }^{168}$. On the other hand, beam collapse, originating from self-focusing, ultimately limits the peak power that can be sent into an LMA fiber. Selffocusing is also dependent on the nonlinear refractive index $n_{2}$, i.e. is a $\chi^{(3)}$ effect as well. Therefore, both the nonlinear effects being essentially important for spectral broadening and for material damage scale linearly with irradiance. Importantly, a decoupling of both phenomena is, nonetheless, in principle possible since spectral broadening depends on the temporal gradient of the pulse while self-focusing depends on the spatial gradient of the beam as explained in section 1.4.

To further draw the analogy between temporal and spatial effects, similar to ref. 171, one may speak of "beam compression" as a result of self-focusing. In a comparable way, diffraction, may inhibit beam compression to arbitrarily small areas, i.e. the collapse of the laser beam. Since both, irradiance and diffraction scale with the inverse of a Gaussian beam size ${ }^{268}$, the critical parameter which ultimately causes the collapse is expressed in terms of an instantaneous power, not in terms of irradiance ${ }^{160,269}$ :

$$
P_{c r}=\frac{\pi(0.61)^{2} \lambda^{2}}{8 n_{0} n_{2}}
$$

where $P_{c r}$ is the so-called critical power, $\lambda$ is the vacuum wavelength, $n_{0}$ the linear and $n_{2}$ the nonlinear refractive index. This equation assumes absence of significant dispersion. For FS and $1030 \mathrm{~nm}$ wavelength, $P_{c r}$ is about $4 \mathrm{MW}$. It is remarkable that this peak power has not been reached for any of the tested fibers as Fig. 3.3(c) shows. This is caused by the high peak irradiances inside the fibers which trigger multi-photon and impact ionization in FS, consequently dissipation of heat and ultimately material damage $259,260,264,270$. This, however, does not explain the significantly different peak irradiances which were
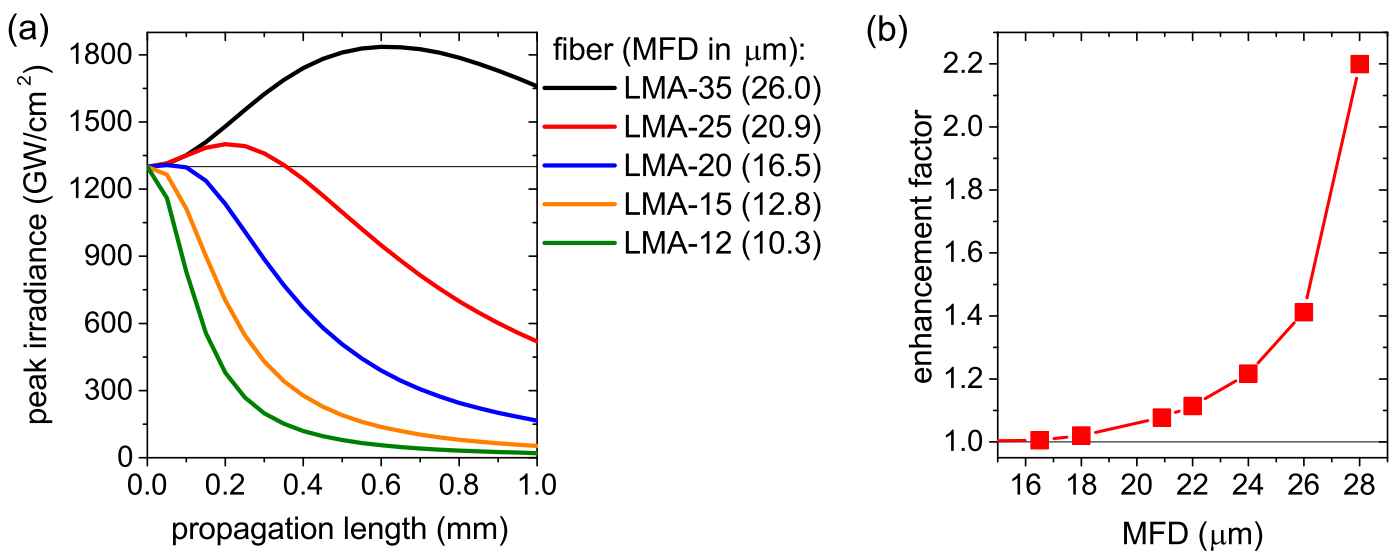

Fig. 3.4. (a) Free beam propagation in $1 \mathrm{~mm}$ FS is simulated with the SISYFOS code 266,267 . The peak irradiance is $1.3 \mathrm{TW} / \mathrm{cm}^{2}$ at the input, the beam size corresponds to the MFDs of the commercially available LMA fibers listed in the plot legend. Propagation length 0 is always at the waist of the Gaussian beam. Pulse energies were adapted to the spot sizes in order to keep the irradiance constant. Sub-critical self-focusing becomes apparent for diameters of more than $16.5 \mu \mathrm{m}$. (b) The enhancement factor, i.e. the ratio between maximal and input irradiance, is plotted over the MFD. 

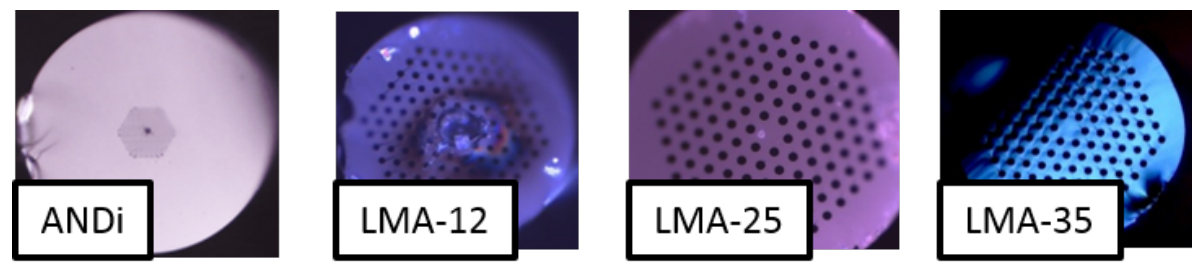

Fig. 3.5. Microscope images of the front fiber facets after damage. While the core of the ANDi and the LMA-12 fibers show severe modifications, the facets of LMA-25 and LMA-35 exhibit no sign of damage.

presented in Fig. 3.3(b). Those are attributed to sub-critical self-focusing which leads to an irradiance enhancement near the front fiber facet ${ }^{159,271}$. The behavior is illustrated in Fig. 3.4. A fixed peak irradiance of $1.3 \mathrm{TW} / \mathrm{cm}^{2}$ has been assumed at the entrance of a FS sample. Upon varying focus diameter and pulse energy, the impact of self-focusing changes strongly. For large beam diameters, the Gaussian beam divergence is too weak to completely suppress self-focusing. Only after nonlinear beam compression, the diffraction overcomes self-focusing and leads to an expanding beam. For small beam diameters, self-focusing cannot overcome beam diffraction at any propagation length. The observed irradiance enhancement is approximated by ${ }^{271}$ :

$$
I_{\max } / I_{p}=\left(1-P_{p} / P_{c r}\right)^{-\kappa_{3}},
$$

where $I_{\max }$ is the maximal and $I_{p}$ the input irradiance, $P_{p}$ is the peak and $P_{c r}$ the critical power. The exponent $\kappa_{3}$ depends on the fiber type. For bulk material, it is $1^{264,271}$. This exponent matches best the determined peak irradiances. The dashed gray line in Fig. 3.3(b) shows the peak irradiances corrected with Eq. (3.5), assuming $P_{c r}=4 \mathrm{MW}$. They are $(1.55 \pm 0.15) \mathrm{TW} / \mathrm{cm}^{2}$ for the LMA fibers. Unexpectedly, the peak irradiance in the ANDi fiber is with about $2 \mathrm{TW} / \mathrm{cm}^{2}$ somewhat higher. The origin is unknown. The difference might arise from reduced thermal effects caused by faster heat transport out of the interaction region due to the very small mode area and correspondingly high temperature gradient. But this is speculation and needs further investigation. To provide additional evidence of sub-critical self-focusing, the fiber front facets were imaged with a light microscope (Fig. 3.5). While the ANDi fiber and LMA-12 exhibit clearly damaged surfaces, damage is not obvious for the larger core fibers. This corresponds to the expectations derived from the enhancement curve of Fig. 3.4. LMA-25 and LMA-35 could, however, be reused after recleaving, i.e. after removing a few millimeters from the front of the fiber. The observation also implies that collapsing the facets of the small core fibers can in-fact increase the damage threshold. By contrast, collapsing large core fibers may prevent long term degradation but does not raise the damage threshold. One may use collapsed ends or end caps and correct Eq. (3.3) for the irradiance enhancement when choosing the proper focal length for coupling the free beam to the fiber mode. But, since both the spot size and the focal length change with input power in the sub-critical self-focusing regime, coupling will become very difficult.

Eventually, the consideration of this section shall be summarized in Fig. 3.6 which can serve as a rough fiber selection guide for spectral broadening of $250 \mathrm{fs}$ pulses. It may, of course, be extended to other pulse durations. It is to note, however, that the uncertainties in the predicted broadening factors are estimated to about $25 \%$ due to the simplicity of the model Eq. (3.1) is derived from. Especially the flat GVD assumption in Eq. (3.1) 


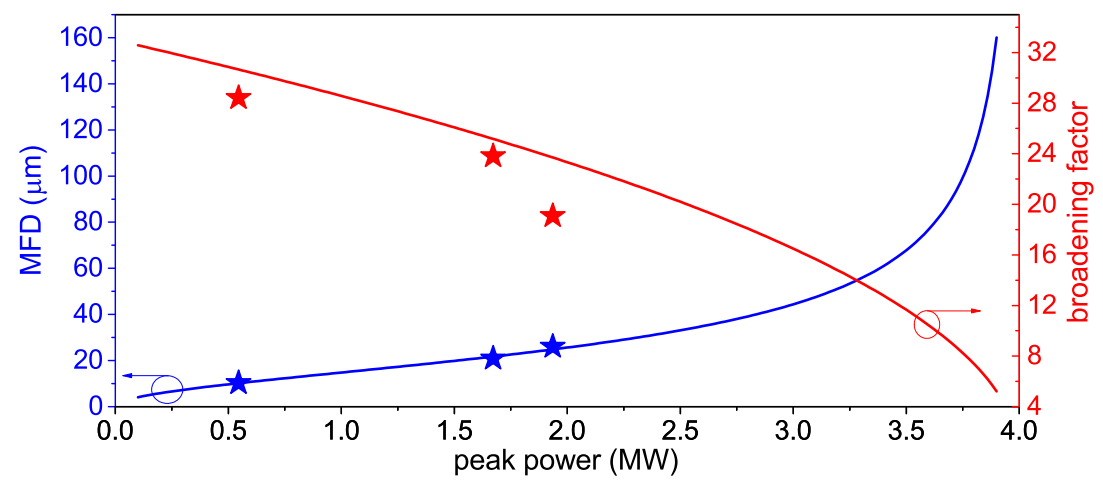

Fig. 3.6. MFDs and broadening factors for various pulse peak powers derived from Eqs. (3.1) and (3.5) where $\kappa_{1}=0.5, t_{p, i n}=250 \mathrm{fs}, \mathrm{GVD}=19 \mathrm{fs}^{2} / \mathrm{mm}, \lambda=1030 \mathrm{~nm}, n_{2}=2.8 \cdot 10^{-16} \mathrm{~cm}^{2} / \mathrm{W}$ and $P_{c r}=4 \mathrm{MW}$. Moreover, $I_{p}$ was determined from $I_{\max }=1.55 \mathrm{TW} / \mathrm{cm}^{2}$ and $\mathrm{sech}^{2}$-pulses were assumed. The stars show the measurement points from Fig. 3.3. The MFDs were used for the derivations of the blue curve, and hence the excellent agreement is not surprising. The broadening factors of Eq. (3.1) are always several percent higher than measured ones.

becomes highly questionable for large broadening factors that may even lead to spectral extension into the anomalous dispersion regime. Moreover, only spectral broadening was discussed in this context so-far. However, pulse compression may become increasingly difficult for long fibers and large broadening factors, resp. Hence, maximizing spectral broadening may not always minimize pulse durations after compression as exemplary described in section 2.2.1 where a strong contribution of OWB was avoided.

\section{Chirped Pulse Broadening}

The previous paragraphs have described the trade-off between peak power and gain of bandwidth in spectral broadening experiments with normally dispersive PCFs. As Fig. 3.6 indicates, spectral broadening vanishes if the peak power approaches the critical power of the fiber material. Consequently, a first ansatz to achieve low FTLs without sacrificing pulse energy is raising the critical power. Jocher et al. realized this by using circular instead of linear polarization ${ }^{272}$. Since the KERR nonlinearity of circularly polarized light is $2 / 3$ of the $n_{2}$ for linear polarized light ${ }^{157}$, the critical power is increased to about $6 \mathrm{MW}$ according to Eq. (3.4). The material damage threshold is likely to be increased as well but the author is not aware of any polarization dependent study of the optical breakdown with fs pulses. The drawbacks of this method are that the critical power increase is limited by the factor 1.5 and additional polarization optics are required after the fiber which introduce chirp and polarization losses.

A second ansatz is to reduce the peak power by increasing the input pulse duration instead of lowering pulse energy. This idea was realized by means of chirping the output of long-cavity Ti:sapph oscillators which deliver peak powers up to $10 \mathrm{MW}^{56}$. With this technique, GANZ et al. increased the pulse duration and energy sent into a LMA-25 fiber compression stage by a factor of four. Nearly the same FTL was reached with both $55 \mathrm{fs}$ unchirped and longer prechirped input pulses. Furthermore, the compression factor was only reduced from 3.8 to $3.4^{206}$. Qualitatively speaking, chirp leads to a reduced temporal gradient on the one hand, resulting in a slower spectral broadening. On the other hand, chirp extends the propagation length that is needed to double the pulse duration, and 
(a)

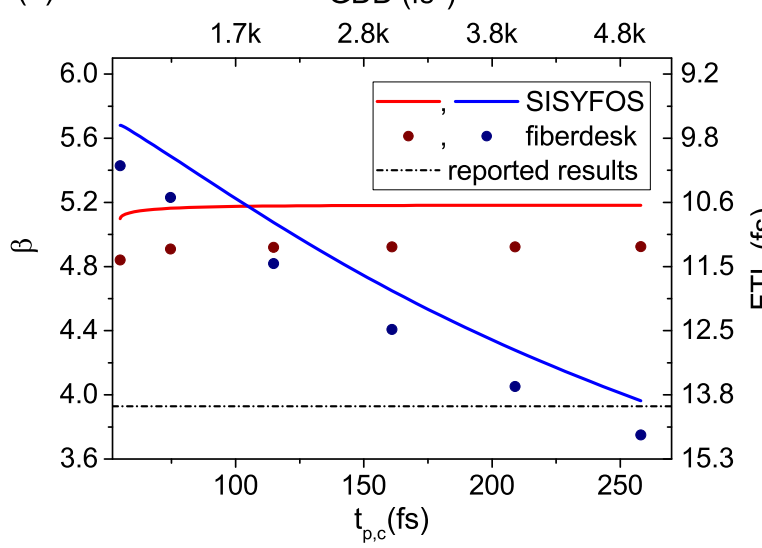

(b)

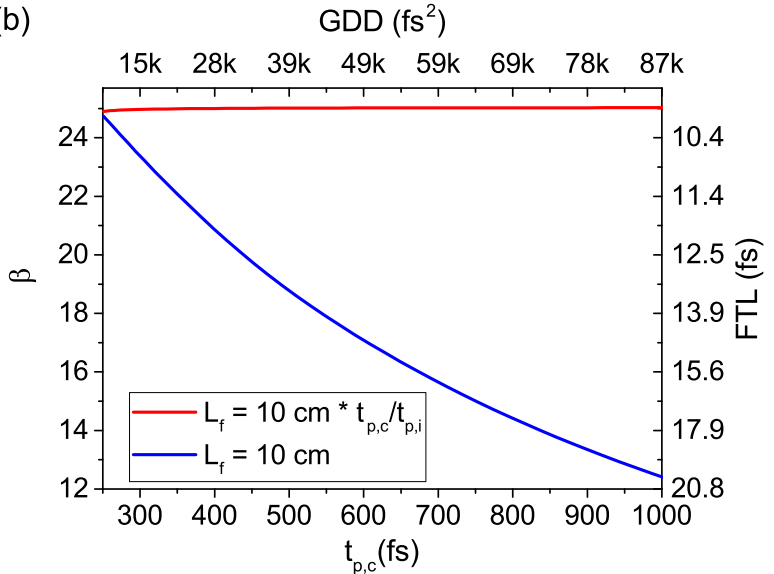

Fig. 3.7. (a) Simulated broadening factors for the data provided in ref. 206 for a fixed fiber length of $L_{f}=3 \mathrm{~cm}$ (stated in the publication, blue line and dots) and for $L_{f}=3 \mathrm{~cm} \cdot t_{p, c} / 55 \mathrm{fs}$. Two simulation packages were used: 50 data points were simulated with SISYFOS and 6 data points with fiberdesk V. 2.0. The results qualitatively agree well, just differ $<10 \%$ in $\beta$. SISYFOS used 4 times the temporal grid points (8192) and the full Sellmeier equation of FS which may explain the slight differences. For comparison, the reported results from GANZ et al. are shown. (b) SISYFOS simulations for the LMA-35 experiments of section 2.2.1, predicting the possibility of increasing the pulse energy inversely proportional to the pulse duration. The FTLs were determined by dividing the initial pulse duration by the broadening factor. Hence, they may not be fully accurate as they refer to FHWM and the broadening factors to the RMS spectral widths in the presented plots. The top x-axes show the GDDs which are needed to achieve the pulse duration on the lower $\mathrm{x}$-axes.

thus also pulse stretching gets slower. Consequently, one may scale both nonlinear and dispersion length by the ratio between chirped and transform-limited duration $t_{p, c} / t_{p, i}$, resulting in the same broadening factor according to Eq. (3.1) if, according to Eq. (3.2), the fiber length is also extended by $t_{p, c} / t_{p, i}$. It is to note that the definition of $L_{n}$ is independent of the pulse duration. This can be attributed to the fact that the broadening factor refers to the initial bandwidth which is, like the temporal gradient, proportional to the inverse of the pulse duration. Therefore, if the pulses are chirped, the temporal gradient is reduced while the bandwidth remains constant and the $t_{p, c} / t_{p, i}$-factor arises. Fig. 3.7 shows simulation results which demonstrate that these simple considerations theoretically in-fact work very well. Both for the experiment of ref. 206 and for the pulse parameters of the KLM TD oscillator utilized in section 2.2.1, a nearly constant broadening factor is predicted. In Supplement 2 at the end of this section, the temporal stretching and spectral broadening factor in absence of nonlinearity and dispersion, resp. have been calculated. They differ from those of FouRIER transform-limited pulses with the same duration $t_{p, c}$. Consequently, it is remarkable that Eqs. (3.1) and (3.2) seem well applicable. A controversy between experimental studies and simulations is the choice of the fiber length. While in ref. 206, the fiber length was kept constant around $3 \mathrm{~cm}$ and in ref. 273 no improvement in spectral broadening was reported for fibers longer than $1.5 \mathrm{~cm}$ (both experiments were performed with an initial 55 fs FTL), the graphs in Fig. 3.7 and the qualitative considerations give clear evidence that longer input pulses require longer fibers. Further investigations will be needed to explain this discrepancy.

The discussions so-far only refer to the broadening factors and do not examine compressibility of the prechirped pulses. The results of refs. 206, 273 imply that compressibility will not significantly change through prechirp. Simulations, however, indicate that higher- 
(a)

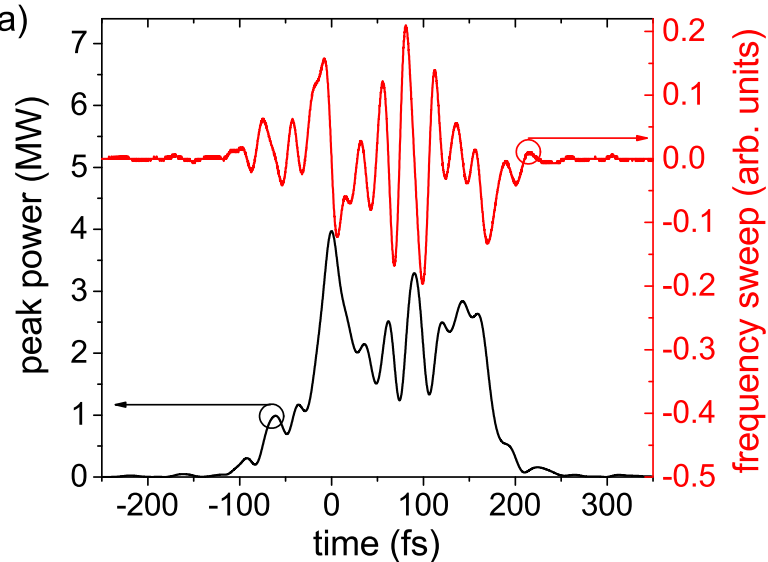

(b)

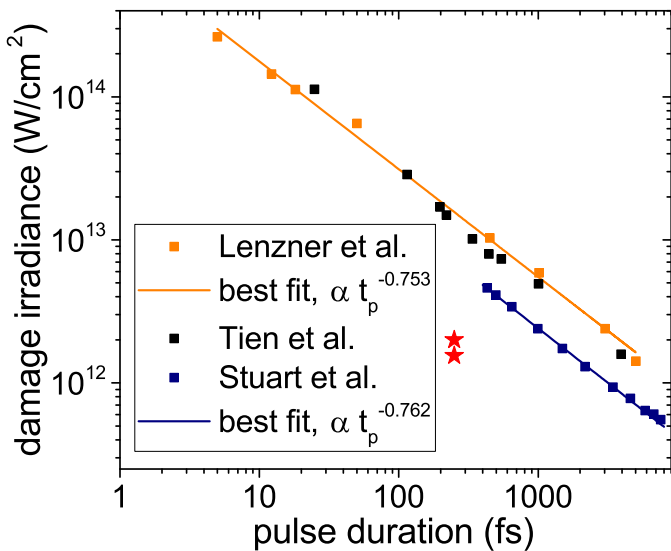

Fig. 3.8. (a) Pulse of Fig. 2.9 chirped to $4 \mathrm{MW}$ peak power by adding $660 \mathrm{fs}^{2}$ GDD (black solid line). Its time derivative ( $1^{\text {st }}$ order numerical) is represented by the red solid line. It shows the strongly modulated instantaneous frequencies generated by SPM. (b) Damage thresholds presented in refs. 259, 260, 270, displayed in an irradiance over time plot. The original data shows fluence which has been multiplied by $1 /\left(\sqrt{\pi / 4 \ln 2} \cdot t_{p}\right)$, assuming Gaussian pulses. The data was fitted by the function $I_{\max }=A \cdot t_{p}^{m}$ where $A$ and $m$ were the free parameters. All three data sets indicate that the threshold nearly scales with $t_{p}^{-3 / 4}$. However, the absolute damage irradiances are different. At $250 \mathrm{fs}$, they are $15.6 \mathrm{TW} / \mathrm{cm}^{2}$ for the orange line (measured at $800 \mathrm{~nm}, 1 \mathrm{kHz}$ repetition rate), $6.9 \mathrm{TW} / \mathrm{cm}^{2}$ (measured at $1050 \mathrm{~nm}, 1 \mathrm{kHz}$ repetition rate) and $1.55 \mathrm{TW} / \mathrm{cm}^{2}$ and $2 \mathrm{TW} / \mathrm{cm}^{2}$ for the measurements presented in the last paragraph (red stars).

order chirp for longer fibers must be compensated in order to compress the pulses close to their FTL. This can be, of course, attributed to the stronger influence of material dispersion. The amount of input GDD is displayed on the top axes in Fig. 3.7. It is already quite challenging to induce several $10^{4} \mathrm{fs}^{2}$ of GDD via chirped mirrors. Stretching with material will induce higher-order dispersion. The use of gratings may reduce beam quality and introduce losses. The chirp needed for compression will be lower than the input chirp ${ }^{273}$ due to the increased bandwidth (cf. Supplement 2). Nevertherless, it will be larger than for transform-limited input pulses, and thus will make compression more challenging.

The need for better dispersion management when going to longer fibers is not the only concern with respect to the generality of the chirped pulse spectral broadening (CPSB) approach. It has got other severe limitations: Firstly, a special property of Gaussian pulses is highly beneficial for CPSB: Adding quadratic phase changes the pulse duration but not the pulse shape, i.e. the pulses remain Gaussian (cf. Supplement 2). Intuitively, CPSB could have been applied to the second compression stage of the setup presented in section 2.2. However, adding linear chirp to the compressed pulses after the LMA35 stage to reduce the peak power below the critical power would have resulted in the strongly modulated temporal shape that is shown in Fig. 3.8(a). Since the instantaneous frequencies which are generated by SPM are proportional to the time derivative of the pulse shape, the very same frequencies would have been generated at multiple instances of time (red solid line in Fig. 3.8(b), the frequency sweep introduced by chirping is not shown). Although four-wave mixing may additionally change the frequency distribution, compression by dispersive optics seems basically impossible.

Secondly, the impact of a pulse duration dependent damage threshold has not been discussed in refs. 206, 273. The studies of StUART et al., LENZnER et al. and Tien 

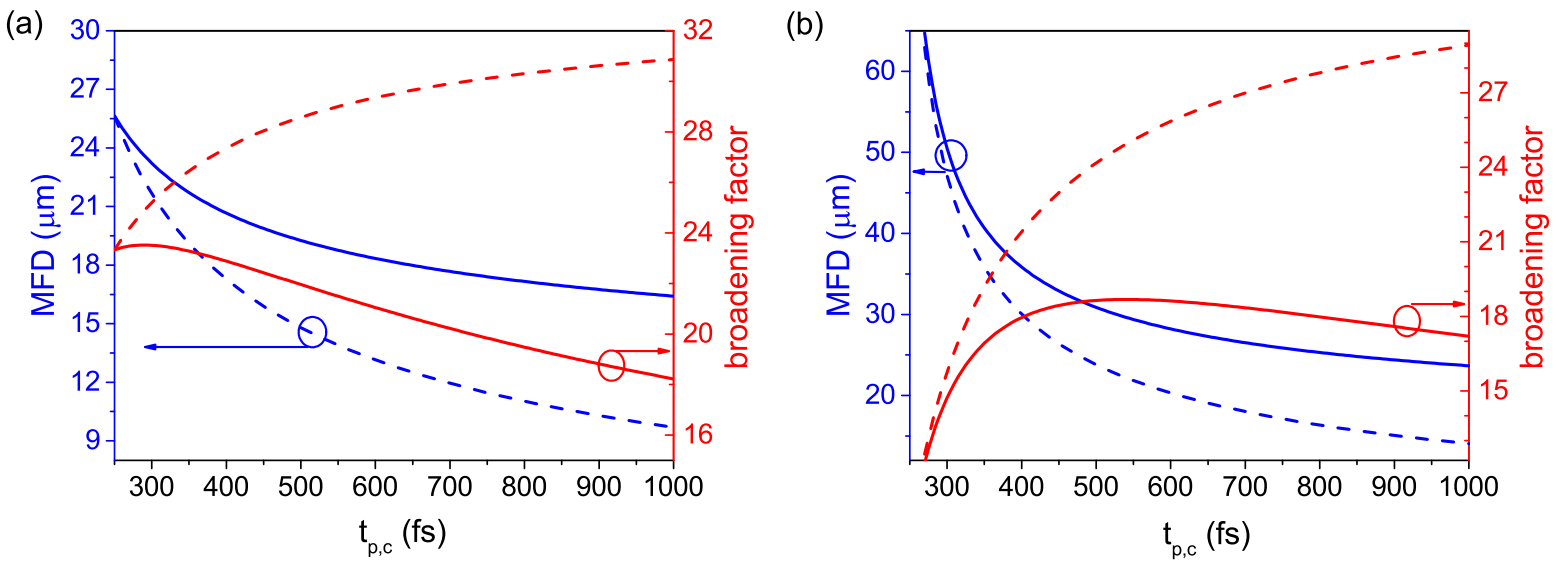

Fig. 3.9. MFDs and broadening factors for various input pulse durations $t_{p, c}$ derived from Eqs. (3.1) and $(3.6)$ where $t_{p, i}=250 \mathrm{fs}, \mathrm{GVD}=19 \mathrm{fs}^{2} / \mathrm{mm}, \lambda=1030 \mathrm{~nm}, n_{2}=2.8 \cdot 10^{-16} \mathrm{~cm}^{2} / \mathrm{W}$ and $P_{c r}=4 \mathrm{MW}$. (a) An initial peak power of $P_{p, i}=2 \mathrm{MW}$ is set. The solid lines show the predicted results. The dashed lines compare these results to a pulse duration- $i n$ dependent damage threshold. The MFDs are different due to the different damage irradiances. Prechirping hardly increases the maximum broadening factor if the damage irradiance scales $\propto t_{p}^{-3 / 4}$. (b) An initial peak power of $P_{p, i} \approx 4 \mathrm{MW}$ is set, being close to the critical power. In this case, an optimal chirp and MFD can be derived, indicating possible applicability of CPSB. Solid lines again represent the pulse duration-dependent damage threshold while dashed lines represent the pulse duration-independent one.

et $a l .{ }^{259,260,270}$ show that the damage irradiance of bulk FS scales approximately with $t_{p}^{-3 / 4}$ for sub-10 ps where the dominant, non-instantaneous impact ionization has not fully evolved (Fig. 3.8(b)). Smith et al. argue that fiber damage underlies the same physical mechanisms ${ }^{271}$. Although it is known that long living trap states decrease the damage threshold if multiple pulses are incident, ${ }^{274}$ the author is not aware of a systematic study which investigates the repetition rate and pulse duration dependence of such cumulative effects in FS. It is, however, not expected that the electron avalanche is accelerated since the mean free path of the free carriers should not be affected. Therefore, a similar pulse duration dependence like in Fig. 3.8(b) is assumed. This implies that strong chirp significantly reduces the fiber damage threshold, and thus the achievable broadening factors. On the one hand, the peak power would scale with $P_{p}=P_{p, i} t_{p, i} / t_{p, c}$, but on the other hand $I_{\max }$ of Eq. (3.5) would also scale with $I_{\max }=I_{\max , i}\left(t_{p, i} / t_{p, c}\right)^{3 / 4}$. The sub-index $i$ refers to the initial, unchirped value. Consequently, the peak irradiance in the fiber is calculated by:

$$
I_{p}=I_{\max , i}\left(\frac{t_{p, i}}{t_{p, c}}\right)^{3 / 4}\left(1-\frac{P_{p, i}}{P_{c r}} \frac{t_{p, i}}{t_{p, c}}\right) .
$$

Two examples are shown in Fig. 3.9. Referring to Fig. 3.6, one could try to establish CPSB with small mode area fibers in order to increase the broadening factor without sacrificing pulse energy. Inserting $I_{p}$ into Eq. (3.1) for $P_{p, i} \approx 2 \mathrm{MW}$ (corresponding to the LMA-35 broadening) yields the plots of Fig. 3.9(a) and demonstrates that CPSB is not applicable in this case. It is to note again that the threshold dependence on pulse duration has been adapted from $\mathrm{kHz}$ bulk measurements and may differ. Upon doubling $P_{p, i}$ to about $4 \mathrm{MW}$, which corresponds to the output of the utilized KLM TD oscillator, an ideal input pulse duration between 500 and $600 \mathrm{fs}$ is predicted (Fig 3.9(b)). 
(a)

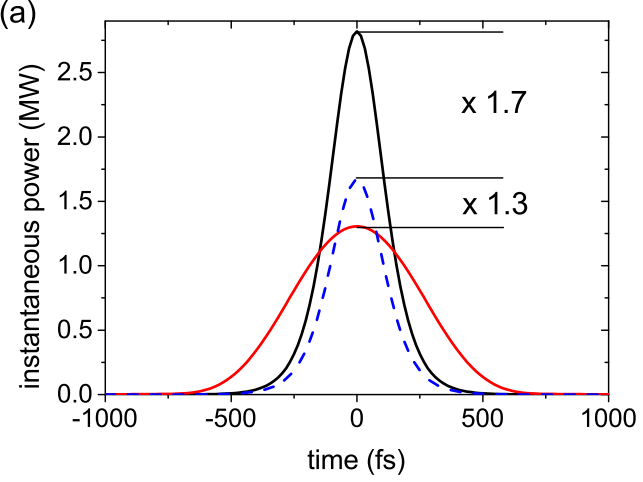

(b)

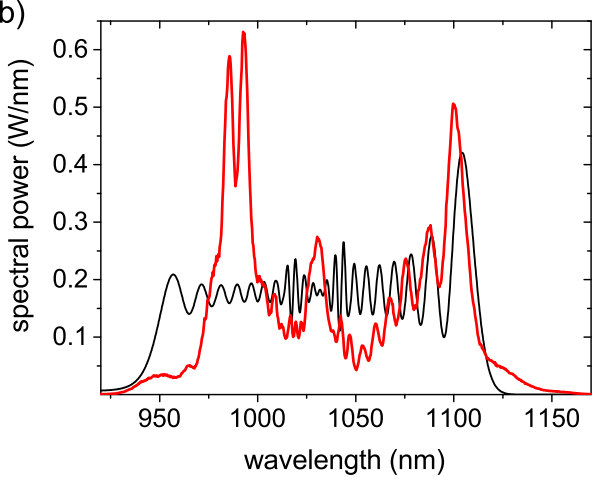

(c)

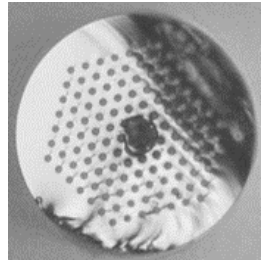

Fig. 3.10. (a) Unchirped pulse derived from about $40 \mathrm{~W}$ average power at the fiber entrance facet (black solid line) and pulse after chirping with $48000 \mathrm{fs}^{2}$ (red solid line). The blue dashed line shows the pulse that caused damage in the unchirped case. All pulses are calculated. The peak powers refer to values inside the fiber. (b) Measured chirped pulse spectrum after $\approx 12 \mathrm{~cm}$ propagation in LMA-25 (red solid line) and comparison to the simulated spectrum (black solid line). The narrow spectral line at $790 \mathrm{~nm}$ is not shown. (c) Fiber front facet after damage. Contrary to the LMA-25 facet in Fig. 3.5, the surface is clearly disrupted.

A experiment utilizing $590 \mathrm{fs}$ pulses was conducted. LMA-25 was used since larger core sizes were not readily available. The $250 \mathrm{fs}$ pulses emerging from the KLM TD oscillator were chirped by 24 bounces off chirped mirrors with $+2000 \mathrm{fs}^{2}$ GDD per bounce. The setup after the stretcher, consisting of 8 chirped mirror pairs and a recollimation mirror with about $1 \mathrm{~m}$ focal length, was practically as sketched in Fig. 3.1(a). An additional FARADAY rotator prevented back reflections from the fiber facet into the oscillator. A fiber length of $11.8 \mathrm{~cm}$ was chosen which is less than the optimal fiber length predicted from Eq. (3.2). It would be about $21 \mathrm{~cm}$ for a time independent damage threshold and $28 \mathrm{~cm}$ for a time dependent damage threshold. The coupling efficiency at $10 \mathrm{~W}$ was about $85 \%$, but dropped to $75 \%$ at $40 \mathrm{~W}$. About $45.5 \mathrm{~W}$ were available. Damage occurred at about $43 \mathrm{~W}$ input power which corresponds to an output peak power of about 1.3 MW. Fig. 3.10(a) shows the unchirped pulse at nearly full power sent into the fiber (black solid line) and the pulse after chirping. For comparison, the transform-limited pulse causing damage of the LMA-25 fiber is shown. About $60 \%$ more pulse energy was transmitted through the fiber while the damage threshold was reduced by about $30 \%$. The enhancement factor (Eq. (3.5)) for a peak power of $1.7 \mathrm{MW}$ is 1.72 , for $1.3 \mathrm{MW}$ it is 1.48 . Hence, the damage irradiance is reduced to $(1.3 \times 1.48) /(1.7 \times 1.72)=66 \%$ of the unchirped value. For comparison, the theoretical value, assuming the scaling of Fig. 3.8(b), would be $(250 / 590)^{3 / 4}=53 \%$. It is to note that only a single experiment was conducted. Nevertheless, it is evidenced that the pulse duration dependence of the damage threshold has to be considered in CPSB. The fiber facet of LMA-25, shown in Fig. 3.10(c), exhibits very clear signs of damage. This is explainable by the coupling efficiency of $75 \%$ and the $3.4 \%$ FRESNEL losses. The corresponding irradiance at the facet $38 \%$ higher than $I_{p}$ in the fiber for perfect mode matching) is close to the $48 \%$ enhancement caused by non-critical self-focusing. This is probably the reason for the shift of the damage location to the surface. Fig. 3.10(b) shows the spectrum measured behind the fiber. Its FOURIER transform-limit is about 16 fs, i.e. the broadening factor is reduced from about 24 to 16 if compared to the experiment with unchirped pulses. Although the low FTLs of the unchirped case are not expected, longer fibers would probably further broaden the spectrum. The FTL is in reasonable agreement with a fiberdesk simulation. However, 
the spectral shapes are different. The measured spectrum exhibits a stronger extension towards longer wavelengths. Furthermore, a narrow spectral feature on the anti-STOKES side at $790 \mathrm{~nm}$ appeared after increasing the input average power above $30 \mathrm{~W}$. This was also not predicted by simulations (including phenomenological quantum noise to allow spontaneous RAMAN scattering). The RAMAN effect was modeled with the theory presented in ref. 256, being insufficient to describe the additional phenomena which was most likely caused by the longer input pulse duration.

\section{Discussion}

The investigations of this section have revealed a general trade-off between the pulse energy which is sent into a normal dispersive PCF and the maximal spectral broadening factor. This results mainly, on the one hand, from irrdiance enhancement in the sub-critical self-focusing regime, ${ }^{159,271}$ and on the other hand, from the pulse duration dependent material threshold $259,260,264,270$. At the moment, no available solid-core fiber technique to overcome the trade-off is in sight. Tapering of LMA fibers could be interesting. But keeping an appropriate air hole structure for maintaining guidance is challenging, ${ }^{275}$ and thus such fibers are not commercially available. Using a sequence of two fibers with different MFDs might be an alternative. However, if the damage threshold increases with $t_{p}^{3 / 4}$, the effect leads, according to simulations, only to minor improvements in the pulse energy-FTL ratio, but to a much more complex setup. Another approach to power-scale solid-core fiber techniques is exploiting higher-order spatial modes ${ }^{276,277}$ which is subject to current research of other groups and might be a future option for high power spectral broadening. Finally, active rod-type fibers have been used in combination with SPM ${ }^{278}$ to obtain spectrally broadened pulses with enhanced pulse energies. Although this presents in principle an interesting approach to manipulate saturation of the SPM effect, the setup is also rather complex and introduces additional noise through the active medium.

In the research presented here, CPSB could not be confirmed to offer good peak power scalability. Apart from the time-dependent damage threshold, it appears restricted to bell-shaped pulses where chirp hardly changes the pulse shape. Furthermore, parasitic nonlinear effects were observed in the experiment with $590 \mathrm{fs}$ pulses which were not predicted by simulations. Although their origin is not fully understood at this point, they present another uncertainty of CPSB. In summary, it was found that the method appears quite restricted to lasers with peak powers close to the critical power of the fiber material, like long-cavity Ti:sapph oscillators. The spectral broadening results presented in refs. 206, 273 at least partly contradict the theoretical considerations as well as the experimental and numerical investigations presented here. Of course, more systematic studies of the pulse duration dependence on the damage threshold for $\mathrm{MHz}$ sources and the fiber length dependence on the broadening factor for chirped pulses would be necessary to draw final conclusions on the capabilities of CPSB. The described initial studies, however, rather motivated to take advantage of alternative pulse compression schemes which will be discussed in the next sections of this chapter.

Nevertheless, there are several applications of solid-core spectral broadening where the results presented here turn out to be very helpful. In section 4.1, the measurements presented in Fig. 3.3 will be considered in order to choose an appropriate seed source for mid-infrared generation via optical parametric amplification. Furthermore, as mentioned in the introduction of this chapter, not all applications require high peak power. Going 
towards $\mathrm{GHz}$ repetition rates is attractive for spectroscopy and has recently been pursued in mode-locked TD oscillator development ${ }^{279}$. Such high repetition rates will most likely come at sub- $\mu \mathrm{J}$ energy levels, and thus will be best suited for spectral broadening in solid-core fiber. Yet, there has been no comprehensive investigation how repetition rate-scalable solid-core fiber broadening is. This will require a better understanding of cumulative effects causing damage (e.g. through studying relaxation times of trap states). Nevertheless, experiments with $250 \mathrm{~W}$ average power and pulse compression down to $23 \mathrm{fs}^{272}$ imply that the average power-scalability of solid-core fiber is good. In addition, multi-photon microscopy of biological tissue usually works best at low $\mathrm{MHz}$ repetition rates and pulse energies about $100 \mathrm{~nJ}^{253}$. Spectral broadening in LMA fibers has been proposed as a tunable tool for such applications ${ }^{280,281}$. Finally, the discussions on the relations between peak power, peak irradiance, broadening factor and choice of MFD can be transferred to other waveguides like fibers suited for the mid-infrared ${ }^{282}$, capillaries $^{283}$ or hollow-core PCFs ${ }^{284}$. The next section will discuss a compression scheme based on such gas-filled fibers.

\section{Supplement 2: Temporal and spectral broadening of intially chirped pulses}

All considerations are made for the mathematically best treatable Gaussian pulses. The results usually agree qualitatively well with other bell-shaped pulses like the sech $^{2}$ pulses emerging from the oscillator. In the FOURIER limited case, the field fulfilling $|E(t)|^{2}=I(t)$ can be described as ${ }^{285}$ :

$$
E(t)=\underbrace{\sqrt{I_{p}} e^{-\frac{\kappa}{2} \frac{t^{2}}{t_{p}^{2}}}}_{A(t)} e^{i \omega_{0} t},
$$

where $I_{p}$ denotes the peak irradiance, $\kappa=$ $4 \ln 2$, the Gaussian shape factor, $t$ the time, $t_{p}$ the pulse duration, $A$ the pulse envelope and $e^{i \omega_{0} t}$ the carrier wave at $\omega_{0}=2 \pi c / \lambda$ where $\mathrm{c}$ is the speed of light and $\lambda$ the wavelength. Chirp is mathematically conveniently added in the frequency domain where the field reads:

$$
\mathcal{E}(\Omega)=\sqrt{2 \pi I_{p} / \kappa} t_{p} e^{-\frac{t_{p}^{2} \Omega^{2}}{2 \kappa}} e^{-i \frac{\mathrm{GDD} \Omega^{2}}{2}},
$$

where $\Omega=\omega-\omega_{0}$ and solely quadratic phase (GDD) was added. Consequently, the power spectrum of a Gaussian pulse has a FWHM of $\Delta \omega_{0}=\kappa / t_{p}$ and $\Delta \omega_{0, \mathrm{RMS}}=\sqrt{\kappa / 2} / t_{p}$ RMS width. Back transformation into the time domain results in the following field:

$$
\begin{aligned}
E(t)= & \sqrt{\frac{I_{p}}{2 \pi \kappa \alpha}} t_{p} e^{-\frac{t^{2}}{4 \alpha}} e^{i\left(\omega_{0} t+\varphi(t)\right)}, \\
= & \sqrt{\frac{I_{p}}{\left(1+\xi^{2}\right)^{1 / 2}}} e^{-\frac{\kappa}{2} \frac{t^{2}}{t_{p}^{2}\left(1+\xi^{2}\right)}} \\
& \times e^{i\left(\frac{\kappa}{2} \frac{t^{2} \xi}{t_{p}^{2}\left(1+\xi^{2}\right)}-\frac{\arctan \xi}{2}+\omega_{0} t+\varphi(t)\right)},
\end{aligned}
$$

where $\alpha=t_{p}^{2} / 2 \kappa+i \mathrm{GDD} / 2$ and $\xi=$ $\kappa \mathrm{GDD} / t_{p}^{2}$. The factor $\sqrt{1+\xi^{2}}$ determines the ratio between the duration of the chirped and transform-limited pulses. Consequently, the temporal stretching or compression factor is determined by the ratio of GDD and the square of the bandwidth. If a chirped pulse with a certain GDD is launched into a fiber with a flat GVD and no nonlinearity, the temporal stretching factor $(\sigma=$ $\left.t_{p, \text { out }} / t_{p, c}\right)$ can be readily calculated as follows:

$$
\begin{aligned}
t_{p, c} & =t_{p, i} \sqrt{1+\xi^{2}}, \\
t_{p, \text { out }} & =t_{p, i} \sqrt{1+\left[\xi+\kappa l / L_{d}\right]^{2}}, \\
\sigma_{c} & =\sqrt{1+\frac{\kappa l / L_{d}+2 \xi}{1+\xi^{2}} \frac{\kappa l}{L_{d}}} .
\end{aligned}
$$


This stretching factor is always larger than the one of an unchirped pulse with the same duration $t_{p, c}$ which is:

$$
\sigma_{u}=\sqrt{1+\frac{\kappa l / L_{d}}{1+\xi^{2}} \frac{\kappa l}{L_{d}}},
$$

where $L_{d}=t_{p, i} / \mathrm{GVD}$ as before.

The term $\varphi(t)$ was added to Eq. (3.9). It represents the SPM term which reads after a propagation of length $l^{165}$ :

$$
\varphi(t)=-e^{-\kappa \frac{t^{2}}{t_{p}^{2}\left(1+\xi^{2}\right)}} \frac{l}{L_{n}} .
$$

Although the transformation of the field in Eq. (3.9) into the frequency domain is analytically not possible, the RMS width of the power spectrum can be determined analytically by:

$$
\begin{aligned}
\Delta \omega_{\mathrm{RMS}}^{2} & =\frac{\int \Omega^{2}|\mathcal{F} \mathcal{T}\{E(t)\}|^{2} d \Omega}{\int|\mathcal{F} \mathcal{T}\{E(t)\}|^{2} d \Omega} \\
& \equiv \frac{\int|d E(t) / d t|^{2} d t}{\int|E(t)|^{2} d t},
\end{aligned}
$$

where $\mathcal{F} \mathcal{T}$ is the FouRIER transform operator. The upper equation assumes that the central frequency (i.e. the first moment of the spectrum) is known. This is the case in Eq. (3.9). The upper identity was first used to calculate the spectral width of unchirped pulses ${ }^{286}$ and in a similar way the temporal width of self-phase modulated pulses ${ }^{287}$. The derivation was, however, only briefly described, and thus it is explicitly shown in Appendix A.2. The result for the field of Eq. (3.9) is:

$$
\begin{aligned}
\Delta \omega_{c, \mathrm{RMS}}= & \sqrt{\frac{\kappa}{2}} t_{p, i}\left[1+\frac{\sqrt{2} \xi}{1+\xi^{2}} \frac{l}{L_{n}}\right. \\
& \left.+\frac{4}{3 \sqrt{3}\left(1+\xi^{2}\right)}\left(\frac{l}{L_{n}}\right)^{2}\right]^{1 / 2} .
\end{aligned}
$$

Consequently, the broadening factor $\beta_{c}=$ $\Delta \omega_{c, \mathrm{RMS}} / \Delta \omega_{0, \mathrm{RMS}}$ reads:

$$
\beta_{c}=\sqrt{1+\frac{\sqrt{2} \xi+4 /(3 \sqrt{3}) \frac{l}{L_{n}}}{1+\xi^{2}} \frac{l}{L_{n}}} .
$$

Comparing the result to an unchirped pulse of the same duration:

$$
\beta_{u}=\sqrt{1+\frac{4 /(3 \sqrt{3}) \frac{l}{L_{n}}}{1+\xi^{2}} \frac{l}{L_{n}}},
$$

demonstrates that initial positive chirp does not only accelerate temporal stretching but also spectral broadening. The simulation results presented in Fig. 3.7 indicate that both effects compensate for each other.

\subsubsection{Kagomé-type Hollow-core Photonic Crystal Fibers}

\section{Parts of this section have been published in:}

K. F. Mak, M. Seidel, O. Pronin, M. H. Frosz, A. Abdolvand, V. Pervak, A. Apolonski, F. Krausz, J. C. Travers, and P. St. J. Russell, "Compressing $\mu$ J-level pulses from 250 fs to sub-10 fs at $38 \mathrm{MHz}$ repetition rate using two gas-filled hollow-core photonic crystal fiber stages," Opt. Lett. 40, 1238 (2015).

In the previous considerations of spectral broadening in solid-core fiber, the critical power of FS was identified as a limiting factor for peak power scaling and achieving the highest possible broadening factors. Obviously, nonlinear materials with lower critical power hold great promise to overcome these limitations. This is practically not possible with other solid state materials because FS with a bandgap of $9 \mathrm{eV}$ is already among the least nonlinear solids (cf. e.g. ref. 290). Therefore, noble gases are usually the broadening media of choice. They exhibit the lowest nonlinearities due to their strongly bound valence 
electrons. However, not only the critical power of gases, but by virtue of MiLLER's rule 291 also the refractive indices are lower than for solids, and thus guiding structures cannot be based on total internal reflection as, for instance, in step-index fibers. The simplest and sofar most widely used approach exploits high FRESNEL reflectivity near grazing incidence in hollow-core capillaries ${ }^{283,292}$. Unfortunately, this technique can only lead to efficient spectral broadening with at least several hundreds of MWs peak power which have not even been reached by the latest generation of TD oscillators. High peak powers are required owing to the need for high irradiances to get significant SPM and the cubic transmission loss dependence on the $\mathrm{MFD}^{292,293}$. In other words, moderate peak powers require tight focusing $\left(n_{2}\right.$ is about 3 orders of magnitudes lower than for FS, depending on gas type, temperature and pressure). This, in turn, gives rise to higher angular frequencies, and hence lower angles of incidence at the interface to the capillary which causes significant reflection losses. Therefore, other guiding mechanisms are needed in the peak power region from $5 \mathrm{MW}$ to $500 \mathrm{MW}$ which is particularly interesting for mode-locked TD oscillators.

Alternative approaches rely on photonic-bandgap (PBG) guiding ${ }^{193,294,295}$, inhibited coupling or anti-resonant guiding in Kagomé-type fibers ${ }^{284,288,296}$ or simplified cladding structures $^{289,297-301}$. The cross-sections of these fiber types are shown in Fig. 3.11. PBG fibers guide only over a narrow bandwidth. They allow FTLs of about $20 \mathrm{fs}$, but exhibit a comparably strong gradient in their waveguide dispersion. By contrast, Kagomé-type fibers are very broadband and enabled few-cycle pulse generation from Ti:sapph amplifier systems $^{302}$. Consequently, pulse compression experiments were conducted with this type of HC-PCFs in collaboration with PhiLIP St. J. Russell's and John C. Traver's group at the Max-Planck-Institute for the Science of Light in Erlangen. KA FAI MAK came to our laboratories in Garching, was mainly in charge of the experiments and has described them in his dissertation ${ }^{303}$. Therefore, they will be only briefly summarized here in the context of developing power-scalable pulse-compression concepts for mode-locked TD oscillators. First, the advantages of HC-PCFs will be highlighted, the pulse compression experiments will be presented afterwards, and thirdly some practical disadvantages of the technique will be explained. Finally, a concluding discussion completes this section.

\section{Properties of Kagomé-type Hollow-core Photonic Crystal Fibers}

While in solid-core fiber nonlinearity and, for LMAs, dispersion is dictated by the fiber material, HC-PCFs offer much more flexibility in tailoring dispersion and nonlinearity at a variety of peak power levels. There are basically three "turning knobs":
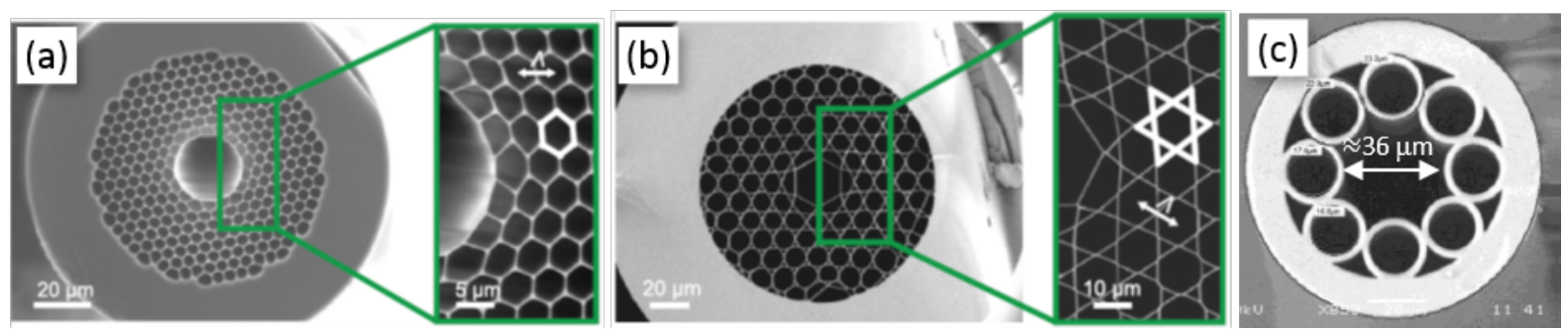

Fig. 3.11. (a) Cross-section of PBG-guiding PCF (taken from ref. 288). (b) Cross-section of Kagométype hollow-core photonic crystal fiber (HC-PCF) (taken from ref. 288). (c) Cross-section of anti-resonant guiding HC-PCF with a simplified cladding structure (taken from ref. 289). Images were taken through scanning electron microscopy. The marked pitch $\Lambda$ is not of interest in this context. 

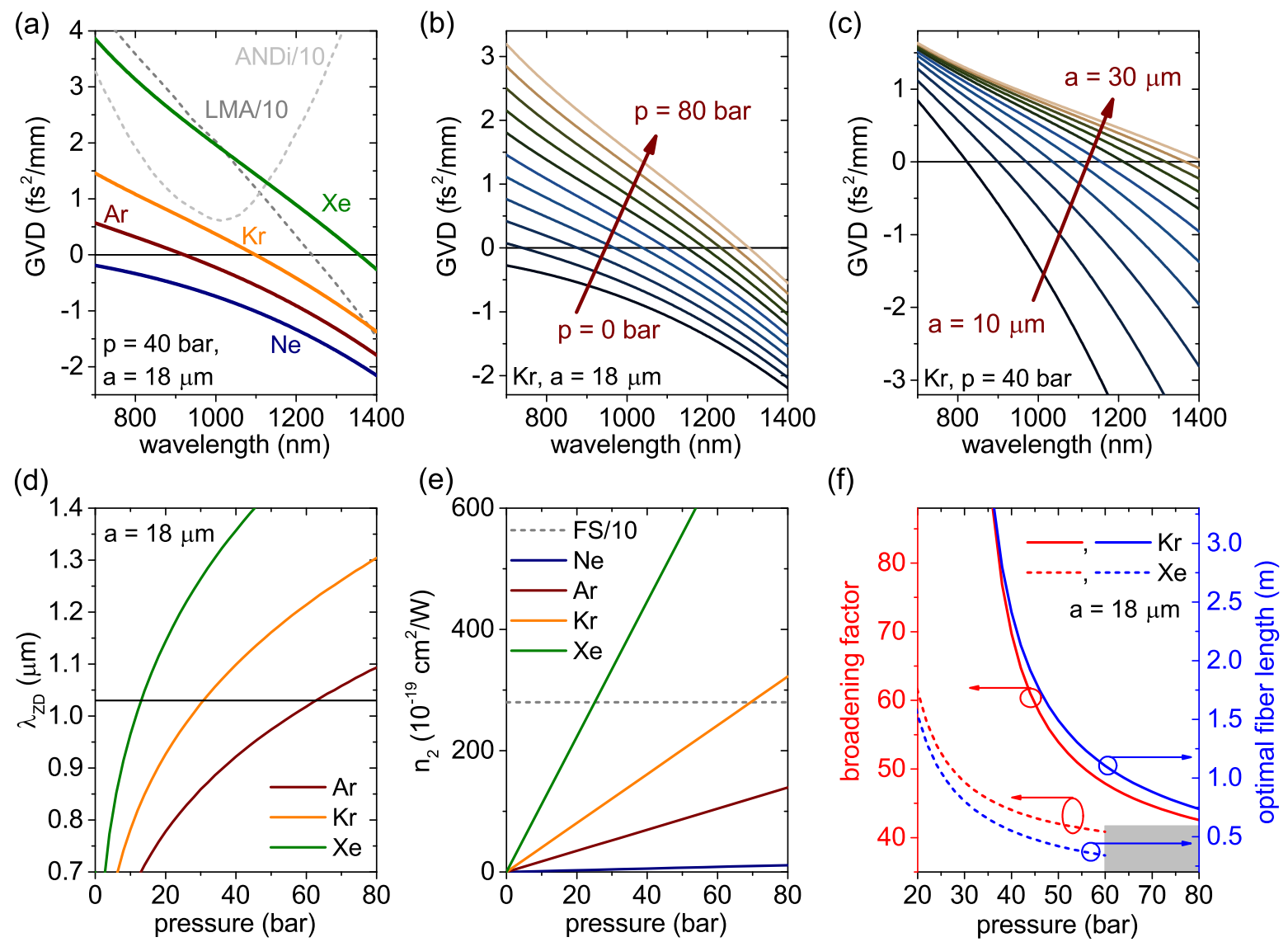

Fig. 3.12. (a) GVDs of a HC-PCF with $a=18 \mu \mathrm{m}$ filled with the noble gases neon (Ne), argon (Ar), krypton $(\mathrm{Kr})$ or xenon $(\mathrm{Xe})$ at a gas pressure of 40 bar. The gas refractive indices have been calculated from the SeLLMeIER equations derived in ref. 304. For comparison, the GVD divided by 10 of the solidcore fibers investigated in the previous section are shown (cf. Fig. 3.1(b)). (b) GVDs of a HC-PCF with $a=18 \mu \mathrm{m}$ filled with Kr. The gas pressures vary in an 8 bar equidistant spacing from 0 to 80 bar. (c) GVDs of a HC-PCF filled with $\mathrm{Kr}$ at a pressure of 40 bar. The bore radii vary in a $2 \mu \mathrm{m}$ equidistant spacing from 10 to $30 \mu \mathrm{m}$. (d) Calculated zero-dispersion wavelengths $\left(\lambda_{\mathrm{ZD}}\right)$ for the gas types of (a), $a=18 \mu \mathrm{m}$ and under varying pressures. The central wavelength of the oscillator $(1030 \mathrm{~nm})$ is shown for reference. The zero-dispersion wavelengths of Ne are always shorter than $700 \mathrm{~nm}$. (e) The nonlinear refractive indices $n_{2}$ under varying gas pressures. The indices under ambient conditions have been taken from refs. 305, 306. The literature values, however, differ by more than an order of magnitude, cf. ref. 307. For comparison, the $n_{2}$ of FS divided by 10 is shown. (f) Derived broadening factors and optimal fiber lengths for $\mathrm{Kr}$ (solid lines) and Xe (dashed lines) filled fibers with $a=18 \mu \mathrm{m}$ under varying pressure (but without gradient inside the fiber). The plots have been calculated from Eqs. (3.1) and (3.2). The gray shaded area indicates the transition of Xe to the supercritical phase. This leads to strong changes of the gas' optical properties ${ }^{308}$.

- bore radius $(a)$,

- gas type,

- gas pressure $(p)$ and temperature $(T)$.

The (linear) refractive index is calculated by ${ }^{284,288,292}$ : 


$$
n_{m k}(\lambda, p, T)=\sqrt{n_{\text {gas }}^{2}(\lambda, p, T)-\frac{\lambda^{2} j_{m k}^{2}}{4 \pi^{2} a^{2}}} \approx 1+\frac{1}{2}\left\{\left[1-n_{\text {gas }}^{2}\left(\lambda, p_{0}, T_{0}\right)\right] \frac{p}{T} \frac{T_{0}}{p_{0}}-\frac{\lambda^{2} j_{m k}^{2}}{4 \pi^{2} a^{2}}\right\}
$$

where $j_{m k}$ denotes the $\mathrm{k}^{\text {th }}$ zero of the $\mathrm{m}^{\text {th }}$-order BESSEL function, $p_{0}$ and $T_{0}$ reference pressure and temperature, resp. Only the fundamental mode $\mathrm{HE}_{11}$ is supposed to be excited, corresponding to $k=1, m=0$. The Taylor expansion of the square root can be stopped after the linear term since the refractive indices will be close to one. The scaling of the $\chi^{(1)}$ polarization term is linear to the number of dipoles according to the LORENTZ "electron-on-a-spring"-model ${ }^{156}$ which leads to $p / T \propto \rho$ (particle density for an ideal gas at constant volume) dependence of the gas refractive index term. The LoREnTz model also explains the linear scaling of $n_{2}$ which is supported by experimental data ${ }^{307,309}$.

Fig. 3.12(a)-(c) shows several examples that evaluate Eq. (3.17) in order to calculate the GVD under the variation of the three tuning parameters. Firstly, it is obvious that the GVD of the gas-filled fibers is generally lower than that of LMA and even that of ANDi fiber near $1030 \mathrm{~nm}$, the central oscillator wavelength. Owing to the anomalous waveguide dispersion, both positive and negative GVD can be adjusted at $1030 \mathrm{~nm}$ (cf. Fig. 3.12(d)). For a HC-PCF with a bore diameter of $36 \mu \mathrm{m}$, which was available in the experiments, it is practically only possible to reach an all-normal dispersion regime in the optical octave from 700 to $1400 \mathrm{~nm}$ with xenon. As the magnitude of waveguide dispersion decreases with $a^{2}$ according to Eq. (3.17), larger bore diameters would also enable ANDi broadening to few-cycle pulses with other gases (cf. Fig. 3.12(c)). However, the nonlinear length would increase quadratically with $a$ for a fixed peak power, too. Due to the lower GVD and $n_{2}$ of the gases in comparison to FS (cf. Fig. 3.12(e)), both dispersion and nonlinear length are usually higher than for solid-core fiber. The consequent use of longer fibers for spectral broadening in the normal dispersion regime leads to non-negligible transmission losses. For comparison, the losses of Kagomé-type HC-PCFs are on the order of $1 \mathrm{~dB} / \mathrm{m}^{288}$ while the solid-core PCFs exhibit losses of less than $10 \mathrm{~dB} / \mathrm{km}^{310}$. Since the GVD reduction in comparison to LMA fiber is stronger than the $n_{2}$ reduction, larger broadening factors are predicted for lossless HC-PCFs than for solid-core fiber (cf. Figs. 3.12(f) and 3.6). At 40 bar, the zero-dispersion wavelength of krypton would be at about $1100 \mathrm{~nm}$ while the GVD at $1030 \mathrm{~nm}$ was only $0.26 \mathrm{fs}^{2} / \mathrm{mm}$. Subsequently, a huge broadening factor of $70 \mathrm{but}$ also a fiber length of $2.4 \mathrm{~m}$ is predicted. It is to note that fiber losses and crossing of $\lambda_{\mathrm{ZD}}$ by the red spectral wing is neglected in this coarse calculation. In comparison, Xe would exhibit a GVD of $1.8 \mathrm{fs}^{2} / \mathrm{mm}$ at $1030 \mathrm{~nm}, \lambda_{\mathrm{ZD}} \approx 1360 \mathrm{~nm}$, a broadening factor of 44 at a fiber length of $55 \mathrm{~cm}$.

\section{Double-stage compression to the few-cycle regime}

The conducted experiment consisted of two compression stages. The setup is shown in Fig. 3.13. The fibers were glued with an epoxy adhesive into gas cells which were connected either to a vacuum pump or a noble gas bottle. Details about the construction of the gas cells can be found in ref. 303. Pressures of up to 40 bar were applied. It is remarkable that the fragile HC-PCFs withstood such high pressures. This was enabled by the tiny gas volume inside the fibers. The gas cell at the front of the fiber had to be evacuated. The interaction with the atoms inside the cell led to very strong fluctuations at the fiber output. This was surprising because of the low peak power of only about $4 \mathrm{MW}$. The critical power 


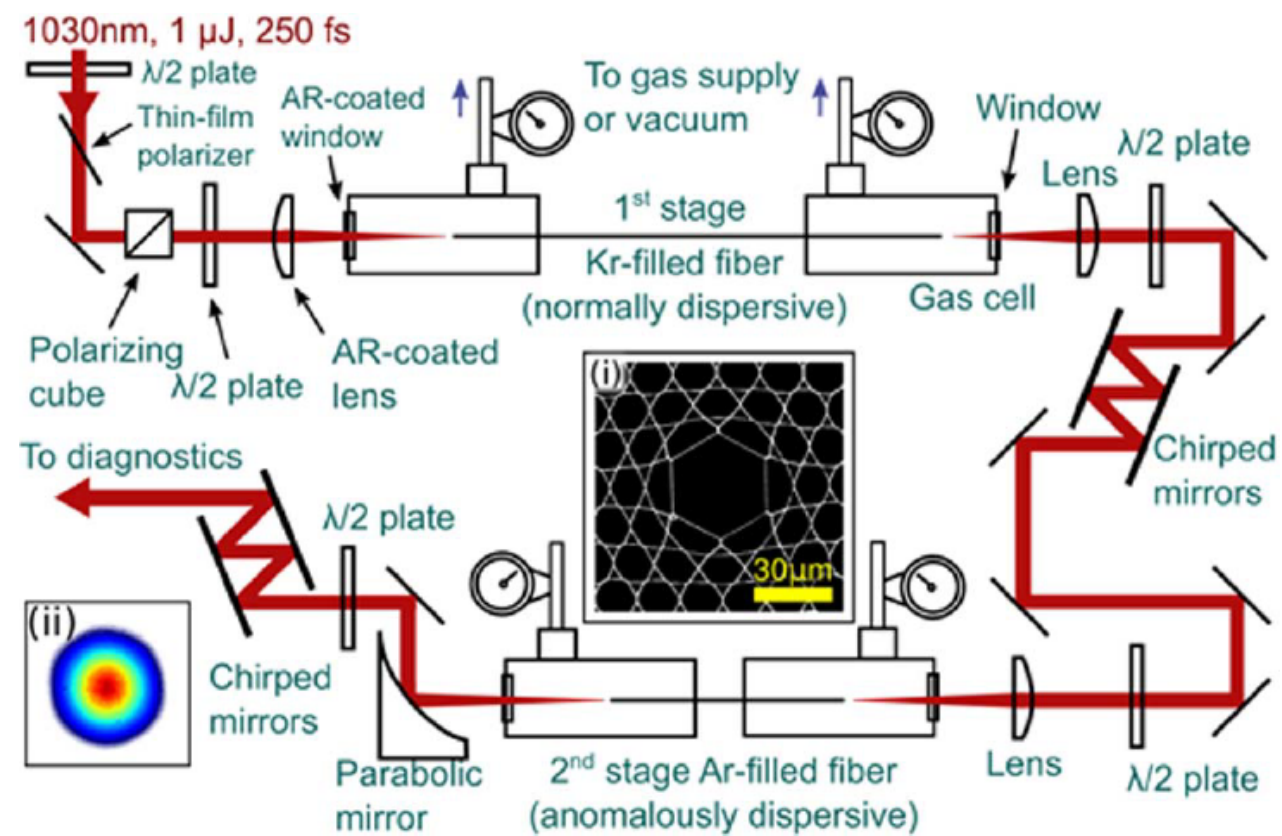

Fig. 3.13. Setup of the double stage Kagomé-type HC-PCF compression which was pumped by the oscillator introduced in section 2.1. Inset (i) shows the cross-section of the fiber with a bore diameter of $36 \mu \mathrm{m}$. It was measured in Erlangen with a scanning electron microscope. Inset (ii) shows the measured beam profile of the compressed output which was measured with a CCD camera.

of $\mathrm{Kr}$ at 40 bar is about $100 \mathrm{MW}$, and hence the peak power enhancement is about $4 \%$ according to Eq. (3.5), hardly distorting the input beam. The peak irradiance in the fiber was about $1.65 \mathrm{TW} / \mathrm{cm}^{2}$, i.e. close to the damage irradiance which was estimated for the solid-core fiber. Since the ionization energy of Krypton is about $14 \mathrm{eV}^{311}$, and thus clearly higher than the FS bandgap $(9 \mathrm{eV})$, the ionization probability is very low. Nevertheless, the pressure gradient approach, initially proposed to suppress self-focusing and ionization ${ }^{312,313}$, could strongly improve the input coupling and reduce the laser fluctuations. Therefore, the first gas cell was continuously evacuated while the second gas cell was pressurized. Due to the small fiber diameter, the gas flow inside the HC-PCF was low enough to create a gradient between the vacuum and the pressure of the second cell. More information about gas flow and pressure distribution within the HC-PCF is presented in ref. 303. Light was coupled into the gas cells through FS windows. Only the first window and focusing lens were AR-coated. The other three windows and the lenses between first and second compression stage were uncoated, yielding total FRESNEL losses of about $30 \%$. The fibers are slightly birefringent, and hence half-wave plates had to be placed in front of them to achieve high polarization extinction ratios. The polarizing cube in front of the first half-wave plate cleaned the input polarization and suppressed cross-phase modulation inside the fiber. Polarization extinction ratios of $14 \mathrm{~dB}$ after the first stage and $13 \mathrm{~dB}$ after the second stage were measured. The chirped mirrors used for compression after the first stage were PC1305 (cf. Fig. 2.8(b)). After the second stage, PC247 mirrors were used (cf. Fig. 2.12(b)). All chirped mirrors required p-polarization. Therefore, additional half-wave plates were required in front of the pulse compressors.

In principle, the first spectral broadening stage was operated in the normal dispersion regime. It is to note that due to the pressure gradient, parts of the optical spectrum nominally experienced anomalous dispersion (cf. Fig. 3.12, simulations in ref. 303, 314). 
(a)

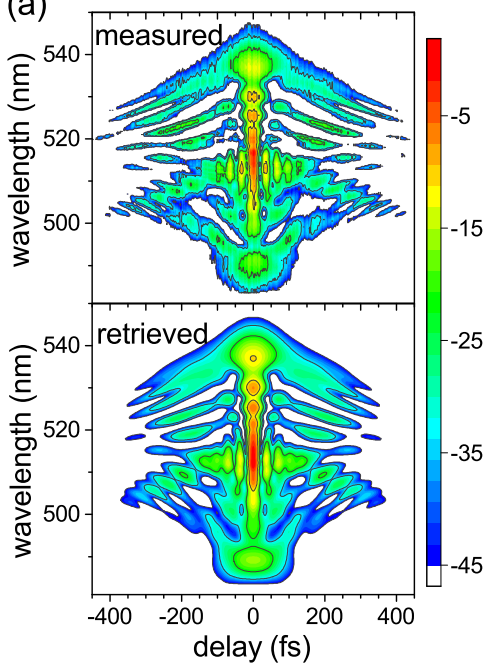

(b)

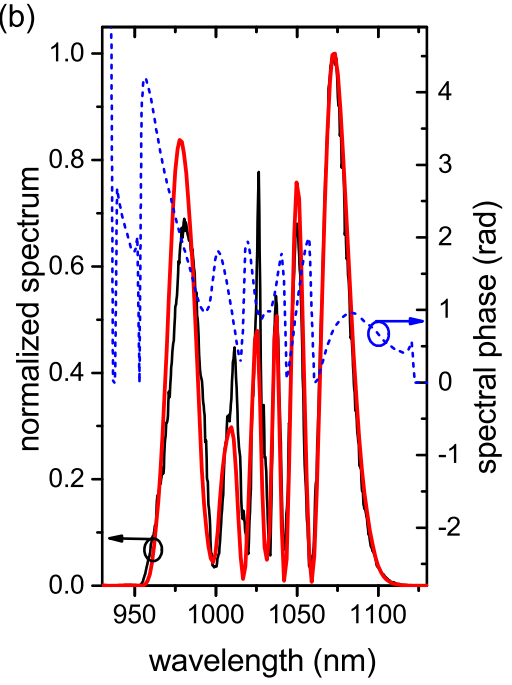

(c)

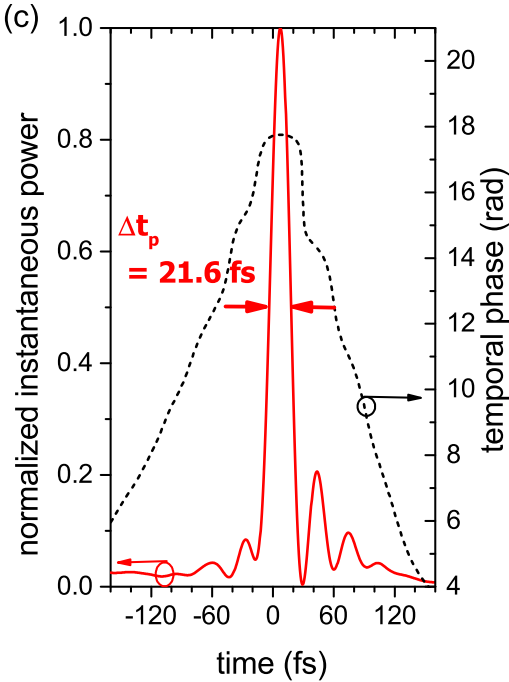

Fig. 3.14. (a) Measured (top) and retrieved (bottom) FROG trace of the pulse after the chirped mirror compressor. The color scale is in units of $\mathrm{dB}$ with respect to the maximum of the trace. The RMS FROG error was $0.50 \%$ for a $1024^{2}$ grid size. (b) Spectrum measured with an OSA (black solid line) and retrieved from FROG (red solid line). The blue dashed line shows the retrieved spectral phase. (c) Retrieved ultrahort pulse of 22 fs duration (red solid line) and corresponding temporal phase (black dashed line).

Its impact was, however, weak, and thus the broadening characteristics can be explained well with normal dispersion spectral broadening. A fiber length of $70 \mathrm{~cm}$ was chosen, being much shorter than the predicted hypothetical fiber length of $2.4 \mathrm{~m}$ for a $40 \mathrm{bar}$ pressurized $\mathrm{Kr}$ filled HC-PCF. Consequently, a FTL of $21 \mathrm{fs}$ was achieved, corresponding to a broadening factor of 12 (Fig. 3.14). A total GDD of $1200 \mathrm{fs}^{2}$ ( 8 bounces off PC1305) was added to the pulses in order to compress them to the nearly transform-limited duration of $22 \mathrm{fs}$. The compressed pulses were measured by means of SH-FROG. They are displayed in Fig. 3.14(c). Remarkably, if a bandwidth of $t_{i}=21 \mathrm{fs}$ and $1200 \mathrm{fs}^{2}$ are plugged into Eq. (3.10), a duration of only about $160 \mathrm{fs}$ is derived. This underlines that due to the very long dispersion length $\left(240 \mathrm{~m}\right.$ for 40 bar and $\left.t_{p}=250 \mathrm{fs}\right)$, spectral broadening was not saturated like in the solid-core fiber compression case (cf. section 2.2.1). There were two reasons why the fiber length was not extended. Firstly, the partly anomalous dispersion arising from the need for a pressure gradient would gain stronger influence in longer fibers, and hence clean compression would have become increasingly difficult. Secondly, the transmission losses would have increased. In the presented setup, the fiber transmission was $80 \%$ which is close to the solid-core fiber transmission of section 2.2.1 ( $85 \%$ ). The power behind the first stage was $28 \mathrm{~W}, 8 \mathrm{~W}$ more than behind the chirped mirrors of the LMA-35 fiber compression stage.

To reach the few-cycle regime, a second broadening stage was set-up. Owing to the large bandwidths required for few-cycle pulses, spectral broadening in the all-normal dispersion regime was practically not possible anymore with bore diameters of $36 \mu \mathrm{m}$ (cf. Fig. 3.12(d)). Therefore, self-compression in the anomalous dispersion regime was targeted. The idea of self-compression is that the SPM broadened spectrum is immediately compressed by the negative GVD of the HC-PCF. In the fundamentals section (section 1.4), it was indicated that negative GDD and SPM can also lead to spectral narrowing, and hence pulse stretching. Consequently, it is obvious that achieving self-compression 
(a)

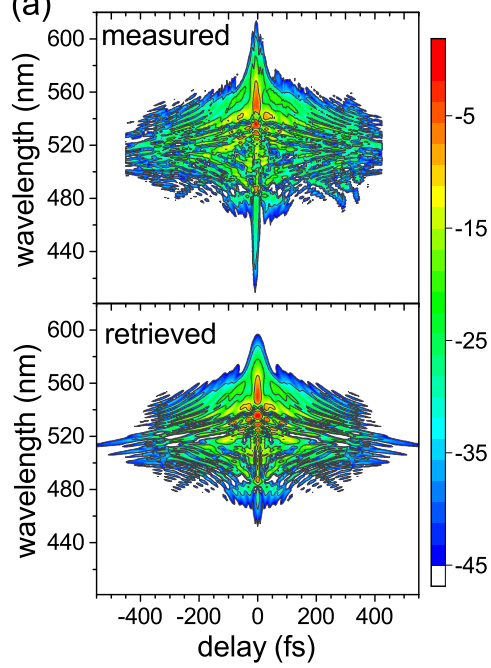

(b)

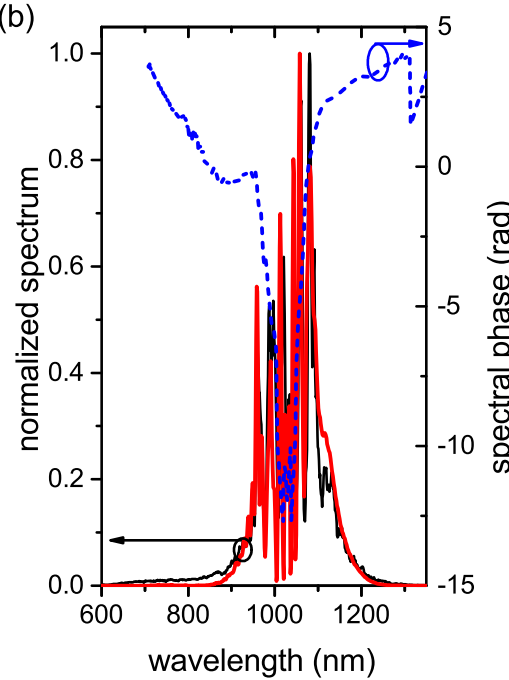

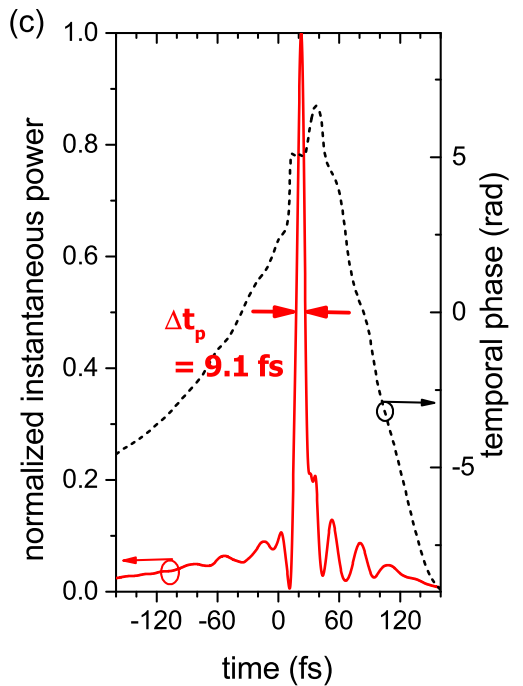

Fig. 3.15. (a) Measured (top) and retrieved (bottom) FROG trace of the pulse after the chirped mirror compressor. The color scale is in units of $\mathrm{dB}$ with respect to the maximum of the trace. The RMS FROG error was $1.08 \%$ for a $1024^{2}$ grid size. (b) Spectrum measured with an OSA (black solid line) and retrieved from FROG (red solid line). The OSA spectrum extends down to about $600 \mathrm{~nm}$ while FROG could only reconstruct a spectrum down to about $700 \mathrm{~nm}$ with strong attenuation of the shortest wavelengths. The blue dashed line shows the retrieved spectral phase. (c) Retrieved ultrahort pulse of 9 fs duration (red solid line) and corresponding temporal phase (black dashed line).

at minimal pulse duration requires a proper balance between dispersion and nonlinear lengths. The theory on the choice of this ratio shall not be treated explicitly within the context of this dissertation. The reader is instead referred to other literature explaining self-compression more detailed, e.g. refs. 165, 257, 303. Since the impact of GVD strongly depends on the bandwidth of the ultrashort pulses (cf. Eq. (3.10)) and owing to the decreasing pulse duration during propagation, the dynamics of self-compression is not nearly as smooth as that of all-normal dispersion spectral broadening. Subsequently, the output spectrum and pulse duration is very sensitive to the input pulse energy.

In the performed experiments, the selection of gas type, pressure and fiber length was derived from simulation results which are presented in refs. 303, 314. A pressure gradient had to be applied again. The second gas cell was pressurized with 26 bar of argon, yielding a maximal zero-dispersion wavelength of about $830 \mathrm{~nm}$ and GVDs between -0.9 and $-0.5 \mathrm{fs}^{2} / \mathrm{mm}$ within the $25 \mathrm{~cm}$ long fiber. Chirped mirrors behind the last gas cell were necessary to compensate for the dispersion of last window and the beam path between fiber and FROG as well as for a pair of glass wedges which was used for fine adjustment of the total GDD. The beam was reflected 6 times from PC247 mirrors. The fiber transmission was again about $80 \%, 18 \mathrm{~W}$ emerged from the last gas cell, $14.5 \mathrm{~W}$ were measured after the chirped mirrors. Spectrum and retrieved pulses are shown in Fig. 3.15. Firstly, sub-10 fs pulse durations could be reached by means of the presented scheme. Even sub-2 cycle pulses would have been possible (FTL of OSA spectrum in Fig. 3.15(b) is only about $5 \mathrm{fs}$ ). The bandwidth of the available mirrors was, however, not sufficient for compressing the very broad spectrum. Secondly, a sharp temporal spike is generated by the selfcompression technique, but the compression quality, i.e. the pulse energy confinement in the main peak, is very low. While $80 \%$ of the power could be confined in the main peak of the few-cycle pulses measured after bulk broadening (cf. Fig. 2.13), only about 

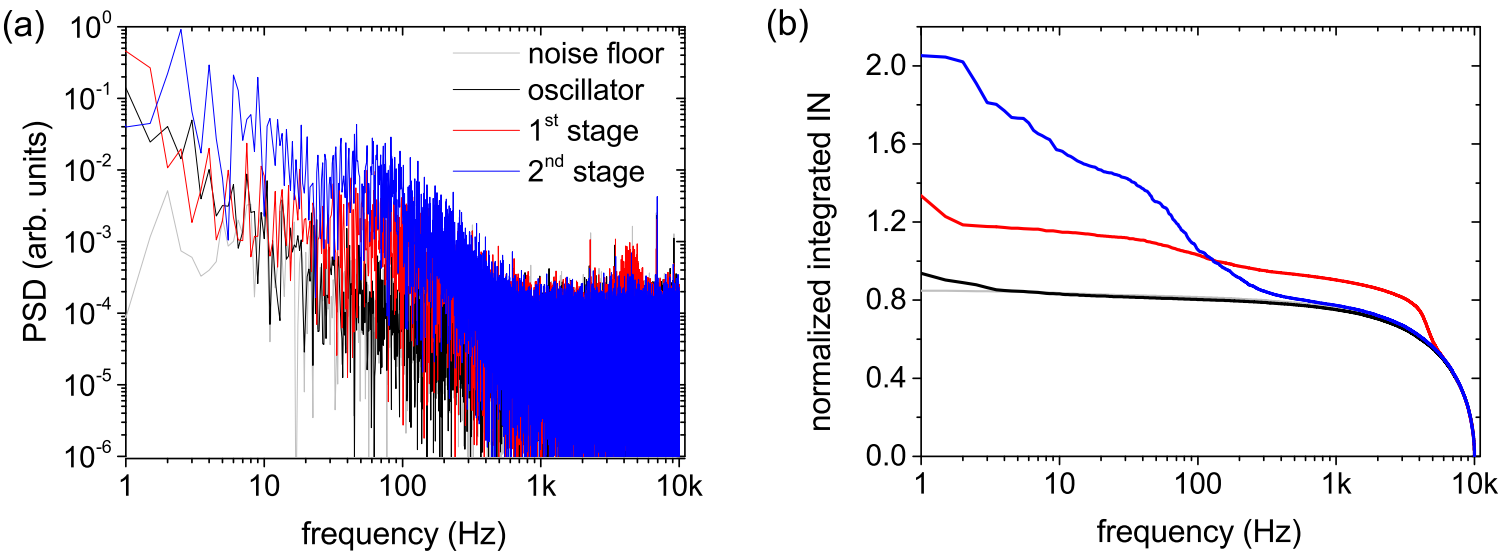

Fig. 3.16. (a) Noise measurements with a fast photodiode and a digital oscilloscope. The time traces were FOURIER transformed to obtain the PSDs. Data was taken in front of the first fiber (black line), behind the first compression stage (red line) and behind the second compression stage (blue line). The noise floor (gray line) is shown for comparison. (b) RMS INs derived from the PSDs from $1 \mathrm{~Hz}$ to $10 \mathrm{kHz}$. The plots are normalized to the measured oscillator intensity noise (black line) over the full bandwidth. Contrary to the graph shown in ref. 314, the square root of the integrated PSDs are taken.

$32 \%$ are confined in the central part of the pulse shown in Fig. 3.15(c). Therefore, the peak power $(13 \mathrm{MW})$ is lower than the one of the $7.7 \mathrm{fs}$ pulses after bulk broadening $(16 \mathrm{MW})$ although the average power is more than two times larger. It is to note that it has not been investigated if the spectral phase shown in Fig. 3.15(b) can be linearized by tailored chirped mirrors. The pulse shape of the few-cycle pulses generated by few-cycle pulse compression is one drawback of the presented Kagomé-type HC-PCF compression scheme. There are a few more as it will be pointed out in the next paragraph.

\section{Drawbacks of Kagomé-type HC-PCF spectral broadening}

It has already been mentioned that the spectral broadening after the first fiber stage was not saturated and that self-compression is very sensitive to the input power. Consequently, the setup appears much more susceptible to spectral and intensity noise than the solidcore fiber compression experiments which have been presented before. In the context of the reported HC-PCF experiments only intensity noise (IN) was measured. No systematic studies on fluctuations of the spectrum were done. The IN measurements were performed with a $\mathrm{GHz}$ photodiode connected to a digital oscilloscope with $50 \Omega$ load resistance. The time traces, measured over $2 \mathrm{~s}$, were FOURIER transformed to calculate the power spectral density within a bandwidth from $1 \mathrm{~Hz}$ to $10 \mathrm{kHz}$ (cf. Fig. 3.16(a)). Three noise measurements are displayed. One was done directly in front of the first fiber, a second one behind the first compression stage and a third one behind the second compression stage. The PSDs have been integrated from $10 \mathrm{kHz}$ to $1 \mathrm{~Hz}$ in order to calculate the total IN (cf. Fig. 3.16(b)). The oscillator noise measurement is basically limited by the high noise floor of the oscilloscope. A spectrum analyzer would give more insights into the noise characteristics for frequencies higher than $10 \mathrm{~Hz}$ (cf. section 2.3.1), but would not be capable of detecting slow fluctuations. These become also visible for the oscillator noise measurement. The data taken after the first compression stage reveals a noise spike around $4 \mathrm{kHz}$. This spike was very sensitive to the coupling into the fiber and could be 


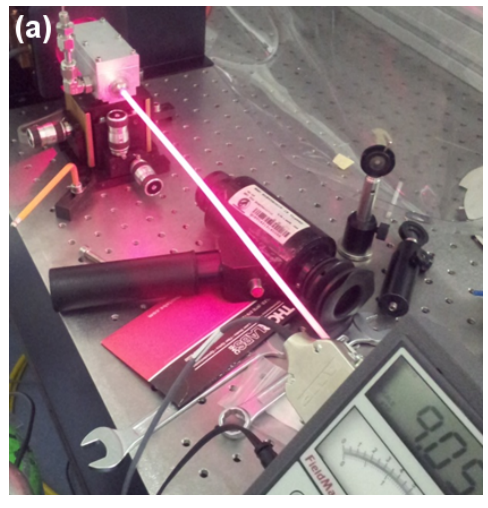

(b)

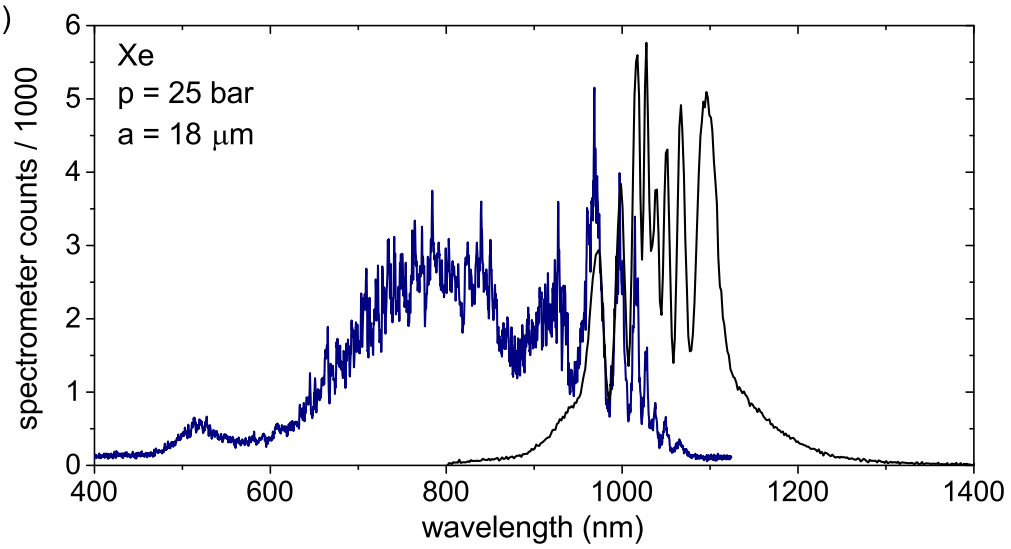

Fig. 3.17. (a) Xe filled fiber for first stage compression. The visible (flickering) glow of the fiber is a clear indicator for ionization and the consequent frequency blue shift. Only $9 \mathrm{~W}$ of average power are transmitted trough the HC-PCF. Fibers were not glued into the gas cells if no pressure gradient was applied. The cells were connected with tubes instead. The fiber was lying inside. (b) Xe filled fiber for second stage compression. The spectrum has been measured with two grating spectrometers (not calibrated) to cover the full range. The spectrum is strongly blue-shifted and extremely unstable. The modulations of the visible spectrum indicate the heavy fluctuations.

removed by careful alignment as the noise measurement after the second stage indicates (which was not done in parallel). In comparison to the oscillator, the IN behind the first stage slightly increased in the acoustic frequency range below $100 \mathrm{~Hz}$. This noise was alignment insensitive and was strongly amplified after the second compression stage where a broad noise enhancement becomes visible between 30 and $100 \mathrm{~Hz}$ and several narrow spikes appear below $10 \mathrm{~Hz}$. This acoustic noise could not be eliminated by improved coupling. The IN was normalized to the integrated value of the oscillator measurement and not to the power in the carrier as usual. Nonetheless, the instability of the compression scheme becomes quite obvious by the relative IN values and was also supported by the flickering of the weak visible light which was generated inside the fibers.

Despite the relatively small available fiber diameter of $36 \mu \mathrm{m}$, the noisy anomalous dispersion regime appears avoidable if Fig. 3.12(d) is considered. The usage of xenon and a pressure of at least 34 bar would lead to a zero-dispersion wavelength of more than $1300 \mathrm{~nm}$. Also, high broadening factors at reasonably short fiber lengths are expected as Fig. 3.12(f) indicates. In-fact, Xe was originally the gas of choice for the first and the second compression stage. However, the unexpected ionization effects, which already enforced employing a pressure gradient, were much more pronounced for Xe than for $\mathrm{Kr}$ or Ar. Two examples are given in Fig. 3.17. Firstly, a photograph of the initial Kagométype HC-PCF setup is shown. The transparent tube with the fiber lying inside emits visible light which is most likely caused by an ionization induced spectral blue-shift. The effect became apperent at about $25 \mathrm{~W}$ pump power. In the shown example only $9 \mathrm{~W}$ are measured directly behind the fiber. Fig. 3.17(b) shows raw data of a spectrum which was generated in the second fiber stage. An ultrabroadband, but unfortunately also highly unstable blue-shifted continuum is generated which is typical for spectral broadening induced by free carriers ${ }^{315}$. Consequently, it is practically not possible to saturate spectral broadening in the presented fiber setup with the available pulse parameters and Kagométype HC-PCFs. 
The need for a pressure gradient also comes with another practical disadvantage. The fiber has to be glued into the gas cells to minimize the gas flow between the cells. Subsequently, the complete fiber had to be replaced after damage of the fragile front facet which occasionally happened during the development of the presented compression scheme. At the time the experiments were conducted, the market price for Kagomé-type HC-PCF was about $1200 \mathrm{EUR} / \mathrm{m}$ (about 10× cost of LMA fiber). Hence, fiber damage would have led to a tedious and expensive replacement procedure. Perhaps simplified guiding structures, as shown in Fig. 3.11(c), will reduce costs and increase robustness in the future. The Kagomé-type HC-PCF also appeared more alignment sensitive than solid-core LMA PCF. The noise spike at $4 \mathrm{kHz}$ in Fig. 3.16 is an indicator. Moreover, higher order spatial modes could be excited in the hollow-core fiber which is not possible with "endlessly single-mode" solid-core PCF.

\section{Discussion}

The presented experiments have revealed the powerfulness of HC-PCF-based pulse compression but also its severe shortcomings. The near-zero GVD, combined with the broad transmission bandwidth has enabled the generation of few-cycle pulses. This was demonstrated for the first time with hollow-core fibers and a $\mathrm{MHz}$ light source. Even singlecycle pulses would have been theoretically possible by means of self-compression ${ }^{314}$. This self-compression technique seems particularly interesting for direct delivery of the ultrashort pulses into a vacuum chamber by means of negative pressure gradients which were employed in HHG experiments with a $\mathrm{kHz}$ system, for instance ${ }^{316}$. Otherwise, selfcompression exhibits two major drawbacks: the low compression quality and the high noise sensitivity. Therefore, in particular if broadband chirped mirrors are available, few-cycle pulse generation in the normal dispersion regime seems clearly preferential. HC-PCFs with larger bore diameters would have enabled to operate the second fiber also with positive GVD. The relatively low peak powers of the utilized oscillator would have resulted, however, in long nonlinear lengths. It is thus expected that light sources with higher peak powers, like the latest generation of mode-locked TD oscillators ${ }^{59,61,139}$, would simplify few-cycle pulse compression. Kagomé-type HC-PCF-based compression experiments at $\mathrm{kHz}$ repetition rates ${ }^{302,316-318}$ were also conducted with higher peak powers, and hence the approach appears scalable with respect to input pulse energies.

The perhaps most surprising finding of the presented study is the strong, detrimental impact of ionization which has not been observed with $\mathrm{kHz}$ systems at similar intensities (cf. e.g. ref. 302). Even more remarkable, they have not even been reported in Kagomé-type HC-PCF compression experiments with other MHz sources ${ }^{319-322}$. Those prior studies were conducted with lower gas pressures, lower $(\mathrm{MHz})$ repetition rates and longer pulses which were hardly susceptible to weakly anomalous dispersion. Only HÄDRICH et al. mentioned "other nonlinear effects" without further specification when compression from 250 fs below 30 fs was targeted with a $10 \mathrm{MHz}$ repetition rate fiber system ${ }^{323}$. The first intensity noise measurement after a Kagomé-type HC-PCF was, to the best of the author's knowledge, shown in the presented study (cf. ref. 314). In a later experiment ${ }^{324}$ another IN measurement after a single fiber stage was reported. However, the data was not fully consistent since the noise increased by $0.16 \%$ in a bandwidth from $100 \mathrm{~Hz}$ to $100 \mathrm{kHz}$ while it only increased by $0.04 \%$ at the full bandwidth from $1 \mathrm{~Hz}$ to $100 \mathrm{kHz}$ which is mathematically impossible. Since the noise of the utilized KLM TD oscillator in the bandwidth 
from $10 \mathrm{~Hz}$ to $500 \mathrm{kHz}$ is as low as $0.1 \%$ (cf. Fig. 3.24 in the next section) and possibly only a factor 2 of noise is accumulated after the second compression stage (note that it is not clear how much noise is hidden below the oscilloscope noise floor), comparing the data of Fig. 3.16 and from ref. 324 is not entirely conclusive. However, the authors of the latter publication attributed "a remarkably low root-mean-square amplitude noise value" to their setup, and hence considered the compression scheme as well applicable. This promises that the encountered Kagomé-type HC-PCF noise issues can be solved although a better understanding of its origin as well as of the origin of the low irradiance ionization is urgently needed. Further research on average power scalability of Kagomé-type HCPCF-based spectral broadening is out of scope of this dissertation. Instead, the following pulse compression studies will focus on bulk material which immediately eliminates the need for mode coupling, and hence comes with much higher applicability than fiber-based techniques. 


\title{
3.2 All Solid-state Spectral Broadening in Bulk Material
}

Section 2.2.2 has already demonstrated that spectral broadening in bulk material allows few-cycle pulse generation, and hence the technique may present a viable alternative to fiber broadening. It circumvents the main issues which have been encountered in the Kagomé-type HC-PCF experiments: It comes with low complexity and costs, is highly robust and exhibits excellent noise properties (as will be shown later). In order to establish a widely applicable ultrashort pulse source, a reliable and easy-to-handle technology is imperative as explained in the introduction section 1.1. The following studies will demonstrate that bulk broadening fulfills these requirements.

Historically, bulk broadening was introduced shortly after the advent of chirped pulse amplification when peak power scalable pulse compression schemes became a topic of intense research ${ }^{54}$. RoLlAND and CORKUM proposed perhaps the simplest broadening technique which is focusing intense light into a bulk plate such that the critical selffocusing length exceeds the physical length of the nonlinear medium ${ }^{325}$. Although the study accomplished pulse compression from $92 \mathrm{fs}$ to $19 \mathrm{fs}$ and was followed by several theoretical papers discussing the method $^{326-328}$, the power efficiency of only about $4 \%$ made the method fairly unattractive. In 2000, MiLOSEviC et al. explicitly evaluated the strengths and weaknesses of pulse compression in bulk media in comparison to hollow core capillaries which have been applied to compress GW peak power pulses for more than 20 years by now ${ }^{54,208,283}$. They employed coupled-mode theory (cf. Suppl. 1) to analyze the spatial losses in both techniques and found that broadening in solid material can only be made efficient if the peak power is much lower than the critical power of the material. This claim, which refers to single-plate broadening, has become questionable owing to the findings of LU et al. ${ }^{329}$ who efficiently broadened ultrashort pulses with GW peak power level.

Two studies on spectral broadening in bulk media are presented: First, optical KERR effect based spectral broadening, which has been already utilized in the experiments of section 2.2.2, shall be discussed. The following section will introduce cascaded $\chi^{(2)}$ nonlinearities which induce an effective $n_{2}$ that can be tuned in sign and magnitude.

\subsubsection{Compression by means of the Optical Kerr Effect of Dielectrics}

\author{
Parts of this section have been published in: \\ M. Seidel, G. Arisholm, J. Brons, V. Pervak, and O. Pronin, "All solid-state spectral \\ broadening: an average and peak power scalable method for compression of ultrashort \\ pulses," Opt. Express. 24, 9412 (2016).
}

This section presents a comprehensive study of bulk broadening by means of the optical KERR effect. First, the parameters which have impact on the broadening performance are discussed qualitatively. An experimental study of these parameters is presented afterwards. Subsequently, the broadened spectra and spectral phases are analyzed. An example of cascaded bulk broadening with intermediate compression follows. Next, the losses due to the coupling of spectral and spatial nonlinear effects are investigated. The experimental part is completed by a check of the noise properties of the spectrally broadened pulses. Simulations follow which investigate the power scalability of the concept. 

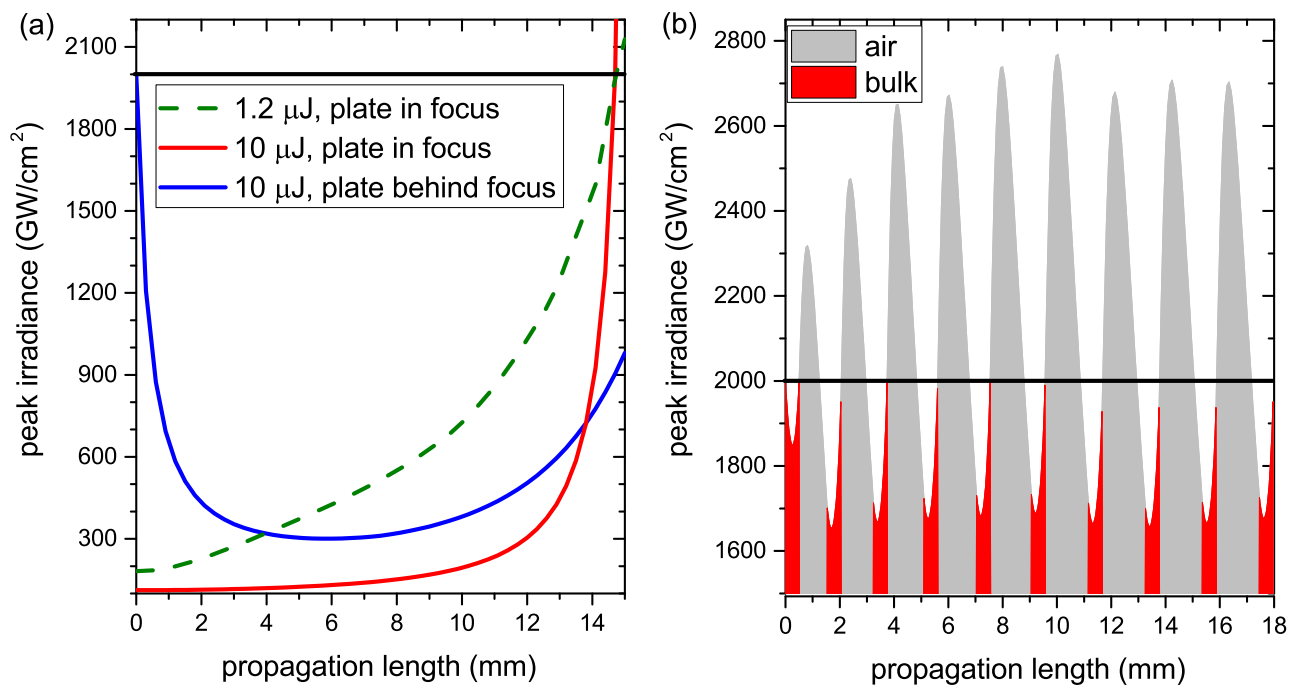

Fig. 3.18. Means of avoiding critical self-focusing within a nonlinear crystal. The plots are taken from simulations which are explained in more detail in the simulation part of this section. A peak irradiance of $2 \mathrm{TW} / \mathrm{cm}^{2}$ is considered as critical (solid black line markers). (a) Single plate approach: critical self-focusing is avoided by choosing a large spot size at the entrance facet (dashed green line: $1.2 \mu \mathrm{J}$, 250 fs input pulses, $d=77 \mu \mathrm{m}$; red line: $10 \mu \mathrm{J}, 250 \mathrm{fs}$ input pulses, $d=283 \mu \mathrm{m}$ ) or by utilizing a strong beam divergence to compensate for self-focusing (blue line: $10 \mu \mathrm{J}, 250$ fs input pulses, $d=67 \mu \mathrm{m}$, $\theta=32 \mu \mathrm{m} / \mathrm{mm}$ ). (b) Multiple plate approach: The beam irradiance is kept below the critical value inside the solid medium while the foci lie in the air gaps between the thin bulk plates (10 $\mu \mathrm{J}, 250 \mathrm{fs}$ input pulses).

Finally, all results are summarized and conclusions on the applicability of bulk broadening are drawn.

\section{General aspects of bulk broadening}

In section 3.1.1, fiber broadening in the sub-critical self-focusing regime was discussed. Bulk broadening works in the critical self-focusing regime, too, since the beam collapse can be avoided if the length of the medium is smaller than the critical self-focusing length $z_{s f}$ which is approximately ${ }^{160,269}$ :

$$
z_{s f}=\frac{\pi d^{2}}{\lambda\left(\sqrt{P_{p} / P_{c r}-1}-\theta\right)},
$$

where $\mathrm{d}$ is the beam diameter at the input facet of the medium, $P_{p}$ the peak power of the laser pulses and $\theta$ the beam divergence. Assuming a $1 \mu \mathrm{m}$ wavelength, the maximal critical power for solid materials is approximately $11 \mathrm{MW}$ (for $\mathrm{LiF}$ ), and thus far below the peak powers of amplifier systems or the latest mode-locked TD oscillators. Therefore, to avoid critical self-focusing, either the beam diameter has to be expanded or the beam divergence has to be adapted to counteract the focusing effect. To visualize these approaches Fig. 3.18(a) shows how the peak irradiance evolves in three exemplary studies. The green dashed line represents the propagation of a $1.2 \mu \mathrm{J}, 250 \mathrm{fs}$ pulse through $15 \mathrm{~mm}$ of crystalline quartz. The peak power $\left(P_{p}=4.2 \mathrm{MW}\right)$ is slightly above the critical power 
of the material $\left(P_{c r} \approx 3.6 \mathrm{MW}\right.$, may slightly vary due to the uncertainties of $\left.n_{2}\right)$. The divergence is set to zero at the front facet of the nonlinear material. In order to stay below the critical irradiance $\left(2 \mathrm{TW} / \mathrm{cm}^{2}\right.$ assumed), the input peak irradiance must not be larger than $180 \mathrm{GW} / \mathrm{cm}^{2}$. For a $10 \mu \mathrm{J}, 250 \mathrm{fs}$ pulse $\left(P_{p}=35.2 \mathrm{MW}\right.$, red line) the input peak irradiance has to be even smaller $\left(110 \mathrm{GW} / \mathrm{cm}^{2}\right)$ in order to avoid damage of the nonlinear material. This is due to the highly nonlinear beam divergence induced by the strong self-focusing inside the material. Although Eq. (3.18) presents a rough estimation of the critical self-focusing length (cf. remark below), it predicts correctly that the peak irradiance at the entrance facet of a nonlinear material, and thus SPM, can be increased by strongly focusing the laser beam in order to apply a high (Gaussian) beam divergence. Fig. 3.18(a) shows that the b-integral (i.e. the area under a curve) of the initially divergent beam (blue line) is higher than that of a beam where $\theta=0$ (red line). Nevertheless, the area under the green dashed line $(1.2 \mu \mathrm{J}, 250 \mathrm{fs}$ pulses $)$ is the largest, and thus the strongest spectral broadening is anticipated in this case.

Ideally, a spectral broadening experiment induces as much SPM as possible while the nonlinear distortions of the beam, caused by self-focusing, are kept as low as possible. However, without additional beam shaping or guiding, the spatial and temporal nonlinearities cannot be decoupled ${ }^{208}$. A promising self-guiding mechanism relies on a sequence of thin plates which are arranged such that the laser beam alternatingly focuses and defocuses $^{330}$. Air has a critical power of $P_{c r} \approx 5 \mathrm{GW}$ at $1030 \mathrm{~nm},{ }^{49}$ and thus exhibits negligible self-focusing at the considered peak power levels. Multi-photon ionization is expected to become relevant, at intensities of about $10^{13} \mathrm{~W} / \mathrm{cm}^{249}$. The principle of alternating bulk and air sequences was recently exploited to avoid multiple filaments in white-light continuum (WLC) generation which led to a strong suppression of spatial losses in the spectral broadening experiment ${ }^{329}$. This multi-plate approach will be studied numerically in order to evaluate if it can be transferred to longer initial pulse durations than in the original publication and MW level peak powers which are common in the latest generation of high power $\mathrm{MHz}$ laser systems. The evolution of the peak irradiance is compared to the single plate approach in Fig. 3.18(b). The graph shows a sequence of ten $0.5 \mathrm{~mm}$ thick quartz plates. The incident pulse has a duration of $250 \mathrm{fs}$ and $10 \mu \mathrm{J}$ energy. The variations of the peak irradiance is much smaller than in the single plate examples. Moreover, the beam is always focused in between the bulk plates. The examples of Fig. 3.18 will be analyzed in detail in the simulation part of this section and shall give for now only basic insights into the beam behavior in the nonlinear sample.

\section{Remark: Shortcoming of Eq. (3.18)}

If Eq. (3.18) is evaluated for $P_{p} \gg P_{c r}$ and $\theta=0$, it is found that $P_{p} / d^{4}=$ $\pi^{2} P_{c r} /\left(z_{s f} \lambda\right)^{2}=$ const. for a fixed $z_{s f}$. Moreover, the peak irradiance $I_{p}$ is proportional to $P_{p} / d^{2}$, and thus $P_{p}^{2} / I_{p}=$ const., i.e. Eq. (3.18) would predict that $I_{p}$ at the entrance facet grows with $P_{p}^{1 / 2}$. By contrast, Fig. 3.18 shows that $I_{p}$ actually decreases with increasing peak power. The discrepancy arises from the derivation of Eq. (3.18) in ref. 269. It expands the beam area in terms of the peak irradiance and truncates the expansion already after the linear term. Self-focusing behaves, however, very nonlinear since the shrinking spot size increases the irradiance, and thus enhances the self-focusing effect. 
(a)

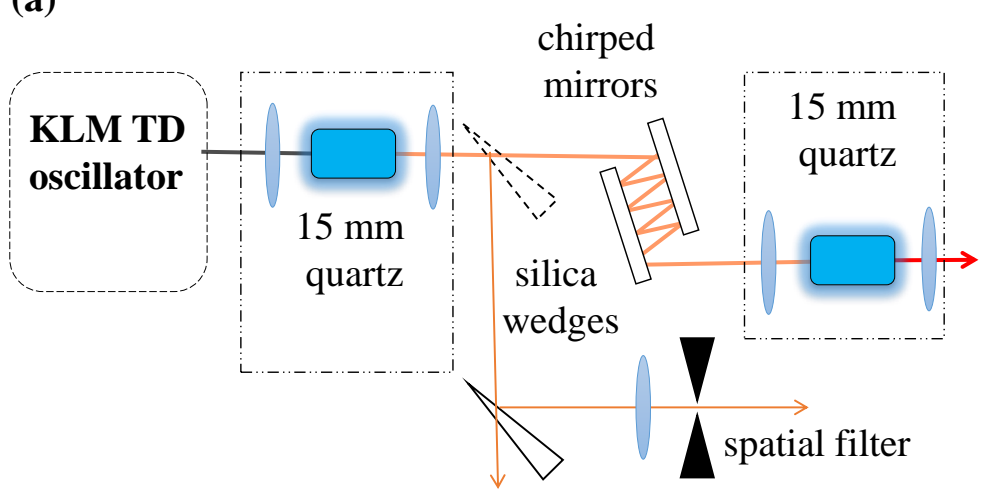

(b)

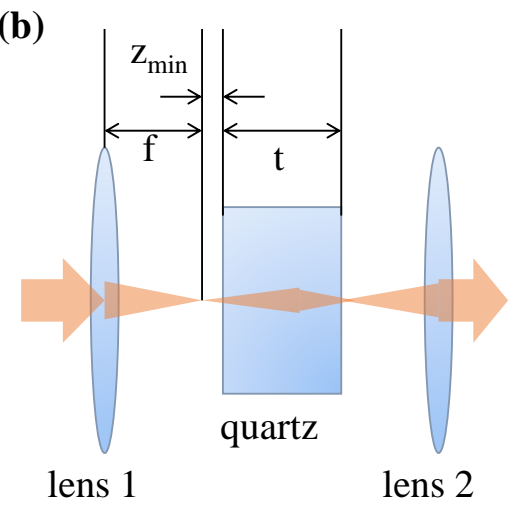

Fig. 3.19. Setup of the bulk broadening experiments. (a) Overview of all performed experiments. The wedge sketched by a dashed line was inserted for characterization purposes and was not present during the compression experiments. (b) Detailed sketch of the spectral broadening stages symbolized by the dotted-dashed lines in (a). The characteristic lengths $\mathrm{t}, \mathrm{f}$ and $z_{\min }$ were varied for optimization purposes.

\section{Spectral broadening under variation of focal length and material}

The experimental setup is shown in Fig. 3.19. Contrary to the experiments presented in section 2.2.2, the direct output of the TD oscillator was utilized, i.e. the $250 \mathrm{fs}$ pulses with about $1.3 \mu \mathrm{J}$ energy.

Several wide bandgap materials were tested. Crystalline quartz performed better than fused silica due to its higher thermal conductivity. Sapphire yielded broadening factors similar to quartz. A YAG crystal was damaged before significant broadening set in. This could be attributed to impurities in the material which were indicated by a violet glow not observable in the other materials. Additionally, several crystal lengths were tested. Best spectral broadening results could be achieved with quartz and sapphire crystals of at least $\mathrm{t}=6 \mathrm{~mm}$ thickness. Eventually, a $15 \mathrm{~mm}$ thick quartz crystal was chosen in the experiments.

To adjust the broadening factor, the sample was slowly moved towards the focus position of lens 1 while the spectrum was monitored after lens 2 with an OSA. The obtained spectra are shown in Fig. 3.20(a). The focal lengths $f$ were varied from $18 \mathrm{~mm}$ to $100 \mathrm{~mm}$. The maximal achievable broadening was not very sensitive to the variation. The location of damage inside the crystal shifted however if $z_{\min }$ was set too small. This is explained by the critical irradiance where ionization sets in. It is reached at the entrance facet if the beam is focused strongly while it is observed inside the crystal if focusing is loose and the self-focusing leads to a beam collapse.

Longer focal lengths also allowed white-light continuum generation ${ }^{49,207}$. The continuum is shown in Fig. 3.20(b). However, ionization, which is essential for WLC generation, was avoided in all other presented experiments. This was because firstly, the WLC was rather unstable and a noticeable heating of the crystal occurred. Therefore, average power scalability of the approach requires strong technical efforts. Secondly, the prominent blue shoulder is $25 \mathrm{~dB}$ below the fundamental, and thus the conversion into the continuum is fairly weak $(<20 \%)$. Thirdly, WLC generation depends on the input pulse duration and gets rather difficult for longer pulses ${ }^{331}$. No ionization losses were detected by means of 

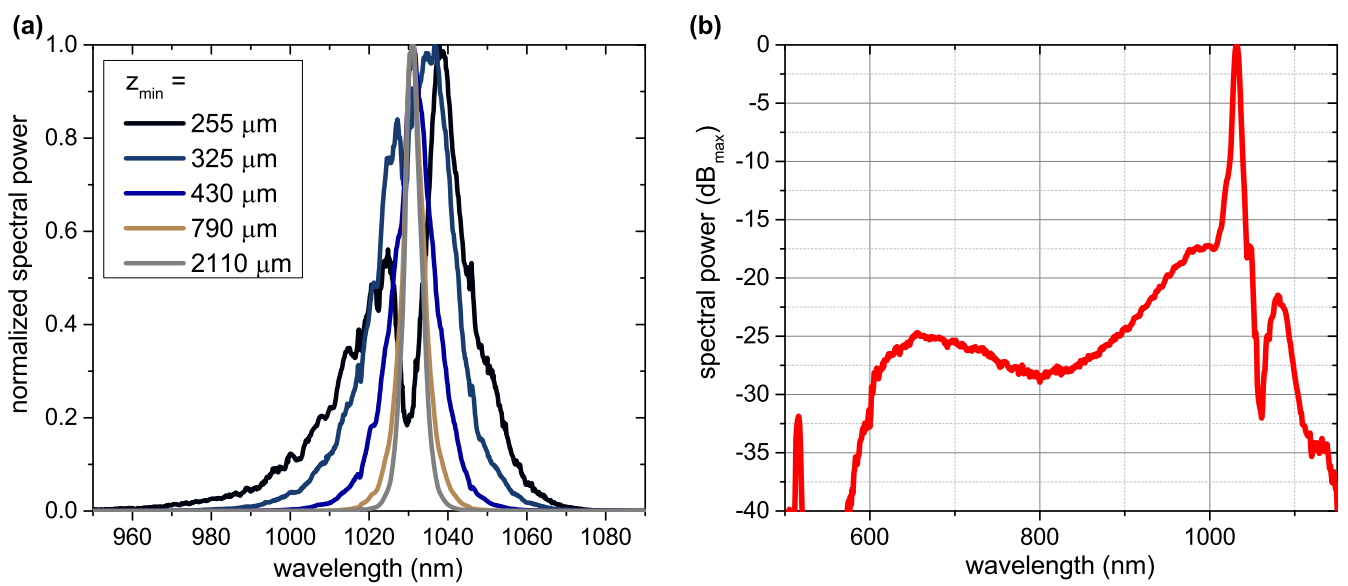

Fig. 3.20. (a) Spectra measured with an OSA in dependence on $z_{\min }$. The focal length was $f=25 \mathrm{~mm}$. The spectrum was filtered such that only the broadened parts were measured. (b) White-light continuum generated in a $15 \mathrm{~mm}$ quartz crystal. The spike at $515 \mathrm{~nm}$ is the second harmonic generated in the quartz crystal due to its $\chi^{(2)}$-nonlinearity.

power measurements for the spectra displayed in Fig. 3.20(a). The spectral broadening is caused solely by SPM.

In this case, the FourIER transform-limit of the pulses could be reduced from 250 fs to $38 \mathrm{fs}$ (black solid line Fig. 3.20(a)). This corresponds to a broadening factor of about 6 which is the largest in a single-stage bulk broadening experiment to the best of the author's knowledge.

\section{Temporal phase behavior under variation of broadening strength}

In order to compress the spectrally broadened pulses, the evolution of the spectral phase under variation of the distance from the focus to the crystal facet $\left(z_{\min }\right)$ was measured by means of SH-FROG (cf. appendix A.1). A $5 \mu \mathrm{m}$ BBO was used as a nonlinear crystal. Fig. 3.21(a) shows the calculated FTL as well as the peak power ratio between pulses with first order chirp compensation only and transform-limited pulses. The quantity is denoted by compression quality. Compensating higher order phase terms, for instance by a tailored chirped mirror design, would lead to shorter pulses and less power in the pedestals (cf. section 2.2.2).

The chirped mirror compressor was set-up such that shortest pulses could be achieved. A GDD of $-1700 \mathrm{fs}^{2}$ was predicted and realized by 4 bounces off mirrors with $-400 \mathrm{fs}^{2}$ GDD and no higher order terms. A pulse duration of $43 \mathrm{fs}$ was measured. It is in good agreement with the predicted pulse duration of $46 \mathrm{fs}$ for a slightly more negative GDD. The pulse in Fig. 3.21(b) shows a significant pedestal structure carrying about $35 \%$ total energy which agrees with the prediction in Fig. 3.21(a). Larger broadening factors come also with an increase of spatial losses due to the coupling of self-focusing and SPM. This has not been considered in this section but will be addressed in detail in the simulation part of this work. 

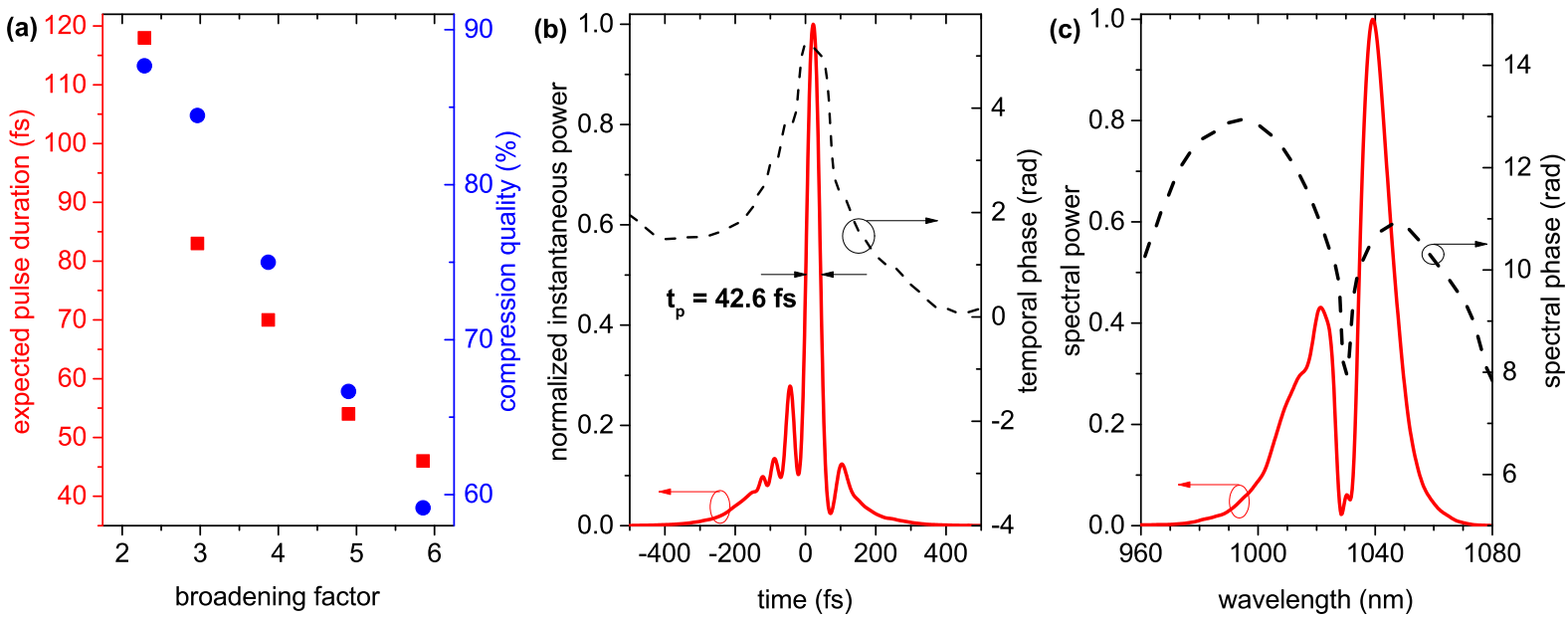

Fig. 3.21. (a) Expected pulse durations in dependence on the broadening factor for compensation of first order chirp only (red squares). The compression quality (blue circles) is the ratio between the peak power of the compressed and the FOURIER transform-limited pulses. (b) Retrieved pulse after compressor. The compression quality is $64 \%$ (c) retrieved spectrum and spectral phase. The spectrum is in good agreement with the black line in Fig. 3.20(a).

\section{Cascading bulk broadening with intermediate compression}

In the previous experiments on high-power bulk broadening, $17 \mathrm{fs}$ input pulses were broadened and compressed down to 7.7 fs (cf. section 2.2.2). To bridge the gap from the experiments reported here to the sub-20 fs regime, a second bulk broadening stage was set-up. After a first bulk stage, the pulses were compressed to $53 \mathrm{fs}$ duration with a total GDD of $-2400 \mathrm{fs}^{2}$ and afterwards focused with an anti-reflection coated lens ( $\mathrm{f}=35 \mathrm{~mm}$ ) into another $15 \mathrm{~mm}$ quartz crystal (Fig. 3.19(a)). After optimizing $z_{\min }$, the FTL could be reduced to 15 fs (Fig. 3.22). The modulations of the spectral power arise from the pedestal structure of the pulse after the first compression stage. The increase in bandwidth of the second stage is clearly larger than in the first stage. This is due to the steeper temporal gradient of the pulse. The broadening factor is about 3.5. The decrease in comparison to the first stage can be attributed to the increasing impact of dispersion. It should be pointed out that the achieved FOURIER transform-limit is comparable to the one which

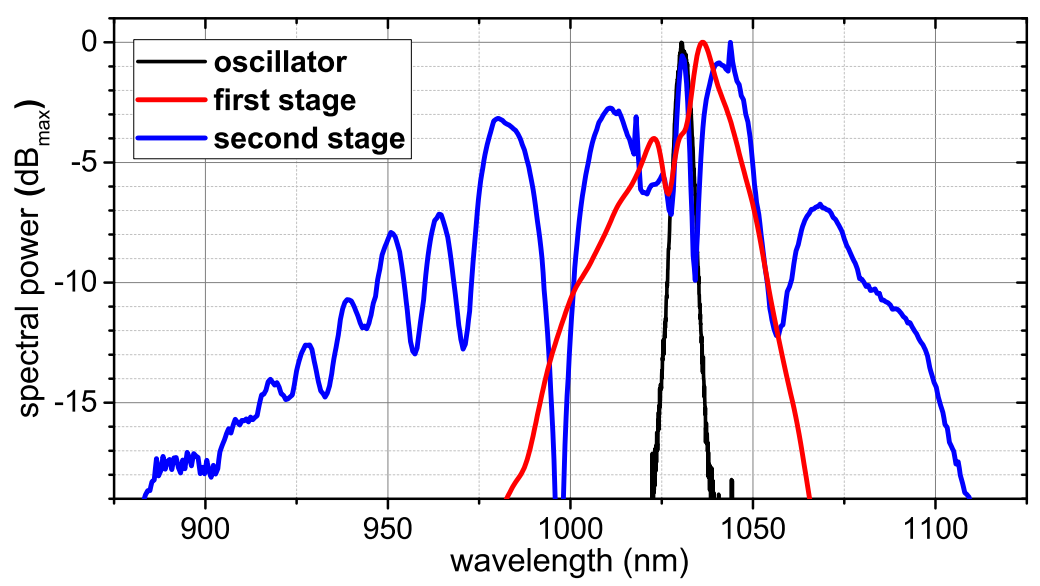

Fig. 3.22. Double stage spectral broadening. The FTL is reduced from $250 \mathrm{fs}$ to $15 \mathrm{fs}$ after the second stage and an intermediate compression by means of chirped mirrors. 
(a)

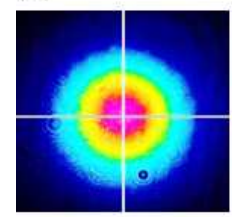

$1010 \mathrm{~nm}$

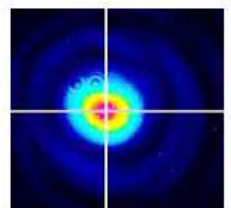

$1030 \mathrm{~nm}$

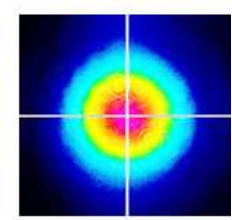

$1050 \mathrm{~nm}$

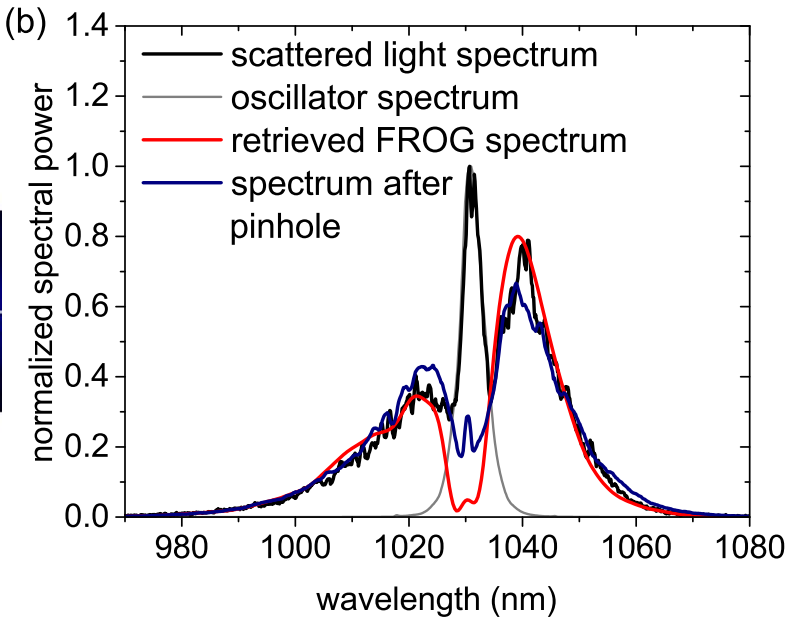

Fig. 3.23. Spatiotemporal effects of bulk broadening. (a) Beam profiles measured behind optical bandpass filters of $10 \mathrm{~nm}$ spectral width. The profiles behind the filters centered at $1010 \mathrm{~nm}$ (left) and $1050 \mathrm{~nm}$ (right) are Gaussian while the profile after the $1030 \mathrm{~nm}$ filter (center) exhibits a ring structure. The profiles were measured about $20 \mathrm{~cm}$ behind the collimation lens. (b) Comparison of the scattered light spectrum (i.e. spatially integrated spectrum, black line), the retrieved FROG spectrum (red line) and the initial oscillator spectrum (gray line). The spatially averaged spectrum presents a superposition of FROG and oscillator spectrum. The blue solid line shows the spectrum measured after spatial filtering which is close to the spectrum retrieved by FROG.

was obtained by fiber compression in section 2.2.1, and hence there is strong evidence that all-bulk broadening enables few-cycle pulse generation at peak power levels way beyond the material's critical power.

\section{Spatial characterization}

Owing to the free beam propagation within the bulk material, temporal and spatial nonlinear effects are coupled and have to be taken into account when the efficiency of the compression stage is calculated. While strong SPM will lead to broad spectra in the beam center, i.e. in the region of high irradiance, the FTL of the beam wings will hardly be changed since the irradiance is too low. The spectrally filtered beam profiles shown in Fig. 3.23(a) have clearly different shapes. The broadened spectral parts (around $1010 \mathrm{~nm}$ and $1050 \mathrm{~nm}$ ) exhibit more desirable Gaussian shapes in contrast to the unbroadened spectral part around $1030 \mathrm{~nm}$ where a ring structure is visible. Therefore, measuring the spectrum emerging from the nonlinear crystal became position sensitive.

In order to spectrally homogenize the beam and to estimate the losses due to spatial nonlinearities, a spatial filtering experiment was set-up. It was performed with about $100 \mathrm{~mW}$ average power, reflected from two wedges (Fig. 3.19(a)). A $10 \mu \mathrm{m}$ pinhole was used and the best focal lengths of the lenses in front of the pinhole $\left(f_{p}\right)$ were calculated by $^{332}$ :

$$
f_{p}=\frac{w_{0} d_{p}}{\lambda}
$$

where $w_{0}$ is the waist of the collimated beam and $d_{p}$ is the pinhole diameter.

First, the unbroadened, collimated oscillator output $\left(M^{2}=1.05\right)$ was sent through the pinhole to verify the spatial filter performance, yielding $87 \%$ transmission of the incident 


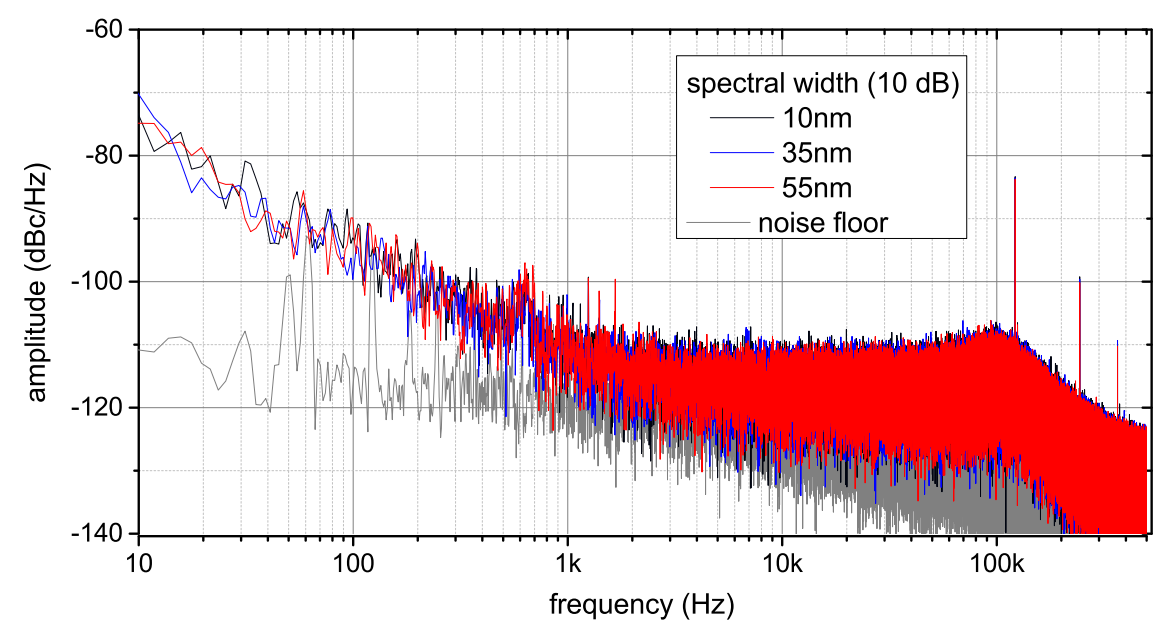

Fig. 3.24. Intensity noise measurements after the first bulk broadening stage for different broadening factors. The $55 \mathrm{~nm}$ spectral full width at $-10 \mathrm{~dB}$ of the maximum corresponds to the black line in Fig. 3.20(a). The excellent noise properties of the oscillator $(0.1 \%$ RMS relative intensity noise in the bandwidth from $10 \mathrm{~Hz}$ to $500 \mathrm{kHz}$ ) are maintained independent of the broadening factor.

power. Afterwards, the $1010 \mathrm{~nm}$ bandpass was inserted into the broadened beam and the lens 2 was adjusted to collimate the wings of the spectrum. After removing the bandpass, the spatial filtering experiment was repeated. A $53 \%$ transmission of the total power was measured which is $40 \%$ less than in the unbroadened case. This is considered as the power loss due to the spatial nonlinear effects. The spectrum behind the pinhole had a FTL of $40 \mathrm{fs}$. The homogeneity was proven by scanning the expanding beam after the pinhole with a multimode fiber coupled to the OSA. Moreover, an $M^{2}$ measurement of the spectrally broadened beam was performed according to ISO Standard 11146. It yielded $M^{2}<1.1$ along both space axes.

The spectrum after the pinhole is similar to the FROG spectrum. Due to the chromatic longitudinal shift of the focus position, the FROG acts like a spatial filter as well. Within the $5 \mu \mathrm{m}$ thin BBO crystal only the spectrally broadened beam is in focus, and thus generates a second harmonic signal. The retrieved FROG spectrum (Fig. 3.21(d)) shows a strong suppression of the $1030 \mathrm{~nm}$ component. The scattering light spectrum (Fig. 3.23(b)) can be decomposed into the part retrieved from the FROG measurement and the initial oscillator output, i.e. the spectral content of the rings at $1030 \mathrm{~nm}$.

\section{Noise properties}

Despite the fact that utilizing kagomé-type HC-PCFs for the compression of the oscillator pulses yielded sub-10 fs pulses in a double-stage setup (section 3.1.2), the applicability of the technique appeared to be restricted owing to the intensity noise accumulation in the broadening stages. Similar power fluctuation measurements were performed after the first bulk broadening stage, i.e. a small fraction of the laser beam was sent onto a fast photodiode to measure the power fluctuations of the pulse train. Fig. 3.24 shows that the accumulated intensity noise is independent of the position $z_{\min }$, and thus also independent of the broadening factor. The noise measurements were performed with an RF spectrum analyzer, and hence the noise floor is clearly lower than in Fig. 3.16, i.e. the oscillator 

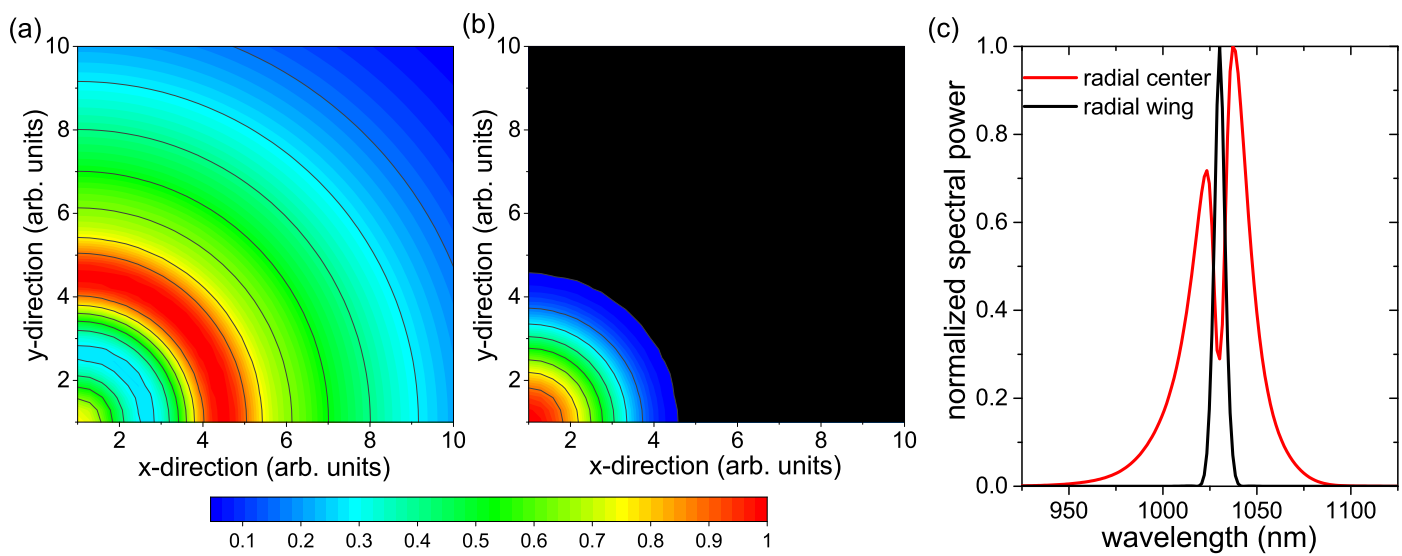

Fig. 3.25. Simulation of the spectral broadening in a $15 \mathrm{~mm}$ crystalline quartz crystal. (a) Near-field pattern of the $1030 \mathrm{~nm}$ filtered beam profile. (b) Near-field pattern of the $1040 \mathrm{~nm}$ filtered beam profile. (c) Radially dependent spectra, the black line shows the spectrum of the unbroadened part located in the wing of the near field-profile, the red line shows the broadened spectrum located in the center of the near-field profile.

noise in the full measurement bandwidth can be discriminated against the intrinsic noise of the measurement device. On the other hand, the slow fluctuations below $10 \mathrm{~Hz}$ are not detected.

\section{Simulations}

Full three-dimensional simulations of nonlinear pulse propagation ${ }^{266,333}$ have been performed to address the question of power-scalability of the bulk broadening approach. The spatial grid is set to $513 \times 513$ points in a quarter of the $\mathrm{x}$-y-plane which is justified by the fairly weak birefringence of quartz and the circular symmetry of the beams. The spacing is set to 10 points within the minimal $1 / \mathrm{e}^{2}$-radius. The temporal grid consists of 256 points with a $10 \mathrm{fs}$ spacing. Simulations were done with the material dispersion derived from the crystalline quartz Sellmeier equation ${ }^{334}$ and a nonlinear refractive index of $2.8 \times 10^{-16} \mathrm{~cm}^{2} / \mathrm{W}$, taken from fused quartz ${ }^{240}$. The beam was treated as collapsed when a critical value of $2 \mathrm{TW} / \mathrm{cm}^{2}$ was reached inside the nonlinear crystal. In this case, the simulation was stopped and rerun with a larger input beam diameter or divergence. A first routine (i) set the front facet of the crystal into the focus and increased the spot size until no collapse occurred (solid red and dashed dark green lines Fig. 3.18(a)). A second routine (ii) set the peak irradiance on the front facet slightly below the damage threshold and increased divergence until the beam collapse was avoided (blue line Fig. 3.18(a)). The waist size, which was the optimization parameter in both routines, was varied in steps of $0.1 \mu \mathrm{m}$.

To benchmark the utilized code, the experimental parameters were used as the simulation input, i.e. a $250 \mathrm{fs}, 1.2 \mu \mathrm{J}$ pulse was focused into a $15 \mathrm{~mm}$ sample. Fig. 3.25 shows simulated spectra and profiles. Near-field profiles were extracted from the simulations while the experimental profiles (Fig. 3.23) were taken after tens of $\mathrm{cm}$ propagation. Qualitatively, the agreement of experiment and simulation is very good. The spectra show the asymmetry with a broader but less powerful blue shoulder. The spectra can be also decomposed into an unbroadened and a broadened part. The $1030 \mathrm{~nm}$ profile shows the ring 

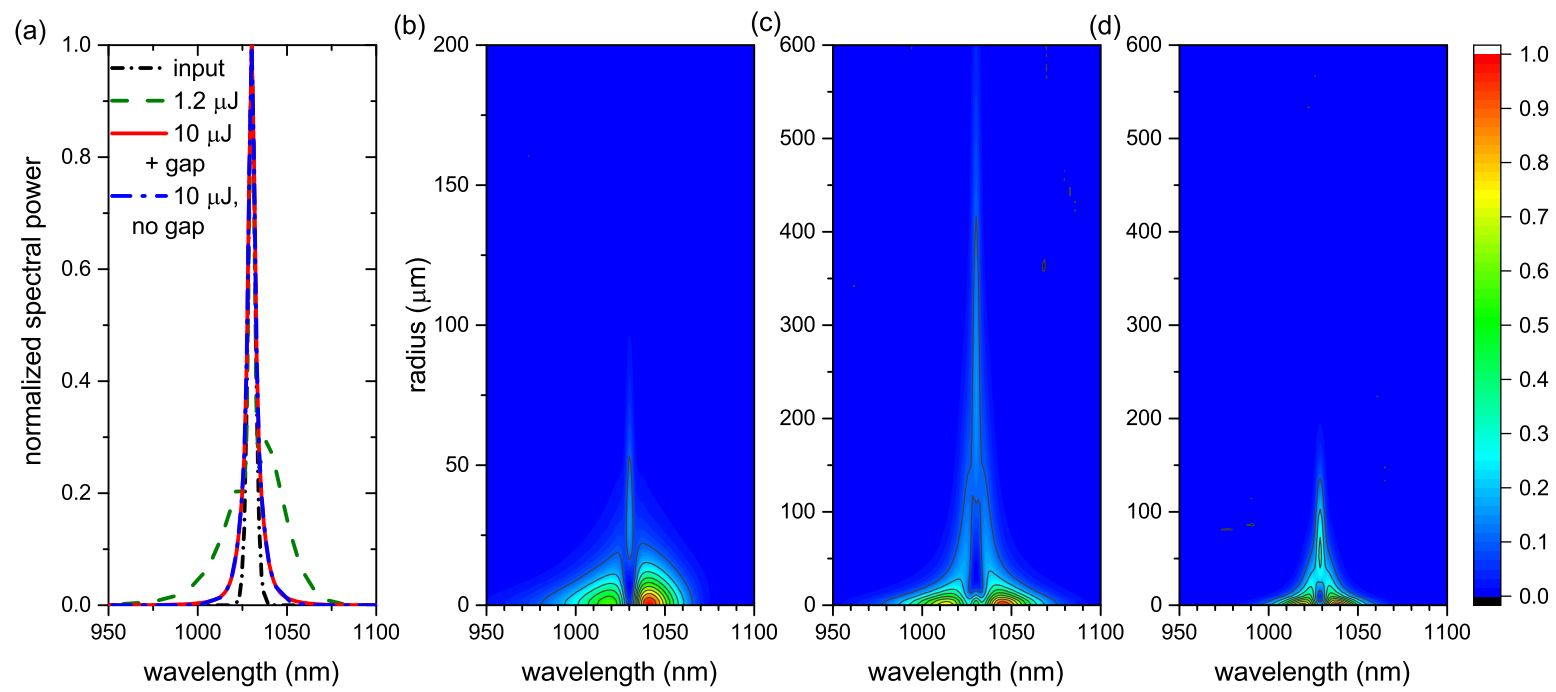

Fig. 3.26. Spatially integrated and radially resolved spectra after propagation through a $15 \mathrm{~mm}$ quartz plate. (a) Normalized spectra for (b) - (d) and the oscillator spectrum. The spectral power is integrated over the whole beam area. (b) $1.2 \mu \mathrm{J}, 250 \mathrm{fs}$ input pulses. A significant part of the input beam is broadened like it was observed in the experiment. (c) $10 \mu \mathrm{J}, 250 \mathrm{fs}$ input pulses with balanced divergence and self-focusing. The broadening in the beam center is comparable to (b) but only a small fraction $(\approx 4 \%$ ) of the input power is broadened. (d) $10 \mu \mathrm{J}, 250 \mathrm{fs}$ input pulses without divergence. The spectral broadening is weaker than in (b) and (c). About $16 \%$ of the total power are contained in the broadened part. All color plots are scaled linearly and are normalized. The units of the radially resolved spectra are $\mathrm{J} / \mathrm{Hz} / \mu \mathrm{m}^{2}$, i.e. the pulse energies $E$ are predicted by $E=2 \pi \Delta \nu \Delta r \sum_{i, j} r_{i} u\left(r_{i}, \nu_{j}\right)$, where $\Delta \nu$ and $\Delta r$ are the simulation grid spacing in frequency and space, $r$ is the radius and $u$ is the plotted energy density. Is is summed over all spatial grid points $r_{i}$ along one axis and all spectral grid points $\nu_{j}$.

structure while the $1040 \mathrm{~nm}$ profile looks Gaussian. The lowest achievable FTL is $31 \mathrm{fs}$. The broadening hardly depends on the focus spot size (i.e. focal length in the experiment) because the pulse peak power is close to the critical power of the material, and thus the nonlinear lens is relatively weak. This is also in good agreement with the experimental observations. Therefore, it is inferred that the utilized code is suited to investigate the power-scalability of the bulk broadening approach.

Subsequently, the simulation was repeated with a $10 \mu \mathrm{J}$ pulse, leaving all other input parameters unchanged. Hence, the critical power is exceeded by about an order of magnitude. Fig. 3.18(a) shows the evolution of the peak irradiance over the propagation distance derived from the routines (i) and (ii). Moreover, the output spectra have been depicted in Fig. 3.26(a). The broadening seems much weaker than in the case of the $1.2 \mu \mathrm{J}$ input. This agrees well with experimental observations ${ }^{335}$. Nevertheless, the computed FTLs are 73 fs for routine (i) and 51 fs for routine (ii). The spatially resolved spectra (Fig. 3.26(b)(d)) reveal that the central parts of the beam are still significantly broadened but they only contain a small amount of the pulse energy in comparison to the $1.2 \mu \mathrm{J}$ case. This shows that balancing divergence and self-focusing increases the broadening factor but also leads to a strong spectral inhomogeneity of the beam. Routine (ii) with $10 \mu \mathrm{J}$ pulses was repeated for $10 \mathrm{~mm}, 5 \mathrm{~mm}$ as well as $3 \mathrm{~mm}$ plates. The FTLs are $62 \mathrm{fs}, 77 \mathrm{fs}$ and $87 \mathrm{fs}$, resp. while the spectrally broadened parts amount $8 \%, 15 \%$ and $18 \%$ of the total input power. 

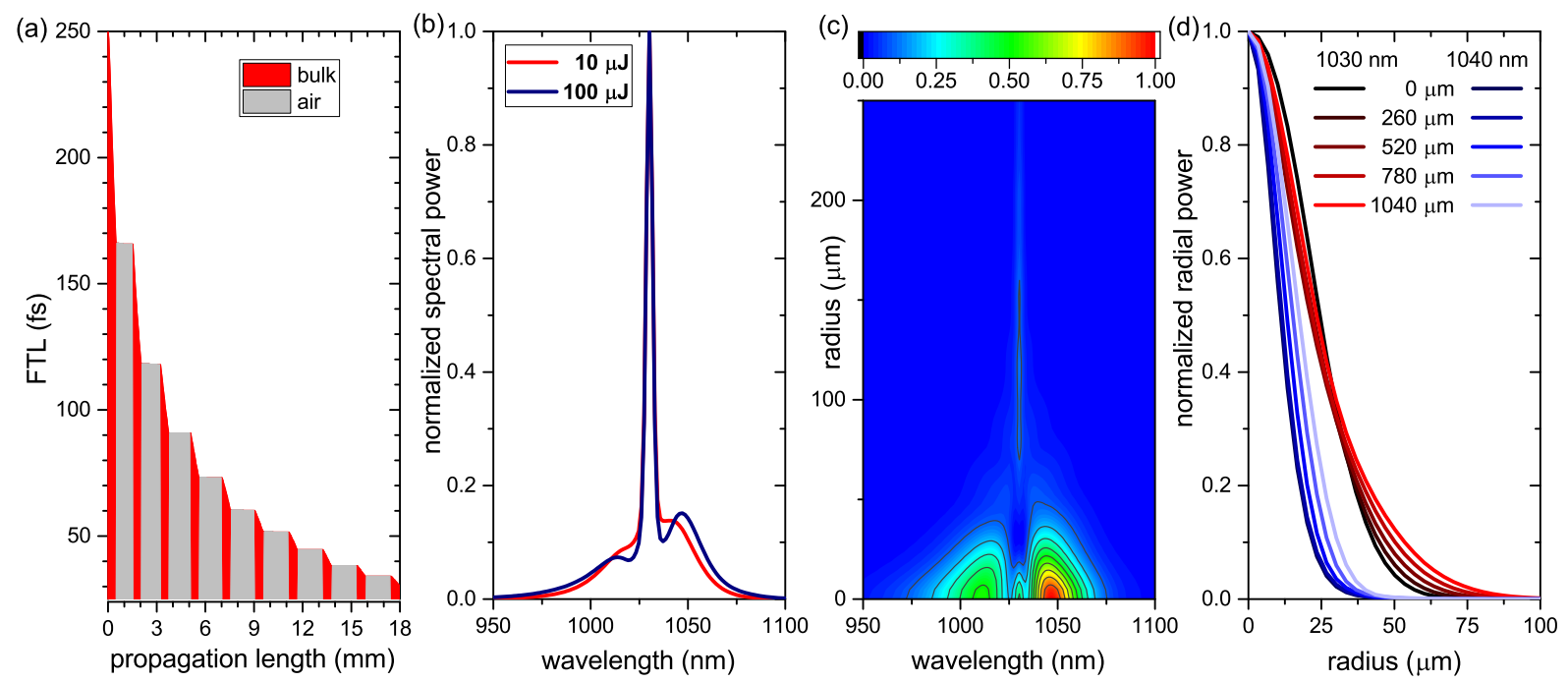

Fig. 3.27. Simulation of propagation of a $10 \mu \mathrm{J}$ pulse through ten $0.5 \mathrm{~mm}$ plates. No Fresnel losses are considered. (a) The FTL is inversely proportional to the plate number. The FTL after 10 plates is $31 \mathrm{fs}$. (b) The spatially integrated output spectrum after ten plates (red solid line). For comparison, the output spectrum of propagating a $100 \mu \mathrm{J}, 250 \mathrm{fs}$ pulse through ten plates (solid blue line) (c) The spatially resolved output spectrum after 10 plates. (d) Spectrally resolved beam profiles for $1030 \mathrm{~nm}$ and $1040 \mathrm{~nm}$ during propagation in the first air gap $(\mathrm{FTL}=165 \mathrm{fs})$. The broadened part emerging from the beam center (bluish lines) diverges quickly while the unbroadened part (reddish lines) is focused first and diverges slowly afterwards. The propagation length inside the gap is stated in the plot legend.

These observations suggest to apply the multiple-plate method which was demonstrated in ref. 329 for spectral broadening of 25 fs pulses with hundreds of MW peak power. A similar method has been recently presented in refs. 62, 336-339 for several hundred Watts of average power. Instead of using multiple plates, the focus behind a single plate (cf. Fig. 3.18(b)) can be self-imaged such that a virtual multiple-plate pattern is created. It is in any case remarkable that in all references fairly small spatial losses are reported although the peak powers lie well above the critical powers of fused silica.

To explain this, another simulation algorithm was written. The simulation of the first plate was done with optimization routine (ii). The simulation of propagation through an air gap follows. The next plate is then set behind the focus in the air gap where the peak irradiance is below the critical value. If the beam collapses in the plate, the length of the air gap is extended. A sequence of ten $0.5 \mathrm{~mm}$ thick plates was simulated for a $10 \mu \mathrm{J}$, $250 \mathrm{fs}$ input pulse. The evolution of the peak irradiance along the plate assembly is shown in Fig. 3.18(b).

The simulation reveals a linear increase of bandwidth per plate. The FTL is inversely proportional to it which is shown in Fig. 3.27(a). Intermediate compression after a few plates would increase the slope $\mathrm{dI}(\mathrm{t}) / \mathrm{dt}$ and would thus lead to a stronger increase in bandwidth per plate. Experimentally, this behavior is indicated in Fig. 3.22. The most remarkable effect of the multiple-plate propagation is that the integrated spectrum resembles the one which was achieved with the $1.2 \mu \mathrm{J}$ pulses. Hence, a significant reduction of the spatial losses in comparison to the single plate approach could be achieved. This is demonstrated in Fig. 3.27(b) and (c). While the broadened parts of the single plate example (Fig. 3.26(c)) amount only $4 \%$ of the total power, about $40 \%$ are broadened in the multi-plate example. This can be attributed to the homogenizing effect of the air 
Table 3.1: Summary of FTLs and Spatial Losses ${ }^{a}$

\begin{tabular}{|c|c|c|c|c|c|}
\hline pulse energy $(\mu \mathrm{J})$ & 1.2 & 1.2 & 10 & 10 & 10 \\
\hline routine & experiment & (i) & (i) & (ii) & (iii) \\
\hline number of plates & 1 & 1 & 1 & 1 & 10 \\
\hline plate thickness (mm) & 15 & 15 & 15 & 15 & 0.5 \\
\hline FTL $\left(f_{s}\right)$ & 38 & 31 & 73 & 51 & 31 \\
\hline estimated spatial losses (\%) & 40 & 60 & 84 & 96 & 60 \\
\hline
\end{tabular}

gaps which is visible in Fig. 3.27(d). The central, broadened parts diverge faster than the unbroadened parts in the wings of the beam. Hence, the unbroadened parts move to the most intense region of the beam and get broadened as well. The multi-plate routine was also executed with $100 \mu \mathrm{J}$ pulses (no other input parameters were changed). Fig. 3.27(b) shows an integrated spectrum similar to one with $10 \mu \mathrm{J}$ input pulses, i.e. the losses do not significantly differ. Furthermore, the $10 \mu \mathrm{J}$ pulse energy simulations were repeated with $0.2 \mathrm{~mm}$ thick plates. After ten plates, the FTL was $68 \mathrm{fs}$, the efficiency about $51 \%$. After 20 plates, the FTL was 35 fs and the efficiency about $44 \%$. Consequently, thinner plates do not necessarily yield better broadening performance. Determining the ideal plate thicknesses has not been subject of this work which mainly highlights the power scalability of the bulk broadening concept to peak power levels far beyond the critical power. The results for $1.2 \mu \mathrm{J}$ and $10 \mu \mathrm{J}$ pulses are summarized again in Table 3.1.

Eventually, the broadening in a single $0.5 \mathrm{~mm}$ plate was investigated. Routine (ii) was repeated with a constant peak power of $40 \mathrm{MW}$ and with input pulse durations of $1 \mathrm{ps,}$ $500 \mathrm{fs}, 250 \mathrm{fs}, 125 \mathrm{fs}, 68 \mathrm{fs}, 34 \mathrm{fs}, 19 \mathrm{fs}$, and 9 fs. The temporal grid spacing was adapted correspondingly. All simulations yielded a broadening factor of about 1.5. Next, simulations with $250 \mathrm{fs}$ input pulse duration and pulse energies of $1 \mu \mathrm{J}, 3 \mu \mathrm{J}, 5 \mu \mathrm{J}, 10 \mu \mathrm{J}, 30 \mu \mathrm{J}$, $50 \mu \mathrm{J}, 100 \mu \mathrm{J}, 300 \mu \mathrm{J}, 500 \mu \mathrm{J}$ and $1 \mathrm{~mJ}$ were executed. Also in this study a broadening factor of about 1.5 was always found. It is inferred that purely SPM based bulk broadening is applicable to a wide range of input pulse durations and energies if the focus sizes and the plate positions are chosen appropriately.

\section{Discussion}

The single stage broadening led to an unprecedented high broadening and compression factor (about 6) and exhibited a much higher efficiency (about 60\%) than the initial experiments of Rolland and CORKUM (compression factor about 5, efficiency about $4 \%)^{325}$. It was shown that a single plate experiment, where the peak power exceeds the critical power, presents a trade-off between efficiency and broadening factor. This agrees with the results of the coupled-mode theory ${ }^{54,208}$. Additionally, it was found that higher broadening factors come at the expense of compression quality if no tailored chirped mirrors are available. In the presented experiments, the peak power of the pulses was increased from 4.2 MW to $9.5 \mathrm{MW}$. With tailored mirrors a peak power of $15 \mathrm{MW}$ would be possible. Similar peak power enhancements of about a factor three could be reached with the simulated multi-plate compression scheme for $10 \mu \mathrm{J}$ pulses. 
The single plate experiment is particularly attractive if only a small broadening factor $(\approx 2)$ is targeted, for instance to reach the few-cycle regime (cf. section 2.2.2). Moreover, transferring the approach to other wavelengths is highly interesting. KREBS et al. performed for example experiments in the $\mathrm{UV}$ at $\mathrm{kHz}$ repetition rates ${ }^{340}$. Transferring the experiment to $\mathrm{MHz}$ rates would require peak irradiances lower than $2 \mathrm{TW} / \mathrm{cm}^{2}$ to avoid ionization but is expected to be also possible due to the increased $n_{2}$ at shorter wavelengths. The bandgap of $\mathrm{CaF}_{2}$ is about $12 \mathrm{eV}$, corresponding to a two-photon absorption edge of about $200 \mathrm{~nm}$. This is expected to be the shortest wavelength where bulk broadening is possible. Similarly, the technique can be employed in the mid-IR range where silica fibers are not applicable at all due to the limited transparency range of quartz and soft glass fibers are limited to $\mathrm{mW}$-level average power handling ${ }^{341}$. Huge broadening factors have been already achieved by means of filamentation in a YAG crystal at about $1 \mathrm{~W}$ average power ${ }^{342}$.

The possibility of WLC generation was shown. However, several drawbacks like the dependence on the input pulse duration, the observed instabilities and the need for cooling, resp., limit the applicability of filamentation for high power sources. Simulations with a $0.5 \mathrm{~mm}$ plate show that purely SPM based broadening is applicable to any MW peak power level and any pulse duration up to the ps order. Moreover, crystals that serve as KERR media in TD oscillators withstand average powers of more than $1 \mathrm{~kW}^{59,335}$ and hence the approach is also average power scalable. Since multiple stage broadening allows to access the few-cycle pulse regime, the method is expected to be employed in seed generation of the third generation of femtosecond technology ${ }^{11}$.

The decoupling of spatial and temporal nonlinear effects by means of introducing a guiding structure was revealed to be essential for peak power scaling of the approach. While first ideas of realizing this were rather complex ${ }^{343}$, the method proposed in ref. 330 is simple and was already successfully applied for GW level pulses ${ }^{329}$. The simulations show that it can be transferred to SPM based spectral broadening and lower peak power levels which match the current generation of fiber and innoslab amplifiers as well as TD oscillators $^{27,32,58-61,335,344}$. The efficiency in the case of $10 \mu \mathrm{J}, 250 \mathrm{fs}$ pulses was increased from $4 \%$ in the single plate geometry to $40 \%$ in an assembly of ten $0.5 \mathrm{~mm}$ thick plates. Further improvements are expected by optimizing peak irradiances, plate thicknesses and distances to maximize the homogenization effect in the gaps. The $40 \%$ efficiency is about a factor of two below the efficiencies of fiber technologies. In the light of the rapidly increasing peak powers of disk, fiber and slab sources, this factor appears compensable. The FTL is inversely proportional to the number of plates if dispersion is negligible. Simulations show that the broadening factor in a single thin plate is constant for a fixed peak power, crystal length and damage threshold, i.e. independent of the input pulse duration. This makes intermediate compression after a few plates attractive for achieving very high compression factors.

In conclusion, spectral broadening in bulk material with average powers of $50 \mathrm{~W}$ was demonstrated. Broadening and compression factors of about 6 with a single plate were reached with a $60 \%$ efficiency. When the results were presented for the first time, this marked a unique combination of unprecedented high compression factors and efficiencies together with an average power which is way beyond those of previously described bulk broadening experiments ${ }^{325,340,343}$. During the past two years, the interest in bulk spectral broadening has been rapidly growing and excellent efficiencies as well as broadening factors have been achieved at $100 \mathrm{~W}$-levels of average power ${ }^{62,336-339}$ 
The presented study has captured the evolution of temporal phase with respect to the broadening factor was analyzed by means of FROG measurements and the coupling between spatial losses and broadening was investigated with respect to peak power by means of full 3D simulations which reproduce the experimental results well. The trade-off between broadening factor and efficiency was pointed out as well as the limited peak power scalability of the single plate approach. Moreover, the experiments showed that multiple bulk broadening stages with intermediate compression allow to go from pulse durations of about 250 fs to the few-cycle regime. Simulations predict that the SPM based approach is also applicable for longer pulse durations which is important for Yb:YAG based amplifiers and SESAM mode-locked TD oscillators.

The multiple thin plate approach is also applicable to pure SPM based broadening of pulses with peak power levels beyond the power for critical self-focusing. It has been demonstrated that the quasi guiding structure, introduced by air gaps between the plates, leads to homogenizing of the spectrum over the beam area, and thus to significantly reduced losses. Moreover, contrary to the single plate approach, simulations with the multiple plate geometry show that the losses do not scale with peak power anymore.

Consequently, ionization-free bulk broadening has proved to be scalable not only in average power but also in peak power. Eventually, the noise properties of the approach have been investigated. The intensity noise remained on the low level of the TD oscillator and was independent of the broadening factor. This is in particular highly favorable in comparison to measurements performed after spectral broadening in kagomé-type HC-PCFs (section 3.1.2). These findings combined with low costs, high robustness and alignment insensitivity, make the approach extremely attractive for pulse compression of high peak and average power light sources.

\subsubsection{Efficient Pulse Compression in Self-Defocusing Bulk Media}

\section{Parts of this section have been published in:}

M. Seidel, J. Brons, G. Arisholm, K. Fritsch, V. Pervak, and O. Pronin, "Efficient HighPower Ultrashort Pulse Compression in Self-Defocusing Bulk Media," Sci. Rep. 7, 1410 (2017).

\section{Why going to self-defocusing nonlinearities?}

In the previous section, it has been demonstrated that high losses are inherent to the propagation of an intense Gaussian beam in a long KERR medium if the peak power of the ultrashort pulses clearly exceeds the critical power of the material. It has been explained that quasi-guided waves overcome this issue and that multi-pass or multi-plate approaches can extend bulk broadening to a huge peak power range with efficiencies of at least $40 \%$ (cf. refs. 329, 330, 336). This section demonstrates a different approach to efficient ultrashort pulse compression in bulk material. To motivate it, Figs. 3.18 and 3.26 shall be reconsidered. On the one hand, through the combination of self-focusing and beam divergence higher b-integrals, and thus higher phase shifts in the beam center could be reached than without applying any divergence to the beam. But on the other hand, this approach also induced increased spectral inhomogeneity since the beam wings were quickly diffracted out of the intense region where SPM happened. This behavior can 
be illustrated by a qualitative ray optics description which is based on a simple model proposed by R.Y. CHIAO et al. ${ }^{160,345}$. The initial model was used to find a threshold for self-trapping of optical beams. It treated the laser beam as a step-index fibre-like waveguide with a cladding of refractive index $n_{0}$ and a core of refractive index $n_{0}+n_{2} I$ where $I$ is the laser irradiance. This led to a good approximation of a medium's critical power. Here, the laser beam is treated as a graded-index waveguide with the refractive index profile

$$
n(r)=n_{0}+n_{2} I_{p} \exp \left\{-2 r^{2} / w^{2}\right\},
$$

where $I_{p}$ is the peak irradiance, $r$ the distance to the beam center and $w$ the beam radius at the front facet of the nonlinear crystal. Typically, ray tracing in graded index optical elements is performed by applying FERMAT's principle. For cylindrical symmetry, this leads to the equation ${ }^{346}$

$$
\frac{d^{2} r}{d z^{2}}=\frac{1}{n(r)} \frac{d n(r)}{d r} \approx \frac{1}{n_{0}} \frac{d n(r)}{d r}=-\frac{4 n_{2} I_{p}}{w^{2} n_{0}} r \exp \left\{-2 r^{2} / w^{2}\right\}
$$

where $z$ is the propagation length inside the crystal. The differential equation was solved numerically with a RUNGE-KUTTA method. It is to note that no $z$-dependence was assigned to $I_{p}$ and $w$ which is a strong approximation since the beam size is changing inside the crystal. Appendix A.3 shows how to transform Eq. (3.21) from a differential to an integral equation where $I_{p}$ and $w$ could be readily recalculated after each propagation step. For the qualitative discussion at this point, the approximation of constant $I_{p}$ and $w$ is, however, sufficient.

Ray packet equivalents (consisting of 100 rays each) of three Gaussian beams ${ }^{161}$ were launched. Their propagation through a $10 \mathrm{~mm}$ thick nonlinear crystal was modeled by means of Eq. (3.21). The rays are plotted in Fig. 3.28. Fig. 3.28(a) presents the modeling of the self-focusing example similar to the one presented in Fig. 3.26(c). The laser beam
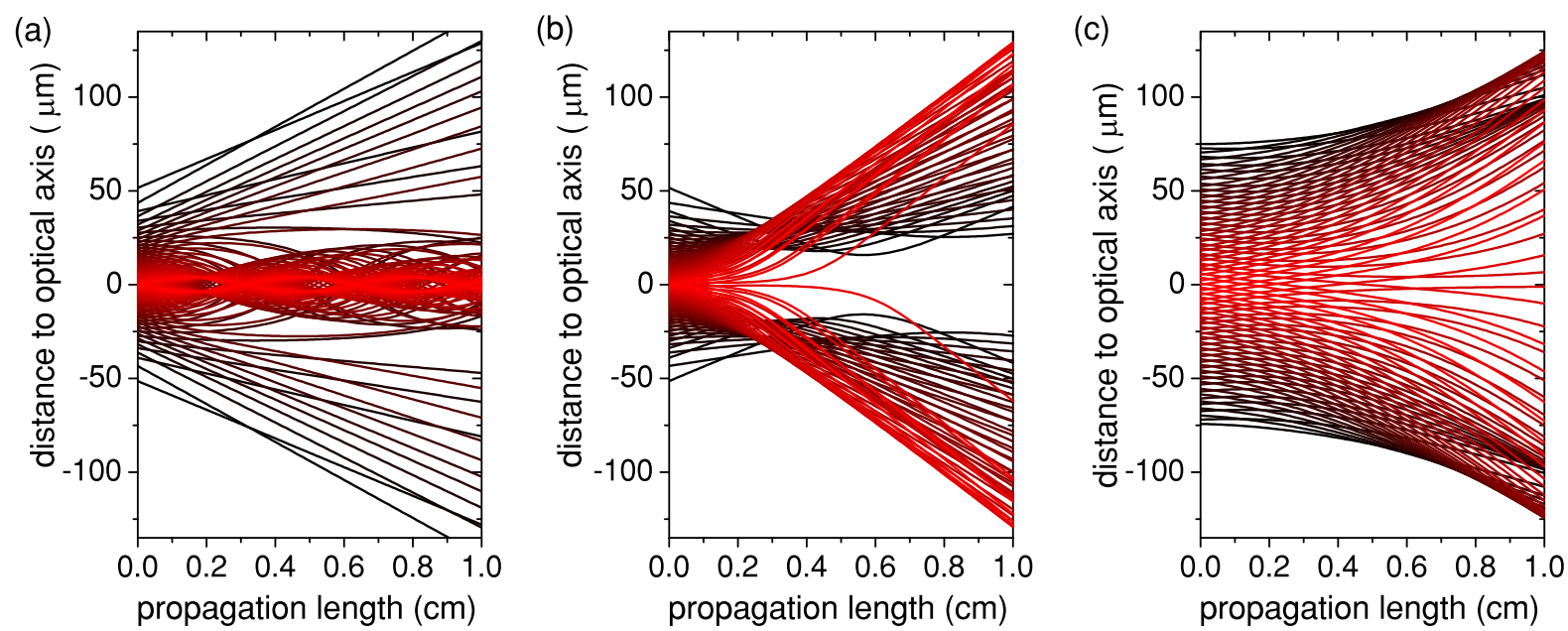

Fig. 3.28. Model for 100 rays describing a Gaussian beam. The darker the rays the larger the initial distance to the optical axis. (a) Self-focusing nonlinearity and beam divergence: The central rays stay close to the optical axis while the outer rays diverge strongly. (b) Self-defocusing nonlinearity and beam convergence: The inner rays are diffracted out of the inner beam region while the outer rays move towards the beam centre. (c) Self-defocusing nonlinearity, no divergence: the mixing of outer and inner rays is clearly reduced in comparison to (b). For all cases, an initial peak irradiance of $200 \mathrm{GW} / \mathrm{cm}^{2}$ was assumed. 
is focused to a waist of $20 \mu \mathrm{m}$. In analogy to the simulation that will be presented later in this section, a BBO crystal with $n_{2}=+8 \cdot 10^{-16} \mathrm{~cm}^{2} / \mathrm{W}$ is placed at $z_{\text {min }}=4 z_{R}$ where $z_{R}$ is the RAYLEIGH length. The rays which are initially in the beam center (red rays) remain in this most intense region due to the self-focusing effect. Therefore, they undergo strong SPM while the rays which are initially in the wing of the beam (black rays) hardly notice the self-focusing effect, diverge strongly, and thus do not undergo SPM. This behavior is qualitatively in excellent agreement with the simulation result of Fig. 3.26(c). The central part of the beam is spectrally broadened while the spectrum of the wings is not affected by the nonlinearity of the crystal.

In the presented model, divergence and nonlinearity can easily be inverted, i.e. beam convergence is combined with self-defocusing. An example is shown in Fig. 3.28(b). The $n_{2}$ is set to $-8 \cdot 10^{-16} \mathrm{~cm}^{2} / \mathrm{W}$ and $z_{\min }=-7 z_{R}$. The rays behave fundamentally different from the self-focusing example. The initially central rays are strongly diffracted and cross the rays from the wings within the defocusing medium, i.e. they undergo SPM only at the beginning of the crystal while the rays from the wings will spectrally broaden towards the end of the crystal. This leads to the homogenization effect in the case of combining self-defocusing and beam convergence. The impression that the rays in the beam center vanish is attributed to the fact that $w_{0}$ and $I_{p}$ are kept constant along the beam path. Full 3D simulations that are presented later in this section will demonstrate that no "donut-shape" beam is generated. Finally, the case of self-defocusing without divergence is studied in Fig. 3.28(c). The peak irradiance is set to $200 \mathrm{GW} / \mathrm{cm}^{2}$ and $n_{2}=-8 \cdot 10^{-16} \mathrm{~cm}^{2} / \mathrm{W}$ like before. Fewer rays cross within the crystal. Basically, only the initially centered rays undergo SPM in the beginning of the nonlinear medium. The rays from the wings diffract slower, but they do not propagate towards the beam center due to the missing convergence. In conclusion, the simple ray model provides an illustrative picture that combining beam convergence with self-defocusing nonlinearity leads to an homogenization effect in bulk broadening. This idea will be supported by more rigid 3D simulations. Beforehand, the question on how to achieve self-defocusing nonlinearities, i.e. negative $n_{2}$ values, will be addressed.

\section{Cascaded quadratic nonlinearities in BBO}

From linear optics, it is known that negative susceptibilities occur on the high frequency side of resonances. For a known absorption spectrum, these can be derived by virtue of the Kramers-Kronig relations. Similar behavior has, for instance, been observed for the optical KERR effect in semiconductors ${ }^{290}$. Although KRAMERS-KRONIG relations can only partially be applied in nonlinear optics ${ }^{156}$, a clear relation between $n_{2}$ dispersion and the two-photon absorption spectrum was found ${ }^{290,347}$. For high-power TD oscillators, the involvement of real absorptions, often causing thermal instabilities (cf. the notes on WLC generation in section 3.2.1), is preferably avoided. However, for achieving effective nonlinear refractive indices, virtual absorptions can be exploited, too. "Virtual" means that light is converted to another frequency but not to a real excitation, e.g. in second harmonic generation (SHG). Supplement 3 will investigate the refractive index change induced by phase-mismatched SHG by means of the Kramers-KroniG relations. The intuitive derivation results in the correct magnitude of $n_{2}$ but misses the $\pi$ phase-shift which occurs when light is converted from the fundamental to the SH (and back, resp.) ${ }^{169}$, and hence predicts the wrong sign. The phase shift is inherently included in the derivations 

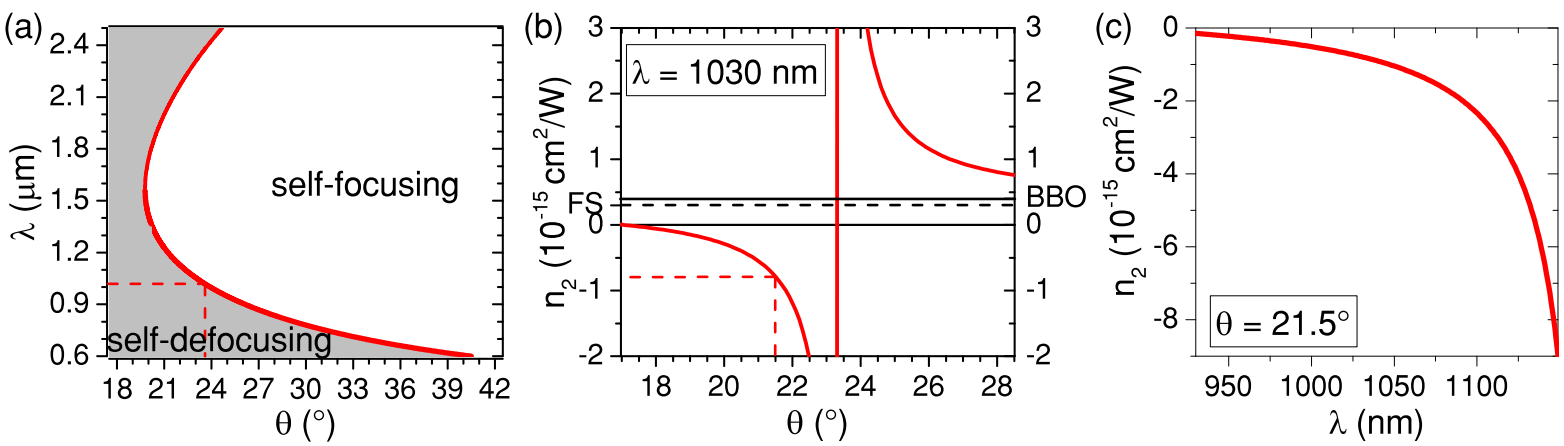

Fig. 3.29. (a) Type I phase-matching curve for BBO. The dashed line shows the phase-matching angle, $\theta=23.5^{\circ}$, for $1030 \mathrm{~nm}$, the pump wavelength. It corresponds to the angle the utilized BBOs were cut at. The grey-shaded area implies a negative $n_{2}^{\text {(cas) }}$. (b) The effective nonlinear refractive index of BBO for $1030 \mathrm{~nm}$ upon phase-mismatching. The red dashed line shows the angle at which the BBOs were operated. It corresponds to $n_{2}=-8 \cdot 10^{-16} \mathrm{~cm}^{2} / \mathrm{W}$. For comparison, $n_{2}^{\text {(Kerr) }}$ of BBO and FS are indicated. (c) The calculated dispersion of the effective nonlinear refractive index for the tuning angle $\theta=21.5^{\circ}$.

of the cascaded quadratic effect by means of the coupled nonlinear wave equations ${ }^{348}$. They result in a cascaded $\chi^{(2)}$ nonlinear refractive index, given by:

$$
n_{2}^{(\mathrm{cas})}=-\frac{4 \pi}{\epsilon_{0} c_{0} \lambda_{F}} \frac{d_{\mathrm{eff}}^{2}}{n_{S H} n_{F}^{2} \Delta k}
$$

where the vacuum permittivity is denoted by $\epsilon_{0}, c_{0}$ is the speed of light in vacuum, $\lambda_{\mathrm{F}}$ the wavelength of the fundamental, $d_{\mathrm{eff}}$ the effective $\chi^{(2)}$-nonlinearity, $n_{\mathrm{SH}}$ and $n_{\mathrm{F}}$ the refractive indices of the second harmonic and the fundamental, resp. Finally, $\Delta k$ denotes the angular wavenumber mismatch or, in other words, the phase-mismatch per unit length. Fig. 3.29 displays magnitude and dispersion of the effective nonlinear refractive index for phase-mismatched quadratic nonlinearities near the type I second harmonic resonance of BBO. Fig. 3.29(a), which is calculated from the SELLMEIER equations in ref. 349, shows where this resonance occurs, i.e. for which tuning angle $\theta$ the SHG process is phase-matched, and indicates the regions of positive and negative $n_{2}^{\text {(cas) }}$. The curves in Figs. 3.29(b) and (c) have been calculated from Eq. (3.22) and a constant KERR nonlinearity which was assumed to be isotropic and was set to $n_{2}^{(\mathrm{Kerr})}=4 \cdot 10^{-16} \mathrm{~cm}^{2} / \mathrm{W}$ if not explicitly stated differently. Literature values, however, vary between 4 and $7 \cdot 10^{-16} \mathrm{~cm}^{2} / \mathrm{W}^{350}$. Fig. 3.29(b) demonstrates that BBO crystals must be tuned near resonance to achieve a defocusing nonlinearity at $1030 \mathrm{~nm}$ which implies dispersion of the $n_{2}$ as Fig. 3.29(c) illustrates. The values $\lambda_{F}=1030 \mathrm{~nm}, d_{\text {eff }} \approx-2 \mathrm{pm} / \mathrm{V}^{351}, n_{S H}=1.658$ and $n_{F}=1.655$ are used to estimate the cascaded quadratic nonlinearity in the experiments and simulations which will be presented afterwards. From these values follows:

$$
\begin{aligned}
n_{2}^{(\mathrm{cas})} & \approx-1.2 \cdot 10^{-15} \frac{\mathrm{cm}^{2}}{\mathrm{~W}} \\
\Delta k & =\frac{4 \pi}{\lambda_{F}}\left(n_{S H}-n_{F}\right) \approx 11.7 \pi / \mathrm{mm}
\end{aligned}
$$

The magnitude of the cascaded nonlinear refractive index induced by cascaded $\chi^{(2)}$ processes is about three times higher than the KERR nonlinearity of BBO at $1030 \mathrm{~nm}$. Con- 
sequently, the effective nonlinear refractive index

$$
n_{2}(\theta, \lambda)=n_{2}^{(\text {Kerr })}+n_{2}^{(\text {cas })}(\theta, \lambda)
$$

is about two times larger in magnitude. Note that KERR and cascaded $\chi^{(2)}$-effects are fundamentally different. Only the latter one can be varied in magnitude and sign via tuning of the crystal angle $\theta$, i.e. the phase-matching of the incoming beam and its second harmonic. Moreover, it exhibits a much stronger wavelength $(\lambda)$ dependence than the KERR effect near the phase-matching angle for SHG.

\section{Simulations investigating spatial properties}

The initial motivation of negative nonlinear refractive indices, explained with the ray optics model, shall be studied in a more quantitative fashion by means of the simulation tool that has been used to investigate the coupling of spatial and temporal nonlinear effects in the previous section on KERR effect-based bulk broadening. As an example, spectral broadening of the KLM TD oscillator output is examined which is later also demonstrated experimentally. The spatial grid of the simulations was set to $128 \times 65$ points with a size of $5 \mu \mathrm{m} \times 5 \mu \mathrm{m}$. Half of the $\mathrm{x}-\mathrm{y}$ plane was simulated. The temporal grid had 512 points with $5 \mathrm{fs}$ spacing and the center frequencies near the fundamental $(300 \mathrm{THz})$ and the second harmonic $(600 \mathrm{THz})$ were factored out. The waves are propagated in frequency domain, and hence the simulations implicitly include self-steepening effects that arise from $\chi^{(2)}$ and $\chi^{(3)}$ effects $^{352}$.

Fig. 3.30 compares the case of a self-defocusing nonliearity and beam convergence ((b) and (c)) with self-focusing in combination with beam divergence $((d)$ and $(e))$. Convergence and divergence refer to the front facet of the nonlinear crystal which is displaced from the focal plane by $z_{\min }$ (Fig. 3.30 (a)). Beam convergence, peak irradiance at the BBO entrance facet and crystal length in the self-defocusing case are similar to the ray model parameters and the experimental conditions which will be explained later in this section. For an incident power of $90 \mathrm{~W}$, a FTL of about $75 \mathrm{fs}$ is reached for both the simulation of positive and negative effective $n_{2}$. A first significant difference is the dependence of the maximal peak irradiance inside the crystal on the incident power. In the selfdefocusing case, the peak irradiance rises sub-linearly (Fig. 3.30(b)). By contrast, the irradiance increases first linearly in the positive $n_{2}$ case (Fig. 3.30(d)), but at about $100 \mathrm{~W}$ of average power rapidly rises, indicating beam collapse which would ultimately lead to crystal damage. The soft optical-limiting behavior in the self-defocusing case is favorable since it makes the broadening less susceptible to irradiance fluctuations and more robust against damage. The most crucial difference becomes apparent in Figs. 3.30(c) and (e). While in the self-focusing case only the central part is spectrally broadened and the incident spectrum around $1030 \mathrm{~nm}$ is spread out in space, in the self-defocusing case such a wavelength dependent spatial distribution is not observed, but the whole beam is spectrally broadened. In both cases, the influence of spatial walk-off becomes visible which is a disadvantage of critically phase-matching the birefringent crystals. That is also why half of the beam plane has been simulated, contrary to Fig. 3.26 where only a quarter of the full plane was considered. Nevertheless, the simulation results clearly point out the advantages of combining self-defocusing with beam convergence. 


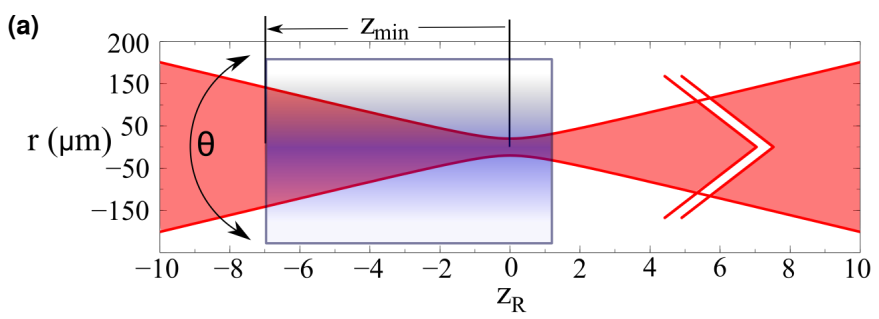

(b)

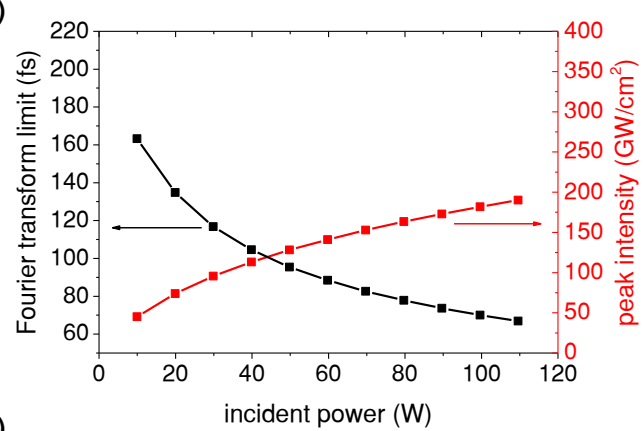

(d)

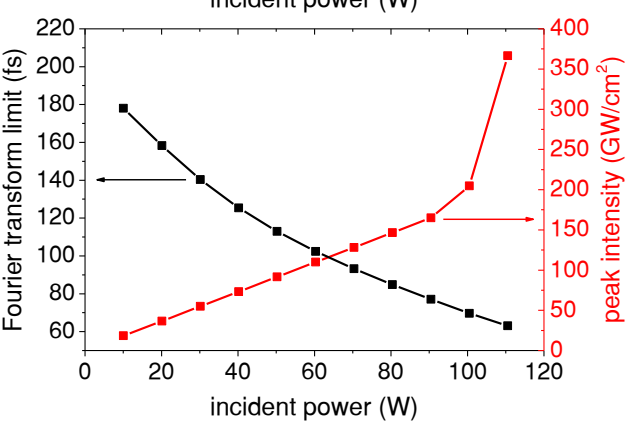

(c)

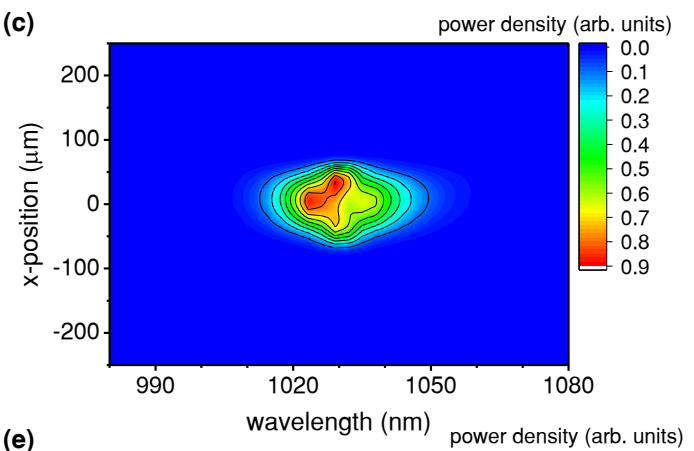

(e)

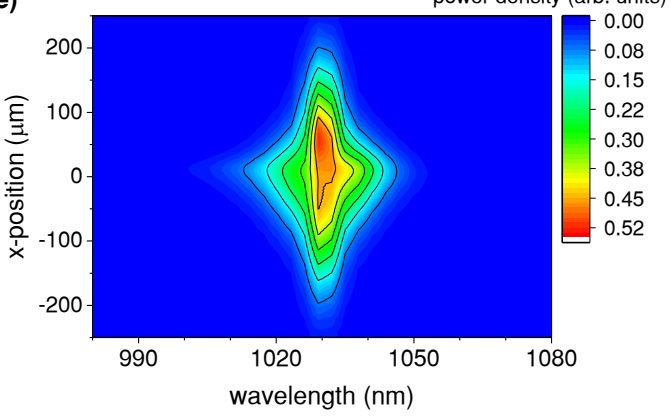

Fig. 3.30. Simulations with an incoming $180 \mathrm{fs} \mathrm{sech}^{2}$-pulse, focused to a beam diameter of $40 \mu \mathrm{m}$ (in absence of nonlinear refraction): (a) Sketch of the simulation parameters. The tuning angle $\theta$ was varied to achieve self-defocusing and -focusing, resp. The effective nonlinear refractive indices were $n_{2}=\mp 8 \cdot 10^{-16} \mathrm{~cm}^{2} / \mathrm{W}$. They resulted from the sum of KERR and cascaded $\chi^{(2)}$-effects in both cases. A hypothetical $n_{2}^{\text {(Kerr) }}=-4 \cdot 10^{-16} \mathrm{~cm}^{2} / \mathrm{W}$ was set in the self-focusing case to match the magnitudes of the contributions to $n_{2}$. The distance from the crystal front facet to the focal plane was $z_{\min }=-7 z_{R}$ $\left(-8.5 \mathrm{~mm}\right.$, illustrated in the figure) in the defocusing and $z_{\min }=4 z_{R}(4.9 \mathrm{~mm})$ in the focusing case. This has been chosen to match FTL and peak irradiance at $90 \mathrm{~W}$ incident power. The RAYLEIGH length $z_{R}$ was about $1.22 \mathrm{~mm}$, the crystal length $10 \mathrm{~mm}$. The beam radius is denoted by $\mathrm{r}$. The red arrows indicate the propagation direction (z-axis). (b) Dependence of the maximal peak irradiance inside the crystal and the FTL of the pulses on the incident power for negative effective $n_{2}$ and beam convergence. (c) Spectral power distribution in dependence on the $\mathrm{x}$-position with respect to the beam center for negative effective $n_{2}$ and beam convergence. (d) Dependence of the maximal peak irradiance inside the crystal and the FTL of the pulses on the incident power for positive effective $n_{2}$ and beam divergence. (e) Spectral power distribution in dependence on the x-position with respect to the beam center for positive effective $n_{2}$ and beam divergence. (c) and (e) show line-outs at $\mathrm{y}=0 \mu \mathrm{m}$ for $90 \mathrm{~W}$ incident power. The average power values refer to the experimental repetition rate of $23.8 \mathrm{MHz}$.

In order to investigate the sensitivity of the pulse compression scheme on the alignment of the BBO crystals, simulations with different phase-matching angles and varied crystal positions were conducted. The simulations refer again to the KLM TD oscillator output which was used for the experimental demonstration of spectral broadening by means of cascaded quadratic nonlinearities. The results are shown in Fig. 3.31. In Fig. 3.31(a) the tuning angle is fixed to $\theta=21.5^{\circ}$, i.e. the effective $n_{2}$ is approximately $-8 \cdot 10^{-16} \mathrm{~cm}^{2} / \mathrm{W}$. The crystal length is $10 \mathrm{~mm}$ which corresponds to about 8 RAYLEIGH lengths. If the 

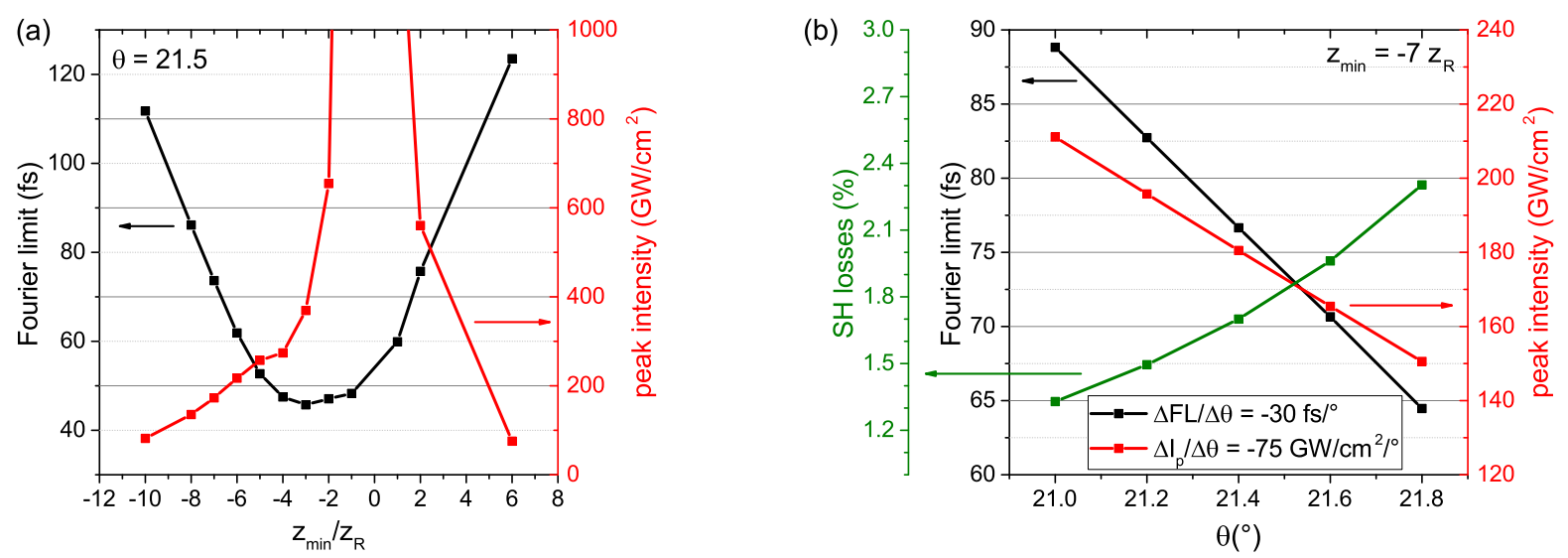

Fig. 3.31. (a) Dependence of the FTL and the maximal peak irradiance inside the $10 \mathrm{~mm}$ thick crystal on $z_{\min }$. The RAYLEIGH length $z_{R}$ is $1.22 \mathrm{~mm}$ for a beam waist of $20 \mu \mathrm{m}$. The tuning angle $\theta$ is fixed to $21.5^{\circ}$. (b) Dependence of the FTL, the maximal peak irradiance inside the crystal and the SH losses on the tuning angle $\theta$ for a fixed $z_{\min }=-7 z_{R}$.

$n_{2}$ was 0 , the maximal peak irradiance inside the crystal would be about $3 \mathrm{TW} / \mathrm{cm}^{2}$ for $z_{\min }$ between -8 and $0 z_{R}$. This would lead to crystal damage. It is to note that the critical irradiance has not been measured for the oscillator parameters. For BBO, it is specified to be $40 \mathrm{GW} / \mathrm{cm}^{2}$ for tens of ps pulse duration ${ }^{353}$ while tens of $\mathrm{TW} / \mathrm{cm}^{2}$ did not damage the crystal when 4 fs pulses were used ${ }^{354}$. For the utilized high-power fs system, a damage threshold of several hundreds of $\mathrm{GW} / \mathrm{cm}^{2}$ is estimated. Crystal damage was observed upon detuning of the crystal angle by a few degrees. The self-defocusing effect strongly reduces the maximal peak irradiance inside the crystal to only about $6 \%$ of the free-space value if $z_{\text {min }}=-7 z_{R}$. This, however, depends on the tuning angle as Fig. 3.31(b) shows. The slope of the maximal peak irradiance in dependence on the tuning angle is about $-75 \mathrm{GW} / \mathrm{cm}^{2} /{ }^{\circ}$ in the vicinity of $\theta=21.5^{\circ}$, i.e. the angle has to be set carefully before power is sent into the broadening crystal in order to avoid its damage. The generated second harmonic provides a good measure for the tuning angle. In the experiment, about $2 \%(1.8 \mathrm{~W})$ of $90 \mathrm{~W}$ were converted to green light in a $10 \mathrm{~mm}$ thick crystal. The FTL of the broadened pulses is also very sensitive to the tuning angle as Fig. 3.31(b) indicates. In the vicinity of $\theta=21.5^{\circ}$, the slope is $-30 \mathrm{fs} /{ }^{\circ}$. It is remarkable that the peak irradiance grows with the FTL. This is due to the tuning angle dependence of $n_{2}$. An increase in $\theta$ leads to a higher absolute value of the nonlinearity, subsequently to a lower FTL as well as to stronger self-defocusing, and thus to a reduced maximal peak irradiance inside the crystal. Peak irradiance and the broadening factor are also strongly dependent on the crystal position with respect to the focal point. In the graph shown in Fig. 3.31(a), crystal damage is expected if $z_{\text {min }}$ is in the interval between $\approx \pm 4$ RAYLEIGH lengths. If the crystal is positioned behind the focus, the broadening factor is relatively low. In the shown example, the best positions for operation are between -8 and $-4 z_{R}$ where sub-100 fs FTLs are predicted whereas the peak irradiances are kept below the damage threshold. In the experiments described in the following, $z_{\min } \approx-10 \mathrm{~mm}$ was chosen which enabled a relatively clean pulse compression by compensating for first order chirp only. The presented simulations helped finding an appropriate setting for the initial measurements. After having established the reported configuration, the setup has been operated for several months on a day-to-day basis without the need of major changes. 

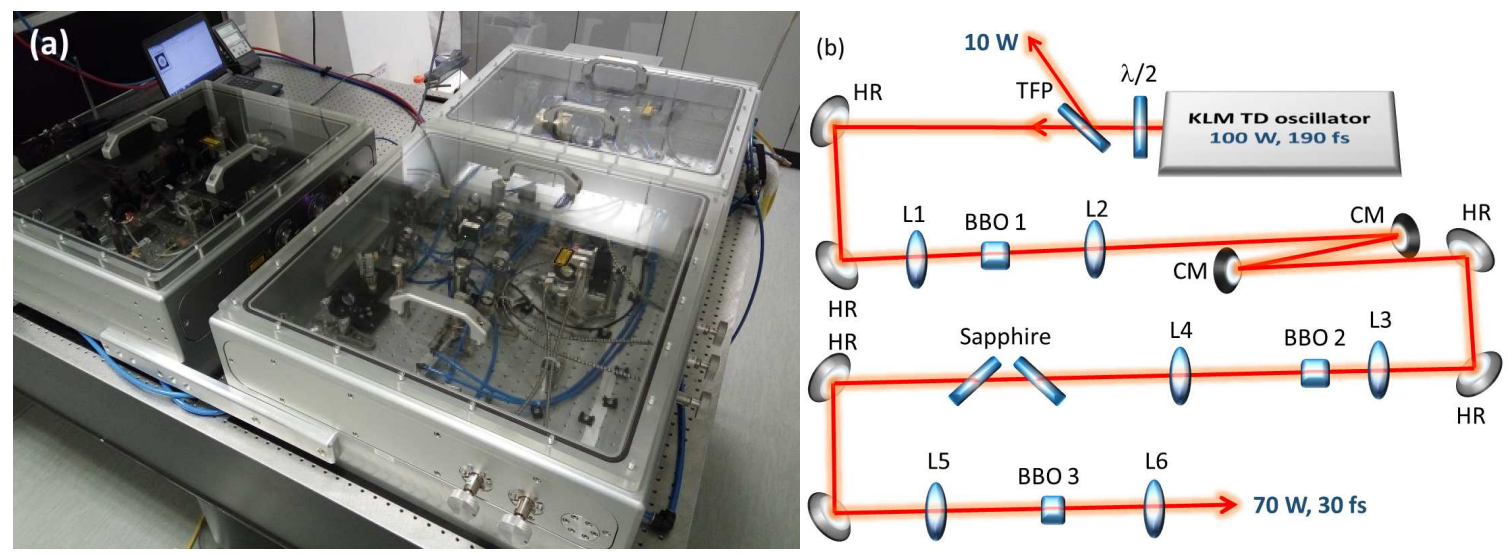

Fig. 3.32. (a) Photo of oscillator and compression chamber. The larger housing contains the KLM TD oscillator. The smaller housing contains the compression setup which is sketched in (b): The power sent to the BBO crystals was controlled by the half-wave plate $(\lambda / 2)$ and the thin-film polarizer (TFP). The beam was steered by pairs of $45^{\circ}$ HR mirrors. All lenses (L1-L6) were plano-convex, AR coated fused-silica lenses. The chirped mirrors (CM) exhibited a GDD of $+2000 \mathrm{fs}^{2}$. The two sapphire plates were $5 \mathrm{~mm}$ thick and placed at BREwSTER's angle. The BBO crystals were mounted on a translation stage and could be rotated in the vertical direction for the purpose of angle-tuning. BBO 1 and $\mathrm{BBO} 2$ were $10 \mathrm{~mm}$ long and BBO $36 \mathrm{~mm}$. The apertures were $7 \times 7 \mathrm{~mm}^{2}$.

\section{Experiments}

The compression experiments were performed with pulses emerging from a commercialgrade KLM TD oscillator (UltraFast Innovations $\mathrm{GmbH}$ ), not with the oscillator used in all other presented experiments. The laser was mainly assembled by JonATHAN BRONs. It was set-up in a monolithic aluminium housing which had a footprint of $145 \mathrm{~cm} \times 70 \mathrm{~cm}$. The housing itself and all optics mounts inside were water-cooled. Moreover, the oscillator could be aligned without opening the housing. This allowed stable operation (power RMS $\approx 0.5 \%$, calculated from 5000 samples, 1 sample/s). The oscillator delivered $190 \mathrm{fs}$ sech $^{2}$-pulses (measured with an autocorrelator) with $4.2 \mu \mathrm{J}$ energy at a repetition rate of $23.8 \mathrm{MHz}$. This corresponds to an average power of about $100 \mathrm{~W}$. The oscillator was set-up according to the principles described in ref. 59. A photograph of the laser is shown in Fig. 3.32(a).

The compression chamber consisted also of a monolithic, water-cooled housing. It had a footprint of $70 \mathrm{~cm} \times 55 \mathrm{~cm}$ and contained three sequential pulse compression stages. The setup is sketched in Fig. 3.32(b). The crystals were water-cooled. Their temperature was between 25 and $30^{\circ} \mathrm{C}$ in thermal equilibrium at $90 \mathrm{~W}$ input power. The spectral broadening was achieved in BBO crystals which were chosen because they have been available at excellent commercial grade from multiple suppliers and they combine high damage threshold with reasonable nonlinearity. The negative uniaxial crystals were cut at the angles $\theta=23.5^{\circ}$ and $\phi=90^{\circ}$. This corresponds to the phase-matching angle for SHG of $1030 \mathrm{~nm}$ with nearly maximized quadratic nonlinearity. By rotating the crystal, the phase-matching angle $\theta$ was tuned to about $21.5^{\circ}$ which resulted in the cascaded nonlinear refractive index calculated in Eq. (3.23).

About $90 \mathrm{~W}$ were focused with a $60 \mathrm{~mm}$ focal length lens into a $10 \mathrm{~mm}$ long BBO crystal whose front facet was placed about $50 \mathrm{~mm}$ behind the lens. The broadened spectrum was compressed by two bounces off chirped mirrors with $+2000 \mathrm{fs}^{2}$ GDD. The semiconductors 
(a)

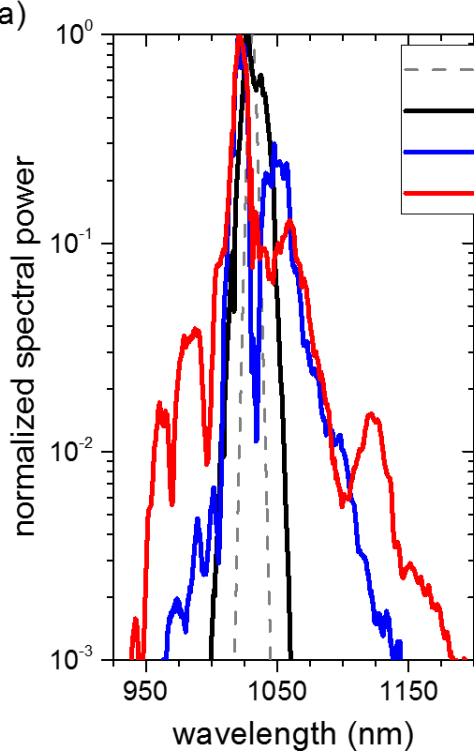

(b)

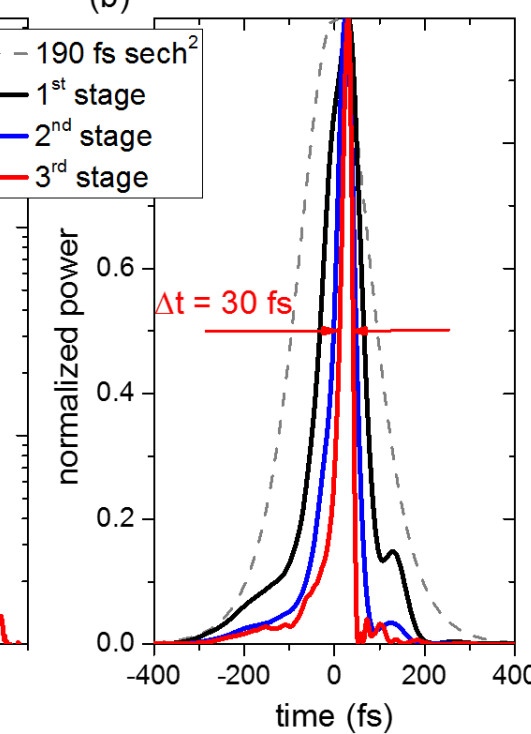

(c)

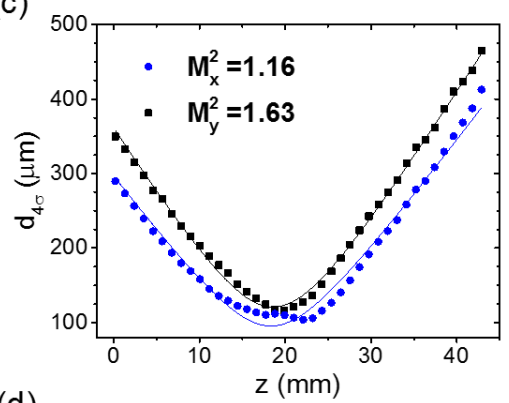

(d)

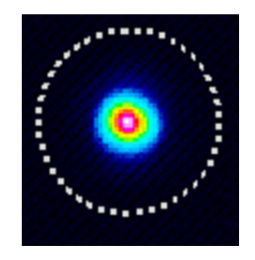

Fig. 3.33. (a) Broadened spectra measured with an OSA. (b) Retrieved FROG traces with 97 fs (black line), $50 \mathrm{fs}$ (blue line), $30 \mathrm{fs}$ (red line) full widths at half maximum. The legend applies to (a) and (b). As a reference a sech ${ }^{2}$-shape spectrum and pulse corresponding to the oscillator input is shown. (c) $\mathrm{M}^{2}$ measurement of the beam behind the third broadening stage. (d) Focused beam profile extracted from the $\mathrm{M}^{2}$ measurement shown in (c).

ZnSe, ZnS and TGG were also tested to compensate the down-chirp of the pulses but they adversely affected the beam profile at high average power. The compression factor was adjusted to about 2 which results in low power in the pulse pedestals if only first order chirp is compensated (cf. Fig. 3.21(a)). In the following stage, the pulses were focused with an $f=50 \mathrm{~mm}$ lens into another $10 \mathrm{~mm}$ BBO crystal. Since the absolute value of GDD required for pulse compression decreases with increasing bandwidth (cf. Suppl. 2), utilizing the normal dispersion of two $5 \mathrm{~mm}$ thick sapphire plates was sufficient to reduce the pulse duration to about $50 \mathrm{fs}$. In this case, no beam distortions were observed. In the final stage, an $f=100 \mathrm{~mm}$ lens and a $6 \mathrm{~mm}$ BBO were utilized. No additional dispersive optics for pulse compression were needed because the positive group velocity dispersion $\left(\approx 48 \mathrm{fs}^{2} / \mathrm{mm}\right.$ at $\left.1030 \mathrm{~nm}\right)$ in combination with the negative nonlinear phase shift self-compressed the pulses to about $30 \mathrm{fs}$. A $10 \mathrm{~mm}$ long BBO was tested as well, but self-compression already happened inside the crystal, resulting in a chirped output. The collimated beam diameters were about $1.8 \mathrm{~mm}, 1.8 \mathrm{~mm}$ and $2.4 \mathrm{~mm}$ in front of the first, second and third broadening stages, resp. and $z_{\min } \approx-10 \mathrm{~mm}$ in all stages. Due to the nonlinear defocusing, the waist sizes could not be measured directly. According to the simulations presented in Fig. 3.30, it is expected that peak irradiances of about $180 \mathrm{GW} / \mathrm{cm}^{2}$ were reached inside the first crystal at full power. Due to the higher peak powers and similar focusing geometries in second and third stage, the peak irradiances were increased correspondingly for the shorter pulses.

The spectra, measured with an OSA, and the pulses, retrieved by SH-FROG measurements, are shown in Fig. 3.33. The compression results are also summarized in Table 3.2. After the third broadening stage, the long wavelength edge of the spectrum was close to the second harmonic resonance. This could be observed through the emergence of weak, visible red radiation. It is inferred that further spectral broadening would require a stronger 

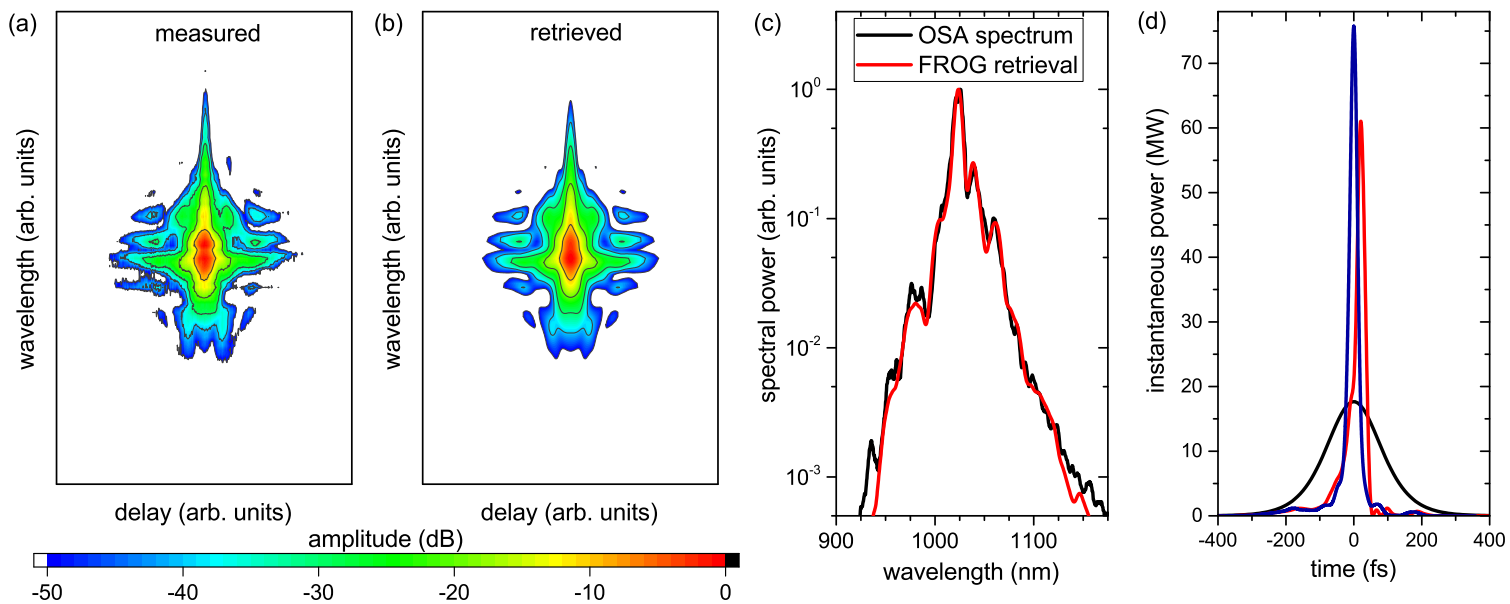

Fig. 3.34. (a) measured and (b) retrieved FROG traces of the compressed pulse after the third broadening stage. The scale is logarithmic. The retrieval grid was $512 \times 512$, the delay spacing $2 \mathrm{fs}$ and the wavelength spacing $0.26 \mathrm{~nm}$. The FROG error of the retrieval was $0.3 \%$. (c) Spectrum measured with an OSA compared to retrieved FROG spectrum of the trace from (b). The scale is also logarithmic. The homogeneity of the beam is evidenced by the excellent agreement between the FROG and OSA spectrum. Since the FROG contains an only $10 \mu \mathrm{m}$ thick BBO crystal, it behaves like a spatial filter (cf. Fig. 3.23(b)). (d) Instantaneous power of the $90 \mathrm{~W}$ average power oscillator output pulses (black line), the $70 \mathrm{~W}$ average power compressed pulses after the third stage (red line) and the simulated pulses which were compressed by two bounces off chirped mirrors with tailored GDD (blue line)

detuning from the second harmonic phase-matching angle, and thus a reduced magnitude of the defocusing nonlinearity. Scanning the far-field beam profile with a multimode fiber connected to an OSA revealed very good beam homogeneity. This was experimentally also confirmed by the good agreement between the spectrum measured with the OSA and the one retrieved from FROG which is shown in Fig. 3.34(a)-(c). About $70 \mathrm{~W}$ of average power were emerging from the third BBO. The remaining $20 \mathrm{~W}$ were partly converted into the second harmonic (about $2 \%$ per stage) which was mainly transmitted through the $45^{\circ} \mathrm{HR}$ mirrors. The majority of the losses arose from the 30 interfaces the beam had to pass. Each stage exhibited an efficiency between 90 and $93 \%$. This resulted in an efficiency of more than $75 \%$ and a compression factor of more than 6 which is akin to fiber broadening results. The polarization extinction ratio was better than 1:30. A peak power of $60 \mathrm{MW}$ was reached. Fig. 3.21(d) shows that additional control of the phase of the output pulses would lead to a further increase of peak power from $60 \mathrm{MW}$ to $75 \mathrm{MW}$. The simulation utilized the FROG retrieval shown in Fig. 3.34(b) and added the GDD of the

Table 3.2: Summary of the presented pulse compression experiments.

\begin{tabular}{ccccc}
\hline & FTL & added GDD & measured pulse duration & peak power \\
\hline $1^{\text {st }}$ stage $^{b}$ & $82 \mathrm{fs}$ & $4000 \mathrm{fs}^{2}$ & $97 \mathrm{fs}$ & $27 \mathrm{MW}$ \\
$2^{\text {nd }}$ stage & $50 \mathrm{fs}$ & $360 \mathrm{fs}^{2}$ & $50 \mathrm{fs}$ & $45 \mathrm{MW}$ \\
$3^{\text {rd }}$ stage & $25 \mathrm{fs}$ & $0 \mathrm{fs}^{2}$ & $30 \mathrm{fs}$ & $60 \mathrm{MW}$ \\
\hline
\end{tabular}

${ }^{a}$ An additional GDD of $\approx 80 \mathrm{fs}^{2}$ is added by the collimation lens after each stage.

${ }^{b}$ For comparison, the simulation of the $1^{\text {st }}$ stage yielding the spectrally resolved profile shown in Fig. 3.30(c) resulted in a 74 fs FouRIER transform-limit. 
tailored mirrors used in KERR effect-based bulk broadening experiments of section 2.2.2 (cf. Fig. 2.13(b)).

In addition to the characterization of the pulses, an $\mathrm{M}^{2}$ measurement in accordance to the ISO Standard 11146 was performed with a WinCamD $\mathrm{M}^{2}$ stage. The $\mathrm{M}^{2}$ factor in horizontal direction was $M_{h}^{2}=1.2$ and clearly better than that in vertical direction $M_{v}^{2}=1.6$ (Fig. 3.33(c)). This is attributed to spatial walk-off in the birefringent crystals. Nevertheless, the beam can be focused well as Fig. 3.33(d) shows. Most remarkable, no self-diffraction rings like observed in positive $n_{2}$-based spectral broadening were detected as predicted by the crude ray model and the simulation results presented in Fig. 3.30.

\section{Discussion}

The initial experiments on spectral broadening in BBO already pointed out in a brief statement that nonlinear beam distortions became only visible in the self-focusing regime ${ }^{355}$. However, these experiments were conducted with a Ti:sapph-based laser system, operating at much lower average power but about $4.7 \mathrm{GW}$ peak power. Hence, the $17 \mathrm{~mm}$ long BBO crystal could be placed in a collimated beam, and thus the RAYLEIGH range clearly exceeded the crystal length. Beam distortions in the self-defocusing regime became apparent and were explicitly stated in experiments with about $100 \mathrm{MW}$ peak power ${ }^{356}$, i.e. in a peak power range where the latest generation of mode-locked TD oscillators operates. The issue was addressed by utilizing flattop beams which do not exhibit a continuous spatial gradient and therefore should be homogeneously spectrally broadened ${ }^{357}$. Although the beam homogeneity improved, adding a beam shaper also added complexity to the setup and introduced losses of about $30 \%$. Moreover, beam shaping will be complicated owing to the average powers on the order of $100 \mathrm{~W}$. Therefore, the proposed method of combining beam convergence and nonlinear self-defocusing presents a novel, elegant alternative to achieve efficient pulse compression in bulk material.

If the bulk broadening technique presented here is compared to KERR effect-based approaches, it shares the previously highlighted advantages over fiber-based methods. In addition, the compression scheme presented here exhibits efficiencies comparable to fiber. Furthermore, the scheme allows to use dielectric materials to compensate chirp or even to achieve self-compression. This has been studied for cascaded quadratic nonlinearities of BBO in more detail in refs. 356, 358. Utilizing the cascaded $\chi^{(2)}$-effect exhibited also a few drawbacks: Firstly, the $\mathrm{M}^{2}$ factor in the direction of the extraordinary crystal axis increased from 1.1 to more than 1.6. It is expected to improve if the crystals are cut for normal incidence, i.e. at $\theta=21.5^{\circ}$ instead of $\theta=23.5^{\circ}$. However, the spatial walk-off is intrinsic due to the need for critical phase-matching for BBO. If periodically poled nonlinear crystals like PPLN or KTP are used, the problem of walk-off could be avoided. Pulse compression in PPLN was predicted for $100 \mathrm{fs}$, nJ-level pulses at $1550 \mathrm{~nm}^{359}$ (type 0 phase-matching) and experimentally demonstrated for $110 \mathrm{fs}, 30 \mu \mathrm{J}$ pulses at $1560 \mathrm{~nm}$ (type I phase-matching) ${ }^{360}$. For more energetic pulses, LBO presents also an alternative to BBO. The walk-off at room temperature is about a factor 7 smaller and non-critical phase-(mis-)matching at $1030 \mathrm{~nm}$ can be achieved by heating the crystal ${ }^{361}$. But the quadratic nonlinearity is also reduced by $60 \%$ in comparison to $\mathrm{BBO}$ and the dispersion of negative effective $n_{2}$ is stronger at $1030 \mathrm{~nm}$. Furthermore, tandem crystal geometries like employed in optical parametric amplifiers or oscillators ${ }^{362}$ could reduce the spatial walk-off in the setup. A second drawback is the relatively complex initial alignment owing 
to the interplay of crystal angle, crystal length and crystal position with respect to the focal point and spot size. Thirdly, the cascaded $\chi^{(2)}$-nonlinearity is dispersive, and thus the generation of few-cycle pulses may require to reduce the absolute value of the effective $n_{2}{ }^{356}$ or the combination with KERR effect-based bulk broadening in a multi-plate assembly ${ }^{62,329,363}$ or multi-pass cell ${ }^{336,337,339}$. However, this also strongly depends on central wavelength and pulse energy as few-cycle pulse generation has already been shown for different laser parameters ${ }^{356,364,365}$.

In summary, spectral broadening based on cascaded $\chi^{(2)}$-nonlinearities was performed at unprecedented high average power levels of $90 \mathrm{~W}$. The previously reported experiments were done at $\mathrm{kHz}$ repetition rates and high average power applications were only subject to simulations ${ }^{366}$. An increase in peak power from $18 \mathrm{MW}$ to $60 \mathrm{MW}$ was demonstrated. This shows that the limited efficiency of single-stage KERR effect-based bulk broadening has been overcome by the cascaded $\chi^{(2)}$ approach.

\section{Supplement 3: Derivation of the cascaded quadratic nonlinearity induced refractive} index by virtue of KRAMERS-KRONIG relations

The equation (3.22) was derived in ref. 348 by solving the coupled wave equations of fundamental (subindex F) and second harmonic (subindex $\mathrm{SH}$ ) propagating through a nonlinear crystal. An alternative, mathematically not as stringent, but physically perhaps more meaningful derivation is shown in the following.

The relation between fundamental and $\mathrm{SH}$ power can be found in many textbooks. The derivation is presented for instance in ref. 183. (Note that the prefactors in the wave equation vary from textbook to textbook and dependent on the definition of the complex electric field.) In the Handbook of Nonlinear Optics ${ }^{367}$, the expression for a weakly depleted (plane) fundamental wave is given by:

$$
\begin{aligned}
P_{\mathrm{SH}} / P_{\mathrm{F}}= & \frac{8 \pi^{2} d_{\mathrm{eff}}^{2} L^{2}}{\epsilon_{0} n_{\mathrm{F}}^{2} n_{\mathrm{SH}} c_{0} \lambda_{\mathrm{F}}^{2}} I_{\mathrm{F}} \\
& \times \operatorname{sinc}^{2}(\Delta k L / 2),
\end{aligned}
$$

where $P_{\mathrm{F} / \mathrm{SH}}$ denotes the power of fundamental/SH, $d_{\text {eff }}$ the effective quadratic nonlinearity, $\epsilon_{0}$ the vacuum permittivity, $n_{\mathrm{F} / \mathrm{SH}}$ the linear refractive indices of fundamental/SH, $L$ the crystal length, $\lambda_{\mathrm{F}}$ the fundamental wavelength, $c_{0}$ the vacuum speed of light and $\Delta k=k_{\mathrm{SH}}-2 k_{\mathrm{F}}$ the wavevector mismatch. In the case of phase-mismatched propagation in a crystal which clearly exceeds the coherence length of the three-wave interaction (like in the presented experiments), the sinc $^{2}$-function is close to 0 , and can thus be approximated by the DIRAC- $\delta$-distribution:

$$
\begin{aligned}
\operatorname{sinc}^{2}(\Delta k L / 2) & =\operatorname{sinc}^{2}\left(\left[k_{\mathrm{SH}} / 2-k_{\mathrm{F}}\right] L\right) \\
& \approx \frac{\pi}{L} \delta\left(k_{\mathrm{F}}-k_{\mathrm{SH}} / 2\right) .
\end{aligned}
$$

By means of Eqs. (3.26) and (3.27), the power of the fundamental can be expressed as:

$$
\begin{aligned}
P_{\mathrm{F}}(L) \approx & {\left[1-\frac{8 \pi^{3} d_{\mathrm{eff}}^{2}}{\epsilon_{0} n_{\mathrm{F}}^{2} n_{\mathrm{SH}} c_{0} \lambda_{\mathrm{F}}^{2}} I_{\mathrm{F}}\right.} \\
& \left.\times \delta\left(k_{\mathrm{F}}-k_{\mathrm{SH}} / 2\right) L\right] P_{\mathrm{F}}(0) \\
= & {\left[1-\alpha\left(I_{\mathrm{F}}, \Delta k\right) L\right] P_{\mathrm{F}}(0) . }
\end{aligned}
$$

Eq. (3.29) exhibits the shape of BEERLAMBERT's absorption law for small depletion, i.e. where $\exp \{-\alpha L\} \approx 1-\alpha L$. Subsequently, the SHG can be interpreted as an irradiance dependent resonance which is tunable by means of phase-matching (cf. red solid line of Fig. 3.29(a)). 
The relation between absorption and refraction is usually described by the KRAMERS-KRONIG relations which are quite common in linear optics, but can, to some degree, be extented to nonlinear optics as well ${ }^{156}$. The nonlinear absorption is related to the nonlinear refractive index change $\Delta n$ by ${ }^{347}$ :

$$
\Delta n(\omega)=\frac{c_{0}}{\pi} \mathcal{P} \int_{0}^{\infty} \frac{\Delta \alpha\left(\omega^{\prime}\right)}{\omega^{\prime 2}-\omega^{2}} d \omega^{\prime},
$$

where $\mathcal{P}$ is the CAUCHY principal value, $\omega$ as well as $\omega^{\prime}$ are angular frequencies and $\Delta$ the induced change in comparison to the linear behavior, i.e. $I_{\mathrm{F}} \rightarrow 0$. With the substitutions

$$
\begin{aligned}
k & =n_{\mathrm{F}} \frac{\omega}{c_{0}}, \\
\kappa & =\frac{8 \pi^{3} d_{\mathrm{eff}}^{2}}{\epsilon_{0} n_{\mathrm{F}}^{2} n_{\mathrm{SH}} c_{0} \lambda_{\mathrm{F}}^{2}} I_{\mathrm{F}},
\end{aligned}
$$

relation (3.30) translates into

$$
\begin{aligned}
\Delta n\left(k_{\mathrm{F}}\right) & =\frac{n_{\mathrm{F}}}{\pi} \mathcal{P} \int_{0}^{\infty} \frac{\kappa \delta\left(k^{\prime}-k_{\mathrm{SH}} / 2\right)}{k^{\prime 2}-k_{\mathrm{F}}^{2}} d k^{\prime} \\
& \approx \frac{n_{\mathrm{F}}}{\pi} \frac{\kappa}{\left(k_{\mathrm{SH}} / 2\right)^{2}-k_{\mathrm{F}}^{2}},
\end{aligned}
$$

where the occurrence of the DIRAC- $\delta$ distribution made the usual integration over a closed loop in the complex plane (cf. e.g. refs. 156, 347) unnecessary. The denominator of the latter equation is further simplified:

$$
\begin{aligned}
\left(k_{\mathrm{SH}} / 2\right)^{2}-k_{\mathrm{F}}^{2} & =\left(k_{\mathrm{SH}} / 2-k_{\mathrm{F}}\right)\left(k_{\mathrm{SH}} / 2+k_{\mathrm{F}}\right) \\
& \approx \Delta k / 2 \cdot 4 \pi n_{\mathrm{F}} / \lambda_{\mathrm{F}} .
\end{aligned}
$$

Finally, Eqs. (3.32) and (3.35) are inserted into Eq. (3.34):

$$
\begin{aligned}
\Delta n(\Delta k) & =\frac{\kappa \lambda_{\mathrm{F}}}{2 \pi^{2} \Delta k} \\
& =\frac{4 \pi}{\epsilon_{0} c_{0} \lambda_{\mathrm{F}}} \frac{d_{\mathrm{eff}}^{2}}{n_{\mathrm{F}}^{2} n_{\mathrm{SH}} \Delta k} I_{\mathrm{F}},
\end{aligned}
$$

which reproduces Eq. (3.22) for $n_{2}^{\text {(cas) }}$ up to a minus sign.

If the cascaded $\chi^{(2)}$ process is considered in the simple picture presented in ref. 169, the result nevertheless appears correct: The $\mathrm{SH}$ is generated in a first step, it travels faster $(\Delta k<0)$ or slower $(\Delta k>0)$ than the fundamental in a second step and is converted back again in the final step. This would imply a focusing behavior for $\Delta k>0$ and a defocusing behavior for $\Delta k<0$ as predicted by Eq. (3.36). However, Stegeman argues that this simple picture ignores the $\pi$ phase shift the back converted fundamental experiences. If $\Delta k=0$, this ensures the depletion of the fundamental, and hence energy conservation of the SHG process. The minus sign of Eq. (3.22) can be explained if this interference effect is included in the simple picture of the process. In a similar way one may argue that the cascaded $\chi^{(2)}$ process cannot be exactly treated like linear absorption, where a single absorbing dipole emits the fundamental light with a $\pi$ phase shift to cancel out the incoming wave. Instead, the cascaded process involves two radiating dipoles: the first one emits the second harmonic and cancels out the fundamental whereas the second cancels out the $\mathrm{SH}$ again and re-emits the fundamental. 


\title{
3.3 Power-scaling Carrier-Envelope-Phase Stabilization
}

\author{
Parts of this section have been published in: \\ M. Seidel, J. Brons, F. Lücking, V. Pervak, A. Apolonski, T. Udem and O. Pronin, "Carrier- \\ envelope-phase stabilization via dual wavelength pumping," Opt. Lett. 41, 1853 (2016).
}

In section 2.3.2 the first CEP stabilization of a high-power TD oscillator was presented. Phase-locking was achieved by means of loss modulation via an AOM. In the experiments presented in the following, the same oscillator was utilized but without any additional intracavity element which may cause thermal effects or adverse self-focusing. Instead, the gain of the active laser medium was modulated. This is conceptually close to the most common CEP stabilization techniques of Ti:sapph (cf. e.g. refs. 216, 218, 221, 226, 368), fiber (cf. e.g. refs. 229, 231, 369) and low-power Yb-ion based solid-state lasers (cf. e.g. refs. 228, 235, 370). Mimicking the stabilization schemes of the low-power oscillators is not straight forward since the higher power levels lead to an increasing trade-off between stability and modulation bandwidth. This trade-off shall be analyzed in more detail in the following.

The experimental setup is sketched in Fig. 3.35. The oscillator and the precompression stage are as before (cf. sections 2.2.1 and 2.3.2). Contrary to the AOM stabilization experiments, the out-of-loop interferometer was not set-up. The in-loop residual noise shown in Fig. 2.21 served as reference instead. A few changes to the f-to-2f interferometer have been made (cf. Fig. 2.19). While a fiber with anomalous dispersion was used before, an ANDi was utilized in the presented experiments to generate the octave spanning continuum. The capability of generating very broadband spectra with this fiber has already been demonstrated in Fig. 3.2 where it was pumped with the KLM TD oscillator output. Unfortunately, a beat note could not be detected when the $250 \mathrm{fs}$ pulses were directly launched into the ANDi fiber. Although full coherence of the octave spanning continuum was computed even for $300 \mathrm{fs}$ input pulses ${ }^{262}$, polarization instabilities may

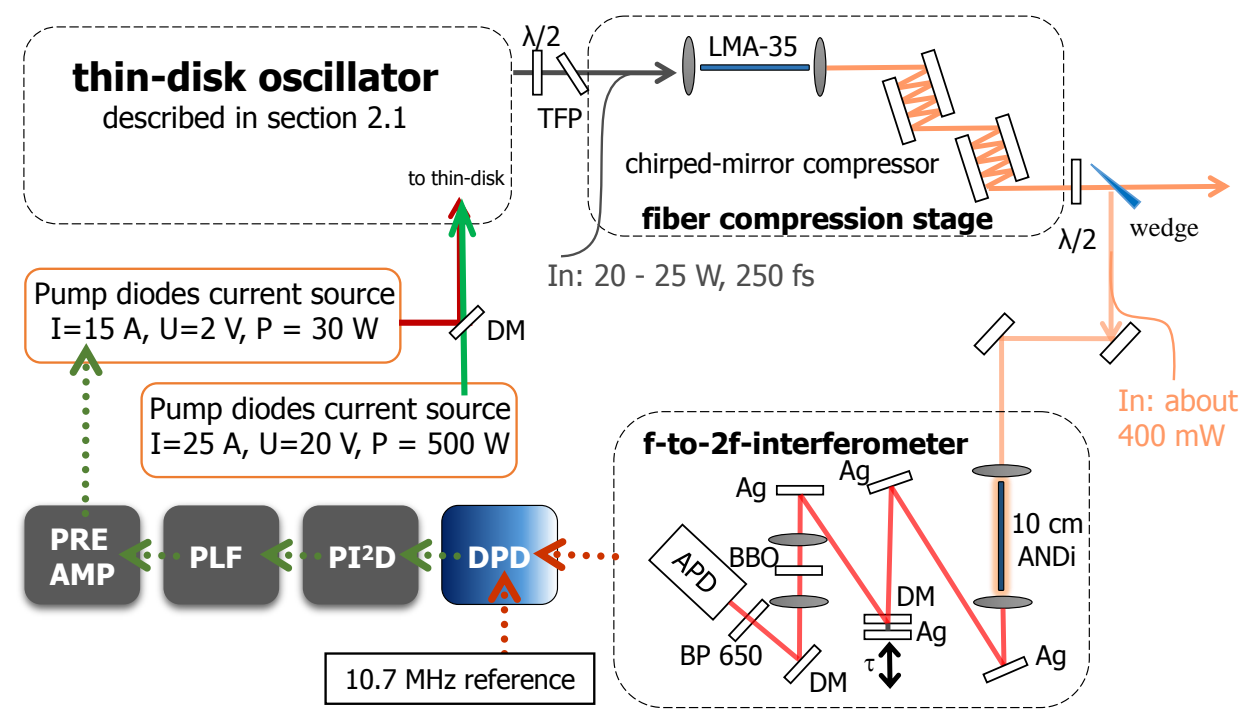

Fig. 3.35. Setup of the CEP stabilization scheme. Solid lines are optical, dotted lines electronic signals; $\lambda / 2$ - half-wave plate, TFP - thin film polarizer, Ag - silver mirror, DM - dichroic mirror, BP 650 - band pass centered at $650 \mathrm{~nm}$ with bandwidth $\Delta \lambda=10 \mathrm{~nm}, \tau$ - delay stage to temporally overlap fundamental and second harmonic, APD - avalanche photo diode, DPD - digital phase detector, $\mathrm{PI}^{2} \mathrm{D}-$ servo controller, PLF - phase lead filter, PREAMP - low noise preamplifier 
have led to the loss of temporal coherence ${ }^{371}$. This manifested itself through a strongly increased noise floor of the RF spectrum analyzer and a resulting SNR of the repetition rate signal of only $40 \mathrm{~dB}$ (at least $60 \mathrm{~dB}$ before). Yet, the reasons for the decoherence are not fully understood. For instance, the spectra shown in Fig. 3.2 and in ref. 263 do not exhibit the typical strong modulation feature of noisy continua. Nonetheless, the ANDi exhibited a better stability during the time in which the servo controller settings were optimized, i.e. over about half an hour. It is also expected that the amplitude-tophase noise coupling is lower than for the anomalously dispersive fiber since the spectral broadening can be saturated. This was, however, not investigated experimentally. Due to the absence of the soliton self-frequency shift ${ }^{372}$ in the normal dispersion regime, a bandpass centered at $650 \mathrm{~nm}$ instead of $680 \mathrm{~nm}$, as used before, was utilized. Subsequently, the PPLN could not be used for SHG anymore owing to the limited availability of poling periods. Instead, a $1 \mathrm{~mm}$ thick, BBO crystal was utilized which could readily be angletuned to double $1300 \mathrm{~nm}$. The crystal has already been used in f-to-2f interferometers ${ }^{19,225}$. Even without an additional polarizer which projects the fundamental and the $\mathrm{SH}$ on the same polarization axis, beat note SNRs of $35-40 \mathrm{~dB}$ (within $10 \mathrm{kHz}$ resolution bandwidth) were measured. Finally, the prism sequence shown in Fig. 2.19 was replaced by a pair of mirrors. The first one was a HR mirror originally designed for the spectrum of Ti:sapph lasers. It reflected the short wavelengths but transmitted the long ones which were delayed and reflected by a silver mirror. This made the f-to- $2 \mathrm{f}$ interferometer much more compact than it was before.

To determine the feed-back loop requirements, the free beat signal was sampled by a fast oscilloscope. The real signal was HILBERT transformed to perform an analytic continuation to the complex plane in order to access the phase. Complex and real signal are related by ${ }^{171}$ :

$$
\tilde{a}(t)=a(t)+i \mathcal{H}\{a(t)\}
$$

where $\mathcal{H}$ is the HILBERT transform operator, $a(t)$ the real and $\tilde{a}(t)$ the complex beat signal. The carrier-envelope-offset frequency is then derived by

$$
f_{\text {ceo }}(t)=\frac{1}{2 \pi} \frac{d}{d t} \Im\{\ln [\tilde{a}(t)]\}
$$

where $\Im$ denotes the imaginary part of the expression in braces. More details on the numerical beat note analysis are presented in Supplement 4 . In the experiment, the oscilloscope sampling rate was set to $25 \times 10^{6}$ samples per second. By virtue of the NYQUIST-SHANNON sampling theorem, instantaneous carrier envelope offset frequencies up to $12.5 \mathrm{MHz}$ could hence be detected. By adjusting the pump current, the $f_{\text {ceo }}$ was set to about $11 \mathrm{MHz}$. The spectral resolution was given by the measurement time of an oscilloscope trace. It was set to $0.5 \mathrm{~s}$ resulting in a $2 \mathrm{~Hz}$ frequency spacing. The residual noise of the locked CEP was determined with this numerical method and the previously used DPD. The agreement was good, as the inset in Fig. 3.36 shows. This proves the accuracy of the numerical method.

The IPN of the free-running beat note is shown in Fig. 3.36. A phase noise of $100 \mathrm{mrad}$ is accumulated after an observation time of $30 \mu \mathrm{s}$. This noise value shall be taken as reference because in many CEP sensitive time-domain applications a sub-radian residual noise is desired. Consequently, the feedback loop requires a bandwidth of at least $30 \mathrm{kHz}$. 


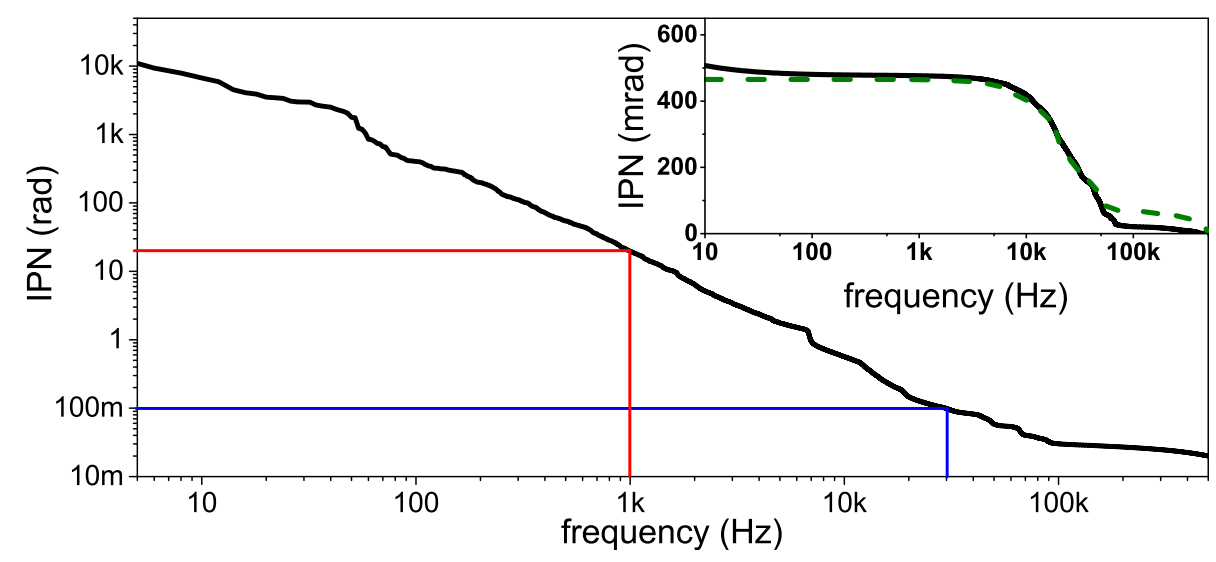

Fig. 3.36. IPN of the free-running beat note. The blue lines display the characteristic frequency where $100 \mathrm{mrad}$ phase noise is accumulated. The red curve displays the characteristic frequency which approximately corresponds to the upper state lifetime of the $\mathrm{Yb}^{3+}$ ions. The inset shows two consecutive measurements of the in-loop phase noise while feedback was applied to the pump diodes. The solid black line presents an analysis with the numerical method, the dashed green line with the digital phase detector.

To implement this is challenging because: (i) the power supply of the pump diodes has to provide more than $400 \mathrm{~W}$ of electrical power and should also exhibit a flat modulation response. Furthermore, (ii) the low-pass filter function of the gain medium due to the $\approx 1 \mathrm{~ms}$ upper state lifetime of the $\mathrm{Yb}$ ions has to be overcome.

Challenge (i) was addressed by a dual-wavelength pumping scheme. In section 2.3.1, strong efforts were taken to pump the KLM TD oscillator with a highly stable power supply. The demand to modulate this supply over a bandwidth of at least $30 \mathrm{kHz}$ is in contradiction to this. However, the modulation depth that was needed to manipulate the carrier-envelope-offset frequency is very low as discussed in section 2.3.2. A frequency shift of $350 \mathrm{kHz}$, which is the standard deviation of the beat note shown in Fig. 2.19(b), was accomplished by a pump power change of only $88 \mathrm{~mW}$ (assuming $40 \%$ slope efficiency at about $45 \mathrm{~W}$ output power), corresponding to only $0.04 \%$ of the total pump power. Subsequently, another supply with less power but with the capability of broadband modulation was added to accomplish CEP stabilization. The combination of both supplies was realized by pumping the zero and the first phonon line of the ${ }^{2} F_{5 / 2}-{ }^{2} F_{7 / 2}$ transition of the $\mathrm{Yb}^{3+}$ ions in parallel. Pump diodes operating at $940 \mathrm{~nm}$ were driven by the stable high-power supply (ILX Lightwave LDX-36000) while a second diode unit, radiating at $969 \mathrm{~nm}$, was driven by a low power supply. Two devices were tested: Thorlabs ITC4020 and ILX Lightwave LDX-32420. The former exhibited a broader modulation bandwidth and was therefore preferred. The pump radiation was combined by an inhouse-coated dichroic mirror before entering the TD head. The transfer function of the $969 \mathrm{~nm}$ pump diodes is depicted by the red lines in Fig. 3.37. A $90^{\circ}$ phase lag was reached at about $60 \mathrm{kHz}$ modulation frequency. The amplitude response was nearly flat up to this point. Therefore, the power supply proves to be suited for providing feedback over the targeted bandwidth. The transfer function looked significantly different when the pump power modulation response of the TD oscillator was monitored with a fast photodiode. It is represented by the black lines in Fig. 3.37. In this case, a $90^{\circ}$ phase lag is already present at frequencies higher than $10 \mathrm{kHz}$. Also, the amplitude response has dropped by $8 \mathrm{~dB}$ at that frequency. This intrinsic low-pass filtering, which presented challenge (ii), was counteracted by an electronic phase lead filter (PLF) ${ }^{231,370}$. Its transfer function is visualized 


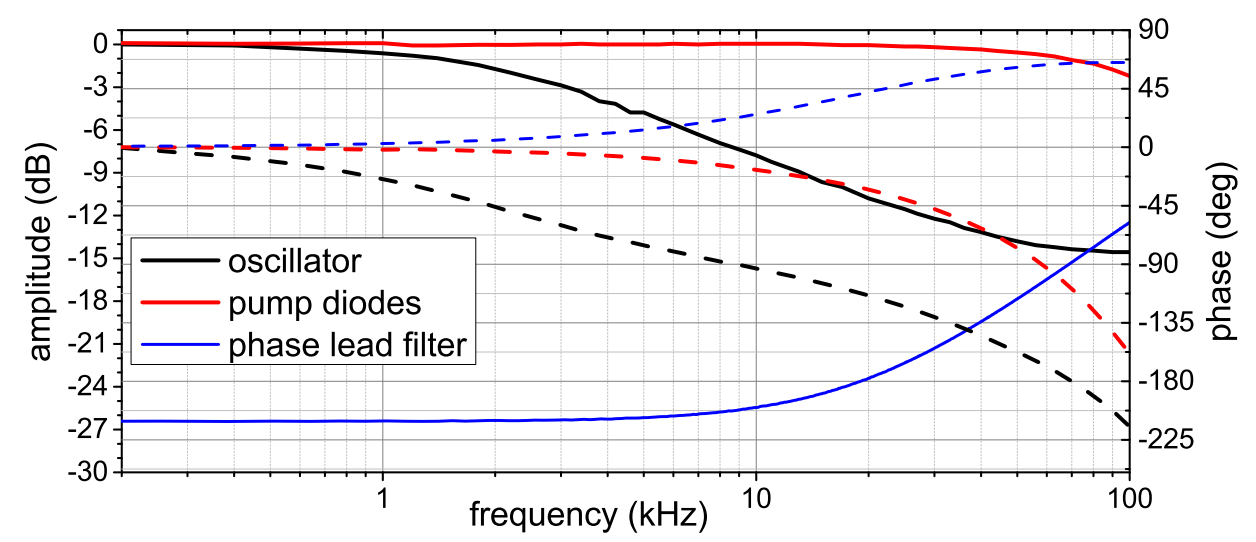

Fig. 3.37. Transfer functions of the $969 \mathrm{~nm}$ pump diodes power (red lines), the TD oscillator power (black lines) and the utilized phase lead filter (blue lines). The solid lines display the amplitudes and the dashed lines display the corresponding phases. The transfer functions of the phase lead filter have been measured with a SRS SR780 network analyzer, the other transfer functions with an SRS SR830 lock-in amplifier.

by blue lines in Fig. 3.37. Due to the positive phase of the first-order high pass, the $90^{\circ}$ phase lag is shifted to higher frequencies and the accessible bandwidth of the feedback loop is extended. Moreover, the slope of the PLF amplitude response is opposite to that of the oscillator. The total attenuation of the feedback signal is compensated by an additional low-noise preamplifier (SRS SR560) which amplifies the signal by a $23 \mathrm{~dB}$ voltage gain over the whole bandwidth.

In order to minimize the residual CEP noise, the cut-off frequencies of the integral parts as well as the cut-on frequency of the derivative part of the laser servo controller were varied as described in section 2.3.2. Again, first experiments were performed with the $5.5 \%$ OC where the in-loop phase noise could be reduced to $235 \operatorname{mrad}(1 \mathrm{~Hz}-500 \mathrm{kHz})$ as shown in Fig. 3.38(a). For the $14 \%$ OC a residual phase noise level of $390 \operatorname{mrad}(1 \mathrm{~Hz}-$

(a)
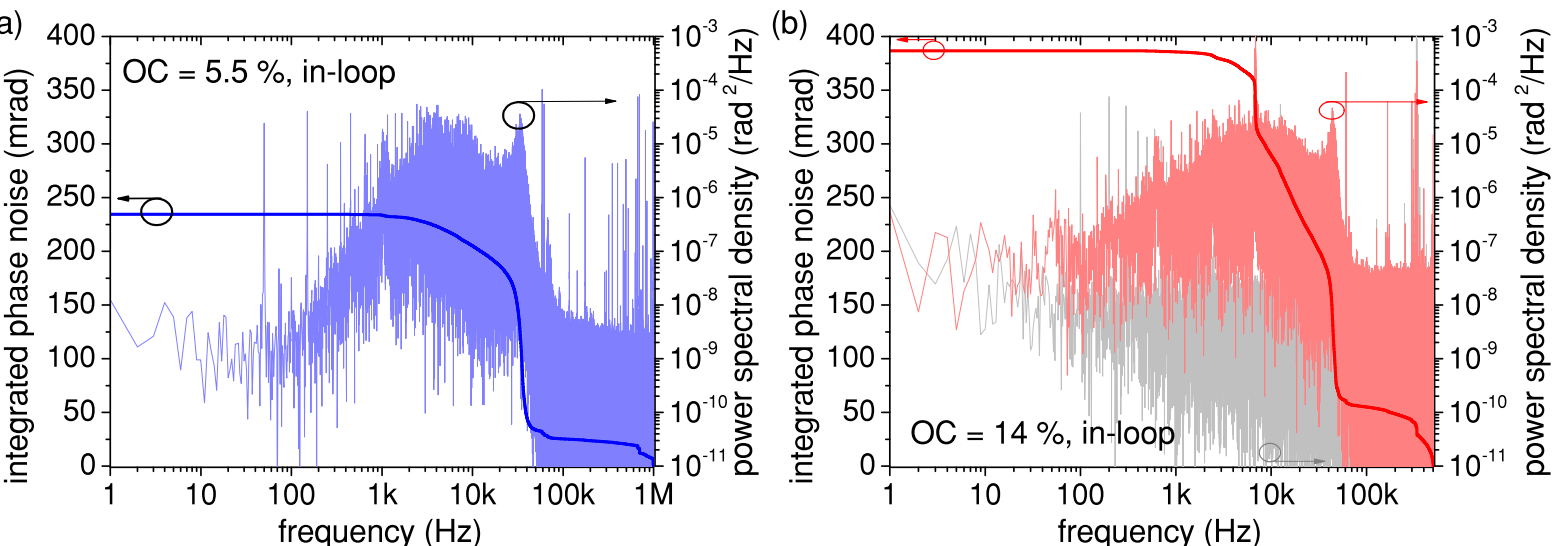

Fig. 3.38. Residual in-loop phase noise for (a) the $5.5 \%$ OC and (b) the $14 \%$ OC in a bandwidth from $1 \mathrm{~Hz}$ to $500 \mathrm{kHz}$. In (b), the noise floor PSD is displayed in gray. Measurements were taken with a DPD. For (a), the cut-off frequencies of the integral parts were 2 and $10 \mathrm{kHz}$, and the cut-on of the differential part was $50 \mathrm{kHz}$. For (b), the cut-off frequencies were $200 \mathrm{~Hz}$ and $5 \mathrm{kHz}$ while the cut-on frequency was set to $20 \mathrm{kHz}$. The same PLF was used in both measurements. 
$500 \mathrm{kHz}$ ) was accomplished (Fig. 3.38(b)), exceeding the value of the lower transmission OC like in the study with the AOM (cf. Fig. 2.21).

\section{Discussion}

The main difference in phase noise is accumulated between the two distinct spikes in the power spectral density of Fig. 3.38(b). One is at $7 \mathrm{kHz}$ and accounts itself for about $50 \mathrm{mrad}$ phase noise. It is also visible in the free-running beat note noise and originates from the oscillator as already pointed out in section 2.3.1 (cf. Fig. 2.18). It is remarkable that this spike has neither been observed in Fig. 3.38(a) nor in the phase noise measurements presented in section 2.3.2. Consequently, it should be removable after an oscillator realignment. It is to note that, although its exact origin is unknown, it can be excluded that the signature stems from the additional pump light at $969 \mathrm{~nm}$ since the feature was also observed in measurements without the use of the second power supply. Another spike is at $44 \mathrm{kHz}$ and accounts for an accumulated phase noise of about $125 \mathrm{mrad}$ in both graphs of Fig. 3.38. This noise is inscribed by the phase locked loop which becomes resonant at this frequency due to the increasing phase lag. These "feedback loop bumps" occur at about half of the frequency of the ones measured in Fig. 2.21. Moreover, in the intracavity loss modulation approach, they added maximal $50 \mathrm{mrad}$ to the total residual noise. Under the assumption that each loop resonance comes with the same frequency noise, a bump at twice the frequency $f$ would only lead to one forth of the phase noise due to the $1 / f^{2}$ scaling $^{212}$. Consequently, it would be favorable to push the servo bandwidth even further although no significant noise was accumulated by the laser system beyond $30 \mathrm{kHz}$ as Fig. 3.36 has demonstrated. One approach to accomplish this was to improve the design of the PLF, for instance by measuring the transfer function of the carrier-envelope-offset frequency and by tailoring the PLF to this function in order to get a flat response over the whole bandwidth the power supply supports. The servo settings for the $5.5 \% \mathrm{OC}$ and the $14 \% \mathrm{OC}$ were different. It would be interesting if the cut-off frequencies cut be increased if the $7 \mathrm{kHz}$ spike in the PSD of the high power oscillator is removed. Probably, the excess noise in the range from 7 to $44 \mathrm{kHz}$ could be strongly reduced as well. This was, however, not investigated experimentally.

It is to conclude that the intracavity loss modulation approach shows clearly lower CEP noise levels due to the extended feedback bandwidth of the AOM. It intrinsically provides a very flat response up to hundreds of $\mathrm{kHz}$ and avoids the stated challenges by modulating the cavity losses and not the round trip gain. Nevertheless, the dual wavelength approach should also be capable of reaching sub-200 mrad CEP noise levels if the phase response is optimally shaped and the oscillator noise is as low as in Fig. 2.4. The utilized power supply is specified with a $100 \%$ modulation depth without the loss of bandwidth at $225 \mathrm{~W}$ maximal electrical power. It is inferred that diodes of up to $100 \mathrm{~W}$ optical power can be driven with the supply. This is about three orders of magnitude more than the modulation amplitude required in the presented experiments. Subsequently, the concept is expected to be scalable to multiple $\mathrm{kW}$ of TD oscillator output power. At such high average power levels, the oscillators will get extremely sensitive to sources of thermal distortions. Subsequently, the benefit of avoiding any additional intracavity elements in the dual wavelength pumping approach would become decisive.

The presented concept can be generalized to the combination of a high power, highly stable signal with a lower power signal that can be modulated over a wide bandwidth. This 
combination was realized by means of optically combining radiation of $940 \mathrm{~nm}$ and $969 \mathrm{~nm}$ wavelength with a dichroic mirror. Both pump diodes and optical elements were readily available, making the presented setup favorable to implement the concept. Another approach was recently presented by KARLEN et $a l .{ }^{373}$. The low power signal modulated the stimulated emission rate of the Er:Yb:glass gain medium. This method could be transferred to TD oscillators. Unfortunately, the pump optics would have to be coated appropriately, i.e. not only for the pump but also for the oscillator wavelength. Due to the very thin disk, single pass absorption with a continuous wave source would certainly not be sufficient, especially since $1030 \mathrm{~nm}$ high power diode units are not readily available. Moreover, an electronic combination of two power supplies is a possible approach if cross-talk between the supplies can be avoided. Furthermore, the high power pump unit consists of multiple diode stacks which are driven by a common power supply. Unfortunately, changing this was not offered by the diode unit supplier. Another method could make use of fiber combiners. A multimode fiber guides the pump light to the the disk. Exchanging this fiber with one that combines the radiation of two sources could be a successful way to implement the stabilization scheme. Finally, it should be noted that the concept presented cannot only be used for CEP stabilization but also for intensity stabilization, i.e. "noise eating", of high-power oscillators.

In conclusion, a concept for CEP stabilizing high-power femtosecond oscillators has been introduced. The power scalable approach has led to a residual in-loop phase noise of $390 \mathrm{mrad}(1 \mathrm{~Hz}-500 \mathrm{kHz})$ in a proof-of-principle experiment with a $45 \mathrm{~W}$ average power KLM TD oscillator. The dual wavelength concept allows both highly stable pumping of the Yb:YAG gain medium and broadband modulation of the CEP. To reach a subradian residual in-loop noise performance, the low pass filtering behavior of the active laser medium was counteracted by a PLF. An optimized shaping of its transfer function promises a further reduction of the CEP jitter.

\section{Supplement 4: Numerical beat signal analysis}

Let $a(t)$ be the real beat signal taken with a fast oscilloscope. Its sampling rate is smaller than the repetition rate of the oscillator but larger than twice the maximal $f_{\text {ceo }}(t)$ within the sampling time. Then $a(t)$ can be written as:

$$
a(t)=A(t) \cdot \cos (\varphi(t))=A(t) \cdot \cos \left(\omega_{\text {ceo }} t+\Delta \varphi(t)\right)
$$

where $A(t)$ and $\varphi(t)$ are the time-dependent amplitude and phase of the beat signal, $\omega_{\text {ceo }}=$ $2 \pi f_{c e o}$, and $\Delta \varphi(t)$ is the time-dependent phase noise. The analytic continuation of the real signal to the complex domain is performed via HILBERT transformation as expressed in Eq. (3.37). The complex signal is

$$
\tilde{a}(t)=A(t) \cdot \exp \left(i \omega_{c e o} t+i \Delta \varphi(t)\right)
$$

Since an instantaneous frequency is defined by the change of phase in time, i.e.

$$
f(t)=\frac{1}{2 \pi} \frac{d}{d t} \varphi(t)
$$

eq. (3.38) follows. 
For the sake of numerical processing, the following identities were used. On the one hand:

$$
\frac{d}{d t} \ln (\tilde{a}(t))=\frac{\frac{d}{d t} \tilde{a}(t)}{\tilde{a}(t)}=\frac{d}{d t} \ln (A(t))+i \frac{d}{d t} \varphi(t) .
$$

On the other hand:

$$
\begin{aligned}
\frac{\frac{d}{d t} \tilde{a}(t)}{\tilde{a}(t)} & =\frac{\Re\left\{\frac{d}{d t} \tilde{a}(t)\right\}+i \Im\left\{\frac{d}{d t} \tilde{a}(t)\right\}}{\Re\{\tilde{a}(t)\}+i \Im\{\tilde{a}(t)\}} \\
& =\frac{\left[\Re\{\tilde{a}(t)\} \Re\left\{\frac{d}{d t} \tilde{a}(t)\right\}+\Im\{\tilde{a}(t)\} \Im\left\{\frac{d}{d t} \tilde{a}(t)\right\}\right]}{|\tilde{a}(t)|^{2}} \\
& +i \frac{\left[\Re\{\tilde{a}(t)\} \Im\left\{\frac{d}{d t} \tilde{a}(t)\right\}-\Im\{\tilde{a}(t)\} \Re\left\{\frac{d}{d t} \tilde{a}(t)\right\}\right]}{|\tilde{a}(t)|^{2}} .
\end{aligned}
$$

Thus, it is found from (3.42) and (3.43) that:

$$
\begin{aligned}
\frac{d}{d t} \varphi(t) & =\frac{\left[\Re\{\tilde{a}(t)\} \Im\left\{\frac{d}{d t} \tilde{a}(t)\right\}-\Im\{\tilde{a}(t)\} \Re\left\{\frac{d}{d t} \tilde{a}(t)\right\}\right]}{|\tilde{a}(t)|^{2}} \\
& =\frac{a(t) \frac{d}{d t}[\mathcal{H}\{a(t)\}]-\mathcal{H}\{a(t)\} \frac{d}{d t} a(t)}{|\tilde{a}(t)|^{2}}
\end{aligned}
$$

This equation has been implemented in the numerical beat note analysis. The time derivatives were taken by means of fast FOURIER transformation and multiplying the factor $i \omega$ in the frequency domain (cf. Appendix A.2). Before transforming back into the time domain, the frequencies were bandpassed to reduce differentiation noise (from $10.5 \mathrm{MHz}$ to $12.5 \mathrm{MHz}$ in the example shown in Fig. 3.36).

The advantages of this method are that HILBERT and FOURIER transformation are relatively fast numeric algorithms. Contrary to short time FOURIER transformation an instantaneous frequency can be determined for every sampling point. Moreover, through the normalization with $|\tilde{a}(t)|^{2}$ in Eq. (3.44), the result is independent of amplitude fluctuations of the beat note signal and, in contrast to a digital phase detector ${ }^{224}$, the method does not only count zero crossings of the signal but utilizes all sampling points to compute the instantaneous carrier-envelope-offset frequency. The following graph will show another example of the numerical analysis. It displays the instantaneous frequency of the beat note measured over a time of about half a second. This graph illustrates well that the beat note is literally locked to the reference frequency of $10.7 \mathrm{MHz}$ by the servo loop.

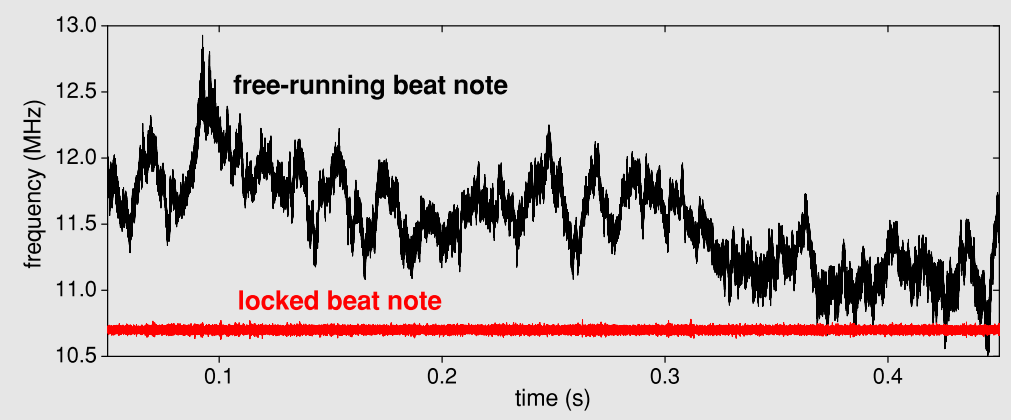




\subsection{Chapter Summary}

In this chapter, four different pulse compression techniques were studied. Two were based on spectral broadening in fiber and two on spectral broadening in bulk material. A summarizing overview of the techniques is provided in Table 3.3. Due to the long interaction lengths of the pulses and the nonlinear material, fiber-based methods usually come with relatively high compression factors, 15 for solid-core and 11 for hollow-core in the reported experiments. In addition, compression efficiencies of more than $70 \%$ can be readily reached. This led to the largest peak power enhancement factor of 10 for the LMA-35 experiment. Solid-core fiber exhibits another advantage which is the saturation of the SPM-based broadening, yielding increased stability of the output spectrum. The OWB contributions can be avoided by a proper fiber length selection. Such a behavior could not be demonstrated for Kagomé-type HC-PCF although it is theoretically possible. The positive pressure gradient, however, leads to rising nonlinearity with propagation length, and hence counteracts the saturation behavior. Furthermore, the very low GVDs of the noble gases would require meters of propagation for saturating the spectral broadening - at least for pulse durations of $\approx 200$ fs that typically emerge from KLM TD oscillators. For the generation of few-cycle pulses, the very low GVDs are actually beneficial because relatively large dispersion lengths allow high broadening factors even for sub-100 fs input pulses. This has been exploited in gas-filled capillaries for more than two decades by now ${ }^{54,283}$. Nevertheless, the reported experiments raise the question if Kagomé-type HC-PCFs are well-suited for few-cycle pulse generation at high average powers. Other groups ${ }^{322,323}$ have reported satisfying performance at tens of Watts, but for longer pulses, lower repetition rates and lower broadening factors. Therefore, further investigations are needed to conclusively demonstrate the average power scaling potential of Kagomé-type HC-PCF. Experiments with higher peak power would allow larger core diameters, consequently the option to operate in the normal dispersion regime even with broad bandwidths, and thus better output stability. While gas-based compression schemes would hence benefit from higher peak powers, solid-core fiber is strongly limited in peak power scalability, and hence does not present a viable platform for compression experiments of pulses exceeding $10 \mathrm{MW}$ of peak power (at about $1 \mu \mathrm{m}$ central wavelength).

The situation is different for bulk materials. Even if the peak power clearly exceeds the critical power, the length of the nonlinear medium can be made shorter than the selffocusing length. Alternatively, for many crystals without inversion symmetry, cascaded quadratic nonlinearities can induce an effective negative nonlinear refractive index which leads to self-defocusing, and hence prevents beam collapse. In comparison to fiber-based schemes, the relatively short interaction lengths in bulk crystals result in low broadening factors. However, resorting to multiple-plate setups can make up for this shortcoming. In the cascaded scheme with intermediate compression of section 3.2.1, a broadening factor of 17 was reached although no particular attention was paid to efficiency and clean pulse compression. Best compression qualities were achieved with small broadening factors of $\approx 2$. The three stage setup utilizing the cascaded quadratic nonlinearities of BBO crystals resulted in a compression factor of more than 6 while the peak power was enhanced from 18 to $60 \mathrm{MW}$ which could be further increased by the use of tailored chirped mirrors. Bulk broadening is particularly attractive because of its practical advantages over fiber-based compression schemes. In the experiments with a $15 \mathrm{~mm}$ quartz crystal, no noise enhancement was observed even for the highest broadening factors of 6 (cf. section 3.2.1) which 
Table 3.3: Comparison compression techniques

\begin{tabular}{|c|c|c|c|c|}
\hline & $\begin{array}{l}\text { solid-core } \\
\text { PCF }\end{array}$ & $\begin{array}{l}\text { Kagomé-type } \\
\text { HC-PCF }\end{array}$ & $\begin{array}{l}\text { KERR-effect } \\
\text { bulk broaden- } \\
\text { ing }\end{array}$ & $\begin{array}{l}\text { cascaded- } \chi^{(2)} \\
\text { bulk broaden- } \\
\text { ing }\end{array}$ \\
\hline $\begin{array}{l}\text { peak power } \\
\text { scalability }\end{array}$ & $\begin{array}{l}\text { limited by criti- } \\
\text { cal power }\end{array}$ & $\begin{array}{l}\text { GW demon- } \\
\text { strated with } \\
\text { Kagomé-type } \\
\text { HC-PCF and } \\
\text { capillaries }^{54,316}\end{array}$ & $\begin{array}{l}\text { requires multi- } \\
\text { pass arrange- } \\
\text { ment }\end{array}$ & $\begin{array}{l}\text { GW demon- } \\
\text { strated }^{355}\end{array}$ \\
\hline $\begin{array}{l}\text { average power } \\
\text { scalability }\end{array}$ & $\begin{array}{l}250 \mathrm{~W} \text { demon- } \\
\text { strated } 272\end{array}$ & questionable & $\begin{array}{l}500 \mathrm{~W} \text { demon- } \\
\text { strated }^{336}\end{array}$ & $\begin{array}{l}90 \mathrm{~W} \text { demon- } \\
\text { strated }\end{array}$ \\
\hline $\begin{array}{l}\text { demonstrated } \\
\text { compression } \\
\text { factors }^{a}\end{array}$ & 15 & $\begin{array}{l}11\left(1^{\text {st }} \text { stage }\right), \\
2.4\left(2^{\text {nd }} \text { stage }\right)\end{array}$ & $\begin{array}{l}6 \quad\left(1^{\text {st }} \quad \text { stage }\right), \\
1.7 / 2.2 \quad\left(2^{\text {nd }}\right. \\
\text { stage } 10 \mathrm{fs} \text { and } \\
7.7 \mathrm{fs} \text {, resp. })\end{array}$ & 6 in 3 stages \\
\hline $\begin{array}{l}\text { demonstrated } \\
\text { compression } \\
\text { efficiencies }^{b}\end{array}$ & $74 \%$ & $\begin{array}{l}70 \%\left(^{\text {st }} \text { stage }\right), \\
52 \%\left(2^{\text {nd }} \text { stage, }\right. \\
\text { Fresnel losses } \\
\text { and mirror } \\
\text { bandwidths } \\
\text { improvable })\end{array}$ & $\begin{array}{l}\approx \\
\left(1^{\text {st }} \quad 50 \%\right. \\
60 \% / 40 \% \quad \text { stage }), \\
\text { stage } \quad 10 \mathrm{fs} \text { and } \\
7.7 \mathrm{fs}, \text { resp. })\end{array}$ & $75 \%$ \\
\hline $\begin{array}{l}\text { peak power } \\
\text { enhancement } \\
\text { factor }\end{array}$ & 10 & 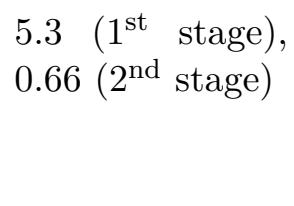 & $\begin{array}{l}2.1 \quad\left(1^{\text {st }} \text { stage }\right), \\
0.76 / 0.64 \quad\left(2^{\text {nd }}\right. \\
\text { stage } 10 \mathrm{fs} \text { and } \\
7.7 \mathrm{fs} \text {, resp. })\end{array}$ & 3.3 \\
\hline $\begin{array}{l}\text { phase shape at } \\
\text { output (at } \approx \\
30 \mathrm{fs})\end{array}$ & $\begin{array}{l}\text { mainly GDD in } \\
\text { all-normal dis- } \\
\text { persion regime }\end{array}$ & $\begin{array}{l}\text { mainly GDD } \\
\text { for all-normal } \\
\text { dispersion, } \\
\text { higher-order } \\
\text { terms for pres- } \\
\text { sure gradient }\end{array}$ & $\begin{array}{l}\text { higher order } \\
\text { terms present, } \\
\text { depending on } \\
\text { broadening } \\
\text { factor per plate }\end{array}$ & $\begin{array}{l}\text { higher order } \\
\text { terms present, } \\
\text { depending on } \\
\text { broadening fac- } \\
\text { tor per crystal } \\
\text { and phase- } \\
\text { mismatch }\end{array}$ \\
\hline $\begin{array}{l}\text { few-cycle } \\
\text { pulse genera- } \\
\text { tion }\end{array}$ & $\begin{array}{l}\text { demonstrated } \\
\text { for low peak } \\
\text { powers } 18,374,375\end{array}$ & $\begin{array}{l}\text { presented in sec- } \\
\text { tion } 3.1 .2\end{array}$ & $\begin{array}{l}\text { presented in sec- } \\
\text { tion } 2.2 .2\end{array}$ & $\begin{array}{l}\text { demonstrated } \\
\text { for longer } \\
\text { wavelengths, } \\
\text { higher peak } \\
\text { powers }\end{array}$ \\
\hline robustness & fair & fragile & high & high \\
\hline
\end{tabular}


is in strong contrast to the Kagomé-type HC-PCF experiments presented in section 3.1.2. Large apertures, easy replacement and cost-efficiency present additional benefits of bulk broadening. Those are especially important for "workhorse-type" laser systems. At the central wavelength of $1030 \mathrm{~nm}$, few-cycle pulse generation with cascaded quadratic nonlinerities is rather difficult due to the dispersion of $n_{2}^{\text {(cas) }}$. The multi-pass arrangement simulated in section 3.2.1 appears to be the best solution to compress efficiently to the sub-10 fs regime. First experiments with KLM TD oscillators resulted in $\approx 30 \mathrm{fs}$ pulses with a peak power enhancement from $60 \mathrm{MW}$ to $230 \mathrm{MW}^{62,376}$. This presents a recordhigh peak power for amplification free systems and is well suited for generating harmonics with tens of $\mu \mathrm{W}$ average powers and sub-50 nm wavelengths ${ }^{323}$.

The chapter has also introduced an alternative approach to carrier-envelope-offset frequency stabilization of high-power mode-locked (TD) oscillators. It takes advantage of the high sensitivity of the CEP to intracavity and pump power modulations, resp. It is based on a dual wavelength pumping scheme where the radiation of high-power laser diodes driven by a very stable power supply is combined with the radiation of low-power laser diodes driven by a power supply with large modulation bandwidth. A sub-400 mrad in-loop residual phase noise was achieved for the full oscillator power. Especially for frequency domain applications where the phase jitter can often be averaged out while the single pulse carrier-envelope-offset frequency is not decisive, this noise value (if also reached out-of-loop) is usable for applications. Nevertheless, the phase locking of the AOM approach was tighter than that of the pump power modulation approach due to the very high modulation bandwidth of the AOM (cf. Suppl. 1). It is, however, expected that the residual phase noise can be further lowered by optimized PLF design or alternative dual wavelength schemes, for instance the one demonstrated in ref. 373 for low-power fiber oscillators. Pump power modulation, being more (average) power-scalable than intracavity loss modulation, may play an important role in future locking experiments of mode-locked oscillators with intracavity powers of several $\mathrm{kW}$.

It is instructive to evaluate if the carrier-envelope-offset frequency stabilization schemes can be transferred from KLM to SESAM mode-locked TD oscillators which have not been stabilized at average power levels above $3 \mathrm{~W}$ so-far. In order to do so, the noise accumulation of the free beat note in Fig. 3.36 is compared to the transfer functions presented in ref. 236. The SESAM mode-locked Yb:YAG TD oscillator shows a strong resonance around $7 \mathrm{kHz}$ as well and an abrupt phase behavior at this frequency which was attributed to the saturable absorber. The combination of high average and peak power makes it difficult to operate the SESAM in the well saturated regime. In particular, multiphoton absorption at the presence of several $\mathrm{kW}$ intracavity average power may lead to strong thermal lensing and rapid degradation. Consequently, SESAM mode-locking high-power TD oscillators generally appears to make CEP stabilization significantly more difficult than KLM where the saturation behavior is determined through the interplay of the KERR-lens with hard and soft apertures. Moreover, the highest power SESAM mode-locked TD oscillator have been operated in a vacuum environment ${ }^{58,61}$ which was, for instance, not necessary for a $270 \mathrm{~W}$ KLM TD oscillator ${ }^{59}$. It can be inferred that high-power SESAM mode-locked TD oscillators are more sensitive to the distortions an intracavity AOM introduces, and thus should be preferentially stabilized by pump power modulations.

In summary, peak and average power scalable concepts for ultrashort pulse compression and CEP stabilization have been demonstrated. The presented schemes are important 
building blocks for upscaling the results of chapter 2 to the latest and the coming generation of mode-locked TD oscillators. 


\section{FROM THE NEAR- TO THE MID-INFRARED}

The previous chapters of this dissertation have demonstrated the progress in the development of KLM TD oscillators towards waveform control and few-cycle operation. So-far all research was concentrated on the optical octave from about 700 to $1400 \mathrm{~nm}$.

In general, ultrashort pulse sources are most sophisticated in the near-IR and visible parts of the electromagnetic spectrum where active laser transitions of electronic nature are located. As mentioned before, the most powerful fs sources emit around 1 micron wavelength. Oscillators directly deliver more than $250 \mathrm{~W}$ of average power ${ }^{58,59}$, while amplifiers even reach $\mathrm{kW}$ levels ${ }^{28,32}$. For comparison, the 2 micron technology has reached $20 \mathrm{~W}$ from mode-locked oscillators ${ }^{151}$ and about $150 \mathrm{~W}$ from amplifiers ${ }^{377}$. At 2.5 microns, lasers stay already well below $10 \mathrm{~W}^{378}$. Finally, broadband gain that allows femtosecond operation has only been demonstrated up to $2.9 \mu \mathrm{m}$ with approximately $300 \mathrm{~mW}$ average power $^{379}$.

Consequently, the availability of mid-IR light sources stands in strong contrast to their tremendous multitude of applications, for example in fundamental physics, chemistry, environmental and life sciences. The spectral region contains the fundamental vibrational transitions of all infrared-active small molecules, the most common functional groups as well as biomolecules like proteins, lipids, nucleic acids and carbohydrates. Importantly, mid-IR absorption results from the specific molecular structure which is exploited in various chemical analysis techniques ${ }^{380}$, to name only a few examples, in trace gas as well as human breath analysis ${ }^{381}$ and in early cancer detection ${ }^{382}$.

Particularly interesting techniques are frequency $\mathrm{comb}^{66}$, in the broadest sense frequency up-conversion ${ }^{383,384}$ and $2 \mathrm{D}$ infrared spectroscopy ${ }^{385}$. Frequency combs allow fast and highly precise data acquisition. Up-conversion spectroscopy avoids the need of mid-IR detectors and, in combination with coherent light sources and cross-correlation methods, enables to extract temporal and phase information of molecular processes ${ }^{386,387}$. $2 \mathrm{D}$ infrared spectroscopy additionally resolves structural information of the samples under test. All these techniques need or at least strongly benefit from fs pulses. Compressing these to few-cycle durations has revealed tremendous strong-field effects, like the gas highharmonic cut-off extension to the x-ray regime ${ }^{388}$ or the occurrence of BLOCH oscillations in semiconductors ${ }^{246,389}$. In these examples, high peak power is required for accessing the regime of extreme nonlinear optics. On the other hand, high average power and photon flux, respectively, results in light source brightness surpassing those available from synchrotron sources ${ }^{198}$, and consequently facilitates the use of (rather noisy) uncooled MIR detectors or detector arrays ${ }^{390}$. Moreover, high average power is important for experimental techniques with low yield such as stand-off gas detection ${ }^{391}$ and may avoid the need for multiple interactions with the sample in resonant cavities or multi-pass cells ${ }^{381}$.

Here, the issue of the scarce availability of high-power fs sources in the mid-IR is addressed by taking advantage of the powerful KLM TD oscillator output and by down-converting it by means of three-wave mixing. 


\subsection{Optical Parametric Amplifiers for Frequency Down-Conversion}

In section 1.4.6 different types of frequency down-conversion schemes have been introduced. The research presented here mainly focuses on the development of OPAs. Seed and signal wavelength are identical $\left(\lambda_{S}\right)$. They are in the near-IR spectral region while the idler wavelength $\left(\lambda_{I}\right)$ is in the mid-IR. The mid-IR irradiance $I_{I}$ for an undepleted pump irradiance $I_{P}$ and under plane-wave assumption is given by ${ }^{184}$ :

$$
\begin{aligned}
I_{I}(L) & =I_{S, 0} \frac{\lambda_{S}}{\lambda_{I}} \frac{\Gamma^{2}}{g^{2}} \sinh ^{2}(g L), \\
g^{2} & =\Gamma^{2}-\left(\frac{\Delta k}{2}\right)^{2}, \\
\Gamma^{2} & =\frac{8 \pi^{2} d_{\mathrm{eff}}^{2} I_{P}}{\epsilon_{0} c_{0} n_{I} n_{S} n_{P} \lambda_{I} \lambda_{S}},
\end{aligned}
$$

where the upper quantities denote the following: $L$ - crystal length, $\Delta k=2 \pi\left(1 / \lambda_{P}-\right.$ $\left.1 / \lambda_{S}-1 / \lambda_{I}\right)$ - phase-mismatch per unit length, $\lambda_{P}$ - pump wavelength, $d_{\text {eff }}$ - effective $\chi^{(2)}$-nonlinearity, $\epsilon_{0}$ - vacuum permittivity, $c_{0}$ - speed of light in vacuum and $n_{P, S, I}$ - linear refractive indices of pump, signal or idler waves. Two important characteristics of these equations shall be pointed out. Firstly, parametric down-conversion via three-wave mixing comes with a so-called quantum defect which becomes visible in the $\lambda_{S} / \lambda_{I}$-term in Eq. (4.1). Every pump photon can only generate a single idler photon which carries $\lambda_{P} / \lambda_{I}$ of the pump photon energy. The remainder $\lambda_{P} / \lambda_{S}$ is converted into signal power. Consequently, even with a $100 \%$ quantum efficiency (every pump photon is down-converted), the power efficiency of the process is limited to $\lambda_{P} / \lambda_{I}=\lambda_{S} / \lambda_{I} /\left(1+\lambda_{S} / \lambda_{I}\right)$. Secondly, the pump irradiance is found in the sinh-function while the mid-IR-power scales only linear with the seed energy. In the high gain limit $\left(g L>>1, \sinh ^{2}(g L) \approx \exp \{2 g L\}\right)$, the idler

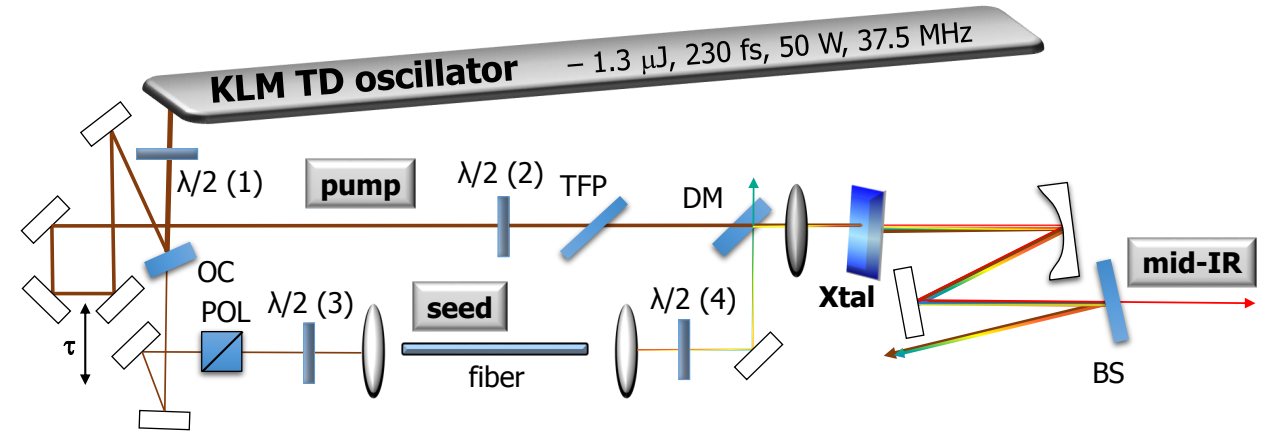

Fig. 4.1. The KLM TD oscillator delivers $1.3 \mu \mathrm{J}$ pulses of $230 \mathrm{fs}$ duration with a $37.5 \mathrm{MHz}$ repetition rate. A dielectric mirror (OC) splits either $3 \%$ or $15 \%$ of the oscillator power. The reflected light can be attenuated by a half-wave plate $(\lambda / 2)$, thin-film polarizer (TFP) sequence and is directly used to pump the nonlinear crystal (xtal). Either PPLN or lithium gallium sulfide, $\mathrm{LiGaS}_{2}$ (LGS) was used. The seed generation is accomplished in either a polarization maintaining ANDi or an LMA-12 fiber. To suppress cross-phase modulation, the polarization is cleaned in front of the fiber with a polarizer (POL). Aspheric lenses were used for coupling and collimation. The long wavelengths of the continuum and the pump are overlapped in space with a dichroic mirror (DM) and in time with a translation stage in the pump arm $(\tau)$. The polarizations of seed and pump are optimized for maximal conversion to the mid-IR. After the nonlinear crystal, the beams are collimated. The mid-IR is separated by means of a beam splitter (BS) which was coated in-house. The mirrors in the pump arm and in front of the fiber were dielectric, the others metallic. 
power may therefore grow with $\exp \left\{\alpha \sqrt{I_{P}} L\right\}$ where $\alpha$ is a constant (assuming no pump depletion and perfect phase-matching). This is explainable by the stimulated emission-like behavior of the down-conversion process (cf. section 1.4.6). Every fundamental process leads to the generation of an additional seed photon which may itself trigger another three-wave mixing process. Subsequently, the seed undergoes a coherent build-up in the nonlinear crystal, and therefore grows together with the mid-IR super-linearly if enough pump photons are available. This is in contrast to the sum-frequency generation process (in particular SHG, c.f. Eq. (3.26)) and the (typical) DFG process where $g L$ is small (or even imaginary) which leads to a linear growth of $I_{I}$ with $I_{P}{ }^{184}$. Consequently, OPAs are usually clearly more efficient than DFG experiments, but slightly less efficient than OPOs due to the weaker seed. Contrary to OPOs, OPAs neither need active cavity length stabilization nor active carrier-envelope-offset frequency stabilization, and are thus much simpler to realize.

Fig. 4.1 shows the generic setup which was used in the experiments presented here. The $50 \mathrm{~W}$ output of the KLM TD oscillator directly serves as the pump source for the downconversion process. The broadband seed is generated in one of the normal dispersion fibers investigated in section 3.1.1. The long-wavelength parts of the fiber continua are collinearly and temporally overlapped with the pump radiation by means of a dichroic mirror and sent into a nonlinear crystal, generating the mid-IR. The setup allows avoiding spatial chirp of the idler and passive stabilization of its $\mathrm{CEP}^{392,393}$. Splitting near-IR and mid-IR radiation at high average powers is non-trivial. Commonly used absorbing materials, in particular germanium, could not be utilized since beam distortions and saturation effects at more than $10 \mathrm{~W}$ average power were observed. Moreover, to the best of the author's knowledge, no standard optics based on non-absorbing materials are commercially available. Therefore, the beam splitters were coated in-house. In general, it is easier to produce an HR coating for the near-IR parts while the mid-IR is transmitted. This is
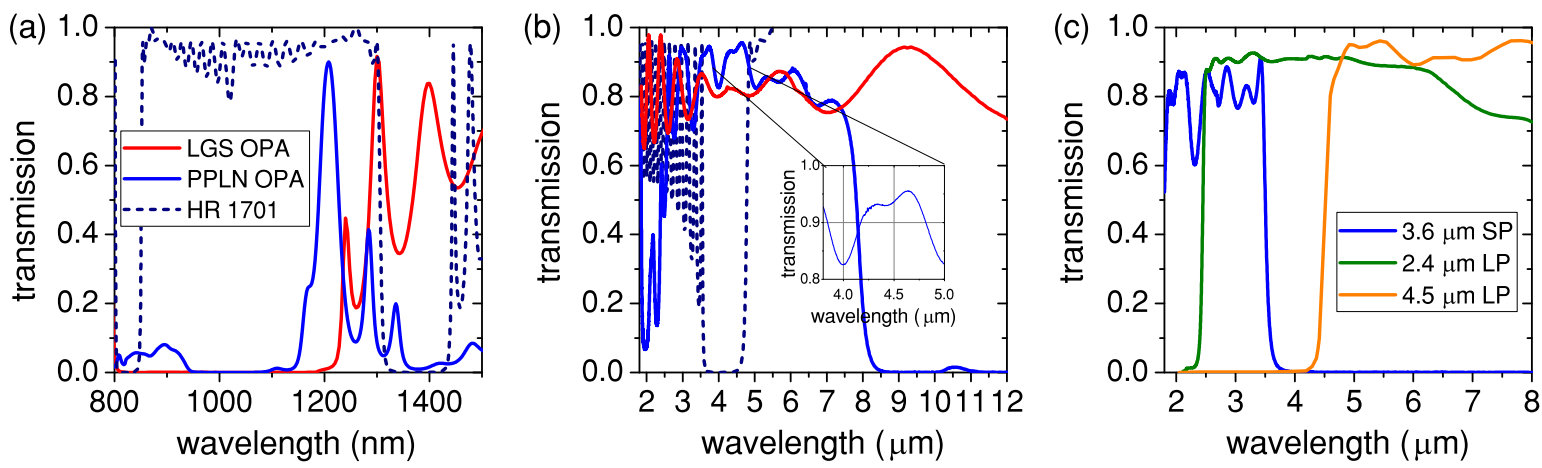

Fig. 4.2. (a) near-IR transmission of the utilzed beam splitters. The solid lines show the beam splitter where residual pump light is almost completely reflected and the signal is also strongly attenuated. The dashed dark blue line shows the transmission of the HR mirror centered at $4.1 \mu \mathrm{m}$. Most of the pump light is transmitted. The signal is unfortunately also strongly reflected. (b) mid-IR transmission of the utilized beam splitters. The mid-IR transmission of the PPLN OPA output was between 83 and $95 \%$ (inset). The transmission of the LGS OPA was between $75 \%$ and $94 \%$. The HR1701 nearly perfectly reflects around the central wavelength, causing hardly any losses of the mid-IR radiation. Absorption in the FS substrate is not considered in this theoretical curve. Actually, the mirror would be opaque beyond $4.5 \mu \mathrm{m}$. (Courtesy: Florian Habel, Vladimir Pervak) (c) Blue and orange solid lines: Transmission of the commercial shortpass (SP) and longpass (LP) filters used for the power measurements that are shown in Fig. 4.25. Green solid line: transmission of a $1 \mathrm{~mm}$ thin longpass filter used after HR1701 to separate the mid-IR from the residual near-IR. 
because shorter wavelengths require thinner coating layers which promises higher robustness. However, the materials should neither absorb the near-IR nor the mid-IR. This is in particular challenging for idler wavelengths longer than $5 \mu \mathrm{m}$ where oxides exhibit strong (multi-)phonon absorptions. Moreover, the GVD of the substrate may severely chirp the short idler pulses. Table 4.1 lists a few examples for typical substrate materials and the used nonlinear crystals. A dispersion controlled HR mirror for the mid-IR would avoid this issue. One example, HR1701, is shown in Fig. 4.2. It is a simple quarter-wave stack (often also called distributed BRAGG reflector) for high reflection of $4.1 \mu \mathrm{m}$ radiation. Unfortunately, it was coated in-house just at the very end of the experimental campaign, and thus beam splitters transmitting the idler were used in most of the experiments. Their curves are also shown in Fig. 4.2. To minimize near-IR absorptions in the dielectric layers, oxides $\left(\mathrm{SiO}_{2}, \mathrm{Nb}_{2} \mathrm{O}_{5}\right)$ were used for the PPLN OPA beam splitter while a combination of a sulfide $(\mathrm{ZnS})$ and a fluoride $\left(\mathrm{LaF}_{3}\right)$ was used for the LGS OPA beam splitter. The substrates were chosen such that the mid-IR only underwent moderate group delay dispersion. For the PPLN OPA $\mathrm{CaF}_{2}$, and for the LGS OPA ZnSe were used. Initial experiments utilized different beam splitters with lower efficiency. They will be described in the context of the respective experiments.

Owing to the very high average power levels, avoiding absorptions was also a major criterion in the choice of the nonlinear crystals. The beams were focused tightly to efficiently down-convert the pump radiation. Therefore, only linear, but also multi-photon absorptions had to be largely suppressed. Consequently, PPLN and LGS with bandgaps of about $4 \mathrm{eV}^{394}$ and $3.8 \mathrm{eV}^{185}$ (values vary slightly in literature), resp. were selected. The bandgaps are more than three times higher than the photon energy at $1030 \mathrm{~nm}$ vacuum wavelength, and hence no sign of detrimental multi-photon absorption was apparent even at maximal pump powers of $42 \mathrm{~W}$ and $30 \mathrm{~W}$, resp. In addition, the nonlinear crystals were fixed in a temperature controlled copper mount which was heated to about $60^{\circ} \mathrm{C}$. This was done to reduce thermal effects in the crystals but also to avoid photorefraction and green induced infrared absorption in PPLN.

Table 4.1: Examples of GVDs at pump and idler wavelengths ${ }^{a}$.

\begin{tabular}{lccc}
\hline material & GVD at $\mathbf{1 . 0 3} \mu \mathbf{m}$ & GVD at $\mathbf{4 . 1} \mu \mathbf{m}$ & GVD at $\mathbf{9} \mu \mathbf{m}$ \\
\hline $\mathrm{FS}$ & $19 \mathrm{fs}^{2} / \mathrm{mm}$ & practically opaque & opaque \\
sapphire $^{b}$ & $32 \mathrm{fs}^{2} / \mathrm{mm}$ & $-1700 \mathrm{fs}^{2} / \mathrm{mm}$ & opaque \\
$\mathrm{LiNbO}_{3}{ }^{b}$ & $251 \mathrm{fs}^{2} / \mathrm{mm}$ & $-1694 \mathrm{fs}^{2} / \mathrm{mm}$ & opaque \\
$\mathrm{CaF}_{2}$ & $18 \mathrm{fs}^{2} / \mathrm{mm}$ & $-304 \mathrm{fs}^{2} / \mathrm{mm}$ & $-5300 \mathrm{fs}^{2} / \mathrm{mm}$ \\
$\mathrm{LGS}^{b, c}$ & $300 \mathrm{fs}^{2} / \mathrm{mm}$ & $-147 \mathrm{fs}^{2} / \mathrm{mm}$ & $-2405 \mathrm{fs}^{2} / \mathrm{mm}$ \\
$\mathrm{ZnSe}$ & $663 \mathrm{fs}^{2} / \mathrm{mm}$ & $67 \mathrm{fs}^{2} / \mathrm{mm}$ & $-820 \mathrm{fs}^{2} / \mathrm{mm}$ \\
$\mathrm{GaAs}$ & $4553 \mathrm{fs}^{2} / \mathrm{mm}$ & $388 \mathrm{fs}^{2} / \mathrm{mm}$ & $-612 \mathrm{fs}^{2} / \mathrm{mm}$ \\
$\mathrm{ZGP}$ & practically opaque $^{b}$ & $185 \mathrm{fs}^{2} / \mathrm{mm}$ & $-1381 \mathrm{fs}^{2} / \mathrm{mm}$ \\
$\mathrm{Si}$ & practically opaque & $375 \mathrm{fs}^{2} / \mathrm{mm}$ & $168 \mathrm{fs}^{2} / \mathrm{mm}$ \\
$\mathrm{Ge}$ & opaque & $1040 \mathrm{fs}^{2} / \mathrm{mm}$ & $425 \mathrm{fs}^{2} / \mathrm{mm}$ \\
\hline${ }^{a}$ Values taken from https://refractiveindex. info/ or SNLO software \\
(http://wwr.as-photonics. com/snlo). URLs accessed 04/2017. \\
Refers to the value of the fast axis. ${ }^{c}$ Refers to the value at phase-matching angle.
\end{tabular}




\subsubsection{Frequency Down-Conversion with Periodically Poled Lithium Niobate}

Parts of this section have been published in:

M. Seidel, X. Xiao, S.A. Hussain, G. Arisholm, A. Hartung, K.T. Zawilski, P.G. Schunemann, F. Habel, M. Trubetskov, V. Pervak, O. Pronin, F. Krausz, "Multi-Watt, multioctave, mid-infrared femtosecond source," Science Advances 4 eaaq1526 (2018).

\section{Seed generation}

For generating idler photons at $\lambda_{I}$ with an OPA, seed photons at $\lambda_{S}=\left(\lambda_{P}^{-1}-\lambda_{I}^{-1}\right)^{-1}$ have to be generated first. If $\lambda_{P}=1030 \mathrm{~nm}, \lambda_{I}=4 \mu \mathrm{m}$ requires a seed wavelength of $1.39 \mu \mathrm{m}$, i.e. very strong spectral broadening of the oscillator output towards longer wavelengths. Alternatively, the pump could be frequency doubled (cf. e.g. ref. 47). However, it would then carry $2 f_{\text {ceo }}$ while the seed would carry only $1 f_{\text {ceo }}$, and hence the idler would not be passively CEP stabilized. Moreover, using the SH would increase the GVD of the pump and reduce the damage irradiance in the nonlinear crystal due to the lower ratio between photon energy and bandgap of the nonlinear crystal. Therefore, continuum generation for seeding the OPA was preferred. Elsewhere, this was realized by exploiting the RAMAN soliton red-shift in anomalously dispersive fibers, ${ }^{395}$ supercontinuum generation (SCG) in $\mathrm{YAG}^{396}$ or in waveguides ${ }^{397}$. In sections 2.3 .2 and 3.2 .1 , the noise issues with anomalously dispersive fibers and with white-light continuum generation in bulk were described. Therefore, these approaches were not favored. Likewise, the typically relatively high nonlinear refractive indices of waveguides in comparison to FS fibers appears also not ideal for high-power pump sources. Consequently, the results of section 3.1.1 were exploited and the continuum generated by an ANDi fiber was utilized.

Initially, a non-PM fiber was used. The output spectra after $10 \mathrm{~cm}$ and $20 \mathrm{~cm}$ of propagation are shown in Fig. 4.3(a). Apparently, the spectrum of the $10 \mathrm{~cm}$ long fiber only
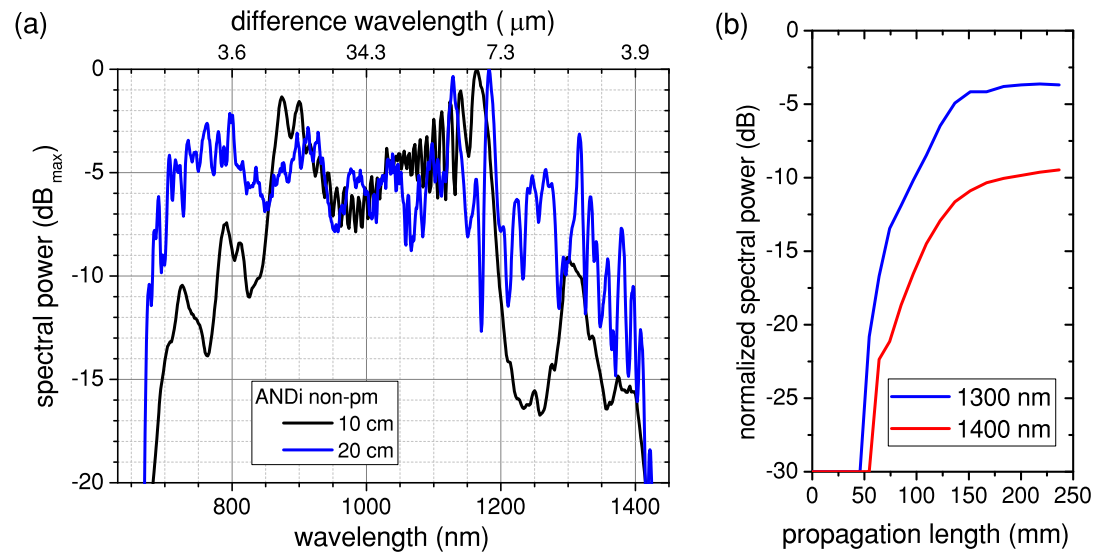

Fig. 4.3. (a) Spectra of non-polarization maintaining (PM) ANDi fibers. A $20 \mathrm{~cm}$ long fiber with collapsed end facets could be pumped with up to $1.5 \mathrm{~W}$ (blue line). For comparison, the broadening result of Fig. 3.2 is shown. It emerged from a shorter $(\approx 10 \mathrm{~cm})$ non-PM fiber (black line). The shorter fiber reveals the typical spectral features of SPM and OWB, while the longer fiber shows a broader, but rather randomly modulated spectrum. The difference wavelength shown on the top horizontal axis corresponds to the idler wavelength which was calculated by $\lambda_{I}=\left|(1.03 \mu \mathrm{m})^{-1}-\lambda_{S}^{-1}\right|^{-1}$. (b) Simulation of the relative power in the range of the seed wavelengths between 1300 and $1400 \mathrm{~nm}$. The numerical model from ref. 262 was used. An incident average power of $750 \mathrm{~mW}$ was assumed which is in good agreement with the coupled power in the experiments. (Courtesy: Alexander Hartung) 
(a)

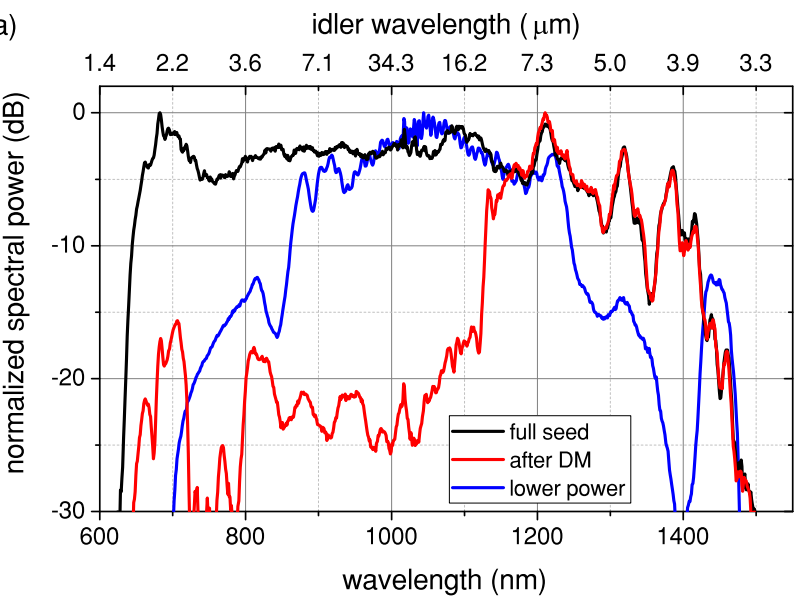

(b)

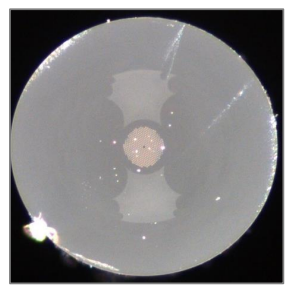

(c)

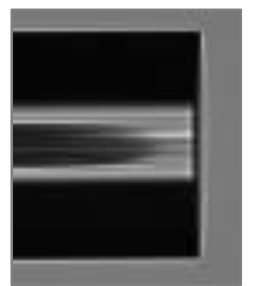

Fig. 4.4. (a) Spectra of PM-ANDi fibres. The black line displays the full spectrum emerging from a $17 \mathrm{~cm}$ long fibre with collapsed end facets. The red line shows the spectrum after the dichroic mirror which enters the nonlinear crystal. The blue spectrum shows a distinct spike between $1400 \mathrm{~nm}$ and $1450 \mathrm{~nm}$ which is not connected to the self-phase modulation broadened spectrum around the central wavelength. It is not predicted in simulations. The red spectrum was used for seeding the OPA. The spectrum is clearly less modulated than the spectrum of the non-PM fibre. (b) Top-view of collapsed ANDi-PM fiber. The fiber diameter is $200 \mu \mathrm{m}$ while the core diameter is only about $3 \mu \mathrm{m}$. The stress rods which lead to an intentionally high linear birefrigence $\left(\approx 4 \times 10^{-4}\right)^{371}$ are clearly visible. (c) Sideview of collapsed ANDi-PM fiber. The air holes are collapsed up to about $50 \mu \mathrm{m}$ distance to the surface. (Courtesy: Alexander Hartung).

contains a small amount of power in the region between $1300 \mathrm{~nm}$ and $1400 \mathrm{~nm}$ which is of interest for seeding. The simulations presented in Fig. 4.3(b) reveal that only after 15 - $20 \mathrm{~cm}$ propagation a maximal power transfer to the seed wavelength has taken place. Longer fibers would introduce additional chirp and, for much longer fibers, also mutual decoherence of the pulses ${ }^{398}$. Consequently, a fiber length of $20 \mathrm{~cm}$ was initially chosen for the experiments. The blue curve in Fig. 4.3(a) displays the output spectrum. Contrary to the shorter fiber, the features of SPM and OWB are not clearly recognizable anymore. Instead, the spectrum is strongly modulated over the full range. LIU et al. explain this observation through the slight birefrigence of the non-PM ANDi fiber which leads to an incoherent supercontinuum for $230 \mathrm{fs}$ input pulses ${ }^{371}$. The polarization rotation effect was not considered in earlier simulations which claimed that highly nonlinear, but also all-normal dispersive fibers maintain full coherence even upon supercontinuum generation with $300 \mathrm{fs}$ input pulses ${ }^{262}$. But the observation agrees well with the inability to detect a beat note in the f-to-2f interferometer being directly pumped with KLM TD oscillator output (cf. section 3.3). Following the studies of DomingUE et al., encountering a similar issue with ultra-high numerical aperture fibres ${ }^{399}$, the authors of ref. 371 could show that using the PM version of the ANDi fiber (NL-1050-Neg-PM, NKT Photonics), improves the coherence of the output spectrum significantly - at least for a spectral extension up to $1300 \mathrm{~nm}$.

The output spectrum after a $17 \mathrm{~cm}$ PM-fiber (shown in Fig. 4.4(a)) exhibited less modulations than that of the non-PM version and led to an about $25 \%$ increase in maximal mid-IR power output of the OPA. Its transverse profile is shown in Fig. 4.4(b). The mode-field diameter was only $2.7 \mu \mathrm{m}$ at $1064 \mathrm{~nm}$. As pointed out in section 3.1.1, the need for tight focusing yielded very high peak irradiances at the entrance surface exceeding $2 \mathrm{TW} / \mathrm{cm}^{2}$. This resulted in degradation after a few weeks of operation. Collapsing 
(a)

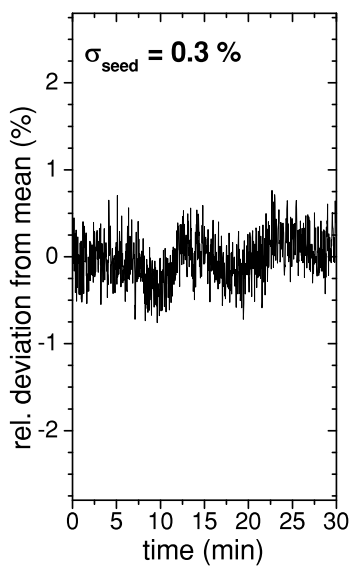

(b)

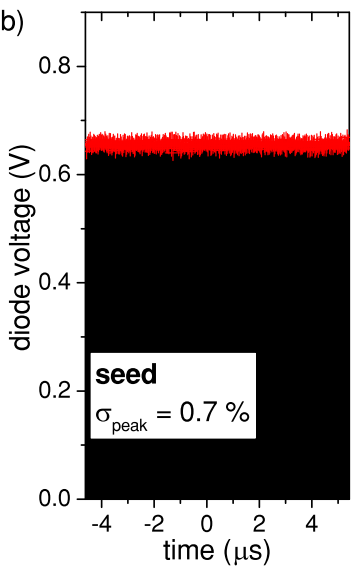

(c)

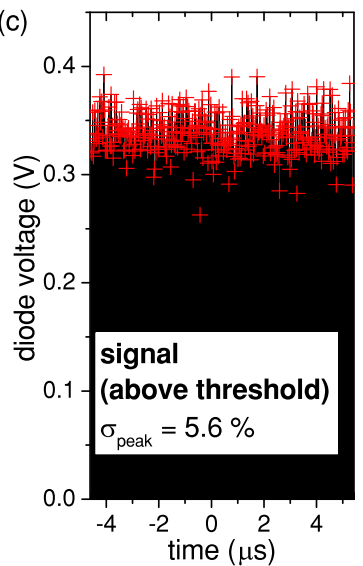

(d)

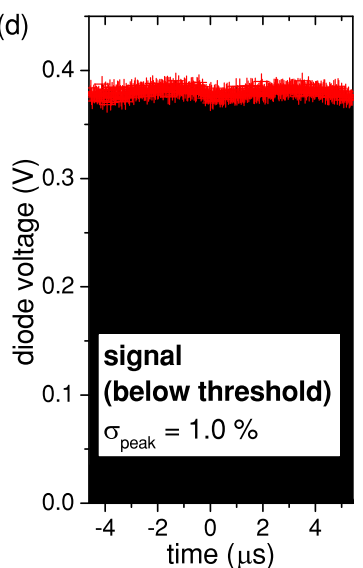

Fig. 4.5. (a) Stability of the fiber continuum emerging from a $17 \mathrm{~cm}$ long ANDi-PM fiber. It was measured with a thermal detector taking one data point every two seconds. (b) - (d) Pulse-to-pulse fluctuations of the fiber-continuum (b), the OPA signal above (c) and below (d) the spectral noise threshold (both measured at full pump power). The traces span over a $10 \mu$ s time window corresponding to a train of 375 pulses. Data was taken with a $\mathrm{GHz}$ bandwidth InGaAs diode (responsive from $900 \mathrm{~nm}$ to $1700 \mathrm{~nm}$ ) and sampled with a digital oscilloscope at a rate of $40 \times 10^{9} \mathrm{~s}^{-1}$. The RMS was calculated from the peak voltages marked with a red cross akin to the characterization in ref. 395 .

the air holes at the front facet eliminated this issue. The length of the collapse was about $50 \mu \mathrm{m}$ (cf. Fig. 4.4(c)). While an $f=3 \mathrm{~mm}$ focal length was used for uncollapsed fibers, an $f=4.5 \mathrm{~mm}$ focal length was used for the collapsed ones. The coupling efficiencies were slightly lower after collapsing (usually between 40 and $50 \%$ measured at $300 \mathrm{~mW}$ input power), but at least $50 \%$ more pump power could be sent onto the facet (up to $1.5 \mathrm{~W}$ tested) resulting in comparable spectral widths. No collapsed fiber was damaged during the course of the experiments.

The stability of the fiber output was characterized by three different measurements ${ }^{395}$. Firstly, slow fluctuations were measured over a time of about 30 minutes (Fig. 4.5(a)). Every $2 \mathrm{~s}$ a data point was taken. The fiber output seems to follow the long-term drifts of the TD oscillator which are indicated in Fig. 4.8(a). Nonetheless, the relative power RMS was only $0.3 \%$, indicating good long-term stability of the seed continuum. Secondly, the pulse-to-pulse fluctuations of the seed was measured with a fast InGaAs diode. It was below a $1 \%$ rms value as well (Fig. 4.5(b)). Thirdly, the pulse-to-pulse fluctuations of the signal were measured which was done for two reasons: The intensity noise of the seed is averaged over the full spectrum. However, typically the noise of the spectral wings is higher than that of the center due to coupling of intensity noise to the spectral broadening in the fiber. The signal after optical parametric amplification therefore provides a measure of the spectrally filtered seed noise under the assumption of a stable pump source. Moreover, no fast photodiode at $4 \mu \mathrm{m}$ was available and hence the pulse-to-pulse fluctuations of the idler could not be directly accessed. Nonetheless, due to the simultaneous decay of the pump photons into idler and signal photons, the fluctuations of the mid-IR can be derived from the signal as well.

In a first measurement of fast signal noise, a spontaneous inset of strong pulse-to-pulse fluctuations was observed when the ANDi-PM fiber was pumped with $1.5 \mathrm{~W}$ of input power (Fig. 4.5(c)). It is inferred, that despite the low relative intensity noise of the seed, it is prone to exhibit strong spectral noise which is akin to the statements of refs. 371 and 
399. Crucially, the observed fluctuations did not continuously increase with input power but exhibited a threshold. Consequently, the OPA was operated below this threshold (Fig. 4.5(d)) where pulse-to-pulse fluctuations of $1 \%$ RMS were determined. Noise suppression was realized by means of the half-wave plates (1) and (3) shown in Fig. 4.1. With half-wave plate (1) the input power at the fiber facet was slightly attenuated. Half-wave plate (3) was used for adjusting the light polarization with respect to the fiber's slow axis. A precise characterization of input polarization and power has not been performed. The optimization was done by direct monitoring of the signal pulse train. The idler power emerging from the OPA was not affected by this routine. The origin of the spontaneous fluctuation onset is unknown. It has neither been reported in ref. 371, nor predicted by very recent simulations ${ }^{398}$. These papers also did not present the pronounced spike in the spectrum between $1400 \mathrm{~nm}$ and $1450 \mathrm{~nm}$ which is visible in blue spectrum of Fig. 4.4(a). The origin of this spectral feature and a connection to the onset of strong fluctuations could not yet be clarified and was not investigated further. The primary interest within the scope of the presented experiments was the stability of the mid-IR which was good after the described adjustments.

\section{OPA simulations and experiments}

The very strong dependence of the idler power on the pump irradiance was indicated in Eq. (4.1). Therefore, it is instructive to investigate the irradiance dependent damage threshold of the nonlinear crystal for optimized focusing in the OPA setup. For $\mathrm{MgO}: \mathrm{LiNbO}_{3}$, the threshold was determined by exposing a $0.5 \mathrm{~mm}$ thick crystal to the pump radiation which was focused to a $100 \mu \mathrm{m}$ spot diameter. Upon increasing the pump power, the beam profile behind the crystal and the crystal temperature were monitored by a CCD and a thermal camera, resp. Damage occurred at an incoming average power of about $24 \mathrm{~W}$ which corresponds to a peak irradiance of about $56 \mathrm{GW} / \mathrm{cm}^{2}$. The usage of a thin crystal was intended to exclude propagation effects like self-focusing. Those were included in simulations with the SISYFOS code ${ }^{266,267}$. It has been already extensively used in the spectral broadening experiments of chapter 3, but was originally designed for frequency mixing experiments. The goal of the simulations was to determine optimal focal sizes, maximal pump powers and best crystal lengths for achieving maximal mid-IR power.

The pump was assumed to exhibit perfect $230 \mathrm{fs}$ width $\mathrm{sech}^{2}$-shape. The seed was extracted from a cross-correlation frequency resolved optical gating (X-FROG) measurement where the fiber continuum was sampled by the oscillator output (cf. Appendix A.1). Both beams were assumed to be of perfect Gaussian shape. Since pump, signal and idler are polarized along the extraordinary axis of PPLN, a single large temporal grid was utilized. It consisted of 2048 points with $1 \mathrm{PHz}$ width and the central frequency of 500 THz. Due to the spherical symmetry of the propagation, only a quarter of beam was simulated within a grid of $65 \times 65$ points and a spacing of about $24 \mu \mathrm{m}$ in horizontal and vertical direction. The simulations included dispersion at $50^{\circ} \mathrm{C}^{400}$, transmission ${ }^{401}$, the largest $\chi^{(2)}$-tensor element $\left(d_{33}=25 \mathrm{pm} / \mathrm{V}\right)^{402}$ and a nonlinear refractive index of $n_{2}=16.5 \times 10^{-16} \mathrm{~cm}^{2} / \mathrm{W}^{403}$. Every domain of the periodically poled crystal was computed individually, i.e. the effective nonlinearity arose from the phase-mismatch of the three waves and not from setting $d_{\text {eff }}=2 d_{33} / \pi$. 

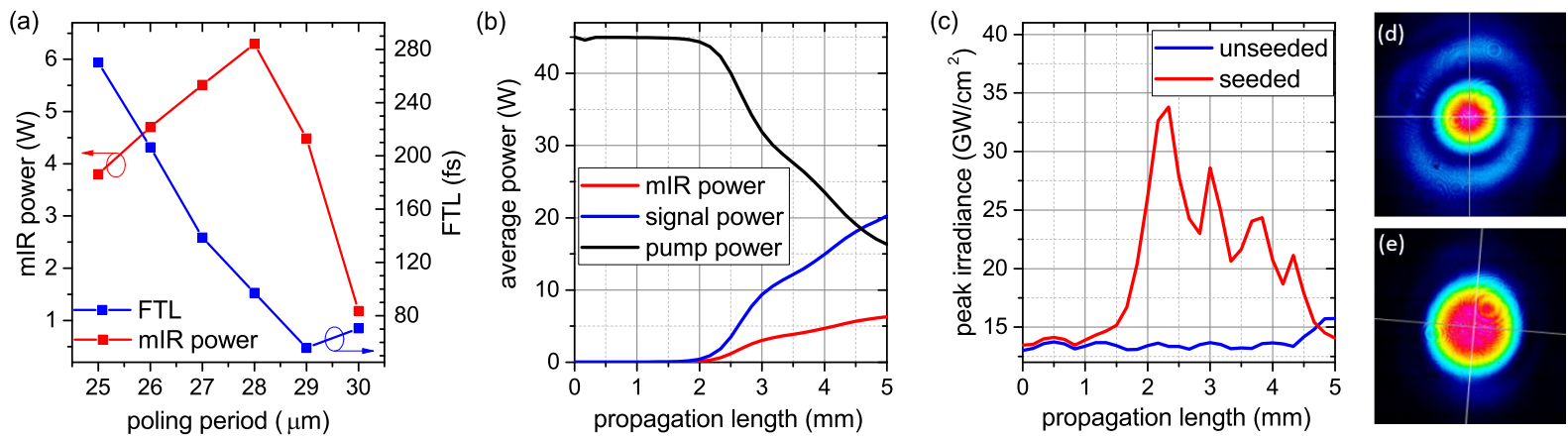

Fig. 4.6. (a) Simulated average powers and FTLs for different poling periods of the PPLN OPA. (b) Evolution of the pump, signal and mid-IR power inside a PPLN crystal with $28 \mu \mathrm{m}$ poling period. (c) Evolution of the peak irradiance inside a PPLN crystal with $28 \mu \mathrm{m}$ poling period for a seeded OPA (red solid line) and solely the pump radiation (blue solid line). The results shown in (a)-(c) were obtained with a $5 \mathrm{~mm}$ thick PPLN crystal and $45 \mathrm{~W}$ of pump power focused to a diameter of $300 \mu \mathrm{m}$. (d) Pump beam profile measured with a CCD camera behind the seeded OPA. (e) Pump beam profile if seed was blocked. The profiles shown in (d)-(e) were obtained with a $5 \mathrm{~mm}$ thick PPLN crystal and $5 \mathrm{~W}$ of pump power focused to a diameter of $120 \mu \mathrm{m}$.

Fig. 4.6(a) shows power and bandwidth dependence of the mid-IR radiation on the poling period of a $5 \mathrm{~mm}$ thick PPLN. The highest average power was reached for a tuning period of $28 \mu \mathrm{m}$. The bandwidth of the mid-IR pulses could be even increased by utilizing longer poling periods. However, this would come at the expense of mid-IR output power. Consequently, the SCG experiments presented in section 4.2 were performed with the poling period set to $28 \mu \mathrm{m}$. Fig. 4.6(b) displays how the power levels of the pump, signal and idler evolve inside the crystal. The conversion process gets saturated after a propagation length of about $3 \mathrm{~mm}$. At this point, the signal power is about one third of the pump power. The simulation predicts a further pump depletion to about $36 \%$ of the initial $45 \mathrm{~W}$. It is remarkable that no sign of back-conversion or temporal walk-off are apparent. LUTHER-DAVIES and YU explain this by the effect of parametric gain guiding ${ }^{404}$. Simulations with thicker crystals did not yield significantly more mid-IR power and hence, a $5 \mathrm{~mm}$ thick crystal was chosen for the experiments.

Recent studies on PPLN OPAs with pump pulses of hundreds of $\mu \mathrm{J}$ energies with sub- $\mathrm{MHz}$ repetition rate $(40$ - $80 \mathrm{~W}$ average power) have revealed strong pump beam distortions which were attributed to high average power and photorefraction in ref. 405 and predominantly to high peak power in ref. 406. The latter distortions were qualitatively also apparent in the presented experiment, but were taken into account when choosing an appropriate spot size for efficient pumping. The simulation results shown in Fig. 4.6(c) demonstrate in accordance with the observations in ref. 406 a strong peak irradiance enhancement in the presence of the weak seed (carries about $1 \%$ of the input power). A typical self-focusing induced beam pattern (like in the studies of section 3.2.1) of the pump radiation was observed when the OPA was seeded at high pump powers (Fig. 4.6(d)). If the temporal overlap of pump and seed was removed, the ring pattern almost completely vanished (Fig. 4.6(e)). This is in contrast to the observations of ref. 405 where the beam distortions were also visible when the seed was blocked. This manifests the instantaneous nature of the distortions in the performed experiments. Since the conducted simulation did neither include thermal lensing, nor photorefraction, nor green induced infrared absorption, it is inferred that the parasitic effect originates from cascaded quadratic non- 
(a)

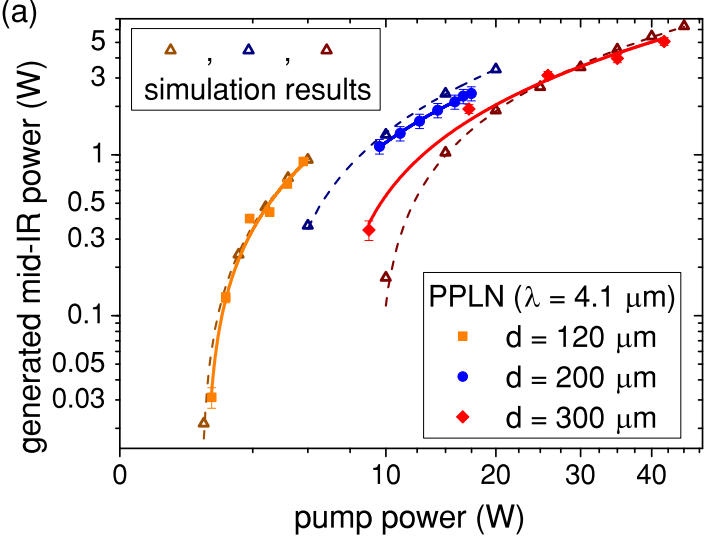

(b)

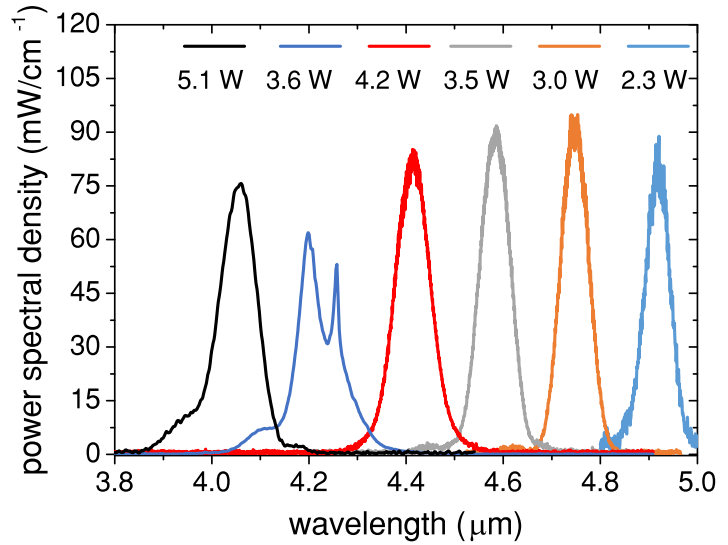

Fig. 4.7. (a) Idler power measurements for different pump spot diameters denoted by d. The x-axis scales with the square root of the pump power while the y-axis is logarithmic. According to Eq. (4.1), this plot would result in straight lines for an undepleted pump. Consequently, the plot reveals saturation of the OPAs. This results in linear growth of the idler power with the pump power. The lines present the best linear fits of the simulation (dashed lines) and measurement (solid lines) points. Maximum idler power values and efficiencies are listed in Table 4.2. All power values have been corrected for reflection losses. For instance, the losses $l_{R}$ for the idler symbolized by the red dots were calculated by $l_{R}=1-0.85 \cdot 0.8544 \cdot 0.46 \cdot 0.98^{3}=69 \%$ where 0.85 results from the beam splitter transmission, 0.8544 from the PPLN back reflection, 0.98 from the silver mirror reflectivity and 0.46 from the transmission of an uncoated $5 \mathrm{~mm}$ thick germanium plate which was inserted in front of the power meter to exclude residual transmission of signal or pump. For the two smaller spot sizes (orange and blue dots) near-IR and mid-IR separation was accomplished only by the germanium plate. These measurements were also done with a non-PM ANDi fiber. (b) Generated mid-IR power for about $42 \mathrm{~W}$ pump power and tuning periods from 28 to $25.5 \mu \mathrm{m}$ (from left to right in $-0.5 \mu \mathrm{m}$ steps) of the PPLN. The total generated idler power is stated in the legend above the curves. The spectrum centered at $4.2 \mu \mathrm{m}$ is shaped through $\mathrm{CO}_{2}$ absorptions. The power was measured $25 \mathrm{~cm}$ behind the nonlinear crystal. For accurate loss corrections, spectra and the transmission of the beam splitter shown in Fig. 4.2(b) were multiplied. The spectra were measured with a Bristol 721 spectrum analyzer which did not allow to measure beyond $5 \mu \mathrm{m}$.

linearities which are accounted for in the propagation code. To stay below the initially determined damage threshold, the peak irradiance at the front facet of the PPLN was always set below $15 \mathrm{GW} / \mathrm{cm}^{2}$, i.e. clearly below the $50 \mathrm{GW} / \mathrm{cm}^{2}$ sent into the $0.5 \mathrm{~mm}$ crystal.

Experiments were performed with three different pump and seed spot sizes to check if thermal nonlinearities adversely affect the OPA performance. An $f=150 \mathrm{~mm}$ lens was chosen first, yielding a $120 \mu \mathrm{m}$ pump spot size. Pump powers up to $5 \mathrm{~W}$ were sent. The orange dots in Fig. 4.7(a) show the experimentally achieved idler powers which were in excellent agreement with simulated powers that are shown in dark orange. Secondly, an $f=300 \mathrm{~mm}$ lens was chosen, resulting in a $200 \mu \mathrm{m}$ pump spot size. Pump powers up to $17.5 \mathrm{~W}$ were tested, yielding the mid-IR powers depicted by the blue dots of Fig. 4.7(a). They are in reasonable accordance with the simulation results (dark blue dots). A good agreement, in particular for higher pump powers, was also achieved for the measurements with an $f=400 \mathrm{~mm}$ lens (red dots Fig. 4.7(a)), yielding a $300 \mu \mathrm{m}$ spot size. In this case the full KLM TD oscillator power could be utilized, resulting in more than $5 \mathrm{~W}$ of average power at around $4 \mu \mathrm{m}$ central wavelength, i.e. about $10 \%$ of the initial near-IR power could be converted to the mid-IR. 
Table 4.2: Generated mid-IR powers and efficiencies for different pump spot sizes.

\begin{tabular}{cccc}
\hline pump diameter & max. mid-IR power & quantum efficiency $^{a}$ & slope efficiency (simulated) \\
\hline $120 \mu \mathrm{m}$ & $0.9 \mathrm{~W}$ & $76 \%$ & $23.6 \%(23.0 \%)$ \\
$200 \mu \mathrm{m}$ & $2.4 \mathrm{~W}$ & $55 \%$ & $16.3 \%(20.3 \%)$ \\
$300 \mu \mathrm{m}$ & $5.1 \mathrm{~W}$ & $48 \%$ & $14.9 \%(17.5 \%)$ \\
\hline
\end{tabular}

$a$ The power efficiency is $25 \%$ of the quantum efficiency. The values were calculated from the ratios of generated idler and reflection loss corrected pump power.

The quantum efficiency of the OPA is generally very high. Up to about $75 \%$ of the pump photons could be down-converted for the tightest focusing configuration. Due to the reduced seed irradiance for the other configurations (seed power is constant while spot size changes), the efficiencies are lower but even for the setup with largest spot sizes quantum efficiencies of nearly $50 \%$ were maintained. This implies, of course, that Eqs. (4.1) - (4.3) are not applicable anymore as Fig. 4.7(a) demonstrates. The idler power rises linear with the pump power as the linearly fitted curves in the plot show. Table 4.2 summarizes the fit results in terms of power slope efficiencies and also provides an overview about achieved power levels and quantum efficiencies. The highest possible slope efficiency is $25 \%$ due to the quantum defect in the down-conversion process. This value is nearly reached for the tight focusing configuration. For loose focusing, the quantum slope efficiency is still about $60 \%$.

The presented power measurements were conducted with a $28 \mu \mathrm{m}$ poling period of the PPLN, resulting in mid-IR radiation at about $4.1 \mu \mathrm{m}$. The utilized PPLN (HC Photonics) was manufactured with a multiple grating structure, containing the poling periods $25.5 \mu \mathrm{m}, 26 \mu \mathrm{m}, 26.5 \mu \mathrm{m}, 27 \mu \mathrm{m}, 27.5 \mu \mathrm{m}$ and $28 \mu \mathrm{m}$. Each grating had a $1 \times 1 \mathrm{~mm}^{2}$ aperture. The shorter the poling period, the longer the idler wavelength. Fig. 4.7(b) shows a tuning curve and the corresponding generated mid-IR power values which were determined at full pump power and a $300 \mu \mathrm{m}$ spot size. More than $2 \mathrm{~W}$ of average power could be reached for all available poling periods. The spectral coverage ranges from $3.8 \mu \mathrm{m}$ to $5 \mu \mathrm{m}$. It could be slightly extended if more poling periods would have been available. It is, however, fundamentally limited by the seed bandwidth on the short wavelength side (cf. Fig. 4.4(a)) and by the crystal transparency on the long wavelength side (cf. ref. 401).

\section{Characterization of the OPA output}

In addition to power and spectrum measurements, stability, pulse and beam of the mid-IR were characterized. The $f=400 \mathrm{~mm}$ lens and the $28 \mu \mathrm{m}$ poling period were utilized to get the most powerful idler output. The stability was measured over approximately 15 minutes with a thermal power meter (Coherent PS19). The result is shown in Fig. 4.8(a). For comparison, the leakage of the oscillator power, which could not be sent into the OPA due to imperfect polarization optics (about $230 \mathrm{~mW}$ ), was monitored with another thermal power meter (Coherent LM-200 XL, designed for up to $200 \mathrm{~W}, 10 \mathrm{~mW}$ resolution). Obviously, the mid-IR fluctuations follow the long-term drifts of the oscillator output. It is not clear where these slow fluctuations come from as they were not observed in Fig. 2.4(b), for instance. A similar behavior was, however, apparent in Fig. 4.3(c). It is to note that the utilized oscillator was not set up on a breadboard which makes it susceptible to external perturbations. The latest generation of KLM TD oscillator (cf. 


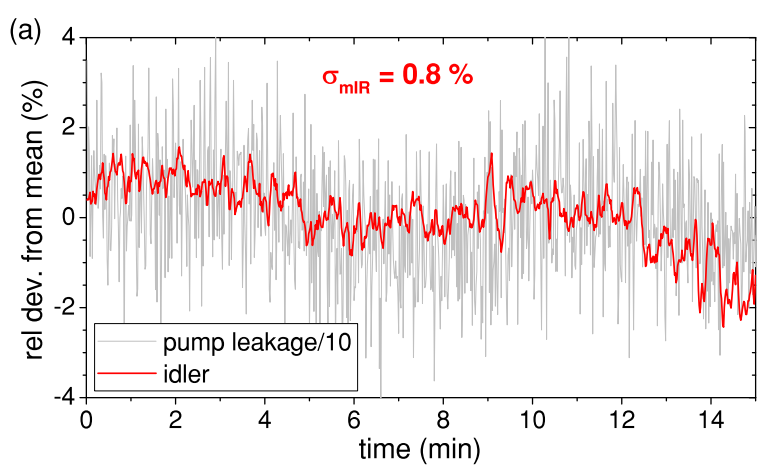

(b)

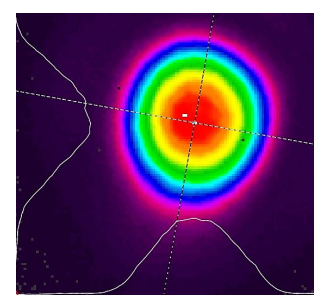

(c)

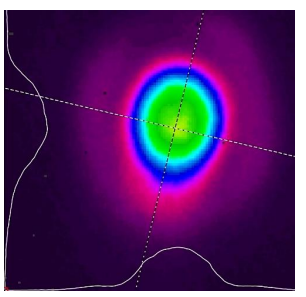

Fig. 4.8. (a) Stability of the mid-IR radiation measured at full pump power, i.e. the OPA generated more than $5 \mathrm{~W}$ of mid-IR power. The RMS was calculated from 946 shots measured at a sampling rate of $1 \mathrm{~Hz}$. The pump leakage (grey solid line) was monitored in parallel with the power meter that usually monitored the full oscillator output. It is clearly visible that the mid-IR fluctuations follow the oscillator fluctuations. (b) Beam profile of the mid-IR measured with about $20 \mathrm{~W}$ pump power. (c) Beam profile of the mid-IR measured at full pump power. An additional neutral density filter was used to not saturate the pyroelectric detector.

e.g. section 3.2.2) is better isolated from its environment and may strongly reduce these long term drifts. Nonetheless, the power stability $(0.8 \% \mathrm{RMS})$ of the mid-IR is good. It is, for instance comparable to the measurement of the soliton seeded double-stage OPA presented in ref. 395 which generated almost an order of magnitude less mid-IR power.

The idler beam profiles, shown in Figs. 4.8(b) and (c), were measured at $20 \mathrm{~W}$ pump power and at full pump power $(42 \mathrm{~W})$ with a pyroelectric detector array. At $20 \mathrm{~W}$ pump power the mid-IR is nearly perfectly bell-shaped which is a consequence of the collinear OPA geometry and the absence of spatial walk-off in the type 0 phase-matching geometry. If pumped with full power, the irradiance enhancement effect, shown in Fig. 4.6, sets in. The impact on the mid-IR profile is, however, clearly weaker than the impact on the pump profile. Although a slight ring formation becomes visible in Fig. 4.8(c), the beam profile is still good and definitely useable for nonlinear experiments as the SCG results presented in section 4.2 demonstrate.

(a)

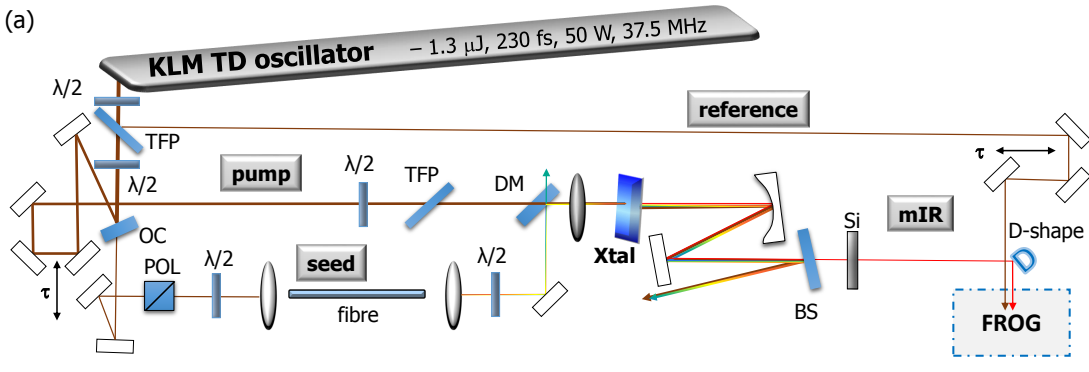

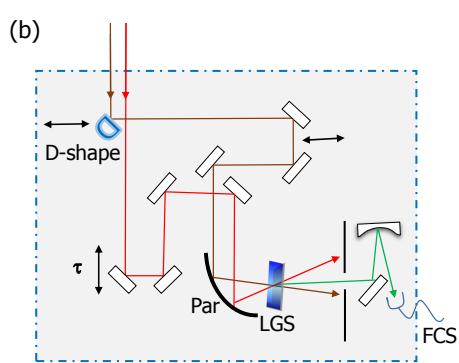

Fig. 4.9. (a) OPA setup with FROG. The power of the reference beam is adjusted between 0.3 to $1 \mathrm{~W}$ to take advantage of the full dynamic range of the grating spectrometer. The abbreviations in the OPA setup are like in Fig. 4.1. (b) Detailed FROG setup. The D-shape mirror is moveable. It may split the wavefront of the reference beam which allows to measure SH-FROG of the reference before conducting $\mathrm{X}$-FROG measurements. In its shown position, it reflects the full reference beam while the mid-IR is transmitted to a piezo-based translation stage. Both beams are noncollinearly overlapped in a $110 \mu \mathrm{m}$ thin LGS crystal. The resulting sum-frequency is focused with a spherical silver mirror into a multimode fiber which is coupled to a grating spectrometer (FCS) with a silicon detector array. All reflective optics, including the parabolic (Par) mirror, are silver coated. 

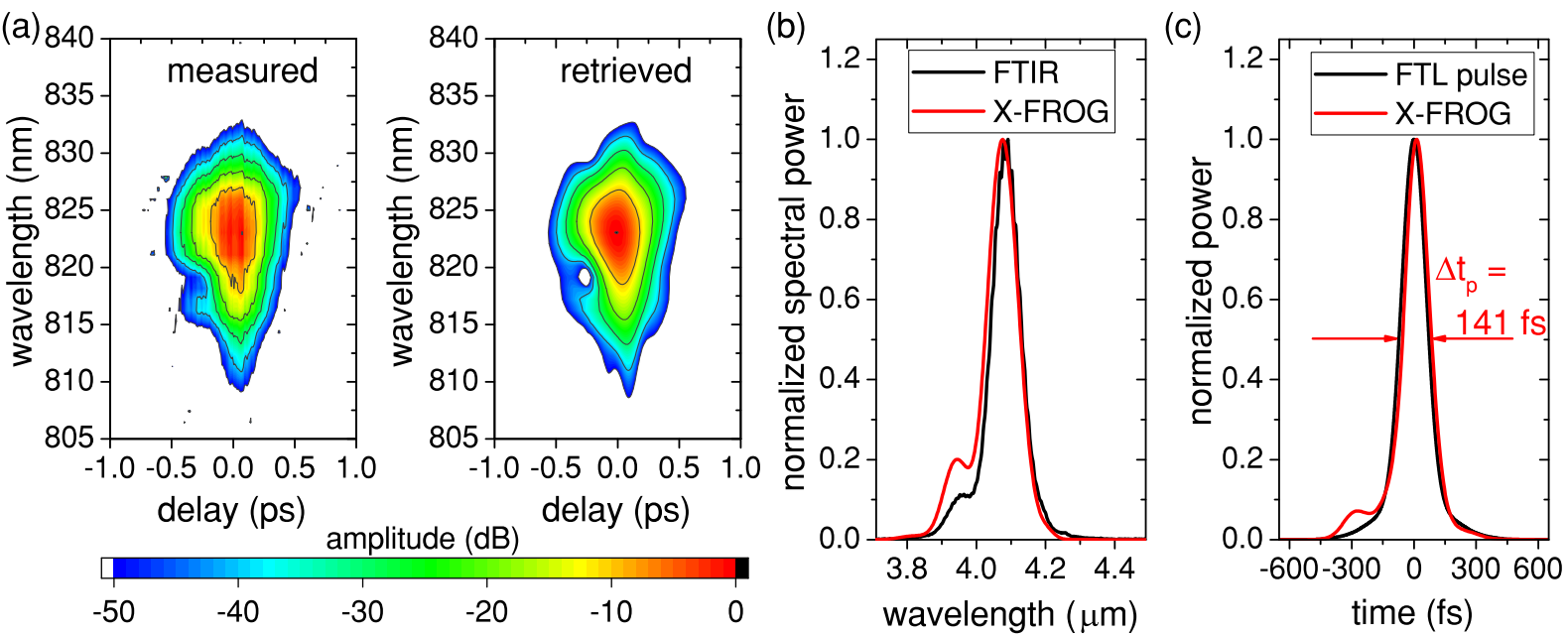

Fig. 4.10. (a) Measured (left) and retrieved (right) X-FROG traces of the idler pulse after beam splitting and chirp compensation. The color scale is in units of $\mathrm{dB}$ with respect to the maximum of the trace. The RMS FROG error was $0.50 \%$ for a $512^{2}$ grid size. (b) Comparison of the spectrum measured with the Bristol 721 spectrum analyzer (black solid line) and the one retrieved from X-FROG (red solid line). (c) Retrieved mid-IR pulse and calculated pulse from the FOURIER transformation of the FTIR spectrum. The FWHM practically corresponds to the transform-limit but the pedestal carries about $10 \%$ of the energy.

To measure the idler pulses, X-FROG measurements were performed. Contrary to selfreferencing techniques, X-FROG, as an up-conversion method, has the advantage that the radiation at $4 \mu \mathrm{m}$ can be characterized with sensitive and cost-efficient silicon detectors which only measure photons energies up to the bandgap of silicon of about $1.1 \mathrm{eV}$ (corresponds to $\approx 1.1 \mu \mathrm{m})^{386}$. The setup is sketched in Fig. 4.9. Essentially a small part of the oscillator output (only $\approx 1 \%$ ) was split in front of the OPA and temporally as well as spatially overlapped in the FROG which was used in the previous chapters in SH configuration and is described in more detail in appendix A.1. The mid-IR radiation was up-converted in a $110 \mu \mathrm{m}$ thick LGS crystal.

The optical layers of the dielectric beam splitter designed for separating near-IR and mid-IR (cf. blue solid line Fig. 4.2(a) and (b)) were coated on YAG, sapphire and $\mathrm{CaF}_{2}$ substrates. To keep the GDD as low as possible the $\mathrm{CaF}_{2}$ substrate was utilized (cf. Table 4.1). Nevertheless, about $-1500 \mathrm{fs}^{2}$ GDD had to be compensated as well as the residual chirp of the OPA. This was accomplished by inserting a $5 \mathrm{~mm}$ thick AR coated Si window $\left(\mathrm{GDD} \approx 1900 \mathrm{fs}^{2}\right.$ ). The pulses were compressed to about $140 \mathrm{fs}$ which is close to the FTL of the spectrum measured with an FOURIER transform infrared spectrometer (FTIR) (cf. Fig. 4.10(c)). The X-FROG RMS error was 0.5\%. After the dielectric HR mirrors for $4 \mu \mathrm{m}$ central wavelength (dark blue dashed lines Fig. 4.2(a) and (b)) became available, the $1 \mathrm{~mm}$ thin $2.4 \mu \mathrm{m}$ longpass filter (Si substrate, dark green solid line Fig. 4.2(c)) was utilized for separating the mid-IR and the residual near-IR.

\section{Discussion}

The most powerful MHz fs sources for wavelengths longer than $3 \mu \mathrm{m}$ were based on OPOs. About $8.9 \mathrm{~W}$ were generated at $3.6 \mu \mathrm{m}$ with longer pulse durations ( $840 \mathrm{fs}$ measured at $1.45 \mu \mathrm{m})$ and without CEP stabilization ${ }^{407}$. Actively CEP stabilized OPOs reached $1.5 \mathrm{~W}$ 
at $3 \mu \mathrm{m}$ and about $1 \mathrm{~W}$ at $4 \mu \mathrm{m}^{408}$. Hybrid fs OPO-OPA systems reached about $1.1 \mathrm{~W}$ between 3.5 and $4.1 \mu \mathrm{m}^{409}$ with excellent power stability while for fiber-seeded fs OPAs about $650 \mathrm{~mW}$ were measured at $3 \mu \mathrm{m}^{395}$. Eventually, a few sub-MHz rate amplifier systems with several tens of Watts output power at about $1 \mu \mathrm{m}$ also reached several Watts of average power at about $3 \mu \mathrm{m}$ wavelength ${ }^{393,405,410}$. The presented PPLN OPA unifies different advantages of the cited examples. It emits multiple Watts of average power with only $140 \mathrm{fs}$ duration, avoids the need for active CEP stabilization, exhibits good power stability and consists of a simple setup. Moreover, the amplified seed, although not explicitly discussed here, presents also an attractive source for two-photon microscopy in biomedical deep tissue imaging ${ }^{253}$.

Spectral powers of several tens of $\mathrm{mW} / \mathrm{cm}^{-1}$ are more than sufficient for spectroscopy applications. Given the repetition rate of $f_{\text {rep }}=37.5 \mathrm{MHz}, 1 \mathrm{~cm}^{-1}$ corresponds to about $800 \times f_{\text {rep }}$, i.e. powers per comb line on the order of $0.1 \mathrm{~mW}$ were reached which would belong to the most powerful mid-IR combs (after referencing $f_{\text {rep }}$ ) that have been realized so-far ${ }^{66}$. The brightness $B$, i.e. the photon flux per unit area and per unit solid angle, of the source can be evaluated by:

$$
B=\frac{\pi}{4 h c_{0} \lambda M_{x}^{2} M_{y}^{2}} \int_{0.1 \% \text { bw }} \operatorname{PSD}(\tilde{\nu}) d \tilde{\nu}
$$

where $\int_{0.1 \% \text { bw }} \operatorname{PSD}(\tilde{\nu}) d \tilde{\nu}$ represents the average power in $0.1 \%$ of the (FWHM) bandwidth of the spectra shown in Fig. 4.7(b). It ranges from about 2 to $4 \mathrm{~mW}$. Moreover, $h$ is the PLANCK constant, $c_{0}$ the speed of light in vacuum, $\lambda$ the vacuum wavelength and $M_{x, y}^{2}$ the Gaussian beam factor. Eq. (4.4) was derived from the formula given in ref. 198 in the following way: The solid angle $\Omega$, being usually small for nearly Gaussian laser beams, was approximated by four times the product of the beam divergence in $\mathrm{x}$ and y direction, i.e. $\Omega=4 \lambda^{2} M_{x}^{2} M_{y}^{2} /\left(\pi^{2} w_{0, x} w_{0, y}\right)$, where $w_{0, x, y}$ is the waist. The beam area was calculated by $A=\pi w_{0, x} w_{0, y}$. Eq. (4.4) resulted in brightnesses on the order of $10^{21}$ photons $\mathrm{s}^{-1} \mathrm{~mm}^{-2} \mathrm{sr}^{-1}(0.1 \% \mathrm{BW})^{-1}$, exceeding synchrotron mid-IR sources by about four orders of magnitude ${ }^{411}$.

The wide transparency range $(0.32 \mu \mathrm{m}-5.5 \mu \mathrm{m})$ and the high figure of merit (FOM) of ${ }^{184,185}$

$$
\mathrm{FOM}=\frac{d_{\mathrm{eff}}^{2}}{n_{I} n_{S} n_{P}} \approx \frac{25^{2} \pi^{2} / 4}{2.2^{3}} \mathrm{pm}^{2} / \mathrm{V}^{2} \approx 24 \mathrm{pm}^{2} / \mathrm{V}^{2},
$$

enabled efficient parametric amplification and made PPLN an ideal choice for the downconversion process. The FOM factors out the crystal-dependent parameters of Eq. (4.3). PPLNs are available in very good quality from multiple suppliers. The utilized crystal has not been damaged a single time during the experimental campaign. Furthermore, no signs of degradation became visible. The peak power scalability of the experiment is, however, limited by the commercially available apertures of typically $1 \times 1 \mathrm{~mm}^{2}$. This enables to send about 5 times more pulse energy. Crystals with apertures of $5 \times 5 \mathrm{~mm}^{2}$ were realized $^{412}$ (but not commercialized). These would allow to utilize several hundreds of MWs for pumping the OPA. The (kHz repetition rate) mid-IR sources with GW peak power levels typically use multiple amplification stages to generate energetic pulses ${ }^{393,406}$. Consequently, there is no fundamental power scaling limit if the down-conversion scheme 
is transferred to higher power oscillators. Multiple amplification stages could also further reduce idler noise, ${ }^{393,395}$ but would complicate the setup.

\title{
4.1.2 Frequency Down-Conversion with LGS
}

Parts of this section have been published in:

M. Seidel, X. Xiao, S.A. Hussain, G. Arisholm, A. Hartung, K.T. Zawilski, P.G. Schunemann, F. Habel, M. Trubetskov, V. Pervak, O. Pronin, F. Krausz, "Multi-Watt, multioctave, mid-infrared femtosecond source," Science Advances 4 eaaq1526 (2018).

\section{Frequency down-conversion beyond the oxygen absorption edge}

While PPLN or periodically poled $\mathrm{LiTaO}_{3}$ are well established crystals and were utilized in all of the mentioned $\mathrm{MHz}$ OPAs and OPOs of the previous discussion, there are basically no commonly used non-oxide crystals which are pumped with fs sources emitting around $1 \mu \mathrm{m}$ wavelength. VALentin Petrov explains in one of his reviews ${ }^{185}$ why this is the case:

\begin{abstract}
"Optical parametric generators, OPAs and synchronously pumped OPOs based on non-oxide nonlinear crystals are difficult to realize in the picosecond regime and even more difficult with femtosecond pulses. The major restriction comes not only from the two-photon absorption. [...] For pumping near $1 \mu \mathrm{m}$ or down to $800 \mathrm{~nm}$, even being below half of the bandgap, the pump wavelength will not be far enough from the bandgap of the nonlinear crystal and consequently strong dispersion will lead to temporal walk-off of the interacting pulses and low parametric gain. Obviously, only thin nonlinear crystals can be used with ultrashort pulse lasers and since the gain will not be sufficient, this is mostly DFG. As an alternative, cascaded schemes could be applied to transform the pump wavelength to spectral ranges of lower dispersion."
\end{abstract}

In-fact, the first down-conversion experiments performed in our group with a KLM TD oscillator ${ }^{198}$ relied on DFG as it was suggested in the quotation above. The results will be briefly reviewed in the next section and compared to the OPA results which are presented here. The investigations generally confirmed what has been stated by V. PETROV when testing a variety of non-oxide crystals. Most of them suffered strongly from multi-photon absorption, in particular AGS which has perhaps been the most commonly used non-oxide crystal. The limited transparency in the UV or visible has been pointed out as the first drawback, which is highlighted, in the quotation above. The experiments with LGS in ref. 198 did not reveal issues with multi-photon absorptions. The bandgap of the crystal, $3.8 \mathrm{eV}^{185}$, is actually very similar to the one of $\mathrm{MgO}^{-\mathrm{LiNbO}_{3}}\left(\approx 4 \mathrm{eV}^{394}\right)$. No detrimental

\section{Remark: Ambiguity of the name "LGS"}

An online search for "LGS crystal" gives two different results. One is an oxide crystal, called langasite or lanthanum gallium silicate $\left(\mathrm{La}_{3} \mathrm{Ga}_{5} \mathrm{SiO}_{14}\right)$. It is piezoelectric but not useable for frequency down-conversion. The LGS which was utilized here is the non-oxide crystal lithium gallium sulfide $\left(\mathrm{LiGaS}_{2}\right)$. 
higher order absorption was encountered in the experiments presented in the previous section. Contrary to PPLN, multi-phonon absorptions of LGS start only at about $8 \mu \mathrm{m}$ and become more prominent at around $11 \mu \mathrm{m}$ which makes the crystal suitable for midIR beyond the phonon absorption edge of oxides at wavelengths shorter than $5 \mu \mathrm{m}$. The transmission of LGS in the mid-IR was determined by FTIR measurements of two crystals (Figs. 4.11(a)-(c)). The absorption coefficients agree well with those presented in ref. 413.

LGS is biaxial, i.e. for given wavelengths of the three-wave mixing process, there are multiple pairs of phase-matching angles $(\theta, \phi)$ and not only a single angle like for uniaxial crystals such as BBO. The phase-matching curves for two main planes are shown in Fig. 4.11(d). The plots reveal turning points of the respective tuning angles, enabling very broad phase-matching bandwidths even for thick crystals. This is a very unique feature of LGS, making it ideal for being pumped with sources around $1 \mu \mathrm{m}$ central wavelength. PPLN, for example, exhibits a similar behavior when an idler at $3.5 \mu \mathrm{m}$ is generated with a pump at $800 \mathrm{~nm}$. But for PPLN, the phase-matching bandwidth for mid-IR is relatively narrow upon pumping with $1 \mu \mathrm{m}$ sources. While only type I phase-matching was used in ref. 198 , yielding $d_{\mathrm{eff}} \approx-4.6 \mathrm{pm} / \mathrm{V}\left(\mathrm{FOM} \approx 2.3 \mathrm{pm}^{2} / \mathrm{V}^{2}\right)$ for the crystal angles $\theta=48.3^{\circ}$, $\phi=0^{\circ}$, also type II phase-matched crystals were used in the presented experiments owing to their higher FOM $\left(d_{\mathrm{eff}} \approx-6.0 \mathrm{pm} / \mathrm{V}, \mathrm{FOM} \approx 3.9 \mathrm{pm}^{2} / \mathrm{V}^{2}\right.$ for $\left.\theta=90^{\circ}, \phi=38.6^{\circ}\right)$. To calculate phase-matching angles and FOMs, the SELLMEIER equations and the $\chi^{(2)}$ tensor from ref. 415 were used.

\section{Seed generation}

Even for type II phase-matching the FOM is a factor of four lower than for PPLN. Moreover, the $\lambda_{I} \lambda_{S}$ factor in the denominator of Eq. (4.3) is about two times larger for the long-wavelength OPA. This results in about a factor 3 less amplifier small signal gain for LGS. Therefore, the three-wave mixing process was not seeded with the ANDi continuum but with the spectrum generated in an LMA-12 fiber. In section 3.1.1, the trade-off between maximal pulse energy and achievable spectral bandwidth for different normally dispersive PCFs has been discussed. Due to the large quantum defect of the
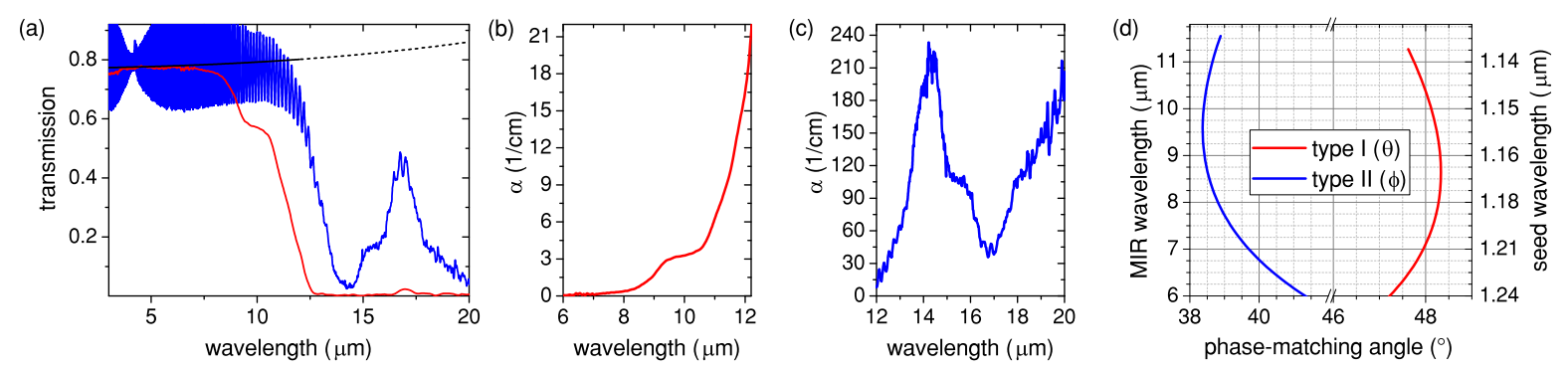

Fig. 4.11. (a) Transmission measurements of a $1 \mathrm{~mm}$ (red solid line) and a $150 \mu \mathrm{m}$ (blue solid line) thick LGS crystal. The thinner crystal exhibits fringes due to the etalon effect. These were used to determine the crystal thickness precisely. The black line shows the computed FrESNEL losses for the slow axis at the crystal cut angles $\theta=48.3^{\circ}$ and $\phi=0^{\circ}$. They were derived from the Sellmeier equations of the crystal ${ }^{414}$ which were, however, only measured for wavelengths up to $12 \mu \mathrm{m}$. (b) Extracted linear absorption coefficient $(\alpha)$ of the $1 \mathrm{~mm}$ thick LGS. (c) Extracted linear absorption coefficient $(\alpha)$ of the $150 \mu \mathrm{m}$ thick LGS. (d) Phase-matching curves based on the SELLMEIER equations ${ }^{414}$ for frequency downconversion of $1030 \mathrm{~nm}$ pump light. For type I phase-matching (idler and seed polarization on the slow axis) $\theta$ is varied while for type II phase-matching (idler and pump polarization on the slow axis) $\phi$ is varied. 
(a)

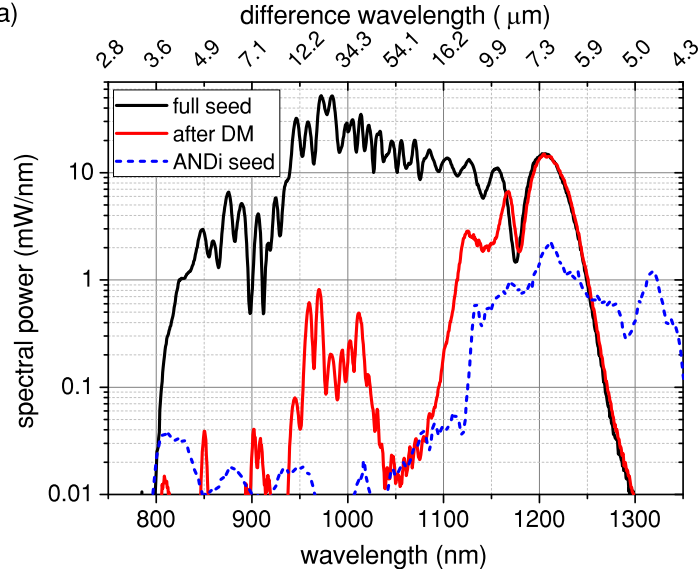

(b)

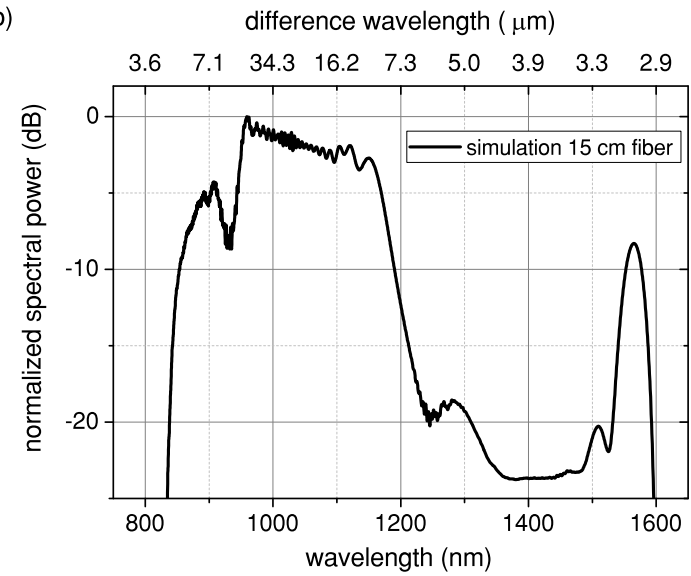

Fig. 4.12. (a) LGS OPA seed spectrum emerging from an $8 \mathrm{~cm}$ long LMA-12 fiber. The black solid line displays the full spectrum, the red solid line shows the spectrum after the dichroic mirror. The ANDi-PM fiber seed (Fig. 4.4) is shown for comparison (blue dashed line). Up to $1250 \mathrm{~nm}$ the spectral power density of the LMA-12 spectrum exceeds that of the ANDi fiber. This reduces the OPA threshold for idler generation at wavelengths of $6 \mu \mathrm{m}$ or longer. (b) Simulation of the output spectrum of a $15 \mathrm{~cm}$ long fiber. The power at $1200 \mathrm{~nm}$ gets reduced in comparison to (a). OWB into the spectral region of anomalous dispersion has set in. The spectral spike at $1560 \mathrm{~nm}$ is not well suited for seeding an OPA due to its strongly enhanced RIN in comparison to wavelengths of normal dispersion ${ }^{416}$. (simulated with fiberdesk V.2.0)

long-wavelength infrared OPA, the seed continuum only has to reach wavelength about $1.2 \mu \mathrm{m}$. Consequently, utilizing LMA-12 instead of an ANDi fiber has proven to be better suited for the down-conversion with the LGS crystal. Fig. 4.12(a) shows that about an order of magnitude higher power spectral density at the relevant seed wavelengths could be achieved with the LMA fiber. An $8 \mathrm{~cm}$ long fiber was chosen. Similar to the situation described in section 2.2.1, OWB was avoided. Fig. 4.12(b) shows that, due to the proximity of the zero-dispersion wavelength (cf. Fig. 3.1(b)), the wave-breaking on the long wavelength side extends into the anomalous dispersion regime and is not as smooth as for the ANDi fiber. Moreover, it introduces significant IN enhancement of the seed spectrum ${ }^{416}$. Consequently, utilizing LMA seed fibers longer than $8 \mathrm{~cm}$ was rather disadvantageous for the OPA operation.

\section{OPA simulations and experiments}

In ref. 198, the LGS damage threshold for $\approx 20 \mathrm{fs}$ laser pulses with $50 \mathrm{~W}$ of average power was determined to be about $1 \mathrm{TW} / \mathrm{cm}^{2}$. Due to the longer pulse durations utilized here and the UV absorption edge comparable to $\mathrm{MgO}: \mathrm{LiNbO}_{3}$, a damage threshold of $50 \mathrm{GW} / \mathrm{cm}^{2}$ was estimated for the $230 \mathrm{fs}$ pump pulses. It was noticed, however, that damage occurred at lower pump powers for $8 \mathrm{~mm}$ thick crystals than for 1 or $2 \mathrm{~mm}$ thick crystals. Moreover, it was observed that the $8 \mathrm{~mm}$ crystal was damaged at its end facet first. This was interpreted as a consequence of critical self-focusing. Since no literature value of the KERR nonlinearity is known by the author, the beam collapse was simulated under varying $n_{2}$ values of the crystal for a spot size of $110 \mu \mathrm{m}$ and a peak irradiance of $12 \mathrm{GW} / \mathrm{cm}^{2}$, the experimental values that caused damage. An $n_{2}$ of $35 \times 10^{-16} \mathrm{~cm}^{2} / \mathrm{W}$ was determined which is twice as large as the $n_{2}$ of $\mathrm{LiNbO}_{3}$ and $50 \%$ higher than the expected value from ref. 290. A direct measurement may determine it more precisely. 

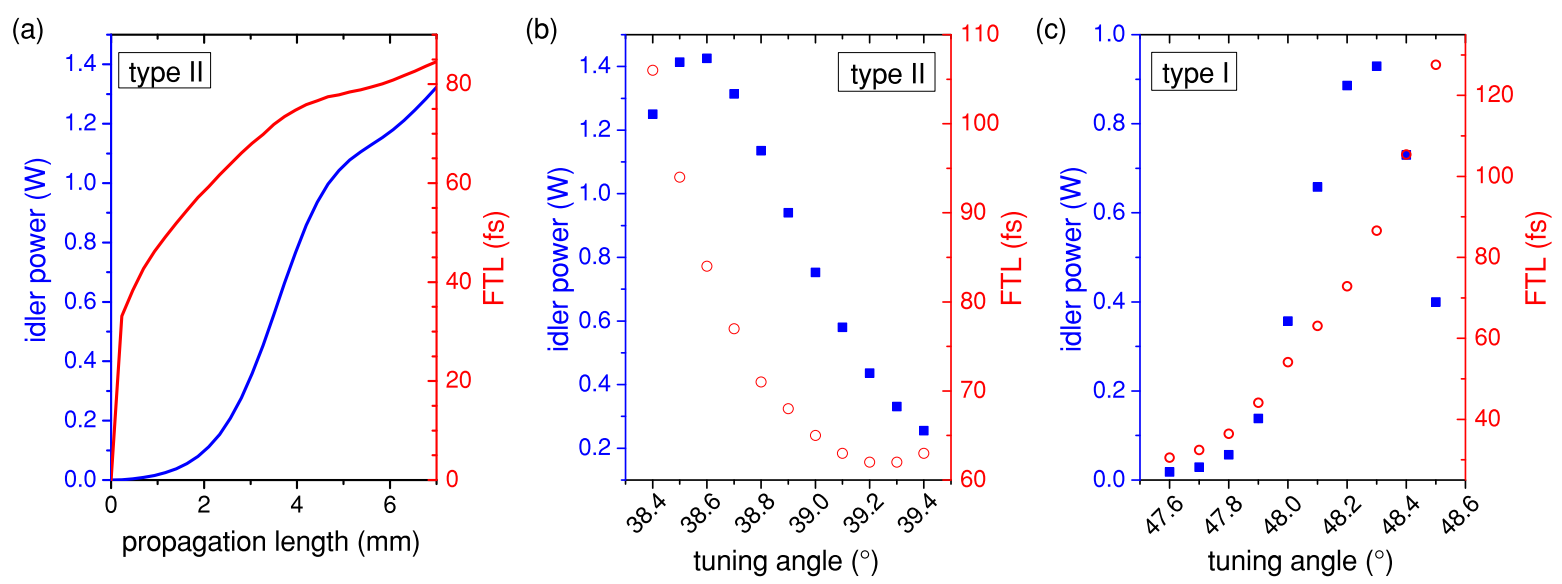

Fig. 4.13. (a) Evolution of idler power and FTL with respect to propagation length. It is demonstrated that, while the idler power strongly rises after $2 \mathrm{~mm}$ of propagation, the slope of the FTL decreases. The central idler wavelength is $9.2 \mu \mathrm{m}$. The evolution of a type I phase-matched OPA yields qualitatively to the same result results. The pump power was $28.8 \mathrm{~W}$, the beam waist in the crystal $115 \mu \mathrm{m}$ and the tuning angle $\phi=38.6^{\circ}$. (b) Angle tuning of a $7 \mathrm{~mm}$ long LGS crystal for type II phase-matching. The simulation parameters are like in (a). (c) Angle tuning of an $8 \mathrm{~mm}$ long LGS crystal for type I phase-matching. The simulation parameters are like in (a). More efficient down-conversion is expected from type II phase-matching due to the higher FOM. Type I phase-matching predicts, however, a slightly lower FTL near the tuning angle for maximal idler power and nearly single-cycle pulse bandwidth upon strong detuning, even for an $8 \mathrm{~mm}$ long crystal (at only about $5 \%$ of maximum conversion).

Under consideration of the damage threshold and the determined $n_{2}$, simulations were conducted to determine optimal focus size and crystal length in a procedure similar to the PPLN OPA. The seed, used in the simulations, was extracted from a SH-FROG measurement. The refractive indices and effective quadratic nonlinearities were extracted from ref. 415. Three frequency grids, each of 512 points and $200 \mathrm{THz}$ width, were factored out. The seed and pump grids were centered at $291 \mathrm{THz}$, while the grid of the idler was centered at $100 \mathrm{THz}$. Due to mirror symmetry, only half of the beam profile was simulated for type I phase-matching. The spatial grid had the size of $128 \times 65$ points with a spacing of $1 / 7$ of the (free beam) pump beam waist in horizontal and vertical direction. Since the spatial grid in the simulation package could not simply be rotated by $90^{\circ}$, the full plane with $128 \times 128$ points was simulated for type II phase-matching. It is to note that the published refractive index data refers to room temperature measurements while the crystals were heated to about $60^{\circ} \mathrm{C}$ in the experiments. This was considered as the reason for the blue-shift of the experimental spectra with respect to the simulated ones. A very recent publication has shown, however, that inaccuracies in the initially reported SELLMEIER equation have also contributed to the discrepancy ${ }^{417}$.

Fig. 4.13 shows that Watt-level mid-IR powers are predicted with bandwidths of less than three optical cycles. This demonstrates that LGS constitutes an important exception from the above cited, general statement about near-IR fs pumped non-oxide nonlinear crystals. Fig. 4.13(a) shows the quasi-exponential increase of idler power with propagation up to $4 \mathrm{~mm}$ where saturation effects become apparent. By contrast, the FTL rises clearly sublinearly after the first $\mathrm{mm}$ of propagation. At the end facet of the crystal, i.e. after $7 \mathrm{~mm}$ (longer crystals were not available for type II phase-matching), the FTL is only about $85 \mathrm{fs}$ while a cycle duration at $9.2 \mu \mathrm{m}$ is about $30 \mathrm{fs}$. Power levels of up to $1.4 \mathrm{~W}$ at $9 \mu \mathrm{m}$ 

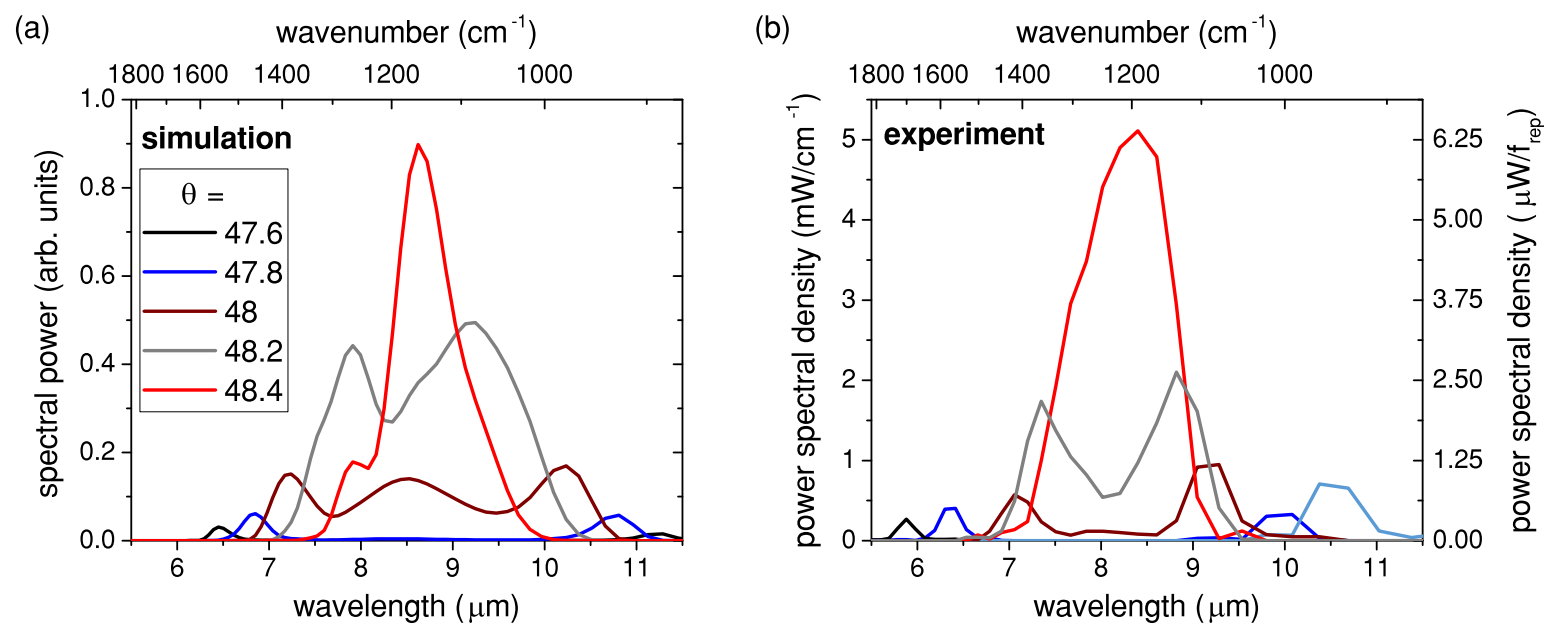

Fig. 4.14. (a) Simulated tuning curve for type I phase-matching and $28.8 \mathrm{~W}$ of pump power. Due to the phase-matching of two idler wavelengths at the same tuning angle (cf. Fig. 4.11(d)), the idler spectrum exhibits two peaks if the tuning angle is $\theta=48.2^{\circ}$ or lower and may become nearly octave-spanning. The spectra of the two perfectly phase-matched wavelengths overlap in the center at around $8.6 \mu \mathrm{m}$. A similar behavior is predicted for type II phase-matching. Due to the idler red-shift in this case, the longer wavelengths are absorbed more strongly, and hence the FTLs are not as low as for type I phase-matching. (b) Experimental tuning curve. It is in good agreement with the simulated one. The FTL of the wine red curve is only about two optical cycles. The black and the light blue curve were measured at the same tuning angle but at different delays. The spectra were measured with an FTIR (Lasnix L-FTS).

are expected (cf. Fig. 4.13(b)) which would correspond to down-conversion efficiencies of about $40 \%$.

Fig. 4.13(b) and (c) compares different phase-matching geometries and crystal cuts, respectively. As expected, type II phase-matching leads to higher mid-IR power (about $40 \%$ more). On the other hand, with type I phase-matching, broader spectra can be generated. The difference is small for maximum power output, but upon detuning even sub-40 fs FTLs are predicted. Unfortunately, the mid-IR converison drops to the sub- $100 \mathrm{~mW}$ level, and hence it is probably more efficient to maximize the OPA output for power and spectrally broaden it by a factor of two. Fig. 4.14 shows simulated (a) and experimental (b) tuning curves which are significantly different from what has been presented in Fig. 4.7(b), the PPLN OPA tuning curve. While there a more typical monotonous relation between poling period and idler wavelength is observed, the LGS idler wavelengths approach upon angle tuning from the long and the short wavelength side a singularity near $8.6 \mu \mathrm{m}$ (for type I) and $9.2 \mu \mathrm{m}$ (for type II), respectively. This is well explainable by the turning points in the phase-matching curves displayed in Fig. 4.11(d).

For the amplification experiments, the dichroic beam splitter characterized in Fig. 4.2 was not available, and hence different means for beam splitting were employed in order to quantify the generated idler power. The simplest approach is to use a germanium plate which transmits only the mid-IR but absorbs the pump and signal. This method only worked poorly since the absorption led to free carrier generation which implied also mid-IR absorption (orange dots Fig. 4.15(a)). This effect was, for instance, controlled to achieve ultrashort $\mathrm{THz}$ pulses from $\mathrm{kHz}$ systems ${ }^{418}$. Here, it was just detrimental. In a type I phase-matching geometry, pump and idler polarization are orthogonal, and thus fairly efficient splitting was achieved by utilizing a ZnSe plate at BREWSTER's angle 
(a)

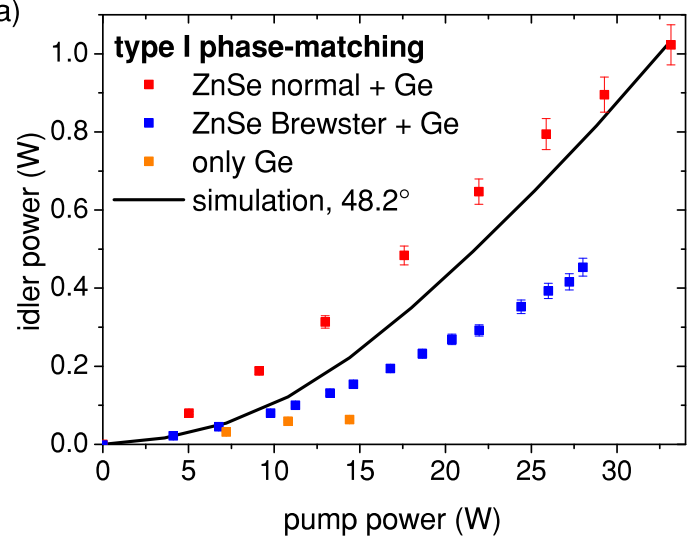

(b)

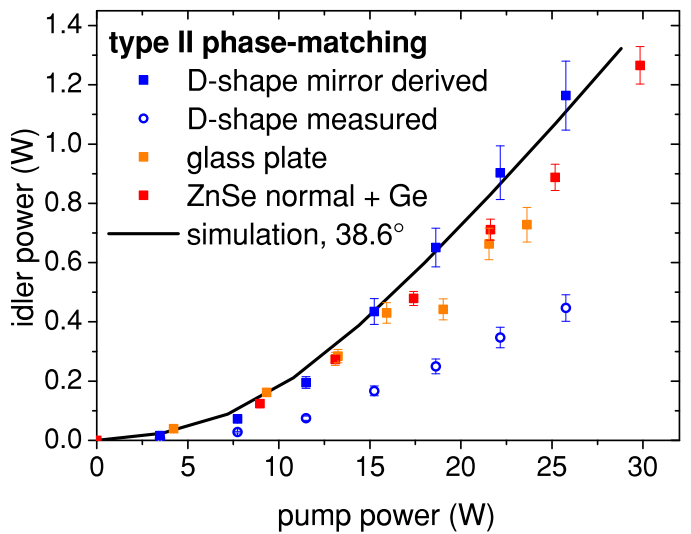

Fig. 4.15. (a) mid-IR generation with type I phase-matched $8 \mathrm{~mm}$ thick LGS crystals. The orange dots show a measurement were pump, seed and idler were sent onto a germanium plate to split the near-IR from the mid-IR. At pump powers above $10 \mathrm{~W}$, the germanium plate becomes increasingly opaque for the mid-IR, most probably due to free carriers in the semiconductor. The blue dots were measured with ZnSe plate at BREwsteR's angle which transmitted the pump and reflected about $60 \%$ of signal and idler. These two beams were split with a germanium plate. At low powers, the agreement with the simulation results is good while the experimental values remain behind their expectations at higher pump powers. For the measurement symbolized by the red dots, an AR coated crystal was used, allowing to pump with up to $33 \mathrm{~W}$. In this case, beam splitting was accomplished by a ZnSe wedge with near-0 AOI (yielding about $17 \%$ reflectivity), an AR-coated ZnSe plate for further attenuation of the near-IR and a Ge plate. The idler powers are partly above the expectations of the simulation which might originate from additional seed power due to excellent coupling into the LMA-12. At full pump power, $1 \mathrm{~W}$ of mid-IR was generated. The graph corrects for the FRESNEL reflection. An mid-IR transmission of $47 \%$ (infinite number of reflections) was assumed for the Ge plate. (b) mid-IR generation with a type II phase-matched $7 \mathrm{~mm}$ thick AR-coated LGS crystal. The red dots have been measured with the same method that yielded $1 \mathrm{~W}$ for the type I crystal. In this case, up to $1.3 \mathrm{~W}$ could be generated at full pump power. The orange dots were derived from measuring first the total transmitted power and then the transmitted near-IR power while the mid-IR was blocked by a $5 \mathrm{~mm}$ thick FS plate. Its power was derived from the difference of both measurements. A maximal pump power of $24 \mathrm{~W}$ could be used due to the detection limit of the thermal power meter. The blue dots were derived from spatially splitting near-IR and mid-IR beams with a D-shape mirror. Due to the at least seven times larger diffraction of the mid-IR, parts of it passed the D-shape mirror which completely reflected the near-IR. The measurement was calibrated at low pump powers by inserting an additional Ge plate and comparing the measured idler power with and without the D-shape mirror. All solid dots symbolize the derived generated idler power. The blue hollow dots show actual power measurements, yielding $450 \mathrm{~mW}$. The error bars refer to the uncertainties of the measurement method, not to a statistical analysis of a measurement set. The dark power (arising e.g. from heating the Ge plate) was subtracted from the shown idler powers. It was measured when pump and seed were delayed such that they were not mixed anymore. Phase-matching was adjusted for maximal output power.

such that the pump is practically fully transmitted while the about $50 \%$ of the idler are reflected at a single surface. The blue dots in Fig. 4.15(a) shows the results achieve by this approach. Signal and idler were still split by a germanium plate. This could have possibly led to the discrepancy of simulation and experiment at higher pump powers. The signal carriers about seven times more power than the idler. In a third technique (red dots Fig. 4.15(a)), the ZnSe wedge was placed in nearly normal angle of incidence such that it reflected only about $17 \%$ per surface of the incoming power. A second, coated ZnSe plate was used to reflect, and hence attenuate the near-IR further while the coating nearly perfectly transmitted the mid-IR. Finally, a germanium plate was set in front of the power meter that measured the remaining idler power which went up to $76 \mathrm{~mW}$. 


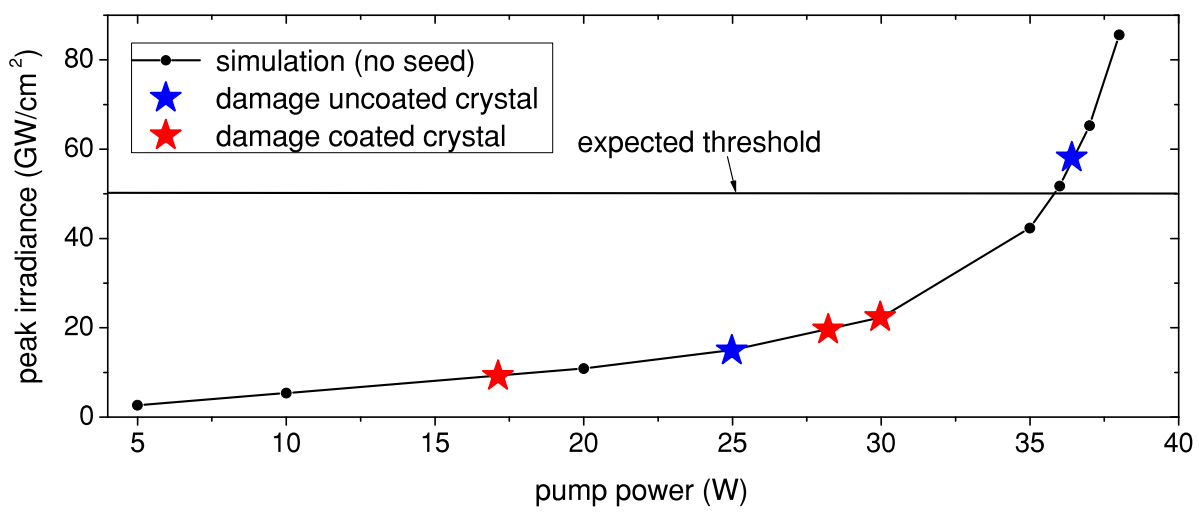

Fig. 4.16. Occurrence of LGS crystal damage at various input powers. The simulated curve that relates the pump power to the peak irradiance inside the crystal is shown as a black line with dots at the simulated points. The blue (red) stars show the pump powers at which the $7 \mathrm{~mm}$ to $8 \mathrm{~mm}$ long uncoated (coated) crystals were damaged. The pump power where damage occurred varies by a factor of two while the simulation predict that the peak irradiance where damage occurs varies even by a factor of six.

However, since the reflection/transmission losses could be precisely determined, it was inferred that a mid-IR power of about $1 \mathrm{~W}$ was generated. In the same way an OPA with type II phase-matched crystal was studied. The red dots in Fig. 4.15(b) show that a mid-IR power of up to $1.3 \mathrm{~W}$ could be generated in this case. To manifest this result and exclude systematic errors, the power measurement was repeated with two other methods. Firstly, only the mid-IR was blocked and the difference to the full output power was determined. Secondly, the near-IR with clearly smaller beam width was reflected from a D-shape mirror while parts of the mid-IR passed the mirror and were measured with a power meter. All approaches led to well comparable results which are also in excellent agreement with simulations as Fig. 4.15(b) demonstrates. The power variations mainly arose from different seed powers, which depended on the coupling into the LMA fiber and the oscillator pulse duration. Also slight variations in the spatial overlap could have led to the differences in the measurement series.

\section{Reliability of the LGS crystal}

The optical properties of LGS were presented for the first time in $2003,{ }^{413}$ although the crystal was reported for the first time in $1947^{415}$. Consequently, LGS is not nearly as established as $\mathrm{LiNbO}_{3}$. To the best of the author's knowledge, the first fs LGS OPA pumped with a 1 micron source has been presented here. The young history of LGS as a nonlinear crystal may explain why the available quality is not comparable to that of PPLN. Whereas the latter was not damaged a single time during the course of the experiments (i.e. for more than two years), multiple LGS crystals were destroyed at a huge range of peak irradiances as Fig. 4.16 illustrates. The lowest encountered damage was only at $1 \%$ of the specified irradiance threshold of ref. 198.

Damage threshold measurements for LGS were conducted elsewhere with ns pulses at $\mathrm{kHz}$ repetition rates ${ }^{419}$. In this case the three tested crystals exhibited a very similar damage fluence ( $<15 \%$ deviation). AR coatings rather slightly increased the fluence where surface damage set in. An OPO containing an $8 \mathrm{~mm}$ thick crystal was pumped six 
times below the damage threshold at a peak irradiance of about $550 \mathrm{MW} / \mathrm{cm}^{2}$. Scaling this value with the square root of pulse duration law, yields $36 \mathrm{GW} / \mathrm{cm}^{2}$ for $230 \mathrm{fs}$ pulses and is in good agreement with the maximal peak irradiance of the experiments presented here. Therefore, the described issue with the strongly varying damage threshold does apparently not stem from fundamental limitations of the crystal itself, but from its scarce availability at constantly high quality. While PPLN can be obtained from multiple suppliers and the most common poling periods can be delivered within a few weeks, the author is only aware of one LGS supplier worldwide at present (ASCUT Ltd \& Co KG) and delivery usually takes several months.

\section{Discussion}

The LGS crystal revealed outstanding properties for direct frequency down-conversion of $1 \mu \mathrm{m}$ radiation to the mid-IR, resulting in the by-far highest power femtosecond source which has been presented for wavelengths longer than $5 \mu \mathrm{m}$. For comparison, $110 \mathrm{~mW}$ of average power were demonstrated around $7 \mu \mathrm{m}$ with the nonlinear crystal cadmium silicon phosphide, $\mathrm{CdSiP}_{2}(\mathrm{CSP})^{420,421}$ where the extraordinary high FOM allowed to keep twophoton absorption losses low. Similar power levels were achieved with more complex cascaded schemes, i.e. a sequence of at least two down-conversion devices ${ }^{409}$. Eventually, with intrapulse DFG about $100 \mathrm{~mW}$ of average power were generated at $10 \mu \mathrm{m}$ central wavelength. The experiment will be briefly presented in the next section. The brightness level of the mid-IR output of the LGS OPA is comparable to that of the PPLN OPA (cf. Eq. (4.4)) and also clearly exceeds those of synchrotron sources ${ }^{411}$.

The presented approach is not only interesting for high average power systems but is also highly attractive for time-domain applications requiring at least hundreds of MW peak, such as high-harmonic generation in gases ${ }^{388}$. To the author's knowledge, LGS crystals are available with about $7 \times 7 \mathrm{~mm}^{2}$ apertures. Picosecond amplifiers at 1 micron deliver up to hundreds of $\mathrm{mJ}^{34}$. They could be utilized for amplifying the mid-IR radiation to mJ levels.

Yet, before LGS can be become a well established nonlinear crystal like $\mathrm{BBO}$ or $\mathrm{LiNbO}_{3}$, a further evolution towards higher reliability and better availability seems imperative. In particular, the $7 \mathrm{~mm}$ to $8 \mathrm{~mm}$ thick crystals seem hard to manufacture in flawless quality. Although the generation of broad spectra was an excellent demonstration of the favorable phase-matching properties of LGS, performance and reliability of the OPA might further improve by using two shorter crystals. These would firstly reduce the crystal volume, facilitating the extraction of high quality pieces from the boule. Secondly, the shorter propagation length would allow higher pump irradiances due to the decreased detrimental impact of self-focusing. Two stage down-converters would unfortunately increase the complexity of the setup, but may lead to very stable infrared output if the down-conversion process is saturated in the individual stages ${ }^{393,409}$. 


\title{
4.1.3 Comparison to Down-Conversion via Difference Frequency Generation
}

\author{
Parts of this section have been published in: \\ I. Pupeza, D. Sánchez, J. Zhang, N. Lilienfein, M. Seidel, N. Karpowicz, T. Paasch-Colberg, \\ I. Znakovskaya, M. Pescher, W. Schweinberger, V. Pervak, E. Fill, O. Pronin, Z. Wei, F. \\ Krausz, A. Apolonski, and J. Biegert, "High-power sub-two-cycle mid-infrared pulses at \\ $100 \mathrm{MHz}$ repetition rate." Nat. Photon. 9, 721 (2015).
}

The first down-conversion experiments with a KLM TD oscillator were based on DFG with thin non-oxide crystals. The setup presented in section 2.2.1 was utilized. The blue and the red wing of the spectrum emerging from the fiber compression stage were mixed in a so-called intra-pulse DFG experiment. The principle is explained by means of Fig. 4.17. Contrary to the sketches of Fig. 1.11, seed and pump cannot be clearly distinguished, but they are convoluted within a single pulse. Due to the ultrahort durations, very high peak irradiances can be employed to drive the three-wave mixing process. For instance, up to $40 \mathrm{TW} / \mathrm{cm}^{2}$ were utilized to generate broadband radiation around $2 \mu \mathrm{m}$ in an intra-pulse DFG experiment with $\mathrm{BBO}^{354}$. On the other hand, the GVD of the nonlinear crystal leads to rapid pulse stretching, and consequently a fast decrease of the peak irradiance driving the three-wave mixing process. Moreover, the spectral wings get delayed with respect to each other during propagation, and hence temporal walk-off limits the interaction lengths. Therefore, the nonlinear crystals must be much shorter than in OPA setups. Typically, intra-pulse DFG comes also with drawback that a single pulse has a single polarization. Subsequently, pump and seed cannot be polarized for proper birefringent phase-matching. Hence, the electric field polarization vector of the incoming beam is projected on both the slow and the fast crystal axis, yielding effectively to the exclusion of half of the photons from the three-wave mixing process. On the other hand, temporal and spatial overlap is always ensured if a homogeneous beam with proper compression is utilized. Furthermore, pump and seed, since originating from a single pulse, typically exhibit common noise which is at least partly canceled by the DFG process (e.g. timing jitter, intensity noise adds up).

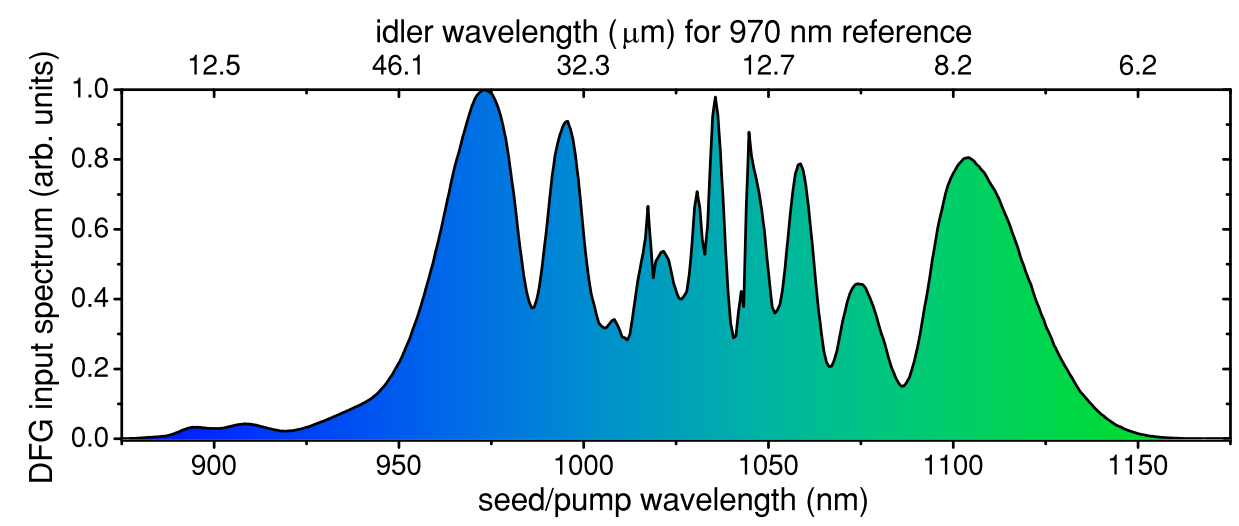

Fig. 4.17. Principle of the intra-pulse DFG setup. The compressed spectrum extracted from the LMA compression stage presented in section 2.2.1 is sent into an LGS crystal. The field polarization vector is rotated by $45^{\circ}$ with respect to fast and slow axis of the crystal for phase-matching. The shorter wavelengths (bluish) serve as pump of the three-wave mixing process while the longer wavelengths (greenish) serve as seed (cf. Fig. 1.11(a)). The top axis shows the idler wavelengths that would be generated from a $970 \mathrm{~nm}$ pump (seed) if the three-wave mixing process was perfectly phase-matched and the nonlinear crystal would not absorb. 
(a)

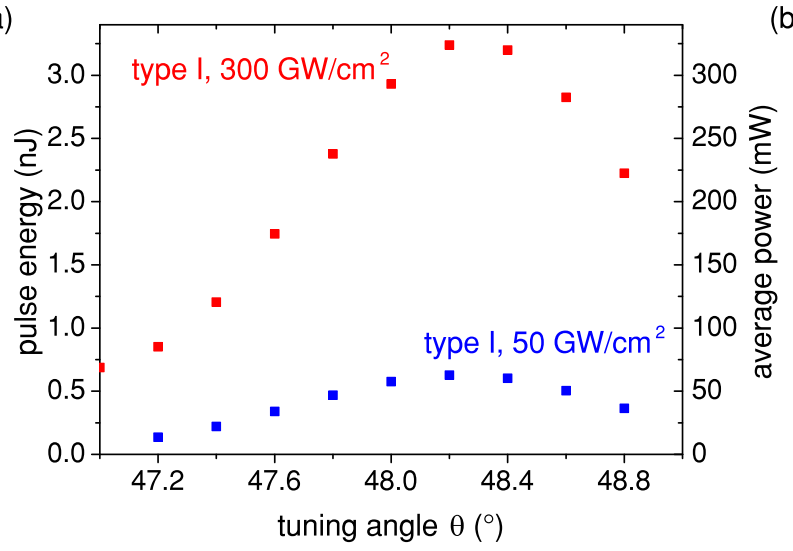

(b)

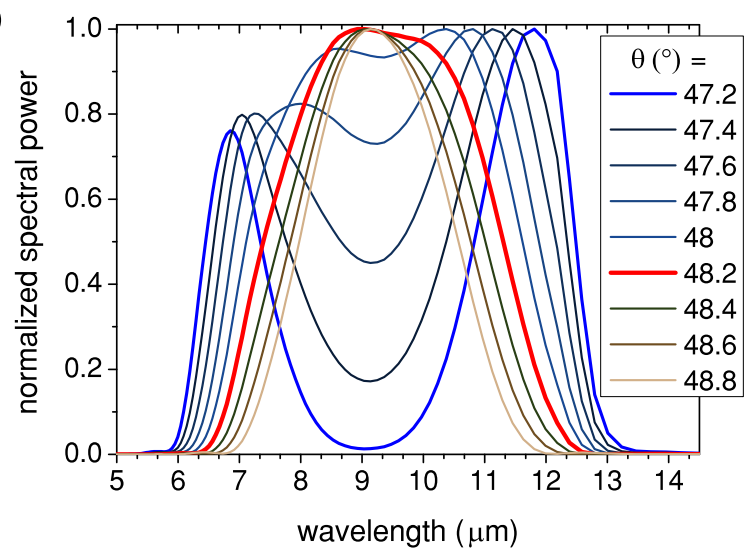

Fig. 4.18. (a) Simulated pulse energies for intra-pulse DFG experiments with the short pulses characterized in Fig. 2.9 and a $1 \mathrm{~mm}$ thick LGS crystal. Peak irradiances of $50 \mathrm{GW} / \mathrm{cm}^{2}$ (blue dots) and $300 \mathrm{GW} / \mathrm{cm}^{2}$ were set, half of the energy was projected on the slow axis (seed) and half on the fast axis (pump). While $50 \mathrm{GW} / \mathrm{cm}^{2}$ was the estimated damage threshold for the OPA, about $300 \mathrm{GW} / \mathrm{cm}^{2}$ were stated to be utilized in the DFG experiments ${ }^{198,422}$. The generated idler power in $\mathrm{mW}$ is calculated from the shown pulse energy in $\mathrm{nJ}$ by multiplying with the repetition rate in $\mathrm{MHz}$. The right axis shows the average powers for the $100 \mathrm{MHz}$ oscillator used in the experiments. To compare with the LGS OPA (37.5 MHz repetition rate oscillator), the power has to be devided by 2.67. The predicted FTLs depend on peak irradiance and tuning angle. For $300 \mathrm{GW} / \mathrm{cm}^{2}$, the FTL in the region of highest conversion efficiency is on the order of a single optical cycle $(30-40 \mathrm{fs})$. (b) Normalized tuning curves for the $50 \mathrm{GW} / \mathrm{cm}^{2}$ simulation results $\left(300 \mathrm{GW} / \mathrm{cm}^{2}\right.$ similar $)$. The splitting of the spectrum upon detuning is visible like in the OPA predictions (cf. Fig. 4.13(d)). The bold red line and the bold blue line are in good agreement with the experimentally reported spectra (cf. Fig. 4.19).

For comparing DFG and OPA based on LGS quantitatively, the simulations which have been presented in Fig. 4.13 where also performed for a $1 \mathrm{~mm}$ thick crystal and the pulses characterized in Fig. 2.9. Half of the energy was assigned to the fast axis (pump) and half to the slow axis (seed). Only type I phase-matching was investigated in accordance with the experiments. The simulation results qualitatively agree with the introductory consideration of this chapter where it was pointed out that the DFG process usually does not allow a coherent build-up process of the signal like in OPA or OPO experiments, making it clearly less efficient. For type I phase-matching and $50 \mathrm{GW} / \mathrm{cm}^{2}$ peak irradiance, only $2.4 \%$ of the maximal idler energy achieved in OPA experiments (also type I) is expected. For $300 \mathrm{GW} / \mathrm{cm}^{2}$, which was approximately the peak irradiance in refs. 198 and $422,22 \%$ of the OPA energy are predicted. On the other hand, the FTLs of the DFG simulations are about a factor two lower than the OPA predictions. Therefore, the OPA is capable of generating mid-IR pulses with energies and peak powers that clearly exceed those attainable by intra-pulse DFG.

The DFG experiments were mainly performed by IoACHIM PUPEzA from MPQ and DANIEL SÁNCHEz from the group of JENS BIEGERT at ICFO in Barcelona. Initially, the compression setup presented in this thesis was used. But later a $100 \mathrm{MHz}$ repetition rate oscillator, otherwise resembling the KLM TD oscillator presented in section 2.1, was built by JinweI ZHANG. Moreover, the compression scheme of section 2.2.1 was mimicked in a compact manner by NiKOLAI LiLIENFEIN. The higher repetition rate led to a higher oscillator average power $(90 \mathrm{~W})$ and a larger spacing of the frequency comb yielding additional power per comb line. Fig. 4.19 shows the generated spectra which have been published in refs. 422 and 198. A generated idler power of $103 \mathrm{~mW}$ was attributed 

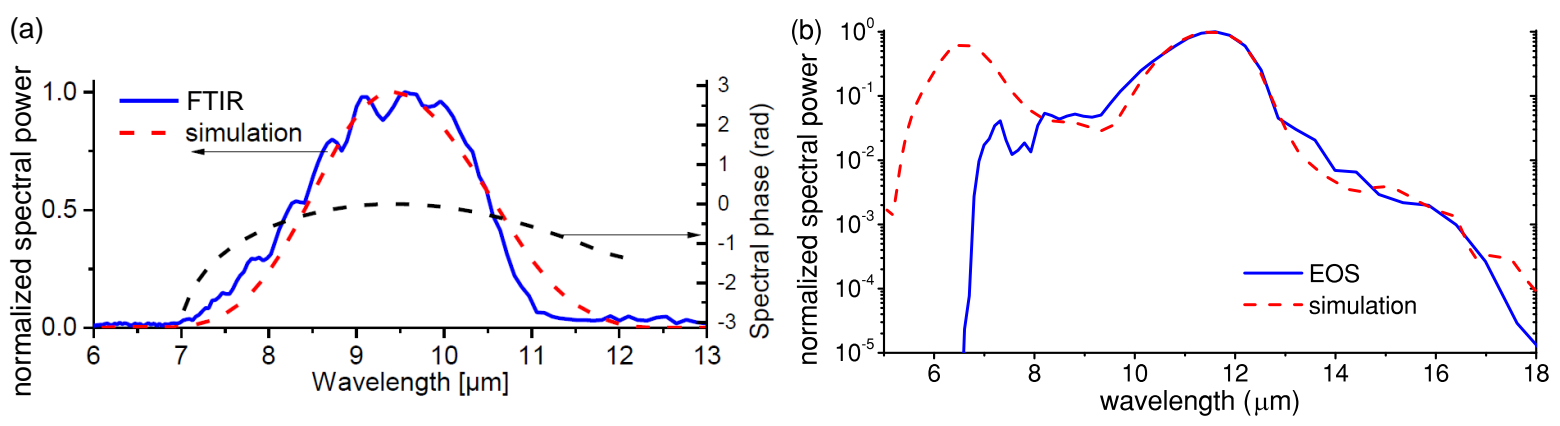

Fig. 4.19. (a) Spectrum published in ref. 422. It was measured with an FTIR (Lasnix) with SNR typically not better than $20 \mathrm{~dB}$. The measured spectrum (blue line) agrees well with the simulation (red line, done by D. SÁNCHEZ) and is in accordance with the red spectrum of Fig. 4.18(b). The flat spectral phase was predicted by the simulation. (b) Spectrum published in ref. 198 (blue line). It is compared to the simulation result for $300 \mathrm{GW} / \mathrm{cm}^{2}$ and the tuning angle $\theta=47.2^{\circ}$ (cf. blue spectrum of Fig. 4.18(b)). The long-wavelength part agrees well while the short wavelengths were likely suppressed by the electrooptic sampling (EOS) measurements. Strong absorptions beyond $10 \mu \mathrm{m}$ (cf. Fig. 4.11) are included in the simulation.

to both spectra. For the spectrum of Fig. 4.19 (a), this is clearly below the simulation prediction of Fig. 4.18(a) $\left(\theta=48.2^{\circ}\right)$, given the peak irradiance of about $280 \mathrm{GW} / \mathrm{cm}^{2}$. Possibly, the utilized uncoated germanium beam splitter blocked parts of the generated mid-IR like it was observed in the OPA experiments (cf. Fig. 4.15(a)). The situation is different for the spectrum shown in Fig. 4.19(b). Here, the simulations would predict a bit lower output power $(85 \mathrm{~mW})$ than what was actually measured although the same Ge beam splitter was used. The agreement between simulated and measured spectrum is very good for wavelengths longer than $8 \mu \mathrm{m}$. However, the short wavelength peak which is expected from the simulation is not present. This may have multiple reasons, such as: (i) Water absorptions that reduce the spectral power between 5 and $7 \mu \mathrm{m}$, (ii) the finite phase-matching bandwidth of the $30 \mu \mathrm{m}$ thin GaSe crystal used for EOS and (iii) the $22 \mathrm{fs}$ reference pulses used for EOS which do not support the full bandwidth of the mid-IR or, in other words, do not exhibit the bandwidth of the $17 \mathrm{fs}$ pulses which were utilized for the simulation.

While a detailed comparison of the experimental output parameters will be given in the summary section of this chapter in Table 4.3, a few general aspects of the comparison between the OPA and intra-pulse DFG approach shall be summarized at the end of this section: While the OPA approach clearly leads to higher mid-IR pulse energies than the DFG approach, the advantage of DFG to generate broadband radiation is apparent, but not very significant due to the large phase-matching bandwidth of LGS. Intra-pulse DFG requires pulse compression in the near-IR which, for instance, allows to apply the advanced chirped mirror technology as done in the fiber compression setup presented in section 2.2.1. By contrast, the OPA approach does not require pulse compression in the near-IR. Instead, the task is shifted to the mid-IR. For the PPLN OPA, this was readily attainable by exploiting material dispersion. Unfortunately, there was no time for compressing the LGS OPA output. In terms of intensity noise, the OPA is advantageous because the pump is typically very stable and by saturation the conversion process, the impact of seed fluctuations decreases. The DFG approach mixes the frequencies of the spectral wings which are typically more noisy the central parts of the spectrum ${ }^{416}$. On the other hand, the intra-pulse DFG approach consists of a common path setup while 
the OPA approach separates the paths of seed and pump. Therefore, the timing jitter of the idler in the DFG setup is better than in the OPA setup. These noise properties have been quantitatively confirmed by a study of Syed Ali Hussain and Wolfgang Schweinberger. Power-scaling the OPA approach seems relatively straight forward as discussed in the previous sections. The seed can be kept as it is while only the pump spot size for higher pulse energies must be adapted. To further scale the DFG approach, the compression scheme in the near-IR must be changed. The investigations in chapter 3 and recent bulk broadening results ${ }^{62,339}$ show that this is possible although some development would be required. Finally, from a pratical point of view, the DFG approach may have the advantage that the thinner LGS crystals seem to be available in better quality at the moment which hopefully changes over the next years. 


\subsection{Supercontinuum Generation in the Mid-Infrared}

\section{Parts of this section have been published in:}

M. Seidel, X. Xiao, S.A. Hussain, G. Arisholm, A. Hartung, K.T. Zawilski, P.G. Schunemann, F. Habel, M. Trubetskov, V. Pervak, O. Pronin, F. Krausz, "Multi-Watt, multioctave, mid-infrared femtosecond source," Science Advances 4 eaaq1526 (2018).

Tunability is an important feature of OPAs and OPOs since it allows to investigate specific (vibrational) transitions at a given frequency. In section 1.1, tunability has thus been claimed to be indispensable for a new generation of ultrafast lasers. However, if a multitude of (unknown) molecular species must be studied, for instance in molecular fingerprinting, ${ }^{66,381}$ broad continua are advantageous. All characteristic spectroscopic signatures may be recorded with a single pulse and no time consuming scans across the mid-IR wavelength range are necessary. Furthermore, large spectral bandwidths are also imperative in time domain applications requiring few-cylce pulses.

For this purpose, cascaded quadratic nonlinearities were exploited. In the context of midIR spectral broadening beyond the oxygen multi-phonon absorption edge, this approach has mainly been discussed for strongly phase-mismatched (non-resonant) crystal orientations which require energetic pulses or waveguides ${ }^{423,424}$. The publications have demonstrated, however, that utilizing self-defocusing nonlinearities resulted in broad, coherent continua with significant spectral extension towards longer wavelengths. The studies presented in section 3.2.2 with near-resonant configurations have indicated that the approach is capable of handling high average powers. Moreover, it has early been recognized, that near-resonant quadratic nonlinearities can give rise to effective $n_{2}$ values which are orders of magnitude larger than pure KERR nonlinearities ${ }^{425}$. This is due to the $d_{\text {eff }}^{2} / \Delta k$ scaling of the effective nonlinear refractive index (cf. Eq. (3.22)). For the chalcopyrite zinc germanium phosphide, $\mathrm{ZnGeP}_{2}(\mathrm{ZGP})^{426}, d_{\mathrm{eff}}=71 \mathrm{pm} / \mathrm{V}$ for type I SHG of $4.1 \mu \mathrm{m}$ results in an effective $n_{2}$ of about $-1 \times 10^{-12} \mathrm{~cm}^{2} / \mathrm{W}$ at the tuning angle $\theta=55^{\circ}$ (Fig. 4.20(b)). For comparison, the KERR effect of ZGP is estimated by $n_{2}^{(\text {Kerr) }}=1.3 \times 10^{-14} \mathrm{~cm}^{2} / \mathrm{W}^{290}$, the KERR effect of FS at 1 micron is about $n_{2}^{(\text {Kerr })}=3 \times 10^{-16} \mathrm{~cm}^{2} / \mathrm{W}^{240}$, i.e. more than three orders of magnitude lower than the near-phase-matched cascaded quadratic nonlinearity
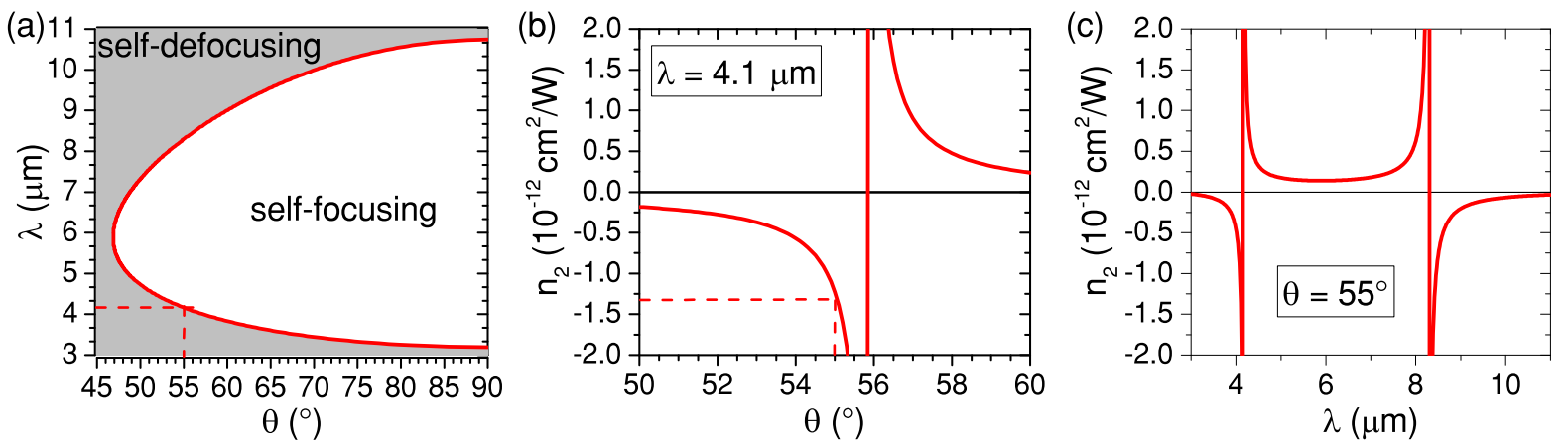

Fig. 4.20. (a) Type I phase-matching curve for ZGP. The dashed line shows the crystal-cut angle, $\theta=55^{\circ}$. The gray-shaded area implies a negative $n_{2}^{\text {(cas) }}$. (b) The effective nonlinear refractive index of ZGP for $4.1 \mu \mathrm{m}$ upon phase-mismatching. Note that the scale is $10^{-12} \mathrm{~cm}^{2} / \mathrm{W}$, contrary to Fig. 3.29 (b) where it was $10^{-15} \mathrm{~cm}^{2} / \mathrm{W}$. At $\theta=55^{\circ}$ (dashed red line), the effective nonlinear refractive index is $n_{2}=-1.2 \cdot 10^{-12} \mathrm{~cm}^{2} / \mathrm{W}$, i.e. more than three orders of magnitudes higher than what was utilized for BBO compression in section 3.2.2. (c) The calculated dispersion of the effective nonlinear refractive index for the tuning angle $\theta=55^{\circ}$. 
(a)

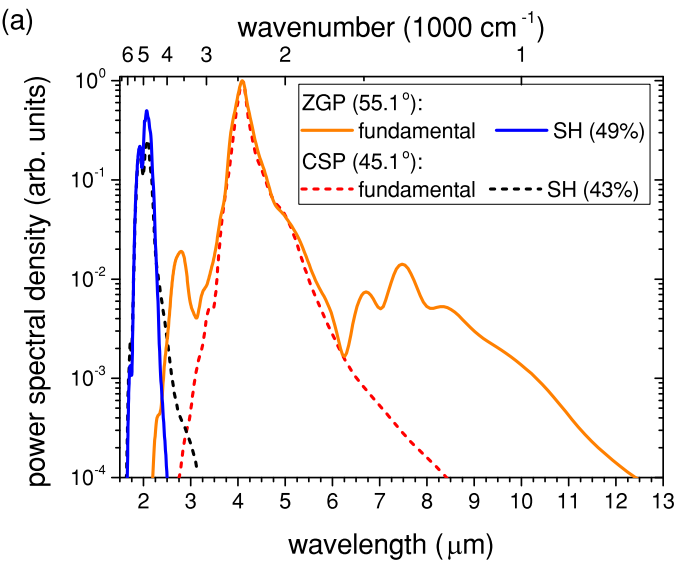

(b)

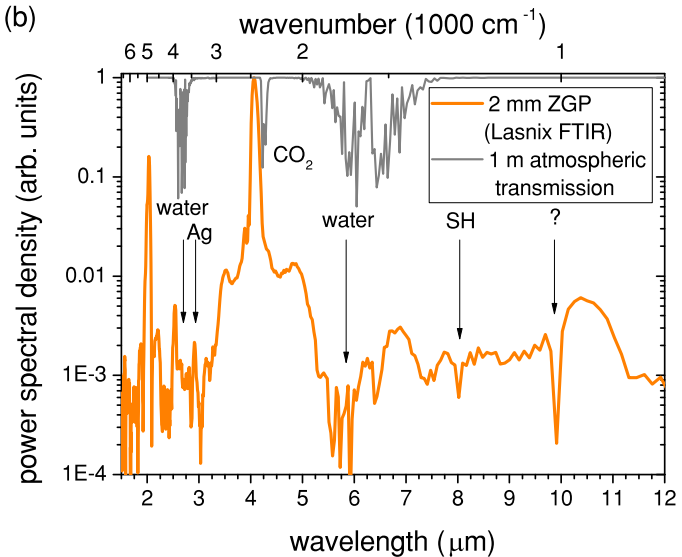

Fig. 4.21. (a) Simulations of continuum generation in ZGP and CSP which motivated the experimental investigation of the approach. The simulations assumed a sech ${ }^{2}$-shaped $100 \mathrm{~nJ}, 100 \mathrm{fs}$ pulse centered at $4.1 \mu \mathrm{m}$ and focused to a peak irradiance of $50 \mathrm{GW} / \mathrm{cm}^{2}$. The crystal thickness was chosen to be $3 \mathrm{~mm}$. SHG in ZGP $\left(d_{\mathrm{eff}} \approx 76.2 \mathrm{pm} / \mathrm{V}\right)$ is phase-matched at about $\theta=56^{\circ}$ (cf. Fig. $\left.4.20(\mathrm{~b})\right)$ while SHG in CSP is phase-matched at about $\theta=46^{\circ}\left(d_{\mathrm{eff}} \approx 38.8 \mathrm{pm} / \mathrm{V}\right)^{428}$. Consequently, the shown spectra were predicted for negative effective $n_{2}$ at the central wavelength. The simulation results for both crystals exhibit significant spectral broadening, but the ZGP spectrum extends much further into the long-wave infrared. The conversion to the $\mathrm{SH}$ is in both cases more than $40 \%$ which is a consequence of the need to tune the crystal close to the resonance in order to achieve large effective $n_{2}$ values. (b) Experimentally obtained spectra from SCG with the compressed idler output of the OPA and a $2 \mathrm{~mm}$ ZGP crystal cut at $\theta=55^{\circ}$. The peak irradiance of the pulse is about $75 \mathrm{GW} / \mathrm{cm}^{2}$. A weak supercontinuum can be inferred from the measured spectrum. It is, however, mostly at about $-30 \mathrm{~dB}$ of the peak power spectral density. Some expected prominent absorption features, which are indicated by the black arrows, can be qualitatively deduced from the spectrum ( $\mathrm{Ag}$ - reduced reflection of protected silver mirrors, $\mathrm{SH}$ phase-matched SHG in the ZGP crystal at $\theta=55^{\circ}$, cf. Fig. 4.20(a)). In particular, the feature at about $10 \mu \mathrm{m}$ is, however, unexpected and may result from some measurement error of the FTIR. Generally, the direct SCG with the OPA output was difficult to detect.

of ZGP. The huge nonlinearity has been very recently identified as the cause for broadband idler generation in a ZGP OPA pumped with mJ pulses at $2.1 \mu \mathrm{m}$ wavelength $^{427}$. Despite its dispersion (Fig. 4.20(c)), simulations predict ultrabroadband continua covering the spectral range from 1.7 to $12 \mu \mathrm{m}$ as the following paragraph will describe.

\section{Direct continuum generation with the compressed OPA output}

The emergence of a supercontinuum from the highly nonlinear ZGP crystal was initially predicted by simulations whose results are shown in Fig. 4.21(a). The simulations were also performed with the SISYFOS package in a very similar manner like those described in section 3.2.2. They included quadratic and cubic nonlinearities, but no plasma term which significantly contributes to SCG based on filamentation ${ }^{49}$. Two nonlinear crystals with nominally ultrahigh quadratic nonlinearities ${ }^{184,185}$ were investigated, namely ZGP and $\mathrm{CSP}^{426}$. The study was not comprehensive and could have included other crystals like gallium selenide, but the predictions for a $3 \mathrm{~mm}$ short ZGP were already highly promising as Fig. 4.21(a) demonstrates. For phase-matching the SHG at $4.1 \mu \mathrm{m}$, ZGP exhibits clearly higher $d_{\text {eff }}$ than CSP or gallium selenide ${ }^{428}$, the transparency range of ZGP extends further into the mid-IR than that of CSP. ZGP can be cut at a desired 
angle and is not a soft material with a layer structure like gallium selenide. Eventually, ZGP has been available from multiple commercial suppliers.

Unfortunately, it has turned out, that the crystal was not necessarily available in good quality. Initially, three $3 \mathrm{~mm}$ thick ZGP crystals were purchased from ASCUT Ltd \& Co KG. (In the meantime, the company does not sell ZGP anymore.) None of them, however, generated any measurable SH. Using the X-FROG (Fig. 4.9) as an interferometer to estimate the crystal axis dependent refractive indices, showed that the three purchased crystals, which were supposed to be identical, exhibited completely different delays. Consequently, their cutting angle seemed random, explaining the absence of SHG. Due to the long delivery times of several months, it should take about a year between the initial simulations shown in Fig. 4.21(a) and starting experiments with a ZGP crystal that worked as predicted. We received two $2 \mathrm{~mm}$ crystals grown by PeTER Schunemann's team at BAE systems in New Hampshire, USA. They were AR coated from $2.04 \mu \mathrm{m}$ to $2.2 \mu \mathrm{m}$ and from $2.8 \mu \mathrm{m}$ to $6.0 \mu \mathrm{m}$ ( $R<1.5 \%$ per side, coated by Twinstar Optics). SHG of the PPLN OPA idler was also successfully tested with some Eksma ZGP crystals, but those had to be returned after testing and could not be used for the SCG experiments.

Fig. 4.21(b) shows the continuum generation results obtained with the compressed PPLN OPA idler focused to about $50 \mu \mathrm{m}$ spot diameter. The spectrum was measured with an FTIR by taking 50 interferograms, correcting their timing jitter and averaging over them before FouRIER transformation to the frequency domain. This postprocessing improved the SNR of the spectrum by about one order of magnitude. The displayed spectrum extends to about $11 \mu \mathrm{m}$ which is comparable to the initial simulations shown in Fig. 4.21(a). However, most of the spectral power is concentrated around the central wavelength of $4.1 \mu \mathrm{m}$ and the spectral wing from $5 \mu \mathrm{m}$ to $11 \mu \mathrm{m}$ is only at a relative power level of $-30 \mathrm{~dB}$. Although some expected absorption features stemming from the propagation through the laboratory, from the used silver mirrors or the nonlinear crystal itself can be recognized, the weaker spectral parts are very close to the noise level and may be distorted from artifacts of the spectrometer. Apart from the low power spectral densities, the spectrum was also hard to reproduce on a daily basis. Therefore, an additional pulse compression stage was introduced. Employing pulses with a steeper temporal gradient accelerates spectral broadening and facilitates SCG.

\section{Precompression of the mid-infrared pulses}

SCG was clearly simplified by employing sub-100 fs pulses. In order to generate them, bulk spectral broadening was utilized to reduce the pulse duration by a factor of two. Despite the moderate peak power of about $750 \mathrm{~kW}$, highly nonlinear materials clearly increased the spectral bandwidth of the mid-IR radiation. Fig. 4.22 shows the measured spectra for several materials. In the experiments, the focal spot diameter was varied between $30 \mu \mathrm{m}$ and $80 \mu \mathrm{m}$. The best results could be achieved with the largest spot size due to the weaker beam diffraction. Nevertheless, the crystals had to be placed into the focus because of the short RAYLEIGH length of only about $1 \mathrm{~mm}$. Fig. 4.22 exhibits similar FTLs for $5 \mathrm{~mm}$ thick Si and GaAs plates as well as for one of the $3 \mathrm{~mm}$ thick ZGP crystals initially purchased from ASCUT Ltd \& Co KG. Among them, the ZGP crystal was the least dispersive (cf. table 4.1), and thus was used for pulse compression. By investigating the spectral broadening of the $2 \mathrm{~mm}$ ZGP crystal, which was cut properly, it turned out that fast axis (ordinary) polarization of the mid-IR led to the strongest broadening at 


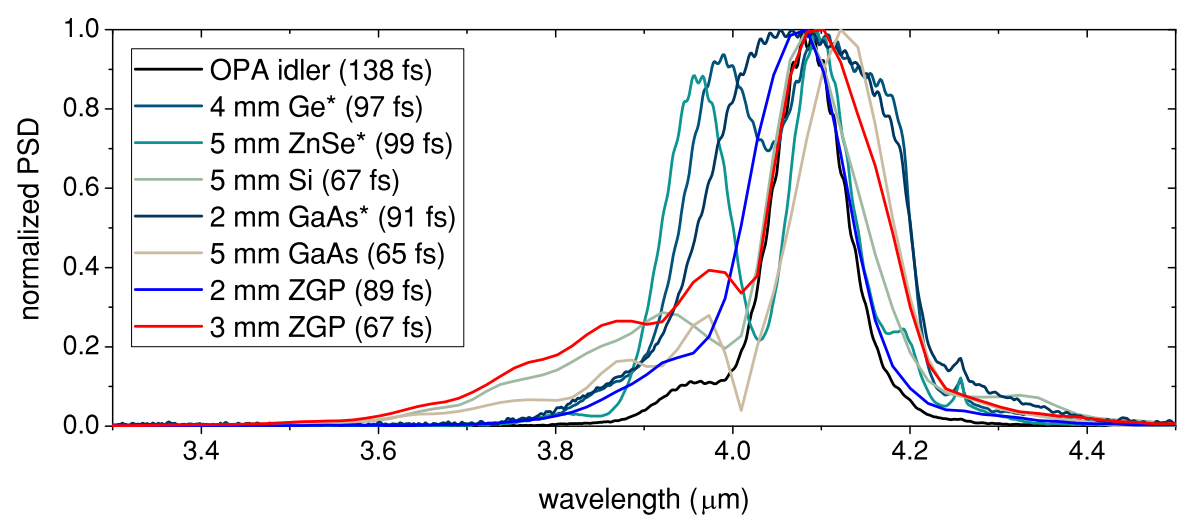

Fig. 4.22. Bulk spectral broadening with the compressed PPLN OPA idler. Several nonlinear materials were tested. Their names, the crystal lengths and the achieved FTLs are given in the figure legend. The asterisk indicates that the material was uncoated. The ZGP crystals were tuned off-resonance and did not produce any measurable SH. Among the tested crystals, the $5 \mathrm{~mm}$ silicon, the $5 \mathrm{~mm}$ gallium arsenide and the $3 \mathrm{~mm}$ ZGP crystal showed the best broadening results. The spectrum of the $3 \mathrm{~mm}$ thick ZGP crystal (red solid line) was utilized for pulse compression. The black solid line shows the input spectrum. All spectra were measured with an FTIR (Lasnix or Bristol).

the $>-10 \mathrm{~dB}$ level. Since ZGP is an uniaxial crystal, it can be inferred that the idler polarization for best spectral broadening in the $3 \mathrm{~mm}$ crystal was also ordinary.

The phase of the uncompressed pulses was measured by X-FROG and afterwards compressed by means of material dispersion. A $2 \mathrm{~mm}$ thick sapphire and a $5 \mathrm{~mm}$ thick $\mathrm{CaF}_{2}$ plate were used for this purpose. The setup was nearly identical to the one which is shown

(a)

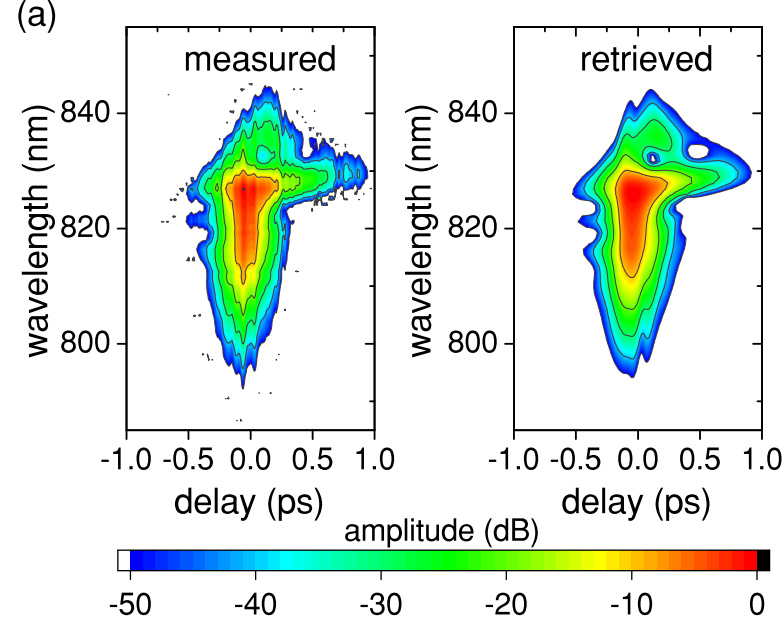

(b)

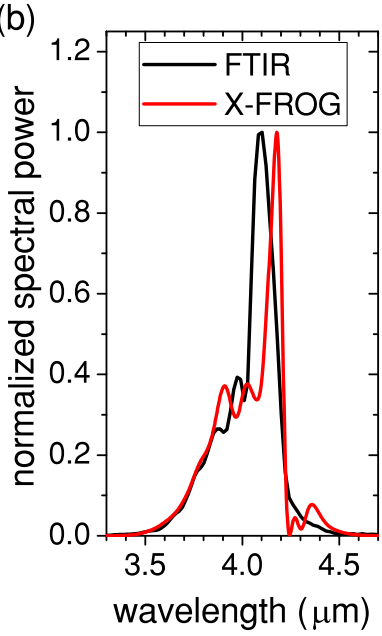

(c)

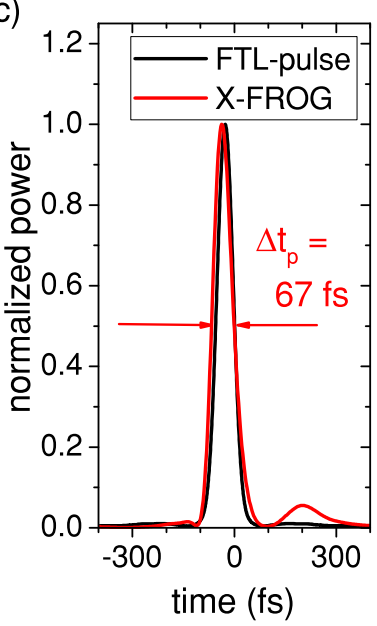

Fig. 4.23. (a) Measured and retrieved FROG traces of the spectrally broadened mid-IR pulses after dispersion compensation. The grid size was $512^{2}$, the residual FROG error $0.5 \%$. No marginal was enforced for the FROG retrieval. The traces show that the central part of the mid-IR spectrum is not ideally in phase with respect to the broadened parts. That is a typical phenomenon of single-stage bulk compression (cf. section 3.2.1) (b) Comparison between the retrieved FROG spectrum and the spectrum measured with an FTIR (Lasnix L-FTS). The peak of the X-FROG spectrum is red-shifted and the $\mathrm{CO}_{2}$ absorptions are more pronounced (due to the longer propagation length to the nonlinear crystal than to the FTIR detector). Otherwise, the agreement is also good and FTLs are nearly identical. (c) Pure material dispersion also led to a good compression of the spectrally broadened pulses which are nearly Fourier limited as the comparison of the red and the black curve shows. 


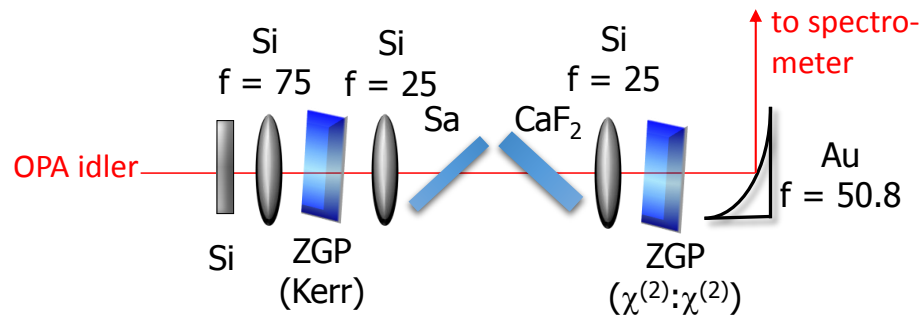

Fig. 4.24. SCG setup. The input beam came from the PPLN OPA (cf. Fig. 4.1). A 5 mm thick, AR-coated silicon plate compressed the OPA idler after a dielectric beam splitter. In an alternative configuration with dielectric mirrors collimating the OPA output and no additional beam splitter, a $1 \mathrm{~mm}$ thin silicon plate with a $2.4 \mu \mathrm{m}$ longpass coating (cf. Fig. 4.2(c)) was utilized. HR mirrors for the $4.1 \mu \mathrm{m}$ were only available at the very end of the experimental campaign. The $3 \mathrm{~mm}$ thick ZGP did not generate SH. Its KERR nonlinearity was utilized to reduce the FTL of the mid-IR pulses by about a factor of two. After collimation with a silicon lens, pulse compression (sapphire and $\mathrm{CaF}_{2}$ plates) and refocusing with another plano-convex Si lens, a supercontinuum was generated in a $2 \mathrm{~mm}$ thick ZGP crystal which was nearly phase-matched for SHG. The emerging beam was coarsely collimated by a parabolic gold mirror and sent to either the FTIR or the monochromator. All focal lengths $\mathrm{f}$ are in mm.

in Fig. 4.9. Only the reference beam path had to be adapted to the additional optical length caused by the high refractive indices of the compression stage optics. The FROG traces are shown in Fig. 4.23(a) and the retrieved, nearly FouRIER transform-limited pulse in Fig. 4.23(c). No particular attention was paid to the beam profile emerging from the broadening stage like in section 3.2. However, owing to the small broadening factor, the beam could be still focused well by a plano-convex silicon lens to a diameter of about $80 \mu \mathrm{m}$ in order to trigger SCG.

\section{Two octave spanning mid-infrared continuum}

For SCG, the compressed pulses were focused into a $2 \mathrm{~mm}$ thick ZGP crystal cut at $\theta=55^{\circ}$ (Fig. 4.24). The nonlinear crystal was mounted such that it could be rotated around the propagation direction axis, tilted in the horizontal direction and translated along the propagation direction. All free parameters were successively optimized under observation of the mid-IR spectrum. Best results were obtained with the nonlinear crystal at near normal incidence and in the focus. The supercontinuum is shown in Fig. 4.25. Including the generated second harmonic, it spans from $1.6 \mu \mathrm{m}$ to $7.1 \mu \mathrm{m}$ (at $-30 \mathrm{~dB}$ ) which corresponds to more than two octaves. If combined with the broadband mid-IR radiation of the LGS OPA, about 2.7 octaves are covered with a power spectral density clearly exceeding $\mu \mathrm{W} / \mathrm{cm}^{-1}$ over the whole range. When utilizing the $67 \mathrm{fs}$ input pulses, SCG was much easier to obtain than without the intermediate pulse compression stage.

The SCG simulations presented in Fig. 4.21(a) were repeated with the retrieved pulses shown in Fig. 4.23. The simulated output spectrum (Fig. 4.26(b)) agrees qualitatively well with the measured one presented in Fig. 4.25. There are two main peaks. One at the central frequency at $4.1 \mu \mathrm{m}$ and the other at its second harmonic. Moreover, the power spectral density exhibits a minimum between $2.5 \mu \mathrm{m}$ and $2.75 \mu \mathrm{m}$. Most importantly, the spectrum broadens also significantly towards longer wavelengths which allows to close the spectral gap to the output of the LGS OPA. The simulations predict an even stronger extension into the long wavelength infrared: at the $-30 \mathrm{~dB}$ level to $8.8 \mu \mathrm{m}$ and at the $-50 \mathrm{~dB}$ level (shown in Fig. 4.26(a)) even over whole transparency range of ZGP ${ }^{426}$. A 


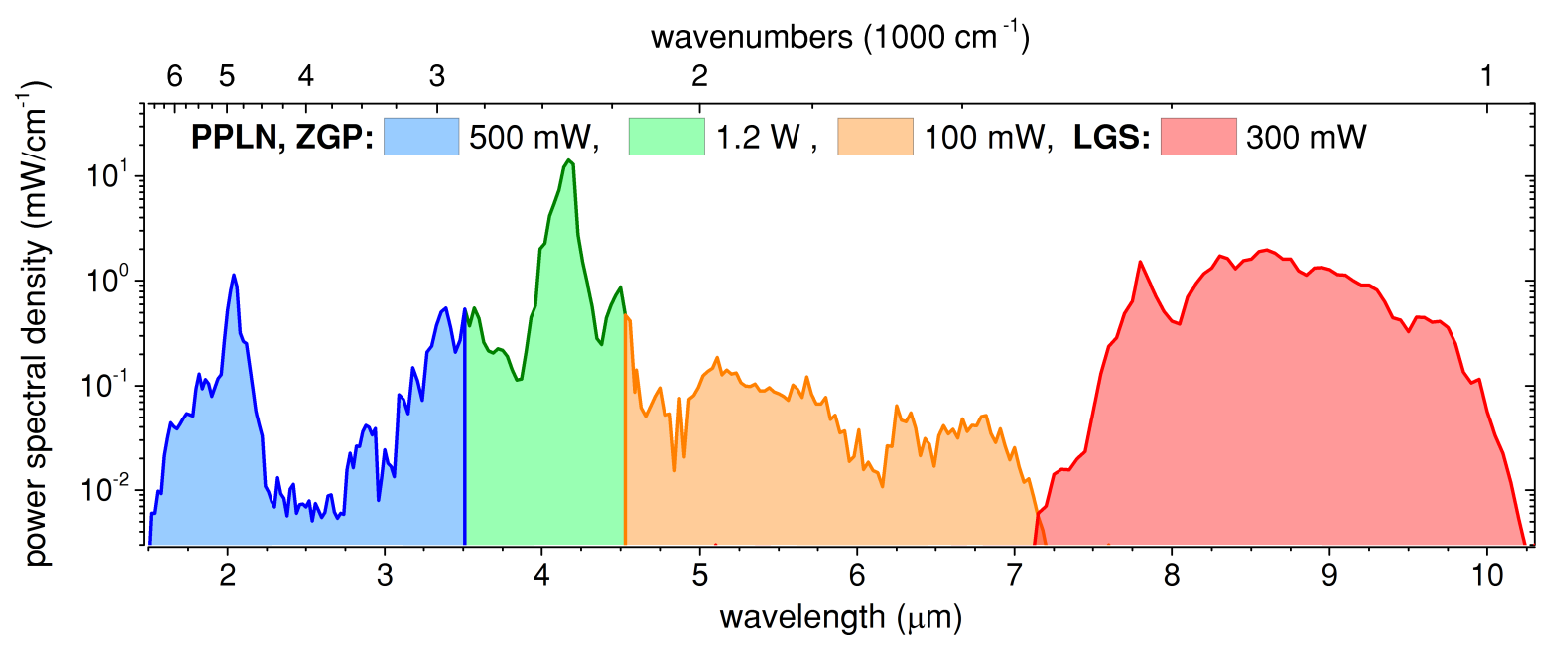

Fig. 4.25. Coverage of the mid-IR spectral region from $1.6 \mu \mathrm{m}-10.2 \mu \mathrm{m}$. Blue, green and orange areas represent a spectrum measurement after SCG in ZGP with a measured total power of $1.8 \mathrm{~W}$. The spectrum was measured about $1.5 \mathrm{~m}$ away from the ZGP crystals and partly shows strong attenuation due to atmospheric absorptions. The average powers were measured behind the collimation parabolic mirror. A short pass (blue area) and a long pass (orange area) were used to measure the power of the individual components. The $-30 \mathrm{~dB}$ width, corresponding to a power spectral density of about $10 \mu \mathrm{W} / \mathrm{cm}^{-1}$, ranges from $1.6 \mu \mathrm{m}-7.1 \mu \mathrm{m}$. The red area shows the spectrum generated from an LGS OPA with a measured output power of $300 \mathrm{~mW}$, extending the spectral coverage to $10.2 \mu \mathrm{m}$, i.e. over 2.7 octaves in total.

reason for this discrepancy could be the unexpected nonlinear absorption of the crystal which amounted in about $20 \%$ transmission loss when ZGP was moved into the focus. There are several reasons for this reduced transmission: Firstly, linear absorption sets in at wavelengths below $2 \mu \mathrm{m}$, overlapping with the spectral range of the broadband $\mathrm{SH}$. Secondly, the crystal was AR coated in the range from $2.04 \mu \mathrm{m}-2.2 \mu \mathrm{m}$ and from $2.8 \mu \mathrm{m}$ - $6 \mu \mathrm{m}$. The continuum, however, exceeded this range which caused additional reflection losses. Thirdly, SCG extended the spectrum to regions of strong water vapor absorptions (cf. Fig. 4.21(b)). Nevertheless, since most of the spectral power is concentrated around $4 \mu \mathrm{m}$, another mechanism must be present resulting in the high transmission losses. GaAs was tested as an alternative material for supercontinuum generation. In this case, a prominent blue shoulder was observed, indicating free carrier generation and consequent self-steepening. A nonlinear transmission loss of about $30 \%$ was determined in this case. Since the laser peak power was on the order of the critical power of GaAs and the photon energy was only about $20 \%$ of the semiconductor bandgap, neither beam collapse nor significant multi-photon absorptions were expected. However, it is known that the carrier lifetime of GaAs is on the ns order which corresponds to the oscillator repetition rate and causes cumulative effects resulting in enhanced sample conductivity and hence absorption ${ }^{255}$. The issue was solved by modifying the GaAs growth process and introducing additional material defects which led to carrier recombination times on the ps order ${ }^{255}$. Similar cumulative effects are suspected to be present in ZGP. Removing those, for instance by also introducing a fast trap mechanism, would be beneficial for $\mathrm{MHz}$ rate SCG generation with respect to a presumably extended spectral coverage of the long wavelength infrared and higher spectral stability of the continuum. 

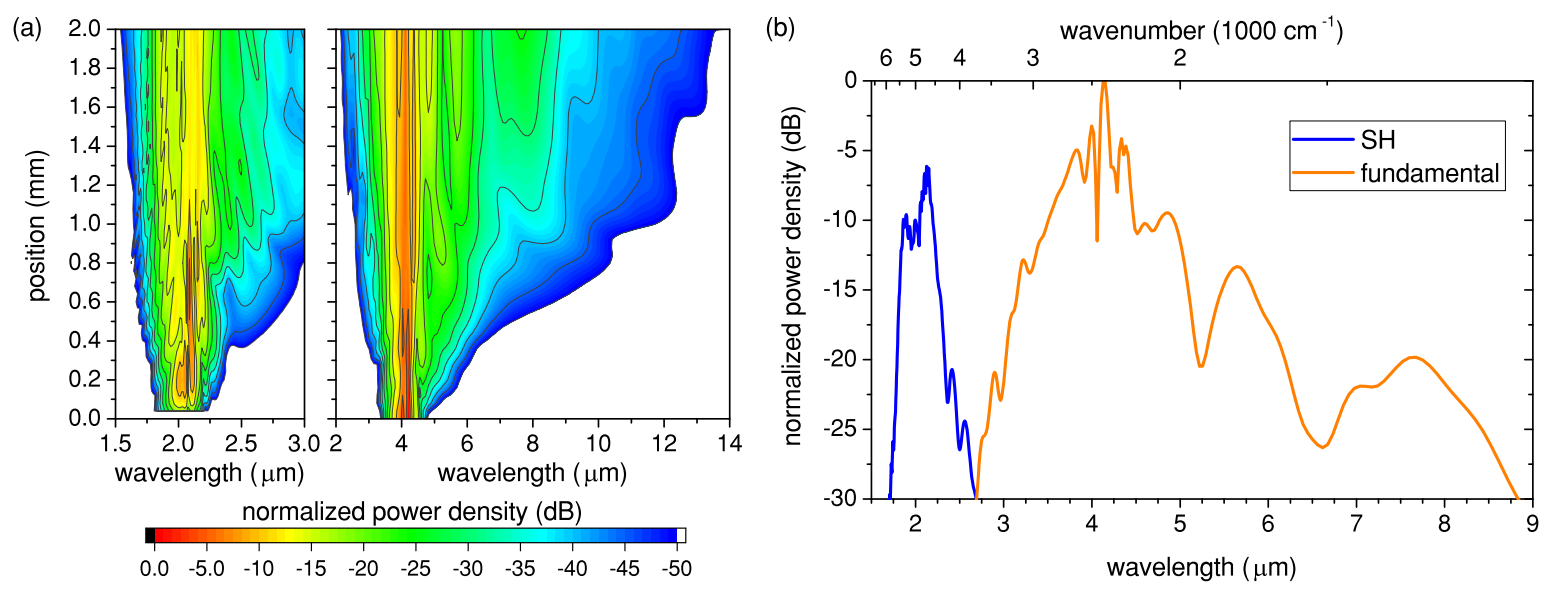

Fig. 4.26. Simulation of continuum generation in ZGP with $67 \mathrm{fs}$ input pulses. (a) Spectral evolution of the continuum (left: SH, right: fundamental). Most of the spectral width is acquired within the first mm of propagation length. The spectrum covers the full transparency range of ZGP. (b) Simulated output spectrum with a width from $1.8 \mu \mathrm{m}$ to $8.8 \mu \mathrm{m}$ at $-30 \mathrm{~dB}$ of the maximum. It is qualitatively comparable to the experimental spectrum shown in Fig. 4.25. The simulation included $\chi^{(2)}-\left(\mathrm{d}_{\mathrm{eff}}=70.8 \mathrm{pm} / \mathrm{V}^{428}\right)$ and $\chi^{(3)}$-nonlinearity $\left(n_{2}=1.3 \times 10^{-14} \mathrm{~cm}^{2} / \mathrm{W}^{290}\right)$, linear absorption and dispersion according to the crystal's SelLmeier equation ${ }^{429}$. The tuning angle $\theta=55^{\circ}$ in the simulation corresponded to the cut angle of the birefrigent crystal that was used in the experiment. As input, the retrieved pulse after the first compression stage was used, carrying an energy of $53 \mathrm{~nJ}$. A Gaussian beam was launched on a spatial grid of $128 \times 65$ points, each $7.5 \mu \mathrm{m} \times 7.5 \mu \mathrm{m}$ in size. Two frequency grids from 0 to $200 \mathrm{THz}$ as well as from $100 \mathrm{THz}$ to $300 \mathrm{THz}$ were factored out. Each contained 512 points. Propagation to the detector was not included in the simulation.

\section{Discussion}

The energy of the pulses with $4.1 \mu \mathrm{m}$ central wavelength, after separating them from amplified seed and pump of the OPA, is about $90 \mathrm{~nJ}$, yielding a combination of peak and average power which was difficult to employ in established SCG schemes that extend to wavelengths beyond $5 \mu \mathrm{m}$. For instance, SCG in chalcogenide fiber yielded spectral coverage from about $1.5-14 \mu \mathrm{m}^{430,431}$. The fibers, however, performed clearly worse when pumped with $\mathrm{MHz}$ sources since small core diameters led to a low power damage threshold $^{341}$ and larger diameters only to moderate spectral broadening ${ }^{432,433}$. Similarly, chalcogenide waveguides ${ }^{434}$ will not be ideal for high average power handling. By contrast, both the ZGP crystal $^{426}$ and spectral broadening based on quadratic nonlinearities (cf. section 3.2.2) are capable of high average power handling. Another common approach to generate mid-IR supercontinua, namely filamentation ${ }^{342,435}$, requires free carriers, i.e. absorption and subsequently heat dissipation. Moreover, the method has only been demonstrated with at least MW peak powers. In addition, filamentation leads, due to self-steeping, to a strong spectral blue shift. This self-steepening effect is controllable by cascaded quadratic nonlinearities ${ }^{352}$ which enabled more pronounced broadening towards longer wavelengths, and subsequently to the overlap of the supercontinuum with the spectrum generated from the LGS OPA. Despite reaching the spectral coverage from $1.6 \mu \mathrm{m}$ to $10.2 \mu \mathrm{m}$ only at $-30 \mathrm{~dB}$, the (combined) continuum still presents a significant advance in power spectral density. For instance, the continuum of comparable extend in ref. 430 was obtained at an average power of less than $1 \mathrm{~mW}$ and measured at $-20 \mathrm{~dB}$. Consequently, the spectrum displayed in Fig. 4.25 presents more than two orders of magnitude increase in power spectral density. SCG in bulk materials also does not require 
any sensitive coupling of the free beam transversal mode to a waveguide mode, and is thus straight forward to employ. On the other hand, the presented continuum exhibits also a few drawbacks. Firstly, the full 2.7 octaves were generated in two OPA channels. State-of-the-art mode-locked thin-disk oscillators ${ }^{58,59,61,139,436}$ readily allow combining the output of LGS and PPLN OPA, and hence to get access to the whole mid-IR range in a single setup. A quasi-third channel results from SHG in the ZGP crystal. The spectrum covers the range from $1.6 \mu \mathrm{m}$ to $2.5 \mu \mathrm{m}$. It is orthogonally polarized with respect to the fundamental. For many spectroscopic applications, polarization is not of particular importance. For nonlinear methods, however, this has to be taken into account. Eventually, the spectral homogeneity of the beam shows the typical features of bulk broadening which are explicitly described in section 3.2.1. Cascaded quadratic nonlinearities allow suppressing these inhomogeneities as shown in section 3.2.2. But this was not explicitly investigated in the experiments presented here.

The multiple channels of the supercontinuum suggest waveform synthesis which has been recently demonstrated in the mid-IR with a $\mathrm{kHz}$ source ${ }^{437}$. Studies on $\mathrm{PHz}$ electronics $^{247,438,439}$ could be significantly extended by compressed pulses. Firstly, because of the long driving wavelength and the associated impact on the electron trajectories in the electronic devices ${ }^{388,389}$. Secondly, the $\mathrm{MHz}$ repetition rate of the presented system would give insights into the reversibility of the current switching effects. Upon attenuating the most powerful parts of the spectrum shown in Fig. 4.25, the source would also become highly interesting for spectroscopy applications in biological matter where Watt average power levels would unnecessarily heat the samples. However, the demonstrated power spectral densities on the $\mu \mathrm{W} / \mathrm{cm}^{-1}$ order are typical for synchrotron radiation ${ }^{440}$. Hence, the demonstrated laser based source presents a compact alternative to the large scale facilities that have found numerous applications in life sciences ${ }^{440}$. 


\subsection{Chapter Summary}

This chapter presented three frequency down-conversion setups: two based on optical parametric amplification and one based on intra-pulse DFG. The OPAs generated Wattclass fs radiation in the range from 4 to $5 \mu \mathrm{m}$ and around $8.5 \mu \mathrm{m}$. The DFG experiment realized a broadband $\mathrm{mW}$-level source in the wavelength region from 6.8 to $16.4 \mu \mathrm{m}$ (at $-30 \mathrm{~dB}$ level). In the meantime, this approach has been further developed towards applications in infrared spectroscopy ${ }^{64,441}$. An overview of the mid-IR sources is given in table 4.3. The most powerful radiation stems from the PPLN OPA owing to the lower quantum defect in comparison to the LGS based schemes and the high FOM of the PPLN crystal. On the other hand, this OPA exhibits the smallest idler bandwidth. Spectral broadening, pulse compression and SCG enabled, however, to extend the initial idler spectrum to two optical octaves (at the $-30 \mathrm{~dB}$ level). It is remarkable that the LGS OPA led to more than an order of magnitude more average power, 25 times higher conversion efficiency and 35 times higher pulse energy than the intra-pulse DFG experiment, but increased the FTL of the mid-IR only by a factor of less than two.

The tuning curves shown in Figs. 4.7(b) and 4.14(b) cover a wide range of the mid-IR spectrum. The highest spectral power densities are achieved in the atmospheric transmission windows from $3-5 \mu \mathrm{m}$ and $8-13 \mu \mathrm{m}$ which is particularly important for trace gas analysis ${ }^{66}$. The high average powers are especially useful for stand-off detection ${ }^{391}$. Utilizing frequency comb spectroscopy for this purpose would require to lock a multiple of the laser repetition rate to a precise frequency reference or to use a second fs laser for dual-comb spectroscopy ${ }^{66,381}$. The passive carrier-envelope-offset frequency stabilization of the idler makes the OPAs attractive for applications in high dynamic range measurements by virtue of electric-field-resolved spectroscopy ${ }^{64}$. This will be briefly discussed in the following outlook chapter of this dissertation.

The tunability of the OPAs could be also extended. The ANDi fiber seed would support radiation down to about $3.3 \mu \mathrm{m}$ (cf. Fig. 4.4(a)). However, the available poling periods only allowed to generate wavelengths longer than $3.8 \mu \mathrm{m}$. ZGP does not just enable SCG but could be also used for efficient frequency doubling. This may give access to the wavelength range from 2 to 2.5 micron while Watt average power levels are maintained. In the range from $5-7 \mu \mathrm{m}$, the LMA-12 seed power is relatively weak. To reach Watt levels in this spectral region a double stage OPA would become necessary since the FOM of LGS is not high enough for amplifying the ANDi seed to multiple Watts in a single crystal. The presented OPA concept is not suited to reach Watt levels beyond $11 \mu \mathrm{m}$. This is due to the transparency range of LGS (cf. Fig. 4.11). For this purpose, cascaded down-

Table 4.3: mid-IR source comparison

\begin{tabular}{|c|c|c|c|}
\hline & PPLN OPA & LGS OPA & LGS DFG \\
\hline output power & $5.1 \mathrm{~W}$ & $1.3 \mathrm{~W}$ & $0.1 \mathrm{~W}$ \\
\hline idler energy & $135 \mathrm{~nJ}$ & $34 \mathrm{~nJ}$ & $1 \mathrm{~nJ}$ \\
\hline total quantum efficiency ${ }^{a}$ & $39 \%$ & $21 \%$ & $1.3 \%$ \\
\hline FOURIER transform limit & $140 \mathrm{fs}$ & $85 \mathrm{fs}$ & $55 \mathrm{fs}$ \\
\hline pulse duration & $140 \mathrm{fs}$ & to be compressed & $66 \mathrm{fs}$ \\
\hline
\end{tabular}

${ }^{a}$ Refers to the ratio between mid-IR photons and all photons emitted from the KLM TD oscillator. 
conversion schemes or longer wavelength pump sources become necessary. One could, for instance, recycle the PPLN OPA signal for frequency down conversion in gallium selenide. For the nonlinear crystal with a $2.1 \mathrm{eV}$ bandgap ${ }^{442}$, the two-photon absorption coefficient at $1.4 \mu \mathrm{m}$ is much lower than at $1 \mu \mathrm{m}$ wavelength. Alternatively, the idler beams of the OPAs could be used for further down-conversion.

For the first time, Watt-level supercontinuum generation in the long-wavelength mid-IR was demonstrated with a table-top fs light source. Other coherent high-power mid-IR continua were limited to wavelengths below $5 \mu \mathrm{m}^{282}$. The power spectral densities reported here are at least as high as those of large scale synchrotron sources ${ }^{440}$. Although synchrotron spectra cover both the mid-IR and the $\mathrm{THz}$ region, the presented table-top laser-based source, which also covers more than two optical octaves, presents a highly attractive alternative. Needless to say, that laser systems are clearly superior to synchrotrons in terms of costs and availability. Furthermore, the presented broadband fs mid-IR source may be also employed in experimental techniques where synchrotron radiation or incoherent light exhibit severe limitations or are not applicable at all. Examples are frequency $\mathrm{comb}^{66}$ or field-resolved spectroscopy ${ }^{64}$. It is to note, however, that the first demonstration of near phase-matched SCG in ZGP will also have to undergo some technical development like the DFG source introduced in section 4.1.3 ${ }^{441}$.

In conclusion, a highly powerful tool for advancing fs mid-IR spectroscopy has been presented. Multiple Watts of average power were generated between 4 and $5 \mu \mathrm{m}$, and, for the first time, a Watt-class fs source operating beyond the multi-phonon absorption edge of oxide crystals was demonstrated. Likewise, the ultrashort pulses may give access to extreme nonlinear optical effects in solids and allow to study ultrafast molecular dynamics. Furthermore, SCG based on near phase-matched cascaded quadratic nonlinearities resulted in significant spectral broadening towards longer wavelengths. With all the presented down-conversion schemes a coverage of the wavelength range from $1.6 \mu \mathrm{m}-10.2 \mu \mathrm{m}$ (at $-30 \mathrm{~dB}$ ) with more than $2 \mathrm{~W}$ total average power as well as from $6.8 \mu \mathrm{m}$ to $16.4 \mu \mathrm{m}$ (at $-30 \mathrm{~dB}$ ) at $\mathrm{mW}$ level was achieved. These results push the frontiers of fs mid-IR sources and with that demonstrate how mode-locked TD oscillators may become essential working horses for research activities in multiple scientific disciplines. 


\section{OUTLOOK AND CONCLUSIONS}

\subsection{Near-Future Applications}

Three application ideas are derived from the knowledge and the achievements which have been accomplished through the experiments and simulations presented in the chapters 2 to 4 of this thesis. The author expects that each of them could be realized on the time scale of about one year.

\subsubsection{The attosecond oscillator}

Of course, an attosecond oscillator cannot be accomplished in the sense that it is based on an even broader gain medium than a femtosecond oscillator. But a laser system which emits attosecond pulses at multiple $\mathrm{MHz}$ repetition rates and does not involve any active or passive amplifiers is in very close reach. Likewise, the applications of such a source stated in the introduction section 1.2 and the outlook of ref. 13 may be finally targeted.

In the introduction section 1.2, the three prerequisites for attosecond pulse generation were stated. Among them, few-cycle pulse generation and waveform control has been demonstrated for the first time with mode-locked TD oscillators as reported in chapter 2 . The few-cycle pulses were obtained at a pulse energy of "only" $150 \mathrm{~nJ}$, and thus it was concluded that further power scaling of pulse compression and CEP stabilization techniques were required. These have been demonstrated in chapter 3. A peak power enhancement of more than a factor of three to $60 \mathrm{MW}$ could be achieved with a bulk broadening technique taking advantage of cascaded $\chi^{(2)}$-nonlinearities. Moreover, a power-scalable KERR-effect based multi-plate approach was simulated which would also allow few-cycle pulse generation. The simulations have been qualitatively confirmed very recently with a $20 \mathrm{~W}$ average power light source delivering more than 300 times higher peak powers than the KLM TD oscillator utilized here ${ }^{443}$. In addition, in a multi-pass and a HERRIOT-cell ${ }^{444}$ type configuration, the multi-plate broadening has been also demonstrated for KLM TD oscillators ${ }^{62,339}$ (certainly also motivated by the work of refs. 32, 336). In the multi-pass geometry ${ }^{62}$, peak powers of $230 \mathrm{MW}$ and pulse durations of $30 \mathrm{fs}$ have been reported, exceeding even the peak powers reached in ref. 323 where a fiber amplifier system which was utilized for generating an unprecedented high XUV photon flux via HHG. Therefore, the KLM TD oscillator based source must be also able to generate high power XUV radiation. Furthermore, the HERRIOT-cell multi-pass spectral broadening approach could reduce the FTL of the KLM TD oscillator by a factor 22 to $10 \mathrm{fs}^{339}$. Consequently, entering the few-cycle regime with the $230 \mathrm{MW}$ source will be also possible. Last but not least, one of the CEP stabilization schemes presented in sections 2.3.2 and 3.3 must be applied to the KLM TD oscillator presented in refs. 139 and 62 . In supplement 1 of section 2.3, the AOM approach was expected to be transferable to such an oscillator with $400 \mathrm{MW}$ intracavity peak power. Very recent (so-far unpublished) studies indicate that CEP stabilization of the latest generation TD oscillator is in-fact possible. Therefore, 
what needs to be done, is firstly combining spectral broadening and CEP stabilization and secondly, generating high-harmonics and measuring their spectrum and temporal shape for instance by attosecond streaking ${ }^{42}$.

\subsubsection{High-speed, high-rate optical switching}

During the past five years few-cycle pulses have opened the perspective to speed up electronic switching to $\mathrm{PHz}$ frequencies ${ }^{247,438,445}$. The reported experiments were performed at $\mathrm{kHz}$ repetition rates and with near-IR laser pulses, and have thus not been able to answer the following questions:

1. How does optical current switching scale with the wavelength of the (driving) laser?

2. What are the electronic switching rates that can be achieved by the optical current injection technique and how reversible is the switching?

Question 1 should not be reduced to the choice of photon energy to bandgap ratio. Tuning the central wavelength is, in-fact, very different from changing the insulator bandgap. This becomes clear by interpreting the ultrafast current switching phenomenon as a twostep process: The first step is to inject electrons into the conduction band (and holes in the valence band). The second steps is to drive the quasi-free carriers within their bands ${ }^{247,445}$. In-fact, pumping semiconductors with ultrashort mid-IR pulses revealed physically fundamentally different effects than observed with few-cycle near-IR pulses, namely the occurrence of HHG and dynamical BLOCH oscillations ${ }^{248,389}$. Essentially, the impact of the laser frequency driving the electron in the conduction band can be inferred from the corresponding cycle period that determines the strength of the light driven excursion of the quasi-free electrons. It is therefore expected that longer driving wavelengths lead to larger charge separation, a stronger material polarization, and thus to higher net currents. For real-world applications, a high photon flux to electronic current conversion would be extremely important to keep the power consumption and the heat generation as low as possible. To the best of the author's knowledge, there has not been any experimental study so-far that has examined ultrafast current switching with a tunable laser, and hence a quantitative scaling relation between laser wavelength and optically induced charge has still to be investigated.

The waveform-stabilized few-cycle pulse sources described in the chapters 2 and 4 present an attractive tool for conducting such a study. About two cycle pulses were reached at about $1 \mu \mathrm{m}$, five cycle pulses at $4 \mu \mathrm{m}$ - that could be compressed by another factor of two - 2 cycle pulses at about $10 \mu \mathrm{m}$ at $\mathrm{mW}$ average power as well as a FTL of three cycles at about $8.5 \mu \mathrm{m}$ and Watt average power level. Pulse compression, possibly by inserting dispersive materials, would be still needed for this source. Efficiently frequency-doubling the $4 \mu \mathrm{m}$ radiation would also give access ultrashort pulses at $2 \mu \mathrm{m}$. Consequently, fieldinduced current injection could be studied with driving lasers that span more than three optical octaves. Due to maximal tens of nJ pulse energies in the long-wavelength infrared, one could also combine strong near-IR pulses for current injection with the weaker mid-IR pulses that drive the electrons.

Question 2 has been partially addressed in current injection studies in GaAs ${ }^{254,255,446,448,449}$. At the beginning of chapter 3, the experiments of HACHÉ et al. have already been briefly described as an example where repetition rate dramatically influenced the experimental 


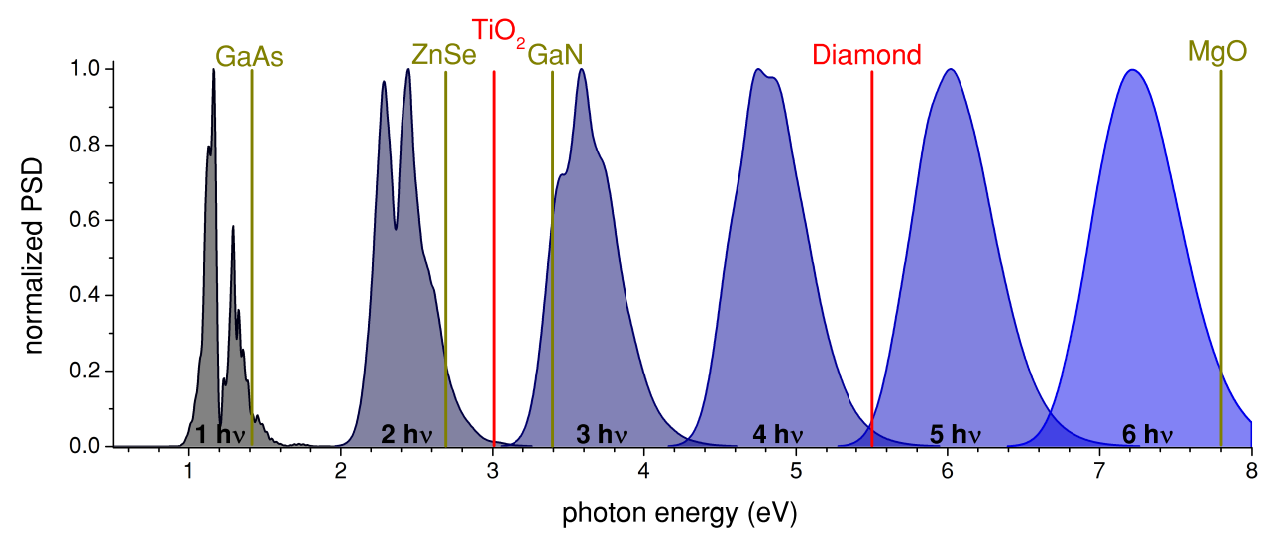

Fig. 5.1. Multi-photon absorption energies of the few-cycle pulse spectrum retrieved in Fig. 2.13(c) and bandgaps of selected materials that have been used in other current control experiments ${ }^{247,446,447}$ or seem suited for the available source. The N-photon absorption energies $(\mathrm{Nh} \nu)$ have been calculated through convolving the fundamental spectrum $\mathrm{N}$ times with itself. The bandgaps are marked through the vertical solid lines. In GaAs for instance, an interference between the one- and two photon absorption channels would be expected ${ }^{255,446}$. There is, however, only a tiny photon energy overlap. Therefore, further spectral broadening is required for utilizing the material. The situation is different for higher order absorption channels. For example, the diamond bandgap is exactly in the overlap region of the 4 and the 5 photon channel.

observations. Growing GaAs at low temperatures was needed to induce trap states which led to ps scale recombination of the excited electron-hole pairs. Only after the introduction of this fast relaxation mechanism, the current control experiments could be transferred from $\mathrm{kHz}$ to $\mathrm{MHz}$ rate sources. The quest for suited materials that allow ultrahigh current switch rates will therefore be an important research area for speeding up electronics by utilizing few-cycle pulses. Low temperature grown GaAs will certainly be a great material to start with. It is known that current control can be achieved with $\mathrm{MHz}$ pulses and by the interference of one and two photon absorption channels ${ }^{446,449}$. Consequently, the few-cycle source described in chapter 2 could be used to establish the experimental setup while the mid-IR sources presented in chapter 4 could significantly extend the studies that have been performed with Ti:sapph oscillators. In-fact such experiments have been prepared already in collaboration with the research group of M.-C. AMANn from the Walther Schotтky Institute in Garching. A low temperature grown GaAs wafer with sputtered electrodes on the top surface was prepared by H. SCHMEIDUCH and G. Boenm. Unfortunately, there has been no time for conducting the experiments so-far.

The search for suited materials is not only a concern of carrier lifetime. Also band structure and -gap play a decisive role as, for instance, demonstrated in refs. 247, 447. Decreasing the bandgap to photon energy ratios from at least 2.9 for FS to at least 1.2 for GaN yielded a two order of magnitude peak irradiance reduction for achieving the same amount of optically induced charge ${ }^{247}$. For the source presented in chapter 2, GaAs appears to be a good material to start with, although it may not be ideal for realizing ultrafast switches. A significant part of the input spectrum could be linearly absorbed. Subsequently, the single-photon would dominate the two-photon channel, resulting in a moderate interference contrast ${ }^{446,450}$. In addition, further broadening to a full octave would be necessary to obtain an overlap between single photon and two photon absorption energies. Fig. 5.1 illustrates this. It shows the photon energies of several absorption channels and the bandgap of several selected materials. For large bandgaps or longer 
wavelength sources, using the electron tunneling picture may be more adequate than the illustrative multi-photon picture, albeit both models worked well for the current injection experiments in $\mathrm{FS}^{445,450}$. Fig. 5.1 shows that the 2 and 3 photon channels overlap such that, for instance the wide bandgap semiconductor $\mathrm{ZnSe}$ or $\mathrm{TiO}_{2}$ (rutile) could be used in combination with the $7.7 \mathrm{fs}$ pulses demonstrated in section 2.2.2. For ZnSe, the twophoton channel would dominate the absorption, and hence $\mathrm{TiO}_{2}$ seems to be the better choice. Also the carrier lifetime of rutile seem appropriate for experiments with $\mathrm{MHz}$ rate source $^{451}$. A larger spectral overlap is present for the 4 and 5 photon channels, making diamond an attractive material to investigate. It is a very robust material with unique thermal properties. On the other hand, high irradiances would be required to trigger absorption which would probably lead to an unfavorable electronic current to photon flux ratio.

The discussion above demonstrates that ultrafast current switching with few-cycle pulses is a research topic with still many things to explore. The widely tunable, high repetition rate sources described in the chapters 2 and 4 of this dissertation, represent an excellent tool to advance the field towards the applications envisioned in ref. 438.

\subsubsection{Field-resolved optical spectroscopy in the mid-infrared}

The most common linear mid-IR spectroscopy techniques rely purely on measuring either transmitted power or spectrum of the utilized light source. This only provides information about the imaginary part of the complex refractive index of the material under test, in other words on (frequency dependent) absorption ${ }^{380,381}$. A coherent light field, however, imprints a complex polarization on the ensemble of quantum mechanical oscillators which results in a characteristic temporal response of the sample. Field-resolved spectroscopy exploits this additional temporal information to exceed the measurement sensitivity of conventional spectroscopy methods ${ }^{64}$. The generic setup of the technique is sketched in Fig. 5.2. The electric field of the free-induction decay of the sample is measured by EOS. More details on the experimental technique and details of the setup can be found for instance in refs. 64, 198, 452. Field resolution may be also extracted from other pulse characterization methods, like $\mathrm{FROG}^{387}$, but EOS does not require a time-consuming

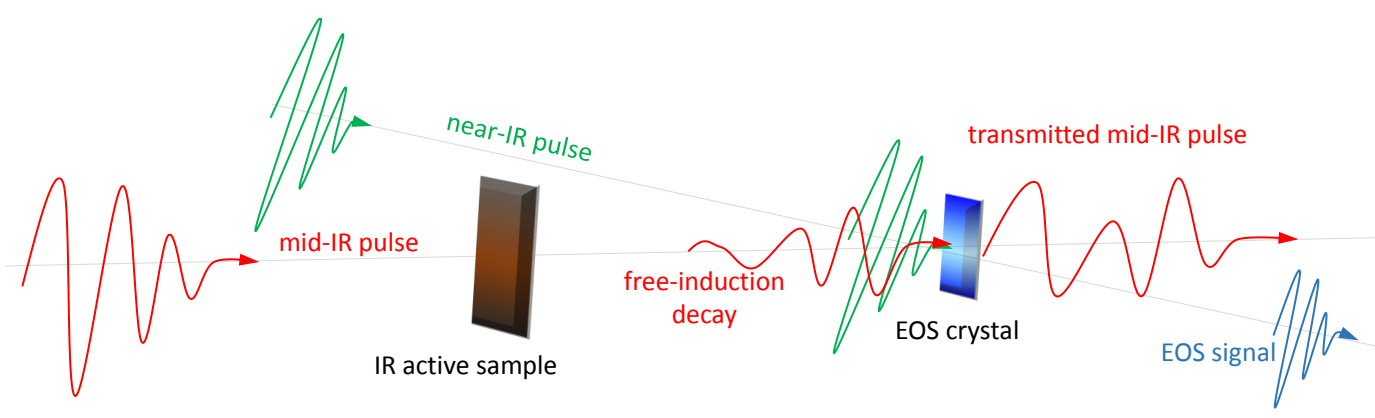

Fig. 5.2. Generic setup of field-resolved spectroscopy. A short mid-IR pulse passes the sample under test and is reshaped through dispersion and absorption. The free-induction decay of the vibrationally excited sample follows. It is up-converted via sum-frequency generation with an ultrashort (known) near-IR pulse. The up-converted signal generates together with the (filtered) near-IR pulse the EOS signal. By varying the delay between mid-IR and near-IR pulses, the full electric field of the free-induction decay is recovered. 
to sample \& EOS

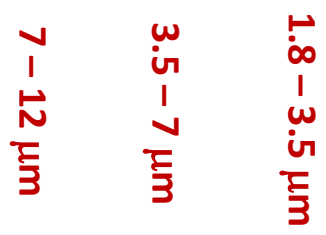

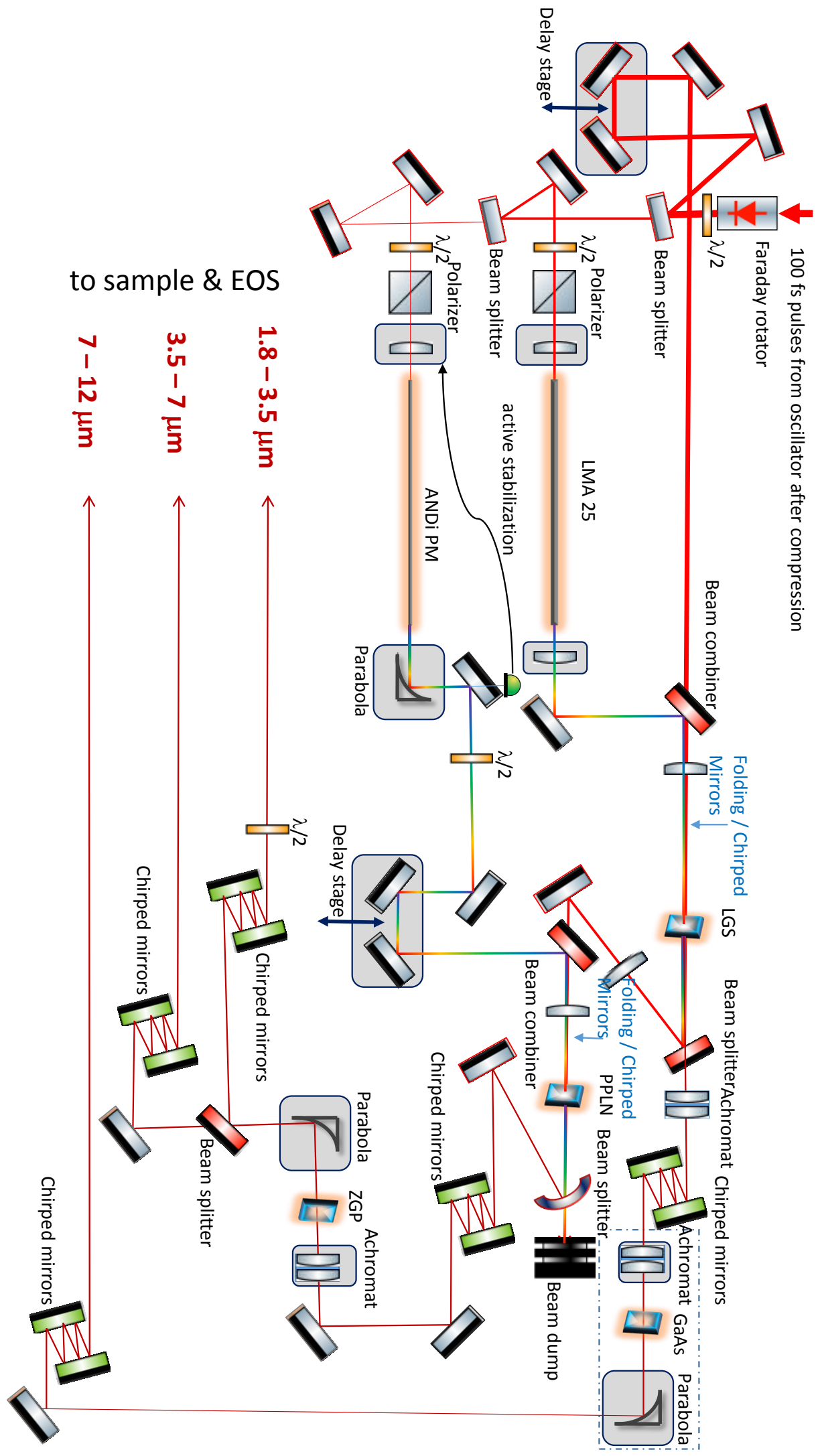

Fig. 5.3. Multi-channel OPA setup for field resolved infrared spectroscopy. Oscillator, near-IR compression stage, sample chamber and EOS setup are not shown. The LGS spectral broadening stage in the box with the blue dashed lines is optional and depends on the targeted extension into the mid-IR. Additional feedback loops for timing control and noise reduction are not shown. They may become necessary, however. ${ }^{441}(\lambda / 2$ : half-wave plate) 
(especially for high resolution) retrieval algorithm and lock-in amplification as well as balanced detection can be readily implemented.

EOS is conventionally understood as a time domain technique ${ }^{453}$ where an ultrashort pulse with a duration of less than half an optical cycle of the measured pulse directly samples field oscillations ${ }^{452}$. Interpreting the method, however, in frequency-domain allowed measuring much higher frequencies, and using clearly longer sampling pulse durations, respectively ${ }^{452}$. In this picture, EOS can be described as a broadband 0-to-f-interferometer with a variable delay. The sum-frequency of the CEP-stabilized probe pulse and the long-wave spectral components of the sampling pulse is generated and interferes after proper polarization adjustment with the short-wave spectral components of the sampling pulse. In this way, the phase of the probe field can be directly accessed which makes the measurement technique fast, but presumably also more vulnerable to fluctuations than characterization methods that rely on measuring up-converted delay-dependent spectra (X-FROG).

In any case, the up-conversion techniques avoid the use expensive mid-IR detectors that have either to be cooled to liquid nitrogen temperatures or suffer from thermal radiation noise $^{386,390}$. The upper frequency-domain description makes EOS intuitively well suited for mid-IR sources which have been presented in section 4.1. In-fact, the down-conversion process of the OPAs and the DFG setup is reversed. Thus, two conditions for EOS are automatically fulfilled, namely the use of a waveform-stabilized probe pulse and the availability of a near-IR bandwidth which is at least as high as the lowest probe frequency (to get interference). The second condition is not fulfilled anymore after spectral broadening of the mid-IR. For instance, the supercontinuum shown in Fig. 4.25 would require a bandwidth of about $190 \mathrm{THz}$ (corresponding to a wavelength of $1.6 \mu \mathrm{m}$ ). For comparison, the $7.7 \mathrm{fs}$ pulse spectrum spanning from about $750 \mathrm{~nm}$ to $1300 \mathrm{~nm}$ (Fig. 2.13(c)) exhibits a bandwidth of about $170 \mathrm{THz}$. This would be enough for sampling all wavelengths longer than $1.75 \mu \mathrm{m}$, i.e. sufficient for tracing the signatures of vibrational transitions. Whereas EOS was implemented early for the DFG source presented in section 4.1.3, an implementation of a field-resolved spectroscopy setup for the OPA source and in particular the mid-IR continuum, which is most attractive for molecular fingerprinting, has not yet been realized.

Fig. 5.3 presents a possible scheme for a field-resolved spectrometer. It is based on the results presented in this dissertation. The input, not shown in the figure, would be the commercial-grade KLM TD oscillator briefly introduced in section 3.2.2. The compression of the full power to $30 \mathrm{fs}$ would not be necessary. But using the first cascaded $\chi^{(2)}$ stage for reducing the pulse duration to about $100 \mathrm{fs}$ would facilitate the seed generation in the normal dispersive fibers. Possibly, a larger core fiber could be used for generating the LGS OPA seed. Consequently, more seed energy would be available for parametric amplification (cf. section 3.1.1). Moreover, the continuum generation in the highly nonlinear ANDi would not need a careful consideration of the pulse-to-pulse noise. An LMA fiber for seeding the PPLN OPA can, however, not be utilized due to the tailored dispersion of the ANDi fiber (cf. Fig. 4.12(b)). Using shorter pump pulses would also increase the idler bandwidth of the PPLN OPA, possibly making the mid-IR compression stage before SCG unnecessary. The LGS OPA idler could also be broadened further to enter the wavelength range from $10 \mu \mathrm{m}$ to $20 \mu \mathrm{m}$. Such a stage is indicated in the box with the blue dashed frames in Fig. 5.3. Whereas in the reported experiments, mid-IR pulse 
compression was achieved only by exploiting material dispersion, chirped mirrors ${ }^{454}$ are utilized in the scheme of Fig. 5.3 to obtain precise phase control and better compression quality.

The near-IR pulse compression stage for obtaining sampling pulses is not included in the setup of Fig. 5.3. An option was to compress the LMA-25 fiber output that is also used for seeding the LGS OPA. Pumping the fiber with $100 \mathrm{fs}$ pulses would lead to a FTL of $10 \mathrm{fs}$ or less (cf. Fig. 3.3). After another bulk broadening stage (cf. section 2.2.2), the bandwidth could be further extended by a factor of two which would be sufficient for EOS of the three mid-IR channels. The setup of Fig. 5.3 recycles the pump pulses after the LGS OPA to use the pump power more efficiently. By doing so, special care should be taken of the beam reshaping resulting from the high nonlinear refractive index of LGS. Pumping the PPLN OPA first would not work due to the strong pump beam distortions (cf. Fig. 4.6). The probably most critical point of the setup is to run the individual channels at the highest possible stability in order to enable ultrasensitive mid-IR detection. An active stabilization of the fiber stage was included in Fig. 5.3. It is commercially available. The timing jitter between the channels might, however, be even more decisive. Active timing synchronization is an issue which has been addressed in OPCPA development ${ }^{455}$, optical waveform synthesis ${ }^{456}$, laser-microwave ${ }^{457}$ and laser-electron synchronization ${ }^{458}$, leading to jitter clearly lower than half of the mid-IR wave cycle period. In particular, the alloptical synchronization techniques may be transferred to the presented setup. A further simplification in this regard could be the usage of only a single seed source, the ANDi fiber output. This would require multiple stage amplification of the LGS OPA which might also reduce the idler power fluctuations if the OPAs are well saturated. The signal derived from the sequential OPA stages might further serve as the basis for ultrashort near-IR pulses that analyze the free induction decay in the EOS setup.

In conclusion, the essential building blocks to realize a broadband field-resolved mid-IR spectrometer for vibrational spectroscopy have been demonstrated in this dissertation, namely the generation of few-cycle pulses in the near-IR and CEP-stable continua in the mid-IR. Nevertheless, there are many technical details which still have to be clarified until such an outstanding tool becomes a working horse which is as reliable as an FTIR. 


\subsection{Have ultrafast thin-disk oscillators matured?}

Yet, mode-locked thin-disk oscillators are still mainly of interest of a few research groups. Nevertheless, during the five years of $\mathrm{PhD}$ work, the technology has been developed with a remarkable pace: In 2012, TRUMPF-laser GmbH presented a $145 \mathrm{~W}$ mode-locked TD oscillator with $41 \mu \mathrm{J}$ pulse energy, beating the previous record by more than $10 \mu \mathrm{J}^{60}$. A few months later, the average power record was nearly doubled by reaching $275 \mathrm{~W}^{58}$. The record with a SESAM mode-locker was practically equalized by a KLM TD oscillator with $270 \mathrm{~W}$ output power in $2014^{59}$. In the same year, a pulse energy of $80 \mu \mathrm{J}$ was reached with a SESAM mode-locked oscillator ${ }^{61}$. The much shorter pulse duration of KLM TD oscillators enabled to achieve nearly the same peak power level of more than $60 \mathrm{MW}$ in $2016^{139}$. In the same year, a first prototype of a commercial KLM TD oscillator was sold in combination with a pulse compression stage ${ }^{436}$. The delivered $60 \mathrm{MW}$ peak power, $30 \mathrm{fs}$ pulse duration is already clearly beyond the parameters that the most powerful Ti:sapph oscillator can provide ${ }^{245}$. Eventually, a first powerful TD oscillator emitting in the midIR has just been presented this year ${ }^{151}$. Beyond the advances in pure laser development, ultrafast thin-disk oscillators have also found an increasing number of applications during the past five years. They became the front-end of energetic regenerative amplifiers ${ }^{34,459}$ and served as sources in preliminary dual-comb ${ }^{63}$ and field-resolved spectroscopy ${ }^{64}$ setup.

Several milestones in the development of the ultrafast laser technology have also been presented in this dissertation:

(i) A first TD oscillator was CEP stabilized. This was also the first CEP stabilized laser oscillator with more than $10 \mathrm{~W}$ of average power.

(ii) For the first time, pulses emitted from a TD laser were compressed to fewcycle durations.

(iii) A first femtosecond Watt-class compact light source operating at a central wavelength longer than $5 \mu \mathrm{m}$ was realized.

(iv) For the first time, a laser-based Watt-level multi-octave spanning femtosecond mid-IR supercontinuum was demonstrated.

Beyond those achievements, power scalable methods have been developed such that pulse compression, CEP stabilization and mid-IR generation schemes truly comply with the demands of a new generation of ultrafast femtosecond oscillators. Therefore, the reported work is expected to serve as a basis for the continuing dynamic development of the laser technology.

From stating the latest advances of mode-locked TD oscillators, it becomes clear that they are progressing towards maturity, i.e. that they become ready for a wide range of applications as well as for the laser market. If applications in fundamental science, photonics and life sciences, like those sketched in section 5.1, are realized within the following years, the interest in the technology will proliferate. Consequently, upcoming breakthroughs may not be restricted to scientific results anymore, but may expand towards commercial success and a (partial) replacement of the Ti:sapph technology. 


\section{APPENDIX}




\section{A.1 FROG measurements}

Pulse characterziation was almost exclusively accomplished via frequency-resolved optical gating (FROG) ${ }^{460}$. While autocorrelators cannot retrieve both amplitude and phase information, ${ }^{80,461,462}$ a tested commercial spectral phase interferometer for direct electric field reconstruction (SPIDER) ${ }^{80,463,464}$ was not flexible enough for measuring very large bandwidths and could not easily be adjusted to measure pulses at central wavelengths other than $1030 \mathrm{~nm}$. Moreover, the FROG setup was clearly more compact and was also sensitive to spatial inhomogeneities that were investigated in section 3.2 .

The FROG setup, which was developed during the course of the PhD studies, is shown in Fig. A.1. It had a small footprint of about $25 \mathrm{~cm} \times 25 \mathrm{~cm}$ and was adapted from the design of Baltuška and coworkers ${ }^{465,466}$ to enable few-cycle pulse measurements. In particular, no dispersive optics other than the nonlinear crystal itself were utilized and, for ultrashort pulse measurements, the generated FROG signal was focused into a fiber coupled spectrometer. The thinnest available nonlinear crystals were utilized, namely $5-10 \mu \mathrm{m}$ thin $\mathrm{BBO}$ and $110 \mu \mathrm{m}$ thin LGS crystals. Due to the high repetition rates, pulse energies of less than $25 \mathrm{~nJ}$ were typically sufficient to saturate the grating spectrometer at its lowest integration time of about $4 \mathrm{~ms}$.

A special feature of the FROG setup was the moveable D-shape mirror. It could either be used to split a single incident beam in order to realize a wavefront dividing interferometer for SH-FROG measurements, or it reflected one beam completely while it allowed another beam to fully pass. In this configuration, X-FROG traces were measured. In particular, this was utilized for mid-IR pulse characterization (c.f. section 4.1.1) where SH-FROG was rather difficult to apply since it would have required a (slow) mid-IR spectrometer. Fig. A.2 shows an example of a broadband X-FROG measurement. The PPLN OPA radiation, which has passed through the dichroic beam splitter, is up-converted to the spectral range between $550 \mathrm{~nm}$ and $850 \mathrm{~nm}$. Seed,

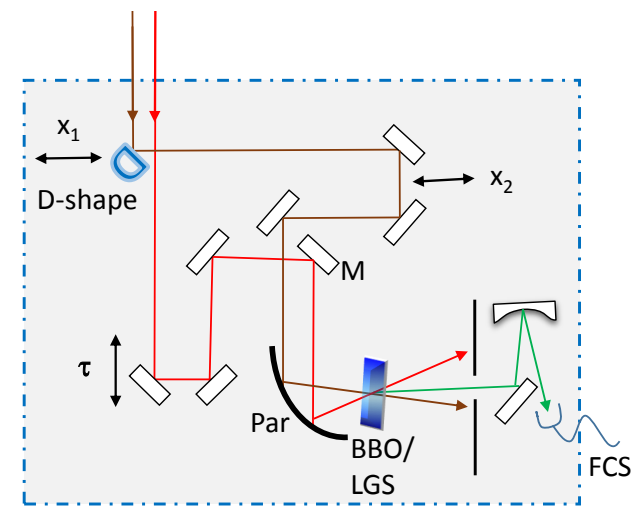

Fig. A.1. FROG setup. The device can be used in auto- or cross-correlation mode. The autocorrelation mode (SH-FROG) divides the waveform of a single incoming beam into two equal parts. This is adjusted by moving the $\mathrm{D}$-shape mirror with a small translation stage $\mathrm{x}_{1}$. With another translation stage $\mathrm{x}_{2}$ the zero delay between the two interferometer arms is adjusted. A piezo stage with $400 \mu \mathrm{m}$ travel range (about $2.2 \mathrm{ps}$ ) sweeps the delay $(\tau)$ between both interferometer arms during the measurement. The separated beams are overlapped again in the nonlinear crystal. BBO was used for measurements in the near-IR while LGS was used for measurements in the mid-IR. The sum-frequency of both beams is separated from the its fundamentals by an aperture and focused into a single- or multimode fiber which is coupled to a grating spectrometer (FCS). In the cross-correlation mode (X-FROG), the D-shape mirror reflects one beam fully and lets the the other beam fully pass. By means of the mirror M, the spatial overlap of the beams has to be readjusted when switching from one to the other mode. If the vertical axes of both beams are properly aligned, this does not require to remove the nonlinear crystal because only a horizontal shift is necessary. All mirrors are coated with silver and a thin protection layer. 


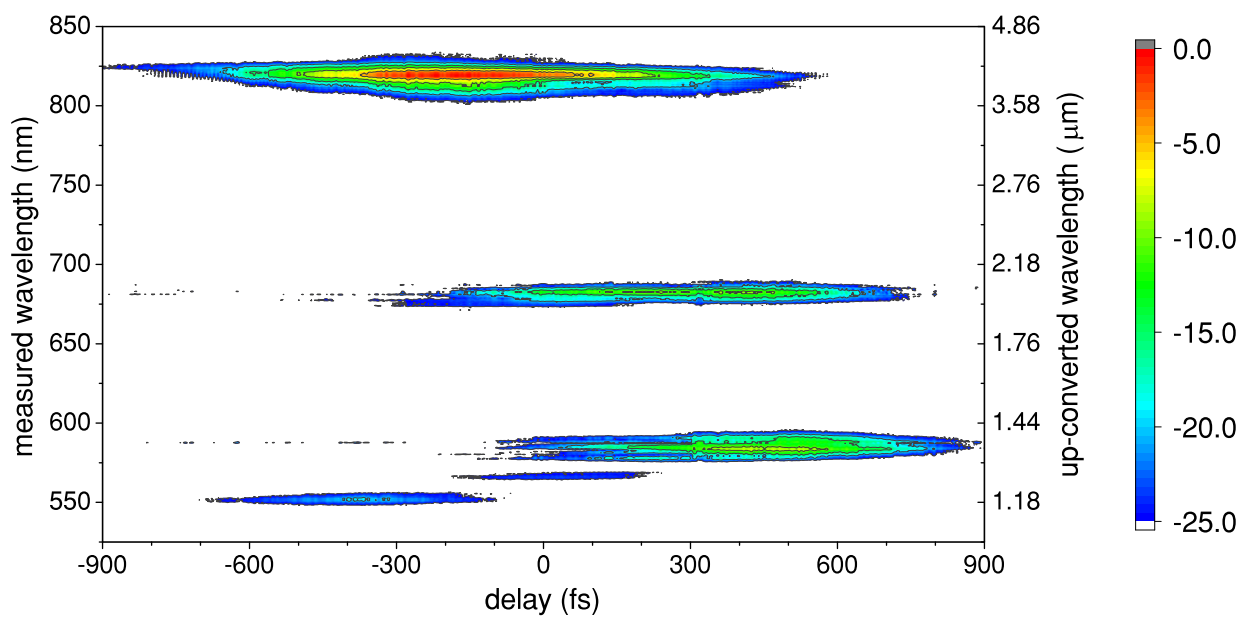

Fig. A.2. Example of an ultrabroaband X-FROG measurement. The trace shows the PPLN OPA output at full pump power after the dichroic beam splitter. The graph clearly shows the seed radiation (around $560 \mathrm{~nm}$ ), the OPA signal (around $590 \mathrm{~nm}$ ), a residual second harmonic of the idler (around $680 \mathrm{~nm}$ ) and the idler radiation (around $820 \mathrm{~nm}$ ). Therefore, a spectral range from $1.15 \mu \mathrm{m}$ to about $4.5 \mu \mathrm{m}$ is captured and time-resolved. The right axis estimates the up-converted wavelengths by $\lambda=\left(\lambda_{\mathrm{FROG}}^{-1}-(1.03 \mu \mathrm{m})^{-1}\right)^{-1}$ where $\lambda_{\text {FROG }}$ is the measured wavelength on the left axis. The scale is in $\mathrm{dB}$ relative to the maximum. A $110 \mu \mathrm{m}$ thin LGS crystal was utilized for sum-frequency generation.

signal, idler and its second harmonic are clearly visible, showing that the X-FROG is sensitive to radiation with wavelengths distributed over about two optical octaves.

An analog controller of the piezo stage (piezosystem jena $\mathrm{GmbH}$ ) and a fast $\mathrm{AD} / \mathrm{DA}$ converter (up to $250 \mathrm{kHz}$ sampling rate, BMC Messysteme $\mathrm{GmbH}$ ) enabled quick scanning of the FROG traces. Typically a few traces per minute could be taken. After every scan, the software automatically calculated the intensity autocorrelation. This was used for optimizing pulse compression, for instance by gently moving the position of the spectral broadening crystal in the few-cycle pulse generation experiment of section 2.2.2. Adding a flip mirror to the setup and detecting the autocorrelation with an avalanche diode or a photo-multiplier could have even sped up the optimization procedure. This has, however, not been implemented. The technique of fine adjusting an essentially known pulse by minimizing its autocorrelation width compensated for the disadvantage of FROG, the time-consuming retrieval algorithm. 


\section{A.2 RMS spectral width of a prechirped Gaussian pulse}

For clarity the FOURIER transform operators are defined first:

$$
\begin{gathered}
\tilde{f}(\omega)=\mathcal{F} \mathcal{T}\{f(t)\}(\omega)=\int_{-\infty}^{+\infty} f(t) e^{-i \omega t} d t, \\
f(t)=\mathcal{F}^{-1}\{\tilde{f}(\omega)\}(t)=\frac{1}{2 \pi} \int_{-\infty}^{+\infty} \tilde{f}(\omega) e^{i \omega t} d \omega,
\end{gathered}
$$

where $f(t), \tilde{f}(\omega)$ are time and frequency domain functions, resp. The integration boundaries $\pm \infty$ will be mostly omitted in the following. The FOURIER transform of a time derivative $f^{\prime}(t)$ can be expressed by Eq. (A.2) as follows:

$$
\begin{aligned}
f^{\prime}(t) & =\frac{1}{2 \pi} \frac{d}{d t} \int \tilde{f}(\omega) e^{i \omega t} d \omega=\frac{i}{2 \pi} \int \omega \tilde{f}(\omega) e^{i \omega t} d \omega . \\
\Rightarrow \quad f^{\prime}(0) & =\frac{i}{2 \pi} \int \omega \tilde{f}(\omega) d \omega .
\end{aligned}
$$

Moreover, the FOURIER transform of a complex conjugated function can be expressed by Eq. (A.1) as follows:

$$
\tilde{f}^{*}(\omega)=\int_{-\infty}^{+\infty} f^{*}(t) e^{i \omega t} d t \stackrel{\tau=-t}{=}-\int_{+\infty}^{-\infty} f^{*}(-\tau) e^{-i \omega \tau} d \tau=\mathcal{F} \mathcal{T}\left\{f^{*}(-t)\right\}(\omega)
$$

Next, the convolution theorem is recalled:

$$
\tilde{f}_{1}(\omega) \cdot \tilde{f}_{2}(\omega)=\mathcal{F} \mathcal{T}\left\{f_{1}(t) * f_{2}(t)\right\}(\omega)=\int d t e^{-i \omega t} \int d \tau f_{1}(\tau) f_{2}(t-\tau) .
$$

With these equations, relation (3.13) from page 71 can be proven:

$$
\begin{aligned}
\int \Omega^{2} \mathcal{E}(\Omega) \mathcal{E}^{*}(\Omega) d \Omega & \stackrel{(\text { A.4) }}{=} 2 \pi \mathcal{F} \mathcal{T}^{-1}\left\{\Omega^{2} \mathcal{E}(\Omega) \mathcal{E}^{*}(\Omega)\right\}(0) \stackrel{(\text { A.5), (A.6) }}{=} 2 \pi \int\left(-i E^{\prime}(\tau)\right)\left(i E^{* *}(\tau-0)\right) d \tau \\
& =2 \pi \int|d E(t) / d t|^{2} d t \\
\int \mathcal{E}(\Omega) \mathcal{E}^{*}(\Omega) d \Omega & =2 \pi \mathcal{F} \mathcal{T}^{-1}\left\{\mathcal{E}(\Omega) \mathcal{E}^{*}(\Omega)\right\}(0)=2 \pi \int E(\tau) E^{*}(\tau-0) d \tau \\
& =2 \pi \int|E(t)|^{2} d t
\end{aligned}
$$

Eq. (A.8) is PARSEvaL's theorem.

To derive Eq. (3.14), the field of Eq. (3.9) can be reduced to its relevant components which are normalized, even and time dependent functions. All other terms do not contribute to Eq. (3.13). The reduced field reads:

$$
e(t)=\exp \left[-\frac{\kappa}{2} \frac{t^{2}}{t_{p}^{2}\left(1+\xi^{2}\right)}+i\left(\frac{\kappa}{2} \frac{t^{2} \xi}{t_{p}^{2}\left(1+\xi^{2}\right)}-\exp \left\{-\kappa \frac{t^{2}}{t_{p}^{2}\left(1+\xi^{2}\right)}\right\} \frac{l}{L_{n}}\right)\right] .
$$

After introducing $u=\frac{\sqrt{\kappa} t}{t_{p} \sqrt{1+\xi^{2}}}$, its time derivative reads:

$$
e^{\prime}(u)=\frac{\sqrt{\kappa}}{t_{p} \sqrt{1+\xi^{2}}} e(u)\left[-u+i\left(\xi u+2 u \exp \left\{-u^{2}\right\} \frac{l}{L_{n}}\right)\right]
$$


and its absolute value squared:

$$
\begin{aligned}
\left|e^{\prime}(u)\right|^{2} & =\frac{\kappa}{t_{p}^{2}\left(1+\xi^{2}\right)}|e(u)|^{2}\left[u^{2}+\xi^{2} u^{2}+4 \xi u^{2} e^{-u^{2}} \frac{l}{L_{n}}+4 u^{2} e^{-2 u^{2}}\left(\frac{l}{L_{n}}\right)^{2}\right] \\
& =\frac{\kappa}{t_{p}^{2}\left(1+\xi^{2}\right)}\left[u^{2}\left(1+\xi^{2}\right) e^{-u^{2}}+4 \xi u^{2} e^{-2 u^{2}} \frac{l}{L_{n}}+4 u^{2} e^{-3 u^{2}}\left(\frac{l}{L_{n}}\right)^{2}\right],
\end{aligned}
$$

where $|e(u)|^{2}=\exp \left\{-u^{2}\right\}$ was used. The remaining task is to solve Gaussian integrals:

$$
\begin{aligned}
\int \exp \left\{-u^{2}\right\} d u & =\sqrt{\int \exp \left\{-u^{2}-v^{2}\right\} d u d v}=\sqrt{\int_{0}^{2 \pi} d \phi \int_{0}^{\infty} r \exp \left\{-r^{2}\right\} d r} \\
& =\sqrt{\pi \int_{0}^{\infty} \exp \left\{-r^{2}\right\} 2 r d r}=\sqrt{\pi \int_{0}^{\infty} \exp \{-x\} d x}=\sqrt{\pi}, \\
\int u^{2} \exp \left\{-\alpha u^{2}\right\} d u & =-\frac{d}{d \alpha} \int \exp \left\{-\alpha u^{2}\right\} d u=-\frac{d}{d \alpha} \sqrt{\frac{\pi}{\alpha}} \\
& =\frac{1}{2} \sqrt{\frac{\pi}{\alpha^{3}}}
\end{aligned}
$$

Now, Eq. (3.13) can be evaluated for the field from Eq. (3.9):

$$
\begin{aligned}
\int\left|e^{\prime}(u)\right|^{2} d u & =\frac{\kappa}{t_{p}^{2}\left(1+\xi^{2}\right)} \frac{\sqrt{\pi}}{2}\left[\left(1+\xi^{2}\right)+\sqrt{2} \xi \frac{l}{L_{n}}+\frac{4}{3 \sqrt{3}}\left(\frac{l}{L_{n}}\right)^{2}\right], \\
\int\left|e^{\prime}(u)\right|^{2} d u / \int|e(u)|^{2} d u & =\frac{\kappa}{2 t_{p}^{2}}\left[1+\frac{\sqrt{2} \xi}{1+\xi^{2}} \frac{l}{L_{n}}+\frac{4}{3 \sqrt{3}\left(1+\xi^{2}\right)}\left(\frac{l}{L_{n}}\right)^{2}\right] .
\end{aligned}
$$

Eq. (3.14) directly follows. 


\section{A.3 Transforming the differential equation (3.21) to an integral.}

The differential equation (3.21) shall be solved up to an integral which in general cannot be evaluated analytically, but can be solved numerically. The equation is of type:

$$
r^{\prime \prime}(z)=f(r[z])
$$

where $r^{\prime \prime}(z)$ denotes the second derivative of $r$ with respect to $z$ and $f(r)$ is some function of $r$. The solution of such a differential equation can be found in math books on differential equations. It will be sketched in the following. Multiplying Eq. (A.16) by $2 r^{\prime}$ gives:

$$
\begin{aligned}
& 2 r^{\prime} r^{\prime \prime}(z)=2 r^{\prime} f(r[z]), \\
\Leftrightarrow \quad & \frac{d}{d z}\left(r^{\prime}\right)^{2}=2 \frac{d}{d z} F(r[z]), \\
\Leftrightarrow \quad & \left(r^{\prime}\right)^{2}=2 F(r[z]),
\end{aligned}
$$

where $F(r[z])$ denotes an antiderivative of $f(r)$, i.e.

$$
\begin{aligned}
F(r[z]) & =-\int_{r_{0}}^{r} \frac{4 n_{2} I_{p}}{w^{2} n_{0}} \xi \exp \left\{-2 \xi^{2} / w^{2}\right\} d \xi, \\
& =\frac{n_{2} I_{p}}{\xi}\left[\exp \left\{-2 r^{2} / w^{2}\right\}-\exp \left\{-2 r_{0}^{2} / w^{2}\right\}\right],
\end{aligned}
$$

for the particular case of Eq. (3.21). Eq. (A.17) is only of first order, and hence it can be solved by separation of the variables:

$$
\int_{r_{0}}^{r(z)} \frac{d \xi}{\sqrt{2 F(\xi)}}=\int_{z_{0}}^{z} d \zeta=\Delta z
$$

If $\exp \left\{-2 r^{2} / w^{2}\right\}$ is approximated by $1-2 r^{2} / w^{2}$, Eq. (A.19) can be solved analytically by means of the integral identity ${ }^{467}$ :

$$
\int \frac{d x}{\sqrt{a x^{2}+b}}=-\frac{1}{\sqrt{-a}} \arcsin \left(\frac{2 a x}{\sqrt{4 a b}}\right) .
$$

This will result in the solution of ray propagation in a graded index fiber with parabolic profile ${ }^{346}$. For the particular problem of self-(de)focusing, Eq. (A.19) can be solved stepwise and $F(r)$ can be reevaluated after each propagation step by means of summing over the ray packet equivalent of the laser beam. This was not done in this work since the simulation package SISYFOS was used to precisely analyze temporal and spatial reshaping of the laser pulse in the nonlinear medium. 


\section{A.4 Publications, etc.}

The following items are related to the PhD work at the Laboratory for Attosecond Physics.

\section{PEER-REVIEWED JOURNAL PUBLICATIONS}

M. Seidel, X. Xiao, and A. Hartung, Solid-Core Fiber Spectral Broadening at its Limits, IEEE J. Sel. Top. Quant. Electron. 24, 5100908 (2018)

M. Seidel, X. Xiao, S.A. Hussain, G. Arisholm, A. Hartung, K.T. Zawilski, P.G. Schunemann, F. Habel, M. Trubetskov, V. Pervak, O. Pronin, and F. Krausz, Multi-Watt, multi-octave, midinfrared femtosecond source, Science Advances 4, eaaq1526 (2018)

J. Zhang, K.F. Mak, N. Nagl, M. Seidel, D. Bauer, D. Sutter, V. Pervak, F. Krausz, and O. Pronin, Multi-mW, few-cycle mid-infrared continuum spanning from 500 to $2250 \mathrm{~cm}^{-1}$, Light: Science \& Applications e17180 (2018)

M. Seidel, J. Brons, G. Arisholm, K. Fritsch, V. Pervak, and O. Pronin, Efficient high-power ultrashort pulse compression in self-defocusing bulk media, Scientific Reports 7, 1410 (2017)

M. Seidel, J. Brons, F. Lücking, V. Pervak, A. Apolonski, T. Udem, and O. Pronin, Carrierenvelope-phase Stabilization via Dual Wavelength Pumping, Optics Letters 41, 1853 (2016)

M. Seidel, G. Arisholm, J. Brons, V. Pervak, and O. Pronin, All solid-state spectral broadening: An average and peak power scalable method for compression of ultrashort pulses, Optics Express $24,9412(2016)$

I. Pupeza, D. Sánchez, J. Zhang, N. Lilienfein, M. Seidel, O. Pronin, N. Karpowicz, T. PaaschColberg, , I. Znakovskaya, M. Pescher, W. Schweinberger, V. Pervak, E. Fill, Z. Wei, F. Krausz, A. Apolonski, and J. Biegert High-power sub-2-cycle mid-infrared pulses at $100 \mathrm{MHz}$ repetition rate, Nature Photonics 9, 721 (2015)

O. Pronin*, M. Seidel*, F. Lücking, J. Brons, E. Fedulova, M. Trubetskov, V. Pervak, A. Apolonski, T. Udem, F. Krausz, High-power multi-megahertz source of waveform-stabilised fewcycle light, Nature Communications 6, $6988(2015) *$ authors contribute equally

K. F. Mak, M. Seidel, O. Pronin, M. Frosz, V. Pervak, A. Apolonskiy, F. Krausz, J. Travers, and P. Russell, Compressing $\mu$ J-level pulses from $250 \mathrm{fs}$ to sub-10 fs at $38 \mathrm{MHz}$ repetition rate using two gas-filled hollow-core photonic crystal fiber stages, Optics Letters 40, 1238 (2015)

I. Znakovskaya, E. Fill, N. Forget, P. Tournois, M. Seidel, O. Pronin, F. Krausz, and A. Apolonski, Dual frequency comb spectroscopy with a single laser, Optics Letters 39, 5471 (2014) 


\section{INVITED CONFERENCE PRESENTATION}

M. Seidel, G. Arisholm, O. Pronin, and F. Krausz $450 \mathrm{~mW}$ femtosecond mid-IR source at $8.5 \mu m$ wavelength, CLEO: Science and Innovations p. STu3I.6 (San José 2016)

\section{POSTDEADLINE CONFERENCE CONTRIBUTIONS}

J. Zhang, K.F. Mak, N. Nagl, M. Seidel, F. Krausz and O. Pronin, 7-W, 2-cycle self-compressed pulses at 2.1 micron from a Ho:YAG thin disk laser oscillator, CLEO/Europe - EQEC 2017, PD-1.5 (Munich 2017)

M. Seidel, G. Arisholm, K. Fritsch, V. Pervak, and O. Pronin Efficient High-Power Pulse Compression in Self-Defocusing Bulk Media, $7^{\text {th }}$ EPS-QEOD Europhoton Conference, p. PD1.2 (Vienna 2016)

J. Brons, V. Pervak, M. Seidel, D. Bauer, D. Sutter, O. Pronin, and F. Krausz Amplificationfree, $145 \mathrm{MW}, 16 \mathrm{MHz}$ Scalable Ultrafast Light-source for XUV and MIR Generation, Advanced Solid-State Lasers, p. ATh3A.1 (Berlin 2015)

M. Seidel, X. Xiao, G. Arisholm, A. Hartung, O. Pronin, and F. Krausz Multi-Watt MHz-rate Femtosecond Mid-Infrared Source, Nonlinear Optics: Materials, Fundamentals and Applications, p. NTh3A.7 (Kauai 2015)

J. Zhang, J. Brons, M. Seidel, V. Pervak, V. Kalashnikov, Z. Wei, A. Apolonski, F. Krausz and O. Pronin 49-fs Yb:YAG thin-disk oscillator with distributed Kerr-lens mode-locking, CLEO/Europe - EQEC 2015, p. PD-A.1 (Munich 2015)

M. Seidel, J. Brons, E. Fedulova, V. Pervak, A. Apolonski, O. Pronin, and F. Krausz HighPower Few-Cycle Pulse Generation by Spectral Broadening in Bulk Material, CLEO: Science and Innovations p. STh5C. 9 (San José 2014)

I. Pupeza, D. Sanchez, O. Pronin, J. Zhang, N. Lilienfein, M. Seidel, I. Znakovskaya, V. Pervak, Z. Wei, F. Krausz, A. Apolonski, and J. Biegert Compact 0.1-W Source of Octave-Spanning MidInfrared Femtosecond Pulses Centered at 10 micron, CLEO: Science and Innovations p. STh5C. 7 (San José 2014)

\section{CONFERENCE PROCEEDINGS}

J. Brons, K. Fritsch, M. Seidel, V. Pervak, F. Krausz and O. Pronin, Efficient, high-power, all-bulk spectral broadening in a quasi-waveguide, CLEO/Europe - EQEC 2017, CF-9.4 (Munich 2017)

M. Seidel, O. Pronin, and F. Krausz, $1.3 \mathrm{~W}$ femtosecond mid-IR source at $8.5 \mu \mathrm{m}$ wavelength, $41^{\text {st }}$ International Conference on Infrared, Millimeter, and Terahertz waves (IRMMW-THz) (Copenhagen 2016)

M. Seidel, X. Xiao, G. Arisholm, A. Hartung, O. Pronin, and F. Krausz, Watt-level Megahertzrate Femtosecond Mid-Infrared Source, Advanced Solid-State Lasers, p. AM3A.7 (Berlin 2015)

J. Zhang, J. Brons, M. Seidel, D. Bauer, D. Sutter, V. Pervak, V. Kalashnikov, Z. Wei, A. Apolonski, F. Krausz, and O. Pronin Generation of 49-fs pulses directly from distributed Kerr- 
lens mode-locked Yb:YAG thin-disk oscillator, Advanced Solid-State Lasers, p. ATh4A.7 (Berlin 2015)

M. Seidel, J. Brons, V. Pervak, A. Apolonski, O. Pronin, and F. Krausz Double-Stage Spectral Broadening in Bulk Crystals of $50 \mathrm{~W}, 1.3 \mu \mathrm{J}, 250 \mathrm{fs}$ pulses to a Fourier transform limit of 15 fs, Nonlinear Optics: Materials, Fundamentals and Applications, p. NM2B.1 (Kauai 2015)

M. Seidel, X. Xiao, A. Hartung, L. Lötscher, A. Apolonski, and F. Krausz Spectral Broadening and Peak Power Limitations of Normally Dispersive Photonic Crystal Fibres for High-Power fs Light Sources, CLEO/Europe - EQEC 2015, p. CD-P.18 (Munich 2015)

M. Seidel, J. Brons, F. Lücking, I. Angelov, Th. Udem, F. Krausz, A. Apolonski, and O. Pronin Carrier-Envelope-Phase Stabilizing High-Power Yb:YAG Thin-Disk Oscillators via Low-Power Pump Diode Modulation, CLEO/Europe - EQEC 2015, p. CA-10.3 (Munich 2015)

M. Seidel, J. Brons, V. Pervak, A. Apolonski, O. Pronin, and F. Krausz Double-Stage Spectral Broadening in Bulk Crystals of $50 \mathrm{~W}, 1.3 \mu \mathrm{J}, 250 \mathrm{fs}$ pulses to a Fourier transform limit of 15 fs, CLEO/Europe - EQEC 2015, p. CD-8.4 (Munich 2015)

J. Zhang, N. Lilienfein, J. Brons, M. Seidel, D. Bauer, D. Sutter, V. Pervak, Z. Wei, A. Apolonski, O. Pronin, and F.Krausz $260 \mathrm{MHz}, \mathrm{MW}$-level thin-disk oscillator, CLEO/Europe EQEC 2015, p. CA-P.12 (Munich 2015)

J. Brons, M. Seidel, V. Pervak, A. Apolonski, O. Pronin, and F. Krausz, Power scalable spectral broadening in bulk crystals with more than $100 \mathrm{~W}$ average power, CLEO/Europe - EQEC 2015, p. CA-P.34 (Munich 2015)

O. Pronin, J. Brons, M. Seidel, J. Zhang, M. Trubetskov, D. Bauer, D. Sutter,V. Kalashnikov, A. Apolonski, V. Pervak, and F. Krausz, High-power femtosecond thin-disk oscillators, CLEO/Europe - EQEC 2015, p. CA-10.5 (invited, Munich 2015)

K. F. Mak, M. Seidel, O. Pronin, M. Frosz, V. Pervak, A. Apolonskiy, F. Krausz, J. Travers, and P. Russell, Compression of $\mu \mathrm{J}$-level pulses from $250 \mathrm{fs}$ to sub-10 fs at $38 \mathrm{MHz}$ repetition rate using two gas-filled hollow-core kagomé-PCF stages, Advanced Solid-State Lasers, p. AF4A.4 (Shanghai 2014)

J. Brons, V. Pervak, E. Fedulova, M. Seidel, D. Bauer, D. Sutter, V. L. Kalashnikov, A. Apolonski, O. Pronin, and F. Krausz, High power Kerr-lens mode-locking of Yb:YAG and Yb:CALGO thin-disk oscillators, Advanced Solid-State Lasers, p. AF1A.5 (Shanghai 2014)

J. Brons, V. Pervak, E. Fedulova, M. Seidel, D. Bauer, D. Sutter, V. L. Kalashnikov, A. Apolonski, O. Pronin, and F. Krausz, Power-scaling a Kerr-lens mode-locked Yb:YAG thin-disk oscillator via enlarging the cavity mode in the Kerr-medium, CLEO: Science and Innovations p. SM4F.7 (San José 2014)

M. Seidel, J. Brons, F. Lücking, V. Pervak, A. Apolonskiy, O. Pronin, T. Udem and F. Krausz, Carrier-envelope phase stabilized, few-cycle, high-power thin-disk oscillator, SPIE Photonics Europe, p. 9135-7 (Brussels 2014)

O. Pronin, J. Brons, M. Seidel, E. Fedulova, A. Apolonskiy, V. L. Kalashnikov, V. Pervak, and F. Krausz, Power and energy scaling of Kerr-lens mode-locked thin-disk oscillators, Proc. SPIE 9135, Laser Sources and Applications II, 91351H (2014)

M. Seidel, O. Pronin, J. Brons, E. Fedulova, F. Lücking, I. Angelov, V. Pervak, A. Apolonski, and F. Krausz Approaching the Few-Cycle Pulse Regime with Thin-Disk Oscillators, Advanced Solid-State Lasers, p. ATu4A.7 (Paris 2013) 
O. Pronin, M. Seidel, J. Brons, F. Lücking, V. Pervak, A. Apolonski, Th. Udem, and F. Krausz, Carrier-envelope phase stabilized thin-disk oscillator, Advanced Solid-State Lasers, p. AF3A.5 (Paris 2013)

J. Brons, V. Pervak, E. Fedulova, M. Seidel, D. Bauer, D. Sutter, V. Kalashnikov, A. Apolonski, O. Pronin, and F. Krausz, $120 \mathrm{~W}, 4 \mu \mathrm{J}$ from a purely Kerr-lens mode-locked Yb:YAG thin-disk oscillator, Advanced Solid-State Lasers, p. AF3A.4 (Paris 2013)

O. Pronin, M. Seidel, J. Brons, F. Lücking, I. Angelov, V. Kalashnikov, V. Pervak, A. Apolonski, Th. Udem, and Ferenc Krausz, Towards CEP stabilized pulses from a KLM Yb:YAG thindisk oscillator, CLEO/Europe - EQEC 2013, p. CFIE-2.2 (Munich 2013)

O. Pronin, J. Brons, M. Seidel, F. Lücking, C. Grasse, G. Boehm, M. C. Amann, V. Pervak, A. A. Apolonskiy, V. L. Kalashnikov, and F. Krausz, High-power Kerr-lens mode-locked thin-disk oscillator in the anomalous and normal dispersion regimes, SPIE 8599, Solid State Lasers XXII: Technology and Devices, 85991N (invited, San Francisco 2013)

\section{PATENT}

J. Brons, M. Seidel, O. Pronin, A. Apolonskiy, Method and device for controlling a carrierenvelope phase and/or an intensity of output pulses of a pulse laser device, US Patent 14/523,456

\section{PRIZE}

Tingye Li Innovation Prize of the Optical Society of America Foundation (2016) 


\section{A.5 Data archiving}

The experimental data, evaluation-files and figures can be found on the data archive server of the Laboratory for Attosecond Physics at the Max Planck Institute of Quantum Optics. The root directory contains folders named after each chapter in the thesis. Each folder contains OriginLab (V. 2015) projects named after the chapter (sub-)sections. The original and the processed data is saved in work books. The figures as included in the thesis are saved as graphs. The nomenclature in the OriginLab projects corresponds to that of the thesis, e.g. Fig32 for Fig. 3.2. Source code for simulations is named after a corresponding figure and is saved in the chapter folders. Source code where only the input parameters were varied (e.g. the same routine was run with different pulse energies) is only saved once. 



\section{Bibliography}

[1] Maiman, T. H. Stimulated optical radiation in ruby. Nature 187, 493-494 (1960).

[2] Einstein, A. Strahlungs-Emission und Absorption nach der Quantentheorie. Verhandlungen der Deutschen Physikalischen Gesellschaft 18, 318-323 (1916).

[3] Lukishova, S. G. Valentin A. Fabrikant: negative absorption, his 1951 patent application for amplification of electromagnetic radiation (ultraviolet, visible, infrared and radio spectral regions) and his experiments. Journal of the European Optical Society - Rapid publications 5, 10045s (2010).

[4] Basov, N. G. \& Prochorov, A. Vorschläge und Rechnungen zu einem Mikrowellen-Oszillator basierend auf stimulierter Emission. $Z h$. Eksperim. i Teor. Fiz. 27, 431 (1954).

[5] Schawlow, A. L. \& Townes, C. H. Infrared and optical masers. Physical Review 112, 1940-1949 (1958).

[6] Overton, G., Nogee, A., Belforte, D. \& Holton, C. Annual laser market review \& forecast: Where have all the lasers gone? Laser Focus World 53, 32-52 (2017).

[7] laserfest.org. Laser Pioneers (accessed: 2017/04/26). URL http://laserfest.org/ lasers/pioneers/nobel.cfm.

[8] Coherent. Laser Materials Processing Introduction to Lasers for Materials Processing (accessed: 2015/11/25). URL https://www . coherent.com/applications/index.cfm? fuseaction=Forms . page\&Page $I D=98$.

[9] Spence, D. E., Kean, P. N. \& Sibbett, W. Sub-100fs pulse generation from a selfmodelocked titanium:sapphire laser. In Conference on Lasers and Electro-optics, CLEO, Techical Digest Series, 619 - 620 (Optical Society of America, 1990).

[10] Spence, D. E., Kean, P. N. \& Sibbett, W. 60fsec pulse generation from a self-mode-locked ti:sapphire laser. Opt. Lett. 16, 42-44 (1991).

[11] Fattahi, H. et al. Third-generation femtosecond technology. Optica 1, 45-63 (2014).

[12] Fattahi, H. Third-generation femtosecond technology (Dissertation, LudwigMaximilians-Universität München, 2015).
[13] Südmeyer, T. et al. Femtosecond laser oscillators for high-field science. Nature Photonics 2, 599-604 (2008).

[14] Hargrove, L. E., Fork, R. L. \& Pollack, M. A. Locking of He-Ne laser modes induced by synchronous intracavity modulation. Applied Physics Letters 5, 4-5 (1964).

[15] Mocker, H. W. \& Collins, R. J. Mode competition and self-locking effects in a Q-switched ruby laser. Applied Physics Letters 7, 270273 (1965).

[16] DeMaria, A. J., Stetser, D. A. \& Heynau, H. Self mode-locking of lasers with saturable absorbers. Applied Physics Letters 8, 174-176 (1966).

[17] Ippen, E., Shank, C. \& Dienes, A. Passive mode locking of the CW dye laser. Applied Physics Letters 21, 348-350 (1972).

[18] Knox, W. H. et al. Optical pulse compression to 8 fs at a $5 \mathrm{kHz}$ repetition rate. Applied Physics Letters 46, 1120-1121 (1985).

[19] Jones, D. J. et al. Carrier-envelope phase control of femtosecond mode-locked lasers and direct optical frequency synthesis. Science 288, 635-639 (2000).

[20] Ell, R. et al. Generation of 5-fs pulses and octave-spanning spectra directly from a Ti:sapphire laser. Opt. Lett. 26, 373-375 (2001).

[21] Aus der $\mathrm{Au}$, J. et al. 16.2-W average power from a diode-pumped femtosecond Yb:YAG thin disk laser. Opt. Lett. 25, 859-861 (2000).

[22] Baer, C. R. E. et al. Femtosecond thin-disk laser with $141 \mathrm{~W}$ of average power. Opt. Lett. 35, 2302-2304 (2010).

[23] Pronin, O. et al. High-power 200 fs Kerrlens mode-locked Yb:YAG thin-disk oscillator. Opt. Lett. 36, 4746-4748 (2011).

[24] Pronin, O. et al. High-power multi-megahertz source of waveform-stabilized few-cycle light. Nat. Commun. 6, 6988 (2015).

[25] Fermann, M. E. \& Hartl, I. Ultrafast fiber laser technology. IEEE Journal of Selected Topics in Quantum Electronics 15, 191-206 (2009). 
[26] Fermann, M. E. \& Hartl, I. Ultrafast fibre lasers. Nature Photonics 7, 868-874 (2013).

[27] Jauregui, C., Limpert, J. \& Tünnermann, A. High-power fibre lasers. Nature Photonics 7, 861-867 (2013).

[28] Müller, M. et al. $1 \mathrm{~kW} 1 \mathrm{~mJ}$ eight-channel ultrafast fiber laser. Opt. Lett. 41, 3439-3442 (2016).

[29] Krauss, G. et al. Synthesis of a single cycle of light with compact erbium-doped fibre technology. Nature Photonics 4, 33-36 (2010).

[30] Giunta, M. et al. Ultra low noise Er:fiber frequency comb comparison. In Conference on Lasers and Electro-Optics, STh4H.1 (Optical Society of America, 2016).

[31] Mourou, G., Brocklesby, B., Tajima, T. \& Limpert, J. The future is fibre accelerators. Nature Photonics 7, 258-261 (2013).

[32] Russbueldt, P. et al. Innoslab amplifiers. Selected Topics in Quantum Electronics, IEEE Journal of 21, 447-463 (2015).

[33] Negel, J.-P. et al. Ultrafast thin-disk multipass laser amplifier delivering $1.4 \mathrm{~kW}(4.7 \mathrm{~mJ}$, $1030 \mathrm{~nm}$ ) average power converted to $820 \mathrm{~W}$ at $515 \mathrm{~nm}$ and $234 \mathrm{~W}$ at $343 \mathrm{~nm}$. Opt. Express 23, 21064-21077 (2015).

[34] Nubbemeyer, T. et al. $1 \mathrm{~kW}, 200 \mathrm{~mJ}$ picosecond thin-disk laser system. Opt. Lett. 42, 1381-1384 (2017).

[35] Baumgartl, M., Lecaplain, C., Hideur, A., Limpert, J. \& Tünnermann, A. $66 \mathrm{~W}$ average power from a microjoule-class sub-100 fs fiber oscillator. Opt. Lett. 37, 1640-1642 (2012).

[36] Krausz, F. et al. Femtosecond solid-state lasers. Quantum Electronics, IEEE Journal of 28, 2097-2122 (1992).

[37] French, P. M. W. The generation of ultrashort laser pulses. Reports on Progress in Physics 58, 169 (1995).

[38] Zewail, A. H. Femtochemistry: Atomic-scale dynamics of the chemical bond. The Journal of Physical Chemistry A 104, 5660-5694 (2000).

[39] Zewail, A. Femtochemistry: Atomic-Scale Dynamics of the Chemical Bond Using Ultrafast Lasers (accessed: 2015/11/25). URL http://www.nobelprize.org/nobel_ prizes/chemistry/laureates/1999/ zewail-lecture.html.
[40] Hentschel, M. et al. Attosecond metrology. Nature 414, 509-513 (2001).

[41] Corkum, P. B. \& Krausz, F. Attosecond science. Nat Phys 3, 381-387 (2007).

[42] Krausz, F. \& Ivanov, M. Attosecond physics. Rev. Mod. Phys. 81, 163-234 (2009).

[43] Wirth, A. et al. Synthesized light transients. Science 334, 195-200 (2011).

[44] Hassan, M. T. et al. Optical attosecond pulses and tracking the nonlinear response of bound electrons. Nature 530, 66-70 (2016).

[45] Clark-MXR, Inc. Clark-MXR Company History (accessed: 2018/01/21). URL http://www.cmxr.com/AboutUs/ CompanyHistory.html.

[46] Wilhelm, T., Piel, J. \& Riedle, E. Sub-20-fs pulses tunable across the visible from a bluepumped single-pass noncollinear parametric converter. Opt. Lett. 22, 1494-1496 (1997).

[47] Cerullo, G. \& De Silvestri, S. Ultrafast optical parametric amplifiers. Review of Scientific Instruments 74, 1-18 (2003).

[48] Dudley, J. M., Genty, G. \& Coen, S. Supercontinuum generation in photonic crystal fiber. Rev. Mod. Phys. 78, 1135-1184 (2006).

[49] Couairon, A. \& Mysyrowicz, A. Femtosecond filamentation in transparent media. Physics Reports 441, 47 - 189 (2007).

[50] Udem, T., Holzwarth, R. \& Hänsch, T. W. Optical frequency metrology. Nature 416, 233-237 (2002).

[51] Hall, J. L. \& Hänsch, T. W. Contributions to the development of laser-based precision spectroscopy, including the optical frequency comb technique (accessed: 2015/11/25). URL http://www.nobelprize.org/nobel_ prizes/physics/laureates/2005/.

[52] Xu, L. et al. Route to phase control of ultrashort light pulses. Opt. Lett. 21, 2008-2010 (1996).

[53] Corkum, P. B. Plasma perspective on strong field multiphoton ionization. Phys. Rev. Lett. 71, 1994-1997 (1993).

[54] Brabec, T. \& Krausz, F. Intense few-cycle laser fields: Frontiers of nonlinear optics. Rev. Mod. Phys. 72, 545-591 (2000).

[55] Cho, S. H., Bouma, B. E., Ippen, E. P. \& Fujimoto, J. G. Low-repetition-rate high-peakpower Kerr-lens mode-locked $\mathrm{Ti}: \mathrm{Al}_{2} \mathrm{O}_{3}$ laser 
with a multiple-pass cavity. Opt. Lett. 24, 417-419 (1999).

[56] Naumov, S. et al. Approaching the microjoule frontier with femtosecond laser oscillators. New Journal of Physics 7, 216 (2005).

[57] Dewald, S. et al. Ionization of noble gases with pulses directly from a laser oscillator. Opt. Lett. 31, 2072-2074 (2006).

[58] Saraceno, C. J. et al. $275 \mathrm{~W}$ average output power from a femtosecond thin disk oscillator operated in a vacuum environment. Opt. Express 20, 23535-23541 (2012).

[59] Brons, J. et al. Energy scaling of Kerr-lens mode-locked thin-disk oscillators. Opt. Lett. 39, 6442-6445 (2014).

[60] Bauer, D., Zawischa, I., Sutter, D. H., Killi, A. \& Dekorsy, T. Mode-locked Yb:YAG thindisk oscillator with $41 \mu \mathrm{J}$ pulse energy at $145 \mathrm{~W}$ average infrared power and high power frequency conversion. Opt. Express 20, 96989704 (2012).

[61] Saraceno, C. J. et al. Ultrafast thin-disk laser with $80 \mu \mathrm{J}$ pulse energy and $242 \mathrm{~W}$ of average power. Opt. Lett. 39, 9-12 (2014).

[62] Brons, J. et al. Efficient, high-power, all-bulk spectral broadening in a quasi-waveguide. In 2017 European Conference on Lasers and Electro-Optics - European Quantum Electronics Conference, CF-9.4 (IEEE, 2017).

[63] Znakovskaya, I. et al. Dual frequency comb spectroscopy with a single laser. Opt. Lett. 39, 5471-5474 (2014).

[64] Pupeza, I. et al. Field-Resolved Spectroscopy in the Molecular Fingerprint Region. In 2017 European Conference on Lasers and ElectroOptics - European Quantum Electronics Conference, $\mathrm{CH}-2.4$ (IEEE, Munich, 2017).

[65] Pronin, O. Towards a compact thin-diskbased femtosecond XUV source (Dissertation, Ludwig-Maximilians-Universität München, 2012).

[66] Schliesser, A., Picque, N. \& Hänsch, T. W. Mid-infrared frequency combs. Nat Photon 6, 440-449 (2012).

[67] Eisele, M. et al. Ultrafast multi-terahertz nano-spectroscopy with sub-cycle temporal resolution. Nat Photon 8, 841-845 (2014).

[68] McClung, F. J. \& Hellwarth, R. W. Giant optical pulsations from ruby. Journal of Applied Physics 33, 828-829 (1962).
[69] Boyd, R. W. Chapter 11 - the electrooptic and photorefractive effects. In Nonlinear $O p$ tics, 511 - 541 (Academic Press, Burlington, 2008), $3^{\text {rd }}$ edn.

[70] Koechner, W. Q-switching. In Solid-State Laser Engineering, 488-533 (Springer New York, New York, NY, 2006), $6^{\text {th }}$ edn.

[71] Svelto, O. 8 Transient Laser Behavior. In Principles of Lasers, 313-373 (Springer, New York, 2010), $5^{\text {th }}$ edn.

[72] Lamb, W. E. Theory of an optical maser. Phys. Rev. 134, A1429-A1450 (1964).

[73] Kärtner, F. X., Aus der Au, J. \& Keller, U. Mode-locking with slow and fast saturable absorbers-what's the difference? IEEE Journal of Selected Topics in Quantum Electronics 4, 159-168 (1998).

[74] Weiner, A. M. Ultrafast Optics (John Wiley and Sons, Inc., 2008).

[75] Diels, J.-C. \& Rudolph, W. Ultrashort Laser Pulse Phenomena (Academic Press, Burlington, 2006), $2^{\text {nd }}$ edn.

[76] Shank, C. V. \& Ippen, E. P. Subpicosecond kilowatt pulses from a mode-locked CW dye laser. Applied Physics Letters 24, 373-375 (1974).

[77] Fork, R. L., Greene, B. I. \& Shank, C. V. Generation of optical pulses shorter than 0.1 psec by colliding pulse mode locking. Applied Physics Letters 38, 671-672 (1981).

[78] Weiner, A. M. Principles of mode-locking. In Ultrafast Optics, 32-84 (John Wiley \& Sons, Inc., 2008).

[79] Weiner, A. M. Ultrafast-pulse measurement methods. In Ultrafast Optics, 85-146 (John Wiley \& Sons, Inc., 2008).

[80] Diels, J.-C. \& Rudolph, W. 9 - diagnostic techniques. In Ultrashort Laser Pulse Phenomena, 457 - 489 (Academic Press, Burlington, 2006), $2^{\text {nd }}$ edn.

[81] Valdmanis, J. A., Fork, R. L. \& Gordon, J. P. Generation of optical pulses as short as 27 femtoseconds directly from a laser balancing self-phase modulation, group-velocity dispersion, saturable absorption, and saturable gain. Opt. Lett. 10, 131-133 (1985).

[82] Zhang, J. et al. 49-fs Yb:YAG thin-disk oscillator with distributed Kerr-lens modelocking. In 2015 European Conference on 
Lasers and Electro-Optics - European Quantum Electronics Conference, PDA.1 (Optical Society of America, 2015).

[83] Paradis, C. et al. Generation of 35-fs pulses from a Kerr lens mode-locked $\mathrm{Yb}: \mathrm{Lu}_{2} \mathrm{O}_{3}$ thindisk laser. Opt. Express 25, 14918-14925 (2017).

[84] Stolen, R. H. \& Lin, C. Self-phasemodulation in silica optical fibers. Phys. Rev. A 17, 1448-1453 (1978).

[85] Nakatsuka, H., Grischkowsky, D. \& Balant, A. C. Nonlinear picosecond-pulse propagation through optical fibers with positive group velocity dispersion. Phys. Rev. Lett. 47, 910-913 (1981).

[86] Treacy, E. Optical pulse compression with diffraction gratings. Quantum Electronics, IEEE Journal of 5, 454-458 (1969).

[87] Fork, R. L., Martinez, O. E. \& Gordon, J. P. Negative dispersion using pairs of prisms. Opt. Lett. 9, 150-152 (1984).

[88] Fork, R. L., Cruz, C. H. B., Becker, P. C. \& Shank, C. V. Compression of optical pulses to six femtoseconds by using cubic phase compensation. Opt. Lett. 12, 483-485 (1987).

[89] Moulton, P. F. Spectroscopic and laser characteristics of Ti: $\mathrm{Al}_{2} \mathrm{O}_{3}$. J. Opt. Soc. Am. B 3, 125-133 (1986).

[90] Haus, H. A. Theory of mode locking with a fast saturable absorber. Journal of Applied Physics 46, 3049-3058 (1975).

[91] Baltuška, A., Wei, Z., Pshenichnikov, M. S. \& Wiersma, D. A. Optical pulse compression to 5 fs at a $1-\mathrm{MHz}$ repetition rate. Opt. Lett. 22, 102-104 (1997).

[92] Lariontsev, E. G. \& Serkin, V. N. Possibility of using self-focusing for increasing contrast and narrowing of ultrashort light pulses. Soviet Journal of Quantum Electronics 5, 796 (1975).

[93] Salin, F., Piché, M. \& Squier, J. Mode locking of Ti: $\mathrm{Al}_{2} \mathrm{O}_{3}$ lasers and self-focusing: a gaussian approximation. Opt. Lett. 16, 1674-1676 (1991).

[94] Piché, M. Beam reshaping and self-modelocking in nonlinear laser resonators. Optics Communications 86, 156 - 160 (1991).

[95] Spinelli, L., Couillaud, B., Goldblatt, N. \& Negus, D. K. Starting and generation of sub-100fs pulses in Ti: $\mathrm{Al}_{2} \mathrm{O}_{3}$ by self-focusing.
In Conference on Lasers and Electro-Optics, CPD7 (Optical Society of America, 1991).

[96] Rausch, S. et al. Controlled waveforms on the single-cycle scale from a femtosecond oscillator. Opt. Express 16, 9739-9745 (2008).

[97] Razskazovskaya, O., Krausz, F. \& Pervak, V. Multilayer coatings for femto- and attosecond technology. Optica 4, 129-138 (2017).

[98] Szipöcs, R., Spielmann, C., Krausz, F. \& Ferencz, K. Chirped multilayer coatings for broadband dispersion control in femtosecond lasers. Opt. Lett. 19, 201-203 (1994).

[99] McPherson, A. et al. Studies of multiphoton production of vacuum-ultraviolet radiation in the rare gases. J. Opt. Soc. Am. B 4, 595-601 (1987).

[100] Ferray, M. et al. Multiple-harmonic conversion of $1064 \mathrm{~nm}$ radiation in rare gases. Journal of Physics B: Atomic, Molecular and $\mathrm{Op}$ tical Physics 21, L31 (1988).

[101] Li, X. F., L'Huillier, A., Ferray, M., Lompré, L. A. \& Mainfray, G. Multiple-harmonic generation in rare gases at high laser intensity. Phys. Rev. A 39, 5751-5761 (1989).

[102] Lewenstein, M., Balcou, P., Ivanov, M. Y., L'Huillier, A. \& Corkum, P. B. Theory of high-harmonic generation by low-frequency laser fields. Phys. Rev. A 49, 2117-2132 (1994).

[103] Goulielmakis, E. et al. Single-cycle nonlinear optics. Science 320, 1614-1617 (2008).

[104] Zhao, K. et al. Tailoring a 67 attosecond pulse through advantageous phase-mismatch. Opt. Lett. 37, 3891-3893 (2012).

[105] Gaumnitz, T. et al. Streaking of 43attosecond soft-X-ray pulses generated by a passively CEP-stable mid-infrared driver. Opt. Express 25, 27506-27518 (2017).

[106] Chini, M., Zhao, K. \& Chang, Z. The generation, characterization and applications of broadband isolated attosecond pulses. Nature Photonics 8, 178-186 (2014).

[107] Apolonski, A. et al. Controlling the phase evolution of few-cycle light pulses. Phys. Rev. Lett. 85, 740-743 (2000).

[108] Seres, E., Seres, J. \& Spielmann, C. Extreme ultraviolet light source based on intracavity high harmonic generation in a mode locked Ti:sapphire oscillator with $9.4 \mathrm{MHz}$ repetition rate. Opt. Express 20, 6185-6190 (2012). 
[109] Chiang, C.-T., Blattermann, A., Huth, M., Kirschner, J. \& Widdra, W. High-order harmonic generation at $4 \mathrm{MHz}$ as a light source for time-of-flight photoemission spectroscopy. Applied Physics Letters 101, 071116 (2012).

[110] Gohle, C. et al. A frequency comb in the extreme ultraviolet. Nature 436, 234-237 (2005).

[111] Pupeza, I. et al. Compact high-repetitionrate source of coherent $100 \mathrm{eV}$ radiation. Nat Photon 7, 608 (2013).

[112] Carstens, H. et al. High-harmonic generation at $250 \mathrm{MHz}$ with photon energies exceeding $100 \mathrm{ev}$. Optica 3, 366-369 (2016).

[113] Lee, J., Carlson, D. R. \& Jones, R. J. Optimizing intracavity high harmonic generation for XUV fs frequency combs. Opt. Express 19, 23315-23326 (2011).

[114] Cingoz, A. et al. Direct Frequency Comb Spectroscopy in the Extreme Ultraviolet. $\mathrm{Na}$ ture 482, 68-71 (2012).

[115] Ozawa, A., Zhao, Z., Kuwata-Gonokami, M. \& Kobayashi, Y. High average power coherent VUV generation at $10 \mathrm{MHz}$ repetition frequency by intracavity high harmonic generation. Opt. Express 23, 15107-15118 (2015).

[116] Yost, D. C., Schibli, T. R. \& Ye, J. Efficient output coupling of intracavity high-harmonic generation. Opt. Lett. 33, 1099-1101 (2008).

[117] Ozawa, A. et al. Non-collinear high harmonic generation: a promising outcoupling method for cavity-assisted XUV generation. Opt. Express 16, 6233-6239 (2008).

[118] Pronin, O. et al. Ultrabroadband efficient intracavity XUV output coupler. Opt. Express 19, 10232-10240 (2011).

[119] Esser, D. et al. Laser-manufactured mirrors for geometrical output coupling of intracavity-generated high harmonics. Opt. Express 21, 26797-26805 (2013).

[120] Lilienfein, N. et al. Enhancement cavities for few-cycle pulses. Opt. Lett. 42, 271-274 (2017).

[121] Salin, F. How to manipulate and change the characteristics of laser pulses. In Rullière, C. (ed.) Femtosecond Laser Pulses: Principles and Experiments, 175-194 (Springer New York, New York, NY, 2005).

[122] Belanger, P. \& Boivin, J. Multigigawatt peakpower generation from a tandem of $\mathrm{TEA}-\mathrm{CO}_{2}$ lasers. IEEE Journal of Quantum Electronics 11, 895-896 (1975). Earlier, not accessible paper in P. A. Belanger and J. Boivin, Phys. Can. 30, No. 3, 47 (1974).

[123] Georges, P. et al. High-efficiency multipass Ti:sapphire amplifiers for a continuous-wave single-mode laser. Opt. Lett. 16, 144-146 (1991).

[124] Strickland, D. \& Mourou, G. Compression of amplified chirped optical pulses. Optics Communications 56, 219 - 221 (1985).

[125] Perry, M. D. \& Mourou, G. Terawatt to petawatt subpicosecond lasers. Science $\mathbf{2 6 4}$, 917-924 (1994).

[126] Perry, M. D. et al. Petawatt laser pulses. Opt. Lett. 24, 160-162 (1999).

[127] Dubietis, A., Jonušauskas, G. \& Piskarskas, A. Powerful femtosecond pulse generation by chirped and stretched pulse parametric amplification in BBO crystal. Optics Communications 88, 437 - 440 (1992).

[128] Russbueldt, P., Mans, T., Weitenberg, J., Hoffmann, H. D. \& Poprawe, R. Compact diode-pumped $1.1 \mathrm{~kW}$ Yb:YAG Innoslab femtosecond amplifier. Opt. Lett. 35, 4169-4171 (2010).

[129] Mikkelsen, A. et al. Photoemission electron microscopy using extreme ultraviolet attosecond pulse trains. Review of Scientific Instruments 80, 123703 (2009).

[130] Aidelsburger, M., Kirchner, F. O., Krausz, F. \& Baum, P. Single-electron pulses for ultrafast diffraction. Proceedings of the National Academy of Sciences of the United States of America 107, 19714-9 (2010).

[131] Gliserin, A., Walbran, M., Krausz, F. \& Baum, P. Sub-phonon-period compression of electron pulses for atomic diffraction. Nature Communications 6, 8723 (2015).

[132] Liu, Y. et al. Towards non-sequential double ionization of Ne and Ar using a femtosecond laser oscillator. Opt. Express 15, 1810318110 (2007).

[133] Bergues, B., Kübel, M., Kling, N. G., Burger, C. \& Kling, M. F. Single-cycle non-sequential double ionization. IEEE Journal of Selected Topics in Quantum Electronics 21, 1-9 (2015).

[134] Giesen, A. et al. Scalable concept for diodepumped high-power solid-state lasers. Applied Physics B 58, 365-372 (1994). 
[135] Giesen, A. \& Speiser, J. Fifteen years of work on thin-disk lasers: Results and scaling laws. IEEE Journal of Selected Topics in Quantum Electronics 13, 598-609 (2007).

[136] Hecht, J. Photonic frontiers: Disk lasers: Higher powers and shorter pulses from thindisk lasers. Laser Focus World 50, 89-91 (2014).

[137] Schad, S.-S. et al. Recent development of disk lasers at TRUMPF. In Proc. SPIE, vol. 9726, 972615-972615-6 (2016).

[138] Schad, S.-S. et al. Near fundamental mode high-power thin-disk laser. In Proc. SPIE, vol. 8959, 89590U-89590U-6 (2014).

[139] Brons, J. et al. Powerful 100-fs-scale Kerrlens mode-locked thin-disk oscillator. Opt. Lett. 41, 3567-3570 (2016).

[140] Kanda, N. et al. High-pulse-energy yb:yag thin disk mode-locked oscillator for intracavity high harmonic generation. In Advanced Solid-State Lasers Congress, AF3A.8 (Optical Society of America, 2013).

[141] Eilanlou, A. A., Nabekawa, Y., KuwataGonokami, M. \& Midorikawa, K. Femtosecond laser pulses in a Kerr lens mode-locked thin-disk ring oscillator with an intra-cavity peak power beyond 100 MW. Japanese Journal of Applied Physics 53, 082701 (2014).

[142] Koechner, W. Properties of solid-state laser materials. In Solid-State Laser Engineering, 38-101 (Springer, New York, NY, 2006), $6^{\text {th }}$ edn.

[143] Wolter, J.-H., Ahmed, M. A. \& Graf, T. Thin-disk laser operation of Ti:sapphire. Opt. Lett. 42, 1624-1627 (2017).

[144] Takagi, S. et al. High-power (over $100 \mathrm{~mW}$ ) green laser diodes on semipolar $\{2021\} \mathrm{GaN}$ substrates operating at wavelengths beyond 530 nm. Applied Physics Express 5, 082102 (2012).

[145] Yanashima, K. et al. Long-lifetime true green laser diodes with output power over $50 \mathrm{~mW}$ above $525 \mathrm{~nm}$ grown on semipolar $\{2021\}$ GaN substrates. Applied Physics Express 5, 082103 (2012).

[146] Akasaki, I. Blue light: A fascinating journey (nobel lecture). Angewandte Chemie International Edition 54, 7750-7763 (2015).

[147] Viana, B. et al. Solid-state lasers and applications. Optical Science and Engineering, chap.
Yb-Doped Solid-State Lasers and Materials, 77-112 (CRC Press, 2006).

[148] Südmeyer, T. et al. High-power ultrafast thin disk laser oscillators and their potential for sub-100-femtosecond pulse generation. $A p$ plied Physics B 97, 281 (2009).

[149] Baer, C. R. E. et al. Frontiers in passively mode-locked high-power thin disk laser oscillators. Opt. Express 20, 7054-7065 (2012).

[150] Diebold, A. et al. SESAM mode-locked $\mathrm{Yb}: \mathrm{CaGdAlO}_{4}$ thin disk laser with $62 \mathrm{fs}$ pulse generation. Opt. Lett. 38, 3842-3845 (2013).

[151] Zhang, J. et al. Multi-mW, few-cycle midinfrared continuum spanning from 500 to $2250 \mathrm{~cm}^{-1}$. Light: Science \& Applications e17180 (2018).

[152] Diels, J.-C. \& Rudolph, W. 5 - ultrashort sources i: Fundamentals. In Ultrashort Laser Pulse Phenomena, 277 - 339 (Academic Press, Burlington, 2006), $2^{\text {nd }}$ edn.

[153] Weiner, A. M. Introduction and review. In Ultrafast Optics, 1-31 (John Wiley \& Sons, Inc., 2008).

[154] Ducasse, A., Rullière, C. \& Couillaud, B. Methods for the generation of ultrashort laser pulses: Mode-locking. In Rullière, C. (ed.) Femtosecond Laser Pulses: Principles and Experiments, 57-87 (Springer New York, New York, NY, 2005).

[155] Koechner, W. Mode locking. In Solid-State Laser Engineering, 534-586 (Springer New York, New York, NY, 2006), $6^{\text {th }}$ edn.

[156] Boyd, R. W. Chapter 1 - the nonlinear optical susceptibility. In Nonlinear Optics, $1-67$ (Academic Press, Burlington, 2008), $3^{\text {rd }}$ edn.

[157] Boyd, R. W. Chapter 4 - the intensitydependent refractive index. In Nonlinear $\mathrm{Op}$ tics, 207 - 252 (Academic Press, Burlington, 2008), $3^{\text {rd }}$ edn.

[158] Sutherland, R. L. Optical Properties of Selected Third Order Nonlinear Optical Materials. In Handbook of Nonlinear Optics, Optical Science and Engineering (CRC Press, 2003), $2^{\text {nd }}$ edn.

[159] Marburger, J. Self-focusing: Theory. Progress in Quantum Electronics 4, Part 1, $35-110$ (1975).

[160] Boyd, R. W. Chapter 7 - processes resulting from the intensity-dependent refractive 
index. In Nonlinear Optics, 329 - 390 (Academic Press, Burlington, 2008), $3^{\text {rd }}$ edn.

[161] Steier, W. H. The ray packet equivalent of a gaussian light beam. Appl. Opt. 5, 1229-1233 (1966).

[162] Herink, G., Jalali, B., Ropers, C. \& Solli, D. R. Resolving the build-up of femtosecond mode-locking with single-shot spectroscopy at $90 \mathrm{MHZ}$ frame rate. Nature Photonics 10, 321-326 (2016).

[163] Svelto, O. 4 Ray and Wave Propagation Through Optical Media. In Principles of Lasers, 131-161 (Springer, New York, 2010), $5^{\text {th }}$ edn.

[164] Oberthaler, M. \& Höpfel, R. A. Special narrowing of ultrashort laser pulses by self-phase modulation in optical fibers. Applied Physics Letters 63, 1017-1019 (1993).

[165] Agrawal, G. Chapter 4 - self-phase modulation. In Nonlinear Fiber Optics, Optics and Photonics, 87 - 128 (Academic Press, Boston, 2013), $5^{\text {th }}$ edn.

[166] Bellini, M. \& Hänsch, T. W. Phase-locked white-light continuum pulses: toward a universal optical frequency-comb synthesizer. Opt. Lett. 25, 1049-1051 (2000).

[167] Cundiff, S. T. \& Ye, J. Colloquium : Femtosecond optical frequency combs. Rev. Mod. Phys. 75, 325-342 (2003).

[168] Boyd, R. W. Nonlinear Optics (Academic, Burlington, 2008), third edn.

[169] Stegeman, G. I. $\chi^{(2)}$ cascading: nonlinear phase shifts. Quantum and Semiclassical Optics: Journal of the European Optical Society Part B 9, 139 (1997).

[170] Wise, F. W. \& Moses, J. Self-focusing and self-defocusing of femtosecond pulses with cascaded quadratic nonlinearities. In Boyd, R. W., Lukishova, S. G. \& Shen, Y. (eds.) Self-focusing: Past and Present: Fundamentals and Prospects, 481-506 (Springer New York, New York, NY, 2009).

[171] Diels, J.-C. \& Rudolph, W. 1 - fundamentals. In Ultrashort Laser Pulse Phenomena, $1-60$ (Academic Press, Burlington, 2006), $2^{\text {nd }}$ edn.

[172] Weiner, A. M. Dispersion and dispersion compensation. In Ultrafast Optics, 147-197 (John Wiley \& Sons, Inc., 2008).

[173] Diels, J.-C. \& Rudolph, W. 2 - femtosecond optics. In Ultrashort Laser Pulse Phenomena,
61 - 142 (Academic Press, Burlington, 2006), $2^{\text {nd }}$ edn.

[174] Kane, S. \& Squier, J. Grating compensation of third-order material dispersion in the normal dispersion regime: Sub-100-fs chirpedpulse amplification using a fiber stretcher and grating-pair compressor. IEEE Journal of Quantum Electronics 31, 2052-2057 (1995).

[175] Szipőcs, R. \& Kőházi-Kis, A. Theory and design of chirped dielectric laser mirrors. $A p$ plied Physics B 65, 115-135 (1997).

[176] Trubetskov, M. K., Pervak, V. \& Tikhonravov, A. V. Phase optimization of dispersive mirrors based on floating constants. Opt. Express 18, 27613-27618 (2010).

[177] Kärtner, F. X. et al. Ultrabroadband doublechirped mirror pairs for generation of octave spectra. J. Opt. Soc. Am. B 18, 882-885 (2001).

[178] Pervak, V., Ahmad, I., Trubetskov, M. K., Tikhonravov, A. V. \& Krausz, F. Doubleangle multilayer mirrors with smooth dispersion characteristics. Opt. Express 17, 79437951 (2009).

[179] Udem, T., Reichert, J., Holzwarth, R. \& Hänsch, T. W. Accurate measurement of large optical frequency differences with a mode-locked laser. Opt. Lett. 24, 881-883 (1999).

[180] Briles, T. C., Yost, D. C., Cingöz, A., Ye, J. \& Schibli, T. R. Simple piezoelectric-actuated mirror with $180 \mathrm{kHz}$ servo bandwidth. Opt. Express 18, 9739-9746 (2010).

[181] Reichert, J., Holzwarth, R., Udem, T. \& Hänsch, T. Measuring the frequency of light with mode-locked lasers. Optics Communications 172, 59 - 68 (1999).

[182] Cundiff, S. T., Ye, J. \& Hall, J. L. Optical frequency synthesis based on mode-locked lasers. Review of Scientific Instruments $\mathbf{7 2}$, 3749-3771 (2001).

[183] Boyd, R. W. Chapter 2 - wave-equation description of nonlinear optical interactions. In Nonlinear Optics, 69 - 133 (Academic Press, Burlington, 2008), $3^{\text {rd }}$ edn.

[184] Petrov, V. Frequency down-conversion of solid-state laser sources to the mid-infrared spectral range using non-oxide nonlinear crystals. Progress in Quantum Electronics 42, 1 - 106 (2015).

[185] Petrov, V. Parametric down-conversion devices: The coverage of the mid-infrared spec- 
tral range by solid-state laser sources. Optical Materials 34, 536-554 (2012).

[186] Svelto, O. 1 Introductory Concepts. In Principles of Lasers, 1-15 (Springer, New York, 2010), $5^{\text {th }}$ edn.

[187] Angelov, I. Development of high-damage threshold dispersive coatings (Dissertation, Ludwig-Maximilians-Universität München, 2014).

[188] Südmeyer, T. et al. Nonlinear femtosecond pulse compression at high average power levels by use of a large-mode-area holey fiber. Opt. Lett. 28, 1951-1953 (2003).

[189] Innerhofer, E. et al. $32 \mathrm{~W}$ of average power in 24-fs pulses from a passively mode-locked thin disk laser with nonlinear fiber compression. In Advanced Solid-State Photonics, TuA3 (Optical Society of America, 2005).

[190] Birks, T. A., Knight, J. C. \& Russell, P. S. Endlessly single-mode photonic crystal fiber. Optics Letters 22, 961-963 (1997).

[191] Knight, J. C., Birks, T. A., Cregan, R. F., Russell, P. S. \& de Sandro, J.-P. Large mode area photonic crystal fibre. Electronics Letters 34, 1347 (1998).

[192] Russell, P. Photonic crystal fibers. Science 299, 358-362 (2003).

[193] Russell, P. S. Photonic-crystal fibers. Journal of Lightwave Technology 24, 4729-4749 (2006).

[194] Alfano, R. R. (ed.) The Supercontinuum Laser Source (Springer, New York, NY, 2016), $3^{\text {rd }}$ edn.

[195] Tomlinson, W. J., Stolen, R. H. \& Johnson, A. M. Optical wave breaking of pulses in nonlinear optical fibers. Opt. Lett. 10, 457-459 (1985).

[196] Marchese, S. V. Towards high field physics with high power thin disk laser oscillators. In Baltes, H. et al. (eds.) Series in Quantum Electronics (Hartung-Gorre, Konstanz, 2008), $1^{\text {st }}$ edn.

[197] NKT Photonics. Application note: Damage threshold of fiber facets (accessed: 2017/03/13). URL http: //www.nktphotonics.com/wp-content/ uploads/2015/02/Application_Note_Damage_threshold_of_fiber_facets.pdf.
[198] Pupeza, I. et al. High-power sub-two-cycle mid-infrared pulses at $100 \mathrm{MHZ}$ repetition rate. Nat Photon 9, 721-724 (2015). Letter.

[199] Spielmann, C., Xu, L. \& Krausz, F. Measurement of interferometric autocorrelations: comment. Appl. Opt. 36, 2523-2525 (1997).

[200] Rothhardt, J. et al. $53 \mathrm{~W}$ average power fewcycle fiber laser system generating soft X-rays up to the water window. Opt. Lett. 39, 52245227 (2014).

[201] Hädrich, S. et al. Energetic sub-2-cycle laser with $216 \mathrm{~W}$ average power. Opt. Lett. 41, 4332-4335 (2016).

[202] Saraceno, C. et al. Sub-100 femtosecond pulses from a SESAM modelocked thin disk laser. Applied Physics B 106, 559-562 (2012).

[203] Papp, S. et al. Self-referencing a CW laser with efficient nonlinear optics. In Nonlinear Optics, NTh3A.6 (Optical Society of America, 2015).

[204] Hanna, M. et al. Coherent beam combining in the femtosecond regime. In Coherent Laser Beam Combining, 277-301 (Wiley-VCH Verlag GmbH \& Co. KGaA, 2013).

[205] Klenke, A. et al. Coherent combination of spectrally broadened femtosecond pulses for nonlinear compression. Opt. Lett. 39, 35203522 (2014).

[206] Ganz, T., Pervak, V., Apolonski, A. \& Baum, P. $16 \mathrm{fs}, 350 \mathrm{~nJ}$ pulses at $5 \mathrm{MHz}$ repetition rate delivered by chirped pulse compression in fibers. Opt. Lett. 36, 1107-1109 (2011).

[207] Alfano, R. R. \& Shapiro, S. L. Emission in the region 4000 to $7000 \AA$ via four-photon coupling in glass. Phys. Rev. Lett. 24, 584587 (1970).

[208] Milosevic, N., Tempea, G. \& Brabec, T. Optical pulse compression: bulk media versus hollow waveguides. Opt. Lett. 25, 672-674 (2000).

[209] Loy, M. \& Shen, Y. Study of self-focusing and small-scale filaments of light in nonlinear media. IEEE Journal of Quantum Electronics 9, 409-422 (1973).

[210] Boyd, R. W., Lukishova, S. G. \& Shen, Y. (eds.) Self-focusing: Past and Present: Fundamentals and Prospects (Springer New York, New York, NY, 2009). 
[211] Saraceno, C. J. et al. Self-referenceable frequency comb from an ultrafast thin disk laser. Opt. Express 20, 9650-9656 (2012).

[212] Paschotta, R. Noise of mode-locked lasers (part i): numerical model. Applied Physics B 79, 153-162 (2004).

[213] Paschotta, R. Noise of mode-locked lasers (part ii): timing jitter and other fluctuations. Applied Physics B 79, 163-173 (2004).

[214] Wittmann, T. et al. Single-shot carrier-envelope phase measurement of few-cycle laser pulses. Nature Physics 5, 357-362 (2009).

[215] Paasch-Colberg, T. et al. Solid-state lightphase detector. Nature Photonics 8, 214-218 (2014).

[216] Vernaleken, A. et al. Carrier-envelope frequency stabilization of a Ti:sapphire oscillator using different pump lasers. Opt. Express 20, 18387-18396 (2012).

[217] Fuji, T., Apolonski, A. \& Krausz, F. Selfstabilization of carrier-envelope offset phase by use of difference-frequency generation. Opt. Lett. 29, 632-634 (2004).

[218] Fuji, T. et al. Monolithic carrier-envelope phase-stabilization scheme. Opt. Lett. 30, 332-334 (2005).

[219] Morgner, U. et al. Nonlinear optics with phase-controlled pulses in the sub-two-cycle regime. Phys. Rev. Lett. 86, 5462-5465 (2001).

[220] Hitachi, K., Ishizawa, A., Nishikawa, T., Asobe, M. \& Sogawa, T. Carrier-envelope offset locking with a $2 \mathrm{f}$-to-3f self-referencing interferometer using a dual-pitch PPLN ridge waveguide. Opt. Express 22, 1629-1635 (2014).

[221] Ye, J. \& Cundiff, S. T. (eds.) Femtosecond Optical Frequency Comb: Principle, Operation, and Applications (Springer US, Boston, MA, 2005).

[222] Grebing, C., Koke, S., Manschwetus, B. \& Steinmeyer, G. Performance comparison of interferometer topologies for carrier-envelope phase detection. Applied Physics B 95, 81-84 (2009).

[223] Tsatourian, V., Margolis, H. S., Marra, G., Reid, D. T. \& Gill, P. Common-path self-referencing interferometer for carrierenvelope offset frequency stabilization with enhanced noise immunity. Opt. Lett. 35, 1209-1211 (2010).

[224] Prevedelli, M., Freegarde, T. \& Hänsch, T. Phase locking of grating-tuned diode lasers. Applied Physics B 60, S241-S248 (1995).

[225] Poppe, A. et al. Few-cycle optical waveform synthesis. Applied Physics B: Lasers and $\mathrm{Op}$ tics 72, 373-376 (2001).

[226] Baltuška, A. et al. Attosecond control of electronic processes by intense light fields. Nature 421, 611-615 (2003).

[227] Witte, S., Zinkstok, R., Hogervorst, W. \& Eikema, K. Control and precise measurement of carrier-envelope phase dynamics. Applied Physics B 78, 5-12 (2004).

[228] Meyer, S. A., Squier, J. A. \& Diddams, S. A. Diode-pumped Yb:KYW femtosecond laser frequency comb with stabilized carrierenvelope offset frequency. The European Physical Journal D 48, 19-26 (2008).

[229] Washburn, B. R. et al. Phase-locked, erbiumfiber-laser-based frequency comb in the near infrared. Opt. Lett. 29, 250-252 (2004).

[230] Lee, C.-C. et al. Frequency comb stabilization with bandwidth beyond the limit of gain lifetime by an intracavity graphene electro-optic modulator. Opt. Lett. 37, 3084-3086 (2012).

[231] McFerran, J., Swann, W., Washburn, B. \& Newbury, N. Suppression of pump-induced frequency noise in fiber-laser frequency combs leading to sub-radian $\mathrm{f}_{\text {ceo }}$ phase excursions. Applied Physics B 86, 219-227 (2007).

[232] Bechhoefer, J. Feedback for physicists: A tutorial essay on control. Rev. Mod. Phys. 77, 783-836 (2005).

[233] Fortier, T. M., Ye, J., Cundiff, S. T. \& Windeler, R. S. Nonlinear phase noise generated in air-silica microstructure fiber and its effect on carrier-envelope phase. Opt. Lett. 27, 445-447 (2002).

[234] Pronin, O. et al. Towards CEP stabilized pulses from a KLM Yb:YAG thindisk oscillator. In 2013 Conference on Lasers and Electro-Optics - International Quantum Electronics Conference, CFIE_2_2 (Optical Society of America, 2013).

[235] Klenner, A. et al. Phase-stabilization of the carrier-envelope-offset frequency of a SESAM modelocked thin disk laser. Opt. Express 21, 24770-24780 (2013). 
[236] Emaury, F. et al. Frequency comb offset dynamics of sesam modelocked thin disk lasers. Opt. Express 23, 21836-21856 (2015).

[237] Koke, S. et al. Direct frequency comb synthesis with arbitrary offset and shot-noiselimited phase noise. Nature Photonics 4, 462465 (2010).

[238] Lücking, F., Assion, A., Apolonski, A., Krausz, F. \& Steinmeyer, G. Long-term carrier-envelope-phase-stable few-cycle pulses by use of the feed-forward method. Opt. Lett. 37, 2076-2078 (2012).

[239] Young, J. E. H. \& Yao, S.-K. Design considerations for acousto-optic devices. Proceedings of the IEEE 69, 54-64 (1981).

[240] Milam, D. Review and assessment of measured values of the nonlinear refractive-index coefficient of fused silica. Appl. Opt. 37, 546550 (1998).

[241] Isomet Corp. Application note: Acousto-Optic Modulation (AN0510) (accessed: 2017/04/06). URL http: //www . isomet.com/App-Manual_pdf /AO\% 20Modulation.pdf.

[242] Kim, J. \& Song, Y. Ultralow-noise modelocked fiber lasers and frequency combs: principles, status, and applications. Adv. Opt. Photon. 8, 465-540 (2016).

[243] Spectra-Physics. Rainbow 2 (accessed: 2017/04/10). URL http: //www.spectra-physics.com/products/ ultrafast-lasers/rainbow2\#3.

[244] Laser Quantum. Venteon Ultra (accessed: 2017/04/10). URL http: //www. laserquantum.com/products/ detail.cfm?id=74.

[245] Spectra-Physics. FemtosourceXL (accessed: 2017/04/10). URL http: //www.spectra-physics.com/products/ ultrafast-lasers/femtosource-xl.

[246] Hohenleutner, M. et al. Real-time observation of interfering crystal electrons in highharmonic generation. Nature 523, 572-5 (2015).

[247] Paasch-Colberg, T. et al. Sub-cycle optical control of current in a semiconductor: from the multiphoton to the tunneling regime. $O p$ tica 3, 1358-1361 (2016).

[248] Ghimire, S. et al. Observation of high-order harmonic generation in a bulk crystal. Nature Physics 7, 138-141 (2011).
[249] Luu, T. T. et al. Extreme ultraviolet highharmonic spectroscopy of solids. Nature $\mathbf{5 2 1}$, 498-502 (2015).

[250] Vampa, G. et al. Linking high harmonics from gases and solids. Nature 522, 462-464 (2015).

[251] Hodgman, S. S. et al. Metastable helium: A new determination of the longest atomic excited-state lifetime. Phys. Rev. Lett. 103, 053002 (2009).

[252] Baum, P. \& Zewail, A. H. 4D attosecond imaging with free electrons: Diffraction methods and potential application. Chemical Physics 366, 2-8 (2009).

[253] Hoover, E. E. \& Squier, J. A. Advances in multiphoton microscopy technology. Nature Photonics 7, 93-101 (2013).

[254] Haché, A. et al. Observation of coherently controlled photocurrent in unbiased, bulk gaas. Phys. Rev. Lett. 78, 306-309 (1997).

[255] Hache, A., Sipe, J. E. \& van Driel, H. M. Quantum interference control of electrical currents in gaas. IEEE Journal of Quantum Electronics 34, 1144-1154 (1998).

[256] Agrawal, G. Chapter 2 - pulse propagation in fibers. In Nonlinear Fiber Optics, Optics and Photonics, 27 - 56 (Academic Press, Boston, 2013), $5^{\text {th }}$ edn.

[257] Diels, J.-C. \& Rudolph, W. 8 - pulse shaping. In Ultrashort Laser Pulse Phenomena, 433 456 (Academic Press, Burlington, 2006), $2^{\text {nd }}$ edn.

[258] Agrawal, G. Chapter 8 - Stimulated Raman Scattering. In Nonlinear Fiber Optics, Optics and Photonics, 295 - 352 (Academic Press, Boston, 2013), $5^{\text {th }}$ edn.

[259] Stuart, B. C., Feit, M. D., Rubenchik, A. M., Shore, B. W. \& Perry, M. D. Laser-induced damage in dielectrics with nanosecond to subpicosecond pulses. Phys. Rev. Lett. 74, 22482251 (1995).

[260] Lenzner, M. et al. Femtosecond optical breakdown in dielectrics. Phys. Rev. Lett. 80, 4076-4079 (1998).

[261] Heidt, A. M. et al. Coherent octave spanning near-infrared and visible supercontinuum generation in all-normal dispersion photonic crystal fibers. Opt. Express 19, 37753787 (2011).

[262] Heidt, A. M. Pulse preserving flat-top supercontinuum generation in all-normal dis- 
persion photonic crystal fibers. J. Opt. Soc. Am. B 27, 550-559 (2010).

[263] Hooper, L. E., Mosley, P. J., Muir, A. C., Wadsworth, W. J. \& Knight, J. C. Coherent supercontinuum generation in photonic crystal fiber with all-normal group velocity dispersion. Opt. Express 19, 4902-4907 (2011).

[264] Smith, A. V. \& Do, B. T. Bulk and surface laser damage of silica by picosecond and nanosecond pulses at $1064 \mathrm{~nm}$. Appl. Opt. 47, 4812-4832 (2008).

[265] Liu, Y., Tu, H. \& Boppart, S. A. Wavebreaking-extended fiber supercontinuum generation for high compression ratio transformlimited pulse compression. Opt. Lett. 37, 2172-2174 (2012).

[266] Arisholm, G. General numerical methods for simulating second-order nonlinear interactions in birefringent media. J. Opt. Soc. Am. $B$ 14, 2543-2549 (1997).

[267] Arisholm, G. \& Fonnum, H. Simulation System For Optical Science (SISYFOS) - tutorial (2012). URL http://www.ffi.no/no/ Rapporter/12-02042.pdf.

[268] Svelto, O. Principles of Lasers (Springer, New York, 2010), $5^{\text {th }}$ edn.

[269] Yariv, A. Quantum Electronics (John Wiley and Sons, Inc., 1989), $3^{\text {rd }}$ edn.

[270] Tien, A.-C., Backus, S., Kapteyn, H., Murnane, M. \& Mourou, G. Short-pulse laser damage in transparent materials as a function of pulse duration. Phys. Rev. Lett. 82, 3883-3886 (1999).

[271] Smith, A. V., Do, B., Hadley, G. \& Farrow, R. L. Optical damage limits to pulse energy from fibers. Selected Topics in Quantum Electronics, IEEE Journal of 15, 153-158 (2009).

[272] Jocher, C., Eidam, T., Hädrich, S., Limpert, J. \& Tünnermann, A. Sub 25 fs pulses from solid-core nonlinear compression stage at $250 \mathrm{~W}$ of average power. Opt. Lett. 37, 4407-4409 (2012).

[273] Dombi, P., Rácz, P., Veisz, L. \& Baum, P. Conversion of chirp in fiber compression. Opt. Lett. 39, 2232-2235 (2014).

[274] Mero, M. et al. On the damage behavior of dielectric films when illuminated with multiple femtosecond laser pulses. Optical Engineering 44, 051107-051107-7 (2005).
[275] Huntington, S. et al. Retaining and characterising nano-structure within tapered airsilica structured optical fibers. Opt. Express 11, 98-104 (2003).

[276] Ramachandran, S. et al. Ultra-large effectivearea, higher-order mode fibers: a new strategy for high-power lasers. Laser $\&$ Photonics Reviews 2, 429-448 (2008).

[277] Wright, L. G., Christodoulides, D. N. \& Wise, F. W. Controllable spatiotemporal nonlinear effects in multimode fibres. Nature Photonics 9, 306-310 (2015).

[278] Saraceno, C. J., Heckl, O. H., Baer, C. R. E., Südmeyer, T. \& Keller, U. Pulse compression of a high-power thin disk laser using rod-type fiber amplifiers. Opt. Express 19, 1395-1407 (2011).

[279] Zhang, J. et al. 260-Megahertz, Megawattlevel thin-disk oscillator. Opt. Lett. 40, 16271630 (2015).

[280] Liu, W., Chia, S.-H., Chung, H.-Y., Kaertner, F. X. \& Chang, G. Energy scalable ultrafast fiber laser sources tunable in 1030-1200 $\mathrm{nm}$ for multiphoton microscopy. In Lasers Congress 2016 (ASSL, LSC, LAC), ATh1A.5 (Optical Society of America, 2016).

[281] Liu, W. Advanced ultrafast fiber laser sources enabled by fiber nonlinearities (Dissertation, Universität Hamburg, 2017).

[282] Swiderski, J. High-power mid-infrared supercontinuum sources: Current status and future perspectives. Progress in Quantum Electronics 38, 189 - 235 (2014).

[283] Nisoli, M., De Silvestri, S. \& Svelto, O. Generation of high energy 10 fs pulses by a new pulse compression technique. Applied Physics Letters 68, 2793-2795 (1996).

[284] Russell, P. S. J., Hölzer, P., Chang, W., Abdolvand, A. \& Travers, J. C. Hollow-core photonic crystal fibres for gas-based nonlinear optics. Nature Photonics 8, 278-286 (2014).

[285] Wollenhaupt, M., Assion, A. \& Baumert, T. Femtosecond laser pulses: Linear properties, manipulation, generation and measurement. In Träger, F. (ed.) Springer Handbook of Lasers and Optics, 937-983 (Springer New York, New York, NY, 2007).

[286] Pinault, S. C. \& Potasek, M. J. Frequency broadening by self-phase modulation in optical fibers. J. Opt. Soc. Am. B 2, 1318-1319 (1985). 
[287] Potasek, M. J., Agrawal, G. P. \& Pinault, S. C. Analytic and numerical study of pulse broadening in nonlinear dispersive optical fibers. J. Opt. Soc. Am. B 3, 205-211 (1986).

[288] Travers, J. C., Chang, W., Nold, J., Joly, N. Y. \& Russell, P. S. J. Ultrafast nonlinear optics in gas-filled hollow-core photonic crystal fibers (invited). J. Opt. Soc. Am. B 28, A11-A26 (2011).

[289] Pryamikov, A. D. et al. Demonstration of a waveguide regime for a silica hollow-core microstructured optical fiber with a negative curvature of the core boundary in the spectral region $>3.5 \mu \mathrm{m}$. Opt. Express 19, 1441-1448 (2011).

[290] Sheik-Bahae, M., Hagan, D. J. \& Van Stryland, E. W. Dispersion and band-gap scaling of the electronic Kerr effect in solids associated with two-photon absorption. Physical Review Letters 65, 96-99 (1990).

[291] Miller, R. C. Optical second harmonic generation in piezoelectric crystals. Applied Physics Letters 5, 17-19 (1964).

[292] Marcatili, E. A. J. \& Schmeltzer, R. A. Hollow Metallic and Dielectric Waveguides for Long Distance Optical Transmission and Lasers. Bell System Technical Journal 43, 1783-1809 (1964).

[293] Renn, M. J., Pastel, R. \& Lewandowski, H. J. Laser guidance and trapping of mesoscale particles in hollow-core optical fibers. Phys. Rev. Lett. 82, 1574-1577 (1999).

[294] Cregan, R. F. et al. Single-mode photonic band gap guidance of light in air. Science 285, 1537-1539 (1999).

[295] Benabid, F. \& Roberts, P. J. Linear and nonlinear optical properties of hollow core photonic crystal fiber. J. Mod. Opt 37-41 (2011).

[296] Benabid, F., Knight, J. C., Antonopoulos, G. \& Russell, P. S. J. Stimulated raman scattering in hydrogen-filled hollow-core photonic crystal fiber. Science 298, 399-402 (2002).

[297] Février, S., Beaudou, B. \& Viale, P. Understanding origin of loss in large pitch hollowcore photonic crystal fibers and their design simplification. Opt. Express 18, 5142-5150 (2010).

[298] Wang, Y. Y., Wheeler, N. V., Couny, F., Roberts, P. J. \& Benabid, F. Low loss broadband transmission in hypocycloid-core kagome hollow-core photonic crystal fiber. Opt. Lett. 36, 669-671 (2011).

[299] Yu, F., Wadsworth, W. J. \& Knight, J. C. Low loss silica hollow core fibers for 3-4 $\mu \mathrm{m}$ spectral region. Opt. Express 20, 1115311158 (2012).

[300] Belardi, W. \& Knight, J. C. Hollow antiresonant fibers with reduced attenuation. Opt. Lett. 39, 1853-1856 (2014).

[301] Hayes, J. R. et al. Anti-resonant hexagram hollow core fibers. Opt. Express 23, 12891299 (2015).

[302] Mak, K. F., Travers, J. C., Joly, N. Y., Abdolvand, A. \& Russell, P. S. J. Two techniques for temporal pulse compression in gas-filled hollow-core Kagomé photonic crystal fiber. Opt. Lett. 38, 3592-3595 (2013).

[303] Mak, K. F. Nonlinear optical effects in gasfilled hollow-core photonic-crystal fibers (Dissertation, Friedrich-Alexander-Universität Erlangen-Nürnberg, 2015).

[304] Börzsönyi, A., Heiner, Z., Kalashnikov, M. P., Kovács, A. P. \& Osvay, K. Dispersion measurement of inert gases and gas mixtures at 800 nm. Appl. Opt. 47, 4856-4863 (2008).

[305] Shelton, D. P. Nonlinear-optical susceptibilities of gases measured at 1064 and $1319 \mathrm{~nm}$. Phys. Rev. A 42, 2578-2592 (1990).

[306] Couairon, A., Chakraborty, H. S. \& Gaarde, M. B. From single-cycle self-compressed filaments to isolated attosecond pulses in noble gases. Phys. Rev. A 77, 053814 (2008).

[307] Börzsönyi, A., Heiner, Z., Kovács, A., Kalashnikov, M. P. \& Osvay, K. Measurement of pressure dependent nonlinear refractive index of inert gases. Opt. Express 18, 25847-25854 (2010).

[308] Azhar, M., Joly, N. Y., Travers, J. C. \& Russell, P. S. J. Nonlinear optics in Xe-filled hollow-core PCF in high pressure and supercritical regimes. Applied Physics B 112, 457460 (2013).

[309] Lehmeier, H., Leupacher, W. \& Penzkofer, A. Nonresonant third order hyperpolarizability of rare gases and $\mathrm{n}_{2}$ determined by third harmonic generation. Optics Communications 56, $67-72$ (1985).

[310] NKT Photonics. Datasheet: LMA-25 (accessed: 2017/03/06). URL http: //www.nktphotonics.com/wp-content/ uploads/2015/01/LMA-25.pdf. 
[311] Kramida, A., Yu. Ralchenko, Reader, J. \& NIST ASD Team. NIST Atomic Spectra Database (ver. 5.3), [Online]. Available: http://physics.nist.gov/asd [2017, March 7]. National Institute of Standards and Technology, Gaithersburg, MD. (2015).

[312] Nurhuda, M., Suda, A., Midorikawa, K., Hatayama, M. \& Nagasaka, K. Propagation dynamics of femtosecond laser pulses in a hollow fiber filled with argon: constant gas pressure versus differential gas pressure. J. Opt. Soc. Am. B 20, 2002-2011 (2003).

[313] Suda, A., Hatayama, M., Nagasaka, K. \& Midorikawa, K. Generation of sub-10-fs, 5mJ-optical pulses using a hollow fiber with a pressure gradient. Applied Physics Letters 86, 111116 (2005).

[314] Mak, K. F. et al. Compressing $\mu$ J-level pulses from 250 fs to sub- 10 fs at $38-\mathrm{MHz}$ repetition rate using two gas-filled hollow-core photonic crystal fiber stages. Opt. Lett. 40, 1238-1241 (2015).

[315] Hölzer, P. et al. Femtosecond nonlinear fiber optics in the ionization regime. Phys. Rev. Lett. 107, 203901 (2011).

[316] Balciunas, T. et al. A strong-field driver in the single-cycle regime based on selfcompression in a kagome fibre. Nature communications 6, 6117 (2015).

[317] Gebhardt, M. et al. Nonlinear compression of an ultrashort-pulse thulium-based fiber laser to sub-70 fs in Kagomé photonic crystal fiber. Opt. Lett. 40, 2770-2773 (2015).

[318] Debord, B. et al. Multi-meter fiberdelivery and pulse self-compression of millijoule femtosecond laser and fiber-aided lasermicromachining. Opt. Express 22, 1073510746 (2014).

[319] Rothhardt, J. et al. $1 \mathrm{MHz}$ repetition rate hollow fiber pulse compression to sub-100-fs duration at $100 \mathrm{w}$ average power. Opt. Lett. 36, 4605-4607 (2011).

[320] Heckl, O. H. et al. Temporal pulse compression in a xenon-filled kagome-type hollowcore photonic crystal fiber at high average power. Opt. Express 19, 19142-19149 (2011).

[321] Emaury, F. et al. Beam delivery and pulse compression to sub-50 fs of a modelocked thin-disk laser in a gas-filled Kagomé-type HC-PCF fiber. Opt. Express 21, 4986-4994 (2013).
[322] Emaury, F. et al. Efficient spectral broadening in the $100-\mathrm{W}$ average power regime using gas-filled gas-filled Kagomé-type $\mathrm{HC}-\mathrm{PCF}$ and pulse compression. Opt. Lett. 39, 68436846 (2014)

[323] Hädrich, S. et al. Exploring new avenues in high repetition rate table-top coherent extreme ultraviolet sources. Light Sci. Appl. 4, e320 (2015).

[324] Emaury, F., Diebold, A., Saraceno, C. J. \& Keller, U. Compact extreme ultraviolet source at megahertz pulse repetition rate with a low-noise ultrafast thin-disk laser oscillator. Optica 2, 980-984 (2015).

[325] Rolland, C. \& Corkum, P. B. Compression of high-power optical pulses. J. Opt. Soc. Am. $B$ 5, 641-647 (1988).

[326] Petrov, V., Rudolph, W. \& Wilhelmi, B. Compression of high-energy femtosecond light pulses by self-phase modulation in bulk media. Journal of Modern Optics 36, 587595 (1989).

[327] Chernev, P. \& Petrov, V. Self-focusing of short light pulses in dispersive media. Optics Communications 87, 28 - 32 (1992).

[328] Chernev, P. \& Petrov, V. Self-focusing of light pulses in the presence of normal groupvelocity dispersion. Opt. Lett. 17, 172-174 (1992).

[329] Lu, C.-H. et al. Generation of intense supercontinuum in condensed media. Optica 1, 400-406 (2014).

[330] Centurion, M., Porter, M. A., Kevrekidis, P. G. \& Psaltis, D. Nonlinearity management in optics: Experiment, theory, and simulation. Phys. Rev. Lett. 97, 033903 (2006).

[331] Bradler, M., Baum, P. \& Riedle, E. Femtosecond continuum generation in bulk laser host materials with sub- $\mu \mathrm{j}$ pump pulses. Applied Physics B 97, 561-574 (2009).

[332] Newport. Spatial Filters (accessed: 2015/11/13). URL http://www. newport. com/Spatial-Filters/144910/1033/ content.aspx.

[333] Arisholm, G. Quantum noise initiation and macroscopic fluctuations in optical parametric oscillators. J. Opt. Soc. Am. B 16, 117127 (1999).

[334] Ghosh, G. Dispersion-equation coefficients for the refractive index and birefringence of 
calcite and quartz crystals. Optics Communications 163, 95 - 102 (1999).

[335] Brons, J. et al. Amplification-free, $145 \mathrm{MW}$, $16 \mathrm{MHz}$ scalable ultrafast light-source for XUV and MIR generation. In Advanced Solid State Lasers, ATh3A.1 (Optical Society of America, 2015).

[336] Schulte, J., Sartorius, T., Weitenberg, J., Vernaleken, A. \& Russbueldt, P. Nonlinear pulse compression in a multi-pass cell. Opt. Lett. 41, 4511-4514 (2016).

[337] Weitenberg, J. et al. Multi-pass-cell-based nonlinear pulse compression to 115 fs at $7.5 \mu \mathrm{j}$ pulse energy and $300 \mathrm{~W}$ average power. Opt. Express 25, 20502-20510 (2017).

[338] Weitenberg, J., Saule, T., Schulte, J. \& Rußbüldt, P. Nonlinear pulse compression to sub-40 fs at $4.5 \mu \mathrm{J}$ pulse energy by multipass-cell spectral broadening. IEEE Journal of Quantum Electronics 53, 1-4 (2017).

[339] Fritsch, K. et al. Fiber free all solid multipass spectral broadening down to 10 fs Fourier limit. In 2017 European Conference on Lasers and Electro-Optics - European Quantum Electronics Conference, PD1.7 (IEEE, Munich, 2017).

[340] Krebs, N., Pugliesi, I. \& Riedle, E. Pulse compression of ultrashort UV pulses by selfphase modulation in bulk material. Applied Sciences 3, 153 (2013).

[341] Møller, U. et al. Multi-milliwatt mid-infrared supercontinuum generation in a suspended core chalcogenide fiber. Opt. Express 23, 3282-3291 (2015).

[342] Silva, F. et al. Multi-octave supercontinuum generation from mid-infrared filamentation in a bulk crystal. Nat Commun 3, 807 (2012).

[343] Reitze, D. H., Weiner, A. M. \& Leaird, D. E. High-power femtosecond optical pulse compression by using spatial solitons. Opt. Lett. 16, 1409-1411 (1991).

[344] Metzger, T. et al. Picosecond thin-disk lasers. In CLEO: 2014, JTh4L.1 (Optical Society of America, 2014).

[345] Chiao, R. Y., Garmire, E. \& Townes, C. H. Self-trapping of optical beams. Phys. Rev. Lett. 13, 479-482 (1964).

[346] Meschede, D. Optics, Light, and Lasers, chap. Light Rays, 1-27 (Wiley-VCH, 2007), $2^{\text {nd }}$ edn.
[347] Hutchings, D. C., Sheik-Bahae, M., Hagan, D. J. \& Van Stryland, E. W. Kramers-Krönig relations in nonlinear optics. Optical and Quantum Electronics 24, 1-30 (1992).

[348] DeSalvo, R. et al. Self-focusing and selfdefocusing by cascaded second-order effects in KTP. Opt. Lett. 17, 28-30 (1992).

[349] Zhang, D., Kong, Y. \& Zhang, J.-Y. Optical parametric properties of 532-nm-pumped beta-barium-borate near the infrared absorption edge. Optics Communications 184, 485 - 491 (2000).

[350] Bache, M., Guo, H., Zhou, B. \& Zeng, X. The anisotropic Kerr nonlinear refractive index of the beta-barium borate $\left(\beta-\mathrm{BaB}_{2} \mathrm{O}_{4}\right)$ nonlinear crystal. Opt. Mater. Express 3, 357-382 (2013)

[351] Eckardt, R. C., Masuda, H., Fan, Y. X. \& Byer, R. L. Absolute and relative nonlinear optical coefficients of KDP, $\mathrm{KD}^{*} \mathrm{P}, \mathrm{BaB}_{2} \mathrm{O}_{4}$, $\mathrm{LiIO}_{3}, \mathrm{MgO}: \mathrm{LiNbO}_{3}$, and KTP measured by phase-matched second-harmonic generation. IEEE Journal of Quantum Electronics 26, 922-933 (1990).

[352] Moses, J. \& Wise, F. W. Controllable selfsteepening of ultrashort pulses in quadratic nonlinear media. Phys. Rev. Lett. 97, 073903 (2006).

[353] Li, H., Zhou, F., Zhang, X. \& Ji, W. Bound electronic kerr effect and self-focusing induced damage in second-harmonic-generation crystals. Optics Communications 144, 75 81 (1997).

[354] Fattahi, H., Schwarz, A., Keiber, S. \& Karpowicz, N. Efficient, octave-spanning difference-frequency generation using fewcycle pulses in simple collinear geometry. Opt. Lett. 38, 4216-4219 (2013).

[355] Liu, X., Qian, L. \& Wise, F. High-energy pulse compression by use of negative phase shifts produced by the cascade $\chi^{(2)}: \chi^{(2)}$ nonlinearity. Opt. Lett. 24, 1777-1779 (1999).

[356] Moses, J. \& Wise, F. W. Soliton compression in quadratic media: high-energy fewcycle pulses with a frequency-doubling crystal. Opt. Lett. 31, 1881-1883 (2006).

[357] Moses, J., Alhammali, E., Eichenholz, J. M. \& Wise, F. W. Efficient high-energy femtosecond pulse compression in quadratic media with flattop beams. Opt. Lett. 32, 24692471 (2007). 
[358] Ashihara, S., Nishina, J., Shimura, T. \& Kuroda, K. Soliton compression of femtosecond pulses in quadratic media. J. Opt. Soc. Am. B 19, 2505-2510 (2002).

[359] Ilday, F. O., Beckwitt, K., Chen, Y.-F., Lim, H. \& Wise, F. W. Controllable Raman-like nonlinearities from nonstationary, cascaded quadratic processes. J. Opt. Soc. Am. B 21, 376-383 (2004).

[360] Ashihara, S. et al. Optical pulse compression using cascaded quadratic nonlinearities in periodically poled lithium niobate. Applied Physics Letters 84, 1055-1057 (2004).

[361] Kato, K. Temperature-tuned $90^{\circ}$ phasematching properties of $\mathrm{LiB}_{3} \mathrm{O}_{5}$. IEEE Journal of Quantum Electronics 30, 2950-2952 (1994).

[362] Armstrong, D. J., Alford, W. J., Raymond, T. D., Smith, A. V. \& Bowers, M. S. Parametric amplification and oscillation with walkoffcompensating crystals. J. Opt. Soc. Am. B 14, 460-474 (1997).

[363] Budriūnas, R., Kučinskas, D. \& Varanavičius, A. High-energy continuum generation in an array of thin plates pumped by tunable femtosecond IR pulses. Applied Physics B 123, 212 (2017).

[364] Zhou, B. B., Chong, A., Wise, F. W. \& Bache, M. Ultrafast and octave-spanning optical nonlinearities from strongly phasemismatched quadratic interactions. Phys. Rev. Lett. 109, 043902 (2012).

[365] Zhou, B. \& Bache, M. Dispersive waves induced by self-defocusing temporal solitons in a beta-barium-borate crystal. Opt. Lett. 40, 4257-4260 (2015).

[366] Bache, M. \& Wise, F. W. Type-i cascaded quadratic soliton compression in lithium niobate: Compressing femtosecond pulses from high-power fiber lasers. Phys. Rev. A 81, 053815 (2010).

[367] Sutherland, R. L. Frequency Doubling and Mixing. In Handbook of Nonlinear Optics, Optical Science and Engineering (CRC Press, 2003), $2^{\text {nd }}$ edn.

[368] Fortier, T. M., Jones, D. J., Ye, J., Cundiff, S. T. \& Windeler, R. S. Long-term carrier-envelope phase coherence. Opt. Lett. 27, 1436-1438 (2002).

[369] Hartl, I., Imeshev, G., Fermann, M. E., Langrock, C. \& Fejer, M. M. Integrated self- referenced frequency-comb laser based on a combination of fiber and waveguide technology. Opt. Express 13, 6490-6496 (2005).

[370] Balčiūnas, T. et al. Carrier envelope phase stabilization of a $\mathrm{Yb}: \mathrm{KGW}$ laser amplifier. Opt. Lett. 36, 3242-3244 (2011).

[371] Liu, Y. et al. Suppressing short-term polarization noise and related spectral decoherence in all-normal dispersion fiber supercontinuum generation. Lightwave Technology, Journal of 33, 1814-1820 (2015).

[372] Agrawal, G. P. Chapter 5 - optical solitons. In Nonlinear Fiber Optics, Optics and Photonics, 129 - 191 (Academic Press, Boston, $2013), 5^{\text {th }}$ edn.

[373] Karlen, L., Buchs, G., Portuondo-Campa, E. \& Lecomte, S. Efficient carrier-envelope offset frequency stabilization through gain modulation via stimulated emission. Opt. Lett. 41, 376-379 (2016).

[374] Heidt, A. M. et al. High quality sub-two cycle pulses from compression of supercontinuum generated in all-normal dispersion photonic crystal fiber. Opt. Express 19, 13873-13879 (2011).

[375] Demmler, S. et al. Generation of high quality, 1.3 cycle pulses by active phase control of an octave spanning supercontinuum. Opt. Express 19, 20151-20158 (2011).

[376] Brons, J. High-power femtosecond laseroscillators for applications in high-field physics (Dissertation, Ludwig-MaximiliansUniversität München, 2017).

[377] Stutzki, F. et al. $152 \mathrm{~W}$ average power Tmdoped fiber CPA system. Opt. Lett. 39, 46714674 (2014).

[378] Vasilyev, S., Moskalev, I., Mirov, M., Mirov, S. \& Gapontsev, V. Multi-watt mid-ir femtosecond polycrystalline $\mathrm{Cr}^{2+}: \mathrm{Zns}$ and $\mathrm{Cr}^{2+}:$ Znse laser amplifiers with the spectrum spanning $2.0 \mu \mathrm{m}-2.6 \mu \mathrm{m}$. Opt. Express 24, 1616-1623 (2016).

[379] Antipov, S., Hudson, D. D., Fuerbach, A. \& Jackson, S. D. High-power mid-infrared femtosecond fiber laser in the water vapor transmission window. Optica 3, 1373-1376 (2016).

[380] Haas, J. \& Mizaikoff, B. Advances in MidInfrared Spectroscopy for Chemical Analysis. Annual Review of Analytical Chemistry 9, 45-68 (2016). 
[381] Cossel, K. C. et al. Gas-phase broadband spectroscopy using active sources: progress, status, and applications (invited). J. Opt. Soc. Am. B 34, 104-129 (2017).

[382] Baker, M. J. et al. Using Fourier transform IR spectroscopy to analyze biological materials. Nature protocols 9, 1771-91 (2014).

[383] Zhu, J., Mathes, T., Stahl, A. D., Kennis, J. T. \& Groot, M. L. Ultrafast mid-infrared spectroscopy by chirped pulse upconversion in $1800-1000 \mathrm{~cm}^{-1}$ region. Opt. Express 20, 10562-10571 (2012).

[384] Tidemand-Lichtenberg, P., Dam, J. S., Andersen, H. V., Høgstedt, L. \& Pedersen, C. Mid-infrared upconversion spectroscopy. J. Opt. Soc. Am. B 33, D28-D35 (2016).

[385] Hamm, P. \& Zanni, M. Concepts and Methods of 2D Infrared Spectroscopy (Cambridge University, Cambridge, UK, 2011).

[386] Lee, K. F., Kubarych, K. J., Bonvalet, A. \& Joffre, M. Characterization of mid-infrared femtosecond pulses (invited). J. Opt. Soc. Am. B 25, A54-A62 (2008).

[387] Lanin, A. A., Voronin, A. A., Fedotov, A. B. \& Zheltikov, A. M. Time-domain spectroscopy in the mid-infrared. Scientific reports 4, 6670 (2014).

[388] Popmintchev, T. et al. Bright coherent ultrahigh harmonics in the $\mathrm{keV} \mathrm{X}$-ray regime from mid-infrared femtosecond lasers. Science 336, 1287-1291 (2012).

[389] Schubert, O. et al. Sub-cycle control of terahertz high-harmonic generation by dynamical Bloch oscillations. Nature Photonics 8, 119123 (2014).

[390] Rogalski, A. History of infrared detectors. Opto-Electronics Review 20, 279-308 (2012).

[391] Maidment, L., Zhang, Z., Howle, C. R. \& Reid, D. T. Stand-off identification of aerosols using mid-infrared backscattering Fouriertransform spectroscopy. Opt. Lett. 41, 22662269 (2016).

[392] Baltuška, A. et al. Controlling the CarrierEnvelope Phase of Ultrashort Light Pulses with Optical Parametric Amplifiers. Physical Review Letters 88, 133901 (2002).

[393] Thiré, N. et al. 4-W, 100-kHz, few-cycle midinfrared source with sub-100-mrad carrierenvelope phase noise. Opt. Express 25, 15051514 (2017).
[394] Thierfelder, C., Sanna, S., Schindlmayr, A. \& Schmidt, W. G. Do we know the band gap of lithium niobate? physica status solidi (c) $\mathbf{7}$, 362-365 (2010).

[395] Steinle, T., Steinmann, A., Hegenbarth, R. \& Giessen, H. Watt-level optical parametric amplifier at $42 \mathrm{MHz}$ tunable from 1.35 to $4.5 \mu \mathrm{m}$ coherently seeded with solitons. Opt. Express 22, 9567-9573 (2014).

[396] Hansel, T., Köhler, W., Assion, A., Bethge, J. \& Büttner, E. Tunable supercontinuumseeded 130fs OPA for NIR and MIR with 25 nJ pulse energy and $5 \mathrm{MHz}$ repetition rate. In CLEO: 2013, 1-2 (2013).

[397] Iwakuni, K. et al. Generation of a frequency comb spanning more than 3.6 octaves from ultraviolet to mid infrared. Opt. Lett. 41, 3980-3983 (2016).

[398] Heidt, A. M., Feehan, J. S., Price, J. H. V. \& Feurer, T. Limits of coherent supercontinuum generation in normal dispersion fibers. J. Opt. Soc. Am. B 34, 764-775 (2017).

[399] Domingue, S. R. \& Bartels, R. A. Overcoming temporal polarization instabilities from the latent birefringence in all-normal dispersion, wave-breaking-extended nonlinear fiber supercontinuum generation. Opt. Express 21, 13305-13321 (2013)

[400] Gayer, O., Sacks, Z., Galun, E. \& Arie, A. Temperature and wavelength dependent refractive index equations for $\mathrm{MgO}$-doped congruent and stoichiometric $\mathrm{LiNbO}_{3}$. Applied Physics B 91, 343-348 (2008).

[401] Ishizuki, H. \& Taira, T. Mg-doped congruent $\mathrm{LiTaO}_{3}$ crystal for large-aperture quasi-phase matching device. Opt. Express 16, 1696316970 (2008).

[402] Shoji, I., Kondo, T., Kitamoto, A., Shirane, M. \& Ito, R. Absolute scale of second-order nonlinear-optical coefficients. J. Opt. Soc. Am. B 14, 2268-2294 (1997).

[403] Ganeev, R. A., Kulagin, I. A., Ryasnyanskii, A. I., Tugushev, R. I. \& Usmanov, T. The nonlinear refractive indices and nonlinear third-order susceptibilities of quadratic crystals. Optics and Spectroscopy 94, 561-568 (2003).

[404] Luther-Davies, B. \& Yu, Y. Efficient Generation of Ultra-short Pulses in the Infrared from a Simple PPLN Optical Parametric Amplifier. In Nonlinear Optics, NTu2A.4 (Optical Society of America, 2017). 
[405] Baudisch, M., Hemmer, M., Pires, H. \& Biegert, J. Performance of MgO:PPLN, KTA, and $\mathrm{KNbO}_{3}$ for mid-wave infrared broadband parametric amplification at high average power. Opt. Lett. 39, 5802-5805 (2014).

[406] Rigaud, P. et al. Supercontinuum-seeded fewcycle mid-infrared OPCPA system. Opt. Express 24, 26494-26502 (2016).

[407] Südmeyer, T. et al. High-power femtosecond fiber-feedback optical parametric oscillator based on periodically poled stoichiometric $\mathrm{LiTaO}_{3}$. Optics letters 29, 1111 (2004).

[408] Adler, F. et al. Phase-stabilized, 1.5 W frequency comb at 2.8-4.8 $\mu \mathrm{m}$. Optics Letters 34, 1330-1332 (2009).

[409] Steinle, T., Mörz, F., Steinmann, A. \& Giessen, H. Ultra-stable high average power femtosecond laser system tunable from 1.33 to $20 \mu \mathrm{m}$. Opt. Lett. 41, 4863-4866 (2016).

[410] Elu, U. et al. High average power and singlecycle pulses from a mid-IR optical parametric chirped pulse amplifier. Optica 4, 1024-1029 (2017).

[411] Cinque, G., Frogley, M. D. \& Bartolini, R. Far-IR/THz spectral characterization of the coherent synchrotron radiation emission at diamond IR beamline B22. Rendiconti Lincei 22, 33-47 (2011).

[412] Ishizuki, H. \& Taira, T. High-energy quasiphase-matched optical parametric oscillation in a periodically poled $\mathrm{MgO}: \mathrm{LiNbO}_{3}$ device with a $5 \mathrm{~mm} \times 5 \mathrm{~mm}$ aperture. Opt. Lett. $\mathbf{3 0}$, 2918-2920 (2005).

[413] Isaenko, L. et al. $\mathrm{LiGaX}_{2}(\mathrm{X}=\mathrm{S}, \mathrm{Se}, \mathrm{Te})$ : new nonlinear crystals for the mid-IR. In Conference on Lasers and Electro-Optics/Quantum Electronics and Laser Science Conference, CWA15 (Optical Society of America, 2003).

[414] Isaenko, L. et al. Growth and properties of $\mathrm{LiGaX}_{2}(\mathrm{X}=\mathrm{S}$, Se, Te) single crystals for nonlinear optical applications in the mid-IR. Crystal Research and Technology 38, 379-387 (2003).

[415] Petrov, V. et al. Second harmonic generation and optical parametric amplification in the mid-IR with orthorhombic biaxial crystals $\mathrm{LiGaS}_{2}$ and $\mathrm{LiGaSe}_{2}$. Applied Physics $B$ 78, 543-546 (2004).

[416] Møller, U. \& Bang, O. Intensity noise in normal-pumped picosecond supercontinuum generation, where higher-order Raman lines cross into anomalous dispersion regime. Electronics Letters 49, 63-65 (2013).

[417] Kato, K. et al. Phase-matching properties of $\mathrm{LiGaS}_{2}$ in the $1.025-10.5910 \mu \mathrm{m}$ spectral range. Opt. Lett. 42, 4363-4366 (2017).

[418] Mayer, B. et al. Sub-cycle slicing of phaselocked and intense mid-infrared transients. New Journal of Physics 16, 063033 (2014).

[419] Tyazhev, A. et al. Singly-resonant optical parametric oscillation based on the wide band-gap mid-IR nonlinear optical crystal $\mathrm{LiGaS}_{2}$. Optical Materials 35, 1612 - 1615 (2013).

[420] Chaitanya Kumar, S. et al. High-power femtosecond mid-infrared optical parametric oscillator at $7 \mu \mathrm{m}$ based on CdSiP2. Opt. Lett. 40, 1398-1401 (2015).

[421] Chaitanya Kumar, S., Schunemann, P. G., Zawilski, K. T. \& Ebrahim-Zadeh, M. Advances in ultrafast optical parametric sources for the mid-infrared based on CdSiP2. J. Opt. Soc. Am. B 33, D44-D56 (2016).

[422] Pupeza, I. et al. Compact 0.1-W Source of Octave-Spanning Mid-Infrared Femtosecond Pulses Centered at $10 \mu \mathrm{m}$. In CLEO: 2014 Postdeadline Paper Digest, STh5C.7 (Optical Society of America, 2014).

[423] Bache, M., Guo, H. \& Zhou, B. Generating mid-IR octave-spanning supercontinua and few-cycle pulses with solitons in phasemismatched quadratic nonlinear crystals. $O p$ tical Materials Express 3, 1647 (2013).

[424] Zhou, B. \& Bache, M. Invited article: Multiple-octave spanning high-energy mid-ir supercontinuum generation in bulk quadratic nonlinear crystals. APL Photonics 1, 050802 (2016).

[425] Sundheimer, M. L., Bierlein, J. D., Bosshard, C., Van Stryland, E. W. \& Stegeman, G. I. Large nonlinear phase modulation in quasiphase-matched KTP waveguides as a result of cascaded second-order processes. Opt. Lett. 18, 1397-1399 (1993).

[426] Schunemann, P. G., Zawilski, K. T., Pomeranz, L. A., Creeden, D. J. \& Budni, P. A. Advances in nonlinear optical crystals for midinfrared coherent sources. J. Opt. Soc. Am. B 33, D36-D43 (2016).

[427] Kanai, T. et al. Parametric amplification of 100 fs mid-infrared pulses in $\mathrm{ZnGeP}_{2}$ driven 
by a Ho:YAG chirped-pulse amplifier. Opt. Lett. 42, 683-686 (2017).

[428] extracted from SNLO software (Version 66). URL http://www.as-photonics.com/snlo.

[429] Zelmon, D. E., Hanning, E. A. \& Schunemann, P. G. Refractive-index measurements and sellmeier coefficients for zinc germanium phosphide from 2 to $9 \mu \mathrm{m}$ with implications for phase matching in optical frequencyconversion devices. J. Opt. Soc. Am. B 18, 1307-1310 (2001).

[430] Petersen, C. R. et al. Mid-IR supercontinuum covering the $1.4 \mu \mathrm{m}$ to $13.3 \mu \mathrm{m}$ molecular fingerprint region using ultra-high NA chalcogenide step-index fibre. Nature Photonics 8, 830-834 (2014).

[431] Zhao, Z. et al. 1.5-14 $\mu \mathrm{m}$ midinfrared supercontinuum generation in a low-loss Te-based chalcogenide step-index fiber. Opt. Lett. 41, 5222-5225 (2016).

[432] Gattass, R. R. et al. All-fiber chalcogenidebased mid-infrared supercontinuum source. Optical Fiber Technology 18, 345 - 348 (2012).

[433] Kedenburg, S., Steinle, T., Mörz, F., Steinmann, A. \& Giessen, H. High-power midinfrared high repetition-rate supercontinuum source based on a chalcogenide step-index fiber. Opt. Lett. 40, 2668-2671 (2015).

[434] Yu, Y. et al. A broadband, quasi-continuous, mid-infrared supercontinuum generated in a chalcogenide glass waveguide. Laser \& Photonics Reviews 8, 792-798 (2014).

[435] Lanin, A. A., Voronin, A. A., Stepanov, E. A., Fedotov, A. B. \& Zheltikov, A. M. Multioctave, $3-18 \mu \mathrm{m}$ sub-two-cycle supercontinua from self-compressing, self-focusing soliton transients in a solid. Opt. Lett. 40, 974-977 (2015).

[436] Seidel, M. et al. Efficient high-power ultrashort pulse compression in self-defocusing bulk media. Scientific Reports 7, 1410 (2017).

[437] Liang, H. et al. High-energy mid-infrared sub-cycle pulse synthesis from a parametric amplifier. Nature Communications 8, 141 (2017).

[438] Krausz, F. \& Stockman, M. I. Attosecond metrology: from electron capture to future signal processing. Nat Photon 8, 205-213 (2014).
[439] Garg, M. et al. Multi-petahertz electronic metrology. Nature 538, 359-363 (2016).

[440] Miller, L. M., Tobin, M. J., Chio-Srichan, S. \& Dumas, P. The Use of Synchrotron Radiation for Biomedical Applications of Infrared Microscopy. In Barth, A. \& Haris, P. I. (eds.) Biological and Biomedical Infrared Spectroscopy (IOS Press, Amsterdam, 2009).

[441] Huber, M. et al. Active intensity noise suppression for a broadband mid-infrared laser source. Opt. Express 25, 22499-22509 (2017).

[442] Edwards, D. F. Gallium Selenide (GaSe). In Palik, E. D. (ed.) Handbook of Optical Constants of Solids, 473 - 487 (Academic Press, Burlington, 1997).

[443] Beetar, J. E., Gholam-Mirzaei, S. \& Chini, M. Spectral broadening and pulse compression of a $400 \mu \mathrm{J}, 20 \mathrm{~W}$ Yb:KGW laser using a multiplate medium. Applied Physics Letters 112, 051102 (2018).

[444] Herriott, D. R. \& Schulte, H. J. Folded optical delay lines. Appl. Opt. 4, 883-889 (1965).

[445] Schiffrin, A. et al. Optical-field-induced current in dielectrics. Nature 493, 70-74 (2013).

[446] Fortier, T. M. et al. Carrier-envelope phasecontrolled quantum interference of injected photocurrents in semiconductors. Phys. Rev. Lett. 92, 147403 (2004).

[447] Keiber, S. et al. Investigation of laser-induced currents in large-band-gap dielectrics. In 19th International Conference on Ultrafast Phenomena, 10.Thu.C.6 (Optical Society of America, 2014).

[448] Roos, P. A., Quraishi, Q., Cundiff, S. T., Bhat, R. D. R. \& Sipe, J. E. Characterization of quantum interference control of injected currents in LT-GaAs for carrierenvelope phase measurements. Opt. Express 11, 2081-2090 (2003).

[449] Roos, P. A. et al. Solid-state carrier-envelope phase stabilization via quantum interference control of injected photocurrents. Opt. Lett. 30, 735-737 (2005).

[450] Kruchinin, S. Y., Korbman, M. \& Yakovlev, V. S. Theory of strong-field injection and control of photocurrent in dielectrics and wide band gap semiconductors. Phys. Rev. B 87, 115201 (2013).

[451] Kazempour, A. Quasiparticle lifetimes in rutile and anatase $\mathrm{TiO}_{2}$ : GW approximation. Physica Scripta 90, 025804 (2015). 
[452] Keiber, S. et al. Electro-optic sampling of near-infrared waveforms. Nature Photonics 10, 159-162 (2016).

[453] Wu, Q. \& Zhang, X.-C. Free-space electrooptic sampling of Terahertz beams. Applied Physics Letters 67, 3523-3525 (1995).

[454] Habel, F. \& Pervak, V. Dispersive mirror for the mid-infrared spectral range of 9-11.5 $\mu \mathrm{m}$. Appl. Opt. 56, C71-C74 (2017).

[455] Prinz, S. et al. Active pump-seed-pulse synchronization for OPCPA with sub-2-fs residual timing jitter. Opt. Express 22, 3105031056 (2014).

[456] Manzoni, C. et al. Coherent synthesis of ultra-broadband optical parametric amplifiers. Opt. Lett. 37, 1880-1882 (2012).

[457] Xin, M. et al. Attosecond precision multikilometer laser-microwave network. Light Sci Appl. 6, e16187 (2017).

[458] Walbran, M., Gliserin, A., Jung, K., Kim, J. \& Baum, P. 5-femtosecond laser-electron synchronization for pump-probe crystallography and diffraction. Phys. Rev. Applied 4, 044013 (2015).

[459] Fattahi, H. et al. High-power, 1-ps, allYb:YAG thin-disk regenerative amplifier. Opt. Lett. 41, 1126-1129 (2016).

[460] Trebino, R. (ed.) Frequency-Resolved Optical Gating: The Measurement of Ultrashort Laser Pulses (Springer US, Boston, MA, 2000).

[461] Trebino, R. \& Zeek, E. The autocorrelation, the spectrum, and phase retrieval. In Frequency-Resolved Optical Gating: The Measurement of Ultrashort Laser Pulses, 6199 (Springer US, Boston, MA, 2000).

[462] Walmsley, I. A. \& Dorrer, C. Characterization of ultrashort electromagnetic pulses. Adv. Opt. Photon. 1, 308-437 (2009).

[463] Stibenz, G. \& Steinmeyer, G. Optimizing spectral phase interferometry for direct electric-field reconstruction. Review of Scientific Instruments 77, 073105 (2006).

[464] Anderson, M. E., Monmayrant, A., Gorza, S.P., Wasylczyk, P. \& Walmsley, I. A. SPIDER: A decade of measuring ultrashort pulses. Laser Physics Letters 5, 259 (2008).

[465] Baltuška, A., Pshenichnikov, M. S. \& Wiersma, D. A. FROG in the single-cycle regime. In Frequency-Resolved Optical Gating: The Measurement of Ultrashort Laser Pulses, 257-303 (Springer US, Boston, MA, 2000).

[466] Baltuška, A., Pshenichnikov, M. S. \& Wiersma, D. A. Amplitude and phase characterization of 4.5 -fs pulses by frequencyresolved optical gating. Opt. Lett. 23, 14741476 (1998).

[467] Bronstein, I. N., Mühlig, H., Musiol, G. \& Semendjajew, K. A. (eds.) Taschenbuch der Mathematik (Harri Deutsch, Frankfurt am Main, 2005). 


\section{Acknowledgements}

First, I would like to thank the Laboratory of Attosecond Physics team at MPQ for their support during the five years of $\mathrm{PhD}$ work. I especially want to thank my advisor Ferenc Krausz, the head of the team, who has created a unique research environment I was strongly benefitting from.

Secondly, I would like to thank Oleg Pronin, "the father of Kerr-lens mode-locked thin-disk oscillators", who has not only developed a beautiful tool that I could work with, but has also advised me in daily discussions and gave me the freedom to try my own ideas. I would also like to thank him and Ferenc Krausz for recommending me for the Studienstiftung des Deutschen Volkes, the Tingye Li Innovation Prize and my current position in Strasbourg.

Moreover, I want to thank the other (former) members of the "AG Pronin", namely Jinwei Zhang, KaFai Mak, Kilian Fritsch, Markus Pötzelberger, Sebastian Gröbmeyer, Nathalie Nagl, Felix Schulze, Johannes Stierle, Tobias Rumpf and Xiao Xiao. A special thanks to Jonathan Brons who has been around since the start of my PhD work. I appreciate the discussions with him and the solid engineering he did (it was almost impossible to loosen a screw that he tightened without special tools).

Many thanks also to the Ioachim Pupeza's group members, for regular exchange of knowledge and equipment, and Vladimir Pervak's team, for providing our bread and butter, the multi-layer mirrors. For very helpful discussions, I would in particular like to thank Nikolai Lilienfein, Simon Holzberger, Florian Habel and Michael Trubetskov.

I also had the pleasure to work with many scientific collaborators. First, I would like to thank Thomas Udem, for advising on our CEP stabilization experiments and for serving as a second reviewer of this dissertation. A special thanks goes to Gunnar Arisholm for developing and providing the beautiful SISYFOS tool as well as for being extremely responsive and helpful with respect to extending his code and interpreting our results. Many thanks also to Alexander Hartung for his contribution to max out the capabilities of solid-core PCF. I would also like to thank Peter Schunemann for providing the ZGP crystals, Daniel Sánchez of Jens Biegert's group for his introduction to SISYFOS, John Travers for supervising the Kagomé fiber project and Jens Bethge from APE for the pulse measurement discussions and the SPIDER trial.

I would like to acknowledge the financial and immaterial support of the Studienstiftung des deutschen Volkes. I especially want to thank my "Vertrauensdozent" Hanno Schäfer for mentoring. I would also like to thank my "personal mentor" Christian Seidel who suggested to apply at the IMPRS for advanced photon science. All the organizer of the MaxPlanck research school, I would like to thank as well. I really enjoyed being part of the graduate school and still enjoy meeting many amiable people of the 2012 selection round.

My PhD work would not have been successful without my former advisors and teachers at the University of Rostock and at CREOL. Therefore, I want to thank Stefan Lochbrunner for being extremely supportive of paving my academic path first to the US and later to MPQ. Furthermore, I thank Eric Van Stryland and David Hagan for teaching me nonlinear optics which has become most essential for my thesis.

Schließlich möchte ich noch einige nichtwissenschaftliche Dankworte in meiner Muttersprache abfassen: Ein ganz herzlicher Dank für die permanente Unterstützung auf meiner Laufbahn (verbunden mit allen Umzügen und den weiten Entfernungen) gebührt meiner Familie, insbesondere meinen Eltern Birgit und Reinhard sowie meinem Bruder Daniel. Auch Doreen möchte ich danken, die besonders in der Schlussphase meiner Promotion oft auf meine Gesellschaft geduldig verzichtet hat und im letzten Sommer trotz eines sehr langen Arbeitswegs mit nach Straßburg gezogen ist. Vielen Dank auch den Münchner Freunden rund um IMPRS sowie Feierabend/Hauskreis. Zum Schluss bleibt mir noch zu schreiben: Gott sei Dank ist's gut gelaufen und Gott sei Dank ist's (endlich) geschafft. 